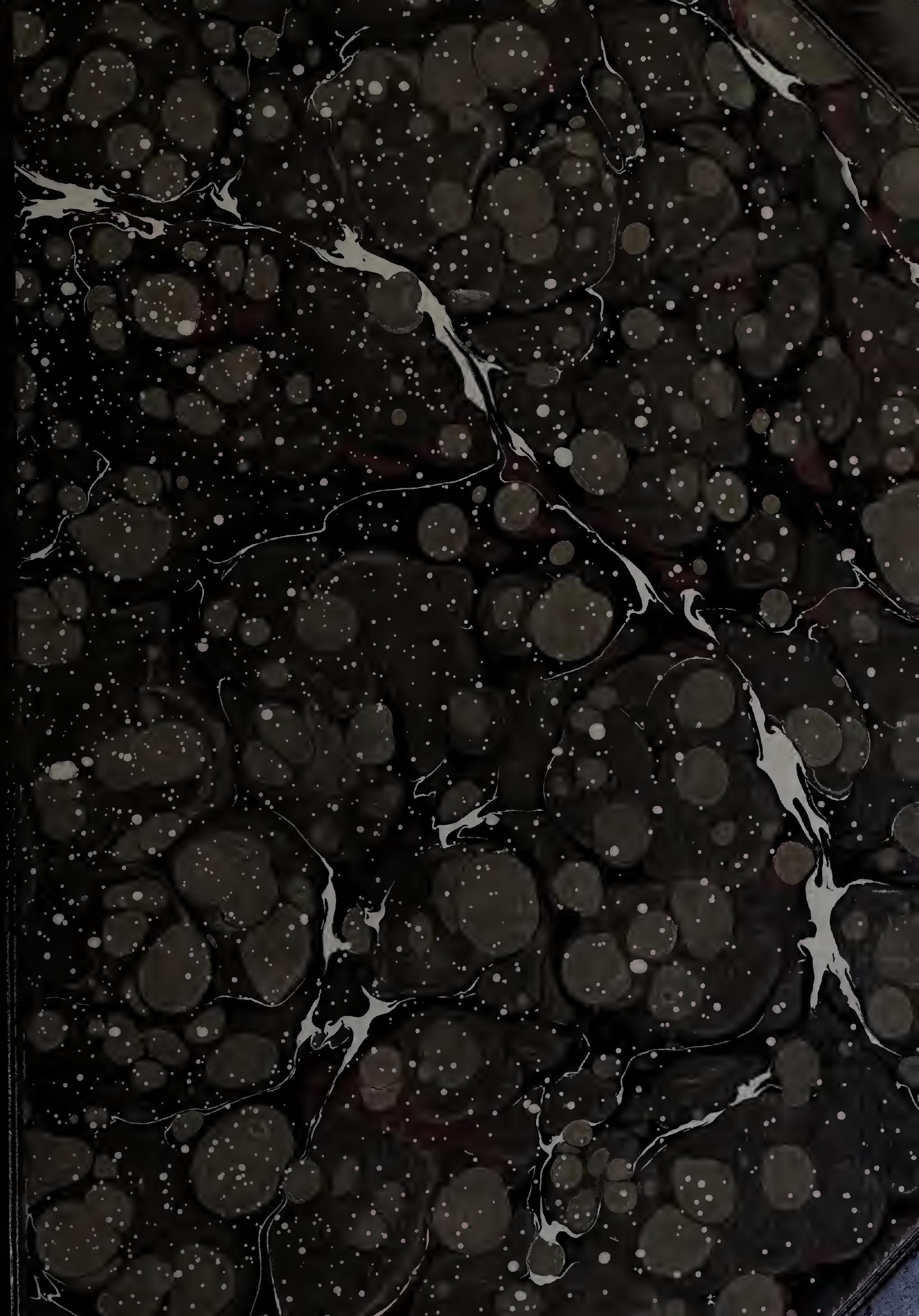




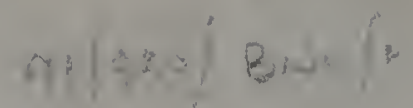

$$
12=
$$




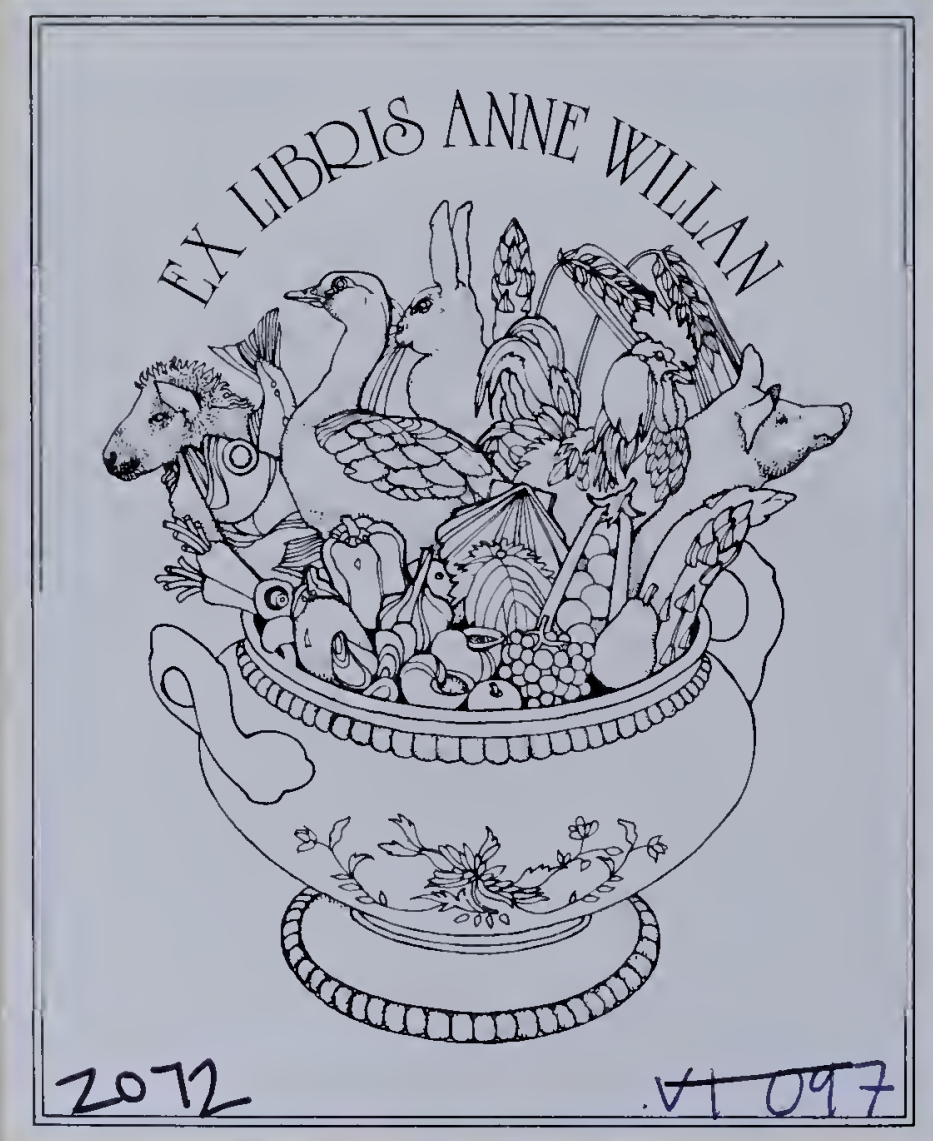




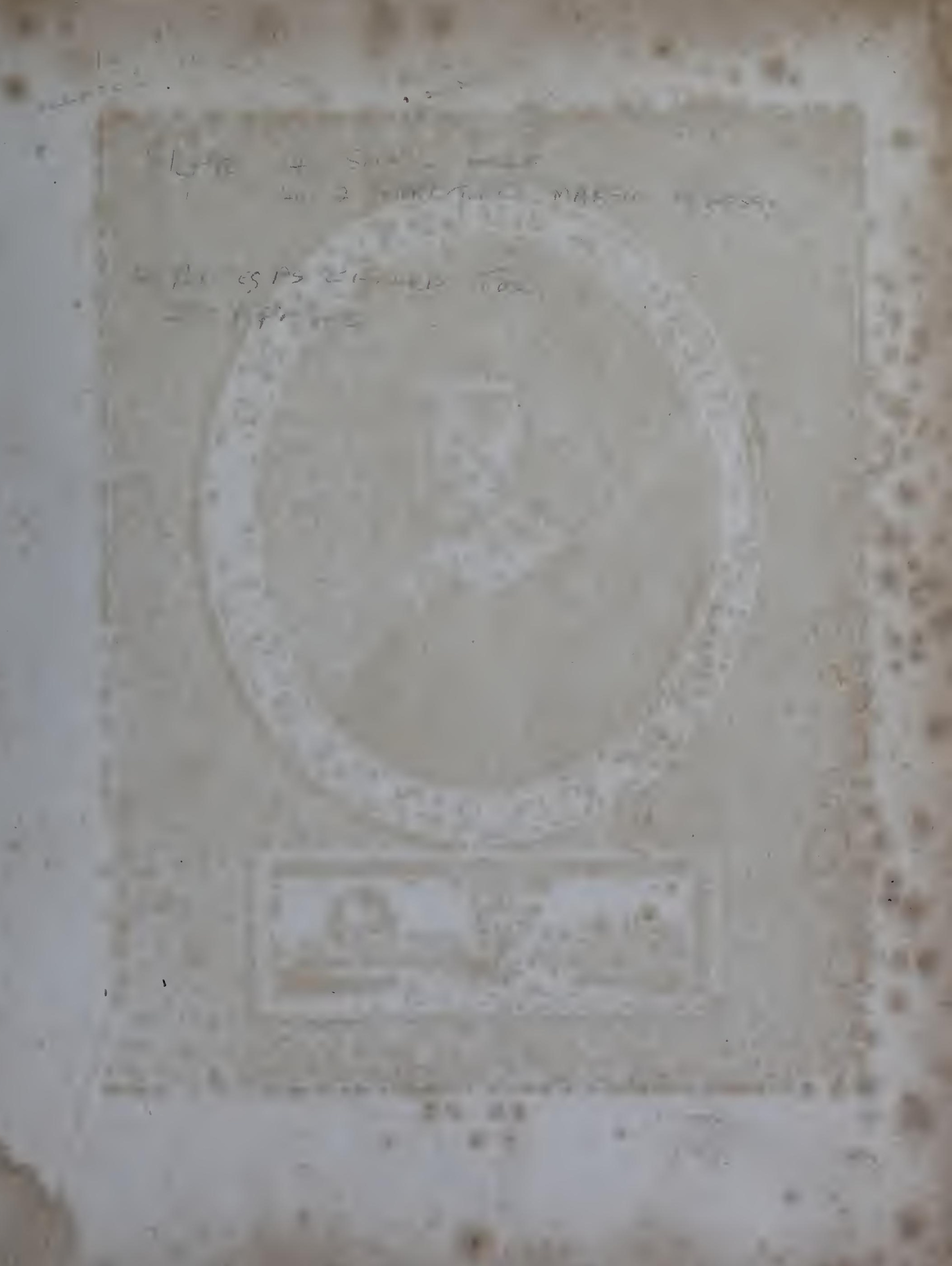




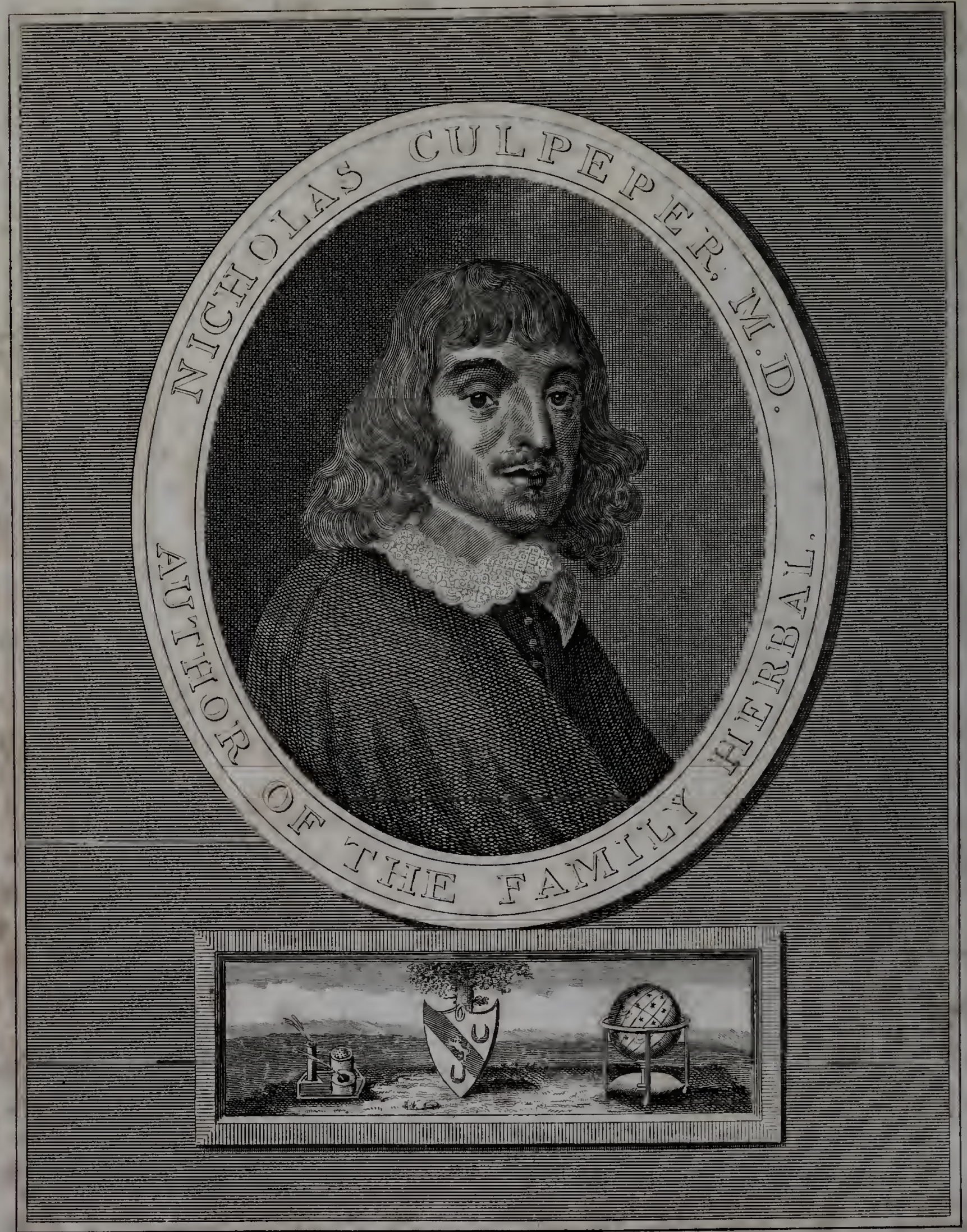

1. (1) $110 \times$

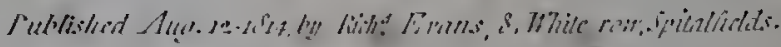




\section{$\mathbb{C} \mathbb{U} \mathbb{P} \mathbb{E} \mathbb{P} \mathbb{R}^{\prime} \mathfrak{S}$ \\ COMPLETE HERBAL, \\ TO WHICH IS NOW ADDED, \\ UPWARDS OF

ONE HUNDRED ADDITIONAL HERBS,

WITH A DISPLAY OF THEIR

Medicinal and Occult Qualities;

Physically applied to

THE CURE OF ALL DISORDERS INCIIDENT TO MANK IND.

To which are now first annexed his

ENGLISH PHYSICIAN ENLARGED,

AND

KEY TO PHYSIC,

WITIX

RULES for Compounding MEDICINE according to the true SYSTEM of NATURE.

NATURAL SYSTEM OF PHYSIC.

TO WHICH IS ALSO ADDED

UPWARDS OF FIFTY CHOICE RECEIPTS, Selected from the Author's last, Legacy to his Wife.

EMBLLLISHED WITH ENGRAVINGS OF UPWARDS OF FOUR HUNDRED DIFFERENT PLANTS, WITH OTHER

SUBJECTS TO ILLUSTRATE THE WORK.

"The Lord hath created Medicines out of the Earth; and he that is wise will not abhor them."-Ecc. xxxviii. 4 .

$$
L O N D O N \text { : }
$$

Published by RICHARD EVANS, No. 8, White's Row, Spitalfieids..

J. HADDON, FRINTEIR, TABERNACLE WALK.

1815. 
- I.t. 


\section{CULPEPER's}

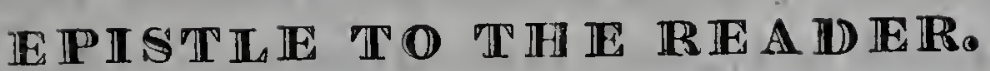

TAKE Notice, That in this Edition I have made very many Additions to every sheet in the book: and, also, that those books of mine that are printed of that Letter the small Bibles are printed with, are very falsely printed; there being twenty or thirty gross mistakes in every sheet, many of them such as are exceedingly dangerous to such as shall venture to use them: And therefore $I$ do warn the Public of them: I can do no more at present; only take notice of these Directions by which you shall be sure to know the True one from the False.

The first Direction.-The true one hath this Title over the head of every Book, Tin Complete Herbal and Englisi Puysician enlarged. The small Counterfeit ones have only this Title, The English Physician.

The second Direction.-The true one bath these words, Government and VirTUES, following the time of the Plants flowering, \&c. The counterfeit small ones have these Words, Virtues and USE, following the time of the Plants flowering.

The third Direction.-The true one is of a larger Letter than the counterfeit ones, which are in Twelves, \&c. of the Letter small Bibles used to be printed on.. I shall now speak something of the book itself.

All other Authors that have written of the nature of Herbs, give not a bit of reason why such an Herb was appropriated to such a part of the body, nor why it cured such a disease. Truly my own body being sickly, brought me easily into a capacity, to know that health was the greatest of all earthly blessings, and truly he was never sick that doth not believe it. Then I considered that all medicines were compounded of Herbs, Roots, Flowers, Seeds, \&c. and this first set me to work in studying the nature of Simples, most of which I knew by sight before; and indecd all the Authors I could read gave me but little satisfaction in this particular, or none at all. I cannot build my faith upon Authors' words, nor believing a thing because they say it, and could wish every body were of my mind in this, - to labour to be able to give a reason for every thing they say or do: They say Reason makes a man differ from a Beast; if that be true, pray what are they that, instead of reason for their judgment, quote old Authors? Perhaps their authors knew a reason for what they wrote, perhaps they did not; what is that to us? Do we know it? Truly, in writing this work first, to satisfy myself, I drew out all the virtues of the vulgar 
or common Herbs, Plants, and Trees, \&c. out of the best, or most approved authors I had, or could get; and having done so, I set myself to study the reason of them. I knew well enough the whole world, and every thing in it, was formed of a composition of contrary elements, and in such a harmony as must needs shew the wisdom and power of a great God. I knew as well this Creation, though thus composed of contraries, was one united body, and man an epitome of it: I knew those various affections in man, in respect of sickness and health, were caused naturally (though God may have other ends best known to himself) by the various operations of the Microcosm; and I could not be ignorant, that as the cause is, so must the cure be ; and therefore he that would know the reason of the operation of the Herbs, must look up as high as the Stars, astrologically. I always found the disease vary according to the various motions of the Stars; and this is enough, one would think, to teach a man by the effect where the cause lies. Then to find out the reason of the operation of Herbs, Plants, \&c. by the Stars went I; and herein I could find but few authors, but those as full of nonsense and contradiction an egg is full of meat. This not being pleasing, and less profitable to me, I consulted with my two brothers; Dr. Reason and Dr. Experience, and took a voyage to visit my mother NATure, by whose advice, together with the help of Dr. Diligence, I at last obtained my desire; and, being warned by Mr. Honestr, a stranger in our days, to publish it to the world, I have done it.

But you will say, What need I have written on this Subject, seeing so many famous and learned men have written so much of it in the English Tongue, much more than I have done?

To this I answer, neither GERRARD nor PARKINSON, or any that ever wrote in the like nature, ever gave one wise reason for what they wrote, and so did nothing else but train up young novices in Physic in the School of tradition, and teach them just as a parrot is taught to speak; an Author says so, therefore it is true; and if all that Authors say be true, why do they contradict one another? But in mine, if you view it with the eye of reason, you shall see a reason for every thing that is written, whereby you may find the very ground and foundation of Physic; you may know what you do, and wherefore you do it; and this shall call me Father, it being (that I know of) never done in the world before.

I have now but two things to write, and then I have done.

1. What the profit and benefit of this Work is.

2. Instructions in the use of it.

1. The profit and benefits arising from it, or that may occur to a wise man from it, are many; so many that should I sum up all the particulars, my Epistle would be as big as the Book; I shall only quote some few general heads. 
First, The admirable Harmony of the Creation is herein seen, in the influence of Stars upon Herbs and the Body of Man, how one part of the Creation is subservient to another, and all for the use of Man, whereby the infinite power and wisdom of God in the Creation appears; and if I do not admire at the simplicity of the Ranters, never trust me; who but viewing the Creation can hold such a sottish opinion, as that it was from eternity, when the mysteries of it are so clear to every, eye? but that Scripture shall be verified to them, Rom. i. 20. "The invisible things of him from the Creation of "the World are clearly seen, being understood by the things that are made, eten his Eternal "Power and Godhead, so that they are without excuse."-And a Poet could teach them a better lesson;

"Because out of thy thoughts God shall not pass,

"His image stamped is on every grass."

This indeed is true, God has stamped his image on every creature, and therefore the abuse of the creature is a great sin; but how much the more doth the wisdom and excellency of God appear, if we consider the harmony of the Creation in the virtuc and operation of every Herb?

Secondly, Hereby you may know what infinite knowledge Adam had in his innocence, that by looking upon a creature, he was able to give it a name according to its nature; and by knowing that, thou mayest know how great thy fall was, and be humbled for it even in this respect, because hereby thou art so ignorant:

Thirdly, Here is the right way for thee to begin the study of Pliysic, if thou art minded to begin at the right end, for here thou hast the reason of the whole art. I wrote before in certain Astrological Lectures, which I read, and printed, intituled, Astrological Judgment of Diseases, what planet caused (as a second cause) every disease, how it might be found out what planet caused it ; here thou hast what planet cures it by Sympathy and Antipathy; and this brings me to my last promise, viz.

Instructions for the right use of the book.

And herein let me premise a word or two, the Herbs, Plants, \&c. are now in the book appropriated to their proper planets. Therefore,

First, Consider what planet causeth the disease; that thou mayest find in my aforesaid Judgment of Diseases.

Secondly, Consider what part of the body is afflicted by the disease, and whether it lies in the flesh, or blood, or bones, or ventricles.

Thirdly, Consider by what planet the afflicted part of the body is governed : that my Judgment of Diseases will inform you also.

Fourthly, You may oppose diseases by Herbs of the planet, opposite to the planet that causes them : as diseases of Jupiter by Herbs of Mercury, and the contrary; 
diseases of the Luminaries by Herbs of Saturn, and the contrary; diseases of Mars by Herbs of Venus, and the contrary.

Fifthly, There is a way to cure diseases sometimes by Sympathy, and so every planet cures his own disease; as the Sun and Moon by their Herbs cure the Eyes, Saturn the Spleen, Jupiter the Liver, Mars the Gall and diseases of choler, and Venus dis. eases in the instruments of Generation.

From my House in Spitalfields, next door to the Red Lion,

September 5, 1653.

NICH. CULPEPER.

TO HIS DEAR CONSORT,

\section{MRS. ALICE CULPEPER.}

MY DEAREST,

THE works that I have published to the world (though envied by some illiterate physicians) have merited such just applause, that thou mayest be confident in proceeding to publish any thing I leave thee, especially this master-piece; assuring my friends and countrymen, that they will receive as much benefit by this, as by my Dispensatory, and that incomparable piece, called, Semiotica Uranica enlarged, and English Physician.

These are the choicest secrets, which I had many years locked up in my own breast. I gained them by my constant practice, and by them I maintained a continual reputation in the world, and I doubt not but the world will honour thee for divulging them; and my fame shall continue and increase thereby, though the period of my Life and Studies be at hand, and I must now bid all things under the sun farewell : farewell my dear wife and child; farewell Arts and Sciences, which I so dearly lóved; farewell all worldly glories, adieu readers.

Nicholas Culpeper. 


\title{
GENERAL INDEX,
}

\author{
TO THE \\ ENGLISH PHYSICIAN ENLARGED,
}

A N D

\section{GALENS KEY TO PHYSIC.}

ADDER's Tongue, or Serpent's Tongue, 3

Agrimony, 4

Water, 5

Alder-Tree, the black, 6

the common, 7

Alehoof, or Ground-Ivy, 5

Alexander, 6

Alkanet, 3, 218

All-Heal, 2

Amara Dulcis, 1

Amaranthus, $\mathbf{9}$

Anemone, 9

Angelica, 8, 218

Anthoræ, 218

Archangel, 11

Arrach, garden, 10

wild and stinking, 10

Ars-smart, 12

Artichokes, 88, 221

Asarabacca, 13, 218

Ash Tree, 14, 222

Asparagus, 13

Asparagu, prickly, 14

Asparagus, or Sperage, 218

Asphodel, Female, or King's Spear, 218

Avens, Colewort, or Herb Bonet, 15

Avens, or Herb Bennet, 219

Balm, 15

Barks, 259

body, 259

Barberry, 16

Barks, 202

Barks, 227

Barley, 16

Bay 'I'ree, 18, 223

Baxil, garden, or Sweet Bazil, 18

Beans, 18

Beans. Frencl, 19

Bearsbreach, or Brankursine, 217.29
Bed-straw, Ladies, 19

Beech Tree, 23

Beets, 20

Beets, black, white, and red, 219

Betony, Water, 21

Wood, ib.

Bifoil, or Twablade, 24

Bilberries, Whorts, or Whortleberries, 23

Birch Tree, 24

Bird's Foot, 24

Birthwort, 218

Bishop's-Weed, 25

Bistort, or Snakeweed, 25, 219

Blade, One, 26

Blites, 27

Blue Bottle, 28

Borrage, 219

Borrage and Bugloss, 28

Bramble, or Black-berry Bush, 27

Brank-Ursine, 29

Briony, or Wild Vine, 30

Briony, white and black, 219

Brook Lime, or Water Pimpernel, 31

Broom and Broom-Rape, 32

Buck's-horn Plantak, 33

Bugle, 33

Bugloss, $219 \cdot 28$

Bulbus Vomitorius, 219

Bur, Clot-bur, or Burdock, 219

Burdock, 36

Burnet, 35, 224, 226

Butcher's Broom, 31

Butter-bur, 224

Butter-bur, or Petasitis, 35

Cabbages and Coleworts, 37.

Calamint, or Mountain Mint, 38

Caltrops, Water, 39, 226

Cammock, or Rest.harrow, 223

Camonile, 39

Campion, Wild, 40

C'aper Roots, 219

Carduus Benedictus, 11
Carraway, 42

Carrots, 41, 221

Celandine, 42

Celesser, 44

Celandine, the greater and lesser, 220

Centaury, ordinary small, 44

Centaury the greater, 219

Cerecloths, 366

Chamelion, white and black, 220

Cherries, Winter, 45

Cherry-'Tree, 45

Chervil, 46

Sweet, or Sweet Cicely, 47

Chesinut-Tree, 47

Chesnuts, Earth, ib.

Chickweed, 48

Chick-pease, or Cicers, ib.

China, 220

Cinquefoil, 224

Cinquefoil, or Five-leaved Grass, 48

Cives, 50

Clary, or Clear Eye, 50

Wild, 51

Cleavers, $5 \mathrm{I}$

Clown's Woodwort, 52

Cock's Head, Red Fitching, or Me. dick Fetch, 52.

Colewort, 219.3y

Coleworts, Sea, ib.

Coltsfoot, 53

Columbines, 53

Comfrey, 54, 220

Compounds, Spirit and Compound Distilled Waters, 283

Conserves, 206

Conserves and Sugars, 315

Coralwort, 54

Costmary, or Alecost, or Balsana Herb, 55.

Costus, both sorts, 220 .

Cowslips, or Peagles, 56

Crabs Claws, 57

Creatures, Living, 252 
Creatures, parts of Living, and Excrements, 253

Cresses, black, 57

- Sciatica, ib.

Crosswort, 56 Water, 58

Crowfoot, 59

Cuckow Point, ib.

Cuckow-points, or Wake-robin, 218

Cucumber roots, wild, 221

Cucumbers, 61

Cudweed, or Cotton-weed, 56

Daisies, 61, 219

Dandelion, vulgarly called Piss-abeds, 62

Darnel, 62

Decoctions, 204, 293

Devil's Bit, 63, 223

Dill, 63

Distilled Waters, Siniples, 278 being digested before hand, 278

Dittany, 221

Dock, 64

Dodder of 'Thyme, Epithymum, \&c. 64

Dog's Grass, or Cough Grass, 65

Doronicum, 221

Dove's-Foot, or Crane's-Bill, 66

Down or Cotton Thistle, 67

Dracunculi, 221

Dragons, 67

Dropwort, 222 ,

Duck's Meat, 66

Dwarf Elder, Walwort, or Danewort, 221

Elder, 225

Elder Tree, 67

- Dwarf, 67

Elecampane, 69, 221

Electuaries, 205

Electuaries, 324

D purging, 331

Elm' Tree, 68

Emplasters, $\varsigma 91$

Eudive, 69

Endive, Garden Endive, 221

Eringo, or Sea Holly, 70, 222

Eyebrighth, 71

Fennel, 73, 222

Fern, 71

$$
\text { Sow, or Hog's Fennel, } 74
$$

Water, or Osmond Royal, 72

Male and Female, 222

of the Oak, 224

Feverfew, or Featherfew, 72

Figwort, 226

Fig-Wort, or Throat-Wort, 74

Fig Tree, 75

Filipendula, or Drop-Wort, 75

Flag, Yellow Water, or Flower-deLiice, 76.
Flax-Weed, or Toad Flax, 76

Flea-Wort, 77

Flower-de-Luce, $78 \cdot \%$.

Flowers, 200, 247

264

- -.- appropriated to certain parts of the body, 265

Fluellin, or Lluellin, 79

Fluxweed, 78

Fox-Gloves, 80

Fruits, 267

- appropriated to the body of man, 267

- by their several operations, bind, \&c. 268 - purging, 268

- and their buds, 248

Fuller's-Thistle, 223

Fumitory, 80

Furze Bush, 81

Galanga, or Galingal, 222

Galanga, English, long and round, 221

Galen's Method of Physic, Key to, 376

Garlick, 82, 218, 227

Gentian, 292

Gentian, Felwort, or Baldmony, 82

Germander, 83

Gilliflowers, Clove, 83

Ginger, 227

Gladon, Stinking, 226

Glad win, Stinking, 84

Golden Rod, S5

Gooseberry Bush, 86

Gout-wort, or Herb Gerrard, 85

Grass, 222

Green, Winter, 86

Gromel, 85

Groundsel, 87

Gums, Rozins, Balsams, and Juices, 270

Hart's Tongue, 88

Hawk-weed,

Hawthorn, $90^{\circ}$

Hazel-Nut, 89

Heart's Ease, 88

Hearts-ease, or Pansies, 226

Hedge Hyssop, 92

Hellebore, black, 93

Hellebore, white and black, 221

Hemlock, 90

Hemp, 91

Henbane, 91

Herb Robert, 94

- 'I'rue-Love, or One Berry, 94

Herbs, 260

appropriated to certain parts of the body of man, 262

alteriag according to pro. perty, 263

purging, 264
Ulerbs or 'Trees, of the leaves of, 200 and their Leaves, 229

Hermodactils, 222

Holly, Holm, or Hulver Bush, 99

Hops, 95

Horehound, 96

Horsetail, 97

Hound's Tongue, 98, 221

Houseleek, or Sengreen, 97

Hyssop, 95

Jacinths, 222

John's Wort, St. 99

Ivy, 99

Juniper Bush, 100

Juices, 202, 252

Juleps, 204

Kidney Wort, or Wall Penny-royal, or Wall Penny-wort, 101

Knapweed, 102

Knotgrass, ib.

Knee-holly, or Butclıer's-broom, or Bruscus, 225

Ladies'-thistles, 226

Lavender, ib.

Leeks, 225

Lettice, 223

Lettuce, 104

Lilies, Water, 223

Lilies, white, 106, 223

Lily, Water, 105

Lithe Valley, ib.

Liquorice, 106, 222

Liverwort, 107

Lohochs, 208

Loosestrife, or Willow-herb, 107 flowers, ib.

Lovage, 108, 223

Lungwort, 109

Madder, 109, 225

Maidenhair, 110

—_ white, or Wall Rue, ib.

Mallows, $223 \quad 272$.

Mándrakes, 223

Maple 'Tree, 112

Marigolds, 114

Marjoram, Wind, 112

- Sweet, 113

Marsh-mallows, $218 . .11$.

Master-wort, 114, 223, 224

Maudlin, Sweet, 114

Mead-sweet, 227

Mechracah, 223

Medicines, method of mixing, $210^{\circ}$

376

'Temperate, 377 ;

Hot, ib.

Hot in the first degree, ib. 
GENERAL INDEX.

Medicines, hot in the second degree, 378

ib. third degree,

ib. fourth degree, Cooling, 378 379

third degree, ib. cool $^{3}$ in the first degree, - cold in the fourth degree, 379 mo istening, ib. drying, ib.

of the appropriation of the several parts of the body, 380

ib.

Brain, ib.

Eyes, ib.

Mouth and Nose, 382

Ears, ib.

Teeth, ib.

Breast and lungs, ib.

Heart, 383

Stomach, 384

Liver, 385

Spleen, ib.

Reins \& Bladder, 386

Womb, ib.

Joints, ib.

tion of, 387

Propriety or opera-

Hardening, ib.

Loosening, 388

Drawing, 389

Discussive, ib.

Repelling, 390

Cleansing, 391

Suppuring, 392

Provoking urine, ib.

Breeding flesh, 393

Glutinative, ib.

Medlar, 115

Resisting poison, ib.

Melilot, or King's Claver, 115

Mercury, French and Dog, 116

Dorg, ib.

Metals, Minerals, and Stones, 254

Metals, Stones, Salts, and other Minerals, 276

Mints, 117

Misselto, 118

Mitlıridate Mustard, IS2

Money-wort, or Herb 'Two-pence, 119

Monks Rhubarb, 225

Moonwort, 120

Mosses, 120
Motherwort, 121

Mouse-ear, 122

Mugwort, 122

Mulberry Tree, 123, 223

Muliein, ib.

Mustard, 124

Hedge, 125

Nailwort, or Whitlow-grass, 126

Nep, or Catmint, ib.

Nettles, 127, 227

Nightshade, 128

Oak, 128

Oats, 129

Oils, 205

Oils, 353

- Simple Oils by expression, ib. tion, 354

- Compound Oils by infusion and decoction, 355

Ointments, 208

Ointments, more simple, $35 \mathrm{~s}$

One Blade, ib. more conipound, 362

Onions, 130, 220

Orchis, 129

Orpine, ib. 130

Orris, or Flower-de-luce, 222

Parsley, 131

- Piert, or Parsley Brealstone, ib.

Parsnips, 192

_- Cow, ib.

- garden and wild, 224

Peach Tree, 133

Pear Tree, 134

Pellitory, of Spain, 134, 225 of the Wall, 135

Peony, male and female, 224

Pennyroyal, 136

Peony, nale and female, 197

Pepperwort, or Dittander, 138

Periwinkle, 138

Peter's Wort, St. 139

Pimpernel, I39

Physic, a Key to Galen's Method of, 376

_- the general use of, ib.

- - of the temperature, ib.

Pills, 209, 339

Pine, Ground, 140

Plaisters, 208, 367

Plantain, 141, 224

Plants, things bred from, 252

Plums, 142

Polypody of the Oak, 149

Poplar 'Tree, 143

Poppy, 144

Poultices, 209

Preserved Rools, Stalls, Barlis, Flowers, Fruits, 31.4

Preserves, 206
Primroses, 146

Privet, 146, 218

Purslain, 116

Queen of the Meadows, or Meadow Sweet, 147

Quince 'Tree, 148

Radishes, garden and wild, 225

Ragwort, 149

Rest-Harrow, or Camonack, 150

Rattle Grass, 150

Receipts, General Caution, 395

to purge the Head, ib.

for a Rlieum in the Head, and the Palsy, ib.

ib. 396.

for the falling off of the

Hair, ib.

to purge the Head, ib.

ib.

- for Eyes that are blasted,

Excellent Water to clear the Sight, ib.

- for a liurt in the Eye with a stroke, ib.

- - to draw Rlieum back from the Eyes, ib.

_ for a Web in the Eye, ib.

_ pain in the Ears, ib.

Ear, ib.

__- Polypus, or a fleshy sub. stance growing in the Nose, ib.

___ bleeding at the Nose, ib.

ib.

ib. another for the Polypus,

Mouth, 397

ib.

to keep Teet! while, ib. fasten the 'Teeth, ib.

for the 'Tuotli-ache, ib. - Scurvy in the Gums, ib.

- rotting and consuning of the Gums, ib.

- the cause of Infirmities ili the Face, ib.

ples, ib.

po take away the marks of the Snall Pox, ib.

Cantion concerning the Infirmities of the Throat, ib. for Hoarseness, 398 the Quinsey, ib.

Sore Breasts, ib.

for, ib.

ib. 


\section{GENERAL INDEX.}

Receipts for the heat of the Stomach, Self-heal, 170 ib. digest, ib.

o cause the Liver well to a Caution, ib. a stoppage of the Liver ib.

the Liver, ib.

Reed, Aromatical, 219

Reeds, common and sugar, 218

Rhadish, or Horse Rhadish, 148

Rhapontick, or Rhubarb of Pontus, 225

Rhubarb, 225

Rhubarb, or Rephontic, 150

_- Monk's, or garden Patience, 157

Service Tree, 171

Seseli, or Hartwort, 226

Shepherd's Purse, 171

Sloe-bush, or Sloe-tree, 225

Smallage, 171, 218

Solomon's Seal, 163, 225

Sope-wort, or Bruise-wort, 172

Sorrel, ib.

Wood, ib.

Sow Thistles, 173

Southern-wood, ib.

Sorrel, 223

Sowbread, 218

Sow-thistles, 226

Species, or Powders, 317

Spignel, 223

Spignel, or Spikenard, 174 leaved Dock, ib.

Rocket, 151

Winter, or Cresses, 152

Roots, 201, 217, 256

- Temperature of, 257 - appropriated to several parts of the body, 258

-_- properties of, ib.

Rosa Solis, or Sun Dew, 155

Rosemary, ib.

Rose Root, 225

Roses, 152

Rue, Meadow, 158

Garden, 159

Rupture-wort, 160

Rushes, 161

- Rye, ib.

Saffron, Meadow, 220

Saffron, 161

Sage, 162

Wood, ib.

Samphire, 164

Sanicle, ib.

Saracen's ('onfound, or Saracen's Wound-wort, 165

Sarsaparilla, or Bind-weed, 225

Satyrion, 226

Sauce-alone, or Jack by the HedgeSide, 165

Saviné, 166

Savory, Winter and Summer, ib.

Saxifrage, the common white, 167

- Burnet, ib. white, or Lady-Smocks, 226

Scabious, ib.

Scabious, three sorts, 168

Scirrets, 226

Scordiun, or Water-Germander, ib.

Scurvygrass, 109

Sea, things belonging to the, 254

Seeds, 201

___ or Grains, 249, 268

- according to their operation, bind, \&c. 209
Spikenard, 223

Spleenwort, Ceterach, or Hart's Tongue, ib.

Spurge, greatcr and lesser, 292

Spurge, Olive, or Widow-wail, 22.3 Squils, 226

Star-Thistle, 175

Stone-Crop, Prick-Madam, or small Houseleek, 177

Strawberries, 175

Swallow-wort, 218, 227

Succory, 220

Sugars, 316

Sulphur-wort, Hogs-fennel, or Horestrange, 224

Syrups, 203, 294

- altering, 294

- purging, 305

- made with Vinegar and Honey, 308

Tamaris, 226

Tamarisk Tree, 178

'Tansie, 226

Tansy, Wild, or Silver Weed, 179

Tears, Liquors, and Rosins, 251

Teazle, 223

Thistles, 179

- Melancholy, 180 our Lady's, ib. -Woollen, or Cotton, $181.6 \%$

- Fuller's, or Teasle, ib.

Thorn, black, or Sloe Bush, 182

Thorough Wax, or Thorough Leaf, 183

Throat-wort, 226

'Thyme, 183 183 ib. Wild, or Mother of Thyme,

Tinctures, 290

Toad-stools, 226

Tobacco, English, 177

Toothwort, 221
Succory, or Chicory, 176

l'ansy, garden, 178
Tormentil, 226

Tormentil, or Septfoil, 184

Treacle Mustard, 181

Trefoil, 226

Trefoil, Meadow, or Honey-suckles, 185

\section{Heart, 186}

Pearl, ib.

Troches, 209, 346

Turbith, 226

Turmerick, 221

Turnsole, or Heliotropium, 185

Tustan, or Park Leaves, 186

Valerian, white and red, 219

Valerian, or Setwell, greater and lesser, 224

Valerian, garden, 186

Vervain, 187

Vine, 188

Vinegars, Physical, 292

Violets, 188

Viper's Bugloss, 189

Viper's Bugloss, or Wild Bugloss, 221

Viper's Grass, 226

Wall Flowers, or Winter Gilli. flowers, 190

Walnut Tree, ib.

Water-flag, or Flower-de-luce, 218

Waters, distilled, 202

Weld, Wold, or Dyer's Weed, 191-

Wheat, 192

Willow 'Tree, ib.

Wines, Physical, 291

Woad, 193, 223

Woodbine, or Honey Suckles, ib.

Woods, 260

Woods, and their Chips or Rasp. ings, 229

Wornıwood, 194

Yarrow, Nose-bleed, Milfoil, or Thousand Leaf, 198

Zedoary, or Setwall, 227 
Sewseteecs

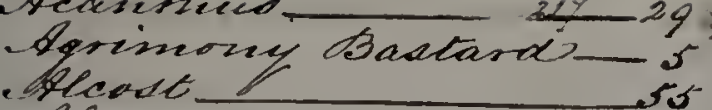

Hlisander

slebral sterculecís

- Anmi Smunara

Aherine

Srages

Aracte Dogi o loaka 53 $-6$ 2 25

Baffeiven

Brildinarey

Baldawnhert

Martar arow-

Bastand Sgrinony

Bastaed, lehubarte

ans Kixiney-

Bear's becche

Geard foot

see heule?

Siprelo

Mishopis teaver.

biver sweet

Blacsebery heeshs

Black pobleer.

chloval uror to

Blase blow

Bonet herb.

Brearetoxe fandey

Browarbugle

Arowze norts

Brusel wart

crudens

Buglodo ffranies

diteclíf 0 ot

siclewort

Butterflowers

Bucceturort

157

$21 \% 29$

9.3

1

3.5

21

Galues frot

Cannurock

barpextexs herb

baseurort.

latmoint

Gaxúfoot:

berefolicus

bleaffuce

Cobansepilas

blecese revreat

bluestruts water

blaivory

blacive

bivid mad ofloweror her $b$.

bivera -.

Giper heits

blisest

13

33

21

182

176

48

88

50
Claver. King's

blodlicer $\$ 6$

bolewort

bonfrey of bonfound hidde is

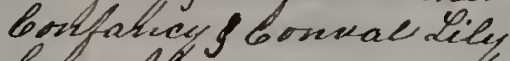

botw flower

Gorsow Devnent or Pelty.

bothow Ifieste?

bottow weed

brughe graes

boleghewart

Craneis hices.

lovesaes Sucine

lonessed Hinter

buckow flower

bulliniss

bull mue to you

bulrage,

Gundminseed Pryal.

Dead necte

Deadicurost

Dentana)

Deuberry buste

Ditsumen

Dock Guat nound leamed

Dogetones

Poqi Srante.

Dequectic ariteb

Braqow erort

Difofe wort

Disndweed.

bartinuts.

buchuse

buglish ferperctary-25

beshogsucen

Gepatoriuse

bue heeb

Feaphery

Helasherpere

Helonis wort

Tielevart

Fenuel Hra's

Ticituring Red

Hicie lemuex graso

Fifoupe const

Finmer gewste betret a

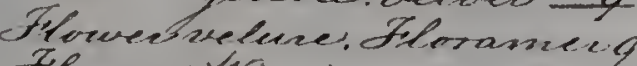

Flowere thínd;

fioalatoued

froquerote

fioolotased

fioxetones

fincelewnter-toldeir

fincapto.t

104

88
Galliond

Cemaralecel ig

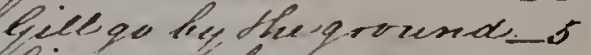

Giclecreet luy arand -5

coats Astranhe 10

contelaned.

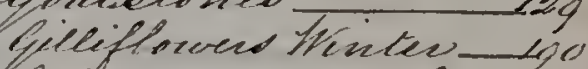

Yonth buepo it kiobs

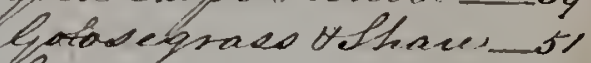

lerz:

lipare atrebof

Graesfine finque?

Graes porey

crals usteitour

Groused duy

Ground Pluts

8

169

Le

152

Hartihorn 33

barpmaido.

5

Heartis tonque?

- bedqescide. Sack by hee 165

- Heleistrofuiesa

182

- benon Hater

- bentlil

$-5$

Hepatoriecsu

Sterba stella. Stellaria 33

Hent. Balaans to

Hesb bonet

15

tient carpenteru_ 3.3

becture or huy

stech of gracel

.23

- Herb' inelfery.

sect livofienees

beet werds arse

159

Sercieles allheal

94

19

Hexculec's Hounderozts-2

Hogis Tunces $y$

Holle Hea

Holne

bones 99

brod theee races in 193

Hooxheal i yo

Hormbean 1

Horee barsley.

Sorde hor $f$

Howele r enzale

53

borcelecti Ifalew.

- bestrendereate.

Hut ificile - iy

Sdlenees Le

hing beab.

lack by the brexpecide 165
53

Sam amed Hray- 6

fazee: TI If

Jiving blamez

divas Knob- is of

dieec g hti "Prnd water woy 
Ludié Lear

30

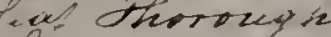

Liver ofaxis

18.3

Laeks Dessh _ 50

186

Lisicl in démed

82

Louret Gouliond _ _ is

Lofetey onafor ____ I 6

maiderefair

19

Gnaiden hair thite, Goldew IIO

suisedonias hardey des _o

male lily

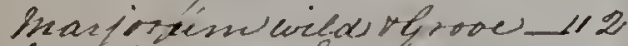

hpiofum bacturard 112

huend or heedow Lweet lif

Snedicte ferehe it 2

meddlubonfrey. Gonfound 33

hilfoil 198

Sulint fperer or Heant ist

Buertia? hirrtio

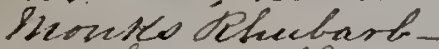

Bupther of Shemw.

motherrort Itinscing - 10

bertal.

heoutacie brint

hitte Bued of Dead

heqfetefeade woody

stovelfeed.

.

kets trater

Cnebruy sterb

Dpopalie wort

Corach

2

orchanet

Oreqanuma

Gatericks

Panne

25

Pancolio

Parrleaved - 186

Direley Brearestow- 131

Dasoley troxerartiled - 6

Pasdle seed fuacedoniaus b

Pascisind

Peachuort

25

Peagled

Pexperayal thale 12

Pensing utot 101

Befifuer Hater. 107

Descematín

Pesmaneter whoremanósy

Petasitis

Pettiquee

35

Dedrimunget

Aickpecare

sillevart

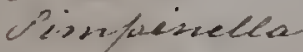

(y)

44
西

mpenuectrates

Pis guets

Slumbago

Dolts

Pond weed

Pot-hent Black

Prt harjirum

Prumee

$2 y 0$

Pamb

Red fircting

Reaboutic

Phubach Bastard

Phubact heovetís

Prfins loaste,

Rusemacy wild

Preach Leers

Sanquieorbeil

Latarend cround

Latiricase

dea-hally

Seal Labieís.

Sper

Len

Lestareese reater

Sebetore

Als therde onuce or Seeip

eleppentary bseqliete

Leepento Tonquee

litterk qraelo or tort.

Seirel heurt

Licklewort

33

Lilierweed

Horbecto

35

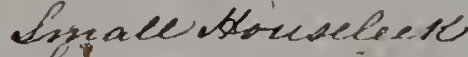

Amakceveeds ala. 25

Lolbequella 1

35

- oldieir. Frestevater - 5y

Dopnite Burybacd.

L faraqus of becage- 13

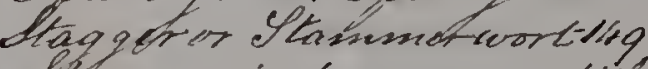
Luear enint.

Starchuror to

184

Linting hoshenwort-19

Lecuededer-

dereine-Credsed

Lueste 50

Gasueco

30

Fenales

$|8|$

Thialle sleserdi or bloly

Fleprough lea

Thousand leaf.

Thesefares in a \$ood

18.3

198

If
Hume. Buosher of _ 183

Toud flax

- Tonque teactó

Tonque cerpentis 174

- Iotsheriolle or wort 54 Fowewort of

Itinity. Herb of the of

Tribirleut.

.39

Troilfterves

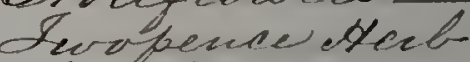

Lubeblade:

- Leerrehaof

line bis ar trild 30

listectoosk or Doqteeste 54

lielicaria

10

Wane Robin?

trace bensury ro al

59

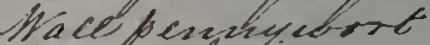

101

Hacle cluciesucts.

101

Haker fegu

39

fated howeleen

12

Hiater facus

Hater heets

ifalce bepber

trater blisubeswels

Mated desígesere

Weled

Hhine $5 \%$

theillow

theistow-y rado 126

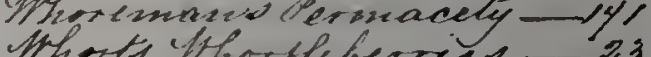

Hild, Rosemany

Hild or this devive

19

Irind Llower

Truder Lelliflowers 190

Hilleams.terb

Arilloir bect

Hort. Blood,

25

Hort Brown

liot Bruede

fiont buel

Hout Bulter

Hort Dead

Hort blanfouse?

Howt pcarte

Hort fierese?

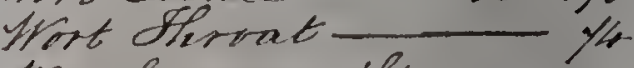

Hort Ltaqger or Lfammeriag

Hort Lit laness.

Horendurot is eceseles

$\operatorname{ling}$

Hindevort Saracenis-165

Hody kighishade

Nortltredses

33

Hort Hocertio'

155

trray and lawn b2

Spouthis wort 155 

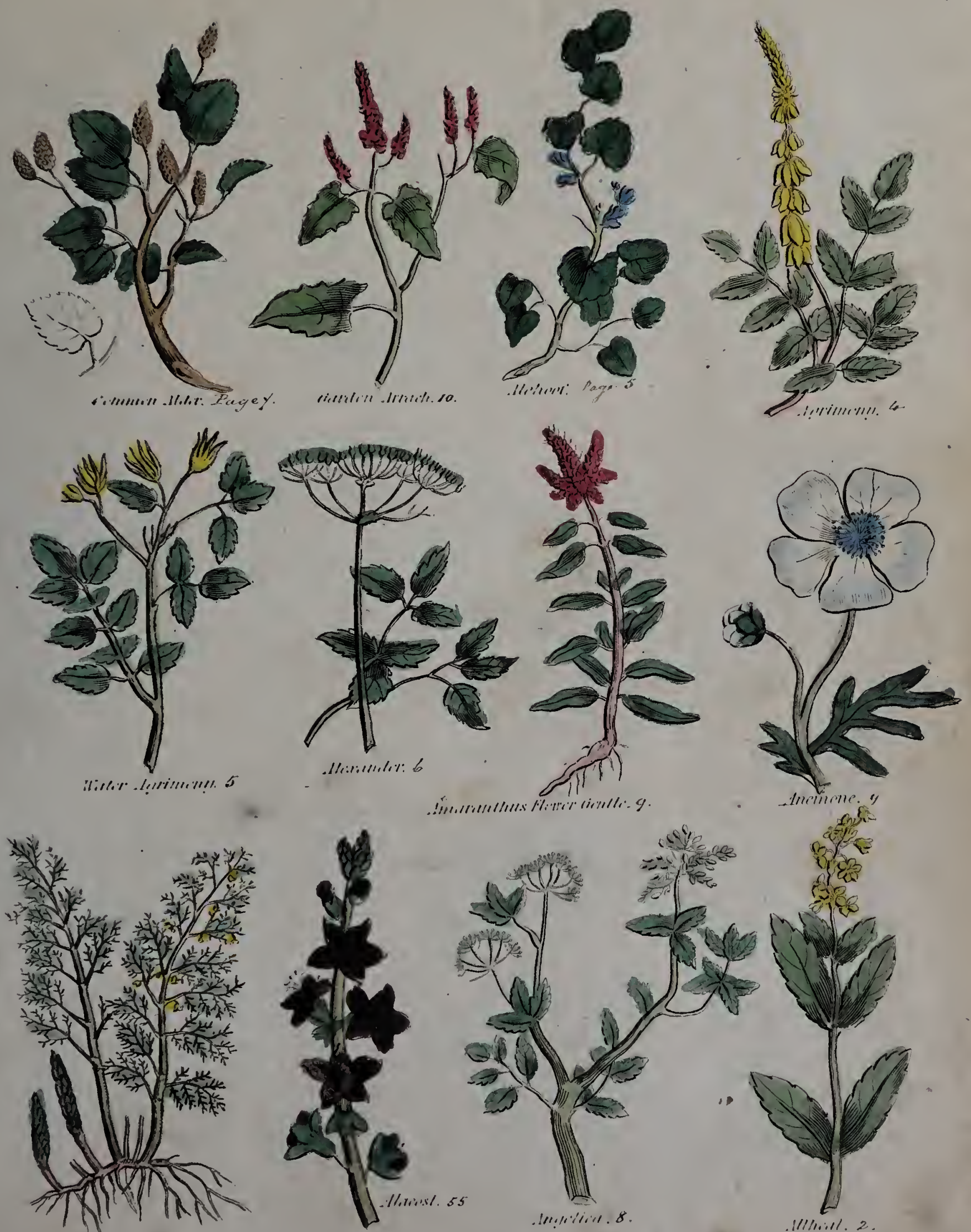

$.1 .271161 .1115 . \quad \therefore 3$ 


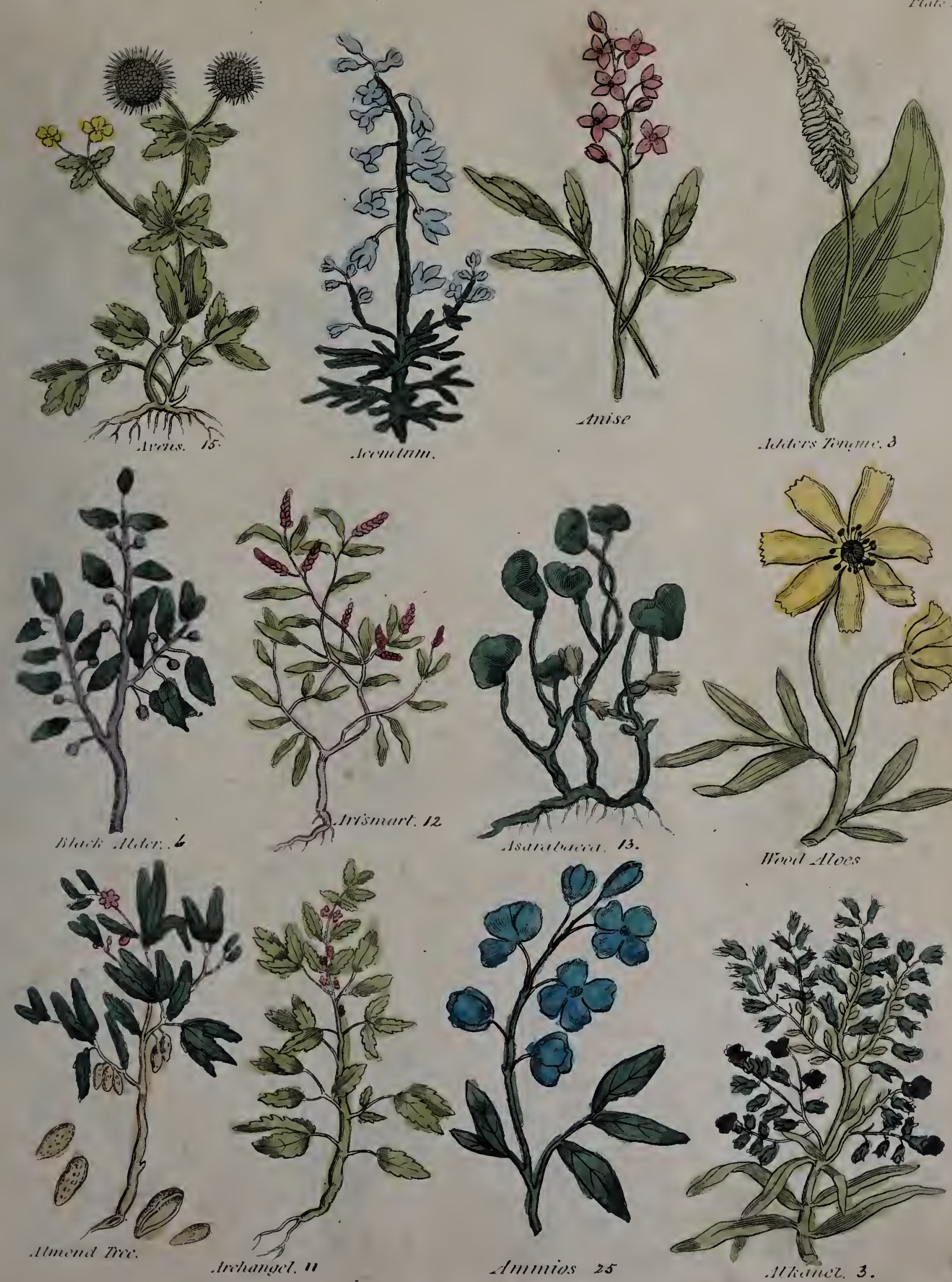

IN
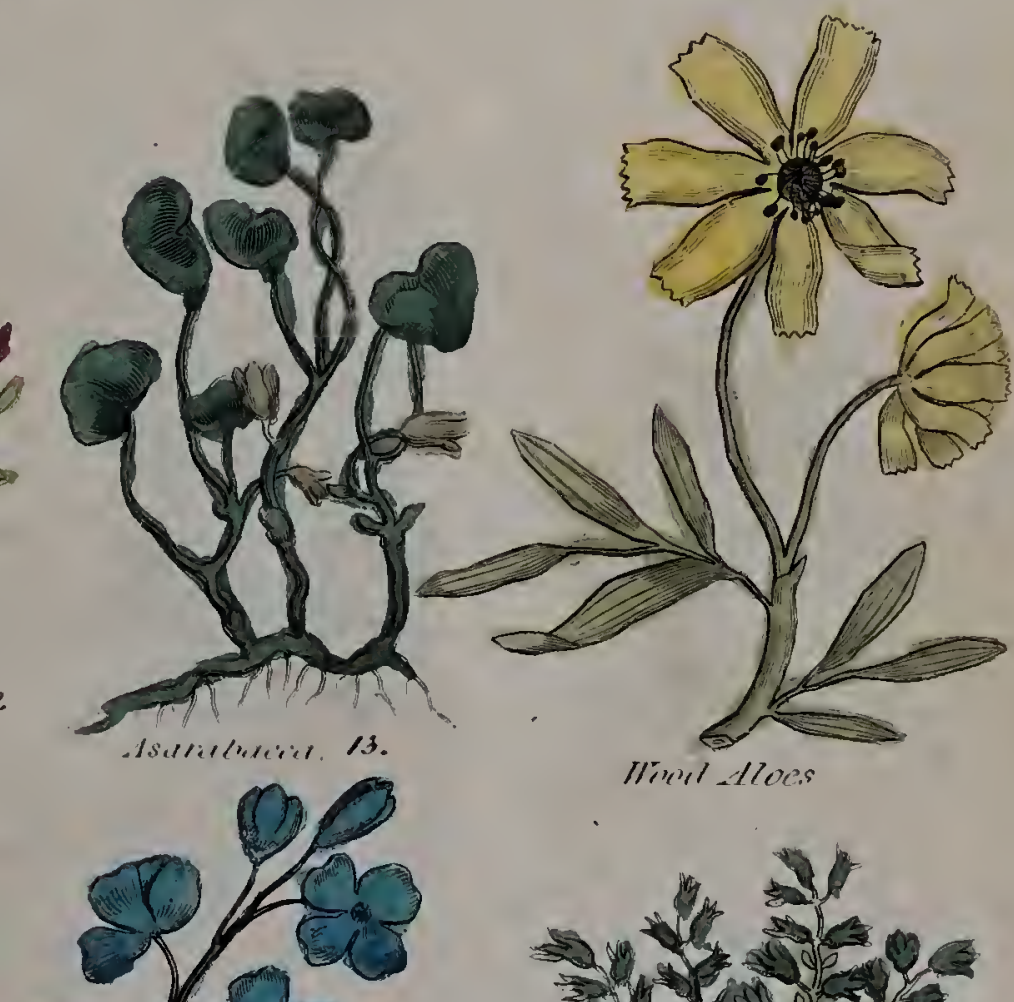

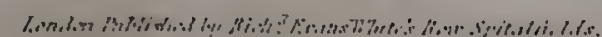




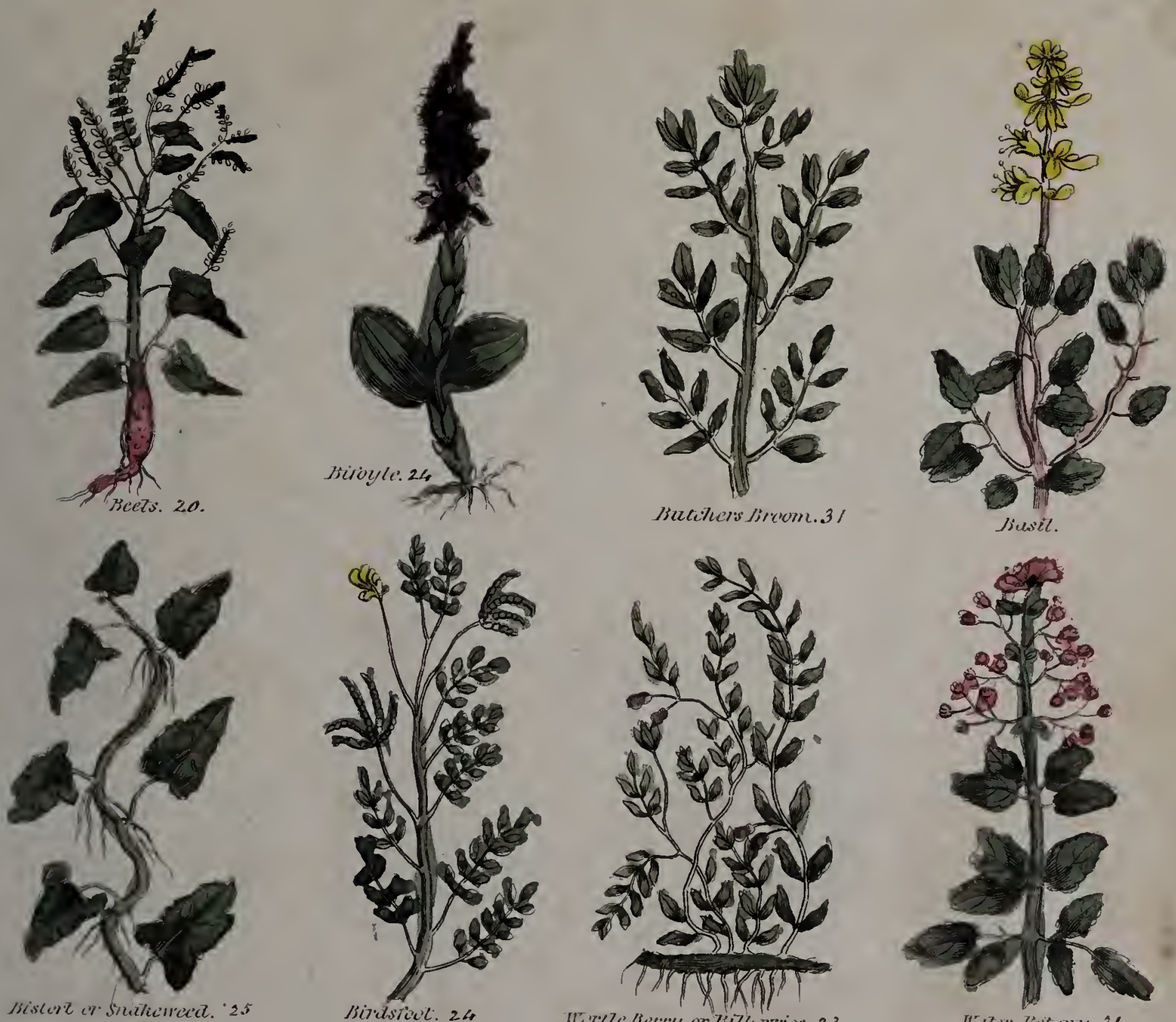

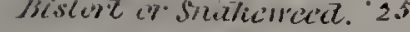
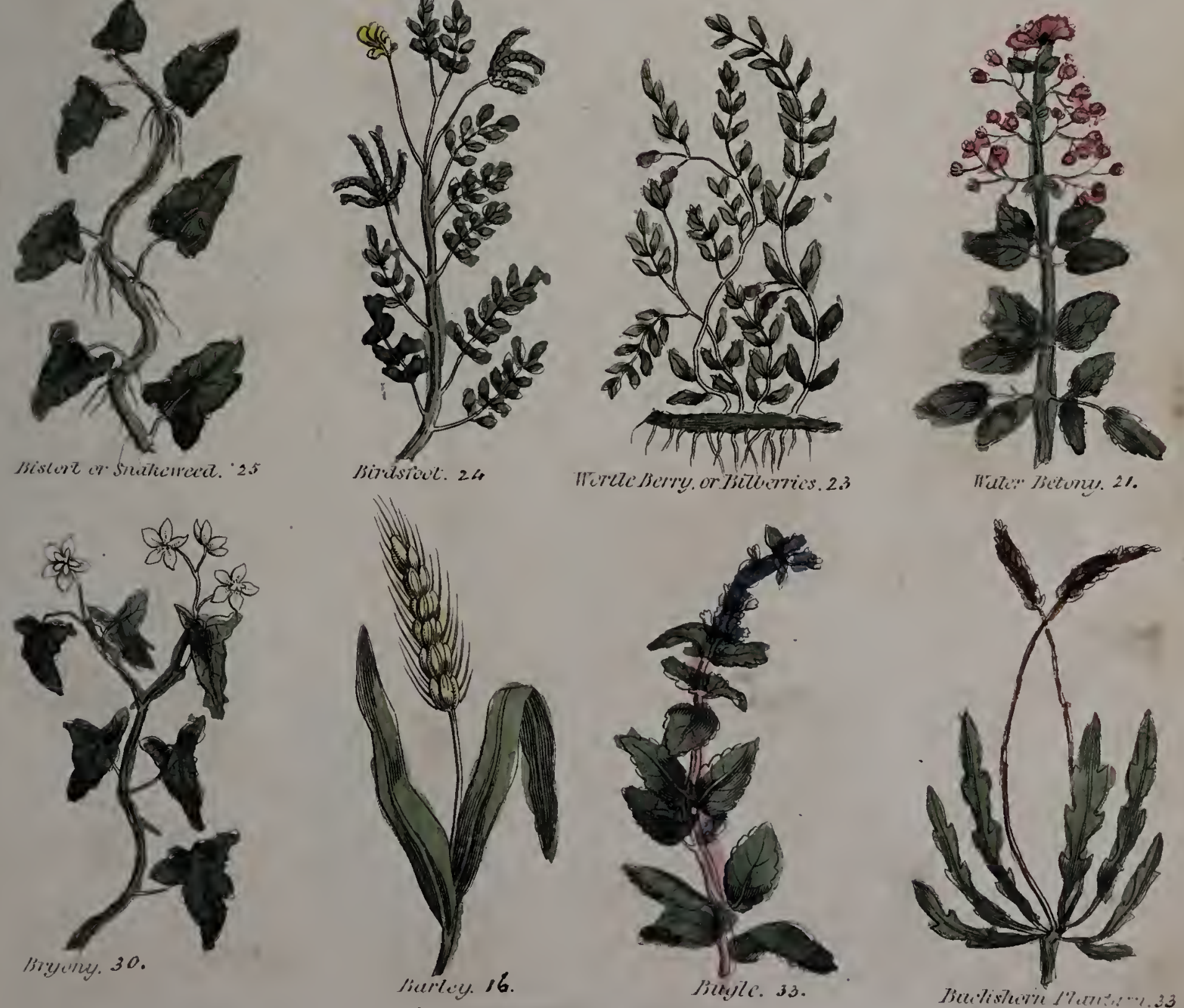


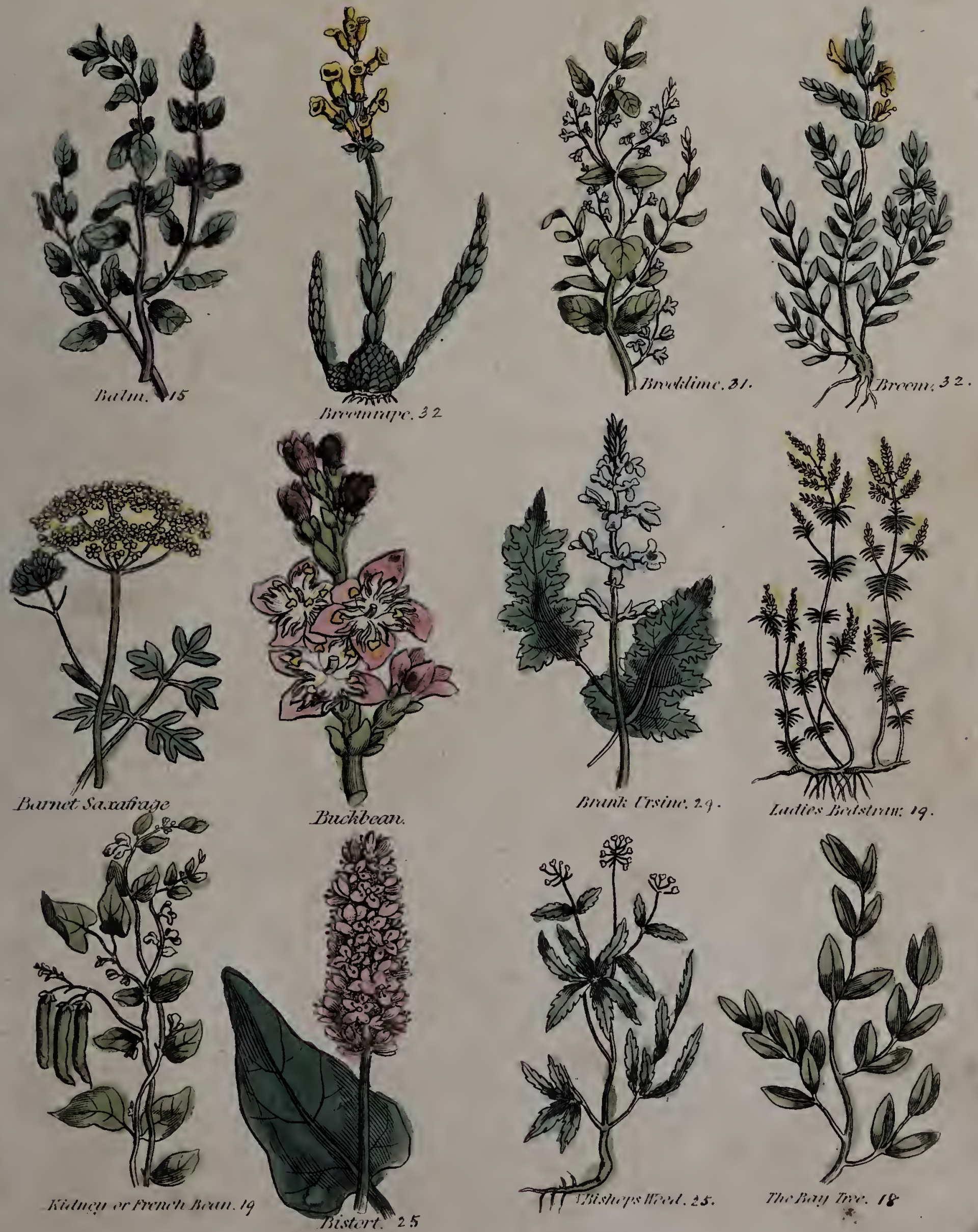



$$
\begin{aligned}
& \$ \\
& -
\end{aligned}
$$
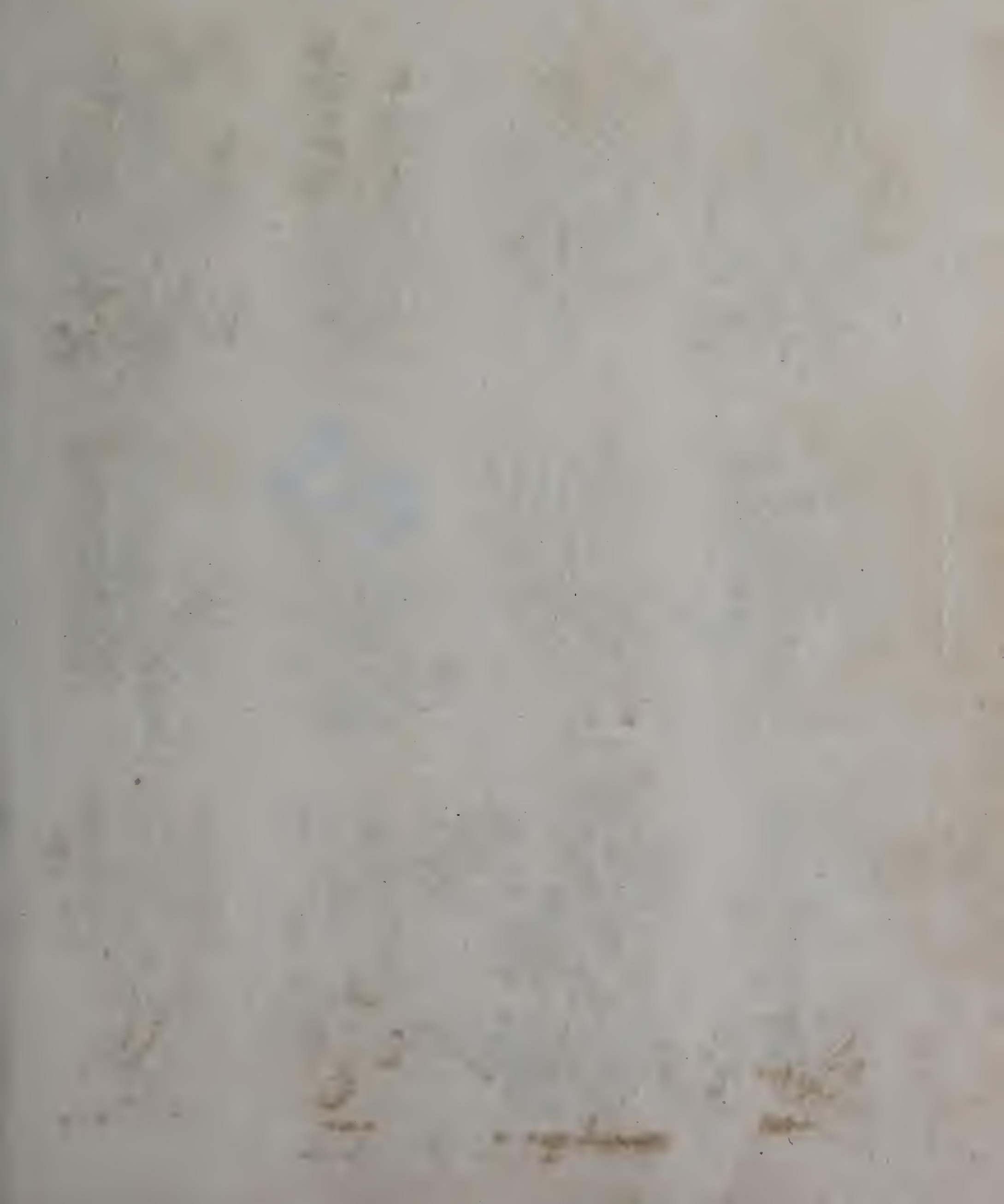

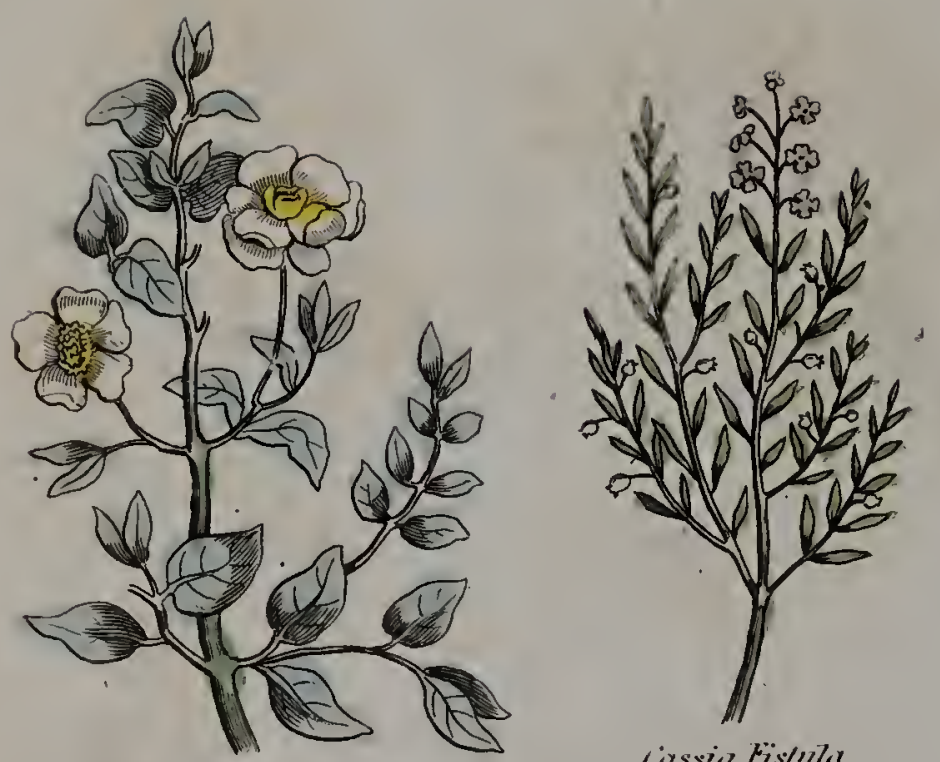

commonfimate cöstus.
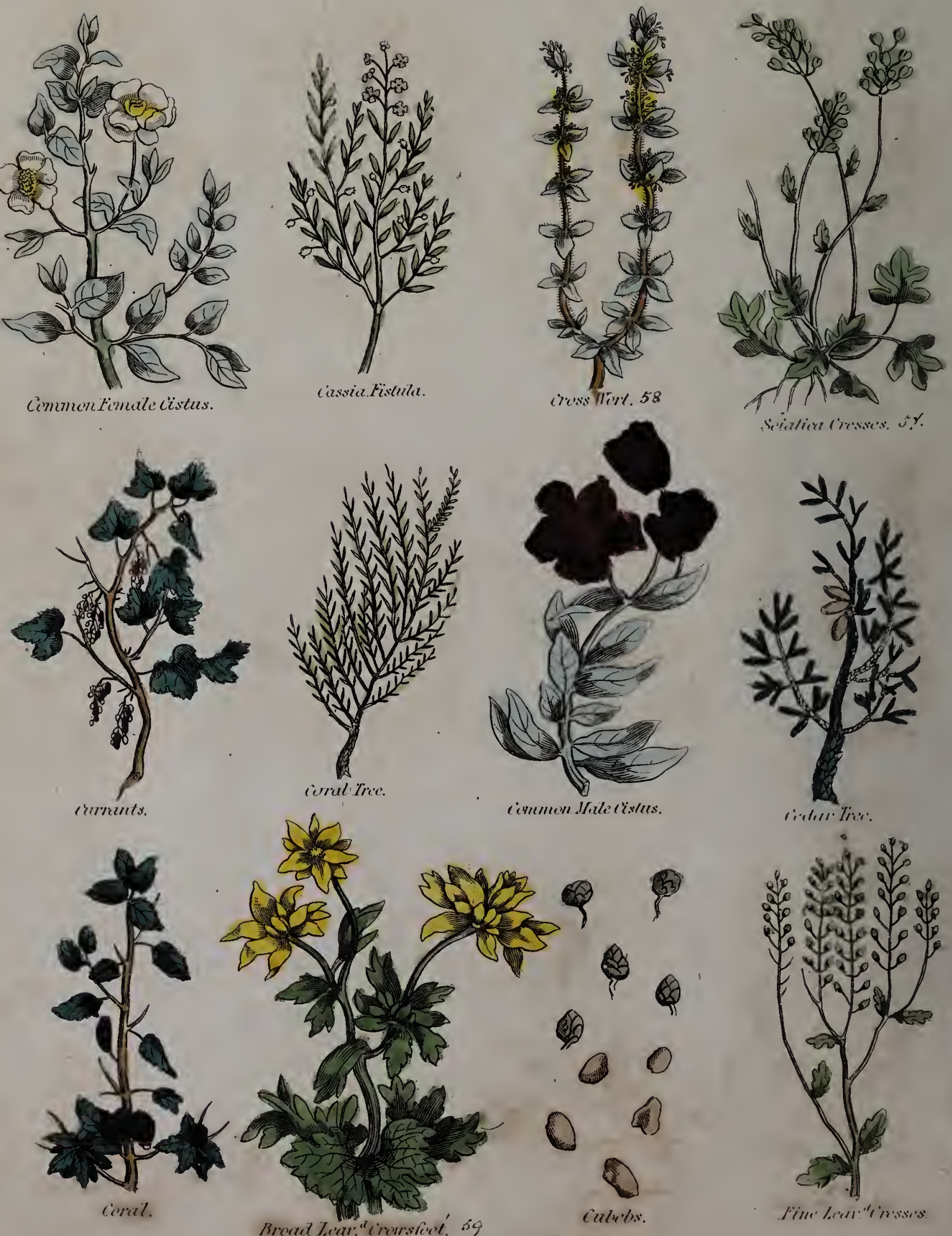

lieterr Trien.

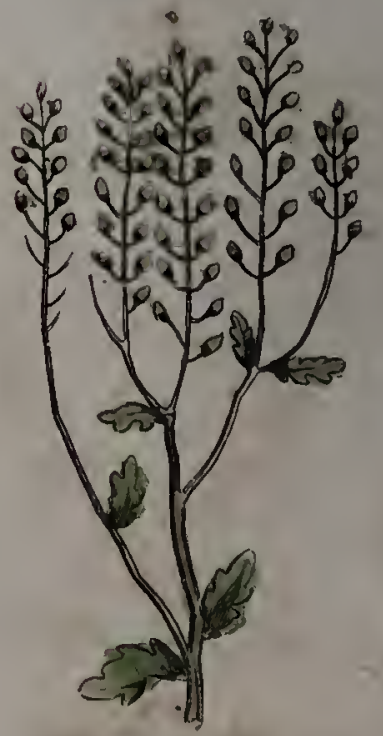

fine lons: "rirsises. 


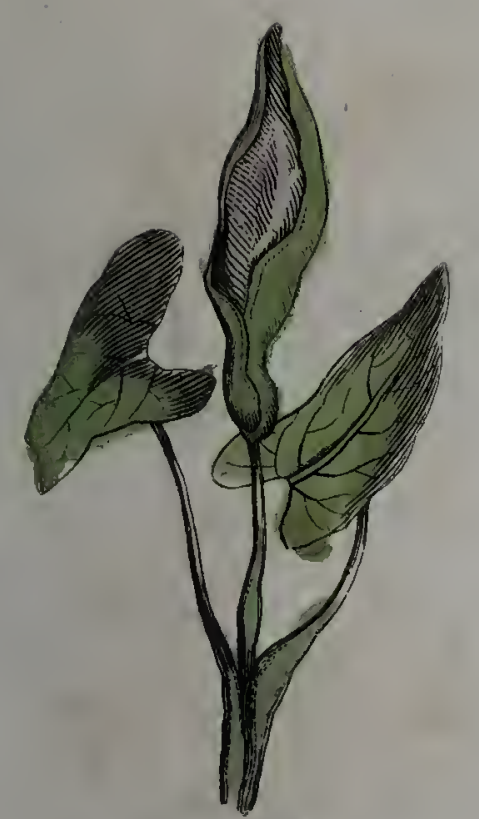

incionlint. 59 .

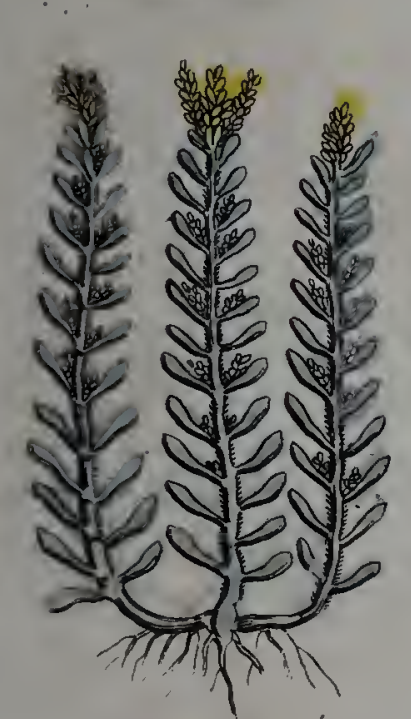

cinlweret. 506

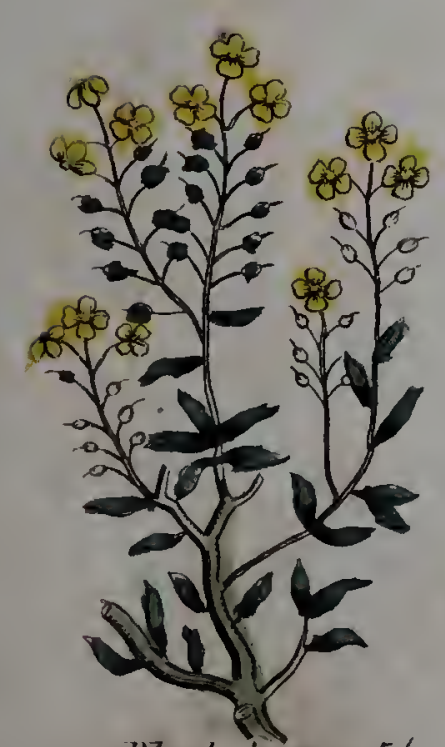

M7arli ciressics. $5 \%$
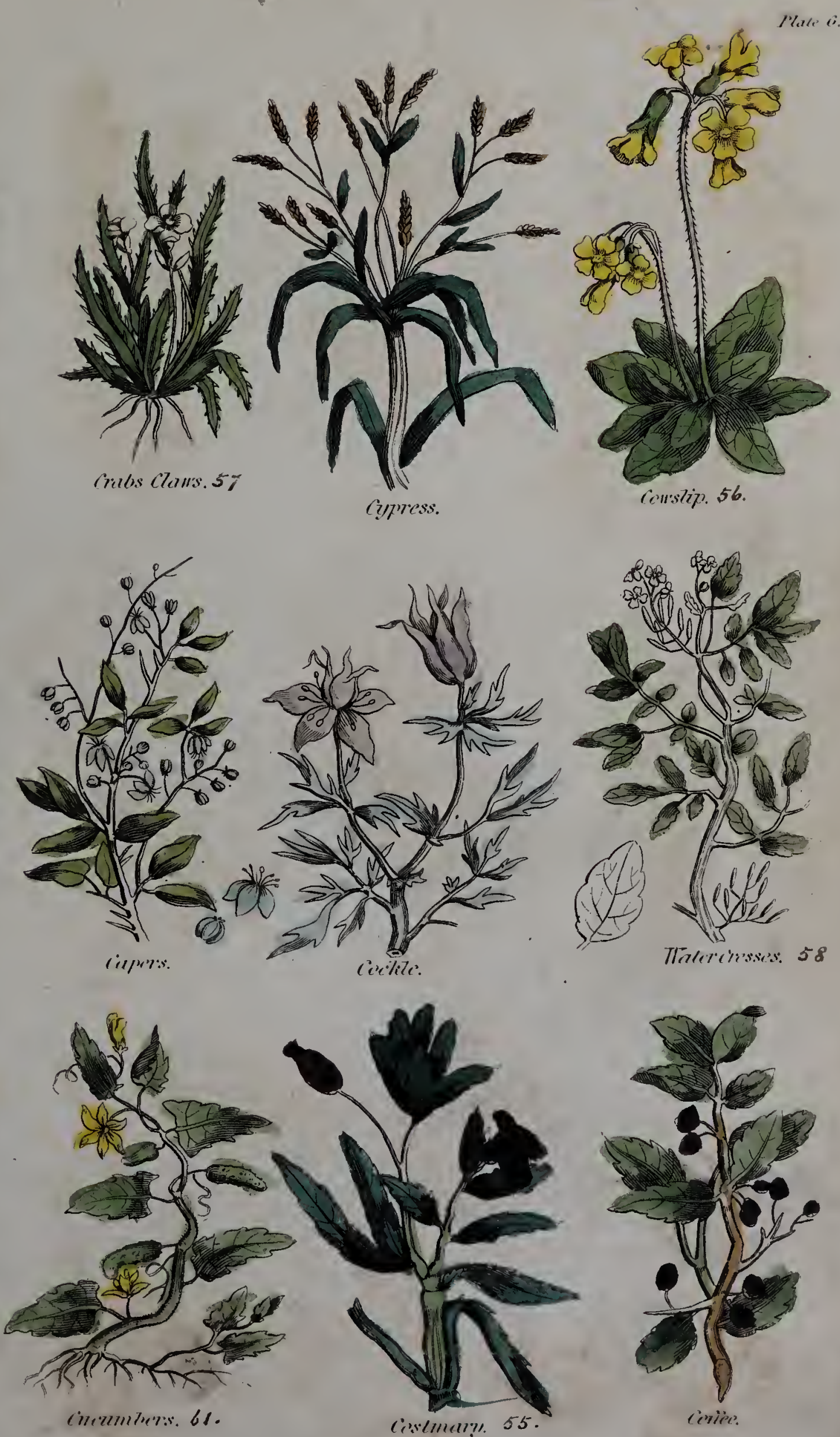
$=$

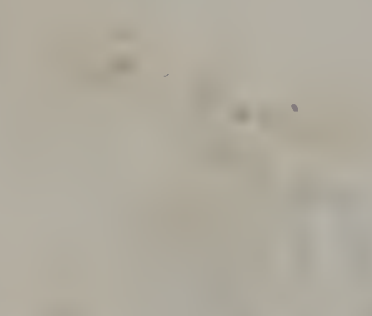

c.

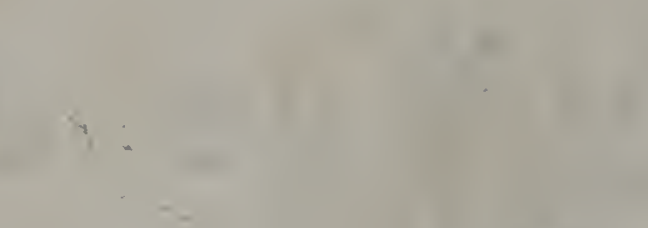

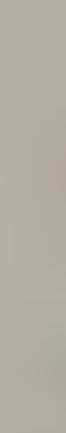

$$
\text { 25: }
$$

1

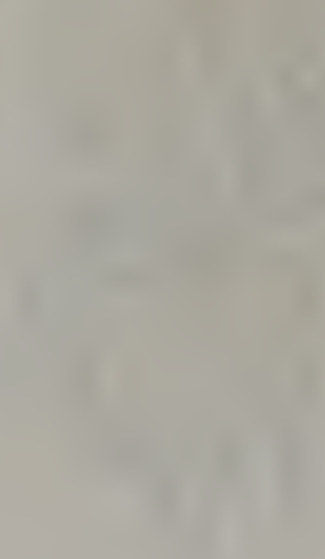

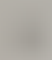
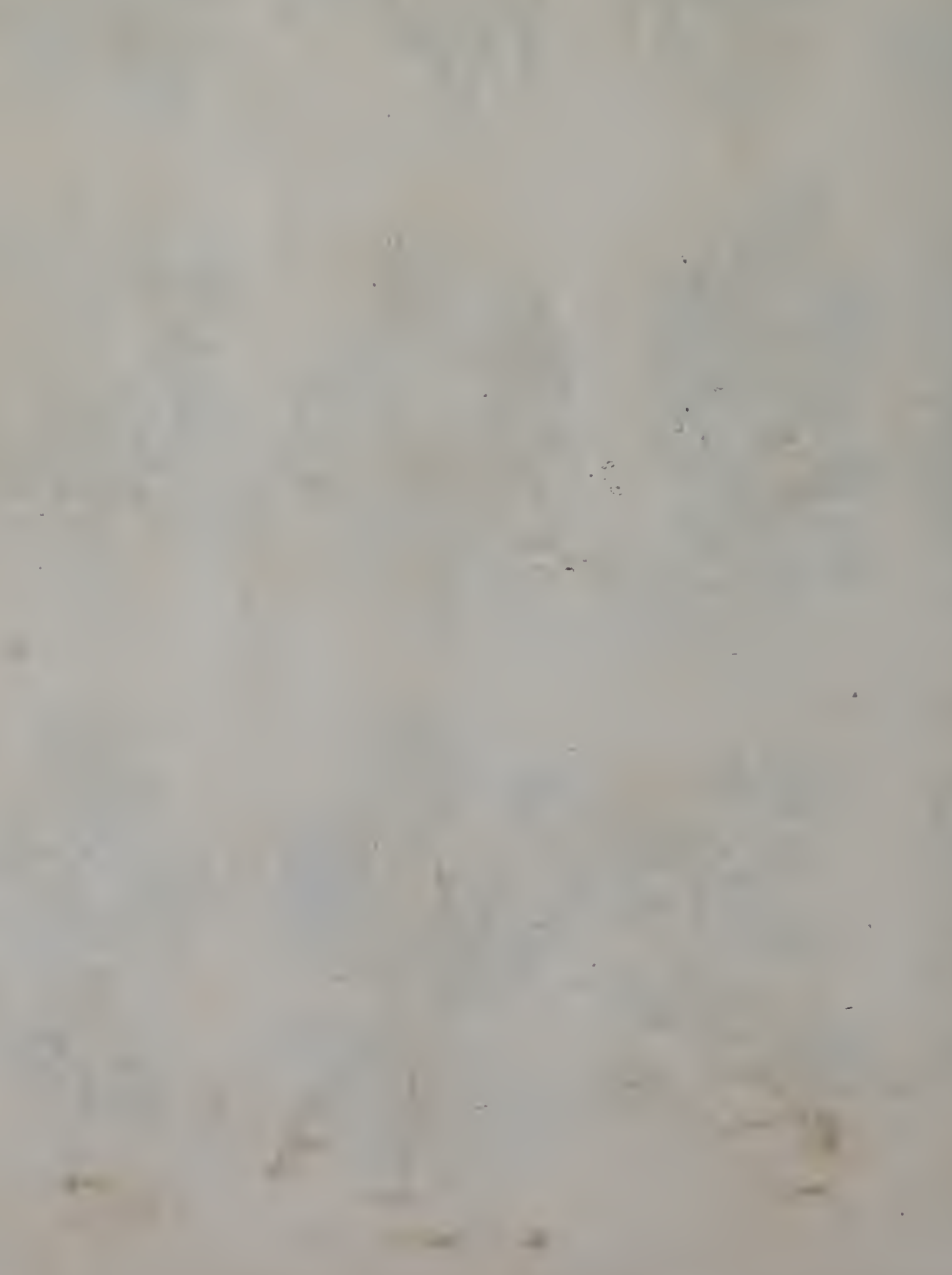

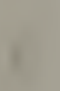

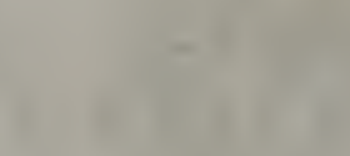
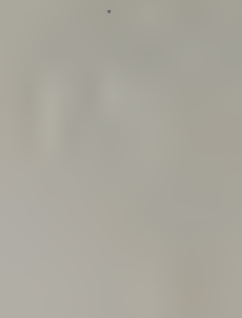

$$
\text { - }
$$

1
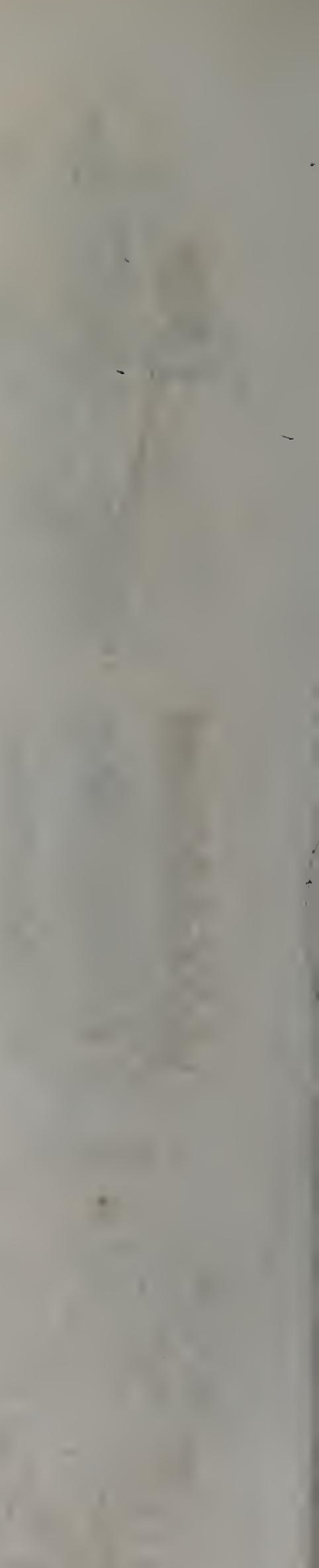

(n)

$x^{2 x}$
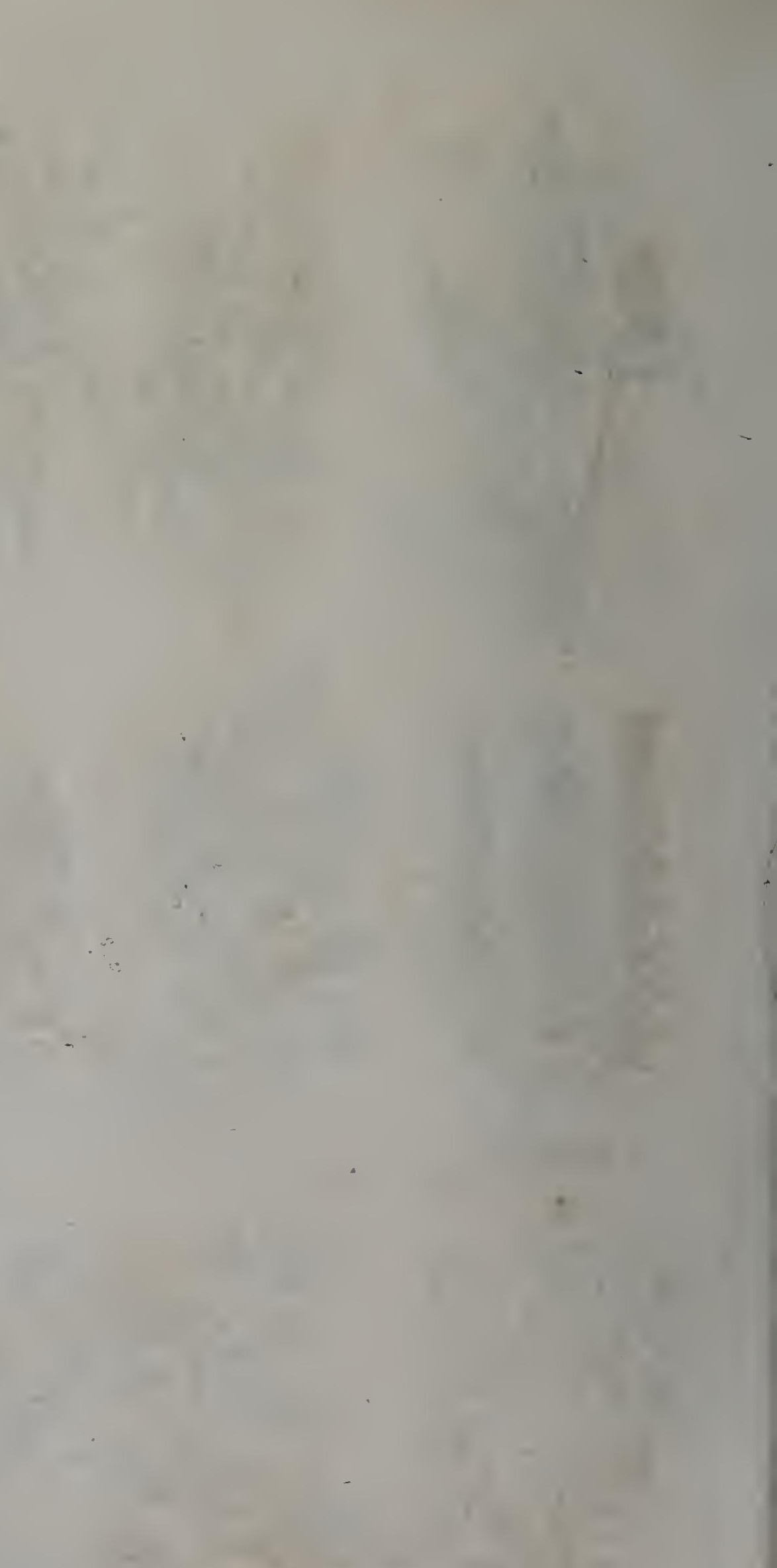

$\sqrt{2}+x^{2}$

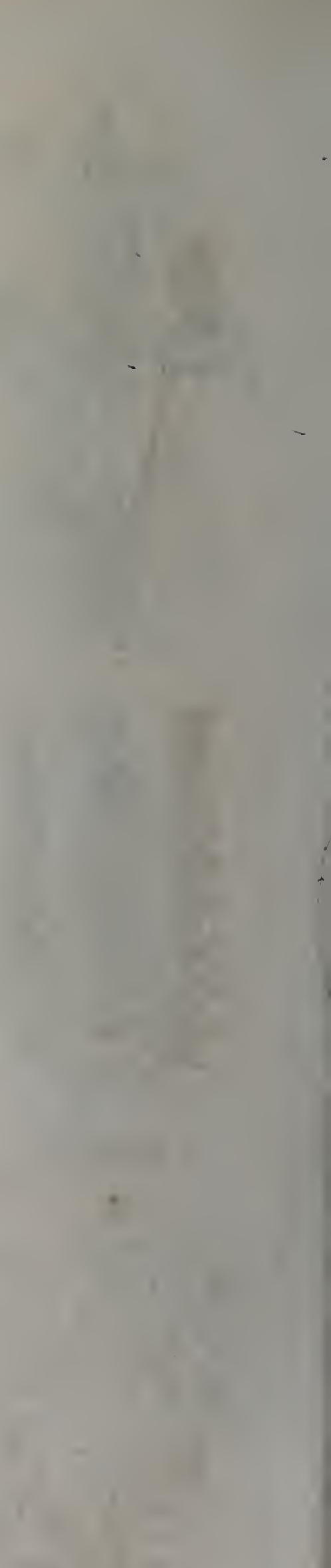

xas

$-7 \quad+6$ 


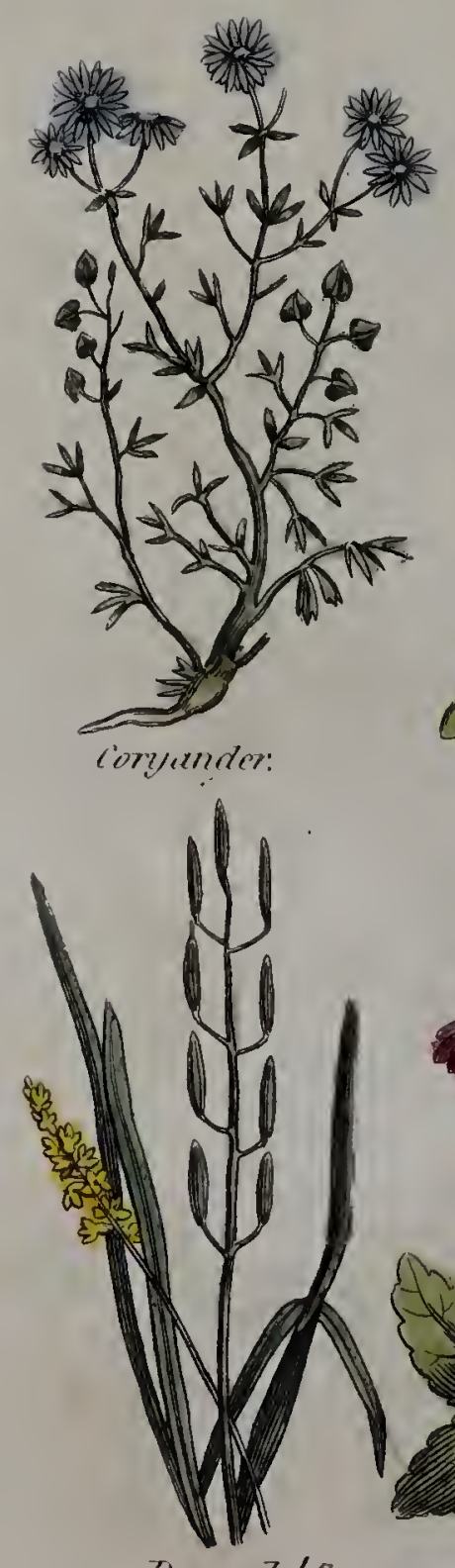

Damez.62

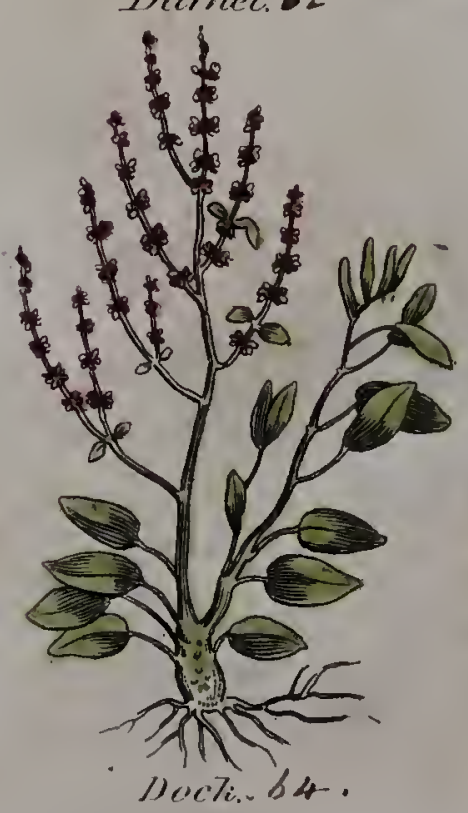

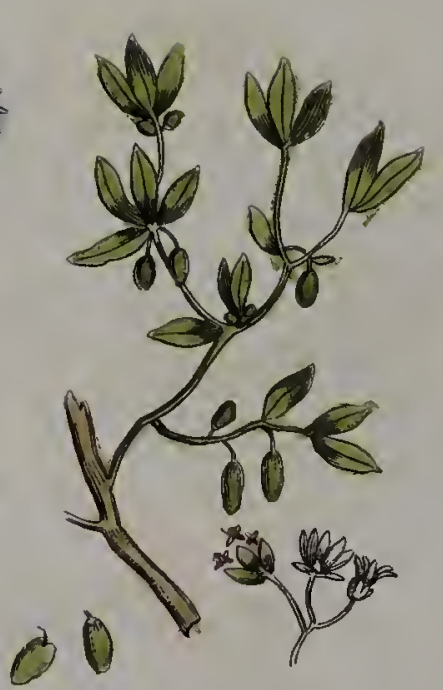

cormal iree.

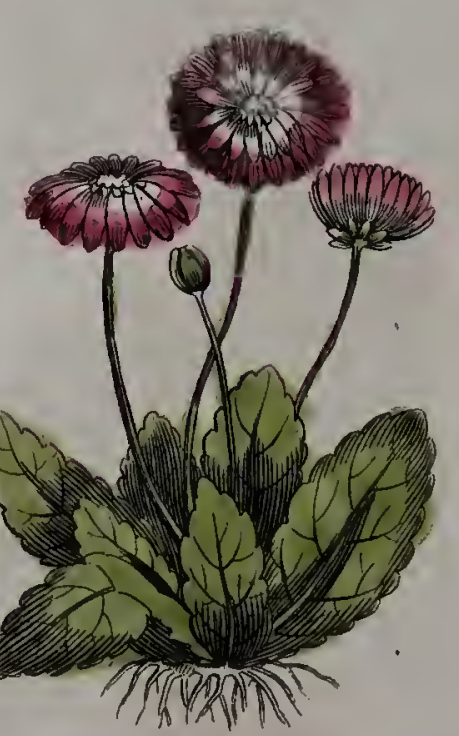

Daiscy. 61.

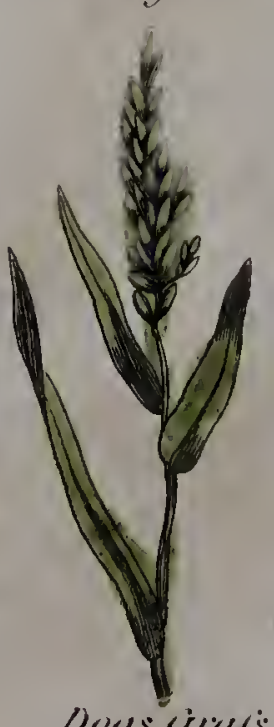

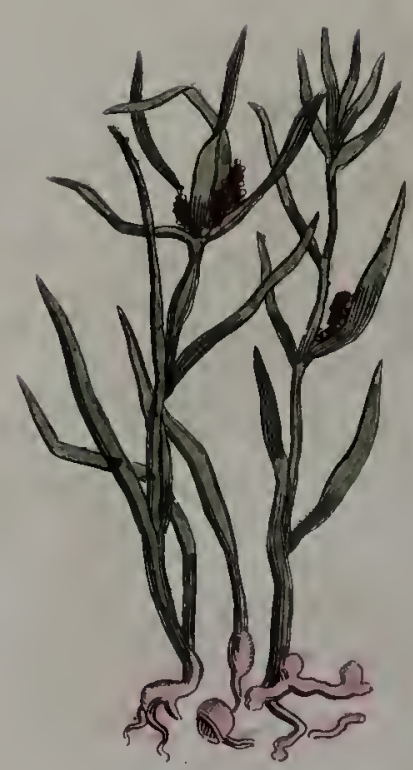

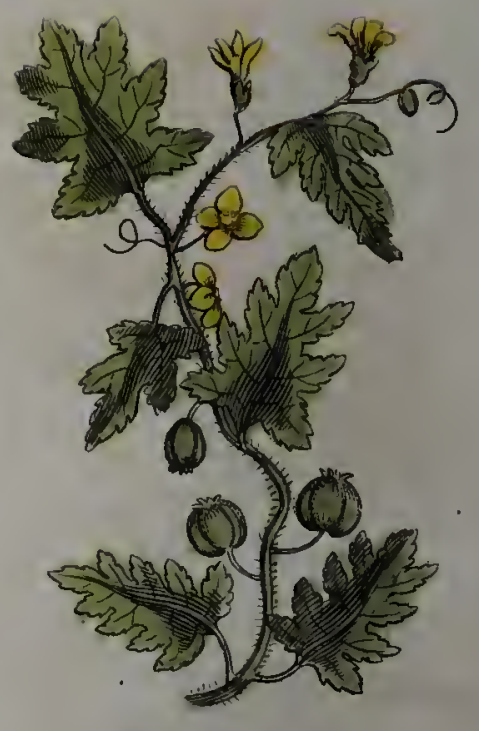

coloquintider.

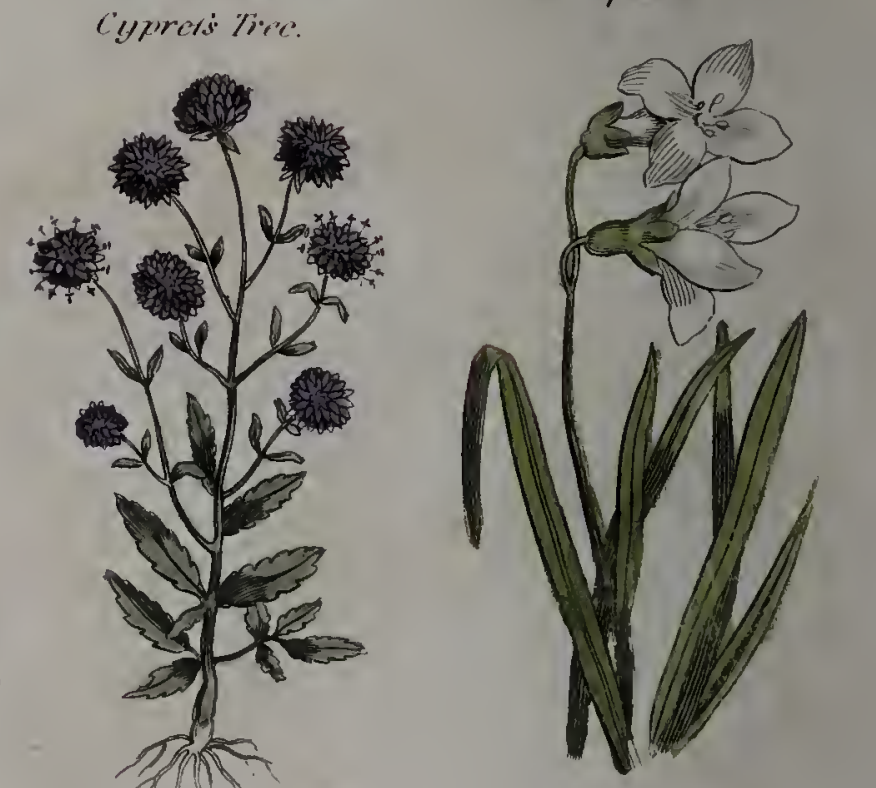

1) rite like 63.

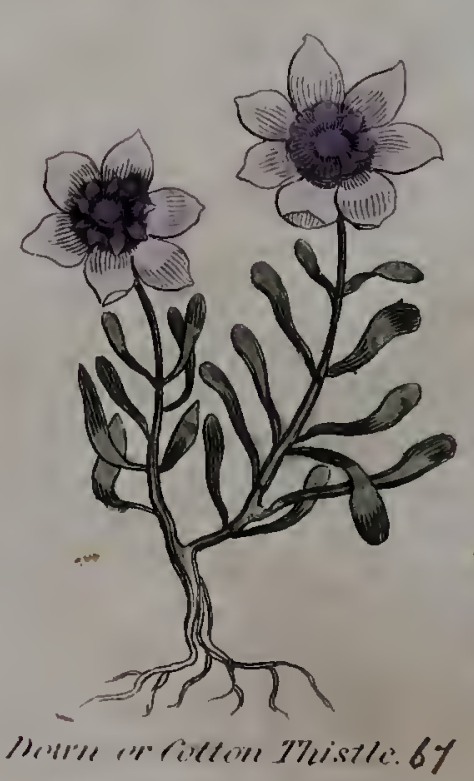

fileise Nestentill

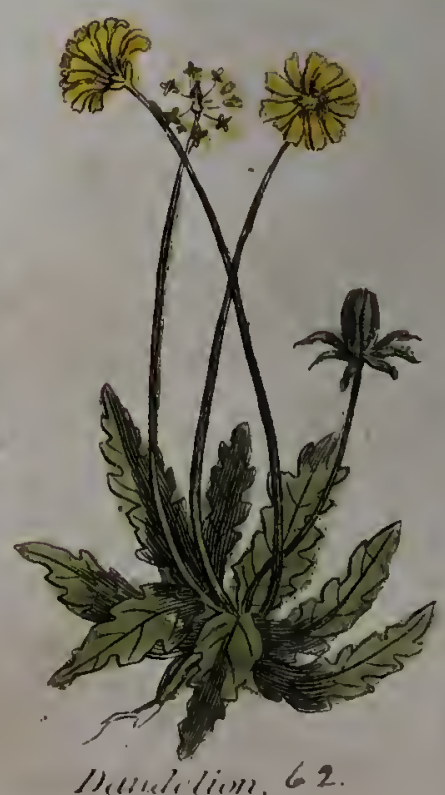

rlee 


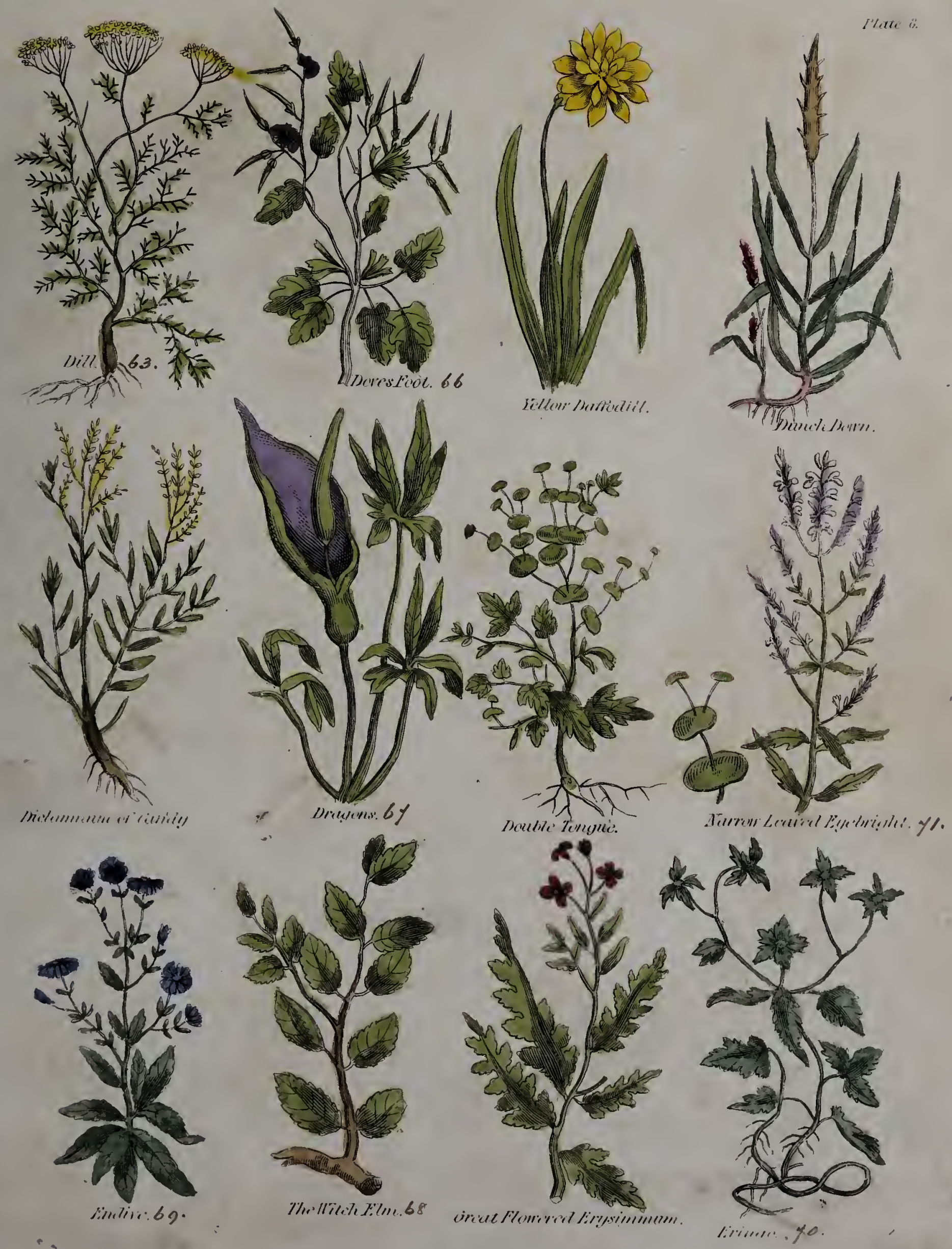





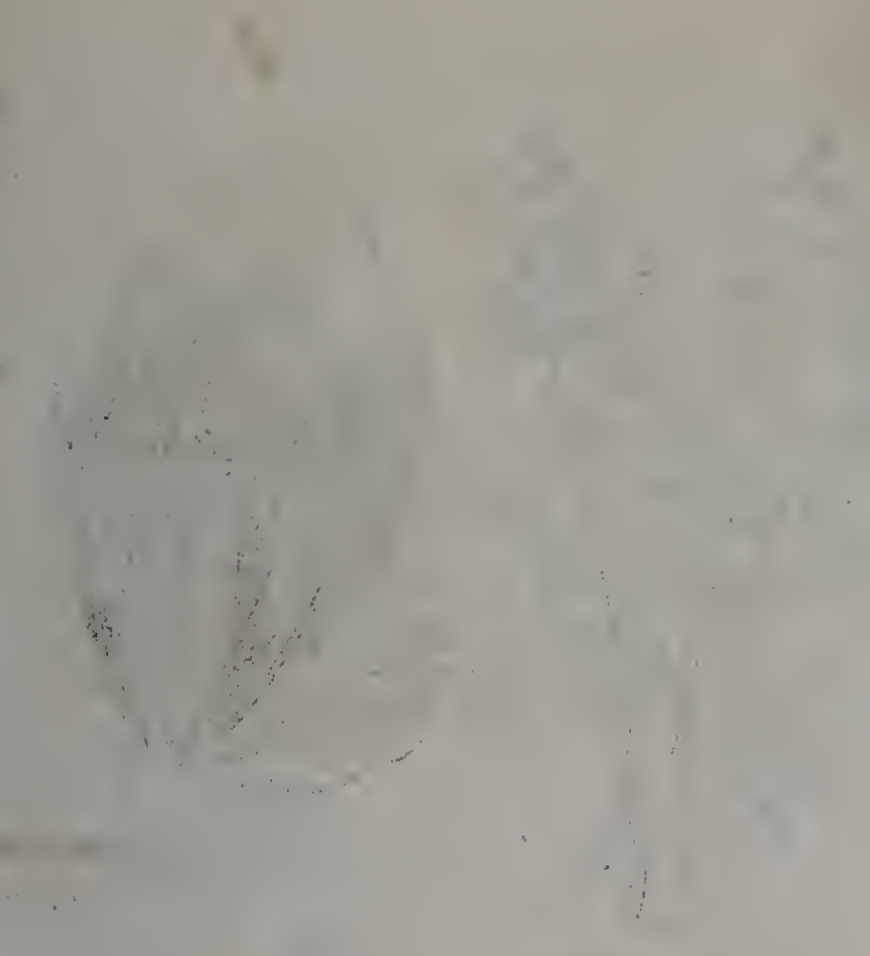



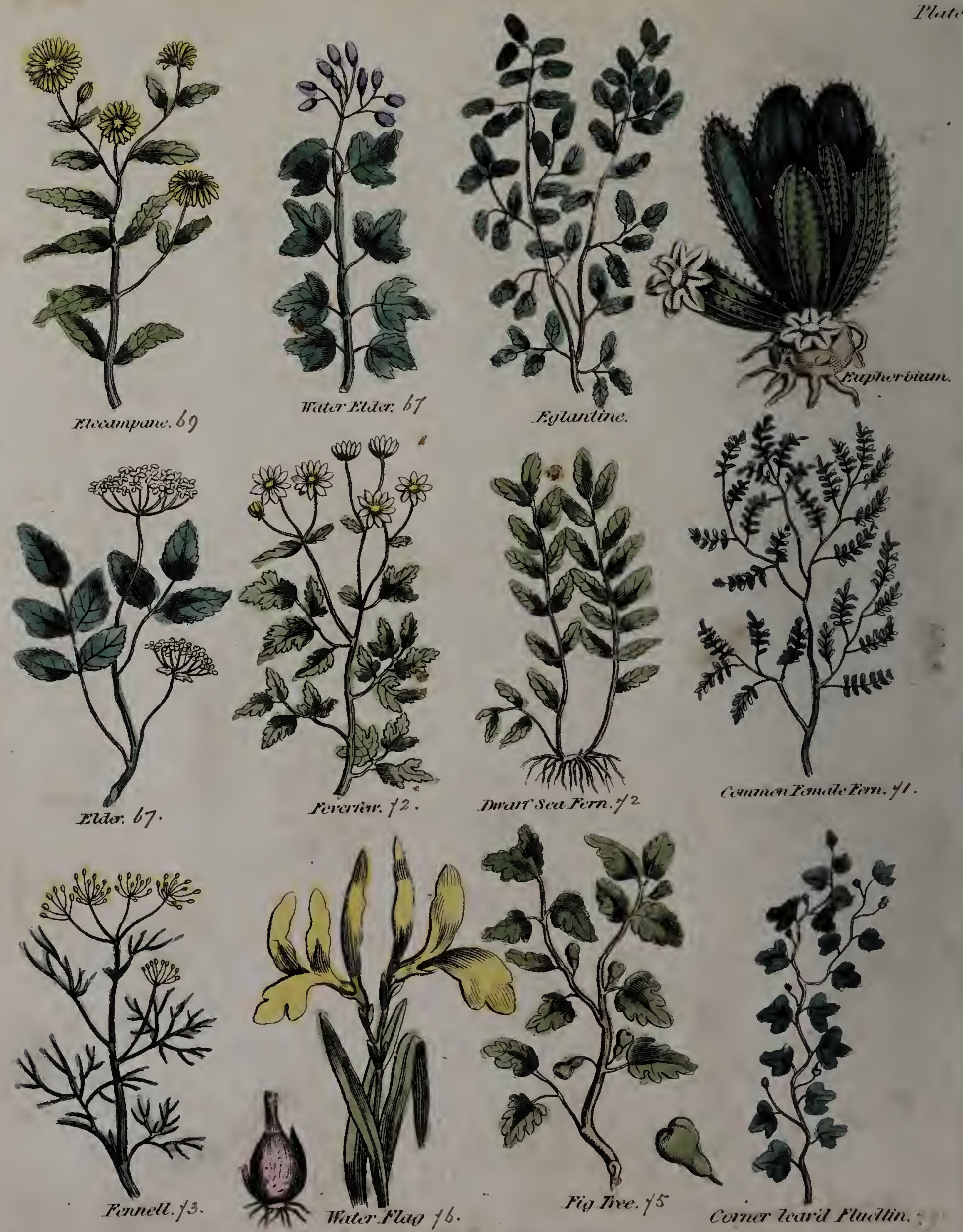

coming leavid fluethin. : 
Mlerent:

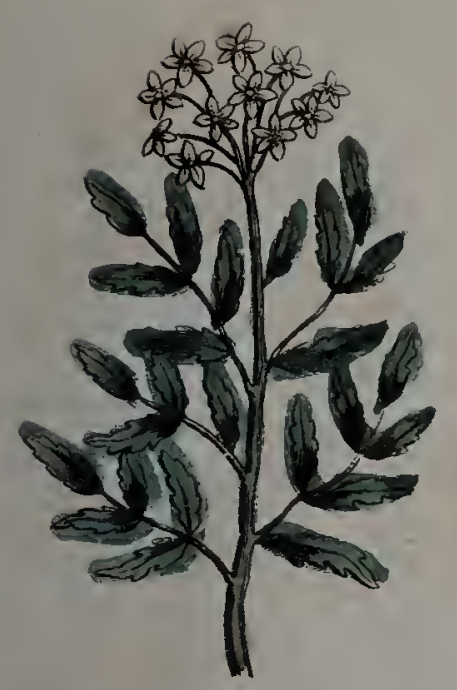

Finizinitule. 15

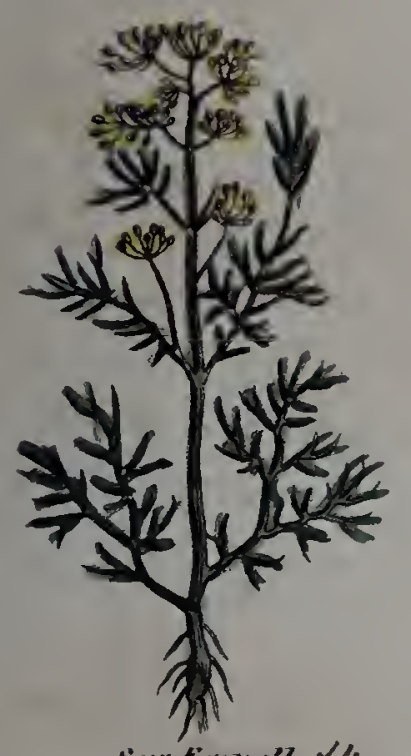

sivrfinuled 14 .

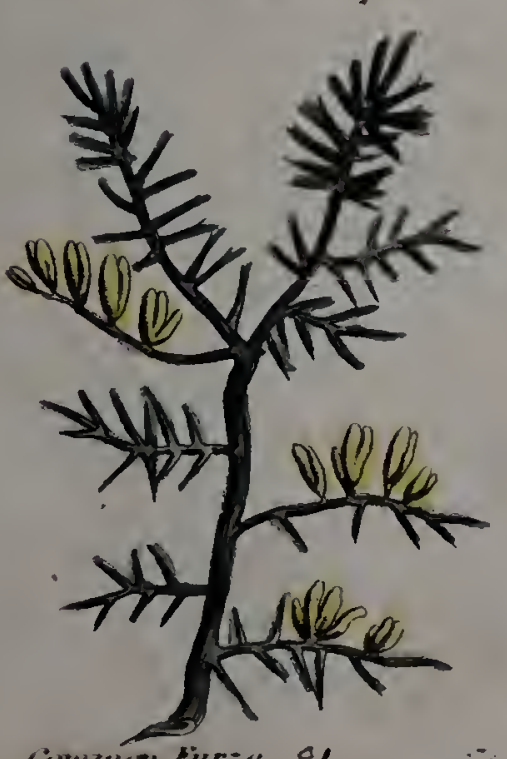

㢣 $y^{2}$
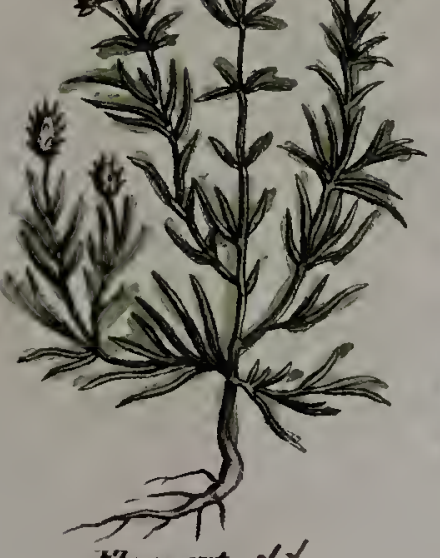

Fenurert. $Y \%$

19

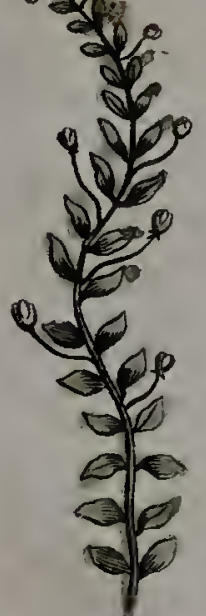

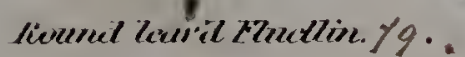

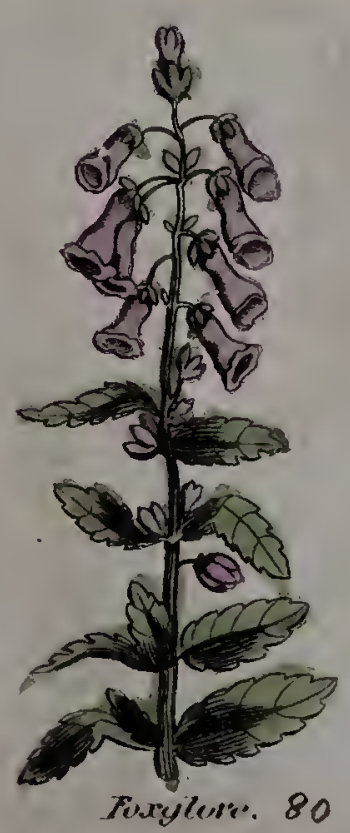

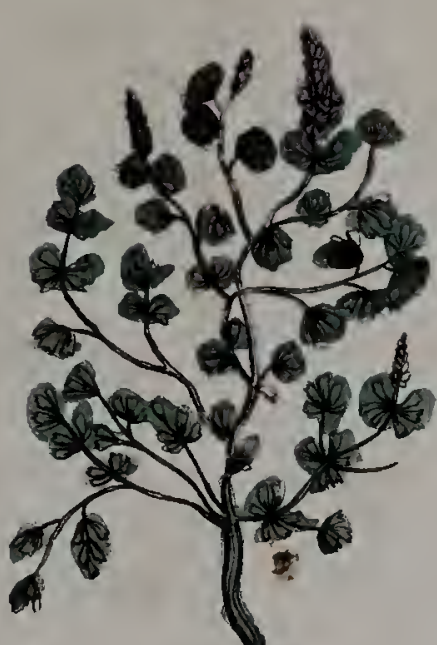

Finntitery. 80.

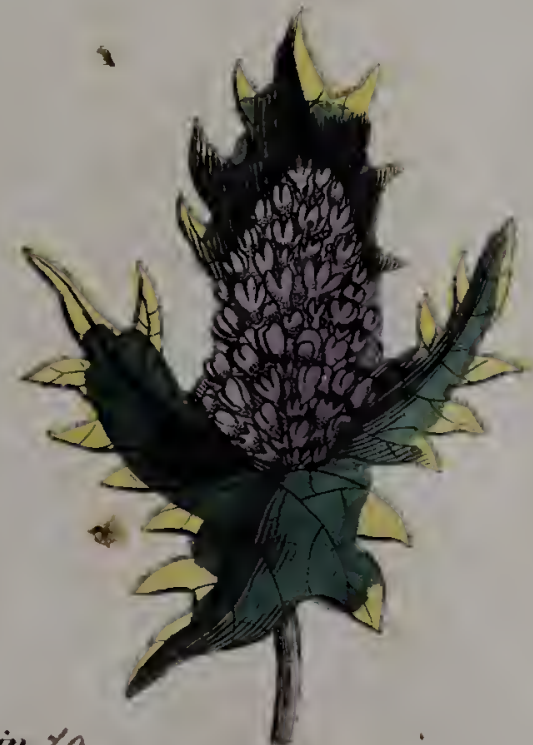

Tringo. yo.

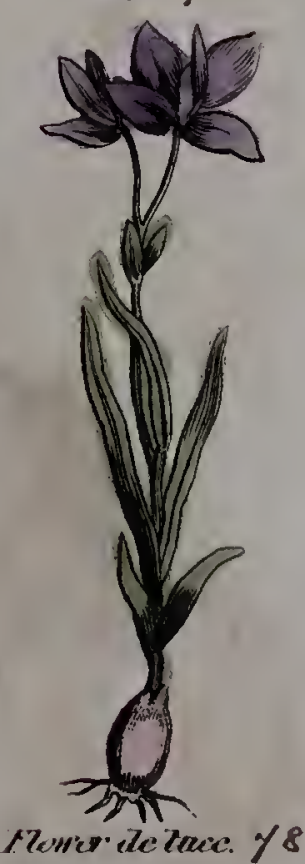

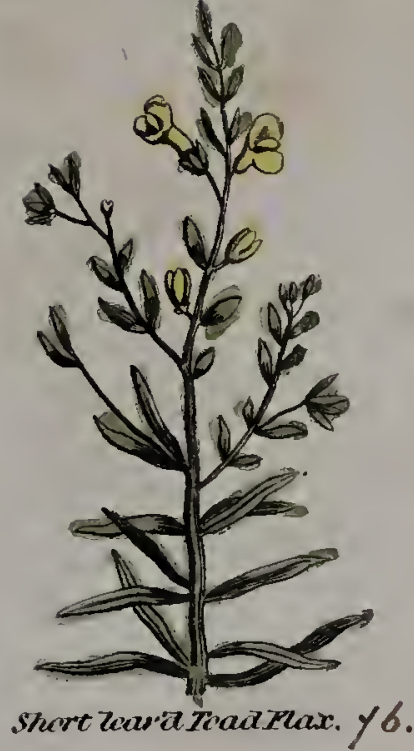

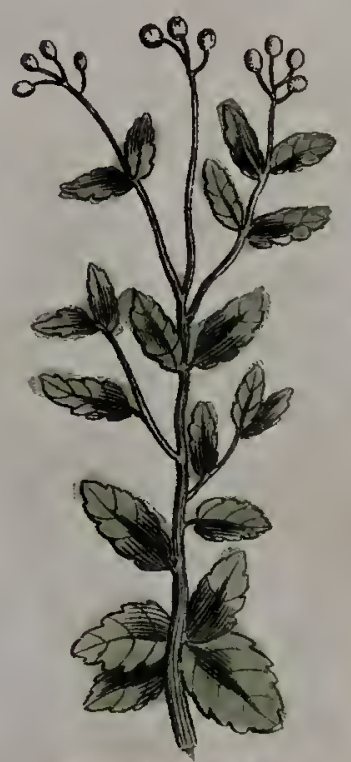

cestessuph figurere. $y 4$.

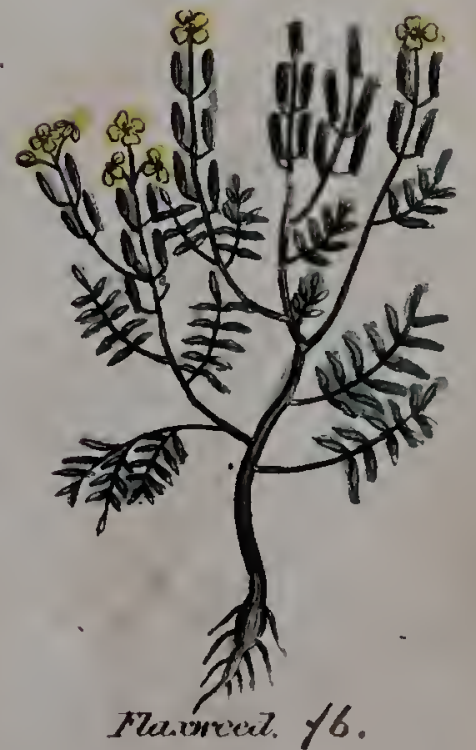





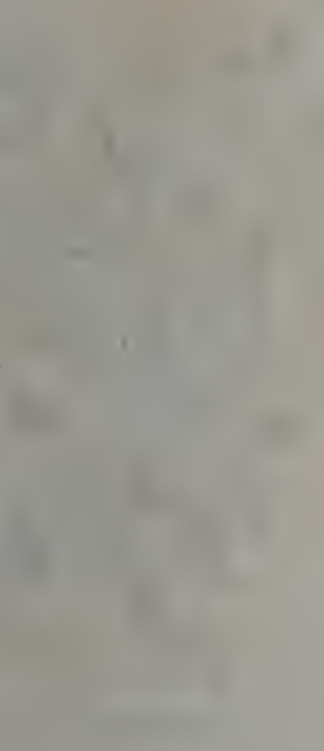

3

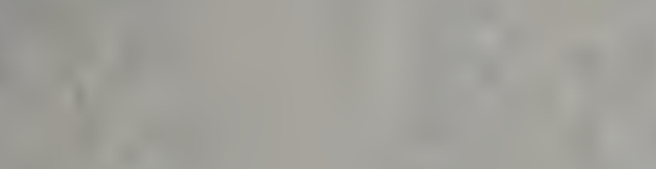

(1)

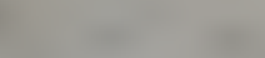

4.
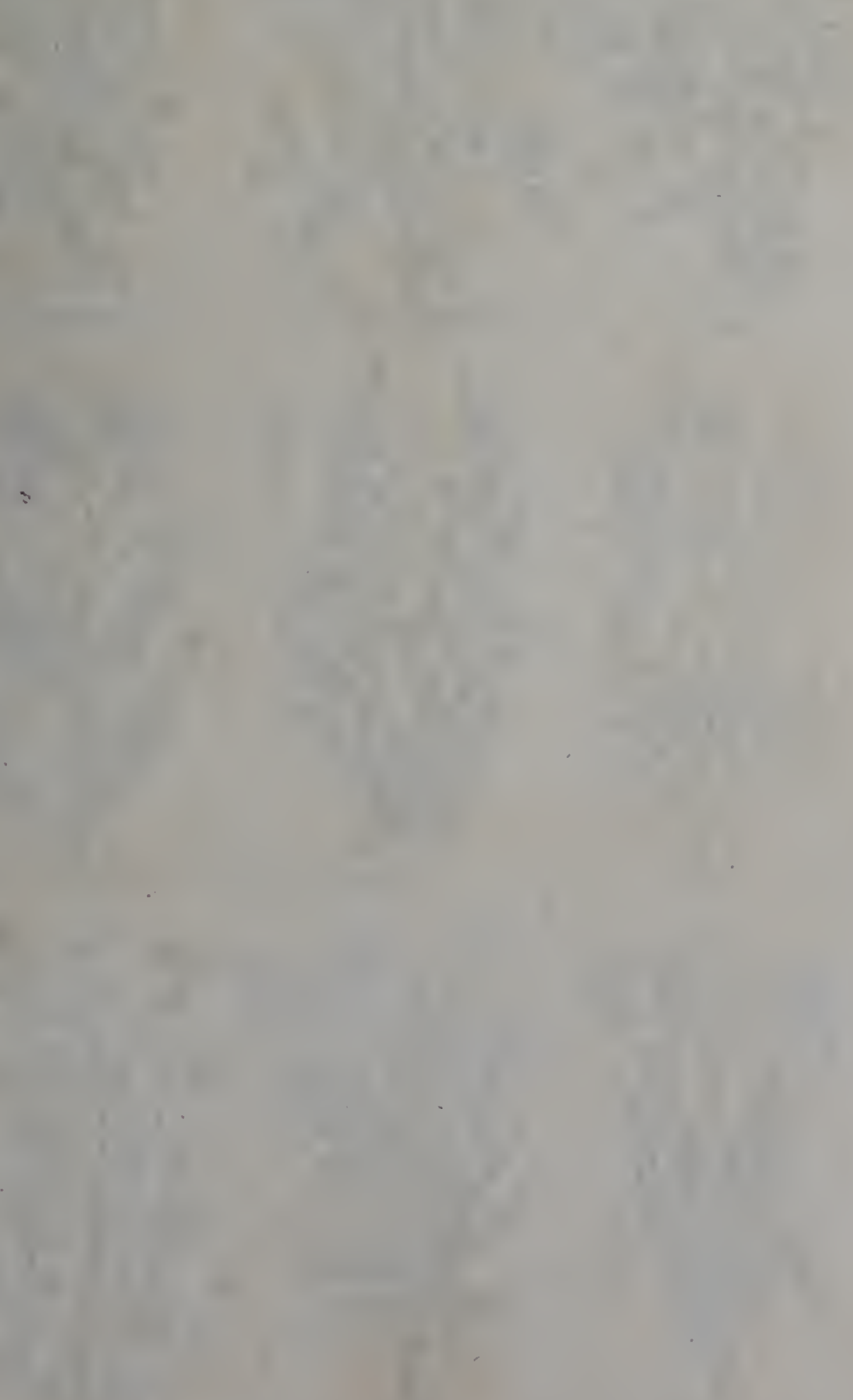

$x$
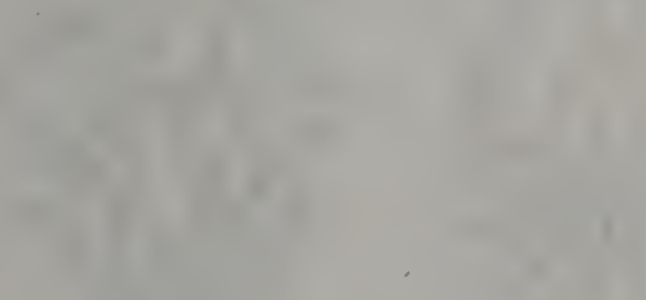

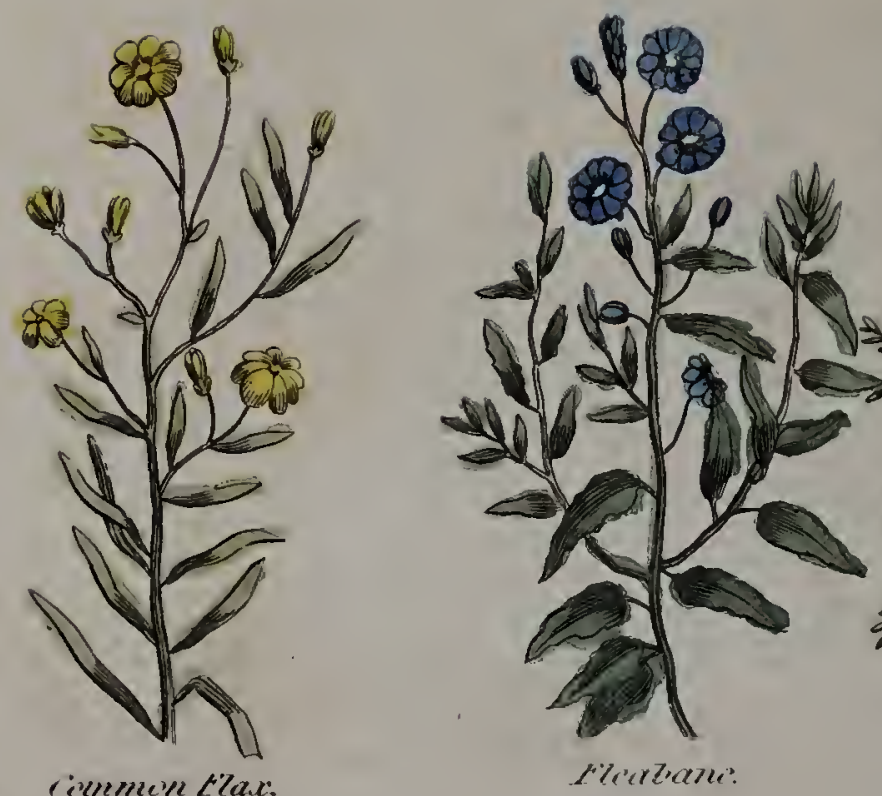

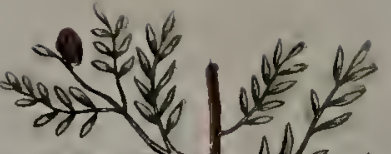

H"tet

$$
\text { cinementrax. }
$$
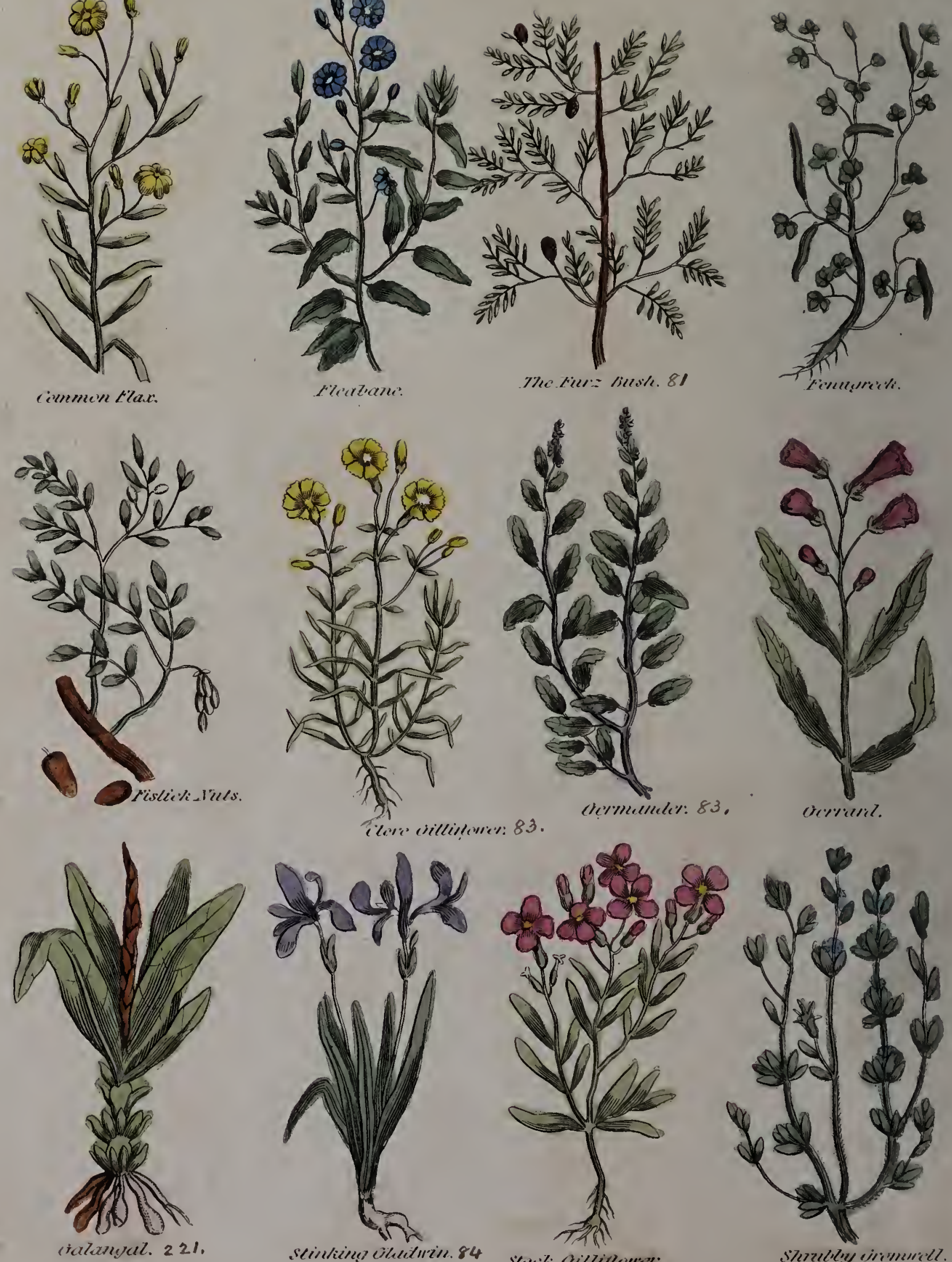

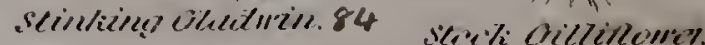
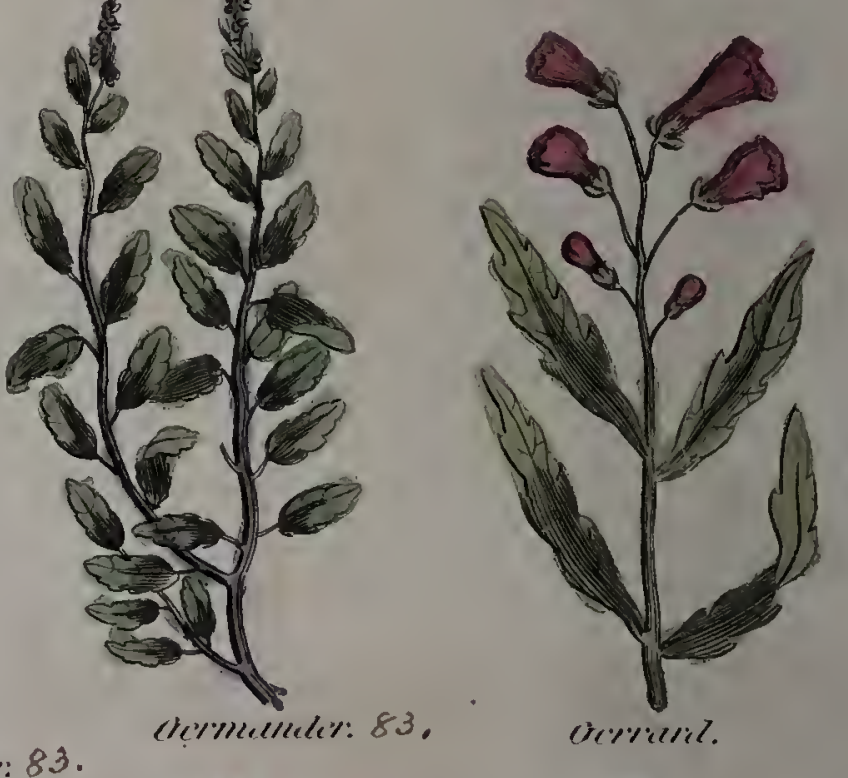


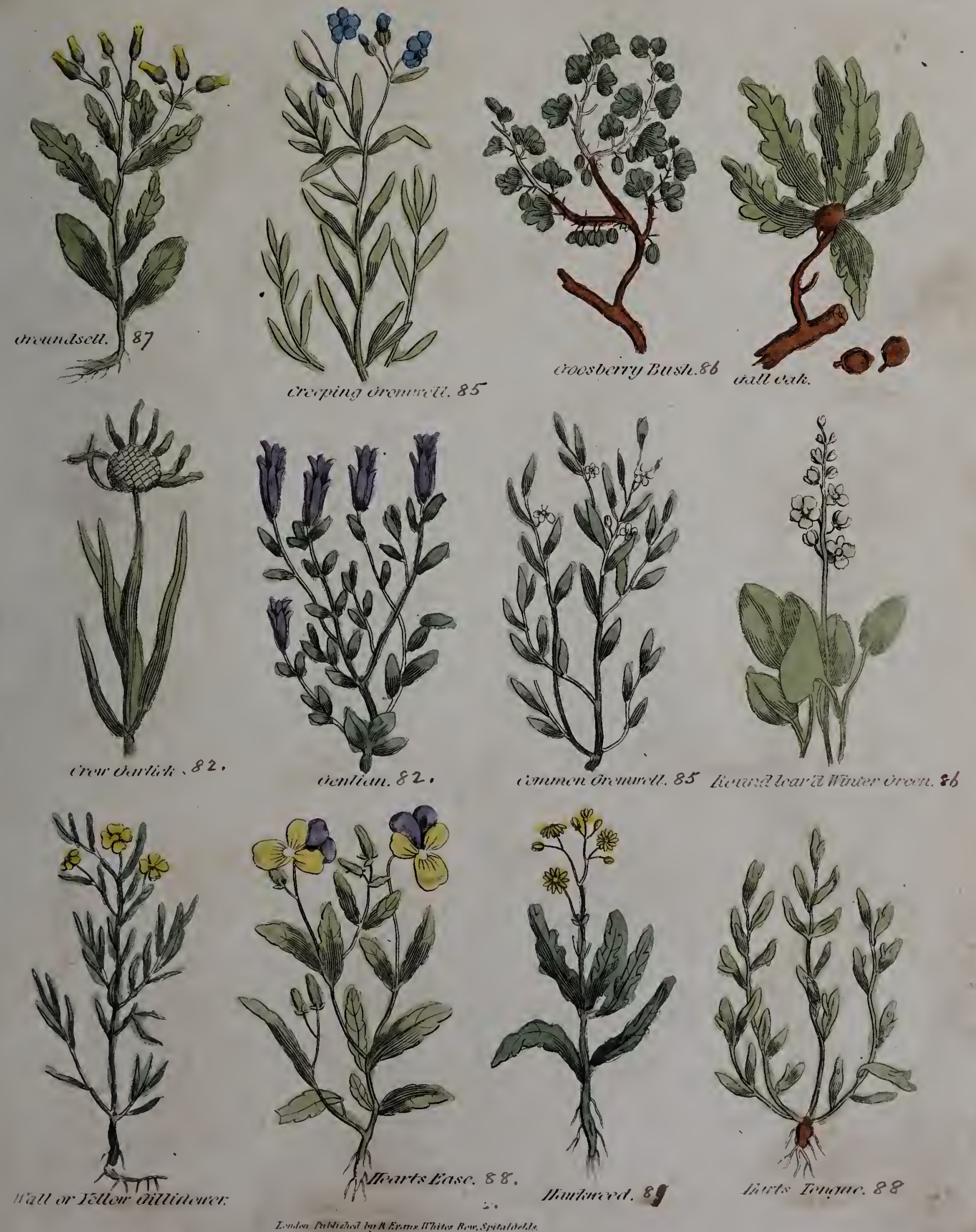




Lutr M.
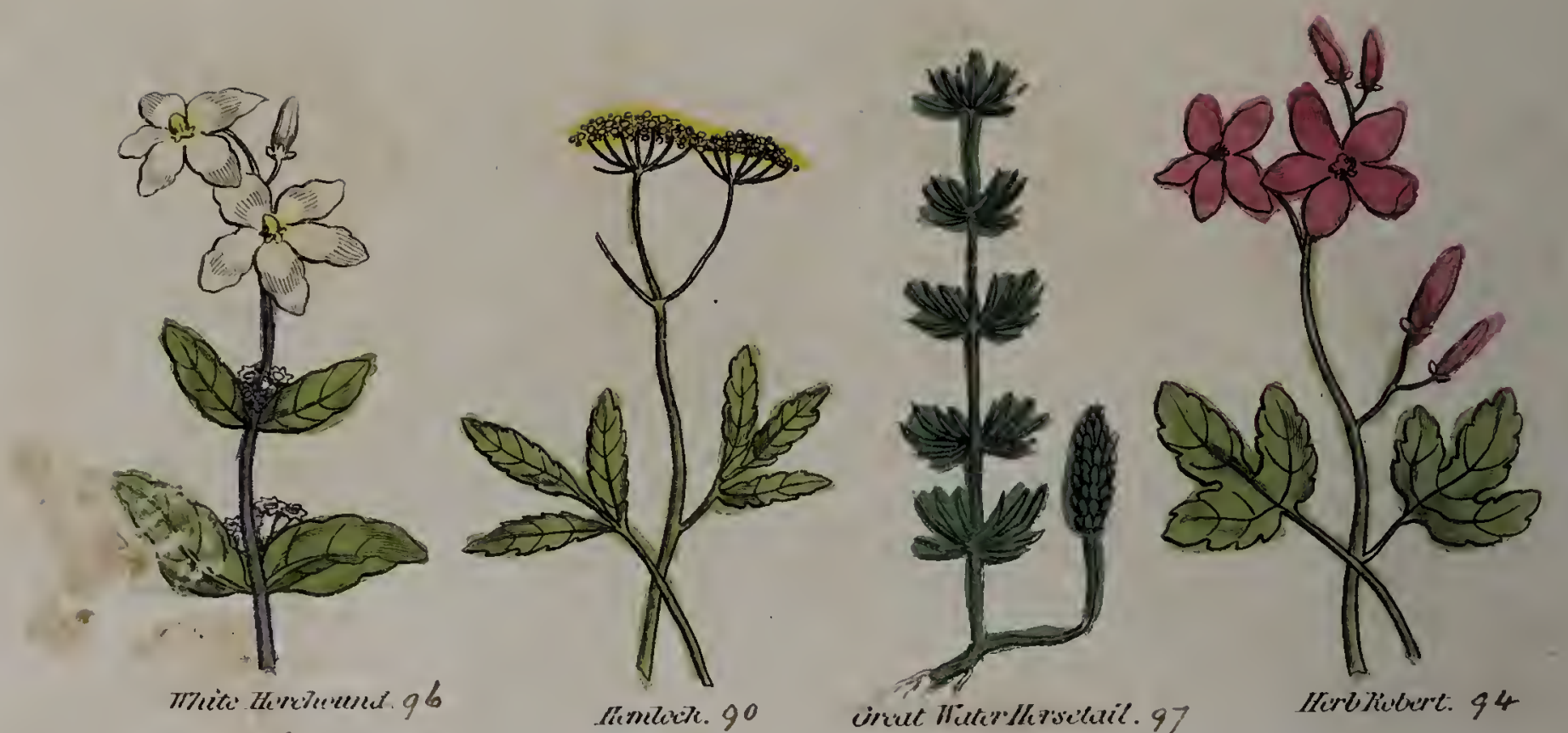

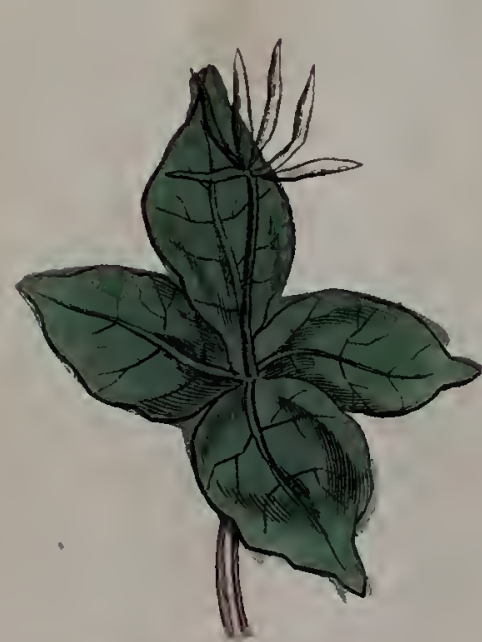

Ilorb Tructure. 94

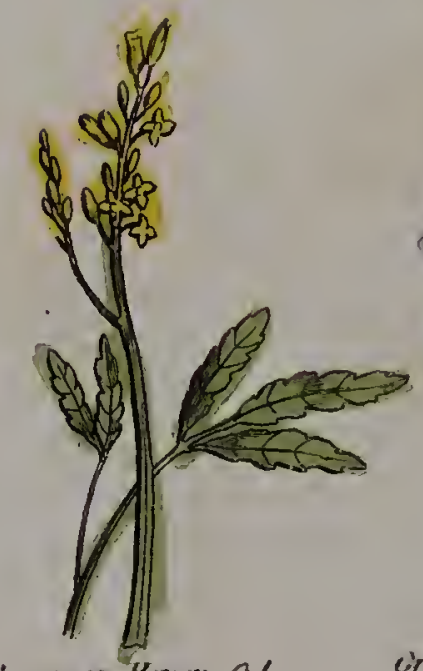

cimemare Harew. 9 '.

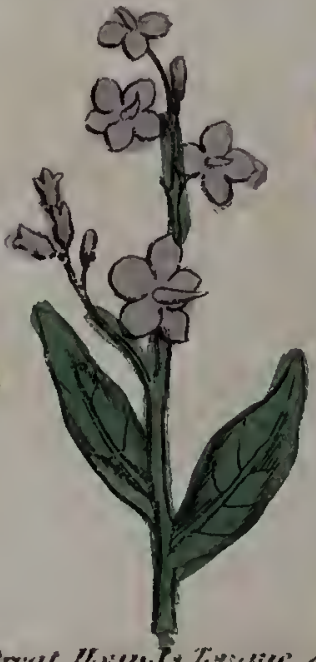

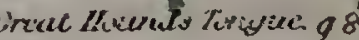

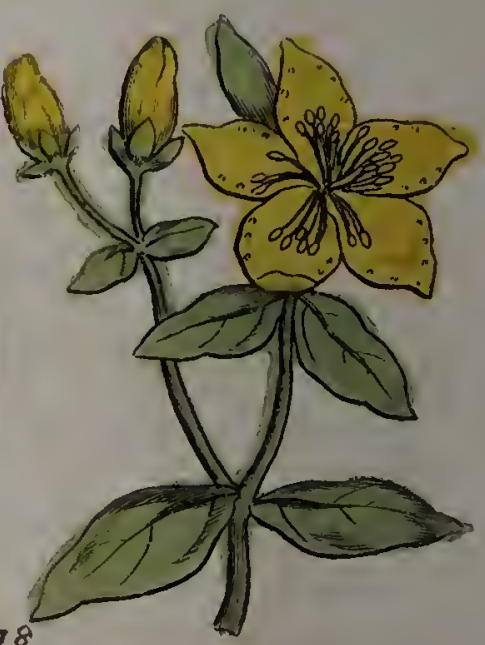

st Jolus Hüt. 99 .

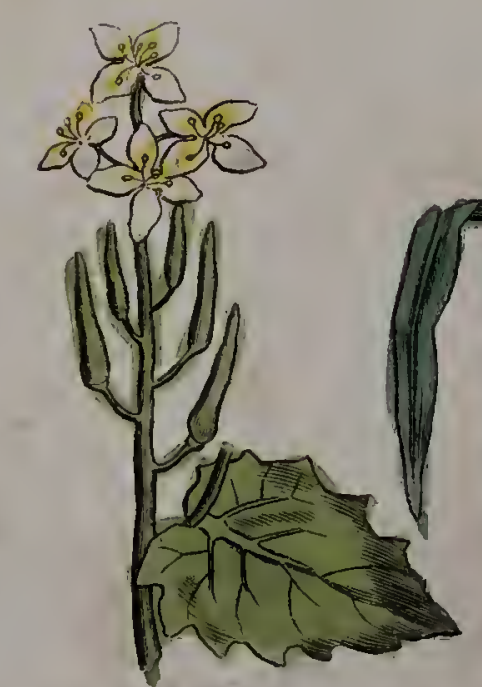

Jack by thelleriye.
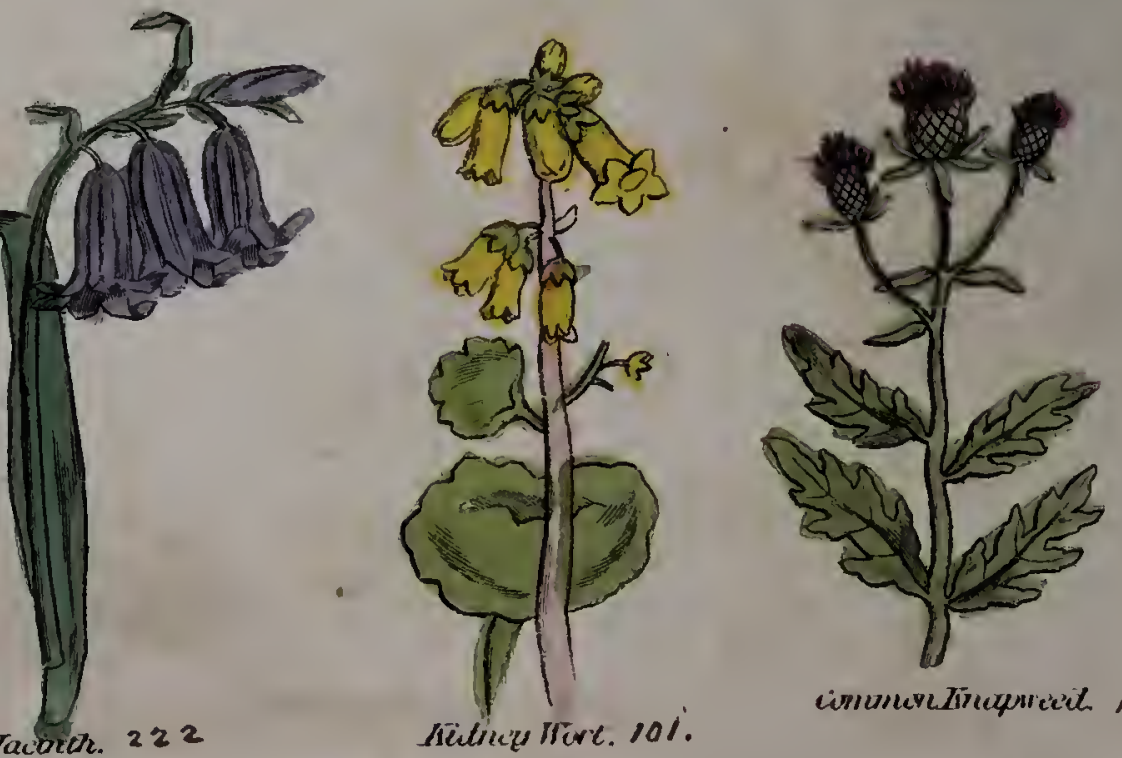

cimmanchinceypriceith 102. 
1Kate 71.

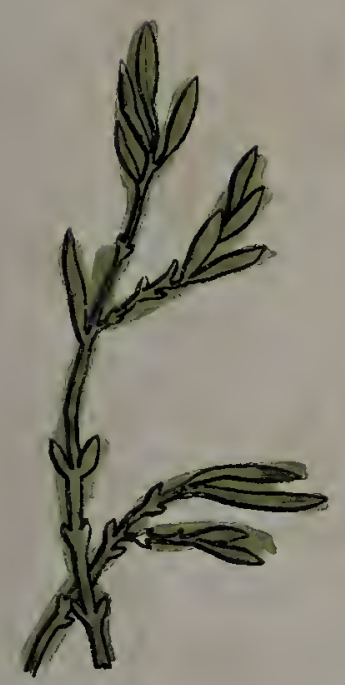

cirrmon finct Gruss. 102.

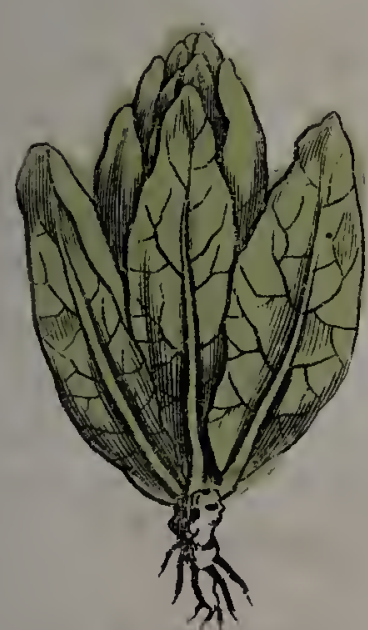

Tetuces. 104

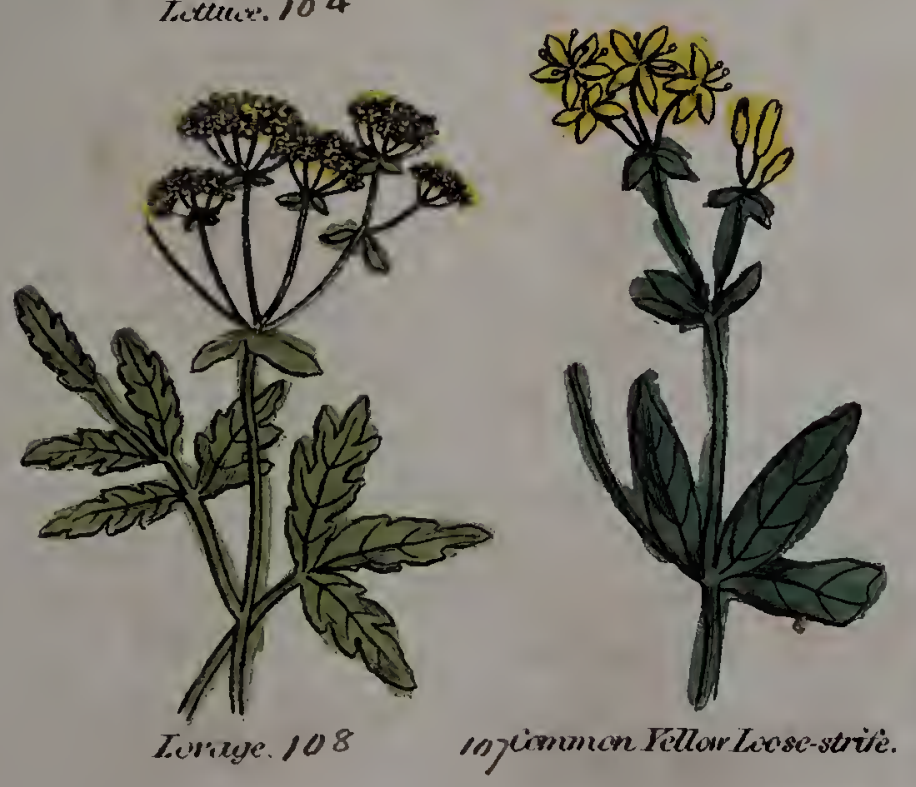

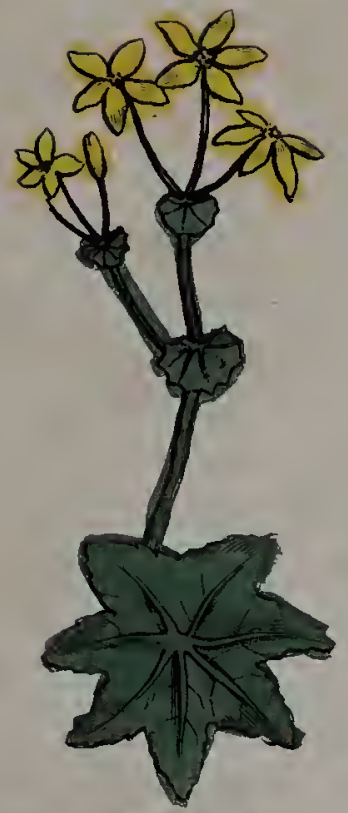

Common Tacties Dlantle 1133

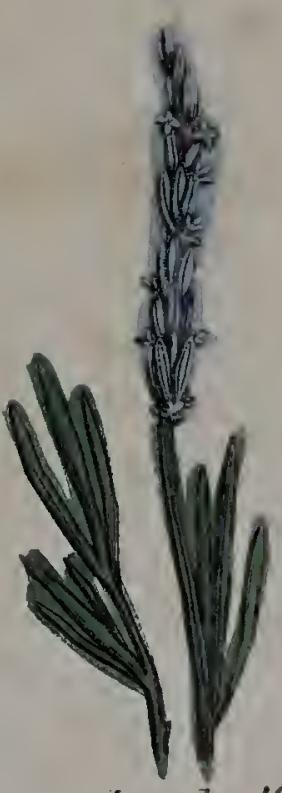

casender: 103.

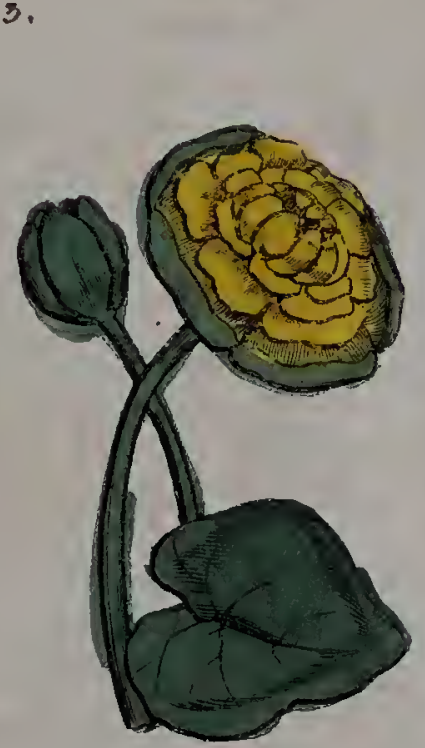

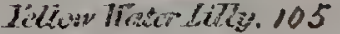

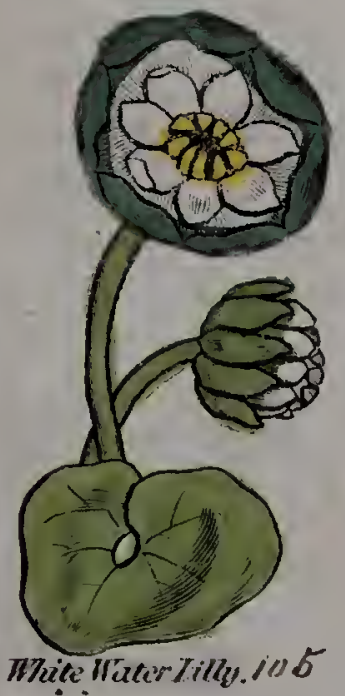

Whice Huter Tilly in of

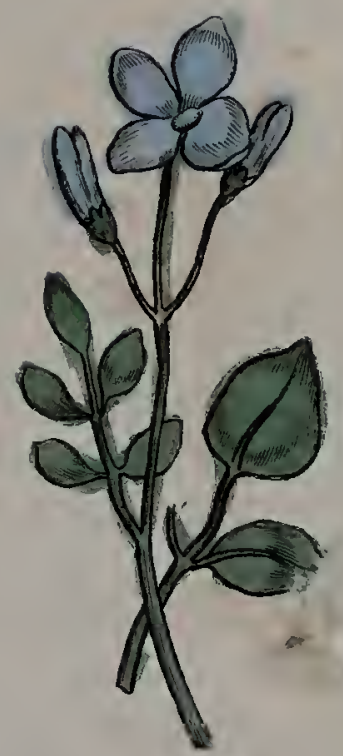

Tauics Sincck.

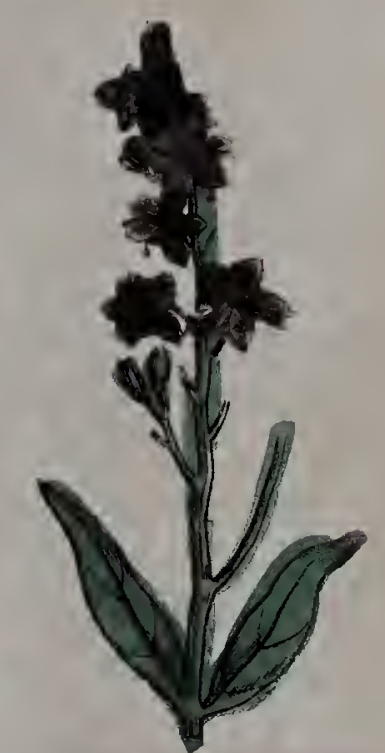

FadFarded Locseserive. 107

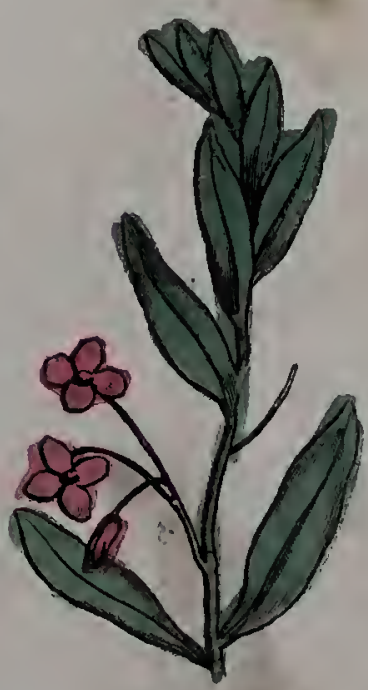

Zinden.Tiee. 



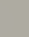

8

. 

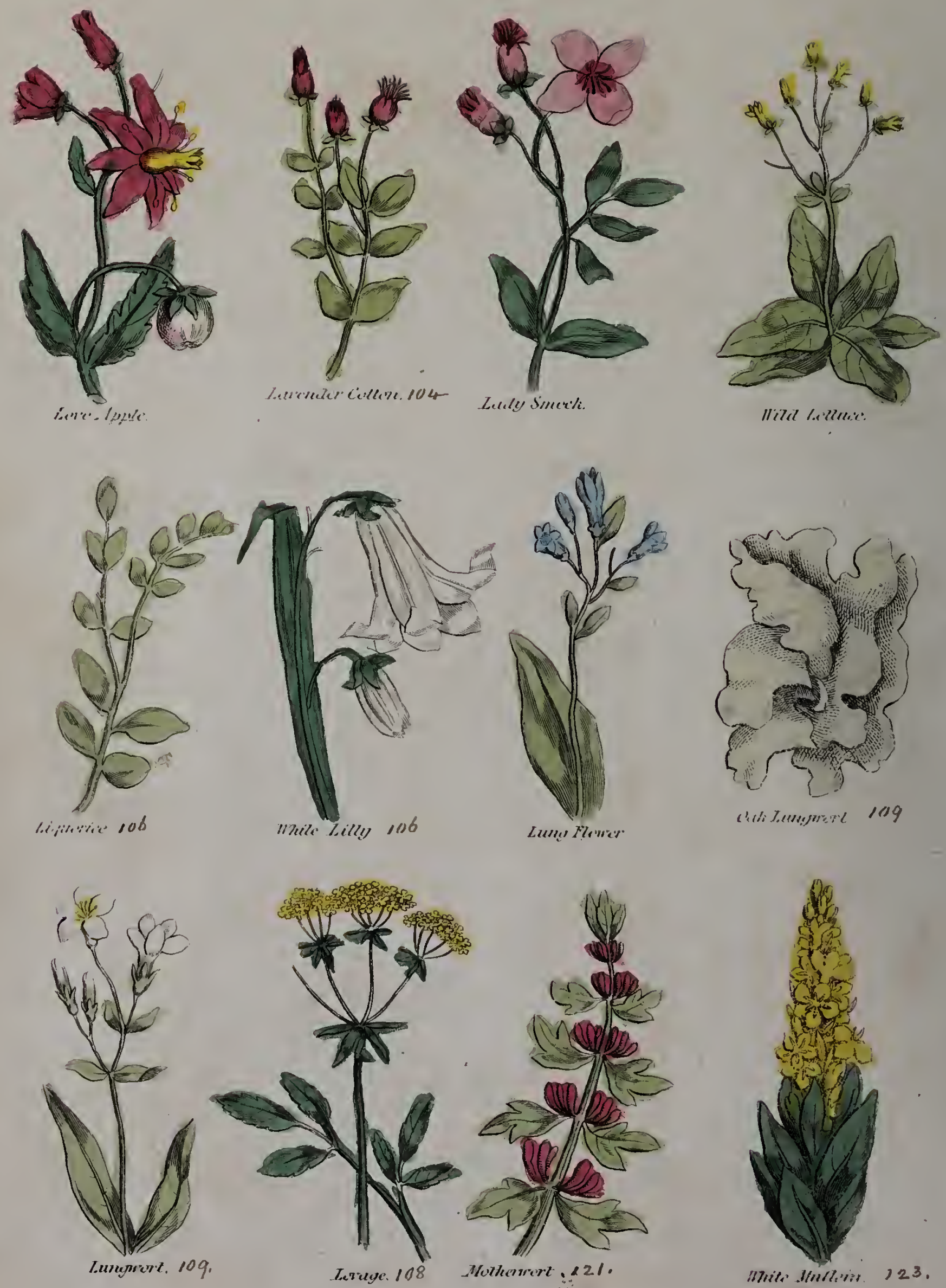

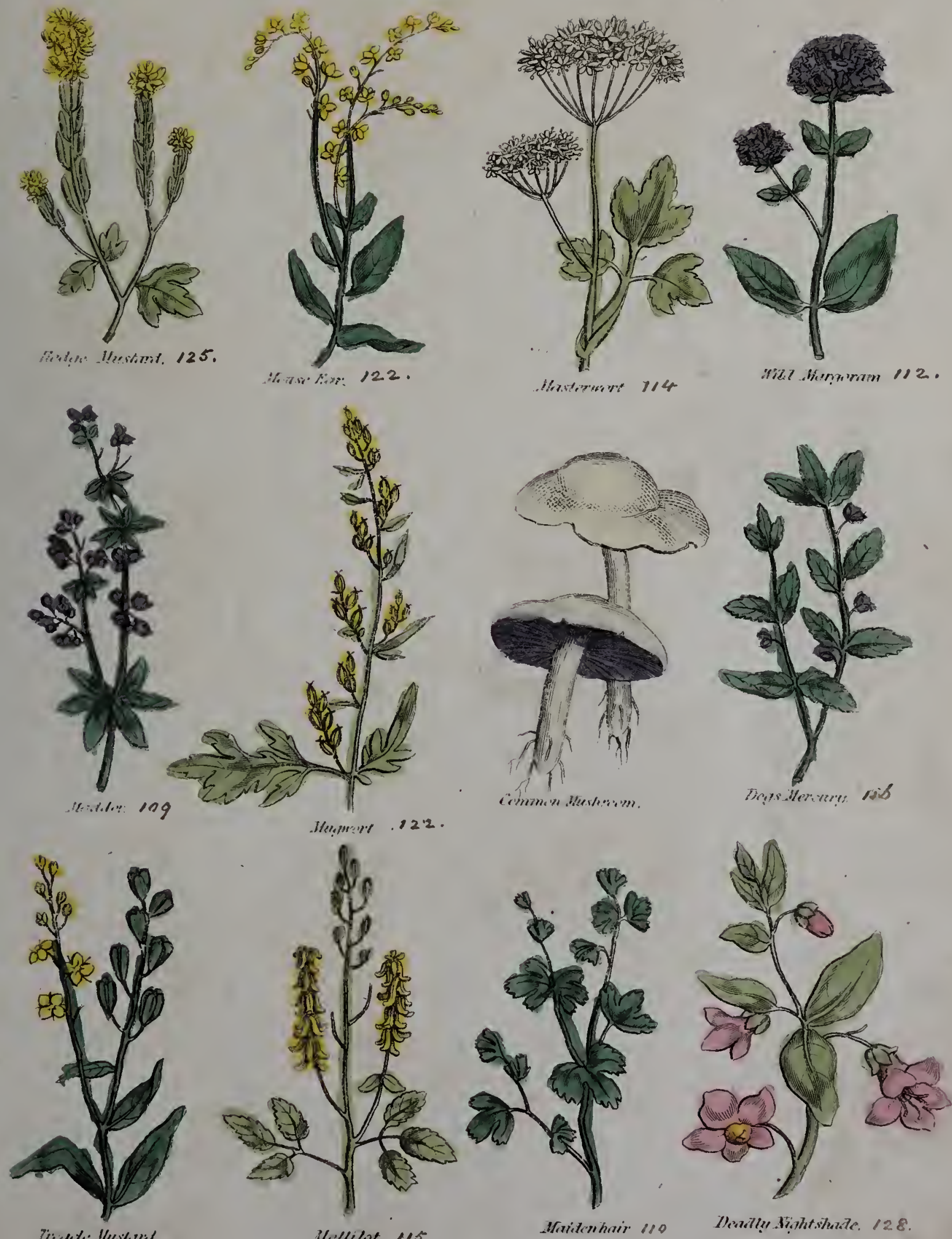

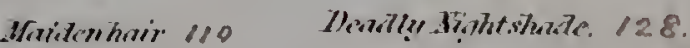




Plate 17 .

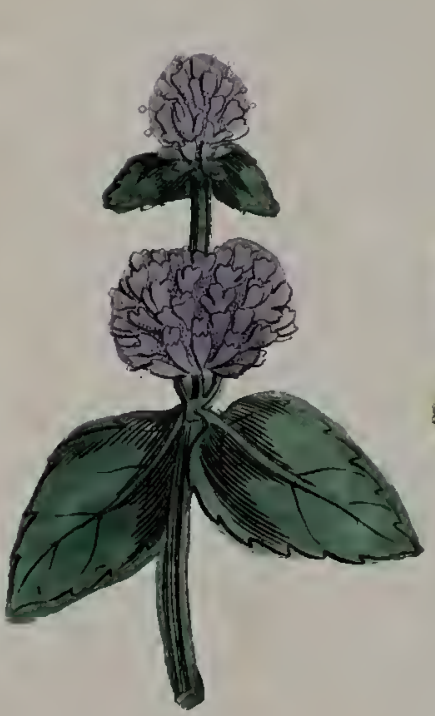

iut Ifoit. H7. 126
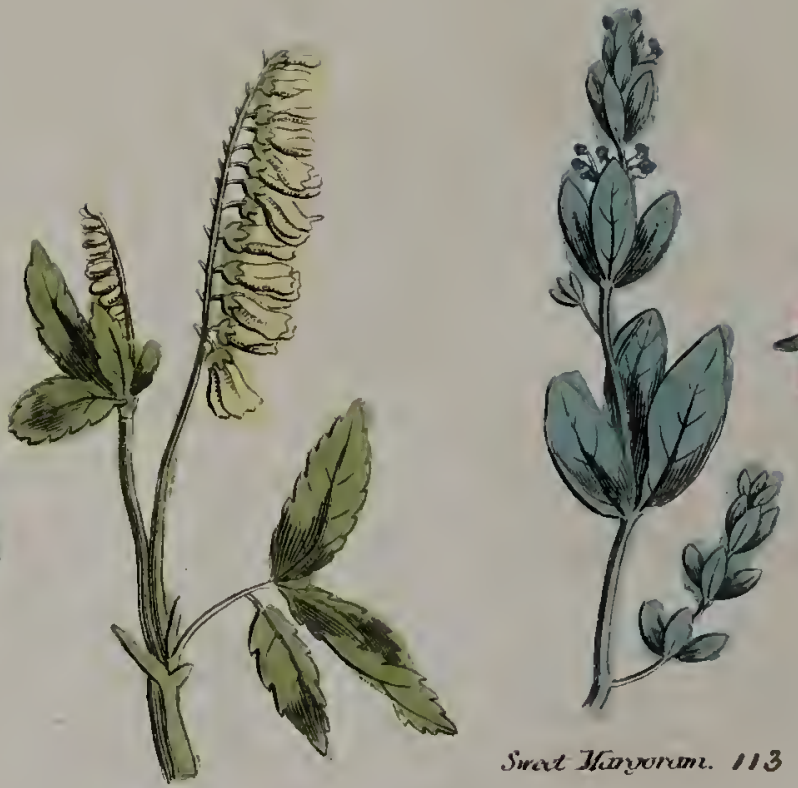

Sircet Jranowame. 113

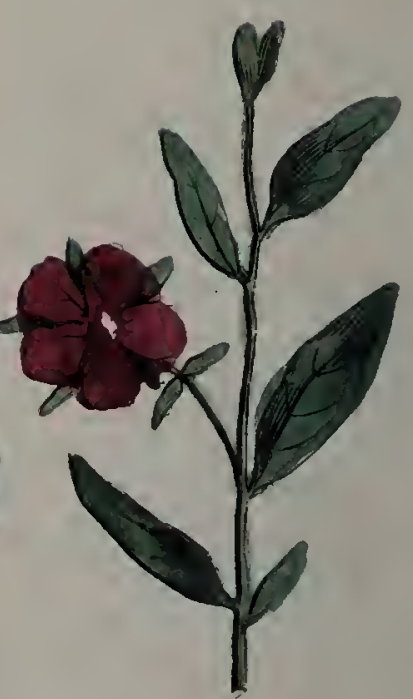

aniler 115

White Brviler. 115
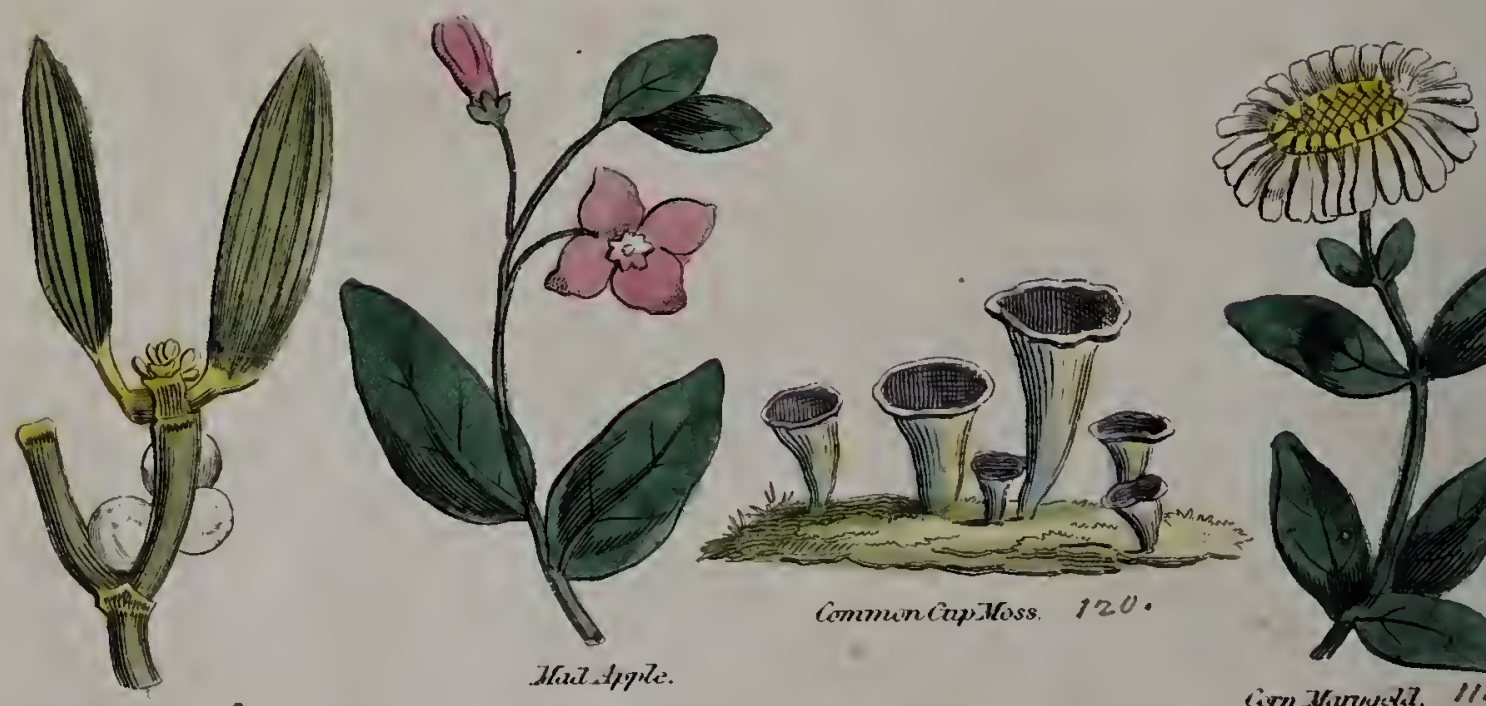

Messctte. $1 / 8$
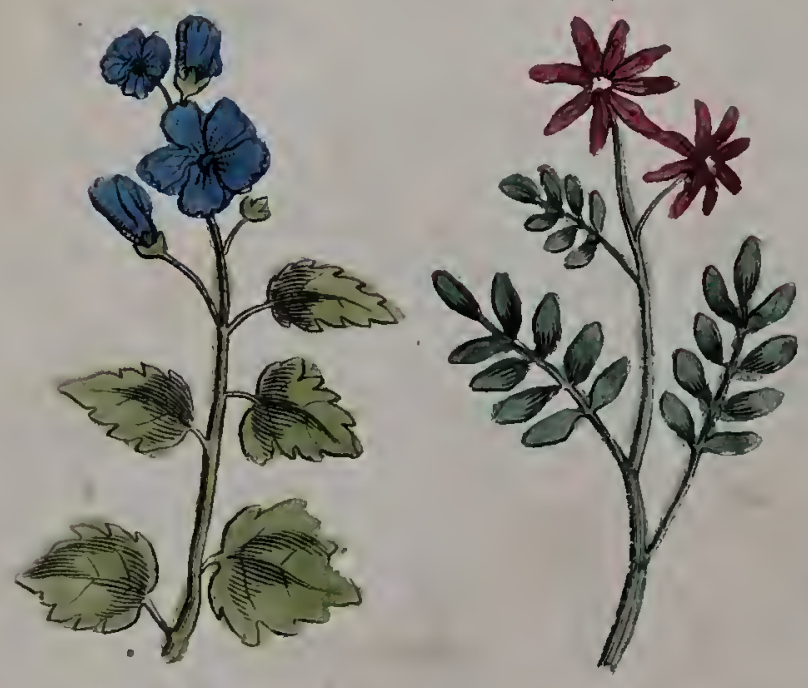

Hurve Nullew $2 / 8$

Nexumered
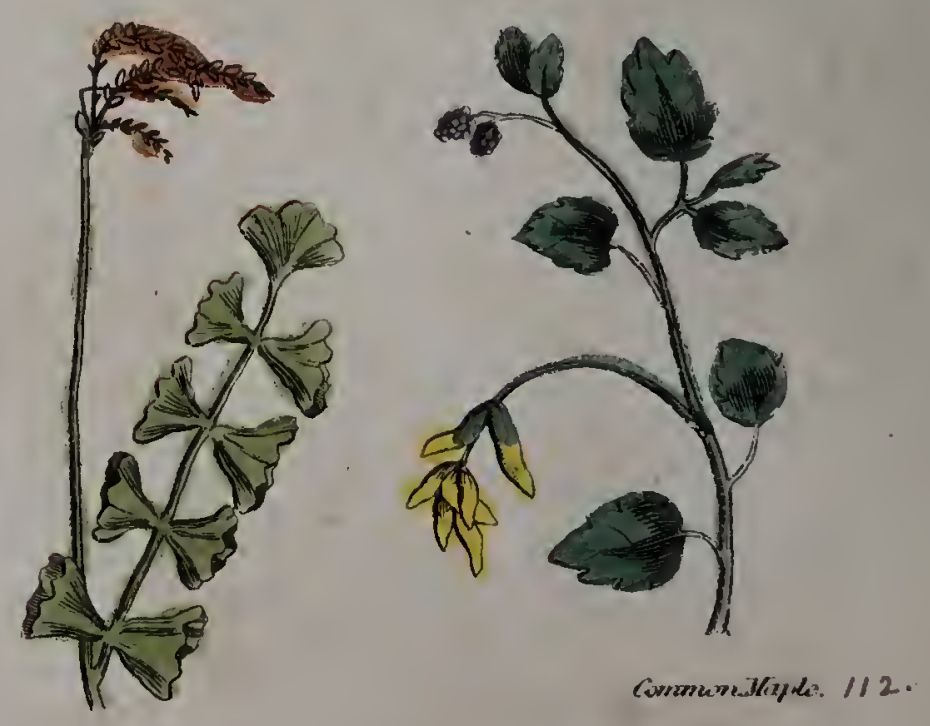


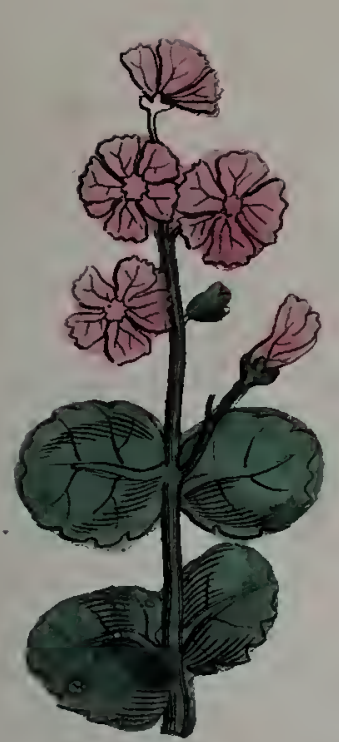

cimeman. Thille 223.

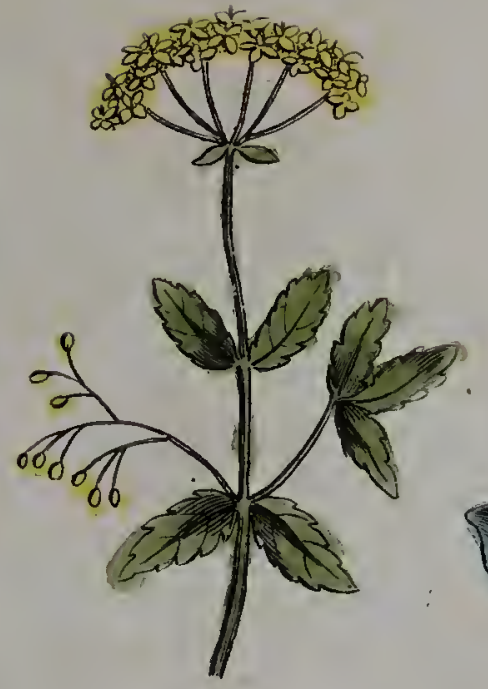

Heatus Tree.

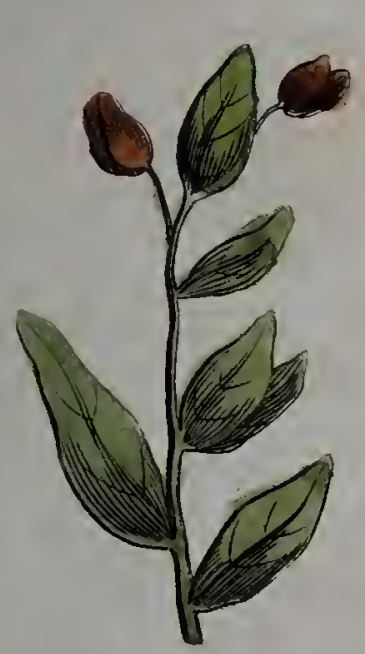

Tutmey Tice

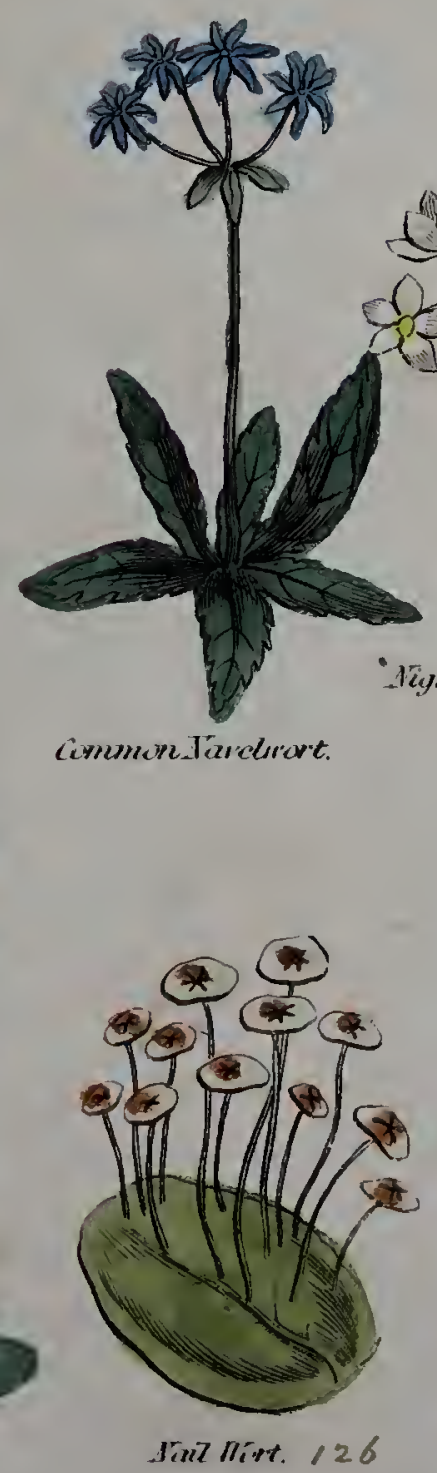

Sipple llivt.

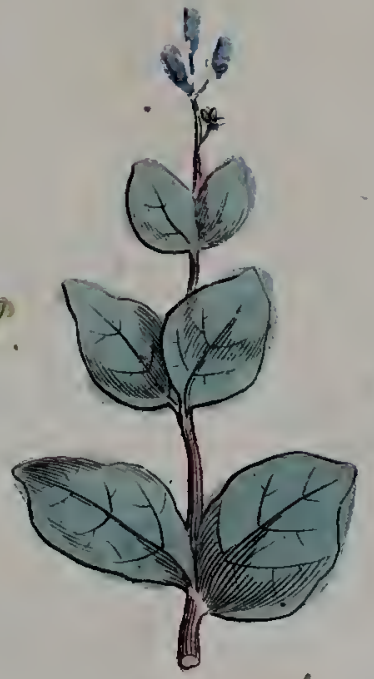

Tep. . 126.

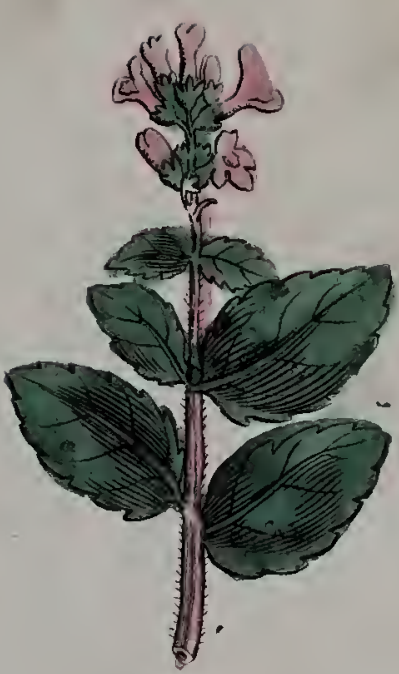

Tetule. 127. :

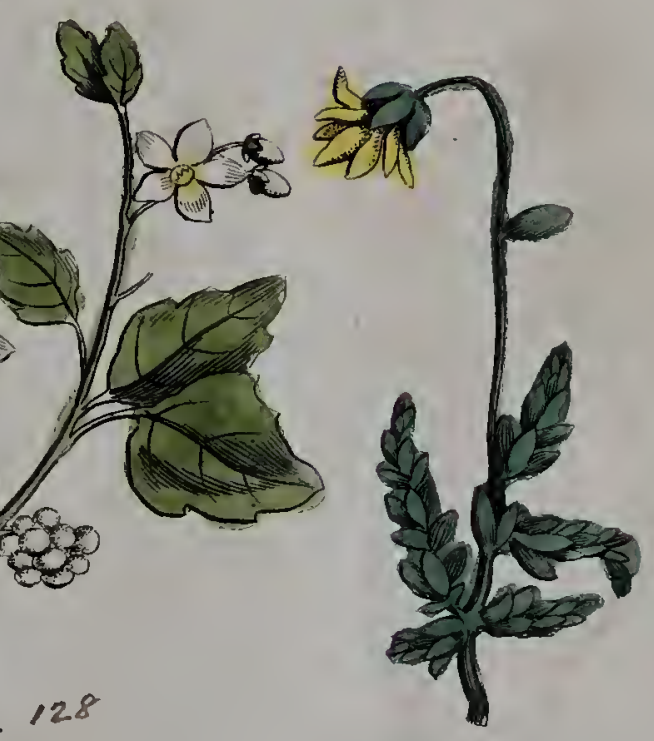

Tarrow Leaved Tarelwart.
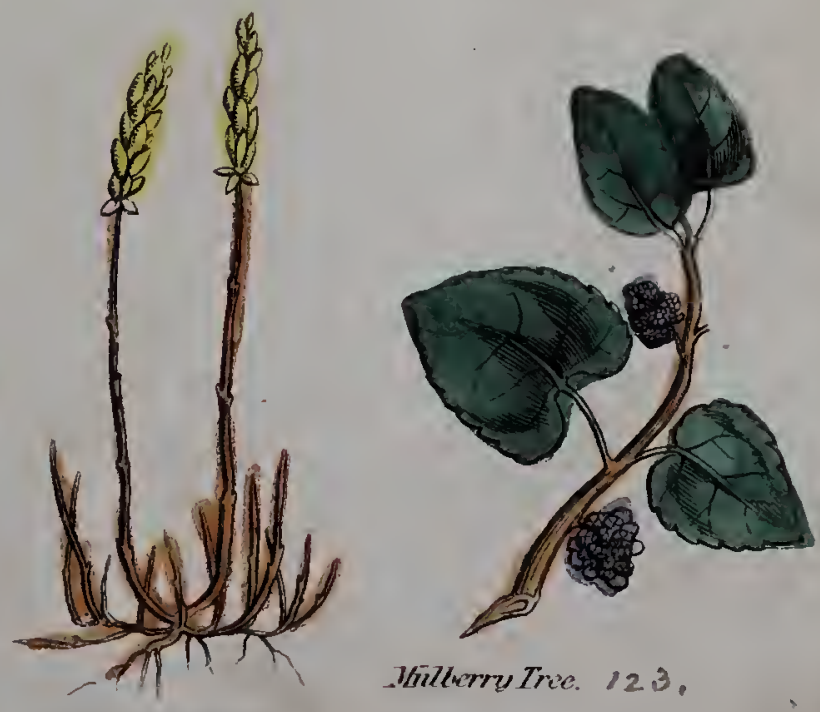

Jintherntrue. 123.

intiten Ilavidenthair: 110 



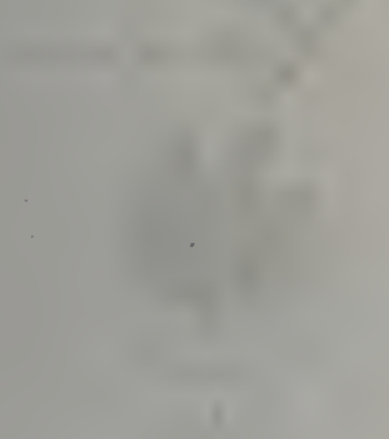

I
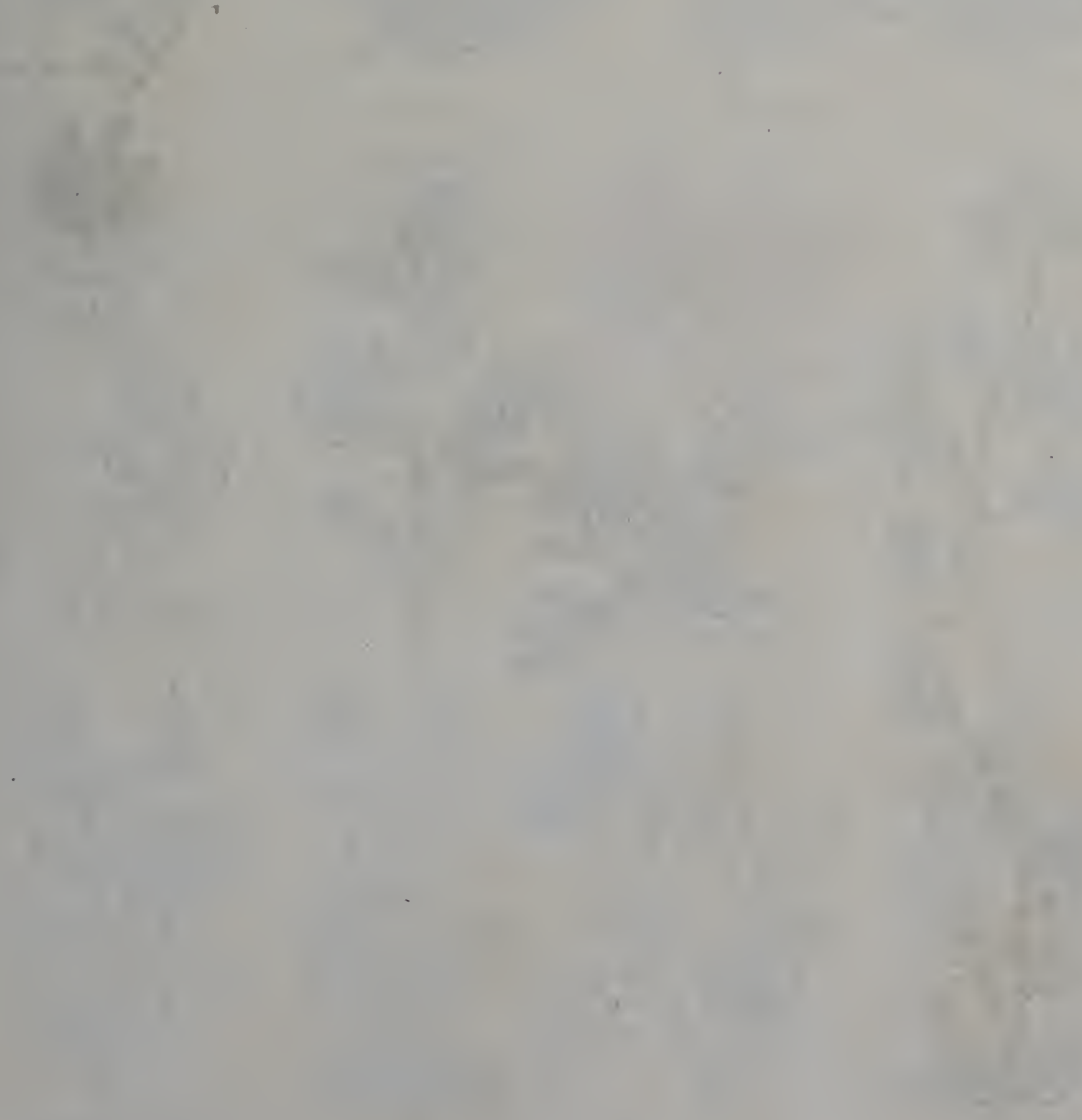

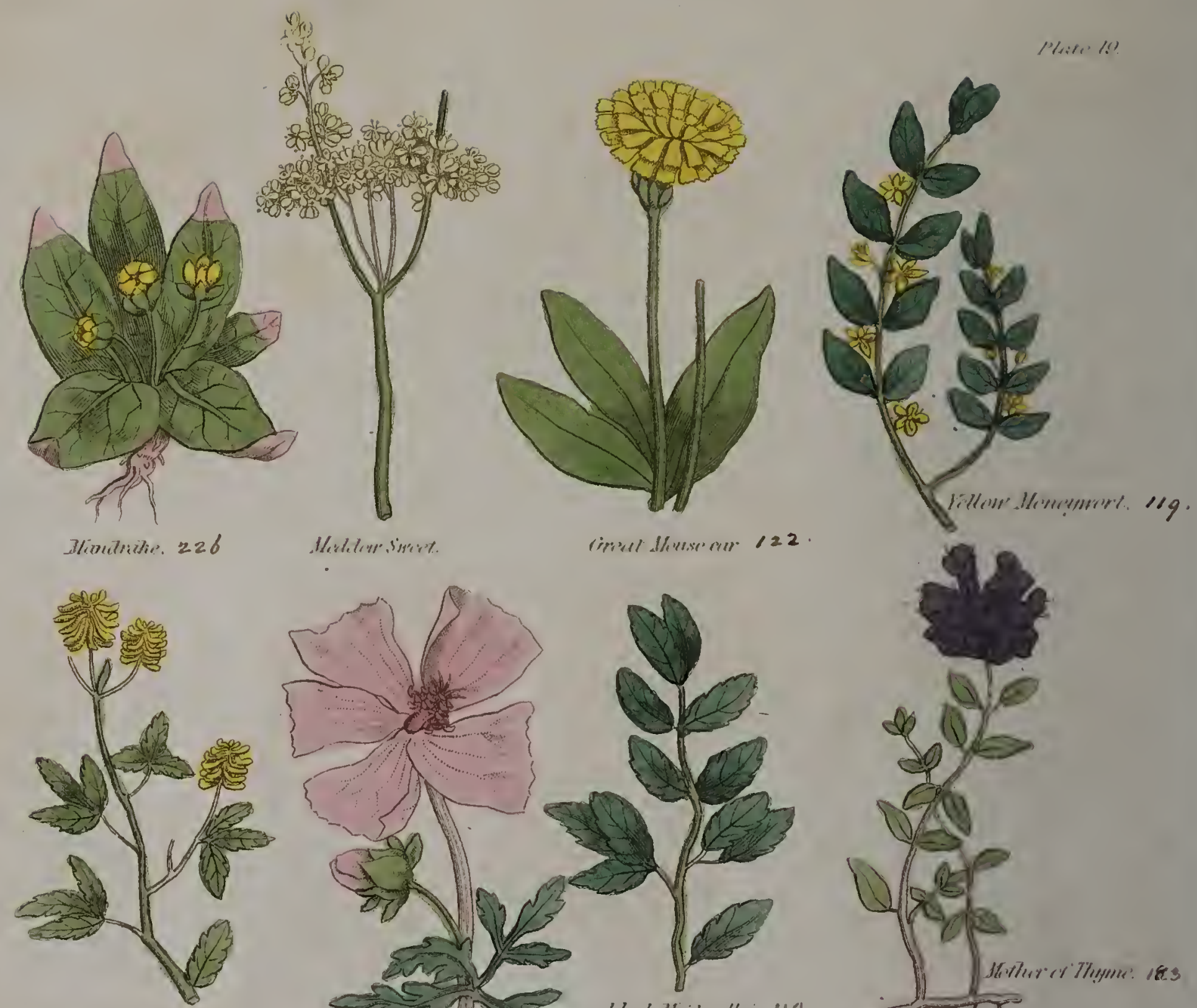

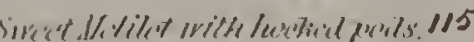
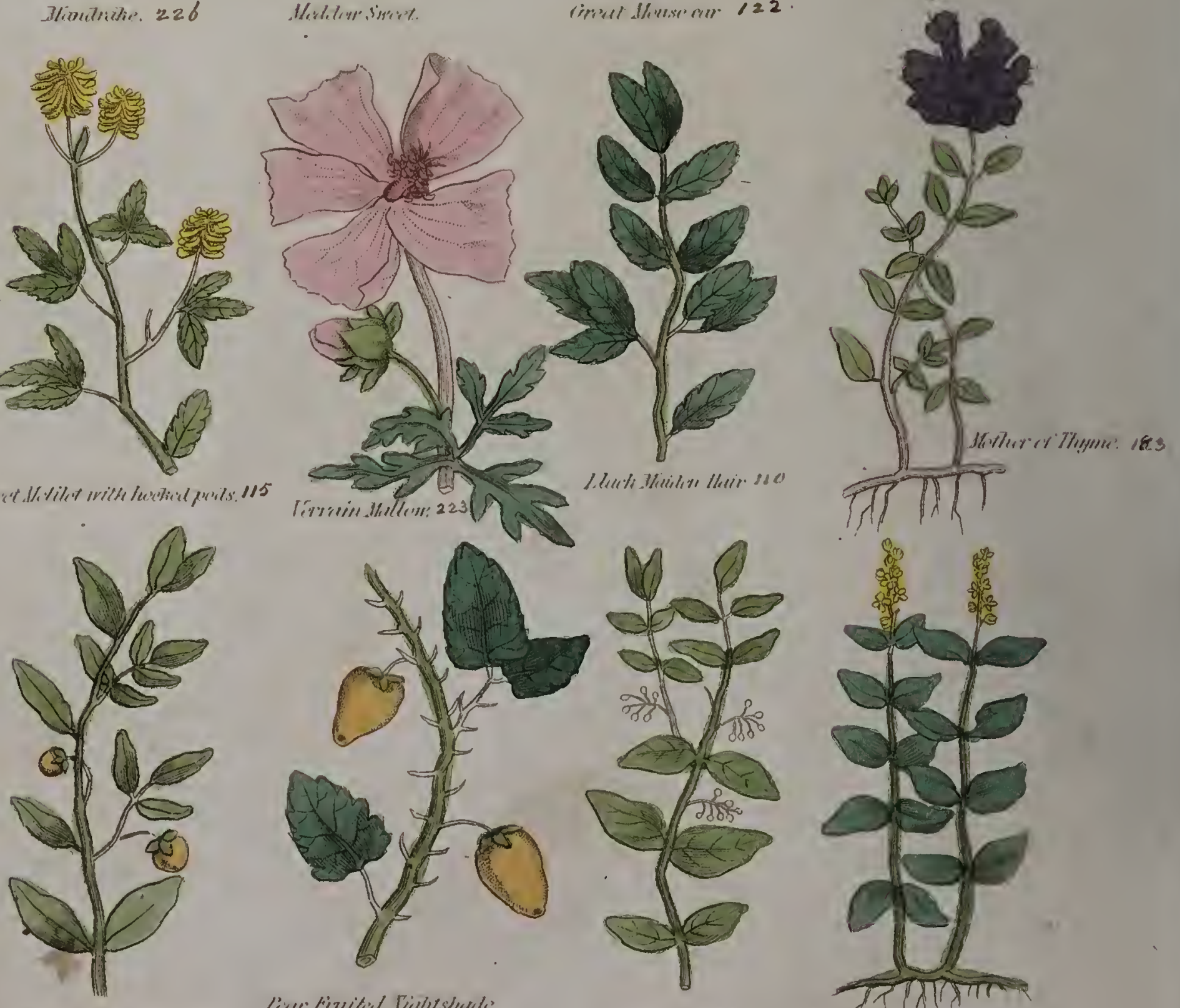

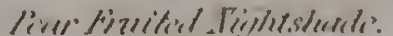

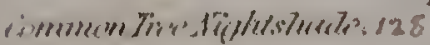

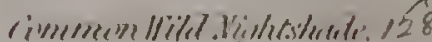

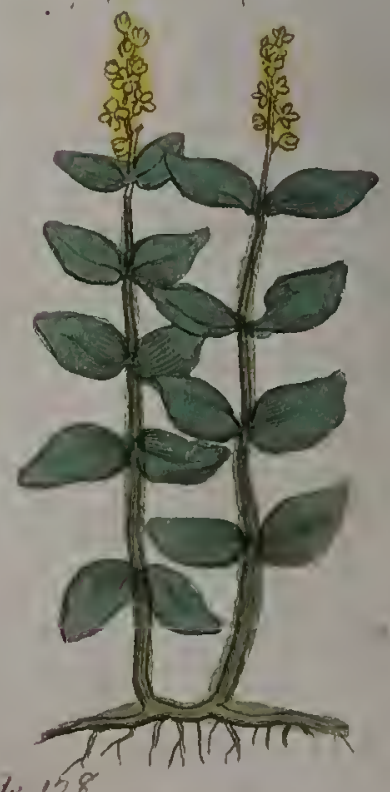

retir lieml. 

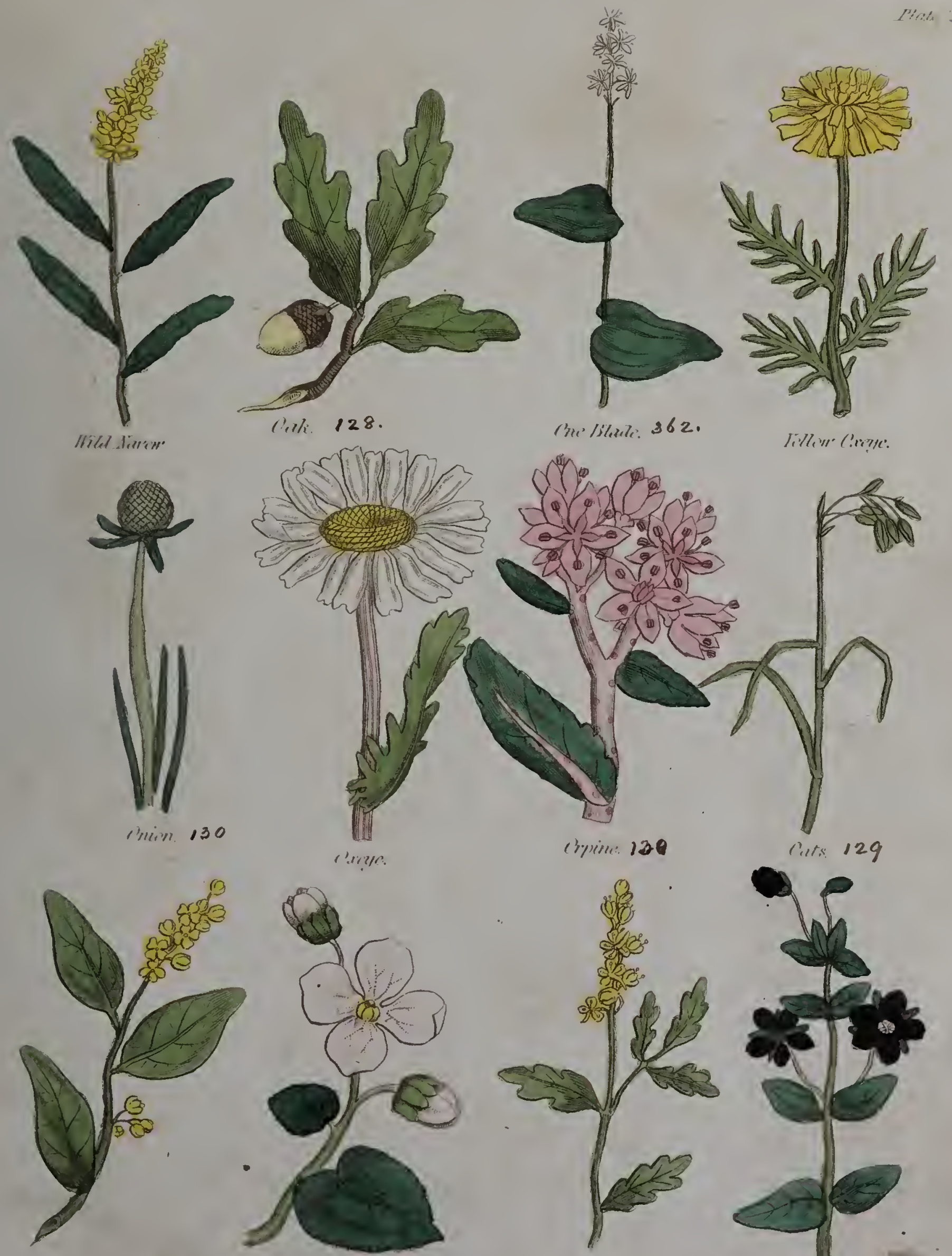

ris'.
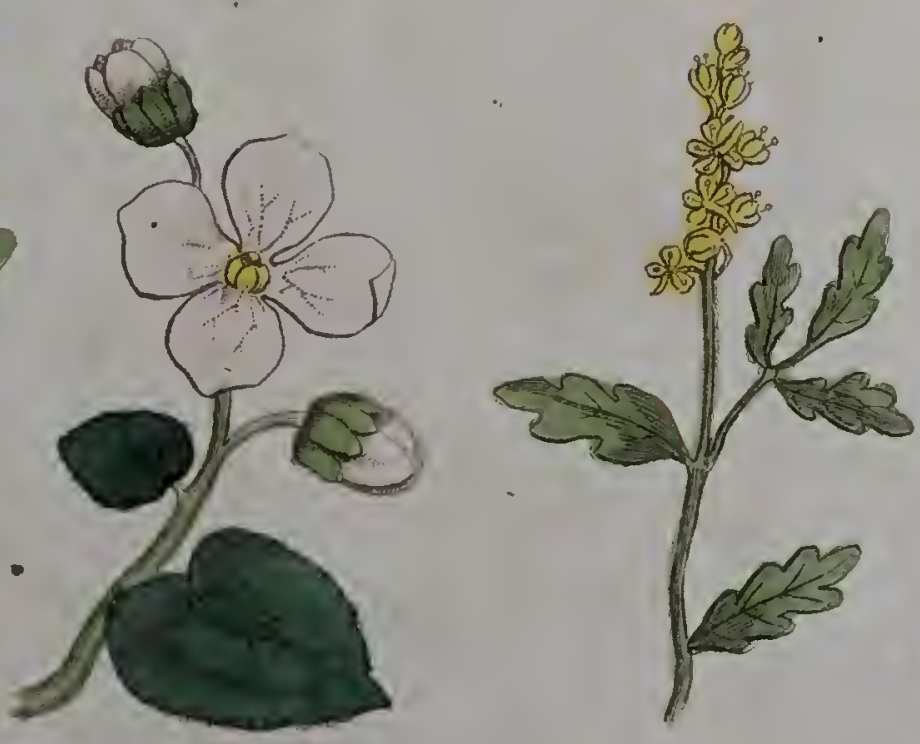

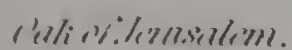

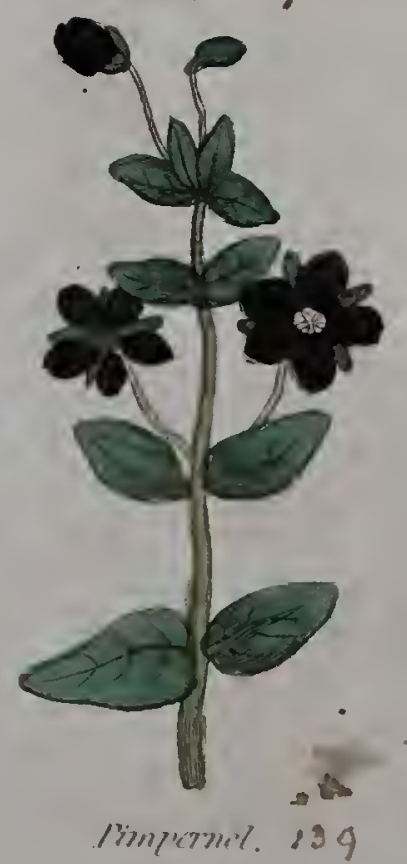




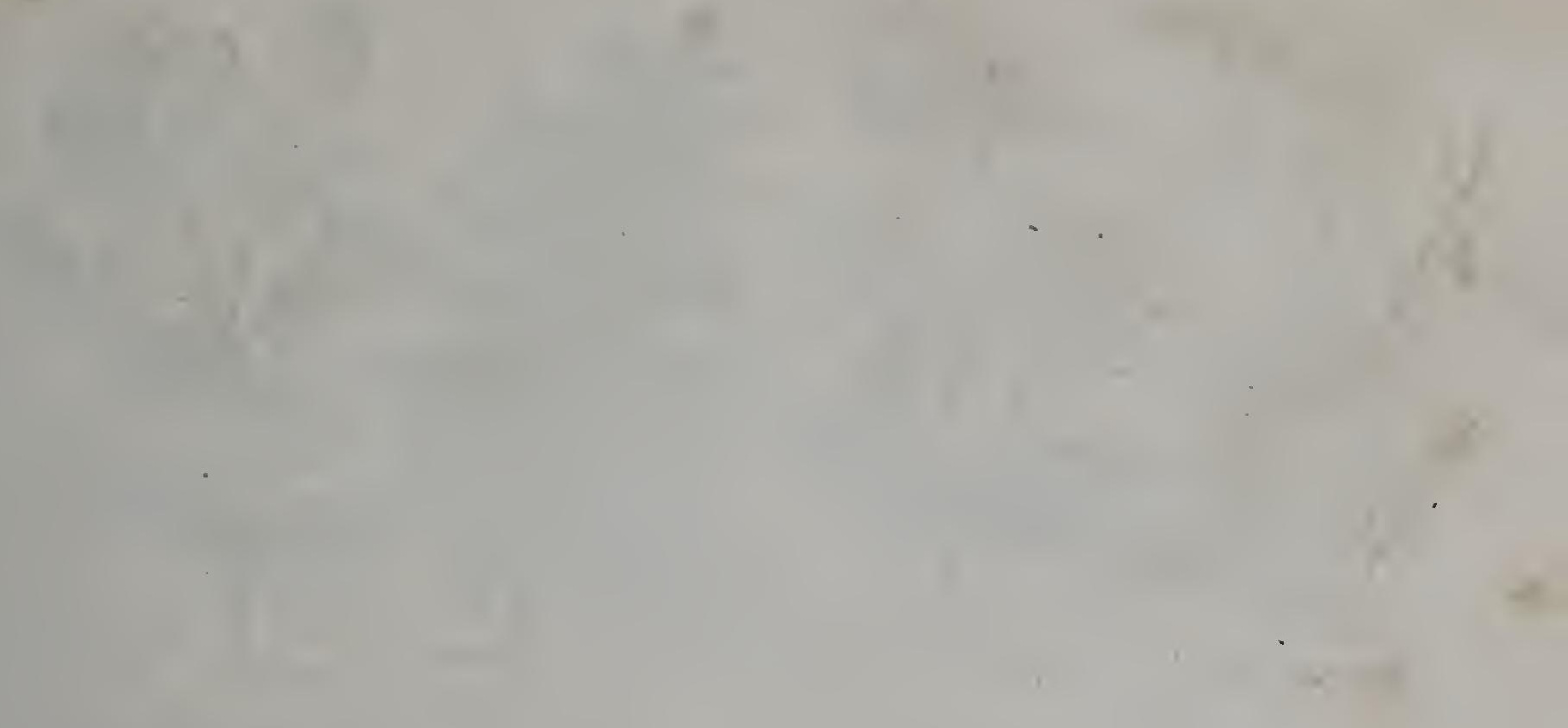

. .

$$
\text { 、 }
$$

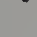




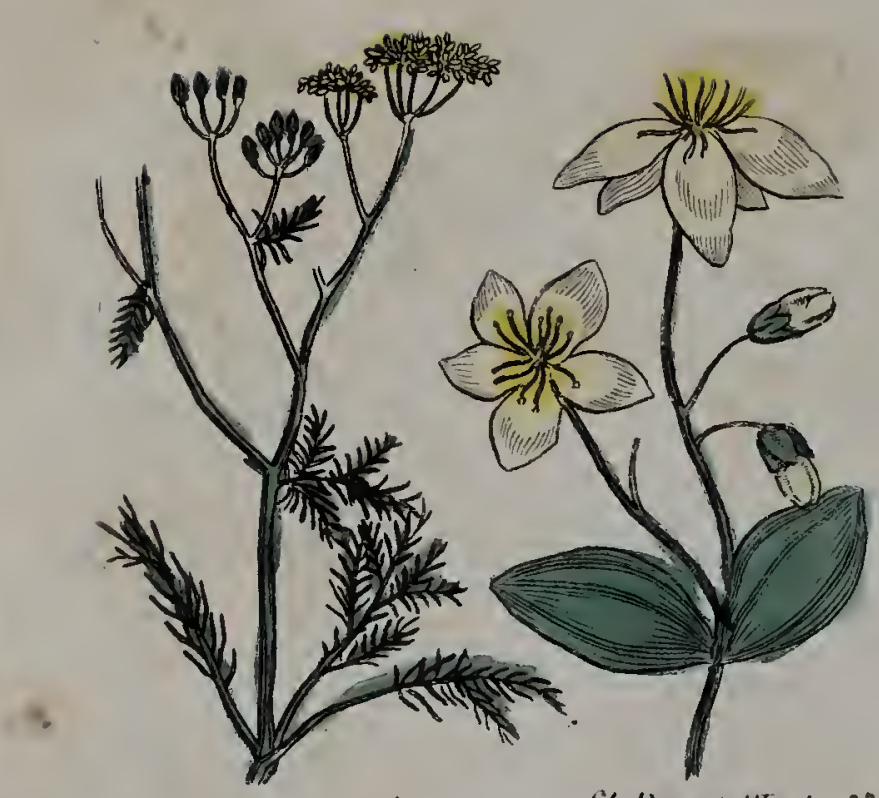

Sprignct. 223

S. Liters llort. 139.

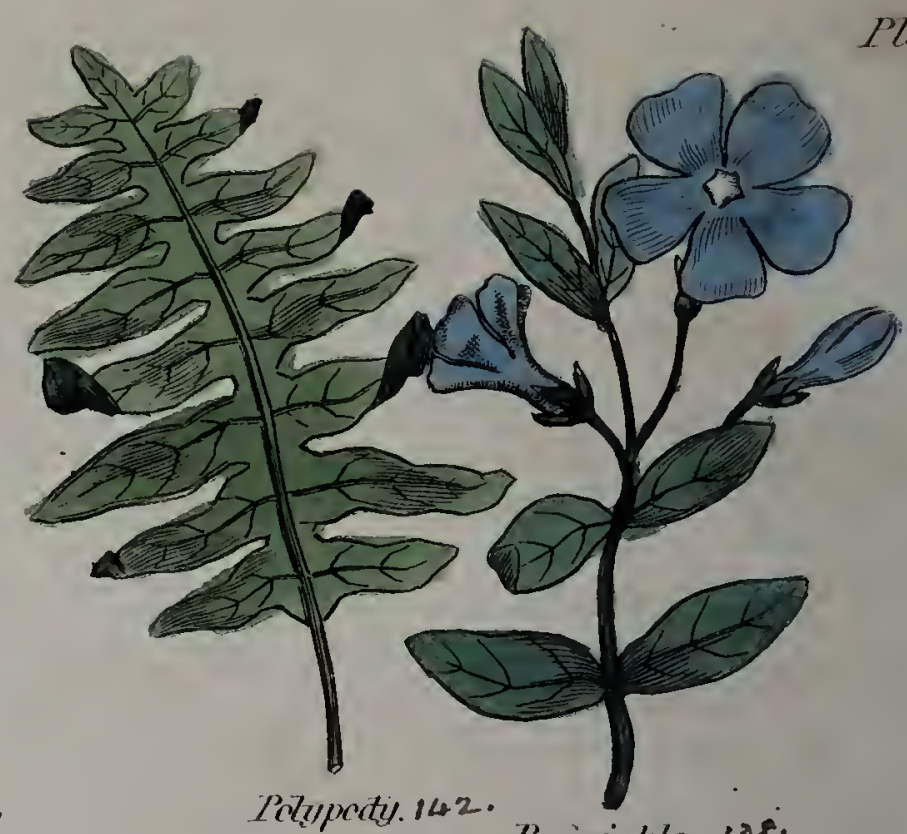

Perinintile. 138.

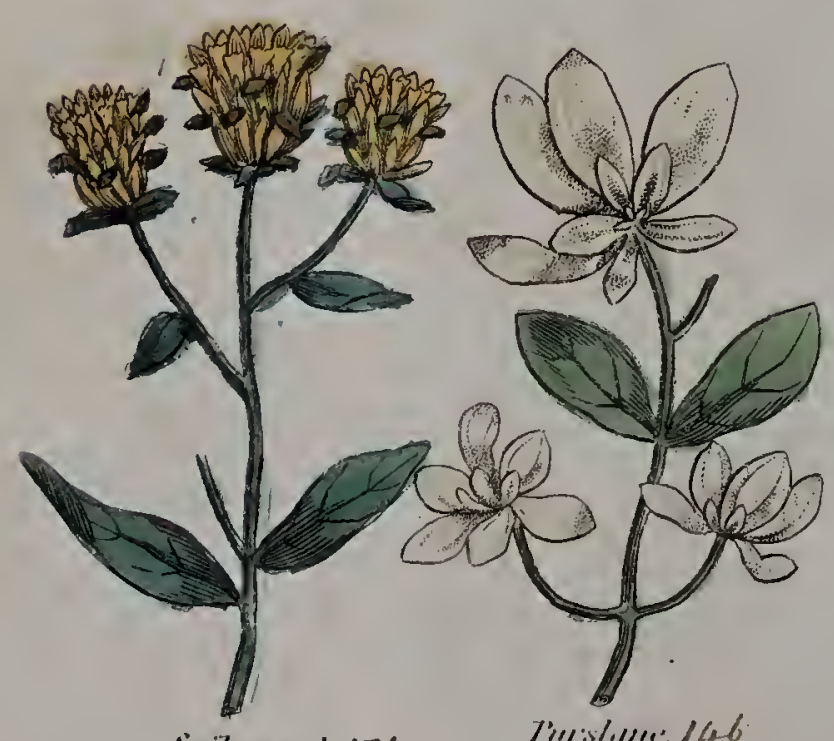

spitenard. $17^{4}$

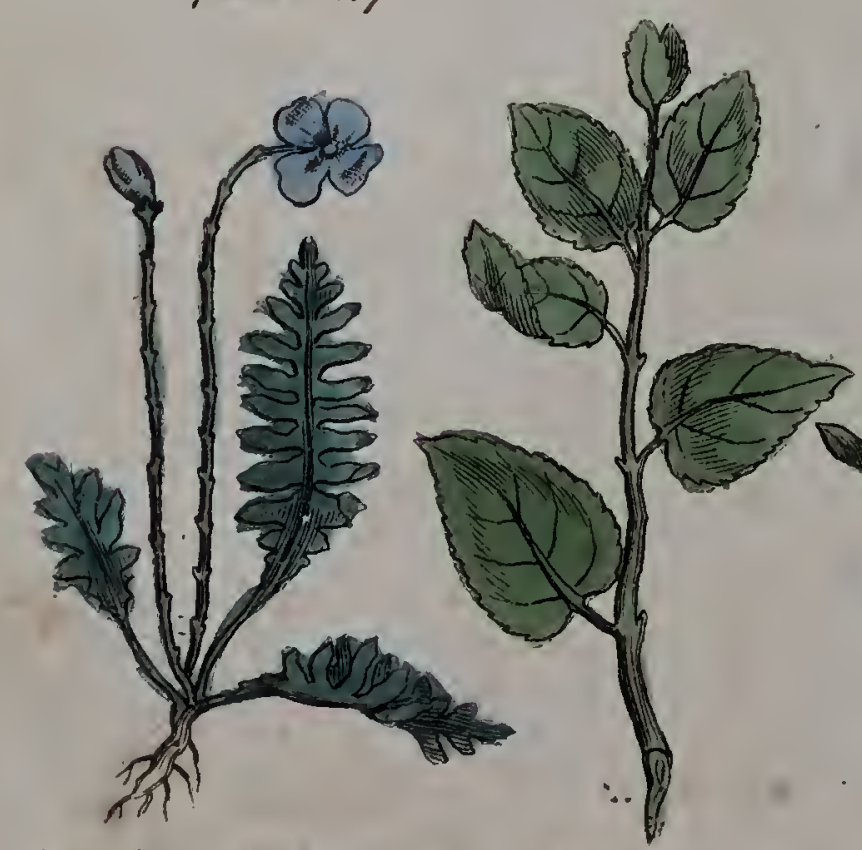

imath Splecrumt. 174

intikclivatar: 143.

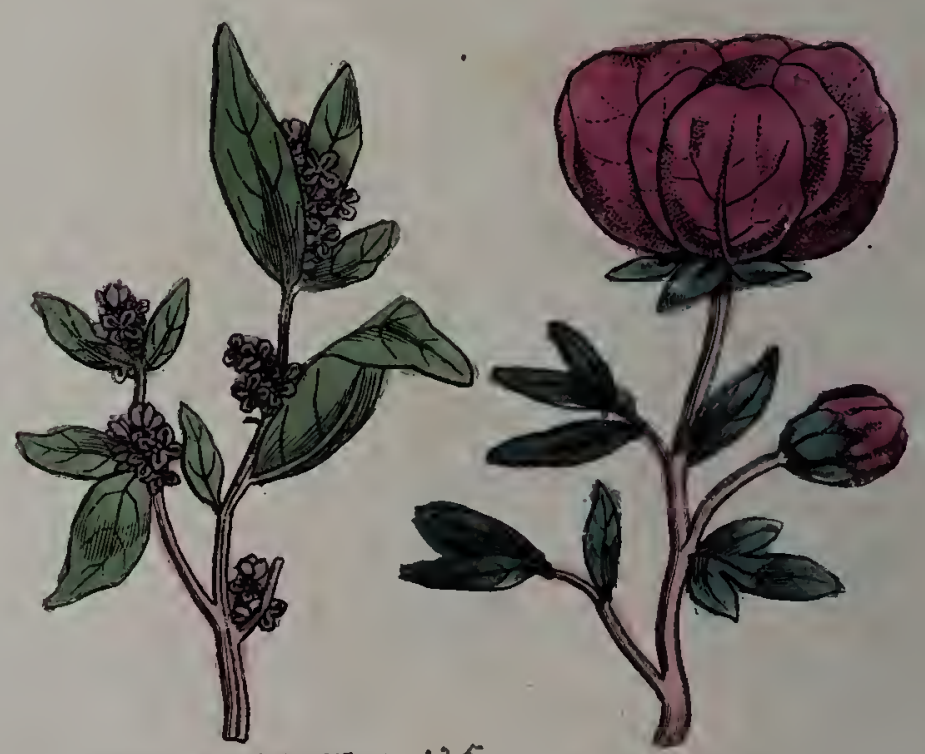

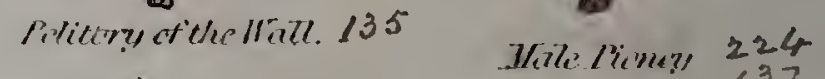
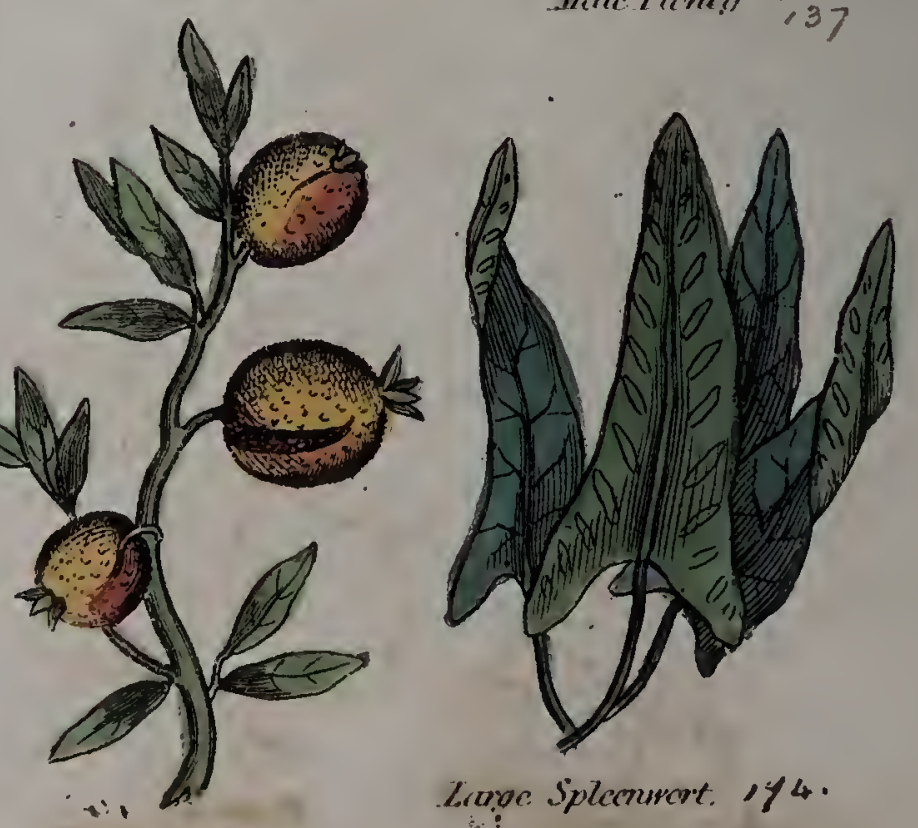

Romermativire. 
Plate..."
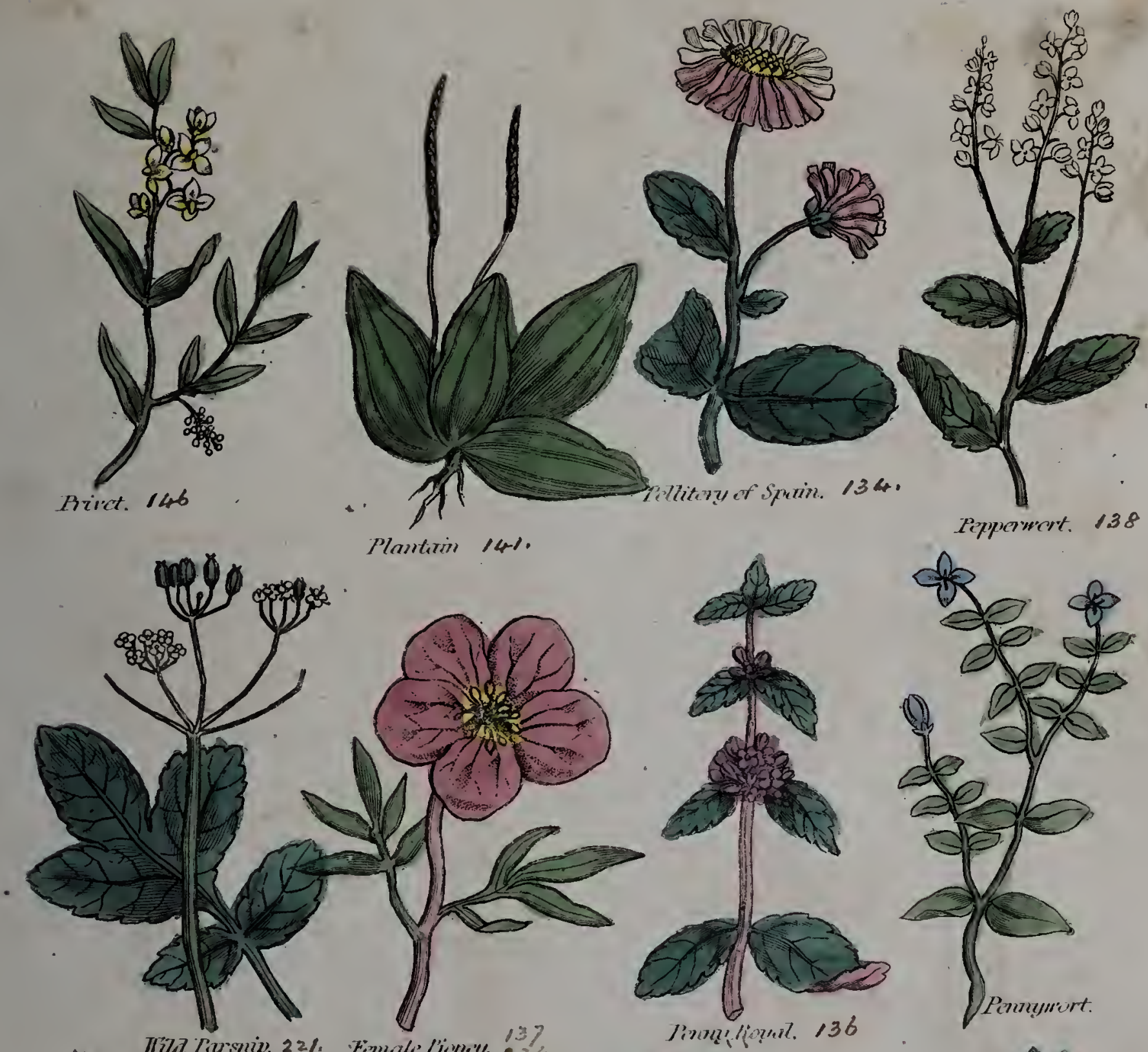

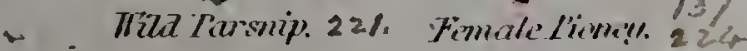

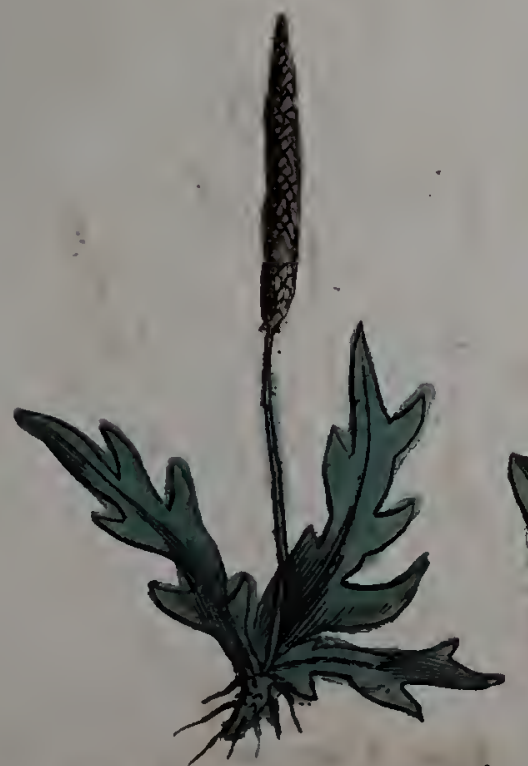

Buthshome ITuntain. 141

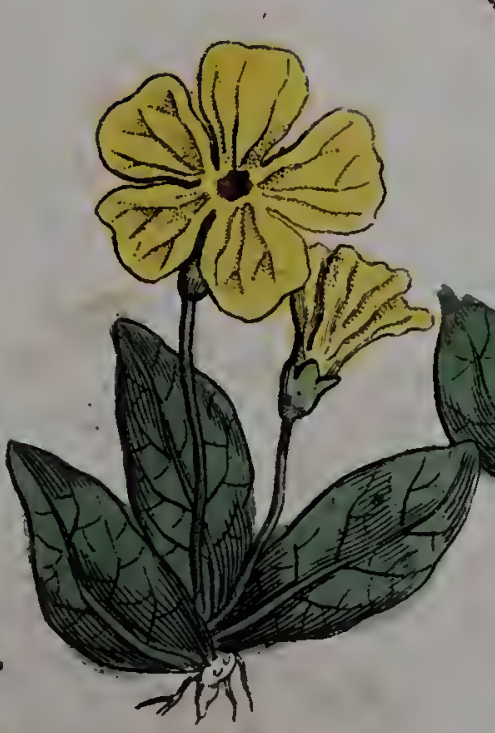

Primeses. 146

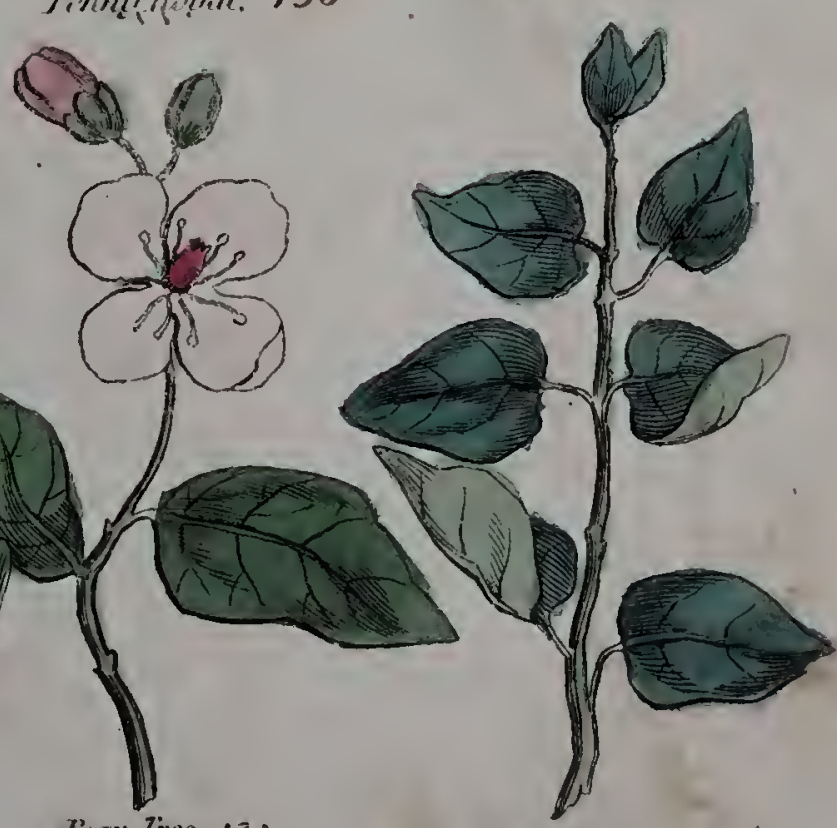

Jilati Piplar 143. 

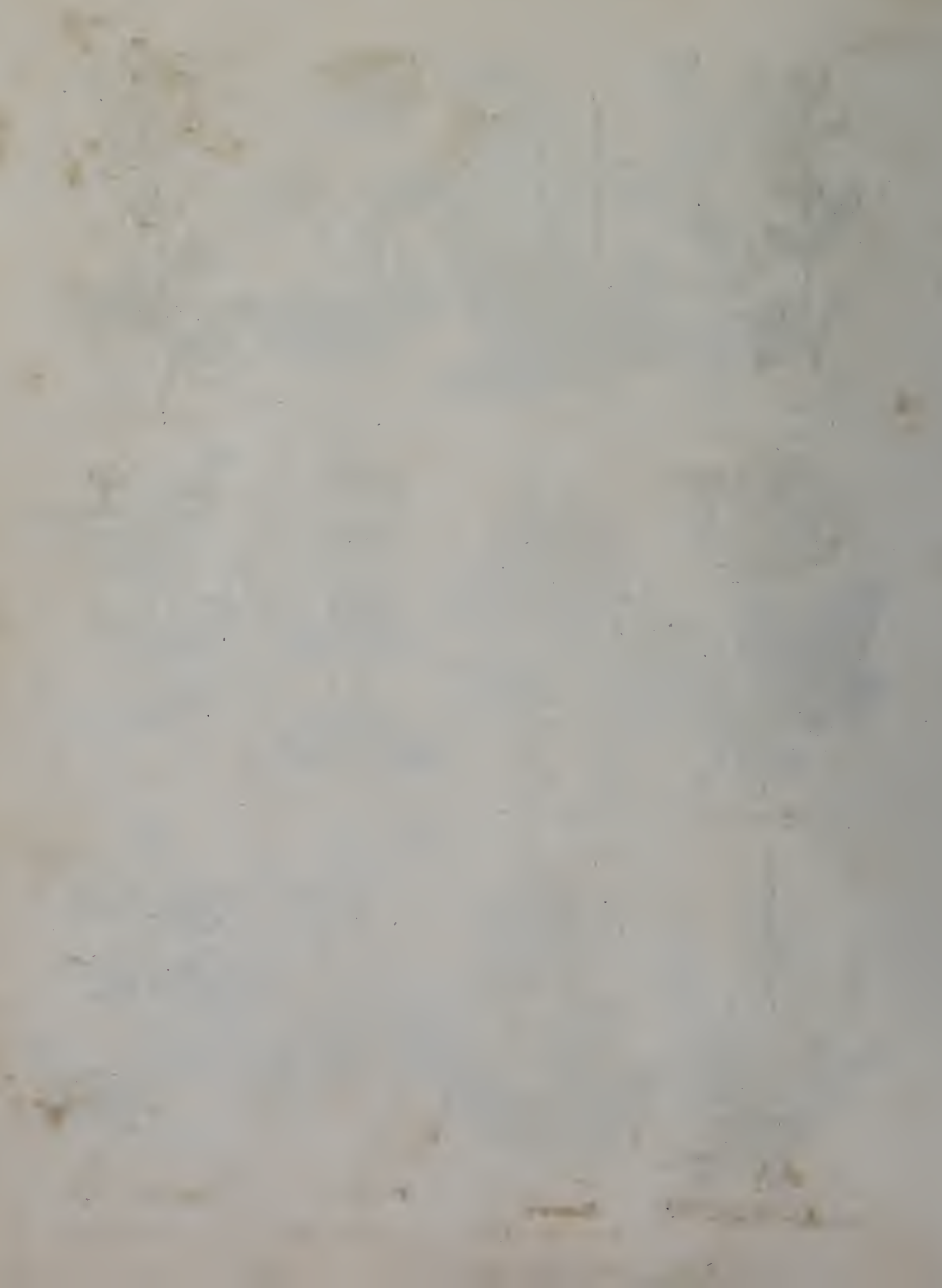



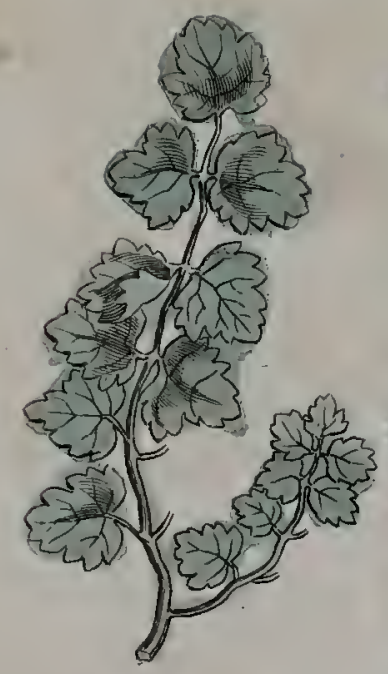

Trestry Prot. 181
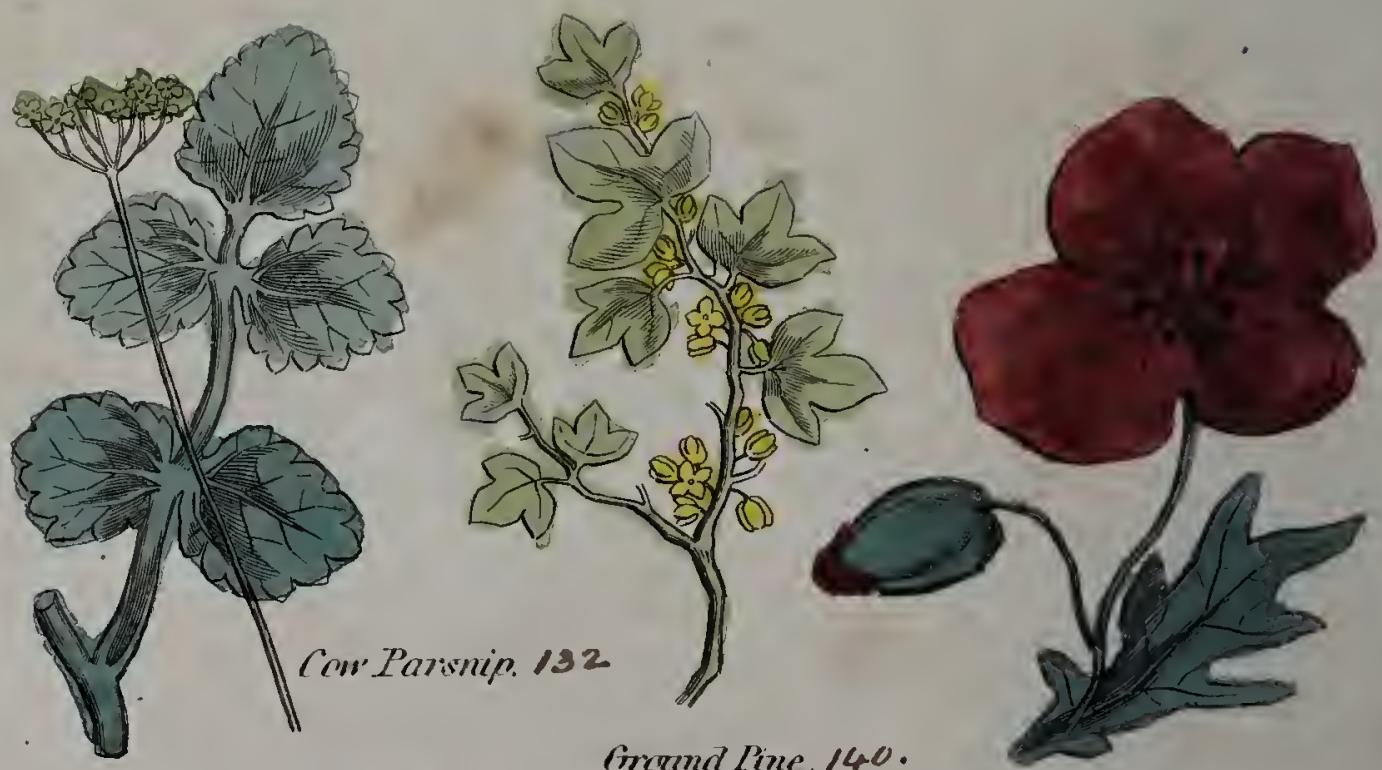

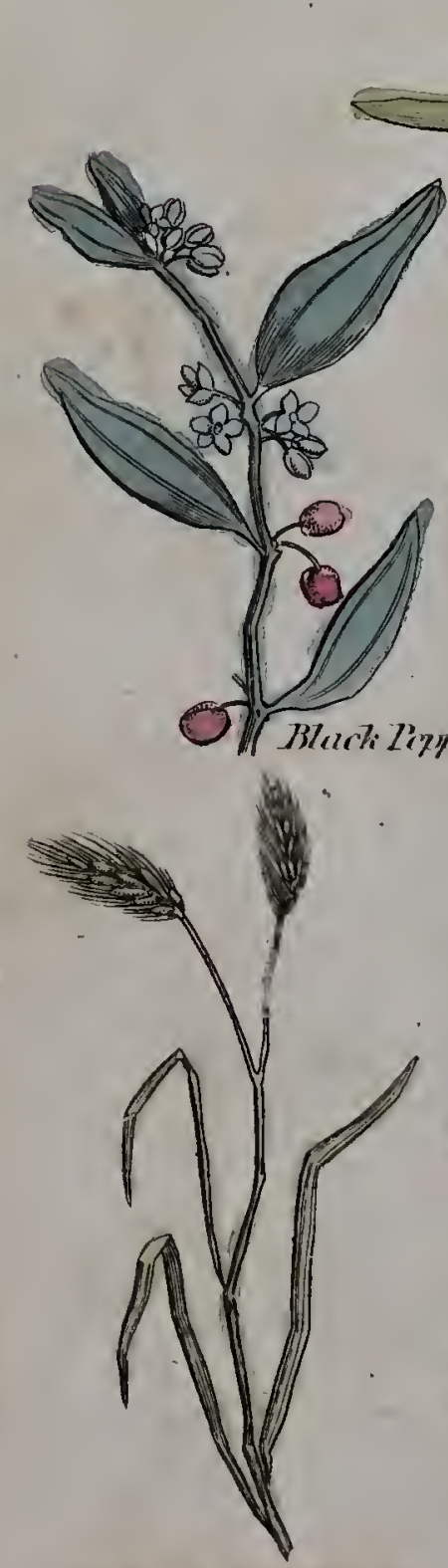

Quick Cirass.

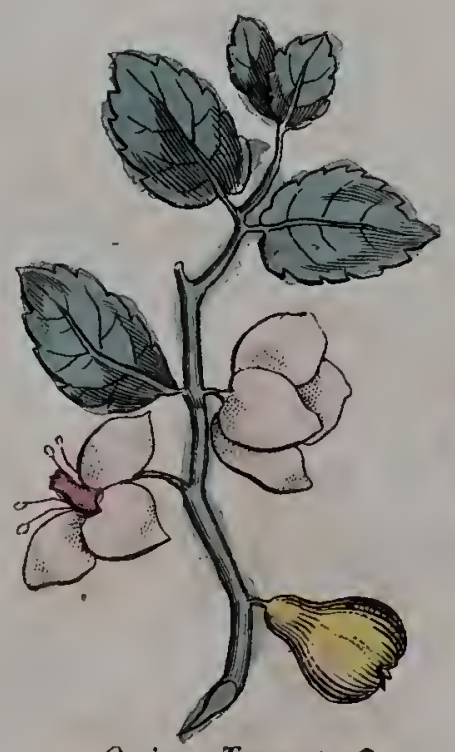

Sinine Tive. 108

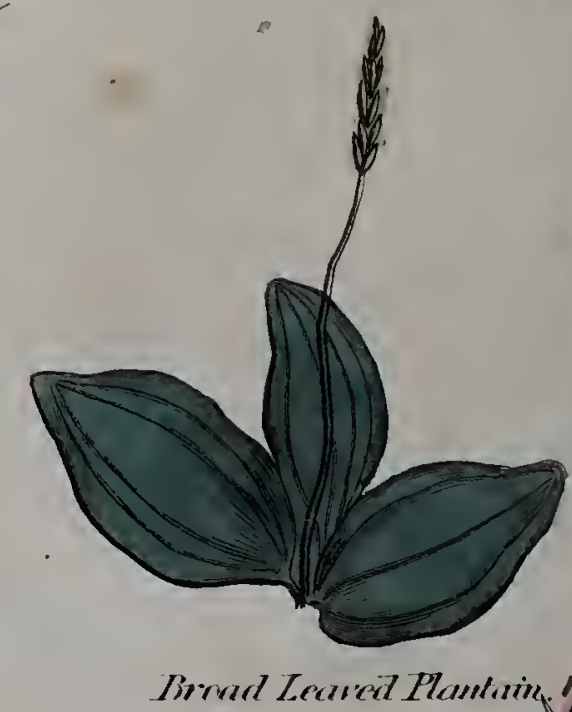

Wild Ponpr. 144.

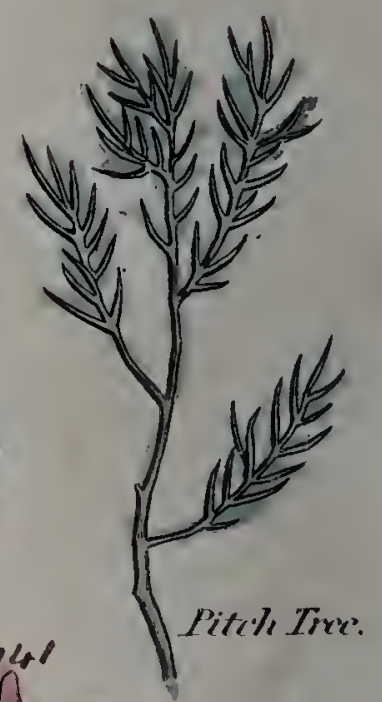

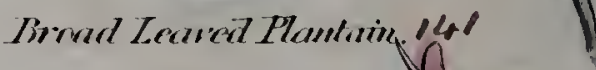
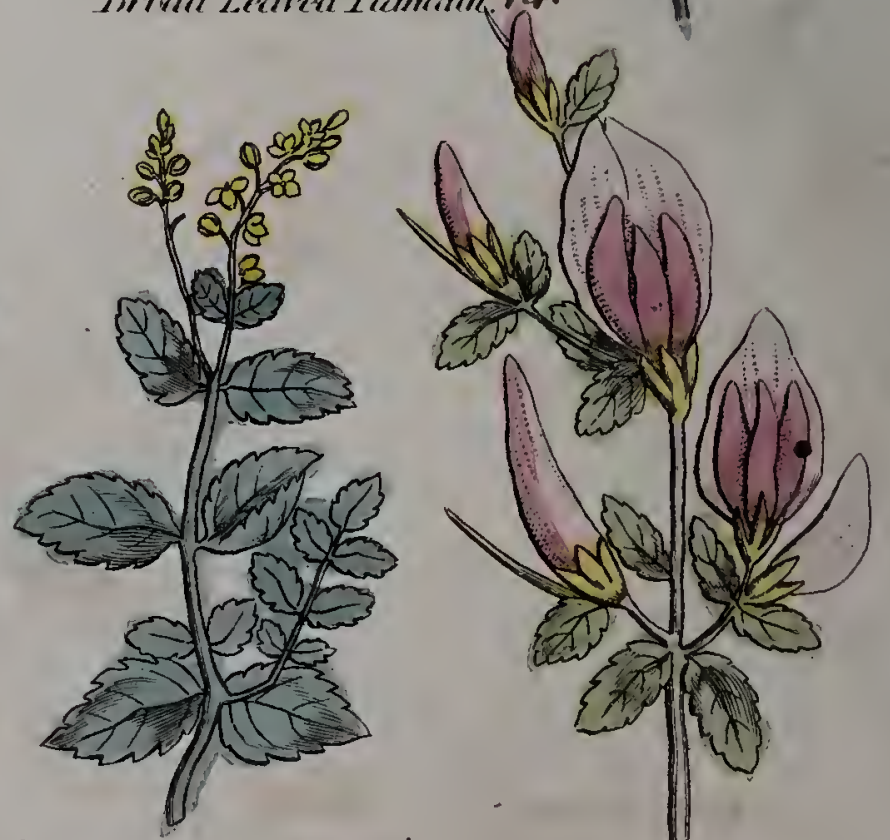

Shermotilie Headem: 14\%.

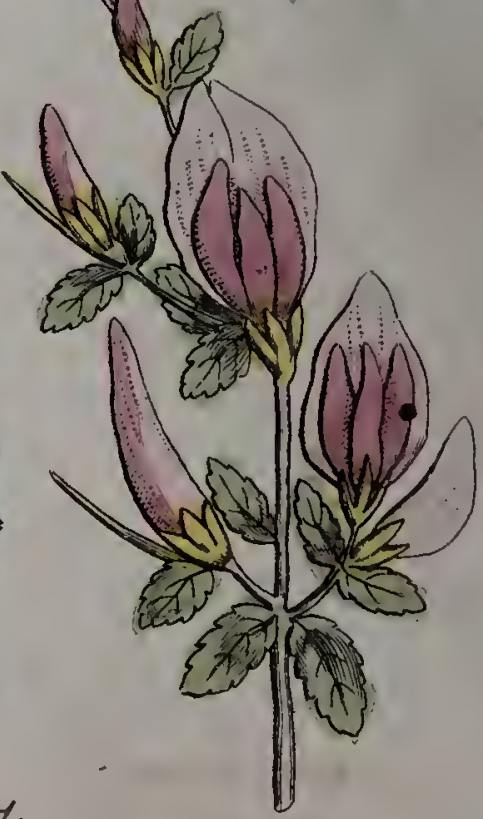

Ress Arros: 150. 


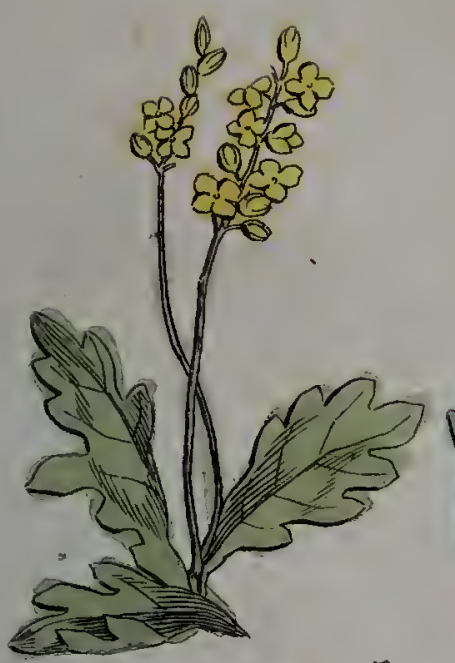

IIITh Ruritisle. 225

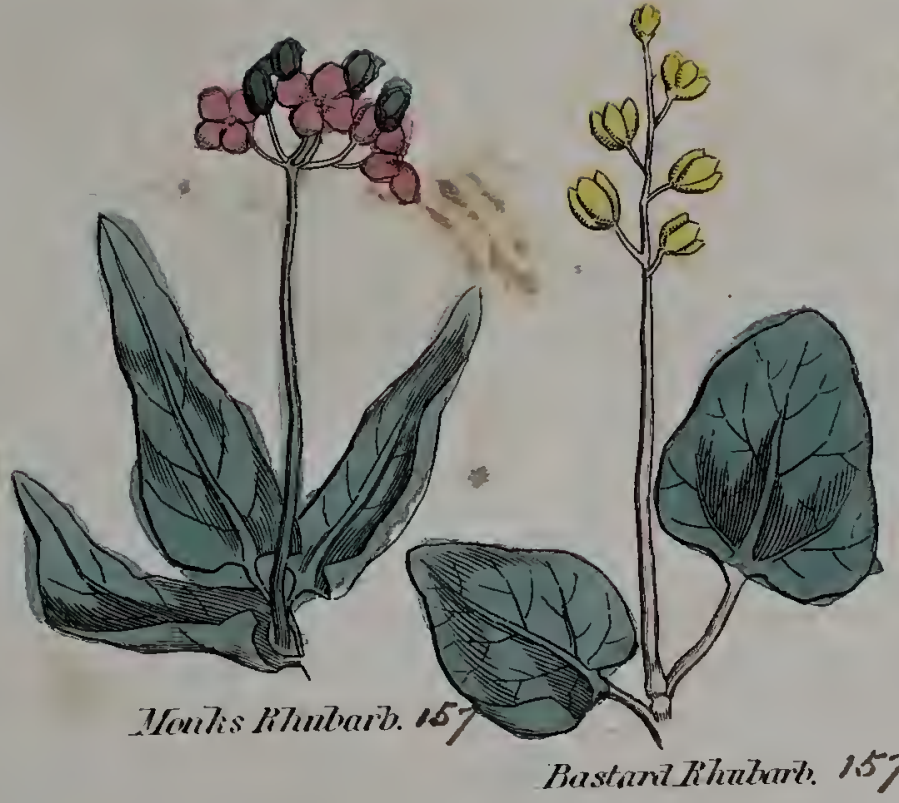

Bastantiluaturt. 1.57

Lune 21.

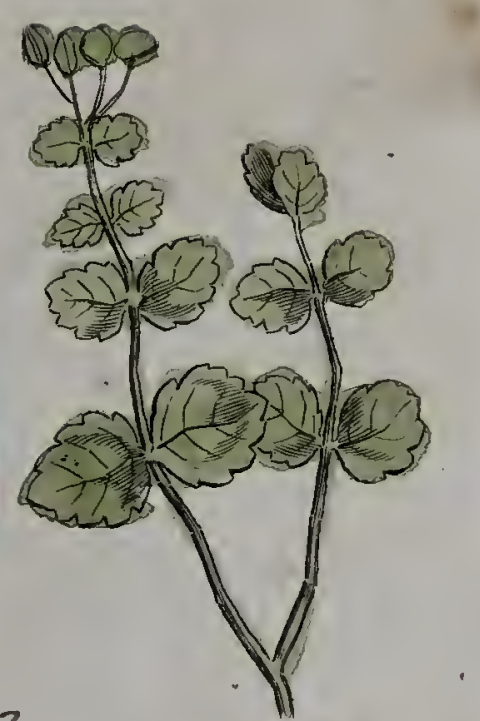

Tratcur lune. 158

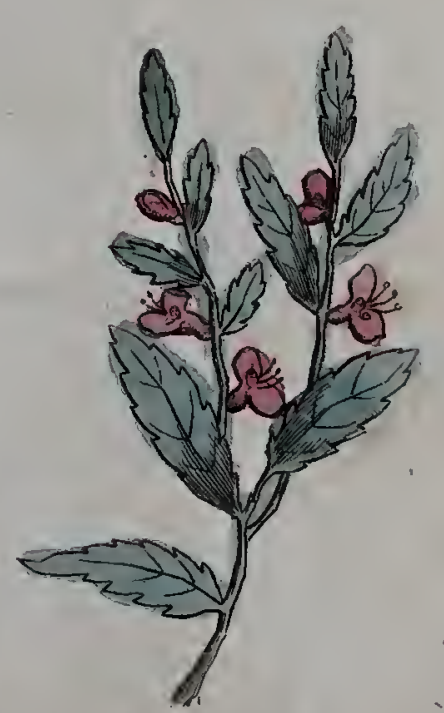

Real Retil. (ivass. 150

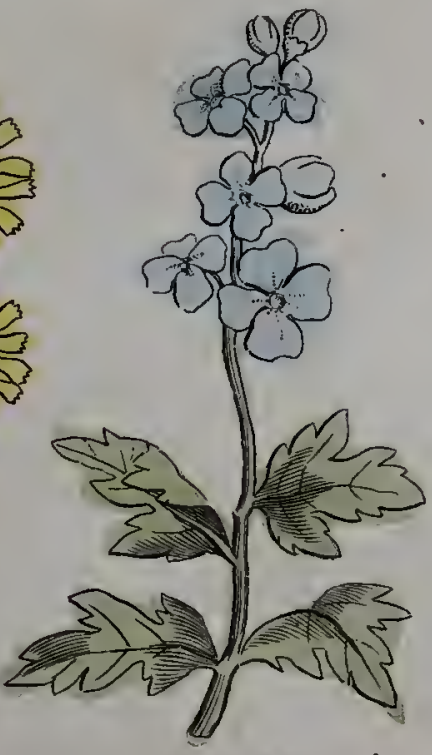

fian tiont.14\%,

Bastard Fiontiet. 151.
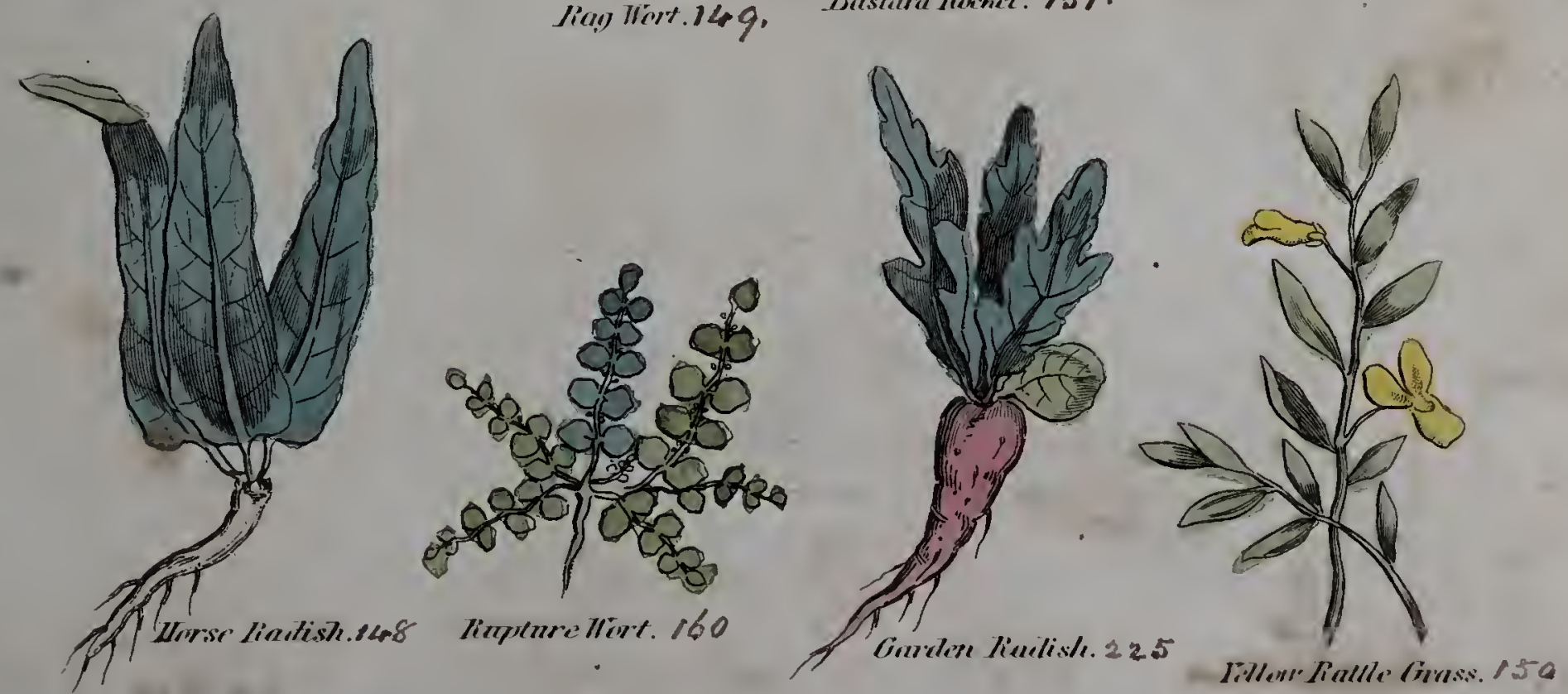




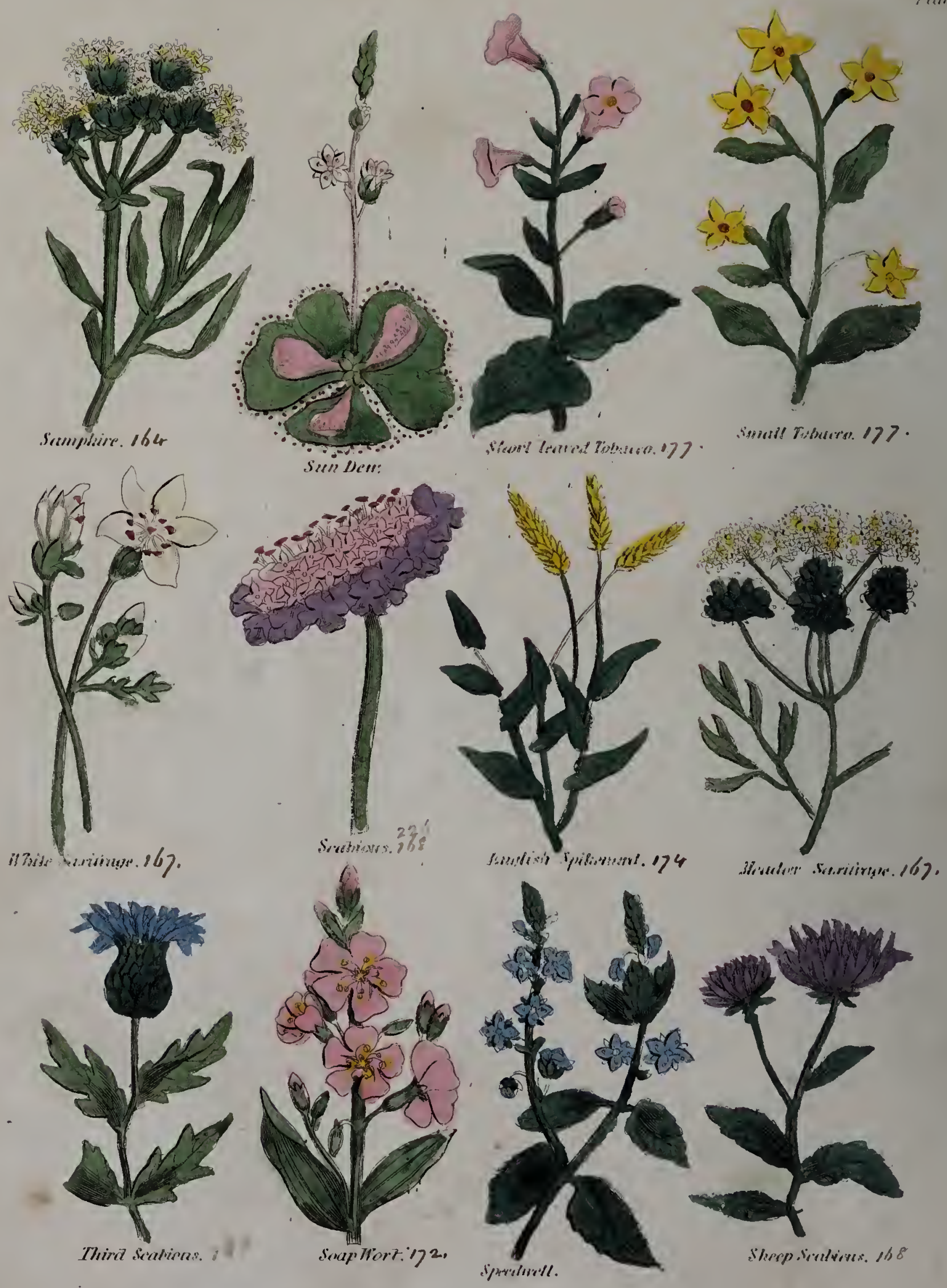



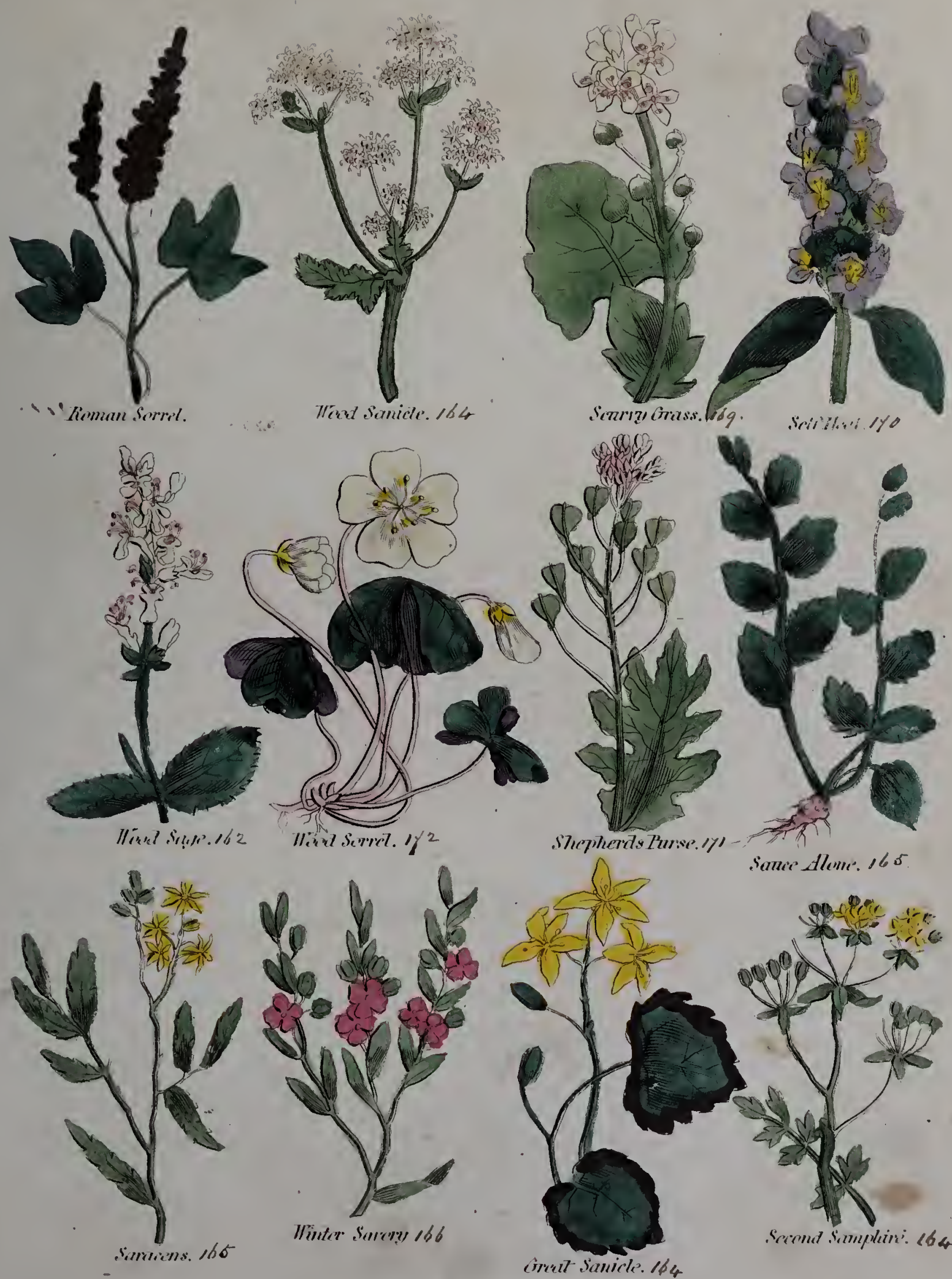

Santer Aleme. 05

serrmar cirasn. lúg.
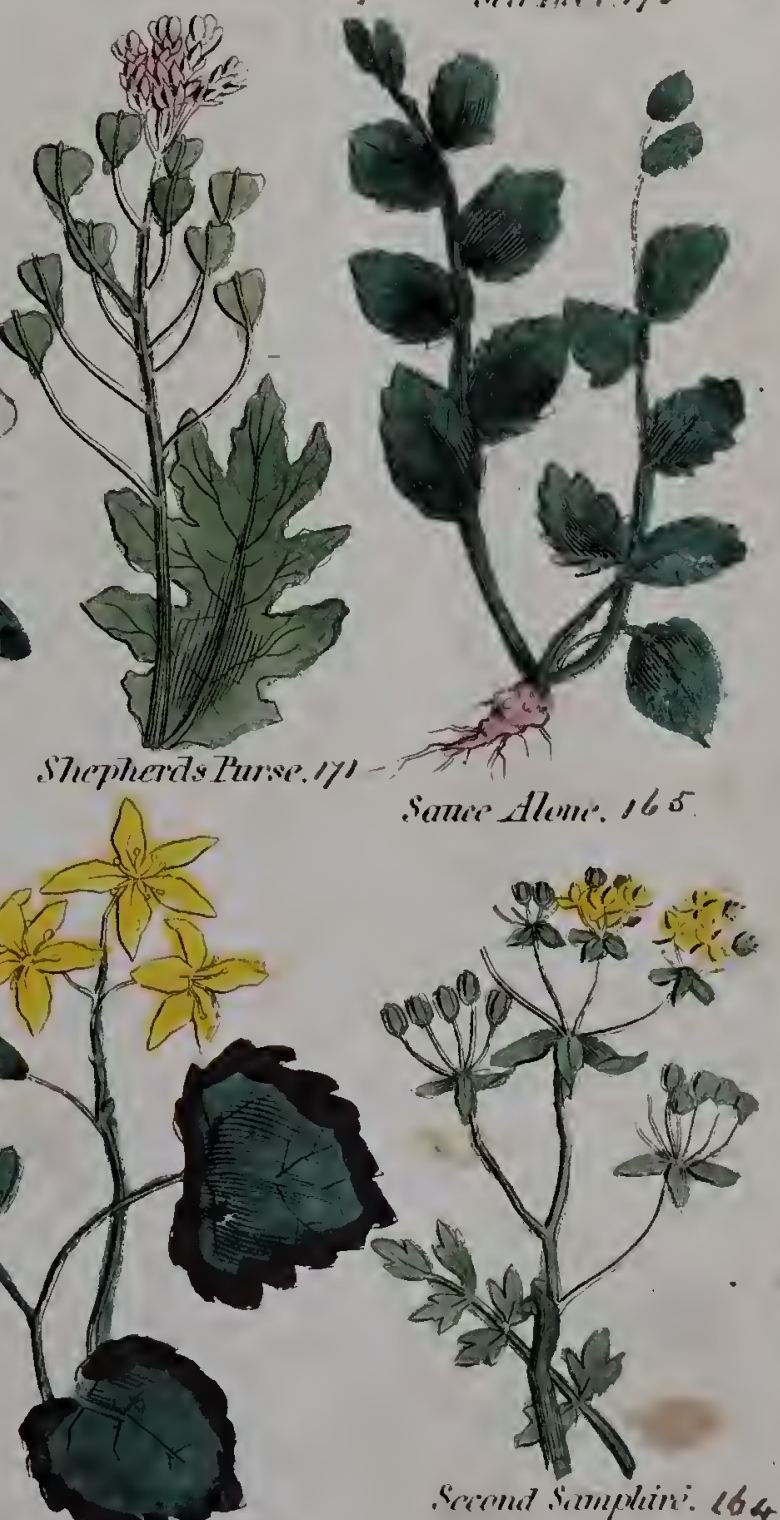

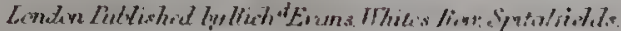





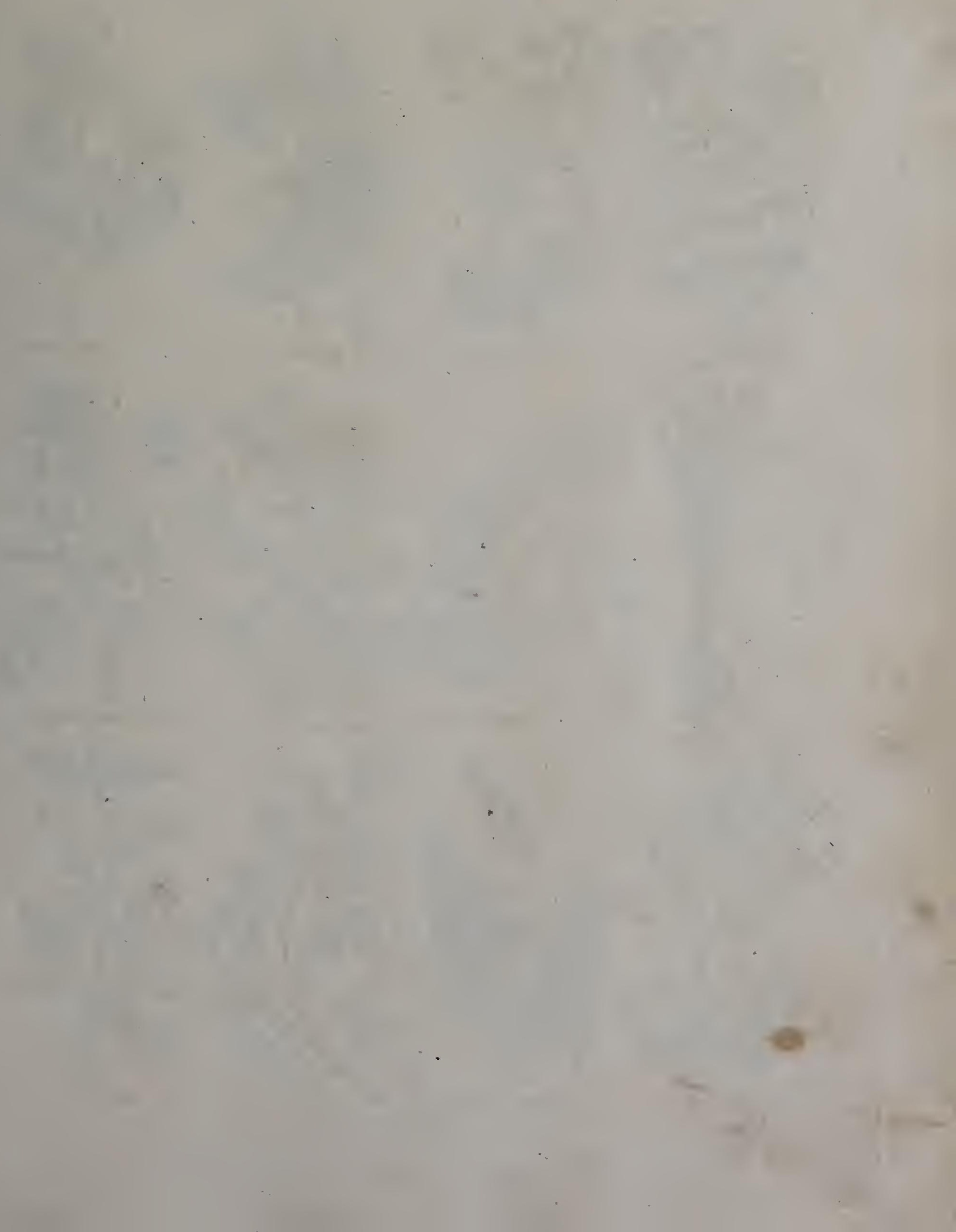


Mestr

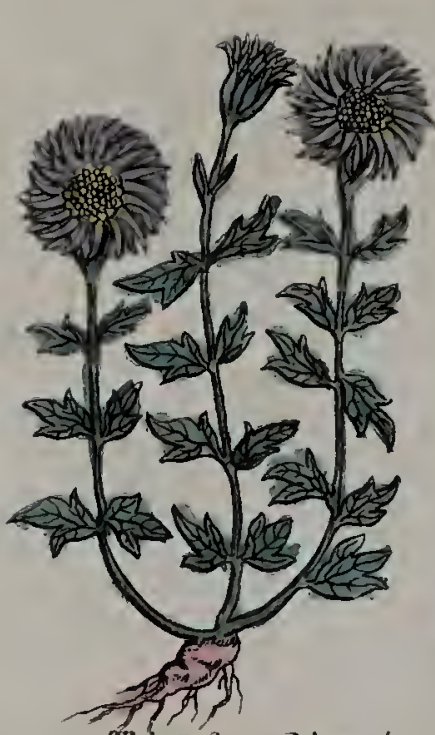

minas samminire. 164
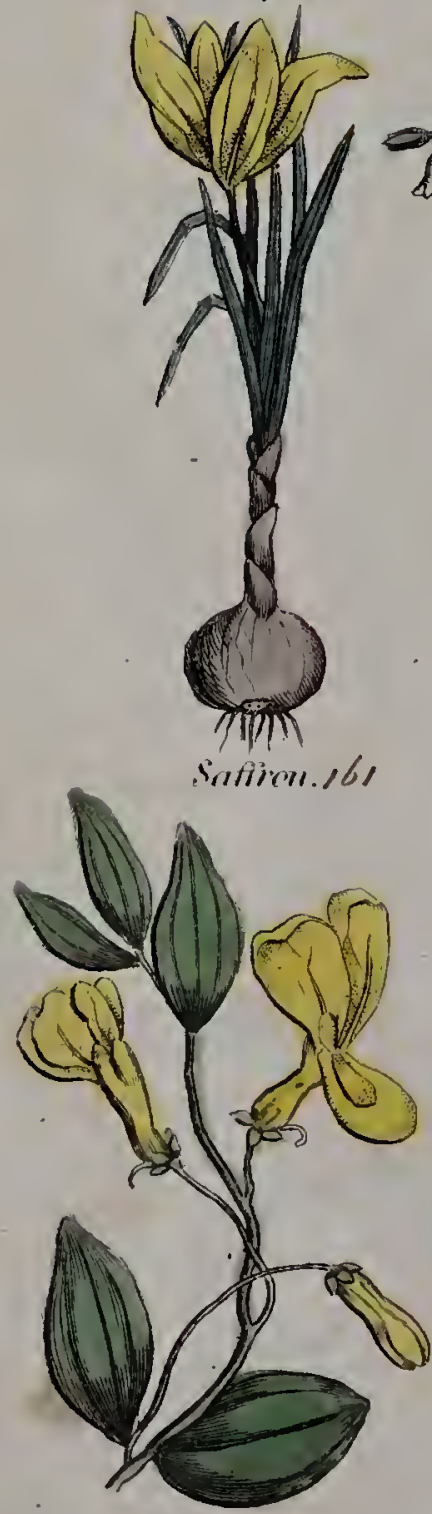

sena shrubl.

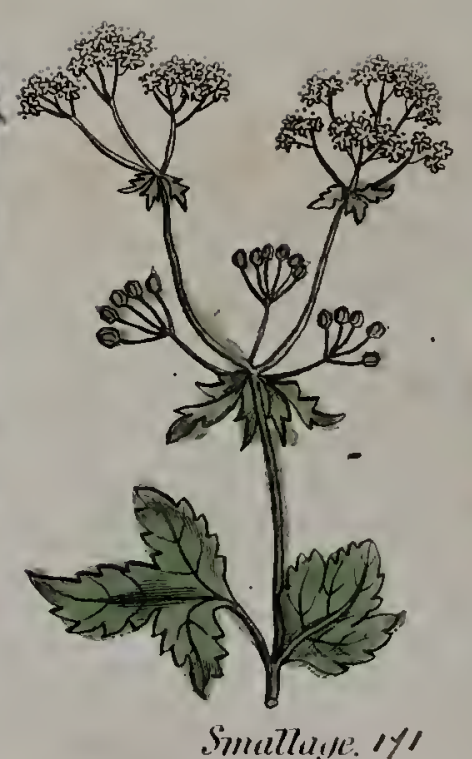

Simcllarke.1Y1
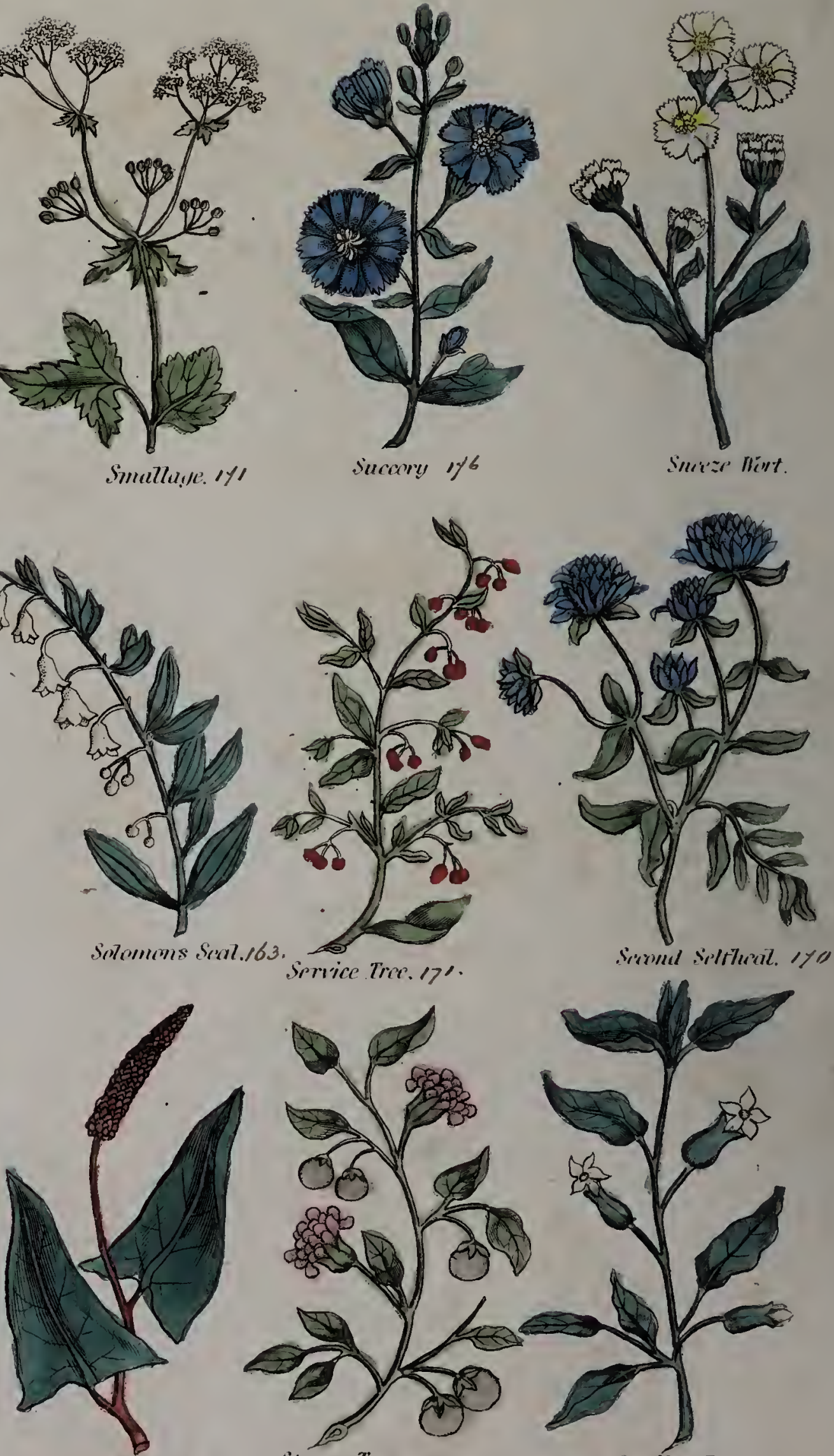

strimer Trer.

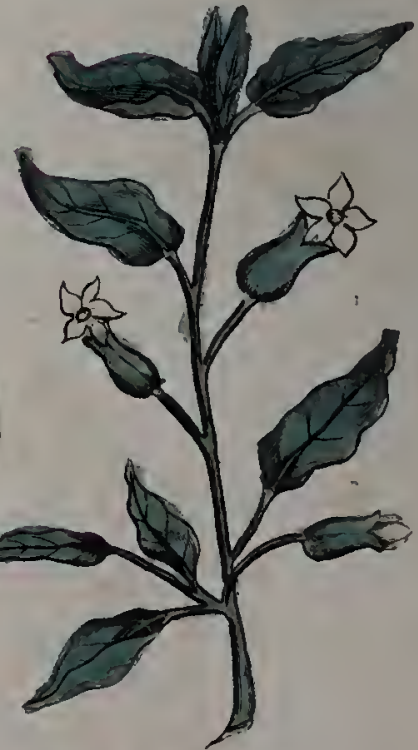

swallar Thent. 218 

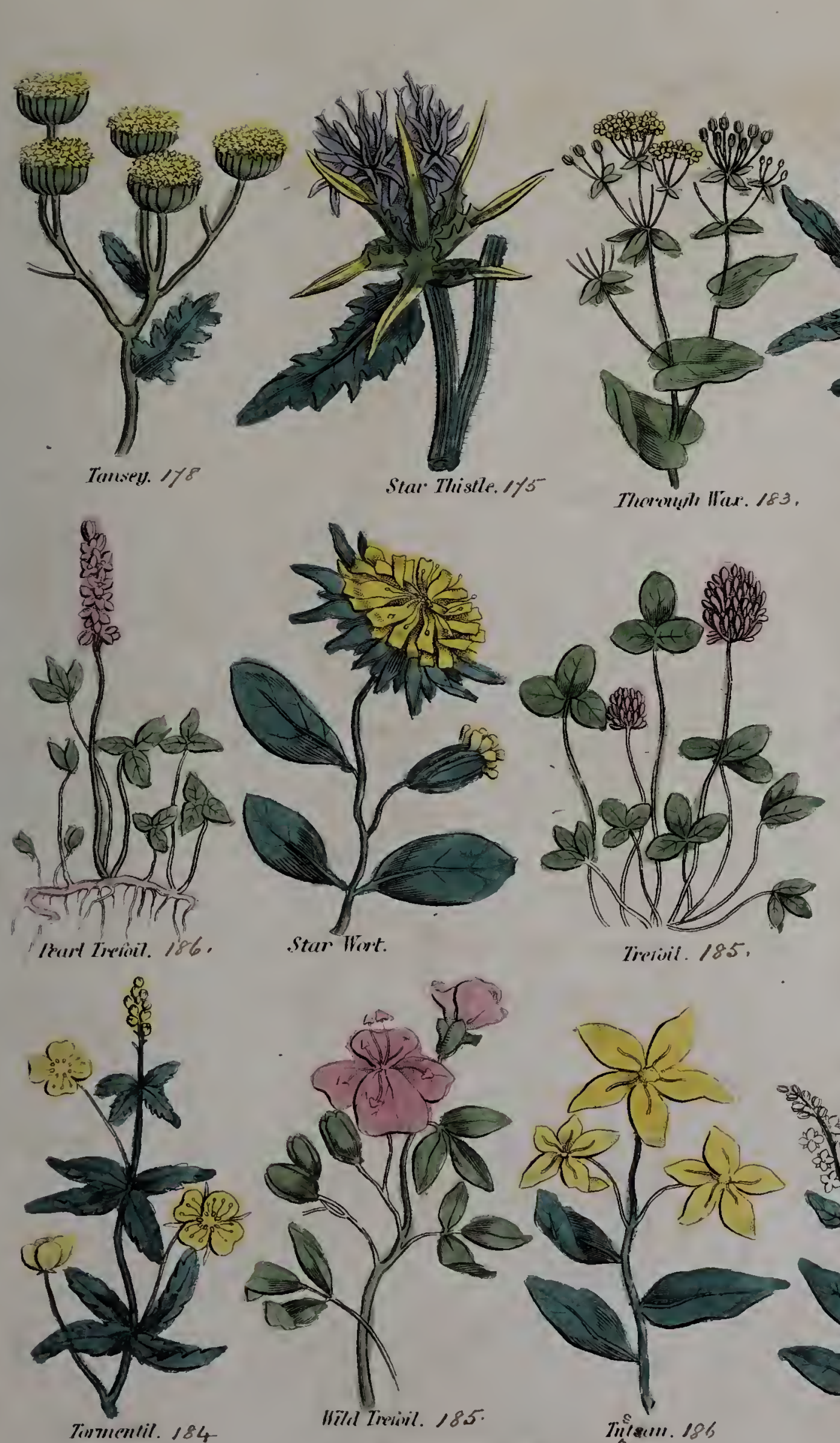

Tiifseriा. 186

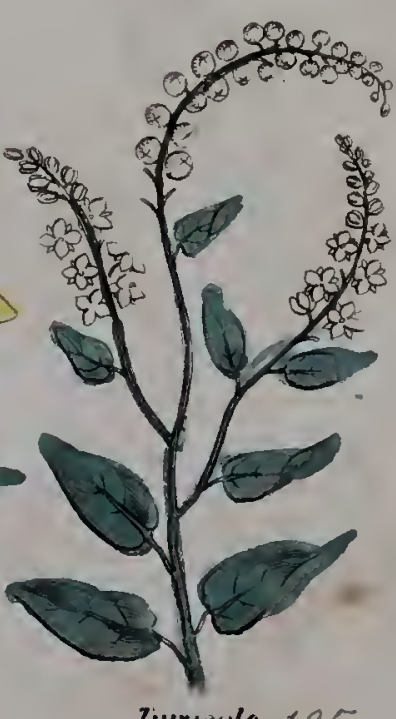

-

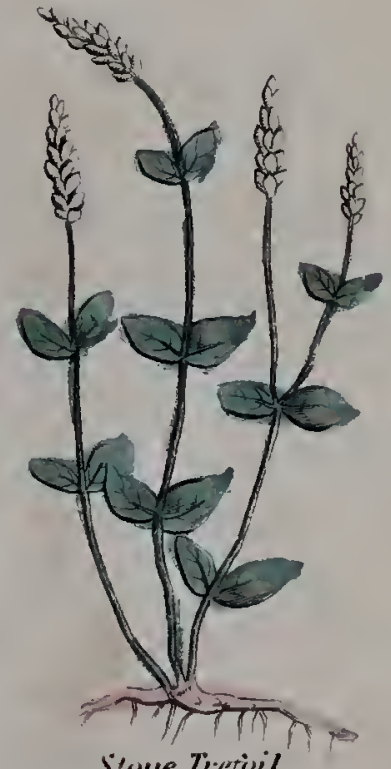



,

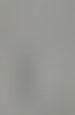

1

(15)

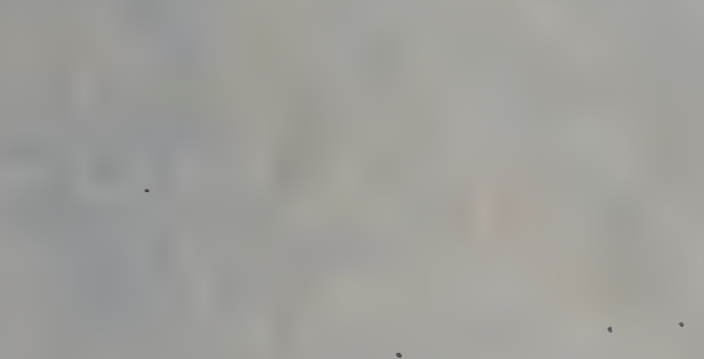

$y^{4}$

1. 

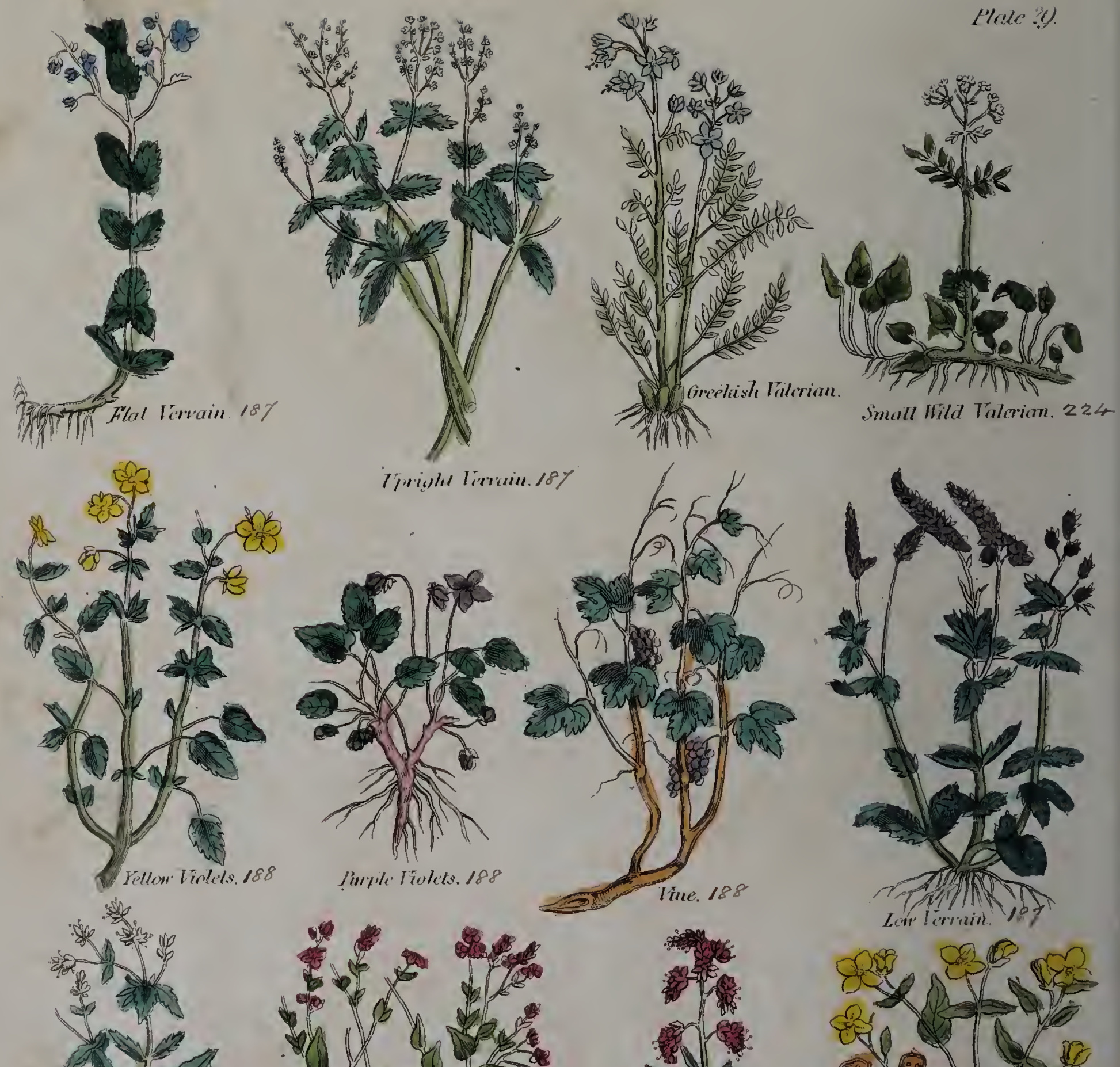

limpile Tolets. 188
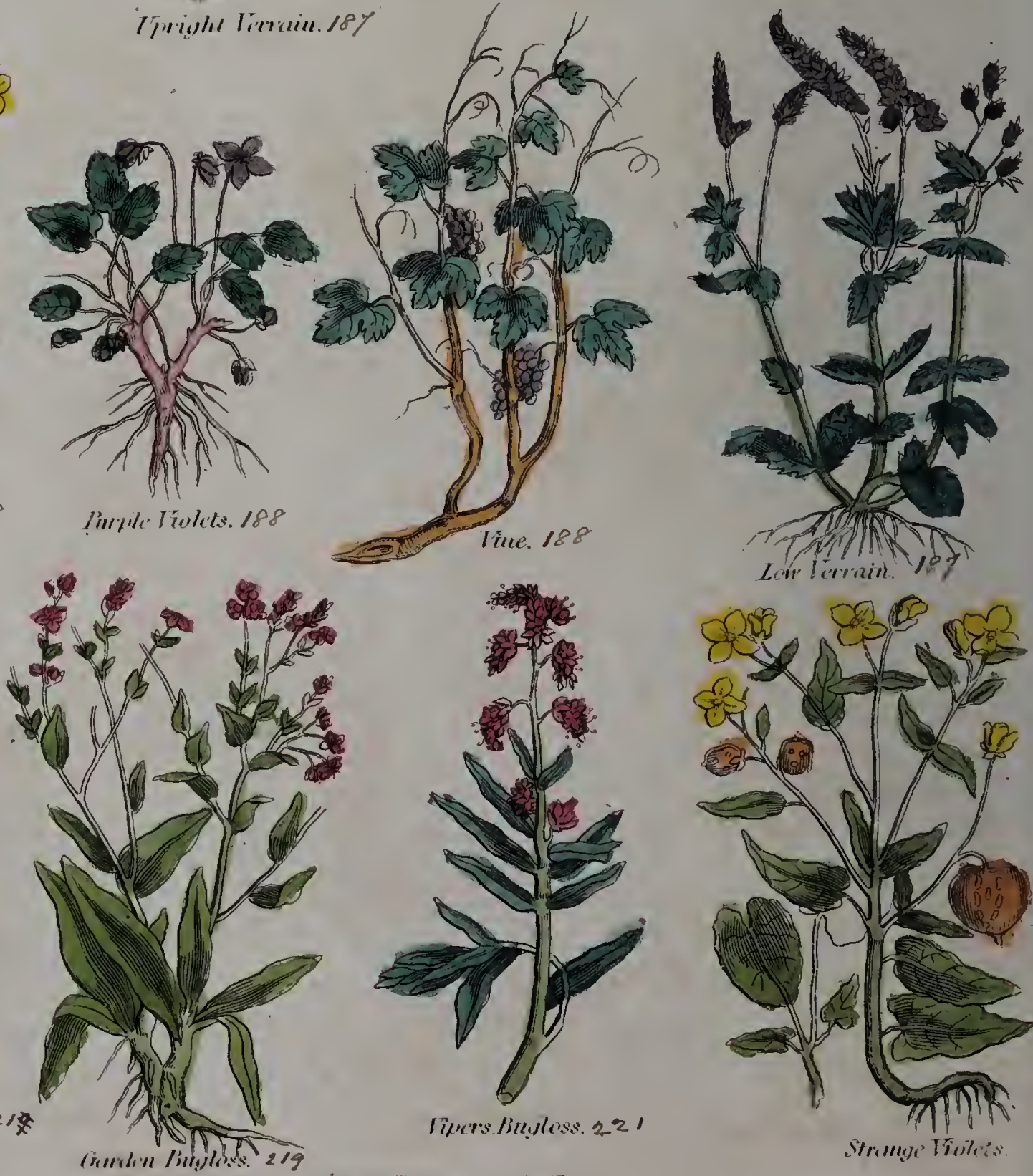

lipers Busless. 221
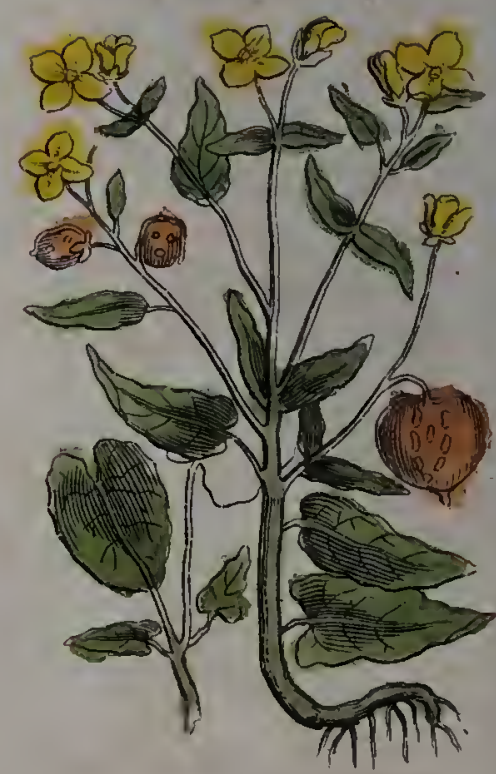

strelluge Thesters 

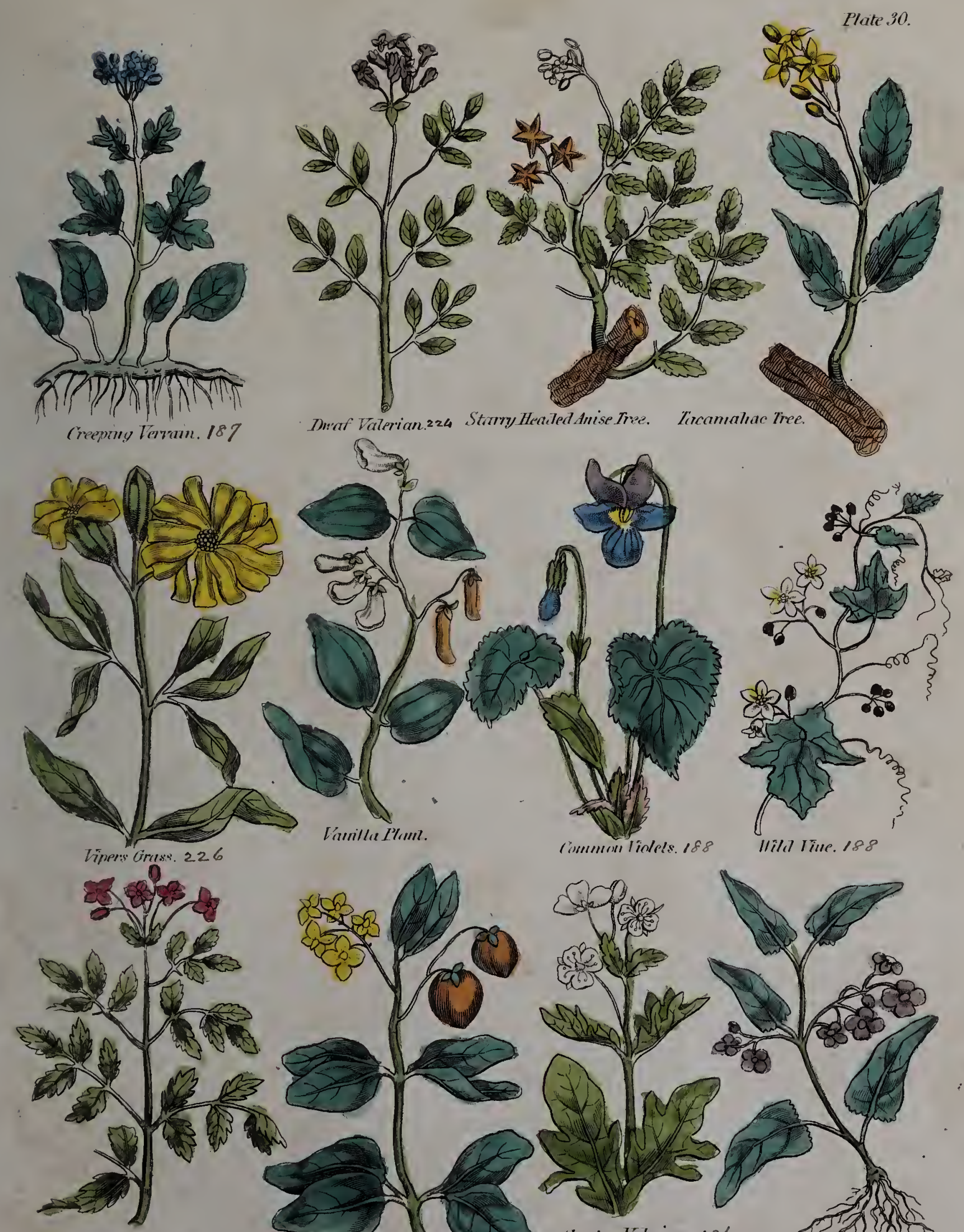

Great Inikd listerimen 224
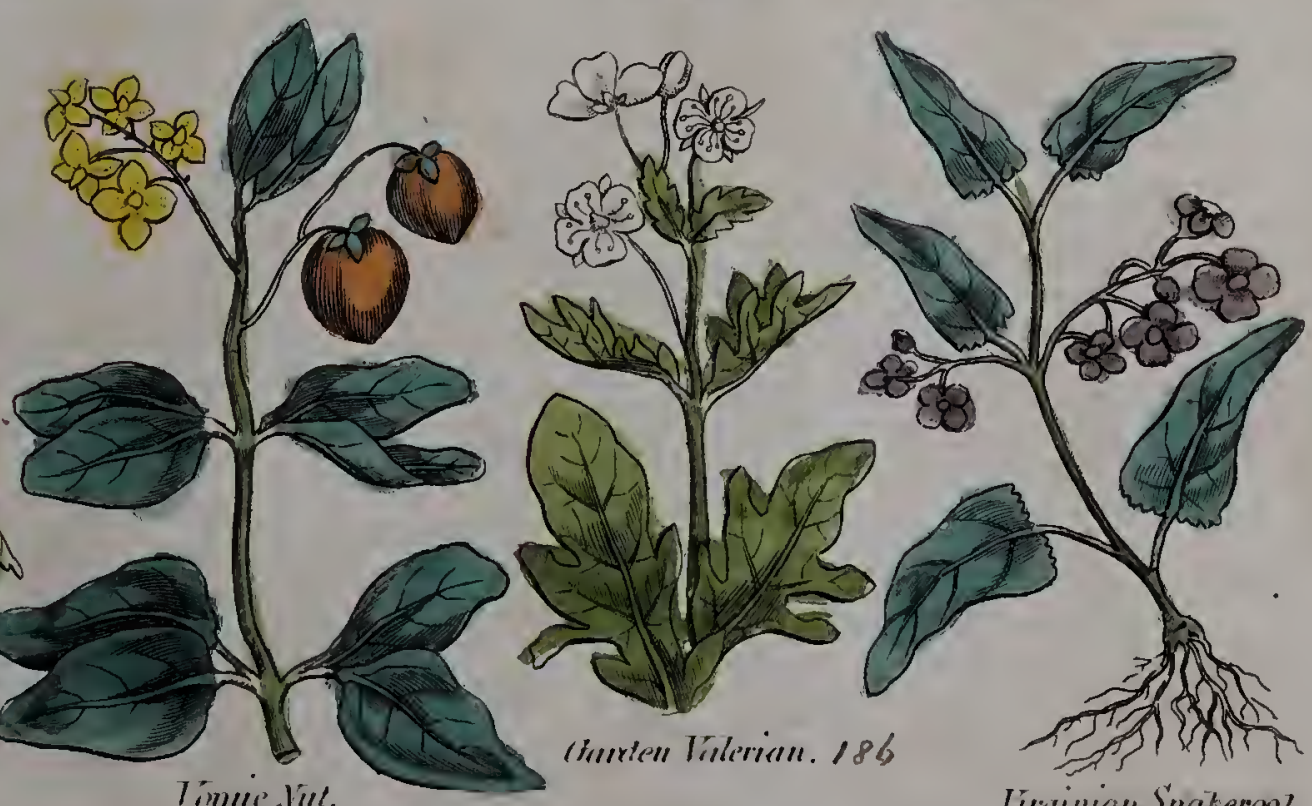

Iirginian Snatiement. 




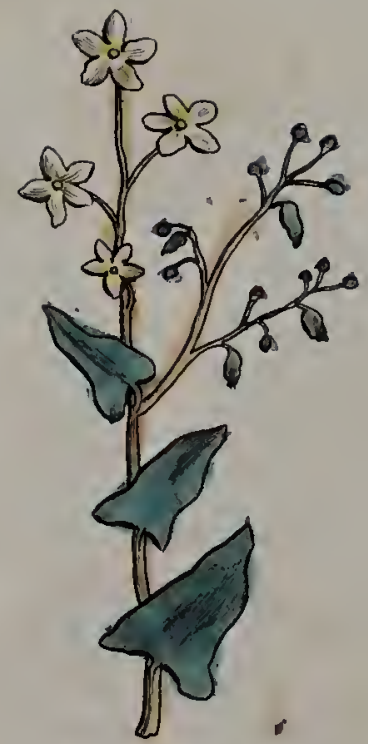

Spanve Taure?.
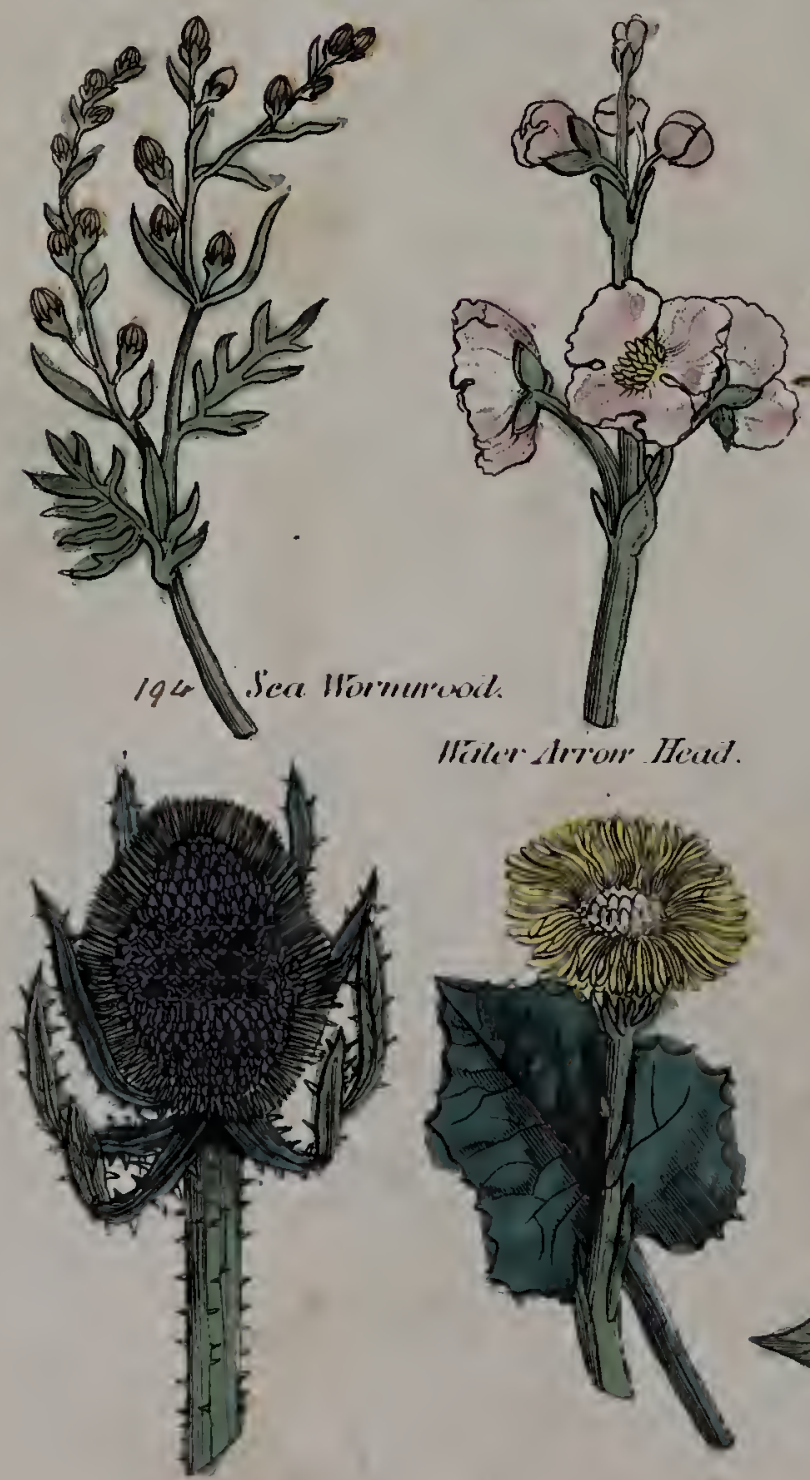

Tea $=7 e^{\circ} \cdot 181$

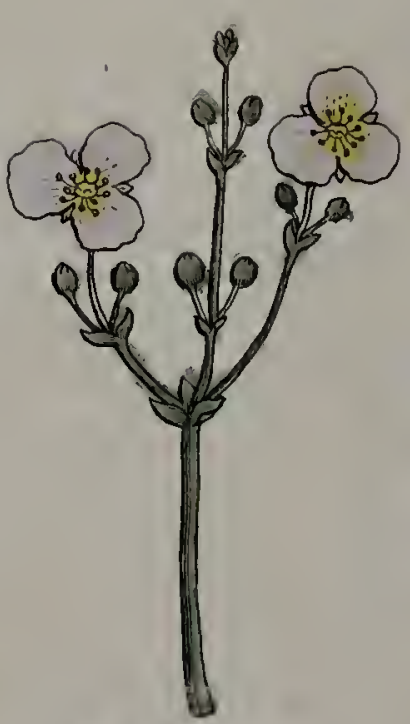

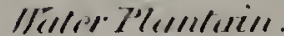

Hêler drvon Head.

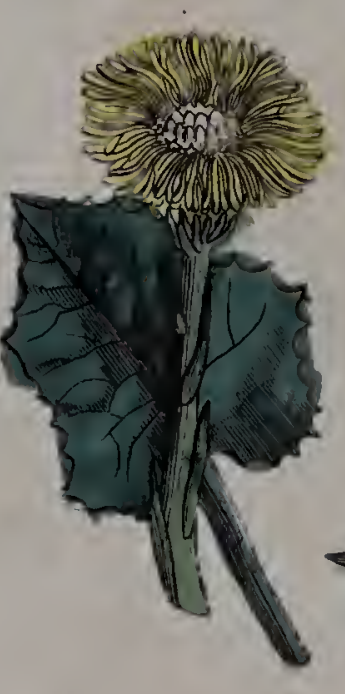

Cirecil collstiout: os

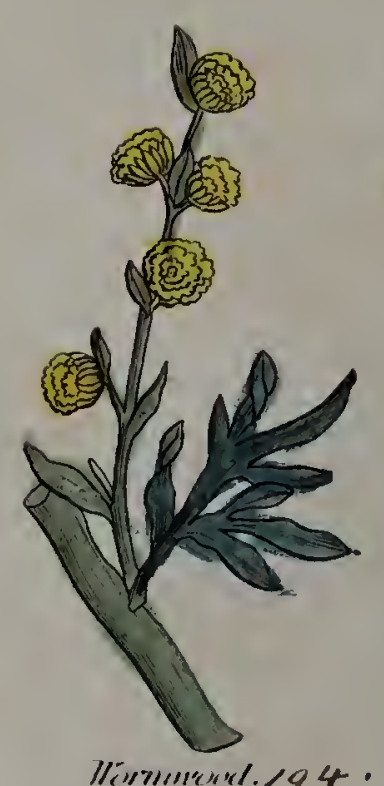

Minemenentel.194.

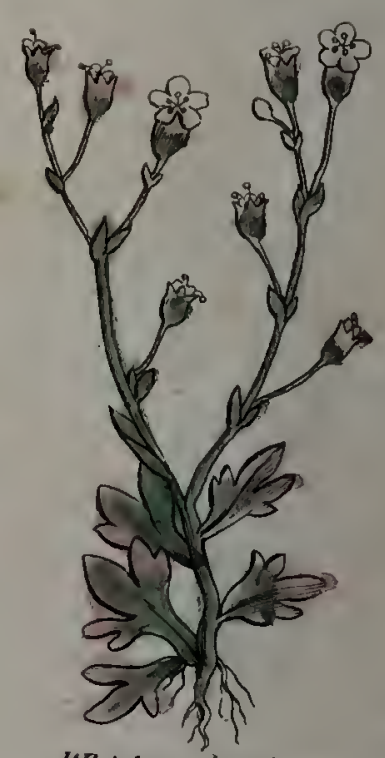

Ihirlent irrelsis.

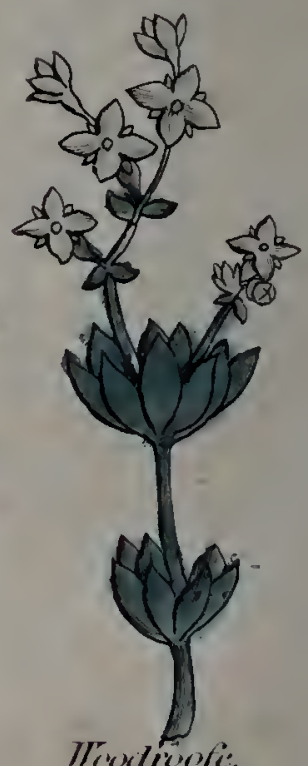

Ilitli Thisitle.

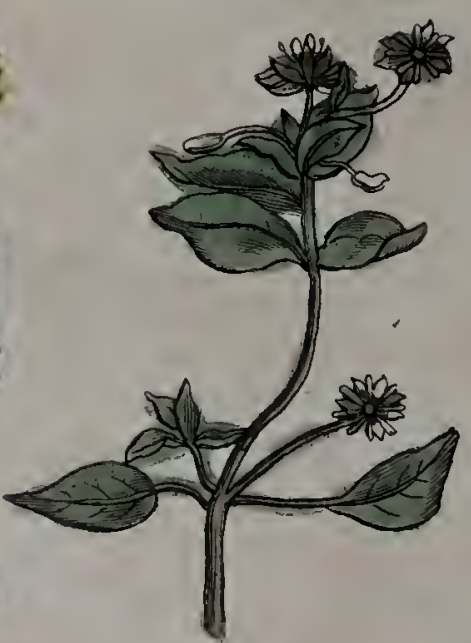

Chictireced. 48

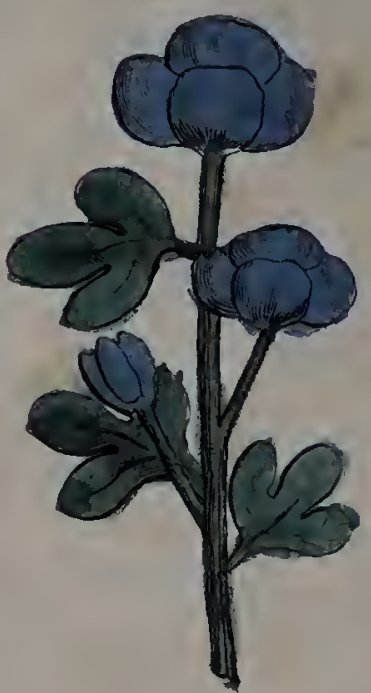

columbine. 53 

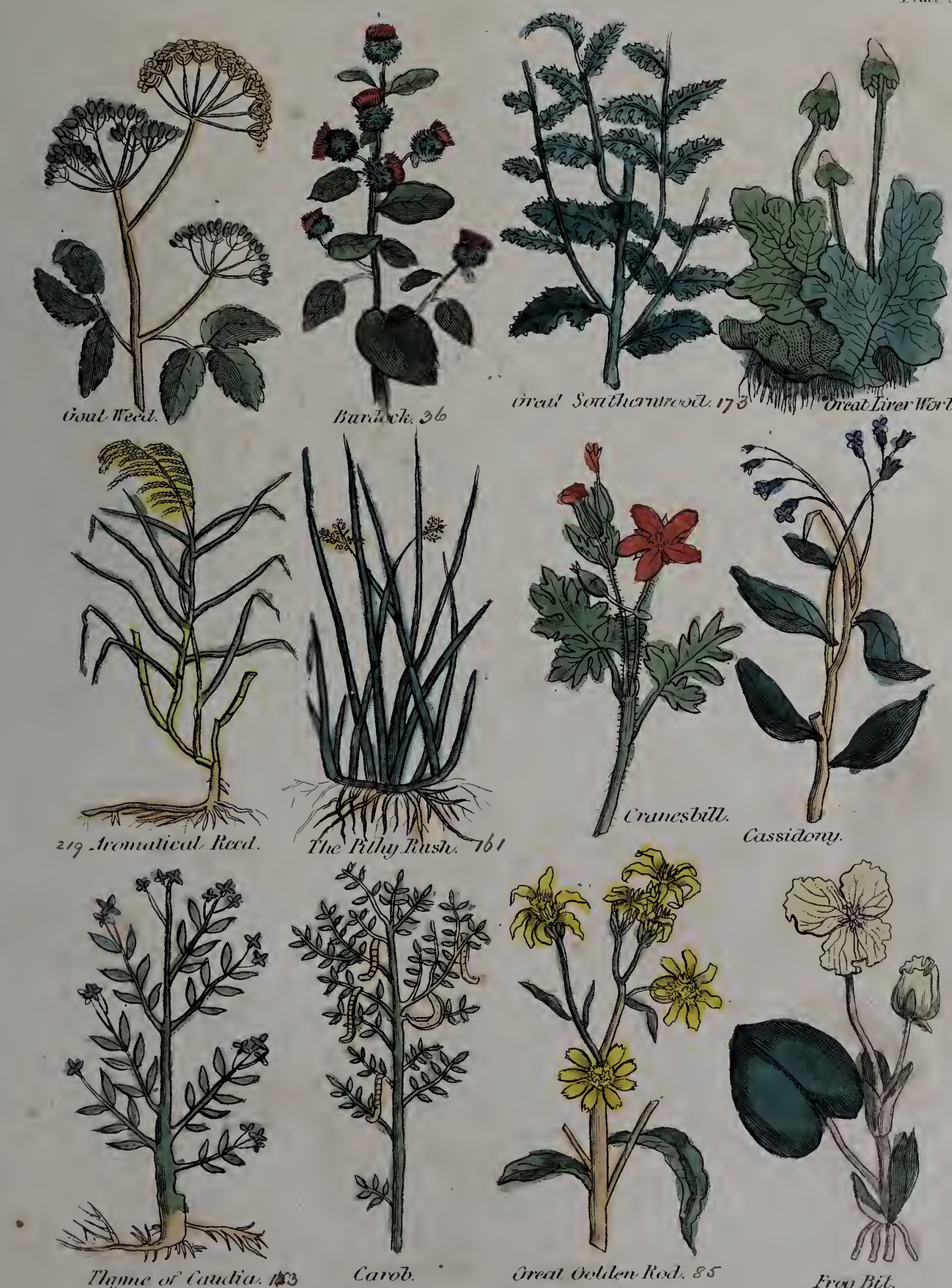



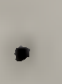

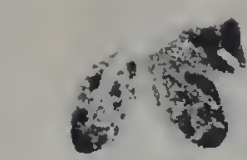

at:

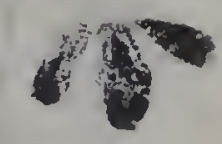

教展

งุด: 


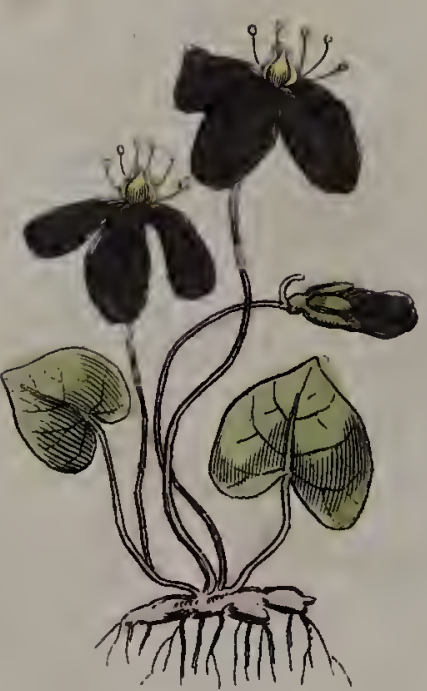

Scarlet Thotet. 188

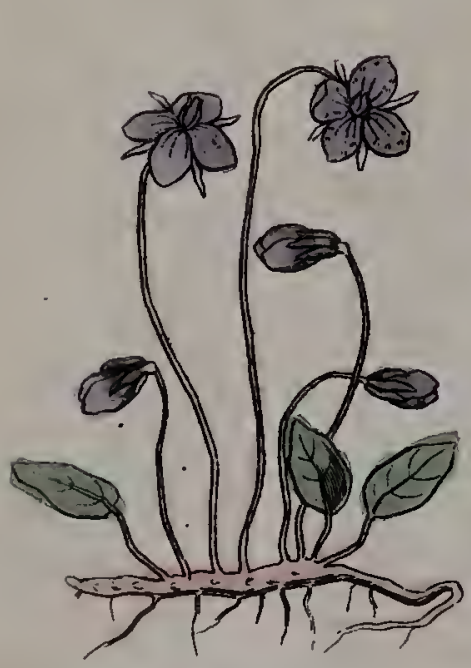

crepping Violets 188

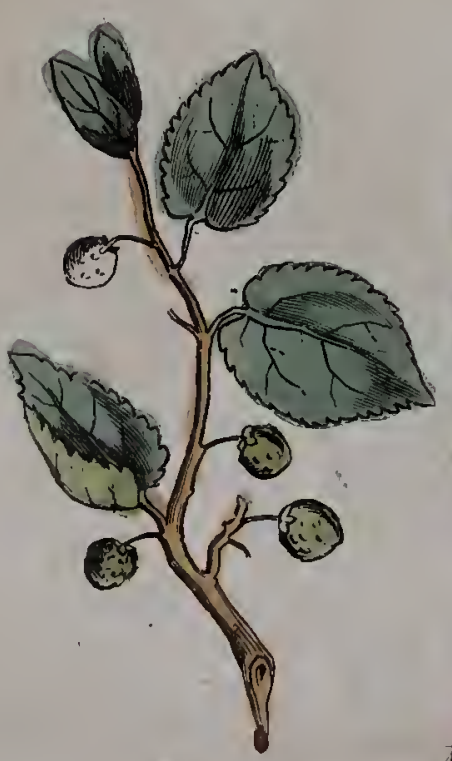

IIalnut Treesos.

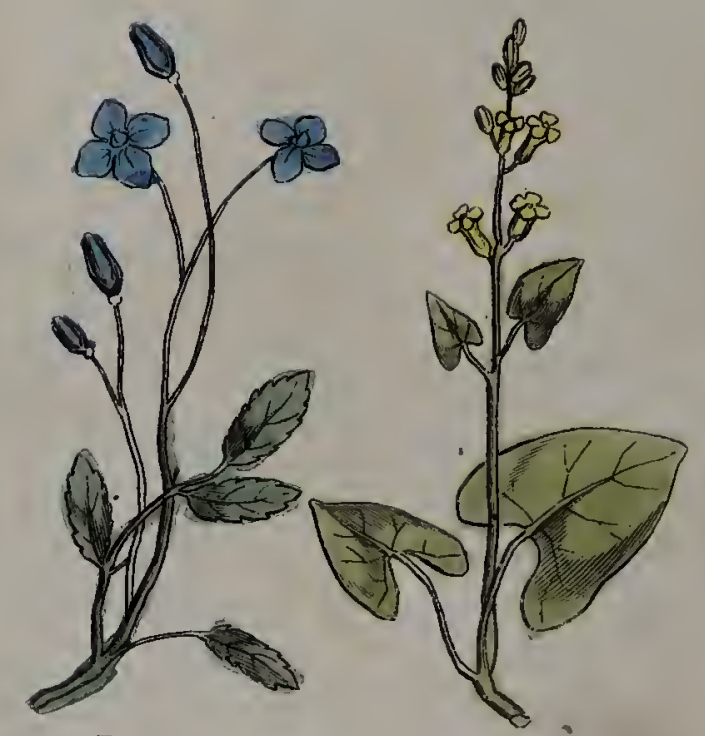

Vipers Tongue.
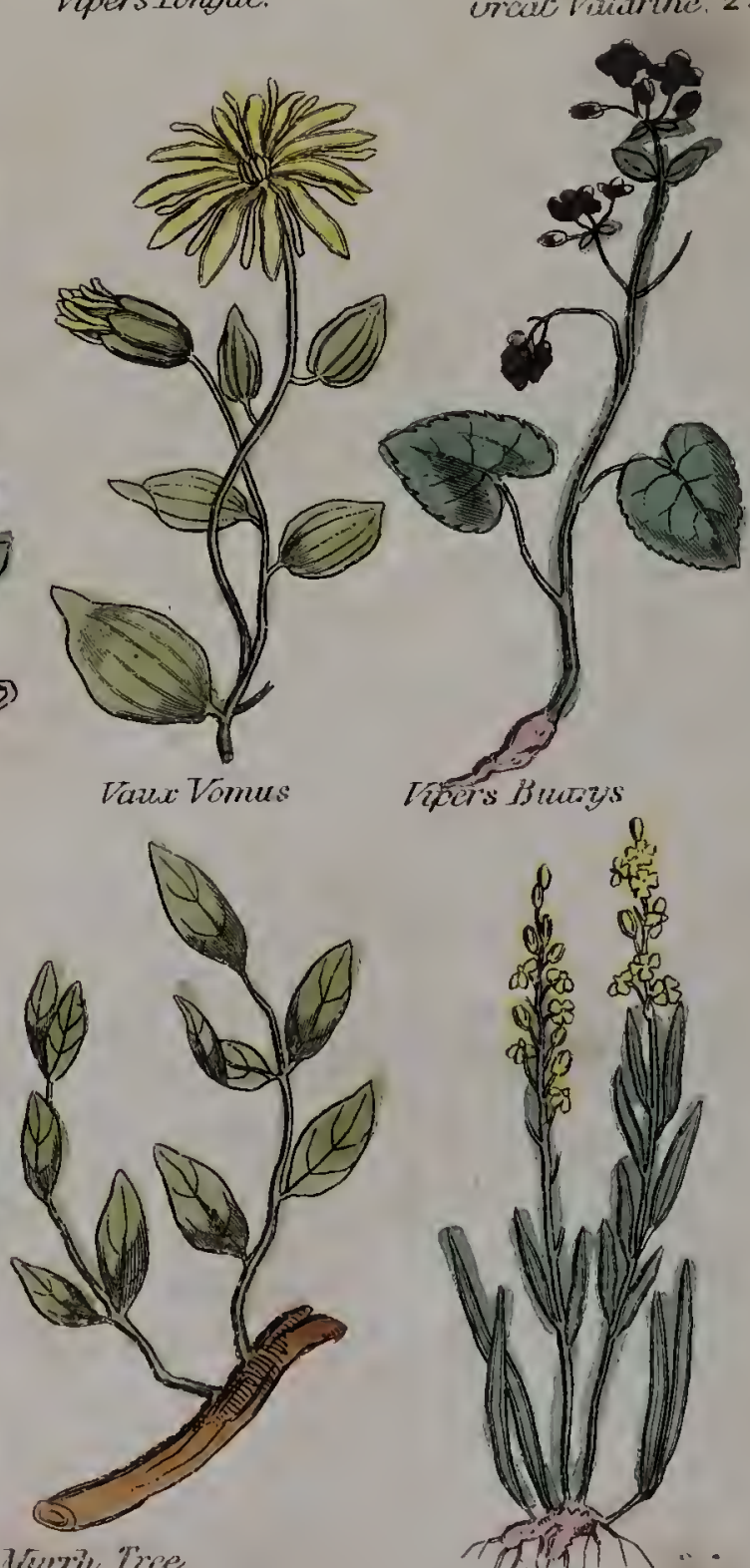

Myrria Tree

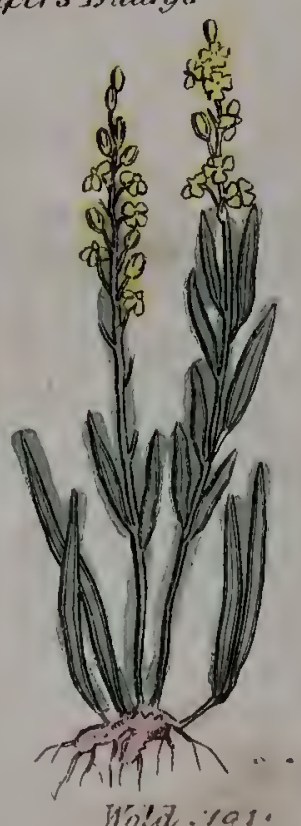

Plake 33.

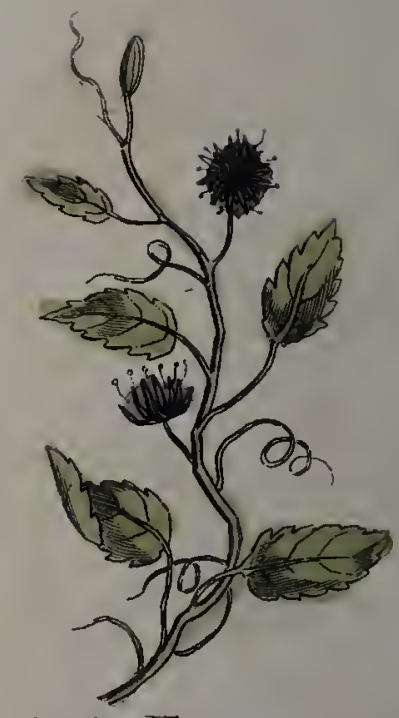

Grooti Tine.
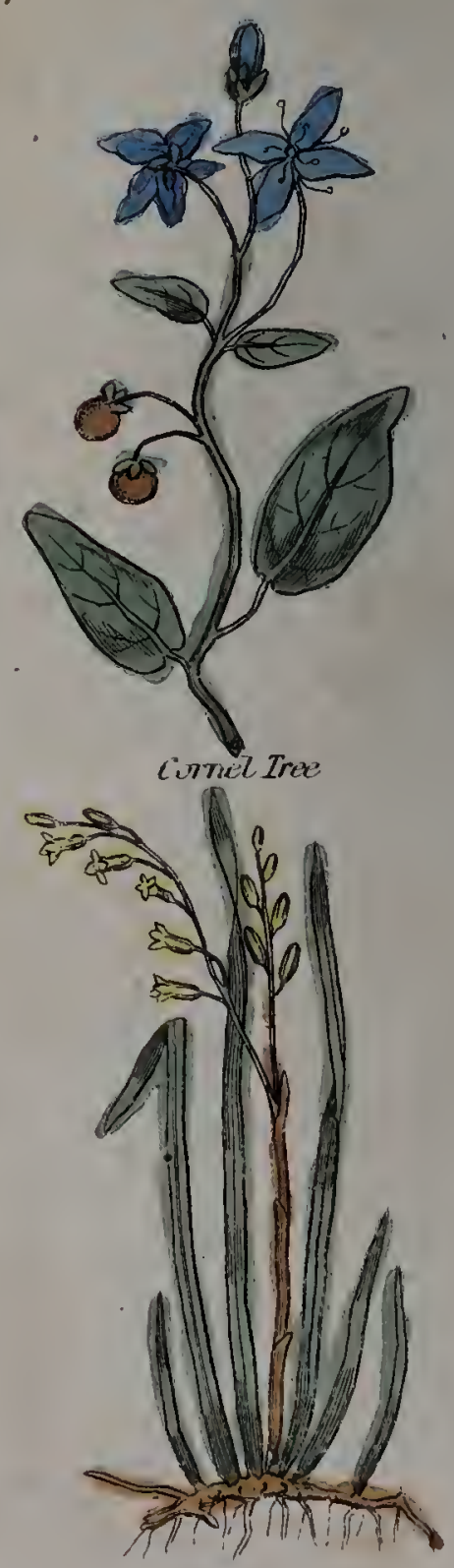

"IJad 193 

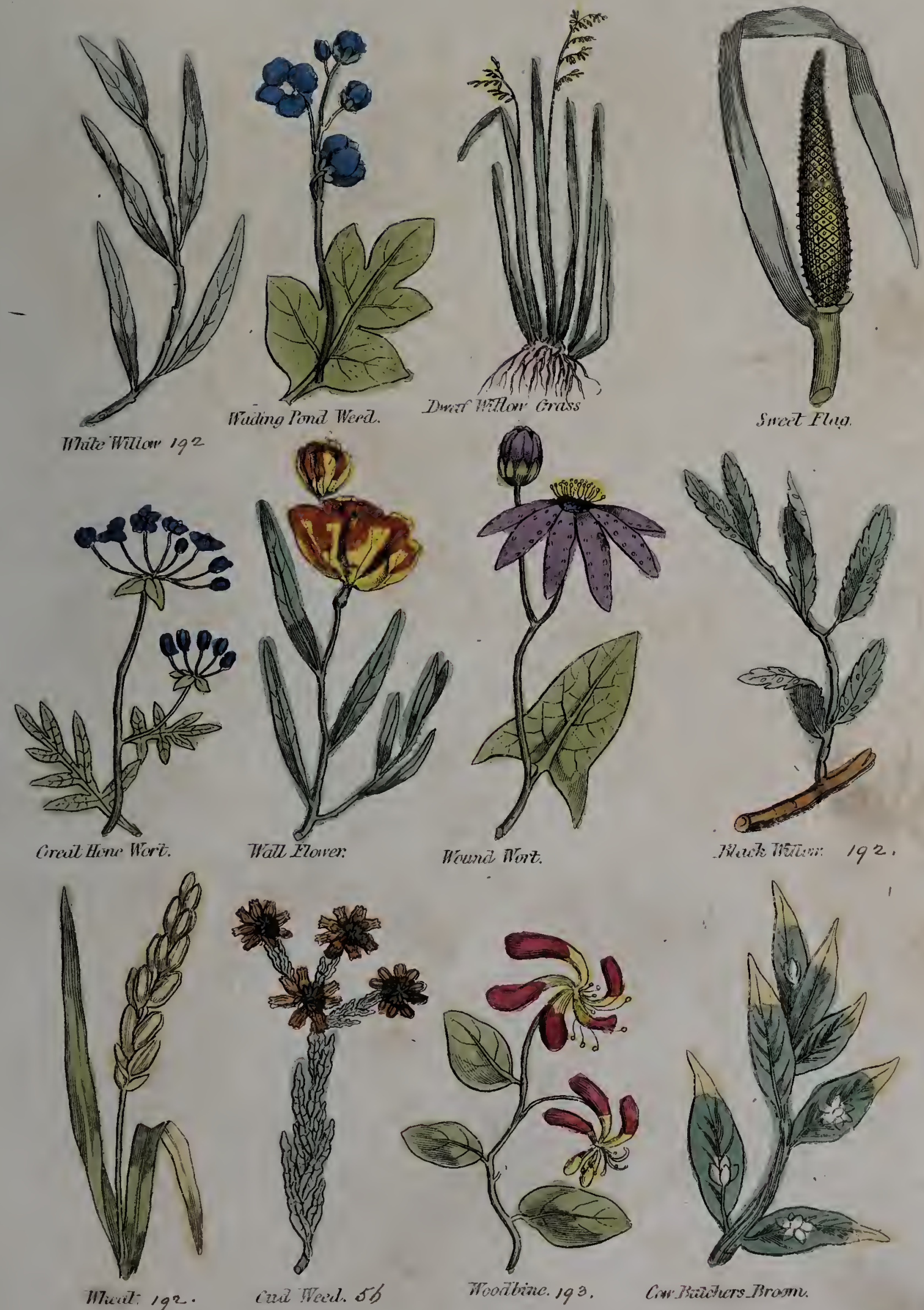



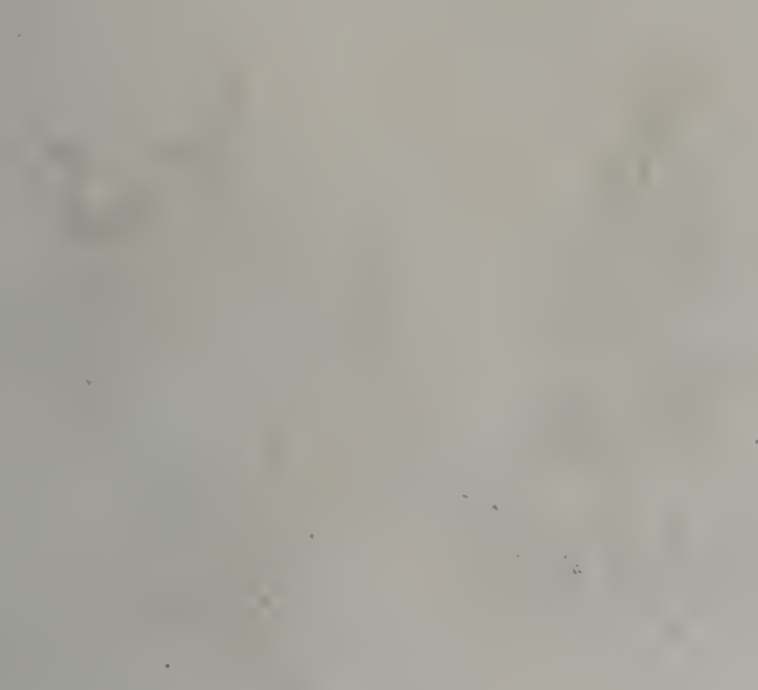

.

,

1
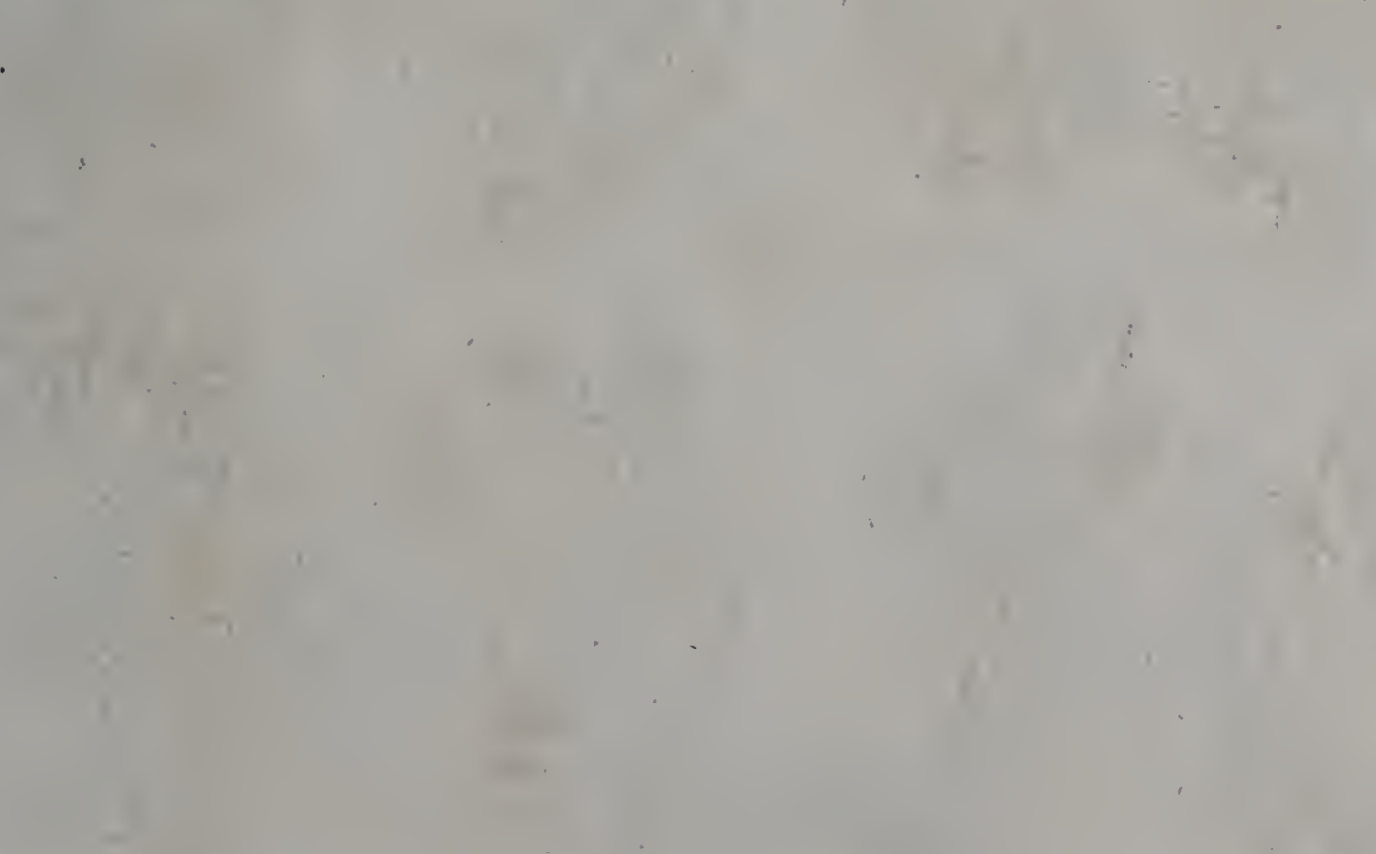

.
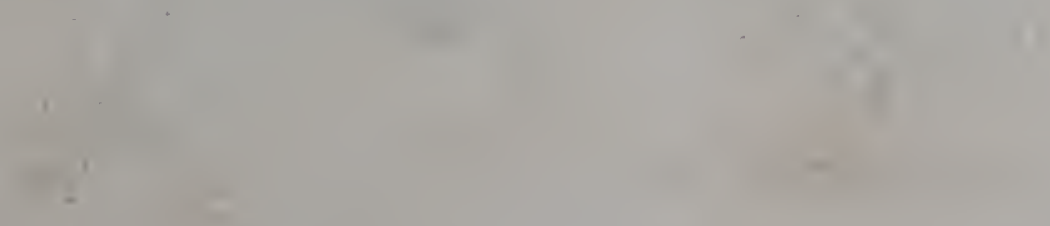


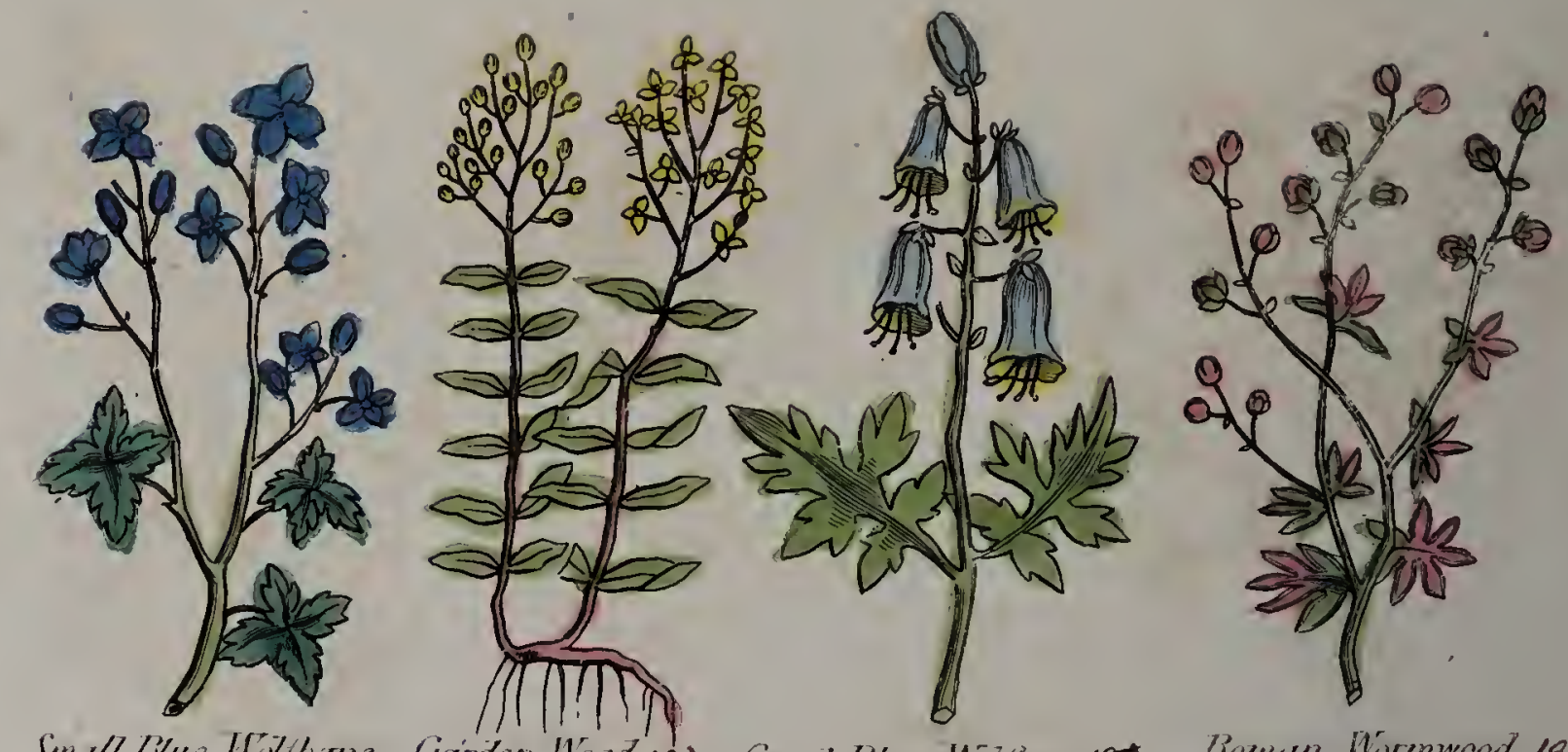

Simall Dlue Totrbane

Garden lloudigs Great Blue Vothianot.

Pamen bormwood 194.
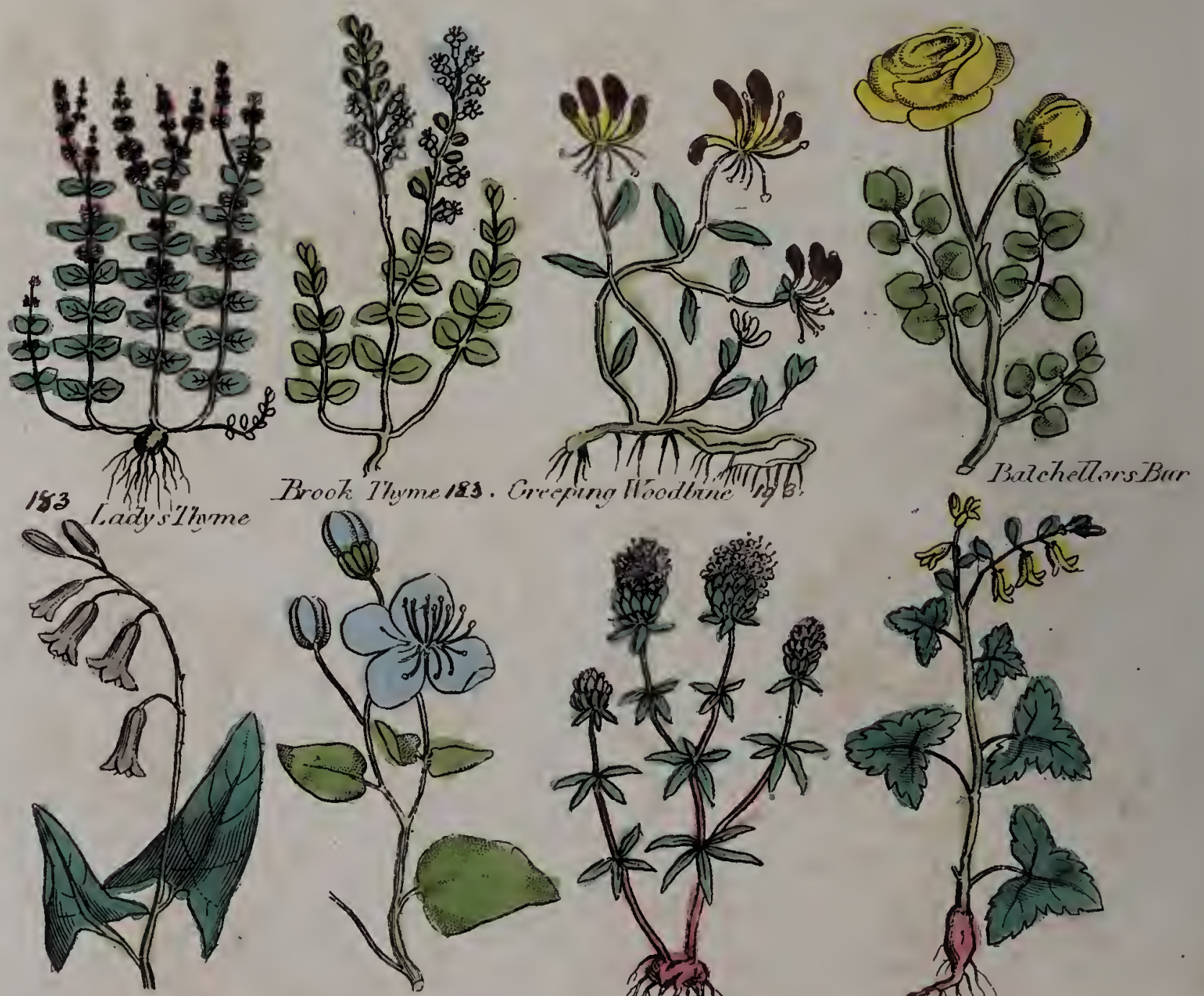

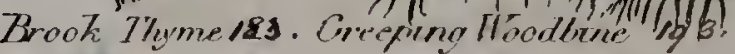
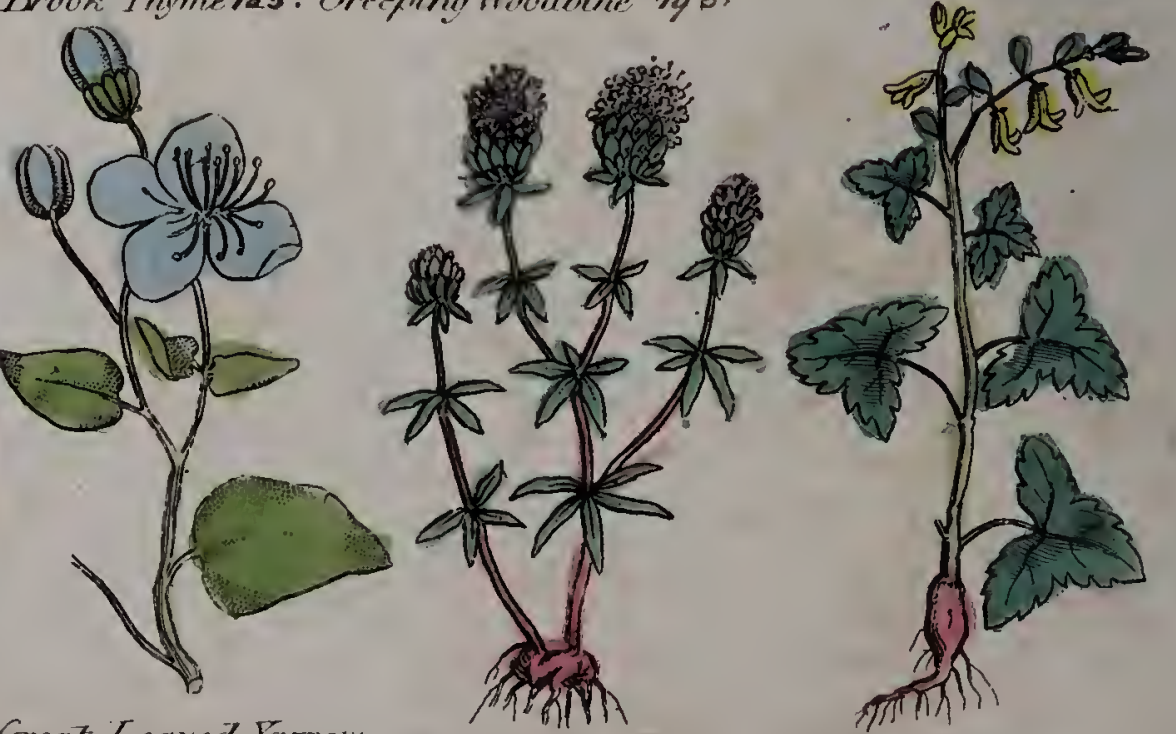

Widdew Weed Great Leaved Yarrow Thyme or Condia Kellow Niblibane. 


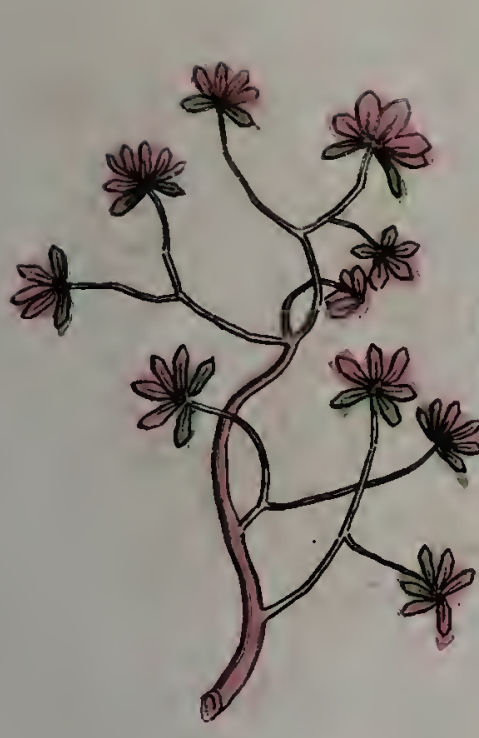

Irecer

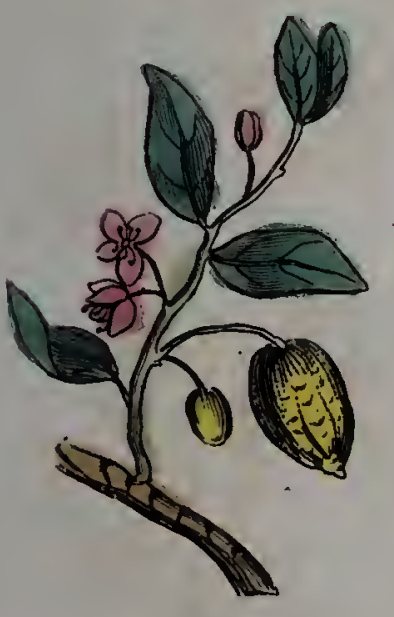

Lernon Trees

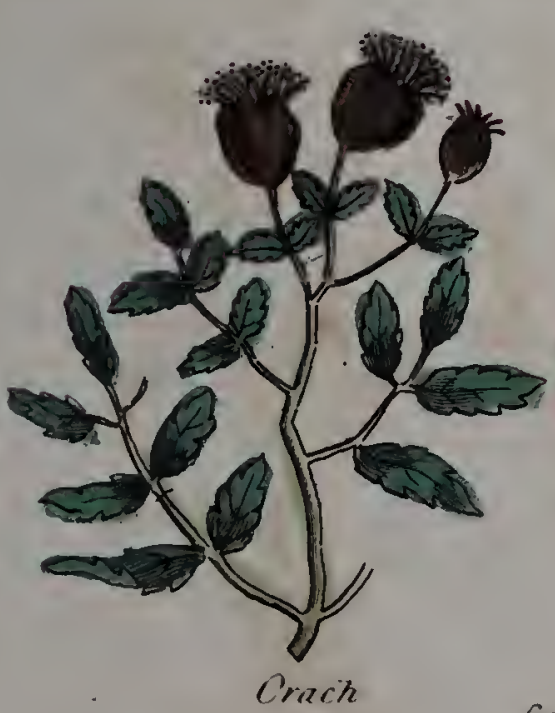

Good King John

Globe Thishle
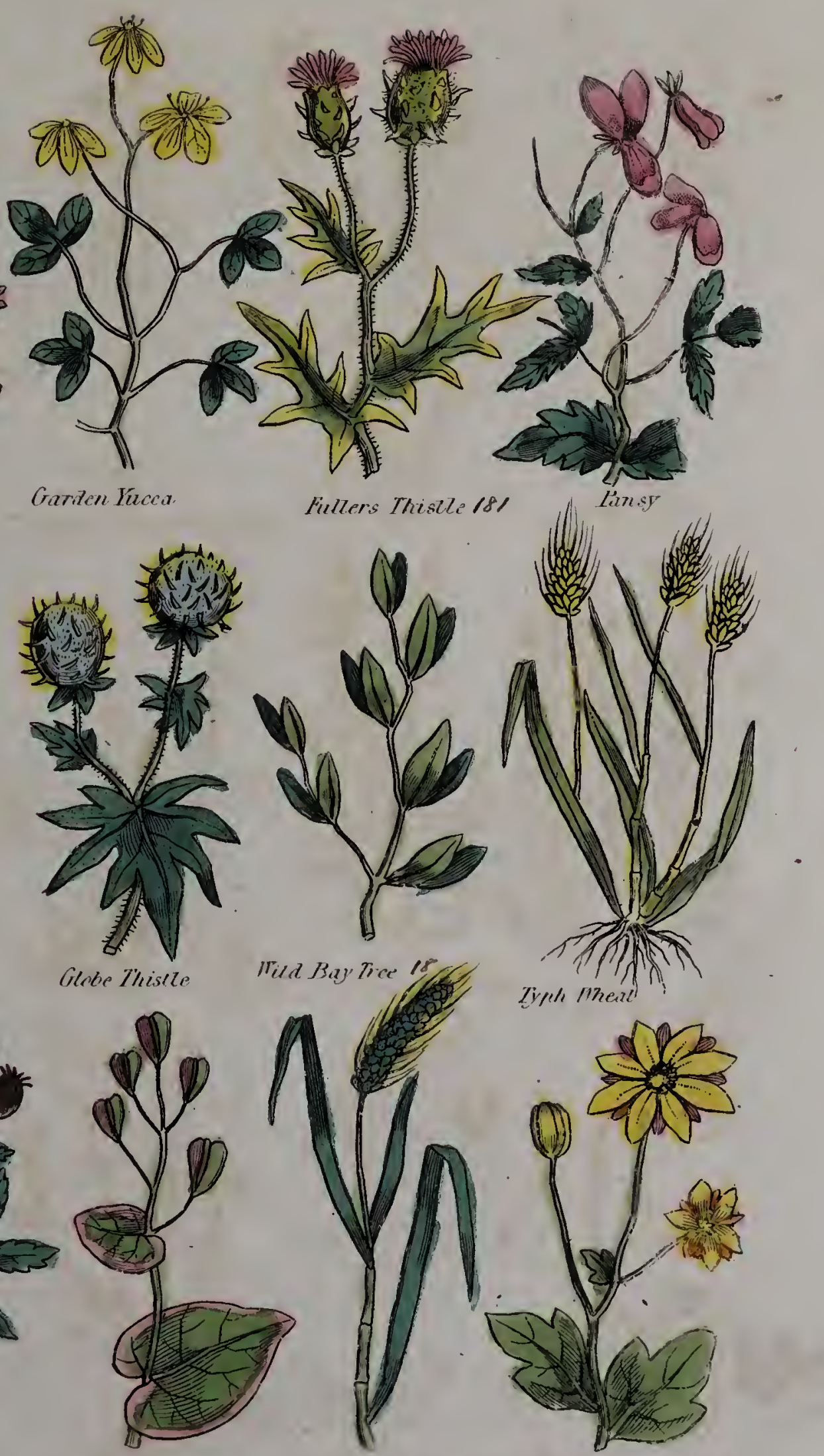

Iroman IIheal
Typh meat?

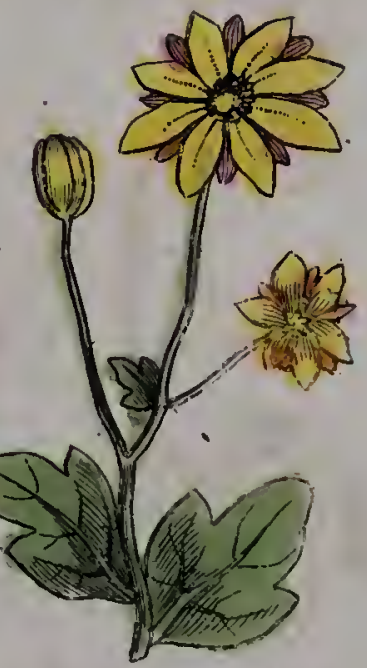

like thert 



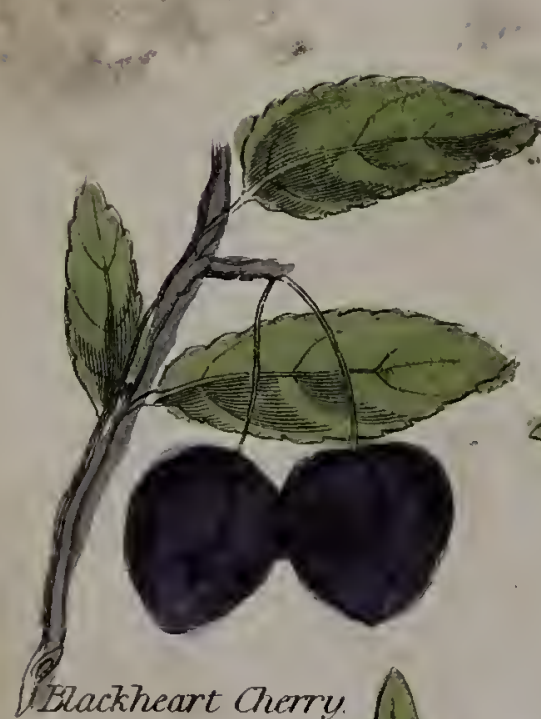

Silackheart Cherry.

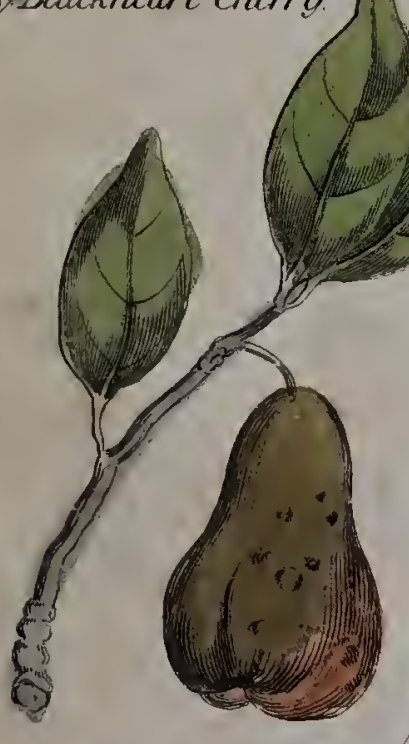

PearTree.

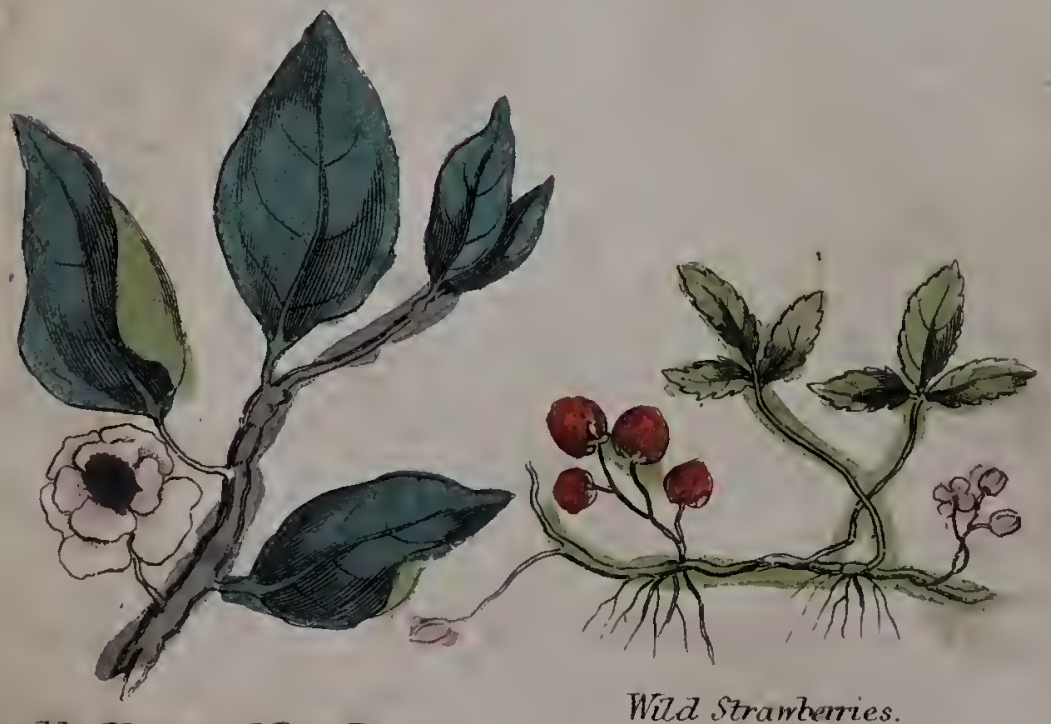

Double Blorsomid PearTree.
Wild Strawharies

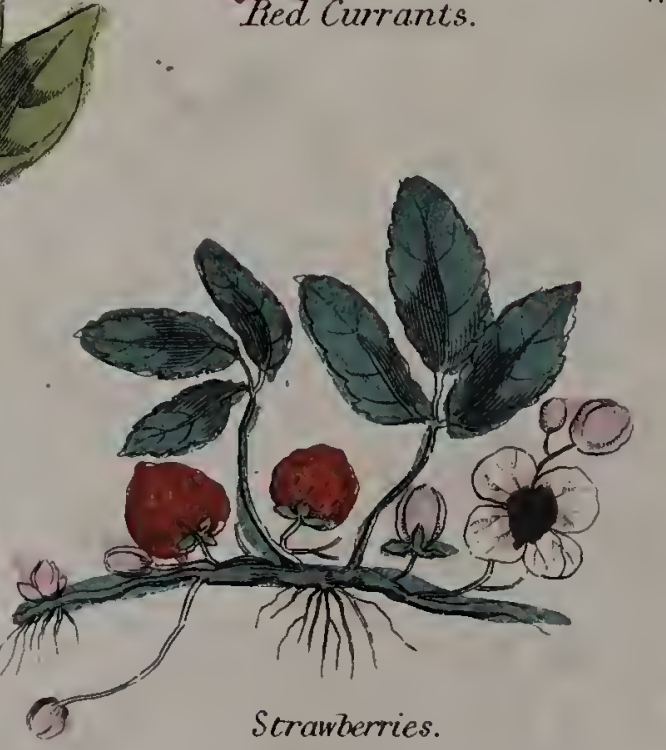

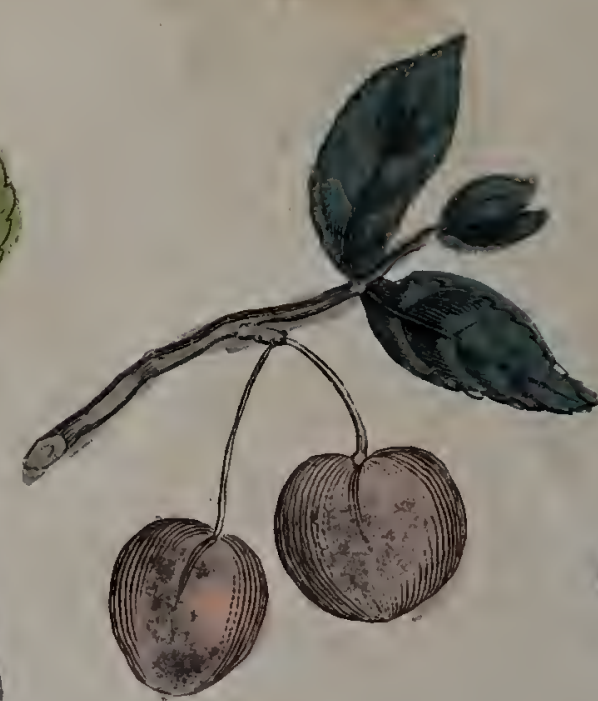

Whiteheart Cherry.

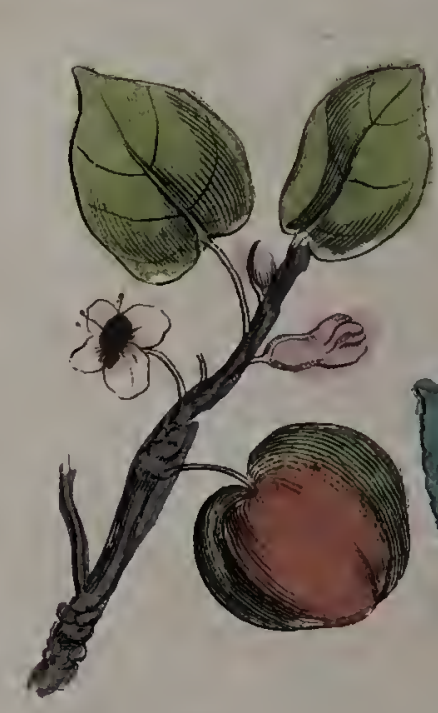

Crabb Apple.

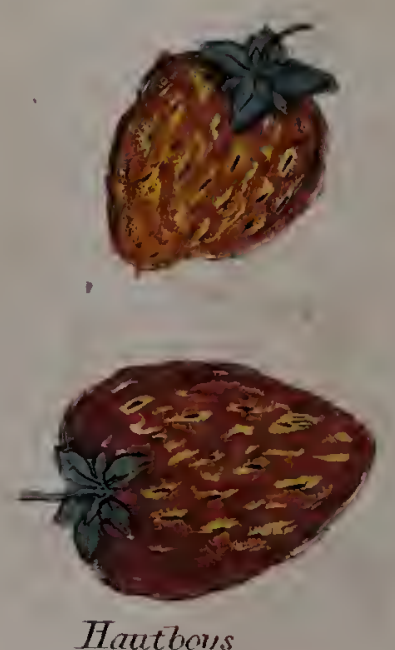

Hautboyss

Lrale 3.\%

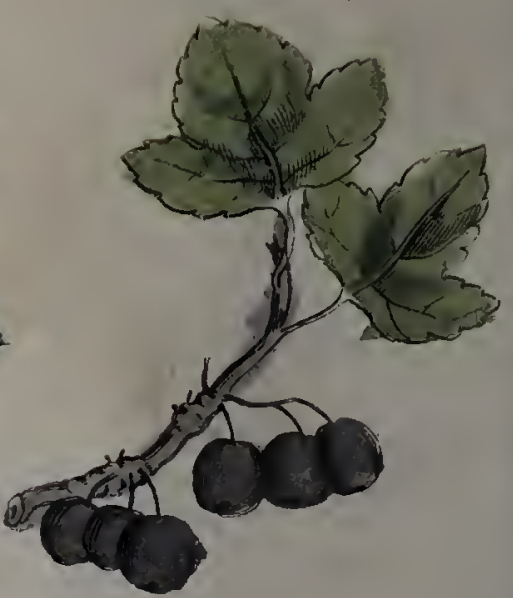

Back Currunts.

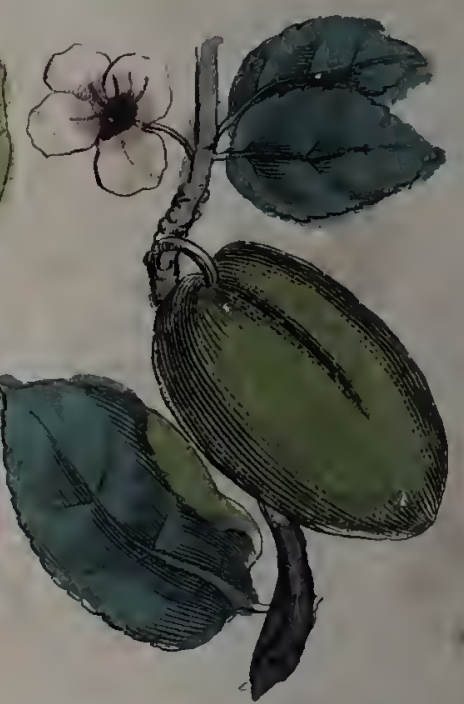

Log Plumb.

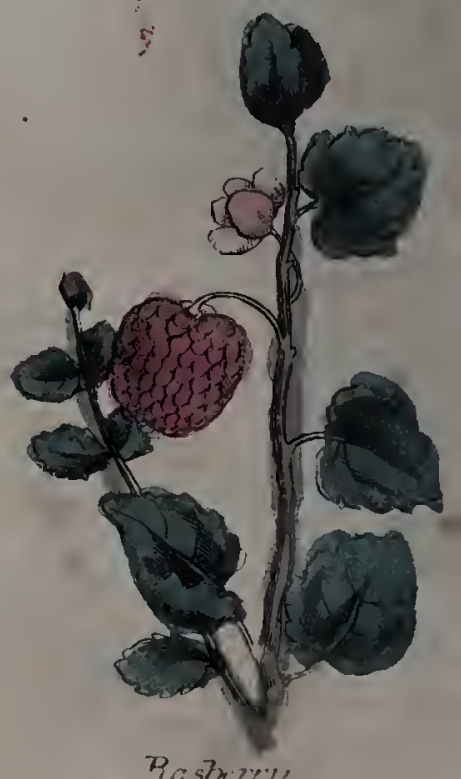




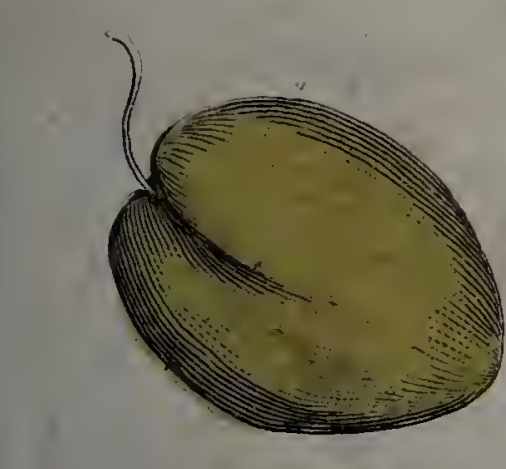

Grest Gage.

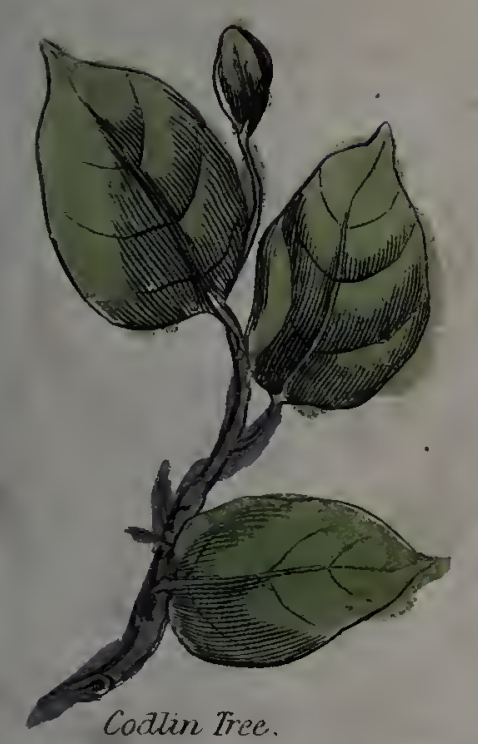

Codlin Free.
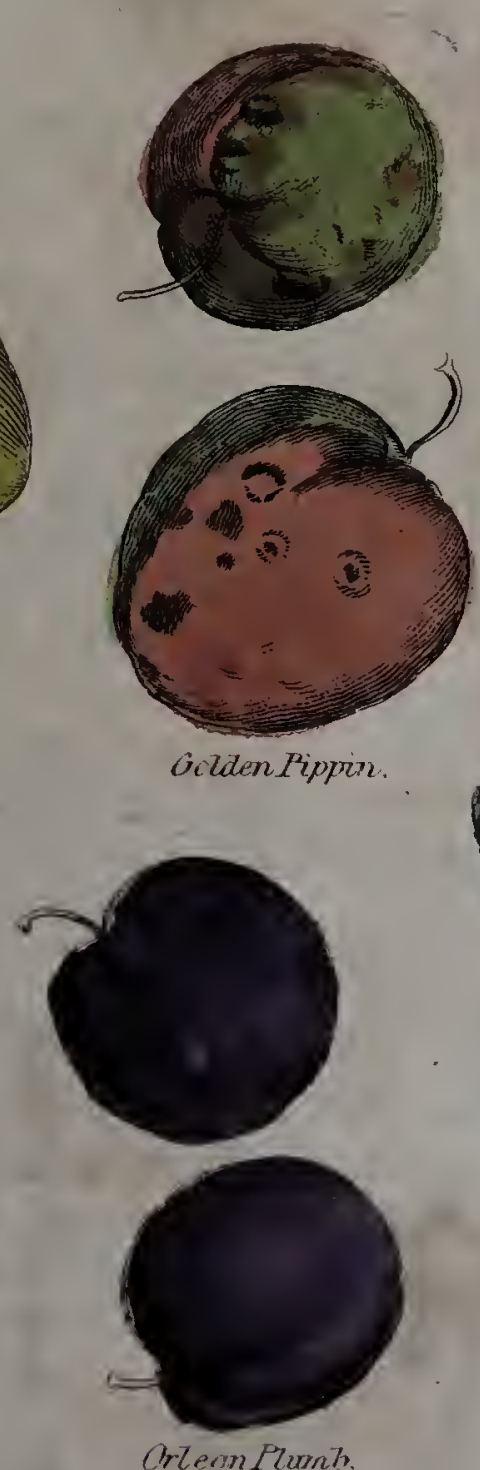

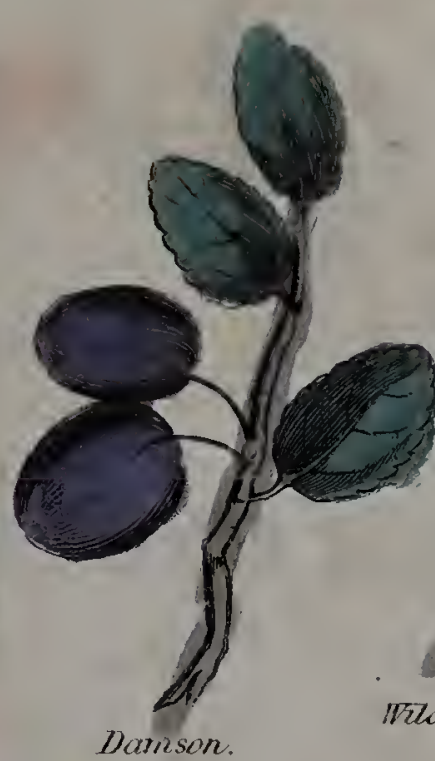

Ind Chery.

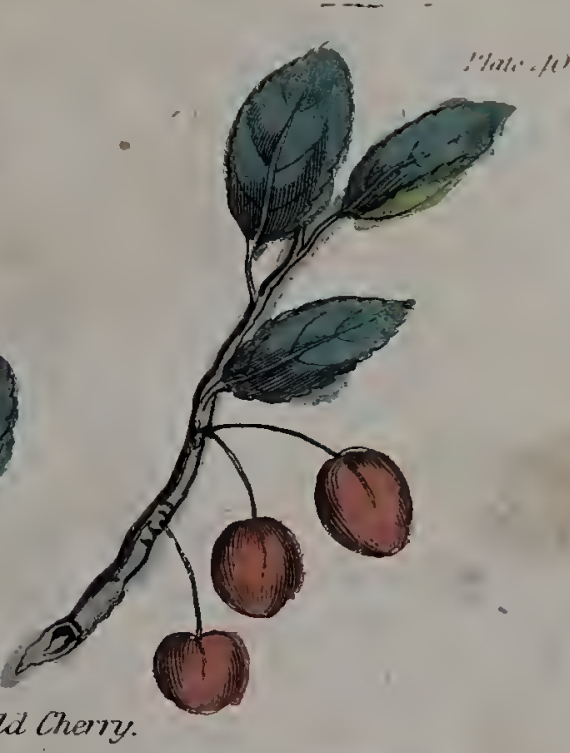

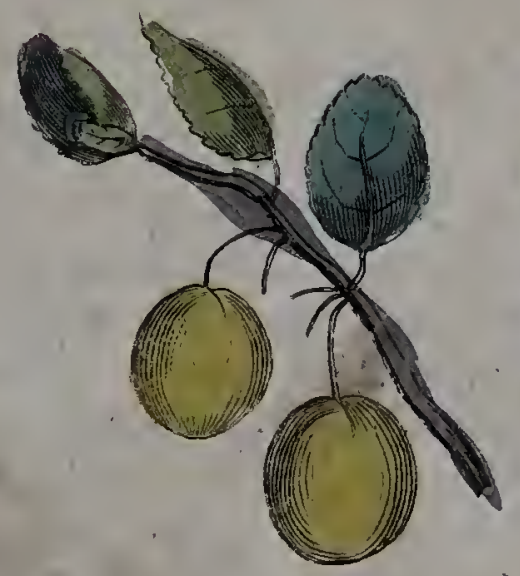

Buzís Mursis.

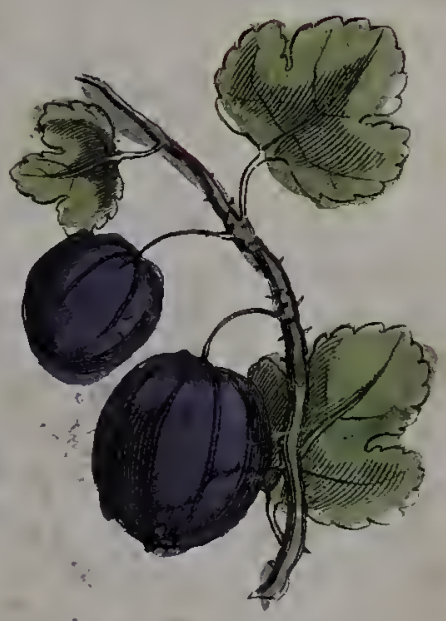

Champian conseberry.
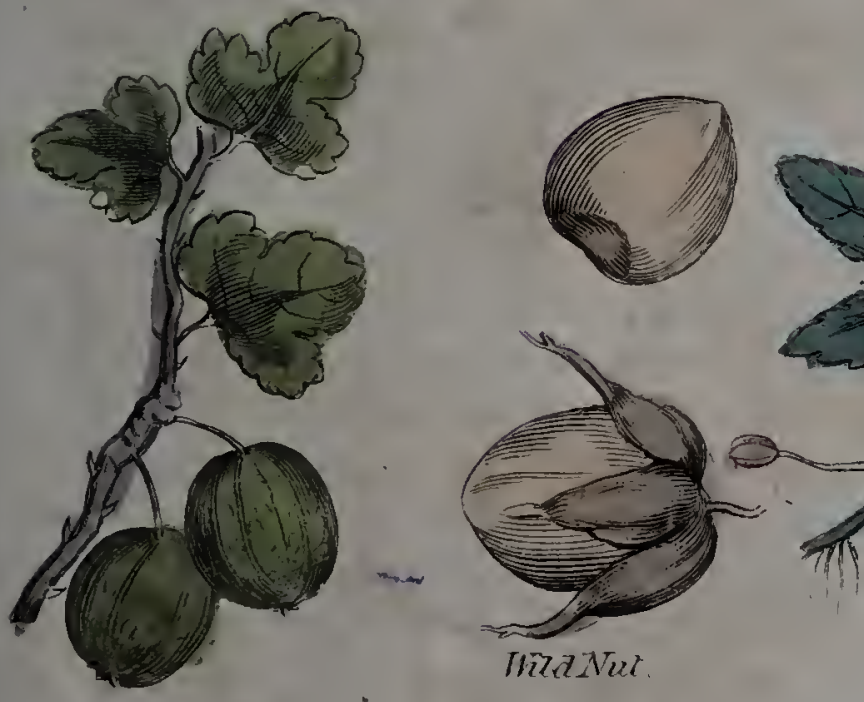

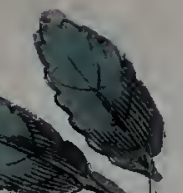

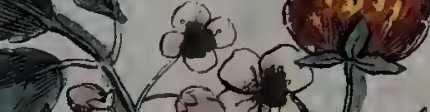

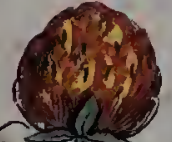

$\sqrt{2}$

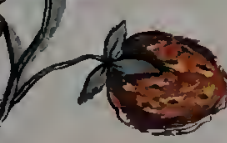

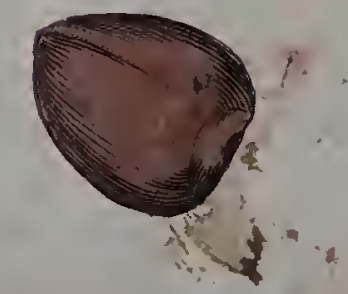

Common Gooseberru. 



\section{ENGLISH PHYSICIAN}

\section{$E N L A R G E D$}

\section{AMARA DULCIS.}

CONSIDERING divers shires in this nation give divers names to one and the same herb, and that the common name which it bears in one county, is not known in another; I shall take the pains to set down all the names that $I$ know of each herb: pardon me for setting that name first, which is most common to myself. Besides Amara Dulcis, some call it Mortal, others Bitter-sweet; some Woody Night-shade, and others Felon-wort.

Descript.] It grows up with woody-stalks ? latter end of March, if the temperature of even to a man's height, and sometimes the air be ordinary; it flowers in July, and higher. 'The leaves fall off at the approach of the seeds are ripe soon after, usually in the winter, and spring out of the same stalk at next month. spring-time: the branch is compassed about Government and virtues.] It is under the with a whitish bark, and has a pith in the planet Mercury, and a notable herb of his middle of it: the main branch branches also, if it be rightly gathered uncler his initself into many small ones with claspers, fluence. Il is excellently good to remove laying hold on what is next to them, as witchcraft both in men and beasts, as also vines do: it bears many leaves, they grow all sudden diseases whatsoever. Being tied in no order at all, at least in no regular order: the leaves are longish, though somewhat broad, and pointed at the ends : many of them have two little leaves growing at the end of their foot stalk; some have but one, and some none. The leaves are of a pale round about the neck, is one of the most admirable remedies for the vertigo or dizziness in the head; and that is the reason (as Tragus saith) the people in Germany commonly hang it about their cattle's necks,
when they fear any such evil hath betided 
them: Country people commonly take the they are something hairy, each leaf conberries of it, and having bruised them, ap- sisting of five or six pair of such ivings set ply them to felons, and thereby soon rid one against the other upon foot-stalks, broad their fingèrs of such troublesome gucsts.

Wè have now shewed you the external use of the herb; we shall speak a word or two of the internal, and so conclude. Take notice, it is a Mercurial herb; and therefore of very subtile parts, as indeed all Mercurial plants are; therefore take a round in form great and strong in magn pound of the wood and leaves together, tude, five or six feet in altilude, with many bruise the wood (which you may easily do, joints, and someleaves thereat; towards the for it is not so hard as oak) then put it in top come forth umbels of small yellow a pot, and put to it three pints of white flowers, after which are passed avay, you wine, put on the pot-lid and shut it close; may find whitish, yellow, short, fiat seeds; and let it infuse hot over a gentle fire twelve hours, then strain it out, so have you a miost excellent drink to open obstructions of the liver and spleen, to help difficuliy of breath, bruises and falls, and congealed blood in any part of the body, it helps tle yellow-jaundice, the dropsy and black jaundice, and to cleanse women newly brought to bed. You may drink a quarter of a pint of the infusion every morning. It purges the body very gently, and not churlishly as some hold. And when you find good by this, remember me.

They that think the use of these medicines is too brief, it is only for the cheapness of the book; let them read those books of mine, of the last edition, viz. Reverius, Vestingus, Riolanus, Johnson, Sennertus, and Physic for the Poor.

\section{A L L-HEA L.}

I $\mathrm{T}$ is called All-heal, Hercules's All-heal, and Hercules's Wound-wort, because it is supposed that Hercules learned the herb and its virtues from Chiron, when he learned physic of him. Some call it Panay, and others Opopane-wort.

Descript.] Its root is long, thick, and exceeding full of juice, of a hot and biting taste, the leaves are great and large, and venomo
winged almost like ash-tree leaves; but that gently.

Place.] Having given you a description of the herb from bottom to top, give me leave to tell you, that there are other herbs called by this name; but because they are strangers in England, I give only the description of this, which is easily to be had iin the gardens of divers places.

Time.] Although Gerrard saith, that they flower from the beginning of May to the end of December, experience teaches them that keep it in their gardens, that it flowers not till the latter end of the Summer, and sheids its seed presently after.

Government and virtues.] It is under the dominion of Mars, hot, biting, and clooleric; and remcdies what evils Mars inflicts the body of man with, by sympathy, as vipers' flesh attracts poison, and the loadstone iron. It kills the worms, helps the gout; cramp, and convulsions, provokes urine; and helps all joint-aches. It helps all cold griefs of the head, the vertigo, falling-sickness, the lethargy, the wind cholic, obstructions of the liver and spleen, stone in the kidneys and bladder. It provokes the terms, expels the dead birth: it is excellent good for the griefs of the sinews, itch, stone, and tooth-ache, the biting of mad dogs and venomous beasts; and purges choler very gently. 


\section{A LKANET. Platez.}

BEsIDes the common name, it is called Orchanet, and Spanish Bugloss, and by apothecaries, Enchusa.

Descript.] Of the many sorts of this herb, there is but one known to grow commonly in this nation; of which one take this description: It hath a great and thick root, of a reddish colour, long, narrow, hairy leaves, green like the leaves of Bugloss, which lie very thick upon the ground; the stalks rise up compassed round about, thick with leaves, which are less and narrower than the former; they are tender, and slender, the flowers are hollow, small, and of a reddish colour.

Place.] It grows in Kent near Rochester, and in many places in the West Country, both in Devonsliere and Cornwall.

Time.] They flower in July, and the beginning of August, and the seed is ripe soon after, but the root is in its prime, as carrots and parsnips are, before the herb. runs up to stalk.

Govermment and virtues.] It is an herb under the dominion of Venus, and indeed one of her darlings, though somewhat hard to come by. It helps old ulcers, lot inflammations, burnings by common fire, and $S t$. Anthony's fire, by antipatlyy to Mars ; for these uses, your best way is to make it into an ointmen!; also, if you make a vinegar of it, as you make vinegar of roses, it helps the morphew and leprosy; if you apply the herb to the privities, it draws forth the dead child. It helps the yellow jaundice, spleen, and gravel in the kidneys. Dioscorides saith, it helps such as are bitten by a vonomous beast, whethcr it be iaken inwardly, or applied to the wound; nay, he saith further, if any one that hath newly eaten it, do but spit into the mouth of a serpent, the with casting, vomiting, or bleeding at the serpent instantly dies. It stays the flux of mouth oi nose, or otherwise downwards. the belly, kills worms, helps the fits of the The said juice given in the distilled water inotluer. Its decoction made in wine, and of Oaken-buds, is very good for women who

drank, strengthens the back, and eases the pains thereof: It helps bruises and falls, and is as gallant a remedy to drive out the small pox and measles as any is ; an ointment made of it, is excellent for green wounds, pricks or thursts.

ADDER'S TONGUE, OR'SERPENT'S TONGUE.

Descript.] THIs herb has but one leaf, which grows with the stalk a finger's length above the ground, being flat and of a fresh green colour; broad like Water Plantain, but less, without any rib in it; from the bottom of which leaf, on the inside, rises up (ordinarily) one, sometimes two or three slender stalks, the upper half whereof is somewhat bigger, and dented with small dents of a yellowish green colour, like the tongue of an adder serpent (only this is as useful as they are formidable.) "The roots continue all the year.

Place.] It grows in moist meadows, and sucl like places.

Time.] It is to be found in May or April, for it quickly perishes with a little heat.

Government and virtues.] It is an herb under the dominion of the Moon and Cancer, and therefore if the weakness of the retentive faculty be caused by an evil influence of Saturn in any part of the body governed by the Moon, or under the dominion of Cancer, this herb cures it by sympathy: It cures these diseasesafter specified, in any part of the body under the influence of Saturn, by antipathy.

It is temperate in respect of heat, but dry in the second degree. The juice of the leaves drank with the distilled water of Horse-tail, is a singular remedy for all manner of wounds in the breast, bowels, or other parts of the body, and is given with good success to those that are troubled (1.) 
have their usual courses, or the whites fow - At the top thercof grow niany small yellow ing down tooabundantly. It helps soreeyes. fiowers, one above another, in long spikes ; Of the leaves infused or boiled in oil, om-after which come rough heads of seed, hangphacine or unripe olives, set in the sun four.ing downwards, which will cleave to and certain days, or the green leaves sufficiently stick upon garments, or any thing that shall boiled in the said oil, is made an excellent rub against them. The knot is black, long, green balsam, not only for green and fresh and somewhat woody, abiding many year's, wounds, butalso forold and inveterate ulcers, and shooting afresh every Spring; which especially if a little fine clear turpentine be root, thongh sniall, hath a reasonable good dissolved thercin. It also stays and re-scent.

freshes all inflammations that arise upon pains by hurts and wounds.

What parts of the body are under each planet and sign, and atso what disease may be found in my astrological judgment of diseases; and for the internal work of nature in the body of man; as vital, aninial, natural and procreative spirits of man; the apprehension, jud , onder Saturn, Mars senses, viz. Sceing, hearing, smelling, tast- and Mercury by antipathy, if they. happen ing and feeling; the virtuous, attractive, in any part of the body governed by Jupiretentive, digestive, expulsive, \&c. under ter, or under the signs Cancer, Sagitarius or the dominion of what planets they are, may Pisces, and therefore must needs be good be found in my Ephemeris for the year 1651. for the gout, either used outwardly in oil or In both which you shall find the chaff of ointment, or inwardly in an electuary, or authors blown away by the fame of Dr. syrup, or concerterl juice: for which sce the Reason, and nothing but rational truths left latter end of this book.

for the ingenious to feed upon

Lastly. To avoid blotting paper with one thing many times, and also to ease your? purses in the price of the book, and withal to make you studious in physic; you have at the latter end of the book, the way of preserving all herbs either in juice, conscrve, oil, ointuient or plaister, electuary, pills, or troches.

AGR INONY. Phali. 1 .

Descript.] 'TuIs has divers long leaves It is of a cleansing and cutting faculty without any manifest heat, moderately drying and binding. It opens and cleanses the liver, helps the jaundice, and is very beneficial to the bowels, healing all inward wounds, bruises, hurts, and other distempers. 'The decoction of the herb made with wine, and drank, is good against the biling and stinging of serpents, and helps them that make foul, troubled or bloody water.

'This herb also helps the cholic, cleanses (some greater, some smaller) set upon a the breast, and rids away the cough. stalk, all of them dented about the edges, A draught of the decoction taken warm be.green above, and greyish underneath, and fore the fit, first removes, and in time rids a little hairy withal. Among which arises away the tertain or quartan agues. 'The up usually but one strong, round, hairy, leaves and seeds taken in wine, stays the brown stalk, two or three feet high, with bloody flux; outwardly applied, being smaller leaves set here and there upon it. stamped with old swines' grease, it helps 
old sores, cancers, and inveterate ulcers, and therefore they are not so frequently found draws forth thorns and splinters of wood, in the southern parts of England as in the nails, or any other such things gotten in the? northern, where they grow frequently: You flesh. It helps to strengthen the members may look for them in cold grounds, by pond that be out of joint: and being bruised and 'and ditches' sides, and also by running applied, or the juice dropped in it, helps waters; sometimes you shall find then grow foul and imposthumed cars.

The distilled water of the herb is good to all the. said purposes, either inward or outward, but a great deal weaker.

It is a most admirable remedy for such Jupiter, as well as the other Agrimony, only wnose livers are annoyed either by heat or this belongs to the celestial sign Cancer. cold. The liver is the former of blood, and It heals and dries, cuts and clcanses blood the nourisher of the body, and Agri- thick and tough humours of the breast, and mony a,strengthener of the liver.

for this I hold it inferior to but few lierbs

I cannot stand to give you a reason in that grow. It helps the cachexia or evil every herb why it cures such diseases; but disposition of the body, the dropsy and if you please to pursue my judgment in the herb Wormwood, you shall find them there, and it will be well worth your while to consider it in every lierb, you shall find them true throughout the book.

WATER AGRIMONY.Flader.

IT is called in some countries, Water yellow-jaundice. It opens obstructions of the liver, mollifies the hardncss of the spleen, being applied outwardly. It breaks imposthumes away inwardly: It is an excellent remedy for the third day ague. It provokes Urine and the terms; it kills worms, and . cleanses the body of sharp humours, which are the cause of itch and scabs; the herb Hemp, Bastard Hemp, and Bastard Agrimony, Eupatorium, and Hepatorium, because it strengthens the liver.

Descript.] The root continues a long time, having nuany long slender strings. The stalk grows up about two feet high, sometimes higher. They are of a dark purple? colour. The branches are many, growing at distances the one firom the other, the one from the one side of the stalk, the other from the opposite point. The leaves are winger, and much indented at the edges. The flowers grow at the top of the branches, of a brown yellow colour, spotted with black spots, having a substance within spreads and creeps upon the ground, the midst of them like that of a Daisy: If shoots forth roots, at the corners of tendet you rub them between your fingers, they jointed stalks, set with two round leaves ar smell like rosin or cedar when it is burnt. every joint somewhat hairy, crumpled and The seeds are long, and easily stick to any unevenly dented about the edges with round woollen thing they touch.

being burnt, the smoke thereof drives away Hies, wasps, \&c. It strengthens the lungs exceedingly. Country people give it to their cattle when they are troubled with the cough, or broken-winded.

\section{ALEHOOF, OR GROUNDIVY.P.I.}

SEVER A L counties give it different namcs, so that there is scarcely an herb growing of that bigness that has got so many: It is called Cat's-foot, Ground-ivy, Gill-go byground, and Gill-creep-by-ground, 'T'unhoof, Haymaids, and Alehoof.

Descript.] This well known herb lies, dents; at the joints likewise, with the leaves Place.] They delight not in heat, and towards the end of the branches, come forth 
hollow, long flowers, of a blueish purple out in any part of the body. The juice of colour, with small white spots upon the lips Celandine, Field-daisies, and Ground-ivy that hang down. The root is small with clarified, and a little fine sugar dissolved strings.

Piace.] It is commonly found under hedges, and on the sides of ditches, under watering of them ; as also for the pin and houses, or in shadowed lanes, and other web, skins and films growing over the sight; waste grounds, in almost every part of this it helps beasts as well as men. The juice land.

Time.] They flower somewhat early, and abide a great while; the leaves continue green until Winter, and sometimes abicle, except the Winter be very sharp and cold.

Government and virtues.] It is an heib of Venus, and therefore cures the diseases she causes by sympathy, and those of Mars by antipathy; you may usually find it all the year long except the year be extremely frosty; it is quick, sharp, and bitter in taste, and is thereby found to be hot and dry; a singular herb for all inward wounds, exulcerated lungs, or other parts, either by itself, or boiled with other the like herbs; and being drank, in a short time it eases all griping pains, windy and choleric humours in the stomach, spleen or belly; helps the yellow jaundice; by opening the stoppings of the gall and liver, and melancholy, by opening the stoppings of the spleen; expels venom or poison, and also the plague; it provokes urine and women's courses; the together, procures ease to them that are troubled with the sciatica, or hip-gout: as also the gout in hands, knees, or feet; if you put to the decoction some honey and a little burnt allum, it is cxccllently good to gargle any sore mouth or throat, and to wash the sores and ulcers in the privy parts of man or woman; it speedily helps green wounds, being bruised and bound thereto. The juice of it boiled with a little honey and verdigrease, doth wonderfully cleanse fistulas, ulcers, and stays the spreading or eating of cancers and ulcers; it helps the itch, scabs, wheals, and other breakings. dropped into the ears, wonderfully helps the noise and singing of them, and helps the hearing which is decayed. It is good to tun up with new drink, for it will clarify it in a night, that it will be the fitter to be drank the next morning; or if any drink be thick with removing, or any other accident, it will do the like in a few hours.

\section{A LEX A NDER. Ohate-}

$I_{T}$ is called Alisander, Horse-parsley, and Wild-parsley, and the Black Pot-lierb; the seed of it is that which is usually sold in apothecaries' shops for Macedonion Parsley-seed.

Descript.] It is usually sown in all the gardens in Europe, and so well known, that it needs no farther description.

Time.] It.flowers in June and July; the seed is ripe in August.

Government and virtues.] It is an herb of Jupiter, and therefore friendly to nature, for it warms a cold stomach, and opens a stoppage of the liver and spleen; it is good to nove woniens' courses, to expel the afterbirth, to break wind, to provoke urine and helps the stranguary; and these things the seeds will do likewise. If either of them be boiled in wine, or being bruised and taken in wine, is also effectual against the biting of serpents. And you lunow what Alexander pottage is good for, that you may no longer eat it out of ignorance but out of knowledge.

THE BLACK ALDER-TREF. Diakaz.

Descript.]. Tuis tree seldom grows to any great bigness, but for the most part 
abideth like a hedge-bush, or a tree spreading its branches, the woods of the body being white, and a dark red colet or heart; the outward bark is of a blackish colour; with many whitislı spots therein; but the inner bark next the wood is yellow, which being chewed, will turn the spittle near into a satfron colour. The leaves are somewhat like those of an ordinary Alder-treet or the Fenale Cornet, or Dogberry-tree, called in Sussex Dog-wood, but blacker, and not so long. The flowers are white, coming forth with the leaves at the joints, which turn into small round berries, first green, afterwards red, but blackish when they aie thorough ripe, divided, as it were, into two parts, wherein is contained two small round and flat seeds. The root runneth not deep into the ground, but spreads rather under the upper crust of the earth.

Place.] 'This tree or shrub may be found plentifully in St. John's wood by Hornsey, and the woods upon Hampstead-Heath; as also a wood called the Old Park in Barcomb in Essex, near the brook's sides.

Time.] It flowers in May, and the berries are ripe in September.

Government and virtues.] It is a tree of Venus, and perhaps under the celestial sign Cancer. The inner yellow bark hereof purges downwards both choler and phlegm, and the watery humours of such that liave the dropsy, and strengthens theinward parts again by binding. If the bark liereof be boiled with Agrimony, Wormwood, Dodder, Hops and some Fennel, with Smallage, Endive, and Succory-roots, and a reasonable draught taken every morning for some time together, it is very effectual against the jaundice, dropsy, and the evil disposition, of the body, especially if some suitable purging medicincs have been taken before, to void the grosser excrements : It purges and strengthens the lirer and splecn, cleansing them from sucli cril humours and hardness as they are afticted with. It is ? (2.) to be understood that these things are performer by the dried bark; for the fresh grcen bark taken inwardly provokes strong vomitings, pains in the stomach, and gripings in the belly; yet if the decoction may stand and settle tro or thrce days, unti the yellow colour be changed l, lack, it will not work so strongly as before, but will stiengthen the stomach, and procure an appetite to meat. 'The outward bark contraliwise do $\mathbf{h}$ bind the borly, and is helpful for all la:ks and fluxes thereof, but this also must be dried first, whereby it will work the het:er. The inner bark thereof boiled in vinegar is an approved remedy to kill lice, to cure the itch, and take away scabs, by drying them up in a short time. It is singularly good to wash the teeth, to take away the pains, to fasten those that are loose, to cleanse them, and to keep them sound. The leaves are good fodder for kine, to make them gire more milk.

If in the Spring-time you use the herbs before mentioned, and will take but a handful of each of them, and to them add an handful of Elder buds, and having bruised them all, boil them in a gallon of ordinary beer, when it is new; and having boiled them half an hour, add to this three gallons more, and let them work together, and drink a dranght of it every morning, half a pint ir thereabouts ; it is an exccllent purge for the Spring, to consume the phlegmatic quality the Winter hath left behind it, and withal to keep your body in health, and consume those evil humours which the heat of Summer will readily stir up. Esteem it as a jewel.

\section{THE COMMON ALDER-TRER.P'schel.}

Descript.] Tins grows to a reasonable height, and spreads much if it like the place. It is so generally known to country people, that I conceive it needless to tell that which is no news.

Place and Time.] It delights to grow in $\mathrm{D}$ 
moist woods, and watry places; flowering three colours. And a certain ointment, an in April or May, and yielding ripe seed in September.

Government and virtues.] It is a tree under the dominion of Venus, and of some watry sign or other, I suppose Pisces; " and therefore the decoction, or dist:iled water of the leaves, is excellent against burnings and inflammations, either with wounds or without, to bathe the place grieved with, and especially for that inflammation in the breast, which the vulgar call an ague.

If you cannot get the leaves (as in Winter it is impossible) make use of the bark in the same manner.

The leaves and bark of the Alder-tree are cooling, drying, and binding. The fresh leaves laid upon swellings dissolve them, and stay the inflammations. The leaves put under the bare feet galled with travelling, are a great refreshing to them. The said leaves gathered while the morning dew is on them, and brought into a chamber troubled with fleas, will gather them thereunto, which being suddendy casi out, will rid the chamber of those troublesome bedfellows.

\section{A NGELICA.}

To write a description of that which is so well known to be growing almost in every garden, I suppose is altogether needless; yet for its virtue it is of admirable use.

In time of Heathenism, when men had found outany cxcellent herh, they dedicated it to their gods; as the Bay-tree to Apollo, the Oak to Jupiter, the Vine to Bacchus, the Poplar to Hercules. These the idolatois following as the Patriarchs they dedicate to their Saints; as our Laty's Thistle to the Blessed Virgin, St. John's Wort to St. John, and another Wort to St, Peter, \&c. Our physicians must imitate like apes (though they cannot come off half so cleverly) for they blasphemously call Phanisies or Hearts-? 'Tlie root also steeped in vinegar, and a little ease, an herb of the Trinity, because it is of " of that rinegar taken sometimes fasting, and of twelve ingredients: Alas, I am sorry for their folly, and grieved at their blasphemy, God send them wisdom the rest of their age, for they have their share of ignorance already. Oh! Why must ours be blasphemous, because the Heathens and infidels were idolatrous? Certainly they have read so much in old rusty authors, that they have lost all their divinity ; for unless it were amongst the Ranters, I never read or heard of such blasphemy. The Heathens and infidels were bad, and ours worse; the icolators give idolatrous names to herbs for their virtues sake, not for their fair looks; and therefore some called this an herb of the Holy Ghost ; others more moderate called it Angelica, because of its angelical virtues and that name it retains still, and all nations follow it so near as their dialect will permit.

Government and virtues.] It is an herb of the Sun in Leo; let it be gathered when he is there, the Moon applying to his good aspect; let it be gathered either in his hour, or in the hour of Jupiter, let Sol be angular; observe the like in gathering the herbs, of other planets, and you may happen to do wonders. In all epidemical diseases caused by Saturn, that is as good a preservative as grows: It resists poison, by dcfending and conforting the heart, blood, and spirits; it doth the like against the plague and all cpidemical discases, if the root be taken in powder to the weight of half a dram at a time, with some good treacle in Carduus water, and the party thereupon laid to sweat in his bed: if treacle be not to be had, take it alone in Carduus or Angelica-water. The stalks or rnots candied and eaten fasting, are good preservatives in time of infection; and at other times to warm and comfort a cold stomach. 
the root smelled unto, is good for the sane it is called Flower Gentle, Flower Velure, purpose. A water distilled from the root Floramor, and Velvet Flower. simply, as steeped in wine, and distilled in a glass, is much more effectual than the water of the leaves; and this water, drank two or three spoonfuls at a time, easeth all pains and torments coming of cold and wind, so that the body be not bound; and taken with soine of the root in powder at the beginning, helpeth the pleurisy, as also all other diseases of the lings and breast, as coughs, phtliysic, and shortness of breath; and a syrup of the stalks do the like. It helps pains of the cholic, the stranguary and stoppage of the urine, procureth womens' courses, and expelleth the after-birth, openeth the stoppings of the liver and spleen, and briefly easeth and discusseth all windiness and inward swellings. The decoction drank before the fit of an ague, that they may sweat (if possible) before the fit comes, wiil, in two or three times taking, rid it quite away ; it helps digestion and is a remedy for a surfeit. The juice or the water being dropped into the eyes or ears, helps dimness of sight and deafness; the juice put into the hollow teeth, easeth their pains. 'I'lie root in powder, made up into a plaister with a little pitch, and laid on the biting of mad dogs, or any other venomous creature, doth wonderfully help. 'The juice, or the water dropped, or tents we! therein, and put root (in want of cither) doth cleanse and cause them to heal quickly, by covering the naked bones with flesh; the distilled water applied to places pained with the gout, or sciatic 1 , doth give a great deal of ease.

The wild Angelica is not so effectual as the garden; although it may be safely used to all the purposes aforesaid.

\section{AMARANTHUS.}

$$
\text { plake }
$$

Besinfs its common name, by which it is best known by the florists of our days, ?

Descript.] It being a garden flower, and well known to every one that keeps it, I inight forbear the description; yet, notwithstanding, because some desire it, I shall give it. It runs up with a stalk a cubit high, streaked, and somewhat reddish toward the root, but very smooth, divided towards the top with small branches, among which stand long broad leaves of a reddish green colour, slippery ; the flowers are not properly flowers, but tuffs, very beautiful to behold, but of no smell, of reddish colour; if you bruise them, they yicld juice of the same colour, being gathered, they keep their beauty a long time; the seed is of a shining black colour.

Time.] They continue in flower from August till the time the frost nip them.

Government and virtues.] - It is under the dominion of Saturn, and is an excellent qualifier of the unruly actions and passions of Venus, though Mars also should join with her. The flowers dried and beaten into powder, stop the terms in women, and so do almost all other red things. And by the icon, or image of every herb, the ancients at first found out their virtnes. Modern writers laugh at them for it; but I wonder in my heart, how the virtues of herbs came at first to be known, if not by their signatures; the moderns have them from the writings of the ancients; the ancients had no writings to liave them from: but to proceed. 'The flowers stop all fluxes of blood; whether in man or woman, bleeding either at the nose or wound. There is also a sort of Amaranthus that bears a white flower, which stops the rlites in women, and the running of the reins in men, and is a most gallant antivenereal, and a singular remedy for the French pox.

$$
\text { A NEMONE. }
$$

$$
P .1 .
$$

$C_{A}$ L.WI also Wind Flower, because they 
say the flowers never open but when the to the throat, or boiled, and in like manner wind blows. Pliny is my author; if it applied, it matters not much, it is excellently be not so, blame him.- The seed also (if it good for swellings in the throat: the best bears any at all) flies away with the wind. "way, I- suppose is to boil it, apply the

Place and.Time.] They are sown usually herb outwardly: the decoction of it besides in the gardens of the curious, and flower in is an excellent remedy for the ycllow jaunthe Spring-time. As for description I shall dice.

pass it, being well known to all those that sow them.

Government and virtues.]" It is under the dominion of Mars, being suipposed to be a kind of Crow-foot: The leaves provoke the terms mightily, being boiled, and the decoction drank. The body being bathed with thedecoction of them, cures the leprosy. The leaves being stamped and the juice snuffed up in the nose, purges the head mightily; so does the root, being chewed in the mouth, for it procures much spitting, and brings away many watery and phlcegmatic humours, and is therefore excellent for the lethargy. And when all is done, let physieians prate what they please, all the pills in the dispensatory purge not the head like to hot things held in the mouth. Being made into an ointment, and the eyelids anointed with it, it helps inflammations of the eyes, whereby it is palpable, that every stronger draws jts weaker like. The same ointment is excellently good to cleanse malignant and corroding ulcers.

GARDEN ARRACH. SinteI

Called also Orach, and Arage; it is cultivated for domestic uses.

Descript.] It is so commonly known to every housewife, it were labour lost to describe it.

Time.] It flowers and seeds from June to the end of August.

Government and virtues.] It is under the easily, safely, and specdily cure any disease government of the Moon; in quality cold thercof; as the fits of the mother, dislocaand moist like unto ler. It softens and tinn, or falling out thereof; cools the lnosens the body of man being eaten, wromb being orer-heated. And let nie tell and fortifies the expulsive faculty in him. you this, and $I$ will tell you the truih, beat The herb, whether it be bruised and applied of the womb is one of the greatest causes 
of hard labour in child-birth. It makes barren women fruitful. It cleanseth the womb if it be foul, and strengthens it exceedingly ; it provokes the terms if they be stopped, and stops them if they flow immoderately; you can desire no good to your womb, but this herb will affect it; therefore if you love children, if you love health, if you love case, keep a syrup always by you, made of the juice of this herb, and sugar, (or honey, if it be to cleanse the? womb) and let such as be rich keep it for their poor neighbours; and bestow it as freely as I bestow my studies upon them, or else let them look to answer it another day, when the Lord shall come to make inquisition for blood.

\section{ARCHANGEL. Phate. 2.}

To put a gloss upon their practice, the physicians call an herb (which country people vulgarly know by the name of Dead. Nettle) Archangè ; whether they favour more of superstition or folly, I leave to the judicious reader. 'There is morc curiosity than courtesy to my countrymen used by others in the explanation as well of the names, as description of this so wcll known herb; which that I may not also be guilty of, take this short description: first, of the Red Archangel. This is likewise called Bee Neltle.

Descript.] This has divers square stalks, somewhat hairy, at the joints whereof grow two sad green leaves lented about the edges, opposite to one another to the lowermost upon long foot stalks, but without any toward the tops, which are somewhat round, yet pointed, and a little crumpled and hairy; round about the upper joints, where the leaves grow thick, are sundry gaping flowers of a pale reddish colour; after which cone the scels three or four in a lusk. The root is small and thready, perishing erery year; the whole plant hath a strong scent but not stinking.

(2.)
White Archangel hath divers square stalks, none standing straight upward, but bending downward, whercon stand two. leaves at a joint, larger and more pointed than the other, dented about the edges, and greencr also, more like unto Nettle lcaves, but not stinking, yet hairy. At the joints, with the leares, stand larger and more open gaping. white flowers, husks round about the stalks, but not with such a bush of leaves as flowers set in the top, as is on the other, wherein stand small roundish black secds; the root is white, with many strings at it, not growing downward but lying under the upper crust of the carth, and abides many years increasing; this has not so strong a scent as the former.

Ycllow Archangel is like the White in the stalks and leaves; but that the stalks are more straight and upright, and the joints with leaves are farther asumder, having longer leaves than the former, and the flowers a little larger and more gaping, of a farr yellow colour in most, in some paler. 'The roots are like the white, only they creep not so much under the ground.

Place.] 'They grow almost cvery where, (unless it be in the middle of the strcet) the ycllow most usually in the wet grounds of woods, and sometimes in the dryer, in diver's counties of this nation.

Time.] They flower from the beginning of the Spring all the Summer long.

Goternment and virtues.] The Archangels are somewhat hot and dricr than the stinging Nettles, and used with better success for the stopping and hardness of the spleen, than they, by using the decoction of the herb in wine, and afterwards applying the herb hot into the region of the spleen as a plaister, or the decoction with spunges. Flowers of the White Archangel are preserved or conserved to be used to stay the whites, and the flowers of the red to stay the reds in women. It makes the lieart merry, drives away molancholy, quicliens 
the spirits is good against quartan agues, stancheth bleeding at mouth and nose, if it be stamped and applied to the nape of the neck; the herb also bruised, and with some salt and vinegar and hog's-grease, laid upon an hard tumour or swelling, or that vulgarly called the king's evil, do help to dissolve or discuss them; and being in like manner applied, doth much allay the pains, and give ease to the gout, sciatica, and other pains of the joints and sinews. It is also very effectual to heal green wounds, and old ul-ers ; also to stay their fretting, gnawing and spreading. It draws forth splinters, and such like things gotten into the Hesh, and is very good against bruises and burnings. But the yellow Archangel is most commended for old, filthy, corrupt sores and alcses, yea although they grow to be hoilow; and to dissolve tumours. The clief use of thom is for women, it being an herb of Venus.

\section{ARSSMART.}

Dilate 2.

Trne hot Arssmart is called also Waterpepper, or Culrage. The mild. Arssmart is called dead Arssmart Persicaria, or Peachwort, because the leaves are so like the leares of a pcach-tree: it is also called Plumbago.

Descriplion of the mild.] This has broad leaves set at the great red joint of the stalks; with semicircular blackish marks on them, usually either blueish or whitish, with such like sced following. 'The root is long, with many strings thereat, perishing yearly; this has no sharp taste (as another sort has, which is quick and biting) but rather sour like sorrcl, or else a little drying, or without taste.

Place.] It grows in watcry places, ditches, and the like, which for the most part are dry in Summer.

Time.] It Howers in June, and the seed is ripe in August.
Govermment and virtues.] As the virtue of both these is various, so is also their government; for that which is hot and biting, is under the cloninion of Mars, but Saturn challenges the other, as appears by that leaden coloured spot he hath placed upon the leaf.

It is of a cooling and drying quality, and very effectual for putrified ulcers in man or beast, to kill worms, and cleanse the putrified places. The juice thereof dropped in, or otherwise applied, consumes all cold swellings, and dissolveth the congealed blood of bruises by strokes, falls, \&c. A piece of the root, or sonie of the seeds bruised, and held to an aching tooth, takes away the pain. The leaves, bruised and linid to the joint that has a felon thercon, takes it away. The juice destroys worms in the ears, being, dropped into tliem; if the hot Arssmart be strewed in a chamber, it will soon kill all the fleas; and the herb or juice of the cold Arssmart, put to a horse, or other cattle's sores, will drive away the fly in the hottest time of Summer; a good handful of the hot biting Arssmart put under a horse's saddle, will make him travel the better, although he were half tired before. The mild Arssmart is good against all imposthumes and inflammations at the beginning, and to heal green wounds. All authors chop the virtues of both scrts of Arssmart together, as nien chop herbs for the pot, when both of then are of contrary qualities The hot Arssmart grows not so ligh or tall as the mild doth, but has many leaves of the colour. of peach leares, very seldom or never spotted; in other particulars it is like the fornier, but may easily be known from it, if you will but be pleased to break a leaf of it cress your tongue, for the hot will make your tongue to smart, but the cold will not. If you sce them both together, you may easily distinguish them, bccause the mild hath far broader leaves. 


\section{ASARABACCA.}

pirate. 2

choler, and therefore docs much help pains

in the hips, and other parts; bcing boiled Descript.] AsaraBAcca appears like in whey, they wonderfully help the ohan evergreen, keeping iis leaves all the structions of we liver and spleen, and thereWinter, but puiting forth new ones in the? fore profitable for the dropsy and jatindice: time of Spring. It has many heads rising being steeped in wine and drank, it helps from the roots, fron whence come many thosecontinualaguesthat come by the plenty smooth leaves, every one upon his foot of stubborn humours; an oil made therenf stalks, which are rounder and bigger than by selting in the sun, with some laudanum Violet, leaves, thicker also, and of a dark, added to it, provokes sweating, (the ridge green shining colour on the upper side, and of the back being anointed therewith) and of a pale yellow green underneath, litle or thereby drives away the shaking fits of the nothing dented about the edges, from among ague. It will not abide any long boiling, which rise small, rould, hollow, brown? for it loseth its chief strength thereby; nor green husks, upun short stalks, about an much beating, for the finer powder proinch long, divided at the brims into five vokes vomits and urine, and the coarser divisions, very like the cups or heads of purgeth downwards.

the Henbine seed, but that they are smaller: and these be all the flower it carries, which are soinewhat sweet, being smelled to and wherein, when they are ripe, is contained small cornered rough seeds, very like the kernels or stones of grapes or raisins. The roots are small and whitish, spreading divers ways in the ground, increasing into divers heads; 'but not running or creeping under the ground, as some other creeping herbs do. They arc somewhat head and brain that is ill affected by taking sweet in smell, resembling Nardus, but cold, and helps the memory.

more when they are dry than green; and of a sharp and not unpleasant taste.

Place.] It grows fiequently in gardens.

Time.] They kerp their leaves green all Winter; but shoot forth new in the Spring, and with them come forth those heads or flowers which give ripe seed about Midsummer, or somewhat after.

Government and virtues.] It is a plant under the dominion of Mars, and therefore inimical to nature. This herb being drank, not only provokes romiting, but purges downward, and by urine also, purges both choler and flegm: If you add to it some spikenard, with the whey of goat's milk, or honered water, it is made more strong, but it purges flegm more manifestly than

The common use hereof is, to take the juice of five or seven leaves in a little drink to cause vomiting; the roots have also the same virtue, though they do not operate so forcibly; they are very cffectual against the biting of serpents, and therefore are put as an ingredient both into Mithridite and Venice treacle. 'The leaves and roots being boiled in lye, and the head often washed therewith while it is warm, comforts the I shall desire ignorant people to forbear the use of the leaves ; the roots purge more gently, and may prove beneficial to such as have cancers, or old putrified ulcers, or fistulas upon their bodies, to take a dram of them in powder in a quarter of a pint of white wine in the morning. The truth is, I fancy purging and vomiting medicines as little as any man breathing doth, for they weaken nature, nor shall crer advise them to be used, unless upon urgent necessity. If a physician be na!ure's servant, it is his duty to strengthen his mistress as much as he can, and weaken her as little as may be.

ASPARAGUS, SPARAGUS, OR SPERACE Descript.] It rises up at furst will divers 
white and green sealy heads, very brittle or more than the wild, yet hath it the same easy to break while they are young, whicl effects in all the afore-mentioned diseases: afterwards rise up in very long and slender 'The decoction of the ront in white wine, green stalks of the bigness of an ordinary and the back and belly bathed therewith, jiding wand, at the bottom of most, or or kneeling or lying down in the same, or bigger, or lesser, as the roots are of growth; sitting therein as a bath, has been found on which are set divers branches of green effectual against pains of the reins and Ieaves shorter and smaller than fennel to the bladrler, pains of the mother and cholic, top; at the joints whereof come forth small and generally against all pains that happen yellowish flowers, which turn into round to the lower parts of the body, and no less berries, green at first, and of an exeellent effeetual against stiff and benumbed sinews, red colour when they are ripe, shewing like or those that are shrunk by cramps and bead or coral, wherein are contained ex- convulsions, and helps the sciatica. ceeding hard black seeds, the roots are dispersed from a spongeous head into many long, thiek, and round strings, wherein is sucked much nourishment out of the ground, and increaseth plentifully thereby.

PRICKLY ASPARAGUS, OR SPERAGE.

Descript.] Tnus grows usually in gar- by the Sun: and the young tender tops, dens, and some of it grows wild in Apple- with the leaves taken inwardly, and some ton meadows in Gloucestershire, where the of them outwardly applied, are singularly poor people gather the buds of young good against the bitings of viper, adder, or shoots, and sell them ebeaper than our gar- any other venomous beast; and the water den Asparagus is sold in London.

Time.] For the most part they flower, and bear their berries late in the year, or medicine for those that are subject todropsy, not at all, although they are housed in or to abate the greatness of those that are Winter.

Government and virtues.] They are both in white wine helps to break the stone, under the dominion of Jupiter. 'The young and expel it, and eures the jaundice. The buds or branehes boiled in ordinary broth, ashes of the bark of the Ash made into make the belly soluble and open, and boiled lyc, and those heads bathed therewith in white wine, provoke urine, being stopped, which are leprous, seabby, or scald, they and is good against the stranguary or diffi- are thereloy eured. The kernels within the culty of making water; it expelleth the husks, commonly ealled Ashen Keys; pregravel and stone out of the kidneys, and vail against stitehes and pains in the sides, helpeth pains in the reins. And boiled in proeeeding of wind, and voideth away the white wine or viregar, it is prevalent for stone by provoking urine. them that have their arteries loosened, or are troubled with the hip-gout or sciatica. this, save only the first, viz. That Ash-tree The deeoction of the roots boiled in wine tops and leaves are good against the bitings and taken, is good to clear the sight, and of serpents and vipers. I suppose this had its being held in the mouth easeth the tooth- rise from Gerrard or Pliny, both whieh hold, ache. The gavden asparagus nourislieth :That there is such an antipathy between an 
adder and an Ash-tree, that if an adder be the most part, and their seed is ripe in July encompassed round with Ash-tree leaves, at the farthest.

she will sconer run through the fire than Government and virtues.] It is governed through the leaves: 'I'he contrary to which by Jupiter, and that gives hopes of a wholeis the truth, as both my eyes are witnesses. some healthful herb. It is good for the dis'I'he rest are virtues something likely, only eases of the chest or breast, for pains, and if it be in Winter when you cannot get the stitches in the side, and to expel crude and leaves, you may safely use the bark instead law humours from the belly and stomach, of them. 'The keys you may easily keep by the sweet savour and warming quality. all the year, gathering them when they are It dissolves the inward congealed blood ripc.

AVENS, CALLED ALSO COTEWORT, AND IERB BONET. 'Phatz, 2 .

Descript.] The ordinary Avens hath many long, rough, dark green, winged leaves, rising from the rool, every one made of many leaves set on each side of the middle rib, the largest three whereof grow at the end, and are snipped or dented round about the edges; the other bcing small picces, sometimes two and sometimes four, standing on each side of the middle rib underneath them. Among which do rise up divers rough or hairy stalks about two feet high, branching forth with leaves at every joint not so long as those below, but almost as much cut in on the edges, some into three parts, some into more. On the tops of the branches stand small, pale, yellow Howers, consisting of fire leaves, like the flowers of Cinquetoil, but large, in the
middle whercof stand a small green herb, which when the flower is fallen, grows to be round, being made of many long greenish purple seeds (like grains) which will stick upon your clothes. 'The root consists of many brownish strings or fibres, smelling somewhat like unto cloves, especially those which grow in the higher, hotter, and drier grounds, and in free and clear air.

Place.] They grow wild in many places under liédge's sides, and by the path-ways in ficlds; yet they rather delight to grow in shadowy than sunny places.

Time.] They flower in May or June for
Lappening by falls or bruises, and the spitting of blood, if the roots, either green or dry, be boiled in wine and drank; as also all manner of inward wounds or outward, if washed or bathed thcrewith. 'The decoction also being drank, comforts the heart, and strengthens the stomach and a cold brain, and therefore is good in the Spring time to open obstructions of the liver, and helps the wind cholic; it also helps those that have fluxes, or are bursten, or have a rupture; it takes away spots or marks in the face, being washed therewith. The juice of the fresh root, or powder of the dried root, has the same effect with the decoction. The root in the Spring-time steeped in wine, gives it a delicate savour and taste, and being drank fasting every morning, comforts the heart, and is a good preservative against the plague, or any other poison. It helps indigestion, and wams a cold stomach, aind opens obstructions of the liver and spleen.

It is very safe.: you need have no dose prescribed; and is vely fit to be kept in every body's liouse.

\section{T.S LI. Plate 4}

'Turs lurb is so well known to be an inliabitant almost in every garden, that I shall not need to write any description thereof, although its virtues, which are many, may not be omitted.

Government and virtues.] It is an herb of Jupiter, and under Cancer, and strengthens nature much in all its actions. Let a syrup 
made with the juice of it and sugar (as you shall be taught at the latter end of this book) be kept in every gentlewoman's house to relieve the weak stomiachs and sick bodies of their poor sickly neighbours"; as also the herb kept dry in - the housc, that so with other convenient simples, you may make it into an clectuary with honey, according as the disease is you shall be taught at the latter end of my book. The Arabian physicians have extolled the virtues thercof to the skies; although the Greeks thought it not worth mentioning. Seraphio says, it causes the mind and heart to become merry, and revives the heart, faintings and swoonings, especially of such who are overtaken in sleep, and drives away all troublesome cares and thouglits out of the mind, arising from melancholy or black choler; which Avicen also confirms. It is very good to help digestion, and open obstructions of the brain, and hath so much purging quality in it (saith Avicen) as to expel those mclancholy vapours from the spirits and blood which are in the heart and arteries, although it cannot do so in other parts of the body. Dioscorides says, That the leaves steeped in wine, and the wine drank, and the leaves externally applied, is a remedy against the stings of a scorpion, and the bitings of mad dogs; and commends the decoction thereof for women to bathe or sit in to procure their courses; it is good to wash aching teeth therewith, and profitable for those that have the bloody-tlux. The leaves also, with a little nitre taken in drink, are good against the surfeit of mushrooms, helps the griping pains of the belly ; and being made into an electuary, it is good for them that cannot fetch their breath: Used with salt, it takes away wens, kernels, or hard swellings in the flesh or throat; it cleanses foul sores, and eases pains of the gout. It is good for the liver and spleen. A tansy or caudle being yearly sown in the
made with eggs, and juice thereof while it $:$ thereof take as follow. is young, putting to it some sugar and rosewater, is good for a woman in child-bed, when the after-birth is not thoroughly voided, and for their faintings upon or in their sore travail. The herb bruised and boiled in a little wine and oil, and laid warm on a boil, will ripen it, and break it.

\section{BA R B ERRY.}

The shrub is so well known by every boy and girl that has but attained to the age of seven years, that it needs no description.

Gorernment and virtues.] Mars owns the slirub, and presents it to the use of $\mathrm{my}$ countrymen to purge their bodies of cholcr. The inner rind of the Barberry-tree boiled in white wine, and a quarter of a pint drank each morning, is an excellent remedy to clearise the body of choleric humours, and free it from such diseases as choler causes, such as scabs, itch, tetters, ringworms, yellow jaundice, boils, \&c. It is excellent for hot agues, burnings, scaldings, heat of the blood, heat of the liver, bloody-flux; for the berries are as good as the bark, and more pleasing: they get a man a good stomach to his victuals, by strengthening the attractive faculty which is under Mars. The hair washed with the lye made of the tree and water, will make it turn ycllow, viz. of Mars' own colour. The fruit and rind of the shrub, the flowers of broom and of heath, or furz, cleanse the body of choler by sympathy, as the flowers, lcaves, and bark of the pcach-tree do by antipathy; because these are under Mars, that under Venus.

\section{BARLEY. $/$.}

TuE continual usefulness hereof liath made all in general so acquainted herewith, that it is altogether needless to describe it, several kinds hereof plentifully growing, being yearly sown in this land. 'The virtues itithereof take as follow. 
Govermment and virtues.] plant of Saturn: if you view diligently it effects by sympathy and antipathy, you may easily perceive a reason of them; as also why barley bread is so unwholesome for melancholy people. Barley in all the parts and compositions thereof (except malt) is more cooling than wheat, and a litthe cleansing: And all the preparations thereof, as barley-water and other things made thereof, give great nourishment to persons troubled with fevers, agues, and heats in the stoniach: A poultice made of barley meal or flour boiled in vinegar and honey, and a few dry ligs put into them, dissolves all impostliunies, and assuages inflammations, being thereto applied. And being boiled with melilot and camomileflowers, and some linseed, fenugreek, and rue in powder, and applied warm, it eases pains in side and stomach, and windiness of the spleen. The meal of barley and fleawort boiled in water, and made a poultice with honey and oil of lilies applied warm, cures swellings. under the ears, throat, neck, and such like; and a plaister made thereof with tar, with sharp vinegar into a poultice, and laid on hot, helps the leprosy; being boiled in red wine with: pomegranate rinds, and myrtles, stays the lask or other flux of the belly; boiled with vinegar and quince, it eases the pains of the gout; barley-tlour, white salt, honey, and vinegar mingled together, takes away the itch speedily and ccrtainly. The water distilled from the green barley in the end of May, is very grood for those that have defluctions of humours fallen into their eyes, and eases the pain, being dropped into them: or white bread steeped therein, and bound on the eyes, does the same.

GARDEN BAZIL, OR SWEET BAZIL.

Deseript.] 'Tu $\mathrm{E}$ greater or ordinary Bazil rises up usually with one upright stalk, divers!y branching forth on all sides, with after-birth; and as it helps the deficiency two leaves at every joint, which are somewhat broad and round, yet printed, of a ed about the edges, and of a strong healthy scent. The flowers are small and white, and standing at the tops of the branches, with two small leaves at the joints, in some places green, in other's brown, after which come black seed. The root perishes at the approach of Winter, and therefore must be new sown every year.

Place.] It grows in gardens.

Time.] It must be sowed late, and flowers in the heart of Summer, being a very tender plant.

Government and virtues.] This is the herb which all authors are together by the ears about, and rail at one another (like lawyers.) Galen and Dioscorides hold it not fit to be taken inwardly; and Chrysippus rails at it with downright Billingsgate rhetoric; Pliny, and the Arabian physicians, defend it.

For my own part, I presently found that speech true;

Non nostrium inter nos tantas componere lites. And away to Dr. Reason went I, who told me it was an herb of Mars, and under the Scorpion, and perhaps therefore called Basilicon, and it is no marvel if it carry a kind of virulent quality with it. Berng applied to the place bitten by venomous beasts, or stung by a wasp or hornct, it speedily draws the poison to it; Everg like drazes his like. Mizaldus affirms, that, being laid to rot in lorse-dung, it will breed venomous beasts. Hilarius, a French physician, affirms upon his own knowledge, that an acquaintance of his, by common smelling to it, liad a scorpion bred in lis brain. Something is the matter ; this herb and rue will not grow together, no, nor near one another: and we know rue is as great an enemy to poison as any that grows.

To conclucie: It expels both brili and pale green colour, but fresh; a little snipp- 
of Venus in one kind, so it spoils all her actions in another. I dare write no more of it.

\section{THE BAY TREE.4 .36}

'T'H Is is so well known that it needs no description: I shall therefore only write the virtues thereof, which are many.

Government and virtues.] I shall but only ald a word or two to what my friend has written, viz. that it is a tree of the sun, and under the celestial sign Leo, and resists witchcraft very potently, as also all the evils old Saturn can do to the body of man, and they are not a few ; for it is the speech of one, and I am mistaken if it were not comfortable in all cold griefs of the joints, Mizaldus, that neither witch nor devil, nerves, arteries, stomach, belly, or womb; thunder nor lightning, will liurt a man in and helps palsies, convulsions, cramp, the place where a Bay-tree is. Galen said, aches, tremblings, and numbness in any that the leaves or bark do dry and heal part, weariness also, and pains that come very much, and the berries more than the by sore travelling. All griefs and pairs leaves; the bark of the root is less sharp proceeding from wind, either in the head, and hot, but more bitter, and hath some astriction withal whereby it is cffectual to break the stone, and good to open obstrictions of the liver, spleen, and other inward parts, which bring the jaundice, dropsy, \&c. The berries are very effectual against all poison of venomous creatures, and the sting of wasps and bees; as also against the pestilence, or other infectious diseases, and therefore put into sundry treacles for that purpose; 'They likewise procure women's courses, and seven of them given to a woman in sore travail of child-birth, do cause a speedy delivery, and expcl the after birth, and therefore not to be taken by such as have not gone out their time, lest they procure abortion, or cause labour too soon. 'They wonderfully help all cold and rlieumatic distillations from the brain to the eycs, lungs or other parts; and being made into an electuary with honey, do help the consumption, old couglis, shoriness of breath, and thin rheums; as also the megrim. They the green husks, is lield to be very effectual mightily expel the wind, and provoke urine; against the stone, and to provolie urine. help the mother, and kill the worms. The? Bean flour is used in poultices to assuage leaves also work the like effects. A bath of the decoction of the leaves and berries, is singularly good for women to sit in, that are troubled with the mother, or the diseases thereof, or the "stoppings of their courses, or for the diseases of the bladder, pains in the bowels by wind and stopping of the urine. A decoction likewise of equal parts of Bay-berries, cummin seed, hyssop, origanum, and euphiorbium, with some honey, and the head 'bathed therewith, wonderfully helps distillations and rheums, and settles the pallate of the mouth into its stomach, back, belly, or womb, by anointing the parts affected therewith: And pains in the ears are also cured by dropping in some of the oil, or by receiving into the ears the fume of the decoction of the berries through a funnel. The oil takes away the marks or the skin and flesh by bruises, falls, \&c. and dissolves the congealed blood in them. It helps also the itch, scabs, and weals in the skin.

\section{B E A N S.}

BотIr the garden and field beans are so well known, that it saves me the labour or writing any description of them. The virtues follow.

Government and virtues.] They are plants of Venus, and the distilled water of the flower of garden beans is good to clean the face and skin from spots and wrinkles, and the meal or flour of them, or the small beans doth the same. The water distilled from . 
inflammations arising from wounds, and 3 is to say, white, yellow, red, blackish, or of the swelling of women's breasts caused by a deeper purple, but white is the most usual ; the curdling of their milk, and represses after which come long and slender flat pods, their milk; Flour of beans and Fenugreek some crooked, some straight, with a string mixed with honey, and applied to felons, running down the back thereof, wherein is boils, bruises, or blue marks by blows, or flattish round fruit made like a kidney; the the imposthumes in the kernels of the ears, root long, spreads witl many strings anhelps them all, and with Rose leaves, nexed to it, and perishes every year.

Frankincense and the white of an egg, being applied to the eyes, helps them that are swollen or do water, or have received any blow upon them, if used with wine. If a bean be parted in two, the skin being taken away, and laid on the place where the leech hath been set that bleeds too much, stays the bleeding. Bean flour boiled to a poultice with wine and vinegar, and some oil put thereto, eases both pains and swelling of the privities. The husks boiled in water to the consumption of a third part thereof, stays a lask; and the ashes of the husks, made up with old hog's grease, helps the old pains, contusions, and wounds of the sinews, the sciatica and gout. The field beans have all the aforementioned virtues as the garden beans.

Beans eaten are extremely windy meat; but if after the Dutch fashion, when they are half boiled you husk them and then stew them, (I cannot tell you how, for I never was a cook in all my life) they are wholesome food.

\section{FRENCH BEANS. T. IV.}

Descript.] THIs French or Kidney Bean arises at first but with one stalk, which afterwards divides itself into many arms or branches, but all so weak that if they be not sustained with sticks or poles, they will be fruitless upon the ground. At several places of these branches grow foot stalks, each with three broad round and pointed green leaves at the end of them; towards? the top comes forth divers flowers made like? to pease blossoms, of the same colour for the most part that the fruit will be of ; that

(3.)

There is another sort of French beans commonly growing with us in this land, which is called the Scarlet flower Bean.

This rises with sundry branches as the other, but runs. higher, to the length of hoppoles, abont which they grow twining, but turning contrary to the sun, having footstalks with three leaves on each, as on the other; the flowers also are like the other, and of a most orient scarlet colour. The Beans are larger than the ordinary kind, of a dead purple colour turning black when ripe and dry; the root perishes in Winter.

Government and virtues.] These also belong to Dame Venus, and being dried and beat to powder, are as great strengtheners of the kidneys as any are; neither is there a better lemedy than it; a dram at a time taken in white wine to prevent the stone, or to cleanse the kidneys of gravel or stoppage. The ordinary French Beans are of an easy digestion; they move the belly, provoke urine, enlarge the breast that is straightened with shortness of breath, engender sperm, and incite to venery. And the scarlet coloured Beans, in regard of the glorious beauty of their colour, being set near a quickset hedge, will much adorn the same, by climbing up thereon, so that they may be discerned a great way, not without adiniration of the beholders at a distance. But they will go near to kill the quicksets by cloathing them in scarlet.

\section{IADIES BED-STRAW.P.TV.}

BESIDEs the common name above written, it is called Cheese-Rennęt, because it performs the same office, as also Gallion, 
Pettimugget, and Maiden-hair; and by some Wild Rosemary.

Descript.] 'This rises up with divers Descrpt.] The rises up ing small brown, and square upright stalks, a or sallad oil, with some wax melted therein, yard high or more; sometines branches after it is strained; either the oil made forth into divers parts, full of joints, and with divers very fine small leaves at every one of them, little or nothing rough at all; at the tops of the branches grow many long tufts or branches of yellow flowers very thick set together, from the several joints which consist of four leaves a piece, which smell somewhat strong, but not unpleasant. The seed is small and black like poppy seed, two for the most part joined together : The root is reddish, with many small threads fastened to it, which take strong hold of the ground, and creep a little: and the branches leaning a little down to the ground, ? take root at the joints thereof, whereby it is easily encreased.

There is another sort of Ladies Bedstraw growing frequently in England, which bears white flowers as the other doth yellow ; but the branches of this are so weak, that unless it be sustained by the hedges, oì other things near which it grows, it will lie down to the ground; the leaves a little bigger than the former, and the flowers not so plentiful as these; and the root hereof is also thready and abiding.

Place.] They grow in meadows and pastures both wet and dry, and by the hedges.

Time.] They flower in May for the most part, and the sced is ripe in July and August.

Government and virtues.] They are both herbs of Venus, and therefore strengthening the parts both interial and external, which she rules. The decoction of the former of those being drank, is good"to fret and break the stone, provoke urine, stays inward bleeding, and heals inward wounds. The herb or flower bruised and put into the nostrils, stays their bleeding likewise: The flowers and herbs being made into an the red Beet being under Saturn and the oil, by being set in the sun, and clianged after it has stood ten or twelve days; or into an ointment being boiled in $A$ mumga thereof, or the ointment, do help burnings with fire, or scalding with water. The same also, or the decoction of the herb and flower, is good to bathe the feet of travellers and lacquies, whose long running causes weariness and stiffness in the sinews and joints. If the decoction be used warm, and the joints afterwards anointed with ointment, it helps the dry scab, and the itch in children; and the herb with the white flower is also very good for the sinews, arteries, and joints, to comfort and strengthen them after travel, cold, and pains.

\section{BEETS. Plates.}

OF Beets there are two sorts, which are best known generally, and whereof I shall principally treat at this time, viz. the white and red Beets, and their virtues.

Descript.] The common white Beet has many great leaves next the ground, somewhat large and of a whitish green colour. The stalk is great, strong, and ribbed, bearing great store of leaves upon it, almost to the very top of it: The flowers grow in very long tufts, small at the end, and turning down their heads, which are small, pale greenish, yellow buds; giving cornered hard, and when it has given seed is of no use at all.

The common red Beet differs not from the white, but only it is less, and the leaves and the roots are somewhat red; the leaves are differently red, some only with red stalks or veins; some of a fresh red, and others of a dark red. The root thereof is red, spungy, and not used to be eaten. Government and virtues.] The government the red Beet being under Saturn and the prickly seed. 'The root is great, long, and 
white under Jupiter; therefore take the branches, being round bellied and open at virtues of them apart, each by itself. The the brins, and divided into two parts, the white Beet much loosens the belly, and uppermost being like a hood, and the loweris of a cleansing, digesting quality, and most like a hip hanging down, of a dark provokes urine. The juice of it opens obstructions both of the liver and spleen, their places small round heads with small and is good for the head-ache and swim- points at the ends, wherein lie small and mings therein, and turnings of the brain; brownish seeds; the root is a thick bush of and is effectual also against all venomous strings and shreds, growing from the head. creatures; and applied to the temples, Place.] It grows by the ditch side, stays inflammations in the eyes; it helps brooks, and other water-courses, generally burnings, being used with oil, and with a through this land, and is seldom found far little alum put to it, is good for St. An- from the water-side.

thony's fire. It is good for all wheals, Time.] It flowers about July, and the pushes, blisters, and blains in the skin : the seed is ripe in August.

herb boiled, and laid upon chilblains or kibes, helps them. 'The decoction thereof is an herb of Jupiter in Cancer, and is apin water and some vinegar, heals the itch, propriated more to wounds and hurts in the if bathed therewith; and cleanses the head breasts than Wood Betony, which follows; of dandruff, scurf, and dry scabs, and does? It is an excellent remedy for sick hogs. It much good for fretting and running sores, ulcers, and cankers in the head, legs, or and applied are effectual for allold and filthy other parts, and is much commended against ulcers ; and especially if the juice of the baldness and shedding the hair.

The red Beet is good to stay the bloodyflux, women's courses, and the whites, and to help the yellow jaundice; the juice of the root put into the nostrils, purges the head, helps the noise in the ears, and the tooth-ache; the juice snuffed up the nose, helps a stinking breath, if the cause lie in ? the nose, as many times it does, if any bruise has been there: as also want of smell coming that way.

WATER BETONY. .3

$\mathrm{C}_{\mathrm{ALLED}}$ also Brown-wort, and in Yorkshire, Bishop's-leaves.

Descript.] First, of the Water Betony, which rises up with square, hard, greenish stalks, sometimes brown, set with broad dark green leaves dented about the edges with notches somewhat resembling the leaves of the Wood Betony, but much larger too, for the most part set at a joint. The flowers has many leaves rising from the root, are many, set at the tops of the stalks and 3 which are somewhat broad and round at 
the end roundly dented about the edges, purc honey is no less available for all sorts standing upon long foot stalks, from among of coughs, or colds, wheesing, or shortness which rise up small, square, slender, but of breath, distillations of thin rheum upon upright hairy stalks, with some leaves there- the lings, which causes consumptions. on to a piece at the joints, smaller than the The decoction made with Mead, and a little lower, whereon are set several spiked heads Pennyroyal, is good for those that are of flowers like Lavender, but thicker and troubled with putrid agues, whether quoshorter for the most part, and of a reddish tidian, tertian, or quartan, and to draw or purple colour, spotted with white spots down and evacuaic the blood and humours, both in the upper and lower part. 'The that by falling into the eyes, do hinder the seeds being contained within the husks that sight; the decoction thereof made in wine hold the flowers, are blackish, somewhat and taken, kills the worms in the belly, long and uncven. The roots are many opens obstructions both of the spleen and white thready strings: the stalk perishes, liver; cures stitches, and pains in the but the roots with some leaves thereon, back and sides; the torments and griping abide all the Winter. The whole plant is pains in the bowels, and the wind cholic; somewhat small.

Place.]. It grows frequently in woods, helps to bring down women's courses, and and delights in shady places,

Time.] And it flowers in July; after? which the seed is quickly ripe, yet in its prime in May.

Government and virtues.] The herb is appropriated to the planet Jupiter, and the sign Aries. Antonius Musa, physician to the Emperor Augustus Caesar, wrote a peculiar book of the virtues of this herb ; and among other virtues saith of it, that it pre- serpents, or mad dogs, being used inwardly
serves the liver and bodies of men from and applied outwardly to the place. A the danger of epidemical diseases, and from dram of the powder of Betony taken with witchcraft also; it helps those that loath a little honey in some vinegar, does wonand cannot digest their meat, those that derfully refresh those that are over wearied have weak stomachs and sour belchings, or by travelling. It stays bleeding at the mouth continual rising in their stomachs, using it or nose, and helps those that void or spit familiarly either green or dry; either the blood, and those that are bursten or have herb, or root, or the flowers, in broth, drink, a rupture; and is good for such as are or meat, or made into conserve, syrup, bruised by any fall or otherwise. The water, electuary, or powder, as every one green herb bruised, or the juice applied to may best frame themselves unto, or as the any inward hurt, or sutward green wound time and season-requires; taken any of in the head or body, will quickly.heal and the aforesaid ways, it helps the jaundice, close it up ; as also any reins or sinews that falling sickness, the palsy, convulsions, or are cut, and will draw forth any broken shrinking of the sinews, the gout and those bone or splinter, thorn or other things got that are inclined to dropsy, those that have into the flesh. It is no less profitable for continual pains in their heads, although it old sores or filthy ulcers, yea, tho' they be turn to plurensy. 'The powder mixed with fistulous and hollow. But some do advise 
to put a little salt for this purpose, being applied with a little hog's lard, it helps a plague sore, and other boils and pushes. The fumes of the deeoetion while it is warm, reeeived by a funnel into the ears, eases the pains of them, destroys the worms and eures the running sores in them. The juiee dropped into them does the same. 'The root of Betony is displeasing both to the taste and stomach, whereas the leaves and flowers, by their sweet and spicy taste, are comfortable both to meat and medieine.

These are some of the many virtues Anthony Muse, an expert physician, (for it was not the practice of Octavius Cesar to keep fools about him) appropriates to Betony ; it is a very precious herb, that is certain, and most fitting to be kept in a man's house, both in syrup, conserve, oil, ointment and plaister. The flowers are usually eonserved.

\section{TIIE BEECII TREE.}

Is treating of this tree, you must understand, that I mean the green mast Beech, which is by way of distinction from that other small rough sort, ealled in Sussex the smaller Becel, but in Essex Horm-bean.

I suppose it is needless to deseribe it, being already too well known to my. countrymen.

Place.] It grows in woods amongst oaks and other trees, and in parks, forests, and ehases, to feed deer; and in other plaees to fatten swine.

Time:] It blooms in the end of April, or beginning of May, for the nost part, and the fruit is ripe in September.

Gorernment and virtues.] It is a plant of Saturn, and therefore performs his qualitics and proportion in these operations. 'The leaves of the Beech tree are enoling and binding, and therefore good to be applied to hot swellings to discuss them; the nuts do much nourish such beasts as feed thereon.
The water that is found in the hollow places of deeaying Beeches will cure both man and beast of any scurf, or rumning tetters, if they be washed therewith; you may buil the leaves into a poultiee, or make an ointment of them when time of year serves.

BILBERRIES, CALLED BY SOME WHORTS, AND WHORTLE-BERRIES. $\mathscr{C} 3$.

Descript.] OF these I shall only speak of two sorts whieh are common in England, viz. 'The black and red berries. And first of the black.

The small bush creeps along upon the ground, searcely rising half a yard high, with divers small green leaves set in the green branches, not always one against the other, and a little dented about the edges: At the foot of the leaves eome forth small, hollow, pale, bluish coloured flowers, the brims ending at five points, with a reddish thread in the middle, which pass into small round berries of the bigness and colour of juniper berries, but of a purple, sweetish sharp taste; the juice of them gives a purplish colour in their hands and lips that eat and handle them, especially if they break them. The root grows aslope under ground, shooting forth in sundry plaees as it ereeps. This looses its leaves in Winter.

The Red Bilberry, or Whortle-Bush, rises up like the former, having sundry hard leaves, like the Box-tree leaves, green and round pointed, standing on the several branehcs, at the top whereof only, and not from the sides, as in the former, come forth divers round, reddish, sappy berries, when they are ripe, of a sharp taste. The root runs in the ground, as in the former, but the leaves of this abide all Winter.

Place.] The first grows in forests, on the heaths, and sucll like barren plaees: the red grows in the north parts of this land, as Lancashire, Yorkshire, \&c.

Time.] They flower in March and Apris, 
and the fruit of the black is ripe in July and August.

Government and cirtues.] They are under the dominion of Jupiter. It is a pity they are used 110 more in physic than they are. The black Bilberries are good in hot agues and to cool the heat of the liver and stomach; they do somewhat bind the belly, and stay vomitings and loatlings; the juice of the berrics made in a syrup, or the pulp made into a conserve witl sugar, is good for the purposes aforesaid, as also for an old cough, or an ulcer in the lungs, or other diseases therein. 'I'he Red W'orts are more binding, and stop women's courses, spitting of blood, or any other flux of blood or humours, being used as well outwardly as inwardly.

BIFOIL, OR TWABLADE. Shatis.

Descript.] TH Is small herb, from a root somewhat sweet, slooting downward many long strings, rises up a round green stalk, bare or naked next the ground for an inch, two or three to the middle thereof as it is in age or growth; as also from the middle upward to the flowers, having only two broad plaintain-like leaves (but whiter) set at the middle of the stalk one against another, compassing it round at the bottom of them.

Place.] It is an usual inlabitant in woods, copses, and in many places in this land.

There is anotlicr sort grows in wet grounds and inarshes, which is somewhat different firm the former. It is a smaller plant, and greener, having sometimes three leaves; the spike of the flowers is less than the former, and the roots of this do run or creep in the ground.

They are often used by many to good purpose for wounds, both green and old, to consoliclate or knit ruptures; and well it may, being a plant of Saturn.

THE BIRCII TREE.

Descript.] This grows a goodly tall strait tree, fraught witl many bouglıs, and slender branclies bending downward: the old being covered with discoloured chapped bark, and the younger being browner by much. The leaves at the first breaking out are crumpled, and afterwards like the beech leaves, but smaller and greener, and dented about the edges. It bears small short cat-skins, somewhat like those of the liazelnut-tree, which abide on the branches a long time, until growing ripe, they fall on the ground, and their seed with them.

Place.] It usually grows in woods.

Government and rirtues.] It is a tree of Venus; the juice of the leaves, while they are young, or the distilled water of them, or the water that comes from the tree being bored with an auger, and distilled afterwards ; any of these being drank for some days together, is available to break the stone in the kidneys and bladder, and is good also to wash sore mouths.

\section{BIRD'S FOOT.}

This small herb grows not above a span high with many branches spread upon the ground, set with many wings of small leaves. The flowers grow upon the branches, many small ones of a pale yellow colour. being set a liead together, which afterwards turn into small jointed cods, wcll resembling the claw of small birds, whence it took its name.

There is another sort of Bird's Foot in all things like the former, but a little larger; the flower of a pale whitish red colour, and the cods distinct by joints like the other, but a little more crooked; and the roots do carry many small white nots or kernels amongst the strings.

Place.] These grow on heaths, and many open untilled places of this land.

Time.] They flower and seed in the end of Summer.

Government and virtues.] They belong to Saturn, and are of a drying, binding quality, 
and thereby very good to be used in wound drinks; as also to apply outwardly for the same purpose. But the latter Bird's Foot is found by experience to break the stone in the back or kidneys, and drives them forth, if the decoction thereof be taken; and it wonderfully helps the rupture, being taken inwardly, and outwardly applied to the place.

All salts have best operations upon the stone, as ointments and plaisters have upon wounds: and therefore you may make a salt of this for the stone; the way how to do so may be found in my translation of the London Dispensatory; and it may be I may give you it again in plainer terms at the latter end of this book.

BISHOP'S-WEED. 4 . I.

Besides the common name Bishopweed, it is usually known by the Greek name Ammi and Ammois; some call it Ethiopian Cummin-seed, and others Cummin-royal, as also Herb William, and Bullwort.

Descript.] Common Bishop's-weed rises up with a round straight stalk, sometimes as high as a man, but usually three or four feet high, beset with divers sinall, long and somewhat broad lcaves, cut in some places, and dented about the edges, growing one against another, of a dark green colour, having.sundry branches on them, and at the top small umbels of white flowers, which turn into small round seeds little bigger than parsley seeds, of a quick hot scent and taste; the root is white and stringy; perishing ycarly, and usually rises again on its own sowing.

Place.] It grows wild in many places in England and Wales, as between Greenhitlie: and Gravesend.

Govermment and virtues.] It is hot and dry in the third degree, of a bitter taste, and somewhat sharp withal; it provokes lust to purpose; I suppose Venus owns it.
It digests humours, provokes urine and women's courses, dissolveth wind, and being taken in wine it eases pain and griping in the bowels, and is good against the biting of serpents; it is used to good effects in those medicines which are given to hinder the poisonous operation of Cantharides, upon the passage of the urine: being mixed with honey and applied to black and blue marks, coming of blows or bruises, it takes them away; and being drank or outwardly applied, it abates an high colour, and makes it pale; and the fumes thereof taken with rosin or raisins, cleanses the mother.

BISTORT, OR SNAKEWEED. 3.

IT is called Snakeweed, English Serpentary, Dragon-wort, Osterick, and Passions.

Descript.] This has a thick short knobbed root, blackish without, and somewhat reddish within, a little crooked or turned together, of a hard astringent taste, with divers black threads hanging there, from whence spring up every year divers leaves, standing upon long footstalks, bcing somewhat broad and long like a dock leaf, and a little pointed at the ends, but that it is of a bluish green colour on the upper side, and of an ash-colour girey, and a little purplish underneath, with divers veins therein, from among which rise up divers small and shender stalks, two feet high, and almost naked and without leaves, or with a very few, and narrow, bearing a spikey bush of pale-coloured flowers; which being past, there abides small seed, like unto sorrel seed, but greater.

There are other sorts of Bistort growing in this land, but smaller, both in height, root, and stalks, and especially in the leaves. 'The root blackish without, and somewhat whitish within; of an austere binding taste, as the former.

Place.] They grow in shadowy moist woods, and at the foot of hills, but are 
chiefly nourished up in gardens. The narrow leafed Bistort grows in the north, in Lancashire, Yorkshire, and Cumberland.

Time.] They flower about the end of May, and the seed is ripe about the beginning of July.

Government and virtues.] It belongs to Saturn, and is in operation cold and dry ; both the leaves and roots have a powerful faculty to resist all poison. The root in powder taken in drink expels the venom of the plague, the 'small-pox, measels, purples, or any other infectious disease, driving it out by sweating. The root in powder, the decoction thereof in wine being drank, stays all manner of inward bleeding, or spitting of blood, and any fluxes in the body of either man or woman, or vomiting. It is also very available against ruptures, or burstings, or all bruises from falls, dissolving the congealed blood, and easing the pains that happen thereupon; it also helps the jaundice.

Whic water distilled from both leaves and roots, is a singular remedy to wash any place bitten or stung by any venomous creature; as also for any of the purposes before spoken of, and is very good to wash any running sores or ulcers. 'The decoction of the root in wine being drank, hinders abortion or miscarriage in child-bearing. The leaves atso kill the worms in children, and is a great help to them that cannot keep their water; if the juice of plaintain be added thereto, and outwardly applied, much helpeth the ghonorrhea, or running of the reins. A dram of the powder of the root taken in vater thereof, wherein some red bot iron or steel hath been quenched, is also an admirable help thereto, so as the body be first prepared and purged from the offensive hunours. The leaves, seed, or roots are all very good in decoctions, drinks or lotions, for inward or outward wounds, or other sores. And the powder strewed upon iny cut or wound in a vein, stays the immoderate blceding thereof. The decoction of the root in water, where unto some pomegranate peels and flowers are added, injected into the matrix, stays the inmoderate flux of the courses. 'T'he root thereof with pelitory of Spain, and burnt allum, of each a little quantity, beaten small and into paste with some honey, and a little piece thereof put into an hollow tooth, or held between the teeth, if there be no hollowness in them, stays the defluction of rheum upon them which causes pains, and helps to cleanse the head, and void much offensive water. The distilled water is very effectual to wash sores or cankers in the nose, or' any other part; if the porrder of the root be applied thereunto afterwards. It is good also to fasten the gums, and to take away the heat and inflammations that happen in the jaws, almonds of the throat: or mouth, if the decoction of the leares, roots, or seeds biuised, or the juice of them be applied; but the roots are most effectual to the purposes aforesaid.

\section{ON E-B L A D F.}

Descript.] This small plant nerer bears more than one leaf, but only when it rises up with its stalk, which thereon bears another, and sèldom more, which are of a blueish green colour, broad at the bottom, and pointed with many ribs or veins like plaintain; at the top of the stalk grow many smatl flowers star-fashion, smelling somewhat sweet; after which comes small reddish berries when they are ripe. The root small of the bigness of a rush, lying and ereeping under the upper crust of the earth, shooting forth in divers places.

Place.] It grows in moist, shadowy, grassy places of woods, in many places of this realm.

Time.] It flowers about May, and the berries are ripe in June, and then quickly perishes, until the next year it springs from the same again. 
Government and virtues.] It is an herb of the Sun, and therefore cordial; half a dram, or a dram at most, of the root hereof in powder taken in wine and vinegar, of each. a little quantity, and the party presently laid to sweat, is held to be a sovereign remedy for those that are infected with the plague, and have a sore upon them, by expelling the poison, and defending the heart and spirits from danger. It is also accounted a singular good wound herb, and therefore used with other herbs in making such bahms as are necessary for curing of wounds, either green or old, and especially if the nerves be hurt.

THE BRAMBLE, OR BLACK-BERRY BUSH.

$\mathrm{IT}_{\mathrm{T}}$ is so well known that it needs no description. 'The virtues thereof are as follows.

Gocernment and virtues.] It is a plant of Venus in Aries. If any ask the reason why Venus is so prickly? Tell them 'tis because she is in the house of Mars. The buds, leaves, and branches, while they are green, are of a good use in the ulcers and putrid sores of the mouth and throat, and of the quinsey, and likewise to heal other fiesh wounds and sores; but the flowers and fruits unripe are very binding, and so profitable for the bloody flux, lasks, and are a fit remedy for spitting of blood. Either the decoction of the powder or of the root taken, is good to break or drive forth gravel and the stone in the reins and kidneys. The leaves and brambles, as well green as dry, are exceeding good lotions for sores in the mouth, or secret parts. 'I'he decoction of them, and of the dried branches, do much bind the belly and are good for too much flowing of women's courses; the berries of the flowers are a powerful remedy against the poison of the most venomous serpents; as well drank as outwardly applied, helpeth the sores of the fundament and the piles; the juice of the berries mixed with the juice of mulberries, do bind more effectually, and help all fretting and eating sores and ulcers wheresoever. The distilled water of the branches, leaves, and flowers, or of the fruit, is very pleasant in taste, and very effectual in fevers and hot distempers of the body, head, eyes, and other parts, and for the purposes aforesaid. The leaves boiled in lye, and the head washed therewith, healeth the itch and running sores thereof, and makes the hair black. The powder of the leaves strewed on cankers and rumning ulcers, wonderfully helps to heal them. Some use to condensate the juice of the leaves, and some the juice of the berries, to keep for their use all the year, for the purposes aforesaid.

\section{BLITES.}

Descript.] Or these there are two sorts commonly known, viz. White and red. The White has leaves somewhat like to beets, but smaller, rounder and of a whitish green colour, erery one standing upon a small long footstalk: the stalk rises up two or three feet high, with such like leaves thereon; the flowers grow at the top in long round tufts or clusters, wherein are contained small and round seed; the root is very full of threads or strings.

'The Red Blite is in all things like the White, but that his leaves and tufted heads are exceeding red at first, and after turn more purple.

There are other kinds of Blites which grow different from the two former sorts but little, but only the wild are smaller in every part.

Place.] They grow in gardens, and wild in many places in this land.

Time.] They seed in August and September.

Government and zirtues.] They are all of them cooling, drying, and binding, serring to restrain the fluxes of blood in either. man or woman, especially the Red; whicl 
also stays the overflowing of the women's reds, as the white Blites stay the whites in women. It is an excellent secret; you cannot well fail in the use. They are all under the dominion of Venus.

There is another sort of wild Blites like the other wild kinds, but have long and spikey heads of greenish secds, seeming by the thick setting together to be all seed.

This sort the fishers are delighted with, and it is good and usual bait; for fishes will bite fast enough at them, if you have wit cnough to catch them when they bite.

BOR AGF AND BUGLOSS.

Trise are so well known to the inhabitants in every garden that I hold it needless to describe them.

'To these I may add a thind sort, which is not so common, nor yet so well known, and therefore I shall give you its name and description.

It is called Langue de Bouff but why then should they call one herb by the name of Bugloss, and another by the name Langue de Bouf? it is some question to me, secing one signifies $\mathrm{Ox}_{\mathrm{x}}$-tongue in Greek, and the other signifies the same in French.

Descript.] The leaves whereof are smaller than those of Bugloss but much rougher ; the stalks rising up about a foot and a half high, and is most commonly of a red colour; the flowers stand in scaly rough heads, bcing composed of many small yellow flowers, not much unlike to those of Dandelions, and the seed flicth away in down as that doth; you may easily know the flowers by their taste, for they are very bitter.

Place.] It grows wild in many places of this land, and may be plentifully found near London, as between Rotherhithe and Deptford, by the ditch side. Its virtues are held to be the same with Borage and Bugloss, only this is somewhat hotter.
Time.] 'They flower in June and July, and the sced is ripe sloortly after.

Gotcrmment and tirtucs.] 'I'hey are all three herbs of Jupiter and under Lco, all great cordials, and great strengtheners of nature. 'I'he leaves and roots are to very good purpose used in putrid and pestilential fevers, to defend the heart, and help to resist and expel the poison, or the renom of other creatures; the sced is of the like effects; and the seed and leaves are good to increase milk in women's breasts; the leaves, flowers, and seed, all or any of them, are good to expel pensiveness and melancholy; it helps to clarify the blood, and mitigate heat in ferers. The juice nlade into a syrup) prevails much to all the purposes aforesaid, and is put with other cooling, opening and cleansing lierbs to open obstructions, and help the yellow jaundice, and mixed with fumitory, to cool, cleanse, and temper the blood thereby ; it lielps the itch, ringworms and tetters, or other spreadnng scabs or sores. 'The flowers candied or made into a conscrve, are helpful in the former cases, but are chiefly used as a cordial, and are good for those that are wcak in long sickness, and to comfort the heart and spirits of those that are in a consumption, or troubled with often swoonings, or passions of the heart. 'The distilled water is no less effectual to all the purposes aforesaid, and helps the redness and intlammations of the eyes, being washed therewith; the lierb dried is never used, but the green; yet the ashes thereof boiled in mead, or honied water, is available against the inflammations and ulcers in the moutl or throat, to gargle it therewith; the roots of Bugloss are effectual, being made into a licking electuary for the cough, and to condensate thick phlegn, and the rheumatic distillations mpon the lungs.

BLUE-BOTTLE.

It is called Syanus, I suppose fiom the 
colour of it: Hurt-sickle, because it turns the edge of the sickles that reap the corn; Bluc-blow, Corn flower, and Bluc-bottle.

Descript.] I shall only describe that which is commonest, and in my opinion most useful; its leaves spread upon the ground, being of a whitish green colour, somewhat on the cdges like those of colnscabions, amongst which rises up a stalk divided into divers branches, beset with long leaves of a grecnish colour, either but very little indented, or not at all; the flowers are of a blucish colour, from whence it took its name, consisting of an innumcrable company of flowers set in a scaly head, not much unlike those of knap weed; the sced is smooth, bright, and shining, wrapped up in a wooly mantle; the root perishes every year.

Place.] They grow in corn fields, amongst all sorts of corn, (pcase, beans, and tares excepted.) If you please to take them up from thence, and transplant them in your garden, especially towards the full of the moon, they will grow more double than they are, and many times change colour.

Time.] They flower from the beginning of May, to the end of harrest.

Government and virtues.] As they are naturally cold, dry, and binding, so they are under the dominion of Saturn. The powder or dried lcaves of the Bluc-bottle, or Corn flower, is given with good success to those that are bruised by a fall, or have broken a rein inwardly, and roid much blood at the mouth; being taken in the water of plaintain, horsetail, or the greater comfrey, it is a remedy against the poison of the scorpion, and resists all venoms and poison. The secd or leaves taken in wine, is very good against the plague, and all infectious discases, and is rery good in pestilential fevers. 'The juice put into fresh or grcen wounds, dotli quickly solder up the lips of them together, and is very effectual to heal all ulcers and sores in the mouth.
The juice dropped into the eyes takes away the heat and inflammation of them. 'The distilled water of this herb, has the same propertics, and may be used for the effer'ts aforesaid.

\section{BRANK URSINE. $\mathcal{Z} . J \mathrm{~V}$}

Besines the common name BrankUrsine, it is also called Bears-brecch, and Acanthus, though I think our English names to be more proper; for the Greek word Acontlus, signifies any thistle whatsoever.

Descript.] This thistle shoots forth very many large, thick, sad green smooth leaves on the ground, with a very thick and juicy middle rib; the leaves are parted with sundry deep gashes on the edges; the leaves remain a long time, before any stalk appears, afterwards rises up a reasonable big stalk, three or four feet high, and brarcly decked with flowers from the middle of the stalk upwards ; for on the lower part of the stalk, there is neither branches nor leaf. 'The flowers are hooded and gaping, being white in colour, and standing in brownish husks, with a long small undivided leaf under cach leaf; they seldom sced in our country. Its roots are many, great and thick, blackish without and whitish within, full of a clanmy sap; a picce of them if you set it in the garden, and defend it from the first Winter cold, will grow and flourish.

Place.] They are only nursed in the gardens in England, where they will grow very well.

Time.] It flowers in June and July:

Government and virtues.] It is an excellent plant under the dominion of the Moon; I. could wish such as are studious would labour to keep it in the gardens. The leaves being boiled and used in glisters, is excellent good to molify the belly, and make the passage slippery. 'I'he decoction drank inwardly, is excellent and good for the bloody-flux: 'The leaves being bruised, 
or rather boiled and applied like a poultice are excellent good to unite broken bones and strengthen joints that have been put out. The decoction of either leaves or roots being drank, and the decoction of leares applied to the place, is excellent good for the king's evil that is broken and runneth; for by the influence of the moon, it revives the ends of the veins which are relaxed. There is scarce a better remedy to be applied to such places as are burnt with fire than this is, for it fetches out the fire, and heals it without a scar. This is an excellent remedy for such as are bursten, being either taken inwardly, or applied to the place. In like manner used, it helps the cramp and the gout. It is excellently good in hectic fevers, and restores radical moisture to such as are in consumptions.

\section{BRIONY, OR WILD VINE. 3.}

$I_{T}$ is called Wild, and Wood Vine, Tamus, or Ladies'Seal. . 'The white is called White Vine by some; and the black, Black Vine.

Descript.] The common White Briony grows ramping upon the hedges, sending forth many long, rough, very tender branches at the beginning, with many very rough, and broad leaves thereon, cut (for the most part) into five partitions, in form very like a vine leaf, but smaller, rough, and of a whitish hoary green colour, spreading very far, spreading and twining with his small claspers (that come forth at the joints with the leaves) very far on whatsoever stands next to it. At the several joints also (especially towards the top of the branches) comes forth a long stalk bearing many whitish flowers together on a long tuft, consisting of five small leaves a-piece, laid open like a star, after which cone the berries separated one from another, more than a cluster of grapes, green at the first, and very red when they are thorough ripe, of no good scent, but of a most loathsome taste provokes vomit. The root grows to be exceeding great, with many long twines or branches going from it, of a pale whitish colour on the outside, and more white within, and of a slarp, bitter, loathsome taste.

Place.] It grows on banks, or under hedges, through this land; the roots lie very deep.

I'ime.] It flowers in July and August, some earlier, and some later than the other.

Govermment and virtues.] They are furious martial plants. The root of Briony purges the belly with great violence, troubling the stomach and bunning the liver, and therefore not rashly to be taken; but being corrected, is very profitable for the diseases of the head, as falling sickness, giddiness, and swimmings, by drawing away much phlegm and rhematic humours that oppress the head, as also the joints and sinews; and is therefore good for palsies, convulsions, cramps, and stitches in the sides, and the dropsy, and for provoking urine; it cleanses the reins and kidneys from gravel and stone, by opening the obstructions of the spleen, and consumes the haidness and swelling thereof. 'The decoction of the root in wine, drank once a week at going to bed, cleanses the mother, and helps the rising thereof, expels the dead child; a dram of the root in powder taken in white wine, brings down their courses. An electuary made of the roots and honey, doth mightily cleanse the chest of rotten phlegm, and wonderfully help any old strong cough, to those that are troubled with shortness of breath, and is good for them that are bruised inwardly, to help to expel the clotted or congealed blood. 'The leares, fruit, and root do cleanse old and filthy sores, are good against all fretting and running cankers, grangrenes, and tetters, and therefore the berries are by sone country people called tetter-berries. The root cleanses the skin wonderfully from all black and blue spots, freckles, 
morphew, leprosy, foul scars, or other deformity whatsoever; also all running scabs and manginess are healed by the powder of the dried root, or the juice thereof, but especially by the fine white hardened juice. The distilled water of the root works the same effects, but more weakly; the root bruised and applied of itself to any place where the bones are broken, helps to draw them forth, as also splinters and thorns in the flesh; and being applied with a little wine mixed therewith, it breaks boils, and helps whitlows on the joints.-For all these latter, beginning at sores, cancers, \&c. apply it outwardly, mixing it with a little hog's grease, or other convenient ointment.

As for the furmer diseases where it must be taken inwardly, it purges very violently, and needs an abler hand to correct it than most country people have.

BROOK-LIME, OR WATER-PIMPERNEL. Descript.] This sends forth from a creeping root that shoots forth strings at every joint, as it runs, divers and sundry green stalks, round and sappy with some branches on them, somewhat broad, round, deep green, and thick leaves set by couples thereon; from the bottom whercof shoot forth long foot-stalks, with sundry small blue flowers on them, that consist of five small round pointed leaves a picce.

There is another sort nothing different from the former, but that it is greater, and the flowers of a paler green colour.

Place.] They grow in small standing waters, and usually near Water Cresses.

Time.] And flower in June and July, giving seed the next month after.

Government and virtues.] It is a hot and biting inartial plant. Brook-lime and Water-Cresses are generally used together in diet-drink, with other things serving to purge the blood and body from all ill humours that would destroy health, and are helpful to the scurvy. They do all provoke urine, and help to break the stone, and pass is away; they procure women's courses, and expel the dead child. Being fried with butter and vinegar, and applied warm, it helps all manner of tumours, swellings, and inflammations.

Such drinks ought to be made of sundry herbs, according to the malaciy. I shail give a plain and easy rule at the latter cnd of this book.

\section{BUTCHER'S BROOM. Dlates.}

IT is called Ruscus, and Bruscus, Kneeholm, Kneeholly, Kneehulver, and Pettigree.

Descript.] The first shoots that sprout from the root of Butcher's Broom, are thick, whitish, and short, somewhat like those of Asparagus, but greater, they rise up to be a foot and a half high, are spread into divers branches, green, and somewhat cressed with the roundness, tough and flexible, whereon are set somewhat broad and almost round hard leares and prickly, pointed at the end, of a dark green colour, two for the most part set at a place, very close and near together; about the middle of the leaf, on the back and lower side from the middle rib, breaks forth a small whitish green flower, consisting of four small round pointed lcaves, standing upon little or no foot-stalk, and in the place whereof comes a small round berry, green at the first, and red when it is ripe, wherein are two or three white, hard, round sceds contained. The root is thick, white, and great at the head, and from thence sends forth divers thick, white long, tough strings. Place.] It grows in copses, and upon heaths and waste grounds, and oftentimes under or near the holly bushes.

Time.] It shoots forth its young buds in the Spring, and the berries are ripe about September, the branches of leaves abiding green all the Winter.

Goterment and virtues.] It is a plant of K. 
Mars, being of a gallant cleansing and or the powder of the seed taken in drink, opening quality. 'The decoction of the? purges downwards, and draws phlegmatic root made with wine opens obstructions, and watery humours from the joints, whereprovokes urine, lielps to expel gravel and by it helps the dropsy, gout, sciatica, and the stone, the stranguary and women's pains of the hips and joints; it also procourses, also the yellow jaundice and the vokes strong vomits, and helps the pains of head-ache; And with some honcy or sugar the sides, and swelling of the spleen, put thereunto, cleanses the breast of phlegm, cleanses also the reins or kidneys and bladand the chest of such clammy humours der of the stone, provokes urine abundantly, gathered therein. The decoction of the and hinders the growing again of the stone root drank, and a poultice made of the in the body. The continual use of the berries and leaves applied, are effectual in powder of the leaves and seed doth cure knitting and consolidating broken bones or the black jaundice. The distilled water of parts out of joint. The common way of the flowers is protitable for all the same using it, is to boil the root of it, and Parsley purposes : it also helps surfeits, and alters and Fennel and Smallage in white wine, and the fit of agues, if three or four ounces drink the decoction, adding the like quan- thereof, with as much of the water of the tity of Grass-root to them: The more of lesser Centaury, and a little sugar put therethe root you boil, the stronger will the de-sin, be taken a little before the fit comes, coction be; it works no ill effects, yet I and the party be laid down to sweat in his hope you have wit enough to give the bed. The oil or water that is drawn from strongest decoction to the strongest bodies. the end of the green sticks heated in the

$$
\text { BROOM, AND BROOM-RAPE. O. } 4 \text {. }
$$

To spend time in writing a description hereof is altogether needless, it being so generally used by all the good housewives almost through this land to sweep their houses with, and therefore very well known to all sorts of people.

The Broom-rape springs up in many places from the rin lice in the head or body of any; places from the roots of the broom (but and is an especial remedy for joint aches, more often in fields, as by hedge-sides and and swollen knees, that come by the falling on heatlis.) The stalk whereof is of the down of humours.

bigness of a finger or thumb, above two feet high, having a shew of leaves on them, and many flowers at the top, of a reddish yellow colour, as also the stalks and leaves are.

The BroOM RAPE also is not without its
virtues.

Tus decoction thereof in wine, is thought to be as effectual to void the stone in the

Place.] They grow in many places of kidney or bladder, and to provoke urine, this land commonly, and as commonly as the Broom itself. 'The juice thereof is spoil all the land they grow in.

Time.] They flower in the Summer wounds, as old and filthy sores and maligmonths, and give their seed before Winter. nant uicers. 'The insolate oil, wherein theie

Govermment and virtues.] The juice or has been three or four repetitions of infusion decoction of the young branches, or seed, of the top stalks, with flowers strained and 
cleared, cleanses the skin from all manner of spots, marks, and freckles that rise either by the heat of the sun, or the malignity of humours. As for the Broom and Broomrape, Mars owns them, and is exceeding prejudicial to the liver; I suppose by reason of the antipathy between Jupiter and Mars, therefore if the liver be disaffected, minister none of it.

\section{BUCK'S-HORN PLANTAIN. C. 3}

Descript.]. THis being sown of seed, rises up at first with small, long, narrow, hairy, dark green leaves like grass, without any division or gash in them, but those that follow are gashed in on both sides the leaves into three or four gashes, and pointed at the ends, resembling the knags of a buck's horn, (whereof it took its name) and being well wound round about the root upon the ground, in order one by another, thereby resembling the form of a star, from among which rise up divers hairy stalks about a hand's breath high, bearing every one a small, long spiky head, like to those of the common Plantain, having such like bloomings and seed after them. The root is single, long and small, with divers strings at it.

Place.] They grow in sandy grounds, as in 'Tothill fields by Westminster, and divers other places of this land.

Time.] They flower and seed in May, June, and July, and their green leaves do ill a manner abide fresh all the Winter.

Government and virtues.] It is under the dominion of Saturn, and is of a gallant, drying, and binding quality. This boiled in wine and drank, and some of the leaves put to the hurt place, is an excellent remedy for the biting of the viper or adder, which I take to be one and the same: The sanre being also drank, helps those that are troubled with the stone in the veins or kidneys, by cooling the heat of the part afflicted, and strengthens them; also weak stomachs that cannot retain, but cast up their meat. It stays all bleeding both at mouth or nose ; bloody urine or the bloodyflux, and stops the lask of the belly and bowels. The leaves hereof bruised and laid to their sides that have an ague, suddenly eases the fit; and the leaves and roots applied to the wrists, works the same effects. The lierb boiled in ale and wine, and given for some mornings and evenings together, stays the distillation of lot and sharp rheums falling into the cyes fiom the head, and lielps all sorts of sore eyes.

\section{BUCK'S HORN.}

IT is called Iart's-horn, Herba-stella, and Herba-stellaria, Sanguinaria, Herb-Eve, Herb-Ivy, Wort-Tresses, and Swine-Cresses.

Descript.]. They have many small and weak straggling branches trailing here and there upon the ground: 'The leaves are many, small and jagged, not much unlike to those of Buck's-horn Plantain, but much smaller, and not so hairy. The flowers grow among the leaves in small, rough, whitish clusters; The seeds are smaller and brownish, of a bitter taste.

Place.] 'They grow in diry, barren, sandy grounds.

Time.] 'They flower and seed when the rest of the Plantains do.

Govermment and virtu:es.] This is also under the dominion of Saturn; the virtues are held to be the same as Buck's-horn Plantain, and therefore by all authors it is joined with it. 'The leaves bruised and applied to the place, stop bleeding. The herbs bruised and applied to warts, will make them consume and waste in a short time.

BUGLI.P.j.

Besines the name Bugle, it is called Middle Confound and Middle Comfrey, Brown Bugle, and by some Sicklewort, and 
Herb-Carpenter ; though in Essex we call for those that are liver-grown (as they ca, another herb by that name.

it.) It is wonderful in curing all manner o

Descript.] This has larger leaves than ulcers and sores, whether new and fresh those of the Self-heal, but else of the same or old and inveterate; yea, gangrenes and fashion, or rather longer ; in some green on fistulas also, if the leaves bruised and apthe upper side, and in others more brown- plied, or their juice be used to wash and ish, dented about the edges, somewhat hairy, bathe the place, and the same made into a as the square stalk is also which rises up to lotion, and some honey and alum cures be half a yard high sometimes, with the all sores in the mouth and gums, be they leaves set by couples, from the middle never so foul, or of long continuance; and almost, whereof upwards stand the flowers, works no less powerfully and effectually for together with many smaller and browner such ulcers and sores as happen in the leaves than the rest, on the stalk below set secret parts of men and women. Being at distance, and the stalk bare between also taken inwardly, or outwardly applied, them; among which flowers, are also small it helps those that have broken any bone, ones of a blueish and sometimes of an ash or have any member out of joint. An colour, fashioned like the flowers of Ground- ointment made with the leaves of Bugle, ivy, after which come small, round blackish Scabions and Sanicle bruised and boiled seeds. The root is composed of many in hog's grease, until the herbs be dry, and strings, and spreads upon the ground.

The white flowered Bugle differs not in occasions as shall require; it is so singularly form or greatness from the former, saving good for all sorts of hurts in the body, that that the leaves and stalks are always green, none that know its usefulness will be withand never brown, like the other, and the out it. flowers thereof are white.

Place.] They grow in woods, copses, and fields, generally throughout England, but the white flowered Bugle is not so plentiful as the former.

Time.] They flower from May until July, and in the mean time perfect their seed. The roots and leaves next thereunto upon the ground abiding all the Winter.

Government and vintues.] This herb be- vapour made thin by excessive drinking longs to Dame Venus: If the virtues of it strong liquor, and so flies up and disturbs make you fall in love with it (as they will the fancy, and breeds imaginations like
if you be wise) keep a syrup of it to take itself, viz. fearful and troublesome. Those inwardly, an ointment and plaister of it I have know cured by taking only two to use outwardly, always by you. spoonfuls of the syrup of this herb after

The decoction of the leaves and flowers supper two hours, when you go to bed. made in wine, and taken, dissolves the con- But whether. this does it by sympathy or gealed blood in those that are bruised in- antipathy, is some doubt in astrology. I wardly by a fall, or otherwise is very know there is great antipathy between effectual for any inward wounds, thursts, Saturn and Venus in matter of procreation; or stabs in the body or bowels; and it is yea, such a one, that the barrenness of an especial help in all wound-drinks, and Saturn can be removed by none but Venus; 
nor the lust of Venus be repelled by none but Saturn; but I am not of opinion this for if the Sun be the preserver of life under is done this way, and my reason is, because God, his herbs are the best in the world to these vapours though in quality melan- do it by. 'They are accounted to be both of choly, yet by their flying upward, secm to one property, but the lesser is more effectual be something aerial; therefore I rather think because quicker and more aromatic: It it is done by antipathy; Saturn being is a friend to the heart, liver, and other exalted in Libra, in the house of Venus.

BURNET.

principal parts of a man's body. Two or three of the stalks, with leaves put into a cup of wine, especially claret, are known to quicken the spirits, refresh and cheer the IT is called Sanguisorbia, Pimpinella, heart, and drive away melancholy : It is a Bipulo, Solbegrella, \&c. The common special help to defend the heart from noigarden Burnet is so well known, that it some vapours, and from infection of the needs no description.---'There is another sort pestilence, the juice thereof being taken in which is wild, the description whereof take some drink, and the party laid to sweat as follows.

Descript.] The great wild Burnet has an astringent quality, whereby they are winged leaves arising from the roots like the available in all manner of fluxes of blood garden Burnet, but not so many ; yet each or humours, to staunch bleedings inward or of these leaves are at the least twice as outward, lasks, scourings, the bloody-flux, large as the other, and nicked in the same? women's too abundant flux of courses, the manner about the edges, of a greyish coloum thites, and the choleric belchings and caston the under side; the stalks are greater, ings of the stomach, and is a singular and rise higher, with many such leaves set wound-herb for all sorts of wounds, both thereon, and greater heads at the top, of a of the head and body, either inward or outbrownish colour, and out of them come ward, for all old ulcers, running cankers, small dark purple flowers, like the former, and most sores, to be used either by the but greater. 'The root is black and long juice or decoction of the herb, or by the like the other, but great also: it has almost powder of the herb or root, or the water of neither scent nor taste therein, like the gar- the distilled herb or ointinent by itself, or den kind.

Place.] It first grows frequently in gar-also no less effectual both to stop fluxes, dens. The wild kind grows in divers and dry up moist sores, being taken in countics of this land, especially in Hunting- powder inwardly in wine, or steeled water, don, in Northamptonshire, in the meadows that is, wherein hot rods of steel have been there: as also near London, by Pancras quenched; or the powder, or the seed church, and by a causeway-side in the middle mixed with the ointments. of a field by Paddington.

Time.] They flower about the end of June, and beginning of July, and their seed is ripe in August.

Goternment and virtues.] This is an herb the Sun challenges dominion over, and with a tlick stalk about a foot high, wherea most precious herb, little inferior to pieces, and at the tops a long spiked head; Betony; the continual use of it preserves flowers of a blue or deep red colour, ac- 
cording to the soil where it grows, and before the stalk with the flowers have abiden a month above ground, it will be withered and gone, and blow away with the wind, and the leaves will begin to spring, which being full grown, are very large and broad, being somewhat thin and almost round, whose thick red foot stalks above a foot long, stand towards the middle of the leaves. The lower part being divided into two round parts, close almost one to another, and are of a pale green colour; and hairy underneath. The root is long, and spreads under ground, being in some places no bigger than ones finger, in others much bigger, blackish on the outside, and whitish within, of a bitter and unpleasant taste.

Place and Time.] They grow in low and wet grounds by rivers and water sides. Their flower (as is said) rising and decaying in February and March, before their leaves, which appear in April.

Government and virtues.] It is under the dominion of the Sun, and therefore is a great strengthener of the heart, and clearer of the vital spirits. The roots thereof are by long experience found to be very available against the plague and pestilential fevers by provoking sweat; if the powder thereof be taken in wine, it also resists the force of any other poison. The root hereof taken with Zedoary and A ngelica, or without them, helps the rising of the mother. The decoction of the root in wine, is singularly good for those that wheese much, or are short winded. It provokes urine also, and women's courses, and kills the flat and broad worms in the belly. The powder of the root doth wonderfully help to dry up the moisturc of the sores that are hard to be cured, and takes away all spots and blemishes of the skin. It were well if gentlewomen would keep this root preserved, to help their poor neighbours. It is fit the rich should heip the poor', for the poor camot help thomselves.

\section{THE BURDOCK.P. 33 .}

They are also called Personata, and Loppy-major, great Burdock and Clod-bur. It is so well known, even by the little boys, who pull off the burs to throw and stick upon each other, that I shall spare to write any description of it.

Place.] They grow plentifully by ditches and water-sides, and by the highways almost every where through this land.

Government and virtues.] Venus challenges this herb for her own, and by its leaf or seed you may draw the womb which way you please, either upwards by applying it to the crown of the head, in case it falls out; or downwards in fits of the mother, by applying it to the soles of the feet; or if you would stay it in its place, apply it to the navel, and that is one good way to stay the child in it. The Burdock leaves are cooling, moderately drying, and discussing withal, whereby it is good for old ulcers and sores. A dram of the roots taken with Pine kernels, helps them that spit foul, mattery, and bloody phlegn. The leaves applied to the places troubled with the shrinking of the sinews or arteries, give much ease. 'The juice of the leaves, or rather the roots themselves, given to drink with old wine, doth wonderfully help the biting of any serpents: And the root beaten with a little salt, and laid on the place, suddenly eases the pain thereof, and helps those that are bit by a mad dog. The juice of the leaves being drank with loney, prorokes uriné, and remedies the pain of the bladker. 'The seed being drank in wine for'y days together, doth wonderfully help the sciatica. The leaves bruised with the white of an esg, and applied to any place burnt with fire, takes out the fire, gives sudden ease, and lieals it up afterwards. 'T'he decoction of them fonmented on any fretting sore or canker, stays the corroding quality, which ! must be afterwards anointed with an oint- 
ment made of the same liquor, hog's-grease, mended, being eaten before meat to keep nitre, and vinegar boiled together. The one from surfeiting, as also from being roots may be preserved with sugar, and drunk with too much wine, or quickly to taken fasting, or at other times, for the same make a man sober again that is drunk bepurposes, and for consumptions, the stone, fore. For (as they say) there is such an and the lask. The seed is much commended antipathy or enmity between the Vine and to break the stone, and cause it to be ex- the Coleworts, that the one will die where pelled by urine, and is often used with the other grows. The decoction of Coleother seeds and things to that purpose.

CABBAGES AND COLEWORTS.

I SHALI spare labour in writing a deseription of these, since almost every one that can but write at all, may describe them from his own knowledge, they being generally so well known, that descriptions are altogether needless.

Place.] They are generally planted in gardens.

Time.] Their flower time is towards the middle, or end of July, and the seed is ripe in August.

Government and virtues.] The Cabbages or Coleworts boiled gently in broth, and eaten, do open the body, but the second decoction doth bind the body. The juice thercof drank in wine, helps those that are bitten by an adder, and the decoction of the flowers brings down women's courses: Being taken with honey, it recovers hoarseness, or loss of the voice. The often eating of them well boiled, helps those that are entering into a consumption. The pulp of
the middle ribs of Coleworts boiled in almond milk, and made up into an electuary with honey, being taken often, is very profitable for those that an often, is very pro- thing more tolerable, and the wholesomer fitable for those that are puffy and short food of the two. 'The Moon challenges the winded. Being boiled twice, an old cock dominion of this herb. boiled in the broth and drank, it helps the ? pains, and the obstructions of the liver and spleen, and the stone in the kidneys. 'The juice boiled with honey, and ding has divers somewhat juice boiled with honey, and dropped into long and broad large and thick wrinkled the corner of the eyes, clears the sight, leaves, somewhat crumpled about the edges, by consuming any film or cloud beginning and growing each upon a thick footstilk, to dim it; it also consumes the canker very brittle, of a greyish green colour, growing therein. They are much com- from among which rises up a strong thick allays the swellings of sores and gouty legs and knees, wherein many gross and watery humours are fallen, the place being bathed therewith warm. It helps also old and filthy sores, being bathed therewith, and heals all small scabs, pushes, and wheals, that break out in the skin. The ashes of Colewort stalks mixed with old hog's grease, are very effectual to anoint the sides of those that have had long pains therein, or any other place pained with melancholy and windy humours. 'This was surely Chrysippus's God, and therefore lie wrote a whole volume on them and their virtues, and that none of the least neither, for he would be no small fool: He appropriates them to every part of the body, and to every disease in every part; and honest old Cato (they say) used no other physic. I know not what metal their bodies were made of; this I am sure, Cabbages are extremely windy, whether you take them as meat or as medicine: yea, as windy meat as can be eaten, unless you eat bag-pipes or bellows, and they are but seldom eaten in our days; and Colewort flowers are somethen

TII: SEA COLTWORTS.

Descript.] Tirs has divers somewhat
ng and broad large and thick wrinkled
aves, somewhat crumpled about the edges,
nd growing each upon a thick footstalk,
wy brittle, of a greyish green colour,
rom among which rises up a strong thick

worts takes away the pain and ache, and 
stalk, two feet high, and belter, with some leaves thereon to the top, where it branches' forth much ; and on every branch stands a large bush of pale whitish flowers, consisting of four leaves a-piece: The root is somewhat great, shoots forth many branches under ground, keeping the leaves green all the winter.

Place.] They grow in many places upon the sea-coasts, as well on the Kentish as Essex shores: as at Lid in Kent, Colchester in Essex, and divers other places, and in other counties of this land.

1 Time.] They flower and seed about the time that other kinds do.

Government and virtues.] The Moon claims the dominion of these also. The broth, or first decoction of the Sea Colewort,
doth by the sharp, nitrous, and bitter qualities therein, open the belly, and purge the body; it cleanses and digests more powerfully than the other kind: The seed hereof bruised and drank kills worms. The leaves or the juice of them applied to sores or ulcers, cleanses and heals them, and dissolves swellings, and takes away inflammations.

CALAMINT, OR MOUNTAIN-MINT.

Descript.] Tris is a small herb, seldom rising above a foot high, with square hairy, and woody stalks, and two small hoary leaves set at a joint, about the height of Marjoram, or not much bigger, a little dented about the edges, and of a very fierce or quick scent, as the whole lierb is: The flowers stand at several spaces of the stalks, from the middle almost upwards, which are small and gaping like to those of the Mints,
of a palc bluisl colour: After which follow small, round blackish seed. The root is small and woody, with divers small strings spreading within the ground, and dies not but abides many years.

Place.] It grows on heaths, and lands, and dry grounds in many places of this land.

Time.] They flower in July, and their seed is ripe quickly after.

Government and virtues.] It is an herb of Mercury, and a strong one too, therefore excellent good in all afflictions of the brain. The decoction of the herb being drank, brings down women's courses, and provokes urine. It is profitable for those that are bursten, or troubled with convulsions or cramps, with shortness of breath, or choleric torments and pains in their bellies or stomach; it also helps the yellow-jaundice, and stays vomiting, being taken in wine. Taken with salt and honey, it kills all manner of worms in the body. - It helps such as have the leprosy, either taken inwardly, drinking whey after it, or the green herb outwardly applied. It linders conception in women, but either burned or strewed in the clramber, it drives away venomous serpents. It takes away black and blue marks in the face, and makes black scars become well coloured, if the green herb (not the dry) be boiled in wine, and laid to the place, or the place washed therewith. Being applied to the hucklebone, by continuance of time, it spends the humours, which cause the pain of the sciatica. The juice being dropped into the ears, kills the worms in them. The leaves boiled in wine, and drank, provoke sweat, and open obstructions of the liver and spleen. It helps them that have a tertian ague (the body being first purged) by taking away the cold fits. The decoction hereof, with some sugar put thereto afterwards, is very profitable for those that be troubled witl the over-flowing of the gall, and that have an old cough, and that are scarce able to breathe by shortness of their wind; that have any cold distemper in their bowels, and are troubled with the hardness or the spleen, "for all which purposes, both or the spleen, for all which purposes, both
the powder, called Diacaluminthes, and the 
compound Syrup of Calamint are the most comfort both it and the brain. The oil effectual. Let no women be too busy with made of the flowers of Camomile, is much it, for it works very violent upon the feminine part.

C A IOMI LE.

used. against all hard swellings, pains or aches, shrinking of the sinews, or cramps, or pains in the joints, or any other part of the body. Being used in clysters, it helps to dissolve the wind and pains in the belly ;

$\mathrm{I}_{\mathrm{T}}$ is so well known every where, that it is but lost time and labour to describe it. The virtues thereof are as follow.

A decoction made of Camomile, and drank, takes away all pains and stitches in the side. The flowers of Camomile beaten, and made up into balls with Gil, drive away all sorts of agues, if the part grieved be anointed with that oil, taken from the flowers, from the crown of the head to the sole of the foot, and afterwards laid to sweat in his bed, and that he sweats well. This is Nechessor, an Egyptian's, medicine. It is profitable for all sorts of agues that come either from phlegm, or melancholy, or from an inflammation of the bowels, being applied when the humours causing them shall be concocted; and there is nothing more profitable to the sides and region of the liver and spleen than it. The bathing with a decoction of Camomile takes away weariness, eases pains, to what part of the body soever they be applied. It comforts the sinews that are over-strained, molifies all swellings: It moderately comforts all parts that have need of warmth, digests and dissolves whatsoever has need thereof, by a wonderful speedy property. It eases all pains of the cholic and stone, and all pains and torments of the belly, and gently provokes urine. The flowers boiled in posset-drink provoke sweat, and help to expel all colds, aches and pains whatsoever, and is an excellent help to bring down women's courses. Syrup made of the juice of Camomile, with the flowers in white wine, is a remedy against the jaundice and dropsy. The flowers boiled the top of the water; divided towards the jaundice and dropsy. 'The flowers boiled top into many branches, each carrying (i.) anointed also, it helps stitches and pains in the sides.

Nechessor saith, the Egyptians dedicated. it to the Sun, because it cured agues, and they were like enough to do it, for they were the arrantest apes in their religion that I ever read of. Bachinus, Bena, and Lobel, commend the syrup made of the juice of it and sugar, taken invardly, to be excellent for the spleen. Also this is certain, that it most wonderfully breaks the stone: Some take it in syrup or decoction, others inject the juice of it into the bladder with a syringe. My opinion is, that the salt of it, taken half a dram in the morning in a little white or Rhenish wine, is better than either; that it is excellent for the stone, appears in this which I have seen tried, viz. That a stone that has been taken out of the body of a man being wrapped in Camomile, will in time dissolve, and in a little time too.

WATER-CALTROPS.

They are called also Tribulus Aquaticus, Tribulus Lacusoris, Tribulus, Marinus, Caltrops, Saligos, Water Nuts, and Water Chesnuts.

Descript.] As for the greater sort of Water Caltrop it is not found here, or very rarely. Two other sorts there are which I shall here describe. The first has a long creeping and jointed root, sending forth tufts at each joint, from which joints rise long flat, slender knotted stalks, even to the top of the water, divided towrards the 
inches long, and half an inch broad, thin and almost transparent, they look as though seem to be two a-piece, smelling somewhat they were torn; the flowers are long, thick sweet, and each of them standing in a and whitisl, set together almost like a large green striped laairy husk, large and bunch of grapes, which being gone, there round below next to the stalk: "The seed is succeed, for the most part, sharp pointed small and greyish in the hard heads that grains all together, containing a snall white come up afterwards. The root is white and kernel in them.

The second differs not much from this, save that it delights in more clear water; its stalks are not flat, but round; its leaves are not so long, but more pointed; As for : the place we need not determine, for their name shews they grow in water.

Government and virtues.] They are under the dominion of the Moon, and being made into a poultice, are excellently good for hot inflammations, swellings, cankers, sore mouths and throats, being washed with the decoction; it cleanses and strengthens the neck and throat, and helps those swellings which when people have, they say the almonds of their ears are fallen down. is excellently good for the rankness of the gums, a safe and present remedy for the king's evil. They are exicellent for the stone and gravel, especially the nuts, being dried. They also resist poison, and bitings of venomous beasts.

\section{CAMPION, WILD.}

\section{Descript.] The wild White Campion} has many long and somewhat broad dark white or red wine being drank, doth stay green leaves lying upon the ground, andl inward bleedings, and applied outwardly, divers ribs therein, somewhat like plantain, it does the like; and being drank, helps but somewhat hairy, broader, but not so to expel urine, being stopped, and gravel long: The hairy stalks rise up in the mid- and stone in the reins and kidneys. 'Two dle of them three or four feet ligh, and drams of the seed drank in wine, purges sometimes more, with divers great white the body of choleric humours, and helps joints at several places thereon, and two those that are stung by scorpions, or other such like leaves thereat up to the top, send- venomous beasts, and may be as effectual ing forth branches at several joints also: for the plague. It is of very good use in All which bear on several foot-stalks white? old sores, ulcers, cankers, fistulas, and the flcwers at the tops of them, consisting of like; to cleanse and lieat them, by confive broad pointed leaves, erery one cut in suming the moist humours falling into 
them and correcting the putrefaction of humours offending them.

CARDUUS BENEDICTUS.

IT is called Carduus Benedictus, GARDEN Carrots are so well known, Blessed Thistle, or Holy Thistle. I or that they need no description; but because Blessed Thistle, or Holy Thistle. I sup- they are of less physical use than the wild pose the name was put upon it by some kind (as indeed almost in all herbs the wild that had little holiness themselves.

I shall spare a labour in writing a powerful in operation than the garden description of this as almost every one that kinds,) I shall therefore briefly describe the can but write at all, may describe them? Wild Carrot.

fiom his own knowledge.

Time.] They flower in August, and seed not long after.

Government and virtues.] It is an Thers arge tufts of white flowers, Mars, and under the sign Aries. Now, in with a deep purple spot in the middle, handling this herb, I shall give you a which are contracted together when the rational pattern of all the rest; and if you seed begins to ripen, that the middle part please to view them throughout the book, being hollow and low, and the outward you shall, to your content, find it true. It stalk rising high, makes the whole umbel lielps swimming and giddiness of the head, to show like a bird's nest. The roots small, or the disease called vertigo, because Aries long, and hard, and unfit for meat, being is in the house of Mars. It is an excellent somewhat sharp and strong.

remedy against the yellow jaundice and other infirmities of the gall, because Mars governs choler. It strengthens the attractive faculty in man, and clarifies the blood, because the one is ruled by Mars. The continual drinking the decoction of it, helps red faces, tetters, and ring-worms, because Mars causes them. It helps the plague, sores, boils, and itch, the bitings of mad dogs and venomous beasts, all which infirmities are under Mars; thus you see what it doth by sympathy.

By antipathy to other planets it cures the

Place.] The wild kind grows in divers parts of this land plentifully by the fieldsides, and untilled places.

Time.] They flower and seed in the end of Summer.

Government and virtues.] Wild Carrots belong to Mercury, and therefore break wind, and remove stitches in the sides, provoke urine and women's courses, and helps to break and expel the stone; the seed also of the same works the like effect, and is good for the dropsy, and those whose bellies French pox. By antipathy to Venus, who the stone in the kidneys, and rising of the governs it, it strengthens the memory, and mother; being taken in wine, or boiled in cures deafness by antipathy to Saturn, who wine, and taken, it helps conception. The has his fall in Aries, which rules the head. lleaves being applied with honey to running It cures quartan agues, and other diseases sores or ulcers, do clcanse them. of melancholy, and adust choler, by sympathy to Saturn, Mars being exalted in better than the roots; and though Galen Capricorn. Also provokes urine, the stopp- commended garden Carrots highly to break ing of which is usually caused by Mars or wind, yet experience teaches they breed it the Moon.

lifirst, and we may thank nature for expelling 
it, not they; the seeds of them expel wind people exceedingly, and they need not to indeed, and so mend what the root marrs. make a whole meal of them neither, and are

\section{CARRAWAY.}

IT is on account of the seeds principally that the Carraway is cultivated.

Descript.] It bears divers stalks of fine cut leaves, lying upon the ground, somewhat like to the leaves of carrots, but not bushing so thick, of a little quick taste in them, from among which rises up a square stalk, not so high as the Carrot, at whose joints are set the like leaves, but smaller and finer, and at the top small open tufts, or umbels of white flowers, which turn into small blackish seed, smaller than the Anniseed, and of a quicker and hotter taste. The root is whitish, small and long, somewhat like unto a parsnip, but with more wrinkled bark, and much less, of a little hot and quick taste, and stronger than the parsnip, and abides after seedtime.

Place.] It is usually sown with us in gardens.

Time.] They flower in June and July, and seed quickly after.

Government and virtues.] This is also a Mercurial plant. Carraway seed has a moderate sharp quality, whereby it breaks ? wind and provokes urine, which also the herb doth. The root is better food than the parsnip; it is pleasant and comfortable to the stomach, and helps digestion. The seed is conducing to all cold griefs of the head and stomach, bowels, or mother, as also the wind in them, and helps to sharpen the eye-sight. 'The powder of the seed put into a poultice, takes away black and blue spots of blows and bruises. The herb itself, or with some of the seed bruised and fried, laid hot in a bag or double cloth, to the lower parts of the belly, eases the pains of the wind cholic.

The roots of Carraway eaten as men do parsnips, strengthen the stomach of ancient fit to be planted in every garden.

Carraway comfits, once only dipped in sugar, and half a spoonful of them eaten in the morning fasting, and as many after each meal, is a most admirable remedy, for those that are troubled with wind.

\section{CE L A N D I N E.}

Descript.] Trus hath divers tender, round, whitislı green stalks, with greater joints than ordinary in other herbs as it were knees, very brittle and easy to break, from whence grow branches with large tender broad leaves, divided into many parts, each of them cut in on the edges, set at the joint on both sides of the branches, of a dark blueish green colour, on the upper side like Columbines, and of a more pale blueish green underneath, full of yellow sap, when any is broken, of a bitter taste, and strong scent. At the flowers, of four leaves a-piece, after which come small long pods, with blackish seed therein. The root is somewhat great at the head, shooting forth divers long roots and small strings, reddish on the outside, and yellow within, full of yellow sap therein.

Place.] They grow in many places by old walls, hedges and way-sides in untilled places; and being once planted in a garden, especially some shady places, it will remain there.

Time.] They flower all the Summer, and the seed ripens in the mean time.

Government and virtues.] 'This is an herb of the Sun, and under the celestial Lion, and is one of the best cures for the eyes; for, all that know any thing in astrology, know that the eyes are subject to the luminaries: let it then be gathered when the Sun is in Leo, and the Moon in Aries, applying to this time; let Leo arise, then may you make into an oil or ointment, which you please, to anoint your-sore eyes 
with: I can prove it doth both niy own not only good against the itch, but takes experience, and the experience of those to away all discolourings of the skin whatwhom I have taught it, that most desperate soever : and if it chance that in a tender sore eyes have been cured by this only body it causes any itchings or inflammamedicine; and then, I pray, is not this far tions, by bathing the place with a little better than endangering the eyes by the art vinegar it is helped. of the needle? For if this does nol absolutely take away the film, it will so facilitate the work, that it might be done without danger. The herb or root boiled in white Wine and drank, a few Annisecds being boiled therewith, opens obstructions of the liver and gall, helps the yellow jaundice; and often using it, helps the dropsy and the itch, an .inose who have old sores in their legs, or other parts of the body. The juice thereof taken fasting, is held to be of singularly good use against the pestilence. The distilled water, with a little sugar and a little good treacle mixed therewith (the party upon the taking being laid down to sweat a little) has the same effect. The juice dropped into the eyes, cleanses them from films and cloudiness which darken the sight, but it is best to allay the sharpness of the juice with a little breast milk. It is good in all old filthy corroding creeping ulcers wheresoever, to stay their malignity of fretting and running, and to cause them to heal more speedily: The juice often applied to tetters, ring-worms, or other such like sprcading cankers, will quickly heal them, and rubbed often upon warts, will take thern away. The herb with the ruots bruised and bathed with oil of camonile, and applied to the navel, takes away the griping pains in the belly and bowels, and all the pains of the motl:er; and applied to women's breasts, stays the overmuch flowing of the courses. 'The juice or decoction of the herb gargled between the teeth that ach, cases the pain, and the powder of the dried root laid upon any aching, hollow or loose tooth, will cause it to fall out. 'The juice mixed with some powder of brimstone is ? $(5,6$.
Anotherill-favoured trick have physicians got to use to the eye, and that is worse than the needle; which is to take away the films by corroding or gnawing medicines. That I absolutely protest against.

1. Because the tunicles of the eyes are very thin, and therefore soon eaten asunder.

2. The callus or film that they would eat away, is seldom of an equal thickness in every place, and then the tunicle may be caten asunder in one place, before the film be consumed in another, and so be a readier way to extinguish the sight than to restore it.

It is called Chelidonium, from the Greek word Chelidon, which signifies a swallow; because they say, that if you put out the eyes of young swallows when they are in the nest, the old ones will recover their eyes again with this herb. This I an confident, for I have tried it, 'that if we mar the very apple of their eyes with a needle, she will recover them again; but whether with this herb or not, I know not.

Also I have read (and it scems to be somewhat probable) that the herb, being gathered as I shewed before, and the elements draw apart from it by art of the alchymist, and after they are drawn apart rectified, the earthly quality, still in rectifying them, added to the Terra damnati (as Alchymists call it) or Terra Sacratissima (as some philosophers call it) the elements so rectified are sufficient for the cure of an diseases, the humours offending being known, and the contrary element given: It is an experiment worth the trying, and can do no harm. 
THE LESSER CELANDINE, USUALLY KNOWN BY THE NAME OF PILEWORT AND FOGWORT.

I WONDER what ailed the ancients to give this the name Celandine, which resembles it neither in nature nor form; it acquired the name of Pilewort from its virtues, and it being no great matter where I set it down, so I set it down at all, I humoured Dr. Tradition so much, as to set him down here.

Descript.] This Celandine or Pilewort (which you please) doth spread many round pale green leaves, set on weak and trailing branches which lie upon the ground, and are flat, smooth, and somewhat shining, and in some places (though seldom) marked with black spots, each standing on a long ? foot-stalk, among which rise small yellow flowers, consisting of nine or ten small nar row leaves, upon slender foot-stalks, very like unto Crowsfoot, whereunto the seed also is not unlike being many small kernels like a grain of corn sometimes twice as long as others, of a whitish colour, with fibres at the end of them.

Place.] It grows for the most part in moist corners of fields and places that are near water sides, yet will abide in drier ground if they be but a little shady.

Time.] It flowers betimes, about March or April, is quite gone by May; so it cannot be found till it spring again.

Government and virtues.] It is under the dominion of Mars, and behold here another verification of the learning of the ancients, viz. that the virtue of an herb mày be
known by its signature, as plainly appears known by its signature, as plainly appears
in this; for if you dig up the root of it, you shall perceive the perfect image of the disease which they commonly call the piles. It is certain by good experience, tlat the decoction of the leaves and roots wonderfully helps piles and hæmorrhoids, also kernels by the ears and throat, called the king's evil, or any other hard wens or tumours.

Here's another secret for my countrymen and women, a couple of them together; Pilewort made into an oil, ointment, or plaister, readily cures both the piles, or hæmorrhoids, and the king's evil: 'P'he very herb borne about one's body next the skin helps in such diseases, though it never touch the place grieved; let poor people make much of it for those uses; with this I cured my own daughter of the king's evil, broke the sore, drew out a quarter of a pint of corruption, cured without any scar at all in one wéek's time.

THE ORDINARY SMALI CENTAURY.

Descript.] This grows up most usua!ly but with one round and somewhat crusted stalk, about a foot high or better, branching forth at the top into many sprigs, and some also from the joints of the stalks below; the flowers thus stand at the tops as it were in one umbel or tuft, are of a pale red, tending to carnation colour, consisting of five, sometimes six small leaves, very like those of St. John's Wort, opening themselves in the day time and closing at night, after which come seeds in little short husks, in form like unto wheat corn. The leaves are small and somewhat round ; the root small and hard, perishing every year. The whole plant is of an exceeding bitter taste.

There is another sort in all tlings like the former, save only it bears white flowers.

Place.] They grow ordinarily in fields, pastures, and wonds, but that with the white flowers not so frequently as the other. Time.] They flower in July or thereabouts, and seed within a month after.

Government and virtues.] They are under the dominion of the Sun, as appears in that their flowers open and shut as the Sun either shews or hides his face. This herb, boiled 
and drank, purges choleric and gross the government of the Sun; yet this, if humours, and helps the sciatica; it opens you observe it, you shall fird an excellent obstructions of the liver, gall, and speen, truth; in diseases of the blood, use the red helps the jaundice, and eases the pains in Centaury; if of choler, use the ycllow; the sides, and hardness of the spleen, used but if phlegin or water, you will find the outwardly, and is given with very good white best.

effect in agues. It helps those that have the dropsy, or the green-sickness, being much used by the Italians in powder for that purpose. It kills the worms in the belly, as is found by experience. The decoction thereof, viz. the tops of the stalks, with the leaves and flowers, is good against the cholic, and to bring down women's courses, helps to void the dead birth, and eases pains of the mother, and is very effectual in all old pains of the joints as the gout, cramps, or convulsions. A dram of the powder taken in wine, is a wonderful good help against the biting and poison of an adder. 'The juice of the herb with a little honey put to it, is good to clear the eyes from dimness; mists and clouds that offend or hinder sight. It is singularly good both for green and fresh wounds, as also for old ulcers and sores, to close up the one and cleanse the other, and perfectly to cure them both, although they are hollow or fistulous; the green herb especially, being bruised and laid thereto. The decoction thereof dropped into the ears, cleanses them from worms, cleanses the foul ulcers and spreading scabs of the head, and takes away all freckles, spots, and rnarks in the skin, being washed with it; the herb is so safe you cannot fail in the using of it, only giving it inwardly for inward diseases. It is very wholesome, but not very toothsorne.

the bigness many times of one's little finger,
shooting forth at several joints in several

There is beside these, another small places, whereby it quickly spreads a great Centaury, which bears a yellow flower; in compass of ground. The stalk rises not all other respects it is like the former, save above a yard high, whereon are set many that the leaves are larger, and of a darker broad and long green leaves, somewhat green, and the stalks pass through the midst like nightshades, but larger ; at the joints of them, as it does in the herb Thorowan. whereof come forth whitish flowers made They are all of them, as I told you, under of five leaves a piece, which afterwards 
turn into green berries inclosed with thin skius, which change to be reddish when expel urine and the stone, and to cause tle they grow ripe, the berry likewise being stone not to engender. The decoction of reddish, and as large as a cherry; wherein the berries in wine and water is the most are contained many flat and ycllowish seeds usual way ; but the powder of them taken lying within the pulp, which being gathered in drink is more effectual.

and strung up, are kept all the year to be? used upon occasion.

Place.] They grow not naturally in this land, but are cherished in gardens for their virtues.

Time.] They flower not until the middle or latter end of July; and the fruit is ripe about August, or the beginning of September.

- Goverment and virtues.] This also is a plant of Venus. They are of great use in physic: The leaves being cooling, may be the stalks also; it rises a little above half used in inflammations, but not opening as a foot high, bearing white flowers in spiked the berries and fruit are; which by draw- tufts, which turn into long and round seeds ing down the urine provoke it to be voided pointed at the ends, and blackish when they plentifully when it is stopped or grown hot, sharp, and painful in the passage; it is 作 good also to expel the stone and gravel out? well. The root is small and long, and of the reins, kidneys and bladder, helping perishes every year, and must be sown to dissolve the stone, and voiding it by grit a-new in spring, for seed after July. for or gravel sent forth in the urine; it also Autumn fails.

helps much to cleanse inward imposthumes or ulcers in the reins or bladder, or in those that void a bloody or foul urine. 'The distilled water of the fruit, or the leaves together with them, or the berries, green or dry, distilled with a little milk and drank morning and evening with a little sugar, is effectual to all the purposes before specified, and especially against the heat and sharpness of the urine. I shall only mention one way, amongst many others, which might be used for ordering the berries, to be helpful for the urine and the stone; which is this: 'Take three or four good handfuls of the berries, either green or fresh, or dried, and having bruised them, put them into so many gallons of beer or ale when it is new tunned up: This drink, The wild Chervil grows two or three feet high, with yellow stalks and joints, set with broader and more hairy leaves, divided into sundry parts, nicked about the edges, and of a dark green colour, which likewise grow reddish with the stalks; at the tops whereof stand small white tufts, of flowers, afterwards smaller and longer seed. The root is white, hard, and enduring long. This has little or no scent.

Place.] The first is sown in gardens for a sallad herb; the second grows wild in many of the meadows of this land, and by the hedge sides, and on heaths.

Time.] They flower and seed early, and thereupon are sown again in the end of Summer.

taken daily, has been found to do much Chervil being eaten, doth moderately warm 
the stomach, and is a certain remedy (saith after-birth; procures an appetite to meat, Tragus) to dissolve congealed or clotted and expels wind. The juice is good to blood in the body, or that which is clotted heal the ulcers of the head and face; the by bruises, falls, \&c. The juice or distilled candied root hereof are beld as effectual as water thereof being drank, and the bruised Angelica, to preserve from infection in the leares laid to the place, being taken either time of a plague, and to warm and comin meat or drink, it is good to help to pro- fort a cold weak stomach. - It is so harIIvoke urine, or expel the stone in the kid- less, you cannot use it amiss.

neys, to send down women's courses, and to help the pleurisy and pricking of the sides.

CHESNUT TREE.

The wild Chervil bruised and applied, dissolves swellings in any part, or the marks of congealed blood by bruises or blows, in a little space.

SWEET CHERVIL, OR SWEET CICELY.

Descript.] This grows very like the commendable nourishment to the body; great hemlock, having large spread leaves yet if eaten over-much, they make the cut into divers parts, but of a fresher green colour than the Hemlock, tasting as sweet as the Anniseed. The stalks rise up a yard high, or better, being creased or hollow, having leaves at the joints, but lesser ; and at the tops of the branched stalks, umbels or tufts of white flowers; after which comes long crested black shining seed, pointed at both ends, tasting quick, yet sweet and pleasant. The root is great and white, growing deep in the ground, and spreading sundry long branches therein, in taste and smell stronger than the leaves or seeds, and continuing many years.

Place.] This grows in gardens.

Government and virtues.] These are all three of them of the nature of Jupiter, and under his dominion. This whole plant, besides its pleasantness in sallads, has its physical virtue. 'The root boiled, and eaten with oil and vinegar, (or without oil) do much please and warm old and cold stomachs oppressed with wind or phlegm, or dominion of Venus, they provoke lust those that have the phthisic or consump- exceedingly, and stir up to those sports she tion of the lungs. The same drank with is mistress of ; the seed is excellent good to wine is a preservation from the plague. It provoke urine; and so also is the root, but provokes women's courses, and expels the it doth not perform it so forcibly as the seed $(5,6$. 
doth. The root being dried and beaten the swellings and ease the pains. It helps into powder, and the powder made into an the sinews when they are shrunk by cramps, electuary, is as singular a remed for spitting or otherwise, and to extend and make them and pissing of blood, as the former Chesnut pliable again by this medicine. Boil a was for coughs.

\section{CHICK WEED. $3 \%$}

handful of Chickweed, and a handful of red rose leaves dried, in a quart of muscadine, until a fourth part be consumed;

IT is so generally known to most people, then put to them a pint of oil of trotters or that I shall not trouble you with the descrip- sheep's feet; let them boil a good while, tion thereof, nor myself with setting forth stillstirring them well; which being strained, the several kinds, since but only two or anoint the grieved place therewith, warm three are considerable for their usefulness.

Place.] They are usually found in moist and watery places, by wood sides, and elsewhere.

Time.] They flower about June, and their seed is ripe in July.

Government and virtues.] It is a fine soft pleasing herb under the dominion of the Moon. It is found to be effectual as Purslain to all the purposes whereunto it serves, except for meat only. The herb bruised, or the juice applied (with cloths or sponges dipped therein) to the region of the liver, and as they dry, to have it fresh applied, doth wonderfully temperate the heat of the liver, and is effectual for all impos- or deeper, according as the pease that thumes and swellings whatsoever, for all follow will be, that are contained in small, redness in the face, wheals, pushes, itch, thick, and short pods, wherein lie one or scabs; the juice either simply used, or two pease, more usually pointed at the boiled with hog's grease and applied, helps lower end, and almost round at the head, cramps, convulsions, and palsy. The juice, yet a little cornered or sharp; the root is or distilled water, is of much good use for small, and perishes yearly.

all heats and redness in the eyes, to drop? some thereof into them; as also into the ears, to ease pains in them ; and is of good effect to ease pains from the heat and sharpness of the blood in the piles, and generally all pains in the body that arise of heat. It is used also in hot and virulent ulcers and sores in the privy parts of men and they provoke urine, and are thought to inwomen, or on the legs, or elsewhere. The crease sperm; they have a cleansing faculty, leaves boiled with marsh-mallows, and whereby they break the stone in the kidmade into a poultice with fenugreek and neys. To drink the cream of them, being linseed, applied to swellings or impos- boiled in water, is the best way. It moves thumes, ripen and break them, or assuage the belly downwards, provolses women's 
courses and urine, increases both milk and of Jupiter, and therefore strengthens the seed. One ounce of Cicers, two ounces of part of the body it rules; let Jupiter be French barley, and a small handful of angular and strong when it is gathered; Marsh-mallow roots, clean washed and cut, and if you give but a scruple (which is but being boiled in the broth of a chicken, and twenty grains, of it at a time, either in four ounces taken in the morning, and white wine, or in white wine vinegar, you fasting two hours after, is a good medicine shall very seldom miss the cure of an ague, for a pain in the sides. The white Cicers be it what ague soever, in three fits, as I are used more for meat than medicine, yet have often proved to the admiration both have the same effect, and are thought more of myself and others; let no man despise it powerful to increase milk and seed. The because it is plain and easy, the ways of wild Cicers are so much more powerful God are all such. It is an especial herb than the garden kinds, by how much they used in allinflammations and fevers, whether exceed them in heat and dryness; whereby infectious or pestilential; or among other they do more open obstructions, break the herbs to cool and temper the blood and stone, and have all the properties of cutting, humours in the body. As also for all lotions; opening, digesting, and dissolving ; and gargles, infections, and the like, for sore this more speedily and certainly than the mouths, ulcers, cancers, fistulas, and other former.

corrupt, foul, or running sores. The juice liereof drank, about four ounces at a time,

CINQUEFOIL, OR FIVE-LEAVED GRASS;

CALLED IN SONE COUNTIES, FIVEFINGERED GRASS.

Descript.] I d days together, cures the falling sickness. Descript.] IT spreads and creeps far The roots boiled in milk, and drank, is a upon the ground, with long slender strings most effectual remedy for all fluxes in man like straw berries, which take root again, or woman, whether the white or red, as also and shoot forth many leaves, made of five the bloody flux. The roots boiled in vineparts, and sometimes of seven, dented about gar, and the decoction thereof held in the edges, and somewhat hard. The stalks the mouth, cases the pains of the toothare slender, leaning downwards and bear ach. The juice or decoction taken with many' small yellow flowers thereon, with a little honey, helps the hoarseness of some yellow threads in the middle, standing the throat, and is very good for the cough about a smooth green head, which, when it of the lungs. The distilled water of both is ripe, is a little rough, and contains small roots and leaves is also effectual to all the brownish seeds. The root is of a blackish purposes aforesaid; and if the hands be brown colours, as big as one's little finger, often washed therein, and suffered at every but growing long, with some threads thereat; time to dry in of itself without wiping, it and by the small string it quickly spreads will in a short time help the palsy, or over the ground.

Place.] It grows by wood sides, hedge vinegar, helps all knots, kernels, hard sides, the path-way in fields, and in the swellings, and lumps growing in any borders and corners of them almost through part of the flesh, being thereto applied; all this land.

Time.] It as also inflammations, and St. Ansooner, some later.

Gorernment and virtues.] This is an herb the shingles also, and all other sorts of 
running and foul scabs, sores and itch. has four square stalks, with broad, rough, The same also boiled in wine, and applied wrinkled, whitish, or hoary green leaves, to any joint full of pain, ache, or the gout somewhat evenly cut in on the edges, and in the hands or feet, or the hip gout, called of a strong sweet scent, growing sonle near the Sciatica, and the decoction thereof the ground, and some by couples upon drank the while, doth cure them, and eases stalks. The flowers grow at certain dismuch pain in the bowels. The roots are tances, with two small leaves at the joints likewise effectual to help ruptures or burst- under them, somewhat like unto the flowers ings, being used.with other things available of Sage, but smaller, and of a whitish blue to that purpose, taken either inwardly or colour. The seed is brownish, and someoutwardly, or both; as also bruises or what flat, or not so round as the wild. The hurts by blows, falls, or the like, and to roots are blackish, and spread not far, and stay the bleeding of wounds in any parts perish after the seed time. It is usually inward or outward.

Some hold that one leaf cures a quotidian, three a tertain, and four a quartan ague, and a hundred to one if it be not Dioscorides; for he is full of whimsies. The truth is, I never stood so much upon the number of the leaves, nor whether I give it in powder or decoction: If Jupiter were strong, and the Moon applying to him, or his good aspect at the gathering, I never knew it miss the desired effect.

CIVES.

Calleu also Rush Leeks, Chives, Civet, and Sweth.

Government and virtues.] I confess I had not added these, had it not been for a country gentleman, who by a letter. certified me, that amongst other herbs, I had left hese out ; they are indeed a kind of leeks, it be grown too great. The powder of the hot and dry in the fourth degree as they dried root put into the nose, provokes are, and so under the dominion of Mars; sneezing, and thereby purges the head and if they be eaten raw, ( $I$ do not mean raw, brain of much rheum and corruption. The opposite to roasted or boiled, but raw, seed or leaves taken in wine, provokes to opposite to chymical preparation) they send venery. It is of much use both for men up very hurtful vapours to the brain, caus- and women that have weak backs, and ing troublesome sleep, and spoiling the helps to strengthen the reins: used either eye-sight, yet of them prepared by the art by itself, or with other herbs conducing to of the alchymist, may be made an excel- the same effect, and in tansies often. The lent remedy for the stoppage of the urine. fresh leaves dipped in a batter of flour, CLARY, OR MORE PROPERLY CLEAR-EYE. eggs, and a little milk, and fried in butter, Descript.] Oun ordinary garden Clary to any, but exceedingly profitable for those 
that are troubled with weak backs, and the sater, and casier remedy by a great deal, effects thereof. The juice of the herb put thran to tear it off with a needle. into ale or bear, and drank, brings down women's courses, and expels the atter-birth.

\section{WILD CIARY.}

Wred Clary is most blasphernously called Christ's Eye, because it cures diseases of the eye. I could wish for my soul, blasphemy, ignorance, and tyranny, were happy, and I joyful.

Descript.] It is like the other Clary, but lesser, with many stalks about a foot and a half high. The stalks are square, and somewliat hairy; the flowers of a bluish colour; He that knows the common Clary cannot be ignorant of this.

Place.] It grows commonly in this nation in barren places; you may find it plentifully, if you look in the fields near Gray's Inn, and near Chelsea.

Time.] They flower from the beginning of June to the latter end of August.

Government and virtues.] It is something : hotter and drier than the garden Clary is, yet nevertheless under the dominion of the Moon, as well as that; the seeds of it being beat to powder, and drank with wine, is an admirable help to provoke lust.

A decoction of the leaves being drank, warms the stomach, and it is a wonder if it should not, the stomach being under Canccr, the house of the Moon. Also it helps digestion, scatters congealed blood in any part of the body. 'The distilled water hereof cleanses the eyes of redness, waterishness, and heat: It is a gallant remedy for dimness of sight, to take one of the seeds of it, and put into the eyes, and there lct it remain till it drops out of itself, (the pain will he nothing to speak on,) it will cleanse the eyes of all filthy and putrified matter; and in often repeating it, will take off a film which covers the sight: a handsomer, $(5,6$.

\section{CLEA V ERS.}

IT is also called Aperine, Goose-share, Goose-grass, and Cleavers.

Descript.] The common Clearers have divers very rough square stalks, not so big as the top of a point, but rising up to be two or three yards high sometimes, if it meet with any tall bushes or trces whereon it may climb, yet without any claspers, or else much lower, and lying on the ground, full of joints, and at every one of them shoots forth a branch, besides the leaves thereat, which are usually six, set in a round compass like a star, or a rowel of a spur: From between the leaves or the joints towards the tops of the branches, come forth very small white flowers, at every end, upon small thready foot-stalks, which after: they have fallen, there do shew two sinall round and rough seeds joined together, which, when they are ripe, grow hard and whitish, having a little hole on the side, something like unto a navel. Both stalks, leaves, and seeds are so rough, that they will cleave to any thing that will touch them. The root is small and thready, spreading much to the ground, but dies every year.

Place.] It grows by the hedge and ditchsides in many places of this land, and is so troublesome an inhabitant in gardens, that it ramps upon, and is ready to choak whatever grows near it.

Time.] It flowers in June or July, and the seed is ripe and falls again in the end of July or August, from whence it springs up again, and not from the old roots.

Government and virtues.] It is under the dominion of the Moon. The juice of the herb and the secd together taken in wine, helps those bitten with an adder, by prescrving the heart from the venom. It is familiarly taken in broth to keep them lean 
and lank, that are apt to grow fat. The this land, both north and west, and fredistilled water drank twice a day, helps the quently by path-sides in the fields near yellow jaundice, and the decociion of the about London, and within three or four herb, in experience, is found to do the same, miles distant about it, yet it usually grows and stays lasks and bloody-fluxes. 'J'he in or near ditches.

juice of the leaves, or they a little bruised, Time.]. It flowers in June or July, and and applied to any bleeding wounds, stays the seed is ripe soon after.

the bleeding. 'The juice also is very good Government and vintues.] It is under the to close up the lips of greed wounds, and dominion of the planet Saturn. It is sinthe powder of the dried herb strewed there-gularly effectual in all fiesh and green upon doth the same, and likewise helps wounds, and therefore bears not this name old ulcers. Being boiled in hog's grease, for nought. And it is very available in it helps all sorts of hard swellings or ker-staunching of blood and to dry up the nels in the throat, being anointed there- fluxes of humours in old fretting ulcers, with. The juice dropped into the ears, cankers, \&c. that hinder the healing of takes away the pain of them.

It is a good remedy in the Spring, eaten them.

A syrup nade of the juice of it, is inferior (being first chopped small, and boiled well) to none for inward wounds, ruptures of in water-gruel, to cleanse the blood, and veins, bloody flux, vessels broken, spitting, strengthen the liver, thereby to keep the urining, or vomiting blood: Ruptures are body in health, and fitting it for that change excellent and specdily, even to admiration, of season that is coming.

CLOWN'S WOODWORT.

cured by taking now and then a little of the syrup, and applying an ointment or plaister of this herb to the place. Also, if

Descript.] IT grows up sometimes to any vein be swelled or muscle, apply a two or three feet, high, but usually about plaister of this herb to it, and if you add two feet, with square green rough stalks, a little Comfrey to it, it will not be amiss but slender, joined somewhat far asunder, I assure thee the herb deserves commendaand two very long, somewhat narrow, dark tion, though it has gotten such a clownish green leaves, bluntly dented about the name; and whosoever reads this, (if he try edges thereof, ending in a long point. The it, as I have done,) will commend it; only flowers stand towards the tops, compassing take notice that it is of a dry earthy quality. the stalks at the joints with the leaves, and end likewise in a spiked top, having long and much gaping hoods of a purplish red colour, with whitish spots in them, standing in somewhat round husks, wherein afterwards stand blackish round sceds. The downward, but set with winged leaves, root is composed of many long strings, longer and more pointed than those of with some tuberous long knobs growing Liniels, and whitish underneath; from the among them, of a pale yellowish or whitish tops of these stalks arise up other slender coloui", yet some times of the year these stalks, naked without leaves unto the tops, knobby roots in many places are not seen where there grow many small flowers in in this plant: This plant smelis somewhat manner of a spike, of a pale reddish colour, trong. with some blueness among them; after Place.] It grows in sundry counties of which rise up in therr places, round, rough, 
and somewhat flat heads. The root is tough, and somewhat woody, yet lives and shoots a-new every year.

Place.] It grows upon hedges, and sometimes in the open fields, in divers places of this land.

Time.] 'They flower all the months of .with small yellowish flowers somewhat July and August, and the seed ripen in earlier, which fall away quickly, and after the mean while.

Government and virtues.] It is under the c ominion of Venus. It has power to rarify and digest; and therefore the green leaves bruised and laid as a plaistei, disperse knots, nodes, or kernels in the flesh; and if, when dry, it be taken in wine, it helps the stranguary; and being anointed with oil, it provokes sweat. It is a singular food for cattle, to cause them to give store of milk; and why then may it not do the like, jeing boiled in ordinary drink. for nurses.

\section{COLUMBINF.S. P:3/}

These are so well known, growing almost in every garden, that I think I may save the expence of time in writing a description of them.

Time.] They flower in May, and abide? not for the most part when June is past, perfecting their seed in the mean time.

Government and virtues.] It is also an herb of Verus. The leaves of Columbines are comınonly used in lotions with good causing a cough, for which also the dried success for sore inouths añd throats. 'Tra- leaves taken as tobacco, or the root is very gus saith, that a dram of the seed taken 11 good. 'The distilled water hereof simply, wine with a little saffron, opens obstruc- or with Elder flowers and Nightshade, is a tions of the liver, and is good for the yellow singularly grood remcly againsi all hotagues, jaundice, if the party after the takirgy to drink iwo ounces at a time, and apply thereof be laid to sweat well in bed. The cloths wet therein to the head and stomach, seed also taken in wine causes a speedy which also does much good, being applied delivery of women in childbirtl : if one to any hot swellings and inflammations: draught suffice not, let her drink the It lielps St. Anthony's fire, and burnings, second, and it will be effectual: The and is singularly good to take away wheals Spaniards used to eat a piece of the root and small pusties that arise through heat; thereof in the morning fasting, many days as also the burning heat of the piles, or together, to help then when troubled with privy parts, cloths wet therein being therethe stone in the reins or lidneys.

Place.] It grows as well in wet grounds

Time.] And flowers in the end of
February, the leaves begin to appear in
March.
Gotermment and rirtues. The plant is
under Venus, the fresh leaves or juice, or

as in drier places,
Time.] And flowers in the end of
February, the leaves begin to appear in
March.
Gotermment and rirtues. The plant is
under Venus, the fresh leaves or juice, or

as in drier places,
Time.] And flowers in the end of
February, the leaves begin to appear in
March.
Gotermment and rirtues. The plant is a syrup thereof is good for a hot dry cough, or wheezing, and shortness of breath. The dry leaves are best for those that have thin rheums and distillations upon their lungs, they are past, come up somewhat round much lesser, thicker, and greener than those of butter-bur, with a little down or frieze over the green leaf oin the upper side, which may be rubbed away, and whitish or meally, spreading much under ground, so that where it takes it will hardly be driven away again, if any little piece be abiding therein; and from thence spring fresh leaves. under Venus, the fresh leaves or juice, or 


\section{COMFREY.}

THIs is a very conımon but a very neg- helps the defluction of rheum from the lected plant. It contains very great virtues. head upon the lungs, the fluxes of blood or Descript.] The common Great Com- humours by the belly, women's immoderate fiey has divers very large hairy green leaves courses, as well tlie reds as the whites, and lying on the ground, so hairy or prickly, the running of the reins, happening tuy what that if they touch any tender parts of the cause soever. A syrup made thereof is hands, face, or body, it will cause it to itclı; very effectual for all those inward griefs the stalks that rise from among them, being and hurts, and the distilled water for the two or three feet high, hollow and cornered, same purpose also, and for outward wounds is very hairy also, having many such like and sores in the fleshy or sinewy part of the seaves as grow below, but less and less up body whatsocrer, as also to take away the to the top: At the joints of the stalks it is fits of agues, and to allay the sharpness of divided into many branches, with some humours. A decoction of the leaves hereleaves thereon, and at the ends stand many of is available to all the purposes, though flowers in order one above another, which not so effectual as the roots. The roots are somewhat long and hollow like the being outwardly applied, help fresh wounds finger of a glove, of a pale whitish colour, or cuts immediately, being bruised and laid after which come small black seeds. The thereto; and is special good for ruptures routs are great and long, spreading great and broken bones; yea, it is said to be so tlick branches under ground, black on the powerful to consolidate and knit together, outside, and whitish within, short and easy that if they be boiled with dissevered pieces to break, and full of glutinous or clammy of flesh in a pot, it will join them together juice, of little or no taste at all.

There is another sort in all things like: this, only somewhat less, and bears Howers of a pale purple colour.

Place.] They grow by ditches and to cool the inflammation of the parts therewater-sides, and in divers fields tlat are abouts, and to give ease of pains. The moist, for therein they chiefiy delight to roots of Comfrey talien fresh, beaten snall, grow. The first generally through all the and spread upon leather, and laid upon land, and the other but in some places. By any place troubled with the gout, doth the leave of my authors, I know the furst presently give ease of the pains; and apgrows in dry places.

Time.] They flower in June or July, and give their seed in August.

Government and virtues.] 'Ihis is an herb of Saturn, and I suppose under the sign Capricorn, cold, dry, and earthy in quality. What was spoken of Clown's Woundwort may be said of this. The Great Comfrey belps those that spit blood, or make a bloody urine. 'The root boiled in water or winc, and the decoction drank, helps all inward hurts, bruises, wounds, and ulcers plied in the same manner, gives ease to pained joints, and profits very much for running and moist uleers, gangrenes, nortifications, and the like, for which it hath by often experience been found helpful.

Ir is also called $h_{v}$ some Toothwort, 'Tooth Violet, Dog-Teeth Violet, and

Descript.] Of the many sorts of this herb two of them may be found growing CORALWORT. Dentaria. 
in this nation; the first of which shoots wounds, especially such as are made in the forth one or two winged leaves, upon long breast or lungs, by taking a dram of the brownish foot-stalks, which are doubled powder of the root every morning in wine: down at their first coming out of the ground; the same is excellently good for ruptures, as when they are fully opened they consist also to stop fluxes; an ointment made of it of seven leaves, most commonly of a sad is exceedingly good for wounds and ulcers, green colour, dented about the edges, set for it soon dries up the watery humours on both sides the middle rib one against which hinder the cure.

another, as the leaves of the ash tree; the stalk bears no leaves on the lower half of it ; the upper half hears sometimes three or four, each consisting of five leaves, somctimes of three; on the top stand four or five flowers upon short foot-stalks, with long husks; the flowers are very like the flowers of Stockgilliflowers, of a pale purplish colour, consisting of four leaves ? a-piece, after which come small pods, which contain the seed; the root is very smooth, white and shining; it does not grow downwards, but creeps along under the upper crust of the ground, and consists of divers small round knobs set together; towards the top of the stalk there grows some single leaves, by each of which comes a small cloven bulb, which when it is ripe, if it be set in the ground, it will grow to be a root.

As for the other Coralwort, which grows in this nation, it is more scarce than this, It is astringent to the stomach, and being a very sniall plant, much like Crow-? strengthens the liver, and all the other infoot, therefore some think it to be one of ward parts; and taken in whey works more the sorts of Crowfoot. I know not where effectually. 'Taken fasting in the morning, to direct you to it, therefore I shall forbear the description.

Place.] The first grows in Mayfield in COSTMARY, OR ALCOST, OR BALSAM HERB. P. VZ. $x$.

Tilis is so frequently known to be an inhabitant in almost every garden, that I suppose it necdless to write a description thereof.

Time.]' It flowers in June and July.

Government and virtues.] It is under the dominion of Jupiter. The ordinary Costmary, as well as Maudlin, provokes urine abundantly, and moistens the hardness of the motlier; it gently purges choler and phlegm, extenuating that which is gross, and cutting that which is tough and glutinous, cleanses that which is foul, and hinders putrefaction and corruption; it dissolves without attraction, opens obstructions, and helps their evil effects, and it is a wonderful help to all sorts of dry agues. it is very profitable for pains in the head that are continual, and to stay, dry up, and consume all thin rheums or distillations Sussex, in a wood called Highread, and in from the head into the stomach, and helps another wood there also, called Fox-holes. much to digest raw humours that are

Time.] They flower from the latter end gathered therein. It is very profitable for of April to the middle of May, and before those that are fallen into a continual evil the middle of July they are gone, and not disposition of the whole body, called to be found.

Cachexia, but especially in the beginning Government and virtues.] It is under the of the disease. It is an especial friend and dominion of the Moon. It cleanses the help to evil, weak and cold livers. The bladder, and provokes urine, expels gravel, seed is familiarly given to children for the and the stone ; it eases pains in the sides worms, and so is the infusion of the flowers and bowels, is excellently good for inward ? in white wine given them to the quantity of $(5,6$. 
two ounces at a time; it makes an excellent and the worms, and being either drank or salve to cleanse and heal old ulcers, being injected, for the disease called Tenesmus, boiled with oil of olive, and Adder's tongue which is an often provocation to the stool with it, and after it is strained, put a little without doing any thing. The grecn leaves wax, rosin, and turpentine, to bring it to a bruised, and laid to any green wound, stays convenient body.

CUDWEED, OR COTTONWEED.P.vi the bleeding, and heals it up quickly. The juice of the herb taken in wine and milk, is, as Pliny saith, a sovereign remedy Besines Cudweed and Cottonweed, it is against the inumps and quinsey; and further also Called Chaffweed, Dwarf Cotton, and saith, That whosoever shall so take it, shall Petty Colton.

Descript.] The common Cudweed rises up with one stalk sometimes, and sometimes with two or three, thick set on all sides with small, long and narrow whitish or woody leaves, from the middle of the stalk almost up to the top, with every leaf stands small flowers of a dun or brownish yellow colour, or not so yellow as others; in which herbs, after the flowers are fallen, come small seed wrapped up, with the down therein, and is carried away with the wind; the root is small and thready.

There are other sorts hereof, which are somewhat less than the former, not much different, save only that the stalks and leaves are shorter, so that the flowers are paler and more open.

Place.] They grow in dry, barren, sand $y$, and gravelly grounds, in most places of this land.

Time.] They flower about July, some earlier, some later, and then seed is ripe in August.

Government and virtues.] Venus is Lady of it. The plants are all astringent, binding, or drying, and therefore profitable for defluctions of rhcum from the head, and to stay fluxes of blood wheresoever, the decoction being made into red wine and drank, or the powder taken therein. It also helps the bloody-flux, and eases the torments that come thereby, stays the immoderate courses of womell, and is also good for inward or outward wounds, hurts, and gave them the name Paralysis. The flowers never be troubled with that disease again.

COWSLIPS, OR PEAGLES.P.vi.

Botir the wild and garden Cowslips are so well known, that I neither trouble mysolf nor the reader with a description of them.

Time.] 'They flower in A pril and May. Government and virtues.] Venus lays claim to this herb as her own, and it is under the sign Aries, and our city dames know well enough the ointment or distilled water of it adds beauty, or at least restores it when it is lost. The flowers are held to be more effectual than the leares, and the roots of little use. An ointment being made with them, takes away spots and wrinkles of the skin, sun-burning, and freckles, and adds beauty exceedingly; they remedy all infirmitics of the head coming of heat and wind, as vertigo, ephialtes, false apparitions, phrensies, fallingsickness, palsies, convulsions, cramps, pains in the nerves; the roots ease pains in the back and bladder, and open the passages of urine. The lcaves are good in wounds, and the flowers take away trembling. If the flowers be not well dried, and kept in a warm place, they will soon putrefy and look green: Have a special eye over them. If you let them see the Sun once a month, it will do neither the Sun nor them harm

Because they strengthen the brain and bruises, and helps children both of burstings? preserved or conserved, and the quantity of 
a nutmeg eaten every morning, is a sufficient dose for inward diseases ; but for wounds, spots, wrinkles, and sunburnings; an ointment is made of the Jeaves, and hog's grease.

\section{CRAB'S CLAWS. P. .}

CALLED also Water Sengreen, Knight's Pond Watcr, Water House-leck, Pond Wced, and Fresh-water Soldier.

Descript.] It has sundry long narrow leaves, with sharp prickles on the edges of them, also very sharp pointed; the stalks which bear flowers, seldom grow so high as the leaves, bearing a forked head, like a Crab's Claw, out of which comes a whitc flower, consisting of threc leaves, with divers yellowish hairy threads in the middle; it takes root in the mud at the bottom of the water.

Place.] It grows plentifully in the fens in Lincolnshire:

Time.] It flowers in June, and usually from thence till August.

Government and virtues.] It is a plant under the dominion of Venus, and therefore a great strengthener of the reins; it is excellently good for inflammation which is commonly called St. Anthony's Firc; it assuages inflammations, and swcllings in wounds: and an ointment made of it is excellently good to heal them; there is scarcely a better remedy growing than this is, for such as have bruised their kidneys, and upon that account discharge blood; a dram of the powder of the herb taken cvery morning, is a very good remedy to stop the terms.

\section{BLACK CRESSES. P.v}

Descript.] IT has long leaves, deeply cut and jagged on both sides, not much unlike wild mustard; the stalk small, very limber, though very tough: you may twist them round as you may a willow before they break. The flowers are very small and yel- low, after which comes small pods, which contain the seed.

Place.] It is a common herb, grows usually by the way-side, and sometimes upon mud walls about London, but it delights to grow most among stones and rubbish.

Time.] It flowers in June and July, and the seed is ripe in August and September.

Govermment and virtues.] It is a plant of a hot, and biting nature, under the dominion of Mars. The seed of Black Cresses strengthens the brain exceedingly, being, in performing that office, little inferior to mustard seed, if at all ; they are excellently good to stay those rheums which may fall down from the head upon the lungs; you may beat the seed into powder, if you please, and make it up into an electuary with honey; so you have an excellent remedy by you, not only for the premises, but also for the cough, yellow jaundice and sciatica. This herb boiled into a poultice, is an excellent remedy for inflammations, both in women's breasts, and men's testiclcs.

\section{SCIATICA CRESSES. P.V.}

Descript.] Trusse are of two kinds; The first rises up with a round stalk about two feet high, spreads into divers branches, whose lower leaves are somewhat larger than the upper, yet all of them cut or tom on the edges, somewhat like the garden Cresses, but smaller, the flowers are small and white, growing at the tops of branches, where afterwards grow liusks with small brownish seeds therein very strong and sharp in taste, more than the Cresses of the garslen; the root is long, white, and woody.

The other has the lower leaves whole, somewhat lorig and broad; not torn at all, but only somewhat deeply dented about the edges towards the ends; but those that grow up ligher are smaller. The flowers and seeds are like the former, and so is the 
root likewise, and both root and seeds as sharp as it.

Place.] They grow in the way-sides in untilled places, and by the sides of old walls.

Time.] They flower in the end of June, and their seed is ripe in July:

Government and virtues.] It is a Saturnine plant. The leaves, but especially the root, taken fresh in Summer-time, beaten or made into a poultice or salve with old hog's grease, and applied to the places pained with the sciatica, to continue thereon four hours if it be on a man, and two hours on a woman; the place afterwards bathed witì wine and oil mixed together, and then wrapped with wool or skins, after they have sweat a little, will assuredly cure not only the same disease in hips, knuckle-bone, or other of the joints, as gout in the hands or feet, but all? other old griefs of the head, (as inveterate rheums, and other parts of the body that are hard to be cured. And if of the former griefs any parts remain, the same medicine after twenty days, is to be applied again. The same is also effectual in the diseases of the spleen; and applied to the skin, takes away the blemish thereof, whether which although it ulcerate tle part, yet that a sallad.

is to be helped afterwards with a salve made of oil and wax. Esteem this as another? secret.

\section{WATER CRESSES P.VI.}

\section{Descript.] OuR ordinary Water Cresses} spread forth with many weak, hollow, sappy stalks, shooting out fibres at the joints, and upwards long winged leaves made of sundry broad sappy almost round leaves, of a brownish colour. 'The flowers are many and white, standing on long foot-stalks, after which come small yellow seed, contained in small long pods like horns. The whole plant abides green in the winter, and tastes somewhat hot and sharp.
Place.] They grow, for the most part, in small standing waters, yet sometimes in small rivulets of running water.

Time.] They flower and seed in the beginning of Summer.

Government and virtues. It is an herh under the dominion of the Moon. They are more powerful against the scurvy, and to cleanse the blood and humours, than Brooklime is, and serve in all the other uses in which Brooklime is available, as to break the stone, and provoke urine and women's courses. The decoction thereof cleanses ulcers, by washing them therewith. The leaves bruised, or the juice, is good, to be applied to the fare or other parts troubled witl fieckles, pimples, spots, or the like, at night, and washed away in the morning The juice mixed with vinegar, and the fore part of the head bathed therewith, is very good for those that are dull and drowsy, or have the lethargy.

Water-cress pottage is a good remedy to cleanse the blood in the spring, and help headaches, and consume the gross humours winter has left behind; those that would live in lealth, may use it if they please; if they will not, I cannot help it. If any

\section{Crosswort. $P .-v$.}

THIs herh receives its name from the situation of its leaves.

Descript.] Common Crosswort grows up with square hairy brown stalks a little abovt a foot high, having four small broad and pointed, hairy, yet smooth thin leaves, growing at every joint, each against other one way, which has caused the name. Towards the tops of the stalks at the joints, with the leaves in three or four rows downwards, stand small, pale yellow flowers, after which come small blackish round seeds, four for the most part, set in every husk. The root is very small, and full of 
fibres, or threads, taking good hold of the patience of Socrates himself, but because ground, and spreading with the branches 1 have not yet attained to the spirit of over a great deal of ground, which perish Socrates, I shall but describe the most not in winter, although the leaves die every usual. year, and spring again anew.

Place.] It grows in many moist grounds, as well nieadows as untilled places, about London, in Hampstead church-yard, at Wye in Kent, and sundry other places.

Descript.] The most common Crowfoot has many thin great lcaves, cut into divers parts, in taste biting and sharp, biting and blistering the tongue: It bears many flowers, and those of a bright, resplendent,

Time.] It flowers from May all the Summer long, in one place or other, as they are more open to the sun; the seed ripens soon after.

Government and virtues.]. It is under the dominion of Saturn. This is a singularly good wound herb,-and is used inwardly, not only to stay bleeding of wounds, but to consolidate them, as it doth outwardly any green wound, which it quickly solders up, and heals. The decoction of the herb in wine, helps to expectorate the phlegm out of the chest, and is good for obstructions in the breast, stomach, or bowels, and helps a decayed appetite. It is also good to wash
any wound or sore with, to clcanse and heal it. The herb bruised, and then boiled, applied outwardly for certain days together, renewing it often : and in the mean time the decoction of the herb in wine, taken inwardly every day, doth certainly cure the rupture in any, so as it be not too inveterate; but very speedily, if it be fresh and lately taken.

\section{CROWFOOT. P.v.} yellow colour. I do not remember, that I ever saw any thing yellower. Virgins, in ancient time, used to make powder of them to furrow bride beds; after which flowers come small hends, some spiked and rugged like a Pine-Apple.

Place.] They grow very common every where; unless you turn your head into a hedge, you cannot but see them as you walk

Time.] They flower in May and June, even till September.

Goverment and virtues.] This fiery and hot-spirited herb of Mars is no way fit to be given inwardly, but an ointment of the leaves or flowers will draw a blister, and may be so fitly applied to the nape of the neck to draw back rheum from the eyes. The herb being bruised and mixed with a little mustard, draws a blister as well, and as perfectly as Cantliarides, and with far less danger to the vessels of urine, which Cantharides naturally delight to wrong: I knew the herb once applied to a pestilential rising that was fallen down, and it saved life even beyond hope; it were good Mary are the names this furious biting to kecp an ointment and plaister of it, if it herb has obtained, almost enough to make were but for that.

up a Welshman's pedigree, if he fetch no farther than John of Gaunt, or William the Conqueror; for it is called Frog's-foot, from the Greek name Barrakion: Crowfoot, Gold Knobs, Gold Cups, King's Knọb, Baffiners, 'Troilflowers, Polts, Locket Goulions, and Butterflowers.

Abundance are the sorts of this herb, every one whereof is somewhat large and that to describe them all, would tire the long, broad at the bottom next the stalk, 
and forked, but ending in a point, without Spring, and continue but until the middle a cut on the edge, of a full green colour, of Summer, or somewhat later; their liusks each standing upon a thick round stalk, of appearing before they fall away, and their a hand-breath long, or more, among which, fruit shewing in April.

after two or three months that they begin to Govermment and virtues.] It is under the wither, rises up a bare, round, whitish green dominion of Mars. 'I'ragus reports, that stalk, spotted and streakcd with purple, a dram weight, or more, if need be, of the somewhat higher than the leaves: At the spotted TVake Robin, either fiesli and top whereof stands a long hollow husk, green, or dried, being eaten and takell, close at the bottom, but open from the is a present and sure remedy for poison and middle upwards, ending in a point: in the the plague. The juice of the herb taken to middle whereof stand the small long pestle the quantity of a spoonful has the same or clapper, smaller at the bottom than at effect. But if there be a little vinegar the top; of a dark purple colour, as the busk ?added thercto, as well as to the root aforeis on the inside, though grcen without; said, it somewhat allays the sharp biting which, after it hath so abided for some time, tiste thereof upon the tongue. The green the husk with the clapper decays, and the leares bruised, and laid upon any boil or foot or bottom thereof grows to be a small plague sore, doth wonderfully help to draw long bunch of berries, green at the frist, forh the poison: A dram of the powder of and of a yellowish red colour when they the dried root taken with twice so much are ripe, of the bigness of a hazel-nut ker-- sugar in the form of a licking clectuary, or nel, which abides thereon almost until the green root, doth wonderfully hclp those Winter; the root is round, and somewhat that are pursy and short-winded, as also those long, for the most part lying along, the that have a cough; it breaks, digests, and leares shooting forth at the largest end, irids away phlegm. from the stomach, which, when it bear's its berries, are some- chest, and lungs. The milk whercin the what wrinkled and loose, another growing root has been boiled is effectual also for the under it, which is solid and firm, with many same purpose. The said powder taken in small threads hanging thereat. The whole wine or other drink, or the juice of the plant is of a very sharp biting taste, prick- berries, or the powder of them, or the wine ing the tongue as nettles do the hands, and wherein they have been boiled, provokes so abides for a great while without altera-? urine, and brings down women's courses, tion. The root thereof was anciently used and purges them effectually after childinstead of starch to starch linen with.

'There is another sort of Cuckow-pornt, 'Taken with shecp's milk, it heals the inward with less leaves than the former, and some- ulcers of the bowels. The distilled water times harder, having blackish spots upon thereof is effectual to all the purposes aforethem, which for the most part abide longer said. A sponful taken at a time heals green in Summer than the former, and the itch; an ounce or more taken at a time both leaves and roots are inore sharp and for some days together, doth help the rupfierce than it: In all things clse it is like the ture: 'The leaves either green or dry, or the former.

Place.] These two sorts grow frequently roiten and filthy ulcers, in what part of the almost under crery hedge-side in many hody socver; and heals the stinking sores places of this land.

in the nose, called Polypus. The water Time.] They shoot forth leaves in the wherein the root has been boiled, dropped 
into the eyes, cleanses them from any filn or skin, cloud or mists, which begin to hinder the sight, and helps the watering and redness' of them, or when, by some chance, they beccme black and blue. 'The root mixed with bean-flour, and applied to the throat or jaws that are inflamed, helps them. The juice of the berries boiled in oil of roses, or beaten inio powder mixed with the oil, and dropped into the ears, cases pains in them. The berries or the roots beaten with the hot ox-dung, and applied, eases the pains of the gout. The leaves and roots boiled in wine with a little oil, and applied to the piles, or the falling down of the fundament, eases them, and so doth sitting over the hot fumes thereof. The fresh roots bruised and distilled with a little milk, yields a most sovereign water to cleanse the skin from scurf, freckles, spots, or blemishes whatsoever therein.

Authors have left large commendations of this herb you see, but for my part, I have neither spoken with Dr. Reason nor Dr. Experience about it.

\section{CUCUMBERS. P.VT.}

Government and virtues.] THExe is no dispute to be made, but that they are under the dominion of the Moon, tirough they are so much cried out against for their coldness, and if they were but one degree colder they would be poison. The best of Galenists hold them to be cold and moist in the second degree, and then not so hot as either lettuce or purslain: They are excellently good for a hot stomach, and hot liver; the unmeasurable use of them fills the body full of raw humours, and so indeed the unmeasurable use of any thing else doth harm. The face being washed with their juice, clcanses the skin, and is excellently good for hot theums in the eyes; the seed is excellently goud to proroke urine, and cleanses the passiges thercof when they are stopped; there is not a betier remedy for ulcers in the bladder growing, than Cucumbers are The usual course is, to use' the sceds in emulsions, as they make almond milk; but a fir better way (in my opinion) is this: When the season of the year is, 'Take the Cucumbers and bruise them well, and distil the water from them, and let such as are troubled with ulcers in the bladder cirink no other drink. 'The face being' washed with the sume water, cures the redilest face that is ; it is also excellently good for sun-burning, freckles, and morphow.

$$
\text { DAISIES. P. VIS. }
$$

'T'Hesu are so well knowu almost to every child, that I suppose it needless to write any. description of them. Take therefore the virtues of them as follows.

Govermnent and virtues.] The herb is under the sign Cancer, and under the dominion of Veuus, and therefore excellently good for wounds in the breast, and very fitting to be kept both in oils, ointments, and plaisters, as also in syrup. The greater wild Daisy is a wound herb of grood respect, often used in those drinks or salves that are for wounds, either inward or outward. The juice or distilled water of these, or the small Daisy, doth much temper the heat of choler, and refiesh the liver, and the other inward parts. A decoction made of them and drank, helps to cure the wounds made in the hollowness of the breast. The same also cures all ulcers and pustules in the mouth or tongue, or in the secret parts. The leaves bruised and applied to the privities, or to any other parts thatareswoln and hot, doth dissolve it, and temper the heat. A decoction made thereof, of Wallwort and Agrimony, and the places fomented and bathed therewith warm, gives great ease to them that are troubled with the palsy, sciatica, of the gout: 'I'he same also disperses and dissolves the knots or: liemels that grow in the fesh of any part of the body, and bruises and hurts that come of 
falls and blows; they are also used for rup- dominion of Jupiter. It is of an opening tures, and other inward burnings, with and cleansing quality, and therefore very very good success. An ointment made effectual for the obstructions of the liver, thereof doth wonderfully help all wounds gall and spleen, and the diseases that arise that lave inflammations about them, or by from them, as the jaundice and hypoconreason of moist humours having access unto driac ; it opens the passages of the urine them, are kept long from healing, and such both in young and old ; powerfully cleanses are those, for the most part, that happen to imposthumes and inward ulcers in the joints of the arms or legs. The juice of urinary passage, and by its drying and them dropped into the rumning eyes of any, temperate quality doth afterwards heal doth inuch help them.

\section{DANDELION, VULGARLY CALLED PISS- A-BEDS. P.VH.}

Descript.] IT is well known to have many long and deep gashed leaves, lying on the ground round about the head of the roots; the ends of each gash or jag, on both sides looking downwards towards the roots; the middle rib being white, which being broken, yields abundance of bitter milk, but the root much more; from among the leaves, which always abide green, arise many slender, weak, naked foot-stalks, every one of them bearing at the top one large yellow flower, consisting of many rows of yellow leaves, broad at the points, and nicked in with deep spots of yellow in the middle, which growing ripe, the green husk wherein the flowers stood turns itself down to the stalk, and the head of down becomes as round as a ball : with long
seed underneath, bearing a part of the down on the head of every one, which together is blown away with the wind, or may be at once blown away with one's mouth. The root growing downwards exceedingly deep, which being broken off within the ground, will yet shoot forth again, and will hardly be destroyed where it hath once taken deep root in the ground.

Place.] It grows frequently in all meadows and pasture-grounds.

Time.] It flowers in one place or other almost all the year long.

Government and virtues.] It is under the them ; for which purpose the decoction of the roots or lenves in white wine, or the lcaves chopped as pot-herbs, with a few Alisanders, and boiled in their broth, are very effectual. And whoever is drawing towards a consumption or an evil disposition of the whole body, called Cachexia, by the use hereof for some time together, shall find a wonderful help. It helps also to procure rest and sleep to bodies distempered by the heat of ague fits, or otherwise: The distilled water is effectual to drink in pestilential fevers, and to wash the sores.

You see here what virtues this common herb hath, and that is the reason the French and Dutch so often cat them in the Spring; and now if you look a little farther, you may see plainly without a pair of spectacles, that foreign physicians are not so selfish as ours are, but more communicative of the virtues of plants to peoplc.

\section{DARNEL.P.T.}

IT is called Jam and Wray; in Sussex they call it Crop, it being a pestilent enemy among corn.

Descript.] This has all the winter long, sundry long, flat, and rough leaves, which, when the stalk rises, which is slender and jointed, are narrower, but rough still; on the top grows a long spike, composed of many heads set one above another, containing two or three husks, with a sharp but short beard of awns at the end; the 
seed is easily shaken out of the ear, the? husk itself being somewhat lough.

Place.] know this too well to grow among their? corn, or in the borders and pathways of the other fields that are fallow.

Government and rivtues.] 'It is a malicious part of sullen Saturn. As it is not without some vices! so hath it also inany virtues? The meal of Darnel is very good to stay gangrenes, and other such like fretting and eating cankers, and putrid sores: It also cleanses the skin of all leprosies, morphervs, ringworms; and the like, if it be used with salt and raidish toots. And being used with quick brimstone and vinegar, it dissolves knots and kernels, and breaks those that are lard to be dissolved, being boiled in wine with pigeon's dung and linseed A decoction thercof made with water and honey, and the places bathed therewith, is profitable for the : sciatica.. Darnè néal applied in a poultice draws forth splinters and broken bones in the flesh: 'l'he red Darnel, boiled in red wine and taken, stays the lask and all other fluxes, and women's bloody issues ; and restrains urine that passes away too suddenly.

\section{BILL. P. Virl.}

Descript.] TuE common. Dill grows up with seldom. more than one stalk, neither so high, nor so great usually as Fennel, being round and fewer joints thereon, whose leaves are sadder, and somewhat long, and so like Fennel that it deceives many, but harder in handling, and somewhat thicker, and of a strong unpleasant scent: The tops of the stalks have four branches and smaller umbels of yellow flowers, which turn into small seed, somewhat flatter and thimmer than Fennel sced. 'The root is somewhat small and wondy, perishes every year after it hath borne seed ; and is alsomprofitable, being never put to anly use.

$(7,8$.
Place.] It is most usually sown, in gardens and grounds for the purpose, and is also found wild in many places.

Goverminent and virtues.] Mercury has the dominion of this plant, and therefore to be sure it strengthens the brain. The Dill being boiled and drank, is good to ease sivellings:and pains:; it also stays the belly and stomach from casting. :'The decoction therefore helps women that are troubled with the pains and windiness of the molher, if they isit thercin. It stays the hiccóugh, being boiledin: wine, and but smelled unto being tied in a clotk. :The seed is of more use than the ileaves; and inore effeclual to digest liaw and vicious humours, and is used in medicines that serve to expel ivind, and the pains proceeding therefrom. The seed, being roasted or fried, and used in oils or plasters dissolve the imposthumes in the fundanent; and dries up all moist ulcers, especially in the fundament; an oil made of Dill is effectual to warm or dissolve humours and imposthumes, and the pains, and to procure rest. The decoction of :Dill, be it herb or seed (only if you boil the seed you must bruise it) in white: wine, being drank, it is a gallant expeller of wind, and provoker of the terms.

\section{DEVIL'S-BIT. P VU}

Descript.] This rises up with a round grcen. smooth stalk; about two fcet high, set with divers long and somewliat narrow, smooth, dark green leaves, somewhat nipped about the edges, for the most part, being else all whole, and not divided at all, or but very seldom; : eren to : the $\therefore$ tops of the branches, which yet are smaller than those below, with one rib only in the niddlé. At the end of eaclr branch stands a rounit head of many flowers set together in the same manner; or more neatly, than Scabinins, and of a bluish purple colour, which being past, there follows seed which falls away. The root is somewhat thick, but 
slort and blackish, with many strings, imorphew, or other deformities thereof, abiding after seed time many years. This especially if a little vitriol be dissolved root was longer, until the devil (as the therein.

friars say) bit away the rest of it for spite? envying its usefulness to mankind; for sure he was not troubled with any disease for which it is proper.

There are two other sorts hereof, in nothing unlike the former, save that the one bears white, and the other bluish-coloured flowers.

Place.] The first grows as well in dry meadows and fields as moist, in many places of this land: But the other two are more rare, and hard to be met with, yet they are both found growing wild about Appledore, near Rye in Kent.

[Time.] They flower not usually until August.

Government and virtues.] The plant is venereal, pleasing, and harmless. The herb or the root (all that the devil hath left of it) being boiled in wine, and drank, is very powerful against the plague, and all pestilential diseases or fevers, poisons also, and the bitings of venemous beasts: It helps also those that are inwardly bruised by any casuality, or outwardly by falls or blows, dissolving the clotted blood; and the herb or root beaten and outwardly applied, takes away the black and blue marks that remain in the skin. The decoction of the herb, with honey of roses put therein, is very effectual to help the inveterate tumours and swellings of the proct pot good blood, being as wholesone meterate tumours and swellings of the a pot herb any growing in a garden; yet almonds and throat, by often gargling the such is the nicety of our times, forsooth, mouth therewith. It helps also to procure women's courses, and eases all pains of the mother and to break and discuss wind therein, and in the bowels. The powder of the root taken in drink, drives forth the worns in the body. The juice or distilled water of the herb, is effectual for green wounds, or old sores, and cleanses the body inwardly, and the seed outwardly, that women will not put it into a pot, because it makes the pottage black; pride and ignorance (a couple of monsters in the creation) preferring nicety before health.

DODDER OF 'THYME, EPITHYMUM, AND OTHER DODDERS.

Descript.] 'THus first from seed gives from isores, scurf, itch, pimples, freckles, is threads or strings, grosser or finer, as the 
property of the plant wherein it grows, spleen, and melancholy that arises from the and the climate doth suffer, creeping and windiness of the hypochondria. It purges spreading on that plant whereon it fastens, also the reins or kidneys by urine; it be it high or low. The strings have no leaves at all on them, but wind and in-profits them that have the jaundice; as terlace themselves, so thick upon a small also the leaves, the spleen: Purging the plant, that it takes away all comfort of the veins of the choleric and phlegmatic sun from it; and is ready to choak or stran- humours, and helps children in :agues, a gle it. After these strings are risen to that little worm seed being put thereto.

height, that they may draw nourishment The other Dodders do, as I said before, from that plant, they seem to be broken off parcicipate of the nature of those plants from the ground, either by the strength of ? whereon they grow : As that which hath their rising, or withered by the heat of the been found growing upon nettles..in the Sun. Upon thise strings are found clus-? westrcountry, hath by experience been ters of sniall heads or husks, out of which found very effectual to procure plenty of shont forth whitish Howers, which after- urine where it hath been stopped or hinwards give small pale white coloured seed, slered. And so of the rest.

somewhat Hat, and twice as big as Poppy- Sympathy and antipathy are two hinges seed. It generally participates of the na- upon which the whole mode of physic ture of the plant which it climbs upon; ; turns; and that physician who minds but the Dodder of Thyme is accounted the them not, is like a door off from the hooks, best, and is the only true Epithymum.

Government and virtues.] All Dodders more like to do a man mischief, than to are under Saturn. T'ell not me of phy- causes, this helps by sympathy, and sicians crying up Epithymum, or that strengthens all the parts of the body he Dodder which grows upon Thyme, (most rules; such as be caused by Sol, it helps by of which comes from Hemetius in Greece, antipathy. What those diseases are, see or Hybla in Sicily, because those moun-my judgment of diseases by astrology; and tains abound with 'Thyme, he is a phy- if you be pleased to look at the herb Wormsician indeed, that hath wit enough to wood, you shall find a rational way for it. chrose the Dodder according to the nature of the disease and humour peccant. We confess, Thyme is the hottest leerb it usually DOG'S-GRASS, OR COUGH GRASS. P.'Y

Descript.] IT is well known, that the grows upon; and therefore that which grass creeps far about under ground, with grows upon 'Thyme is hotter than that long white joined roots, and small fibres which grows upon cold herbs; for it draws almost at every joint, very sweet in taste, nourishment from what it grows upon, as as the rest of the herb is, and interlacing well as trom the earth where its root is, and one another, from whence shoot forth many thus you see old Saturn is wise enough to fair grassy leaves, small at the ends, and have two strings to liis bow. This is ac- cutting or sharp on the edges. The stalks counted the most effectual for melancholy are jointed like corn, with the like leaves diseases, and to purge black or burnt choler, which is the canse of many diseases of the long husk in them, and hard rough seed in head and brain, as also for the trembling of them. If you know it not by this desthe heart, faintings and swoonings. It is cription, watch the dogs when they are helpful in all diseases and griefs of the sick, and they will quickly lead you to it. 
Place.] It grows commonly through this by the path-sides in many places, and will land in divers ploughed grounds to the no also be in gardens.

small trouble of the husbandmen, as also of the gardeners, in gardens, to weed it out, if they can; for it is a constant customer to the place it gets footing in.

Government and virtues.] 'Tis under the dominion of Jupiter, and is the most medicinal of all the Quick-grasses. Bcing boiled and drank, it opens obstructions of ? the liver and gall, and the stopping of urine, and eases the griping pains of the belly and inflammations; wastes the matter of the stone in the bladder, and the ulcers thereof also. The roots bruised and applied, do consolidate wounds. 'The seel doth more powerfully expel urine, and stays the lask and vomiting. The dis-
tilled water alone, or with a little wormseed, kills the worms in children.

Time.] It flowers in June, July, and August, some earlier and some later: and the seed is ripe quickly after.

Government and virtues.] It is a very gentle, though martial plant. 'It is found by experience to be singularly good for wind cliolic, as also to expel the stone and gravel in the kidneys. The decoction thereof in wine, is an excellent good cure for those that have inward wounds, hurts, or bruises, both to stay the bleeding, to dissolive and expel the congealed blood, and to heal the parts, as also to cleanse and heal outward sores, ulcers, and fistulas; and for green wounds, many do only bruise the herb, and apply it to the places, and it heals them quickly. 'The sane decoction in wine The way of use is to bruise the roots, and gout, or to joint-aches, or pains of the having well boiled them in white wine, sinews, gives much ease. The powder or drink the decoction': "Tis opening but not decoction of the herb taken for some time purging, very safe: 'Tis a remedy against together, is found by experience to be sinall diseases coming of stopping, and such. are hialf those that are incident to the body of man; and although a gardener be of another opinion, yet a physician holds half an acre of them to be worth five acres of Carrots twice told over.

DOV.F'S-FOOT; OR CRANE'S-BILL.T.8

Descript.] 'Tuis has divers small, round, pale-green leaves, cut in about the edges, the herb, and the Moon will be Lady of it; much like mallow, standing upon long, a word is enough to a wise man. It is reddish, hairy stalks, lying in a round com- effectual to help inflammations, and $\mathrm{St}$ pass upori the ground; among which rise? Anthony's Fire, as also the gout, either up two or three, or more, reddish, jointed, applied by itself, or in a poultice with Barslender, weak, hairy stalks, with some like ley meal. 'The distilled. water by some is leaves thereon, but smaller, and more cut in highly esteened against all inward inflamup to the tops, where grow many very mations and pestilent ferers; as also to small bright red flowers of five leaves a- help the redness of the eyes, and swellings piece; after which follow small heads, with of privities, and of the breasts before they small short beaks pointed forth, as all other be grown too much. The fresli herb apsorts of those heribs do.

plied to the forehead, cases the pains of Place.] It grows in pasture grounds, and the head-ache coming of heat. 
DOWN, OR COTTON-THISTLE.P.VII

Descript.] Tus has large leaves lying on the ground, somewhat cut in, and as it were crumpled on the edges, of a green colour on the upper side, but covered with long hairy wool, or Cotton Bown, set with most sharp and cruel pricks, from the middle of whose head of flowers, thrust forth many purplish crimson threads, and sometimes (although very seldom) white ones. The seed that follows in the heads, lying in a great deal of white down, is somewhat large, long, and round, like the seed of ladies thistle, but paler. The root is great and thick, spreading much, yet it usually dies after seed-time.

Place.] It grows in divers ditehes, banks, and in corn-fields, and highways, generally every where throughout the land.

Time.] It flowers and bears seed about the end of Summer, when other thistles do tlower and seed.

Government and virtues.] Mars owns the plant, and manifests to the world, that though it may liurt your finger, it will help your body ; for I fancy it much for the ensuing virtues. Pliny and Dioscorides write, That the leaves and roots thereof taken in drink, help those that liave a crick in their neck; whereby they cannot turn? their neck but their whole body must turn ? also (sure they do not mean those that have got a crick in their neck by being under the hangman's hand.) Galen saith, that the root and leaves hereof are of a healing yuality, and good for such persons as have their bodies drawn together by some spasm or convulsions, as it is with children that have the rickets.

\section{DRAGONS. P. VIH.}

THEY are so well known to every one that plants them in their gardens, they need 110 description; if not, let them look down $(\boldsymbol{7}, 8$. to the lower end of the stalks, and see how like a snake they look.

Government and virtues.] The plant is under the dominion of Mars, and therefore it would be a wonder if it should want some obnoxious quality or other: In all herbs of that quality, the safest way is either to distil the herb in an alembick, in what vehicle you please, or else to press out the juice, and distil that in a glass still, in sand. It scours and cleanses the internal parts of the body mightily, and it clears the external parts also, being externally applied, from freckles, morphew, and sun-burning: Your best way to use it externally, is to mix it with vinegar ; an ointment of it is held to be good in wounds and ulcers; il consumes cankers, and that flesh growing in the nostrils, which they call Polypus: Also the distilled water being dropped into the eyes, takes away spots there, or the pin and web, and mends the dimness of sight; it is excellently good against pesstilence and poison. Pliny and Dioscorides affirm, that no serpent will meddle with him that carries this herb about him.

THE ELDER TREE. P. $x$.

I IroLD jt needless to write any description of this, since every boy that plays with a pop-gun will not mistake another tree instead of Elder: I shall therefore in this place only describe the Dwarf-Eilder. called also Dead-wort, and Wall-wort.

\section{TIIE DWARE-ELDER: $\mathcal{P} \cdot q$.}

Descript.] Turs is but an lierb every year, dying with his stalks to the ground, and rising afresh every Spring, and is like unto the Elder both in form and quality, rising up with a square, rough, hairy stalk, four feet ligh, or more sometimes. The winged leaves are somewhat narrower than the Elder, but else like them. The Howers are white with a dash of purple, standing in umbels, very like the Elder also, but more 
sweet in scent; after which come small the eyes, assuages them; the juice of the blackish berries, full of juice while they are leaves snuffed up into the nostrils, purges fiesh, wherein is sinall hard kernels, or seed. the tunicles of the brain; the juice of the The root doth creep unerd the upper crust berries boiled with honey and dropped of the ground, springing in divers places, into the ears, helps the pains of them; the being of the bigness of one's finger or decoction of the berries in wine, being thumb somctimes.

Place.] 'The Elder tree grows in hedges, being planted there to strengthen the fences and partitions of ground, and to hold the banks by ditches and water-courses.

The Dwarf Elder grows wild in many places of England, where being once gotten into a ground, it is not easily gotten forth again.

Time.] Most of the Elder Trees, flower in June, and their fruit is ripe for the most part in August. But the Dwarf Elder, or Wall-wort, flowers somewhat later, and his fruit is not ripe until September.

Government and virtues.] Both Elder and Dwarf Tree are under the dominion of Venus. 'The first shoots of the common Elder boiled like Asparagus, and the young leaves and stalks boiled in fat broth, doth mightily carry forth phlegm and choler. The middle or inward bark boiled in water, ings, the wind cholic, (holic, and stone, the and given in drink, works much more difficulty of urine, the cure of old sores and violently; and the berries, either green or fistulous ulcers. Either leaves or bark of dry, expel the same humour, and are often Elder, stripped upwards as you gather it, given with good success to help the dropsy; causes vomiting. Also, Dr. Butler, in a the bark of the root boiled in wine, or the manuscript of his, commends Dwarf Elder juice thereof drank, works the same effects, to the sky for dropsirs, viz. to drink it, but more powerfully than either the leaves being boiled in white wine; to drink the or' fruit. 'The juice of the root taken, doth? decoction I mean, not the Elder. mightily procure vomitings, and purges the watery humours of the dropsy. The decoction of the root taken, cires the biting of an adder, and biting of mad dogs. It mollifies the hardmess of the mother, if women sit thereon, and opens their veins, and brings down their courses: The berries TIIE ELM TREE.P.\&

Tins tree is so well known, growing generally in all counties of this land, that it needless to describe it.

Government and virtues.] It is a cold and boiles in wine perform the same effect: and applied, heal and the hair of the head washed therewith bound thereon with its own bark. The is made black. 'The juice of the green leaves or the bark used with vinegar, cures leaves applied to the hot intlammations of scurf and leprosy very effectually: 'The 
decoction of the leaves, bark, or root, being cools the heat and sharpness of the urine, bathed, heals broken bones. The water and excoriation in the urinary parts. The that is found in the bladders on the leaves, seeds are of the same property, or. rather while it is fresh, is very effectual to cleansc more powerful, and besides are available for the skin, and make it fair; and if cloaths be fainting, swoonings, and passions of the often wet therein, and applied to the rup- heart. Outwardly applied, they serve to tures of children, it heals them, if they be temper the sharp humours of fretting ulcers, weil bound up with a truss. The said hot tumours, swellings, and pestilential water put into a glass, and set into the sores; and wonderfully help not only the ground, or else in dung for twenty-five redness and inflamuations of the eyes, but days, the mouth thereof being close stopped, the dimness of the sight also; they are also and the botton set upon a layer of ordinary used to allay the pains of the gout. You salt, that the foeces may settle and water cannot use it amiss; a syrup of it is a fine become clcar, is a singular and sorcreign cooling medicine for fevers.

balm for green wounds, being used with soft tents: The decoction of the bark of ? the root, fomented, mollifies hardz tunours, and the shrinking of the sinews. "The roots . Descript.] IT shoots forth many large of the Elm, boiled for a long time in water, leaves, long and broad, lying near the and the fat arising on the top thereof, being ground, small at both ends, somewhat soft clean skimmerl off, and the place anointed in handling, of a whitish green on the upper therewith that is grown bald, and the hair side, and grey underneath, each set upon fallen away, will quickly restore them a short footstalk, from among which rise again. The said batk ground with brine? up divers great and strong hairy stalks, three or pickle, until it come to the form of a or four ffet high, with some leaves therepoultice, and laid on the place pained with , upon, compassing them about at the lower the gout, gives great ease, 'The decoc- cnd, and are branched towards the tops, tion of the bark in water, is excellent to bearing divers great and large thowers, like bathe such places as have been burnt with those of the corn marigold, both the border fire. of leaves, and the niddle thrum being yellow, which turn into down, with long, small, ENDIVE. P.VII. brownish secds amongst it, and is carried Descript.] Conmon garden Endive away with the wind. The root is great bears a longer and larger leaf than Succory, and thick, bmonched forth divers ways, and abides but one year, quickly running blackish on the outside and whitish within, up to a stalk and seed, and then perishes; of a very bitter taste, and strong, but good it has blue flowers, and the seed of the scent, especially when they are dried, no ordinary Endive is so like Succory seed, part else of the plant having any sinell. that it is hard to distinguish them.

Pluce.]. It grows on moist grounds and Government and virtues.] It is a fine shedowy places oftener than in the dry and cnoling, cleansing, jovial plant. 'The de- ofren borders of the fields and lanes, and in cortion of the leaves, or the juice, or the other waste places, almost in every county distilled water of Endive, serve well to cool of this iand.

the exressive heat of the liver and stomach, Time.] It flowers in the end of June and and in the hot fits of agues, and all other? July, and the seed is ripe in August. The inflammations in any part of the body; it roots are gathered for use, as well in the 
Spring before the leaves come forth, as in Autumn or Winter.

Government and virtues.] It is a plant under the dominion of Mercury. The fresh roots of Elecampane preserved with sugar, or made into a syrup or conserve, are very effectual to warnı a cold windy stomach, or the pricking therein, and stitches in the sides caused by the spleen; and to help the cougli, shortness of breath, and wheezing in the lungs. The dried root made into powder, and mixed with sugar, and taken, serves to the same purpose, and is also profitable for those who have their urine stopped, or the stopping of women's courses, the pains of the mother, and the stone in the reins, kidneys, or bladder ; it resists poison, and stays the spreading of the venom of serpents, as also putrid and pestilential fevers, and the plague itself. The roots and herbs beaten and put into new ale or beer, and daily drank, clears, strengthens, and quickens the sight of the eyes wonderfully. The decoction of the roots in wine, or the juice taken therein, kills and drives forth all manner of worms in the belly, stomach, and maw; and gargled in the mouth, or the root chewed, fastens loose teeth, and helps to keep them from putrefaction; and being drank is good for those that spit blood, helps to remove cramps or convulsions, gout, sciatica, pains in the joints, applied outwardly or inwardly, and is also good for those that are bursten, or have any inward bruise. The root boiled well in vinegar beaten afterward, and made into an oint-
ment with hog's suet, or oil of trotters, is an ment with hog's suet, or oil of trotters, is an excellent remedy for scabs or itch in young or old; the places also bathed or washed with the decoction doth the same; it also helps all sorts of filthy old putrid sores or cankers whatsoever. In the roots of this The decoction of the root hereof in wine, herb lieth the clief. In the roots of this is very effectual to open obstructions of the herb lieth the chief effect for the remedies spleen and liver, and helps yellow jaunaforesaid. The distilled water of the leaves dice, dropsy, pains of the loins, and wind and roots together, is very profitable to cholic, provokes urine, and expels the cleanse the skin of the face, or other parts, stone, procures women's courses. 'The conn

from any morphew, spots, or blemishes therein, and make it clear.

\section{ERINGO, OR SEA-HOLLY.P. 8 .}

Descript.] 'The first leaves of our ordinary Sea-Holly, are nothing so hard and prickly as when they grow old, being almost round, and deeply dented about the edges, hard and sharp pointed, and a little crumpled, of a bluish green colour, every one upon a long foot stalk; but those that grow up higher with the stalk, do as it were compass it about. The stalk itself is round and strong, yet somewhat crested, with joints and leaves set thereat, but more divided, sharp, and prickly; and branches rising from thence, which have likewise other small branches, each of them having several bluish round prickly heads, with many small jagged prickly leaves under them, standing like a star, and sometimes found greenish or whitish: The root grows wonderfully long, even to eight or ten feet in length, set with rings and circles toward the upper part, cut smooth and without joints down lower, brownish on the outside, and very white within, with a pith in the middle; of a pleasant tasle, but much more, being artificially preserved, and candied with sugar.

Place.] It is found about the sea coast in almost every county of this land which borders upon the sea.

Time.] It flowers in the end of Summer, and gives ripe seed within a month after.

Government and cirtues.] 'The plant is venereal, and breeds seed exceedingly, and strengthens the spirit procreative; it is hot and moist, and under the celestial Balance. 
tinued use of the decoction for fifteen days, taken fasting, and next to bedward, doth help the stranguary, the difficulty and stop- over it. If the herb. was but as much used page of urine, and the stone, as well as all as it is neglected, it would half spoil the defects of the reins and kidneys; and if spectacle maker's trade; and a man would the said drink be continued longer, it is said that it cures the stone; it is found good against the French pox. The roots bruised and applied outwardly, help the kernels of the throat, commonly called the king's evil; or taken inwardly, and applied to the place stung or bitten by any serpent, heal it speedily. If the roots be bruised, and boiled in old hog's grease, or salted lard, and broken bones, thorns, \&c. remaining in the flesh, they do not only draw them forth, but heal up the place again, gathering new flesh where it was consumed. The juice of the leaves dropped into the ear, helps imposthumes therein. The distilled water of the whole herb, when the leaves and stalks are young, is profitably drank for all the purposes aforesaid; and helps the melancholy of the heart, and is available in quartan and quotidian agues; as also for them that have their necks drawn awry, and cannot turn them without turning their whole body.

\section{EYEBRIGHT. I. VMr?.}

Descript.] Common Eyebright is a small low herb, rising up usually but with one blackish green stalk a span high, or not much more, spread from the bottom into sundry branches, whereon are small and almost round yet pointed dark green leaves, finely snipped about the edges, two always set together, and very thick: At the joints with the leaves, from the middle up-: ward, come forth small white flowers, marked with purple and yellow spots, or stripes; after which follow small round heads, with very small seed therein. The root is long, small and thready at the end.

Place.] It grows in meadows, and grassy places in this land.

$(\mathbf{7}, \mathbf{8}$.
Government and virtues.] It is under the think, that reason should teach people to before artificial spectacles; which that they may be instructed how to do, take the virtues of Eyebright as follows.

The juice or distilled water of Eyebright, taken inwardly in white wine or broth, or dropped into the eyes for divers days together, helps all infirmities of the eyes that cause dimness of sight. Some make conserve of the flowers to the same effect. Being used any of the ways, it also helps a weak brain, or memory. This tunned up with strong beer, that it may work together, and drank, or the powder of the dried herb mixed with sugar, a little Mace, and Fennel seed, and drank, or eaten in broth; or the said powder made into an electuary with sugar, and taken, has the same porverful effect to help and restore the sight, decayed through age; and Arnoldus de Villa Nova saitl, it hath restored sight to them that have been blind a long time before.

\section{FERN. P. Ix.}

Descript.] Of this there are two kinds principally to be treated of, viz. the Male and Female. The Female grows higher than the Male, but the leaves thereof are smaller, and more divided and dented, and of as strong a smell as the male; the virtue of them are both alike, and therefore: I shall not trouble you with any description or distinction of them.

Place.] They grow both in heaths and in shady places near the hedge-sides in all counties of this land.

Time.] They flower and give their sced at Midsummer.

The Fentale Fern is that plant which is in Sussex, called Brakes, the seed of which prefer the preservation of their natural 
some authors hold to be so rare: Such a pith in the middle, which is called the heart thing there is I know, and may be easily thereof.

had upon Midsummer Ere, and for ought I know, two or three days after it, if not more.

Government and virtues. dominion of Mercury, both Male and Female. The roots of both these sorts of Fern being bruised and boiled in Mead, or honeyed water, and drank, kills both the broad and long worms in the body, and abates the swelling and hardness of the spleen. The green leaves eaten, purge the belly of choleric and waterish humours that trouble the stomach. They are dangerous for women with child to meddle with, by reason they cause abortions. The roots bruised and boiled in oil, or hog's grease, make a very profitable ointment to heal wounds, or pricks gotten in the flesh. The powder of them used in foul ulcers, dries up their malignant moisture, and causes their speedier healing. Fern being burned, the smoke thereof drives away serpents, gnats, and other noisome creatures, which in fenny countries do, in the night time, trouble and molest people lying in their beds with their faces uncovered; it causes barrenness.

: OSMOND ROYAL, OR WATER FERN.

$$
\text { Plate ix }
$$

Descript.] Thus shoots forth in spring time (for in the Winter the leaves perish) divers rough hard stalks, half round, and yellowish, or flat on the other side, two feet high, having divers blauches of winged yellowish green leaves on all sides, set one against another, longer, narrower, and not nicked on the edges as the tormer. From the top of some of these stalks grow forth a long bush of small and more ycllow, a long bush of small and more ycllow, mands this herb, and has commended it to on the staiks as the succour her sisters (women) and to be a on the slalks as the leaves are, which are general strengthener of their wombs, and accounted the Howers and seeds. The root remedy such infirmities as a careless midis rough, thick and scabby: with a white wife hath there caused; if they will but be

Place.] It grows on moors, bogs, and watery places, in many parts of this land.

Time.] It is green all the summer, and the root only abides in winter.

Government and virtues.] Saturn owns the plant. This has all the virtues mentioned in the former Ferns, and is much more effectual than they, both for inward and outward griefs, and is accounted singularly good in wounds, bruises, or the like. The decoction to be drank, or boiled into an ointment of oil, as a balsam or balm, and so it is singularly good against bruises, and bones broken, or out of joint, and gives much ease to the cholic and splenetic diseases : as also for ruptures or burstings. The decoction of the root in white wine, provokes urine exceedingly, and cleanses the bladder and passages of urine.

\section{FEVERFEW, OR FEATHERFEW. P.}

Descript.] Common Featherfew has large, fresh, green leaves, much torn or cut on the edges. The stalks are hard and round, set with many such like leaves, but smaller, and at the tops stand many single flowers, upon small foot stalks, consisting of many small white leaves standing round about a yellow thrum in the middle. The root is somewhat hard and short, with many strong fibres about it. The scent of the whole plant is very strong, and the taste is very bitter.

Piace.] This grows wild in many places of the land, but is for the most part nourished in gardens.

Time.] It flowers in the months of June and July.

Government and virtues.] Venus com- 
pleased to make use of her herb boiled in plied warm outwardly to the places, helps white wine, and drink the decoction; it the wind and cholic in the lower part of cleanses the womb, expels the after-birth, the belly. It is an especial remedy against and doth a woman all the good she can opium taken too liberally.

desire of an herb. And if any grumble because they cannot get the herb in winter, tell then, if they please, they may make a syrup of it in summer; it is chiefly used for the disease of the mother, whether it be the strangling or rising of the mother, or fashion is not yet left off, viz. to boil Fennel hardness, or intlammation of the same, swith fish; for it consumes that phlegmatic applied outwardly thereunto. Or a decoc- humour, which fish most plentifully afford tion of the flowers in wine, with a little Nut- and annoy the body with, though few that meg or Mace put therein, and drank often in use it know wherefore they do it; I suppose" a day, is an approved remedy to bring the reason of its benefit this way is, because down women's courses speedily, and helps it is an herb of Mercury, and under Virgo, to expel the dead birth and after-birth. and therefore bears antipathy to Pisces. For a woman to sit over the hot fumes of Fennel is good to break wind, to provoke the decoction of the herb made in water or urine, and ease the pains of the stone, and wine, is effectual for the same; and in some helps to break it. The leaves or seed, cases to apply the boiled herb warm to the boiled in barley water and drank are good privy parts. The decoction thereof made, for nurses, to increase their milk, and make with some sugar, or honey put thereto, is it more wholesome for the child. The used by many with good success to help leaves, or rather the seeds, boiled in water, the cough and stuffing of the chest, by stays the hiccough, and takes away the colds, as also to cleanse the reins and loathings which oftentimes happen to the bladder, and helps to expel the stone in stomachs of sick and feverish persons, and them. The powder of the herb taken in sallays the heat thereof. The seed boiled in wine, with some Oxymel, purges both cho- wine and drank, is good for those that are ler and phlegm, and is available for those bitten with serpents, or have eaten poisonthat are short winded, and are troubled with ous herbs, or mushrooms. Tlre seed, and melancholy and heaviness, or sadness of the roots much more, help to open obstrucspirits. It is very effectual for all pains in tions of the liver, spleen, and gall, and the head coming of a cold cause, the herb thereby help the painful and-windy swelbeing bruised and applied to the crown of lings of the spleen, and the yellow jaundice; the head: As also for the vertigo, that is a as also the gout and cramps. The seed is running or swimming in the head. The of good use in medicines to help shortness desoction thereof drank warm, and the of breath and wheezing by stopping of the herb bruised with a few corns of Bay salt, lungs. It helps also to bring down the and applied to the wrists before the coming courses, and to cleanse the parts after of the ague fits, doth take them away. The delivery. The roots are of most use in distilled water takes away freckles, and physic drinks, and broth that are taken to other spots and deformities in the face. cleanse the blood, to open obstructions of The herb bruised and heated on a tile, with the liver, so provoke urine, and amend the some wine to moisten it, or fried with a ill colour in the face after sickness, and to little wine and oil in a frying-pan, and ap- cause a good habit through the body. 
Both leaves, seeds, and roots thereof are little Euphorbium put to the nose, helps much used in drink or broth, to make peo- those that are troubled with the lethargy, ple more lean that are too fat. The dis- frenzy, giddiness of the head, the falling tilled water of the whole herb, or the con-sickness, long and inveterate head-aches, densate juice dissolved, but especially the the palsy, sciatica, and the cramp, and natural juice, that in some counties issues generally all the diseases of the sinews, used out hereof of its own accord, dropped into with oil and vinegar. The juice dissolved the eyes, cleanses them from mists and in wine, or put into an egg, is good for a films that hinder the sight. The sweet cough, or shortness of breath, and for those Fennel is much weaker in pliysical uses that are troubled with wind in the body. than the common Fcnnel. The wild Fen- It purges the belly gently, expels the hardnel is stronger and hotter than the tame, ness of the spleen, gives ease to women and therefore most powerful against the that have sore travail in child-birth, and stone, but not so effectual to encrease milk, eases the pains of the reins and bladder, because of its dryness.

\section{SOW-FENNEL, OR HOG'S-FENNEL.}

Besides the common name in English, Hog's Fennel, and the Latin name Peucidanum, is called Hoar-strange, and Hoarstrong, Sulphur-wort, and Brimstone-wort.

Descript.] The common Sow-Fennel has divers branched stalks of thick and somewhat long leaves, three for the most part joined together at a place, among which arises a crested straight stalk, less than Fennel, with some joints thereon, and leaves growing thereat, and towards the tops some branches issuing from thence; likewise on the tops of the stalks and branches stand divers tufts of yellow flower's, whereafter grows somewhat flat, thin, and yellowish seed, bigger than Fennel seed. The roots grow great and deep, with many other parts and fibres about them of a strong scent like hot brimstone, and yield forth a yellowish milk, or clammy juice, almost like a gum.

Place.] It grows plentifully in the salt low marshes near Feversham in Kent.

Time.] It flowers plentifully in July and Aligust.

Government and virtues.] This is also an herb of Mcrcury. The juice of Sow-Fennel (saith Dioscorides, and Galen,) used with vinegar and rose water, or the juice with a and also the womb. A little of the juice dissolved in wine, and dropped into the ears, eases much of the pains in them, and put into a hollow tooth, eases the pain thereof. The root is less effectual to all the aforesaid disorders; yet the powder of the root cleanses foul ulcers, being put into them; and takes out splinters of broken bones, or other things in the flesh, and heals thein up perfectly : as also, dries up old and inveterate running sores, and is of admirable virtue in all green wounds.

\section{FIG-WORT, OR THROAT-WORT. I. 10.}

Descript.] Common great Fig-wort sends divers great, strong, hard, square brown stalks, threc or four feet high, whereon grow large, hard, and dark green leaves, two at a joint, harder and larger than Nettle leaves, but not stinking; at the tops of the stalks stand many purple flowers set in husks, which are sometimes gaping and open, somewhat like those of Water Betony; after which come hard round heads, with a small point in the middle, wherein lie small brownish seed. 'The root is great, white, and thick, with many branches at it, growing aslope under the upper crust of the ground, which abides many years, but keeps not his green leaves in Winter.

Place.] It grows fiequently in moist 
and shadowy woods, and in the lower parts of the fields and meadows.

Time.] It Howers about July, and the seed will be ripe about a month after the flowers are fallen.

Government and virtues.] Some Latin authors call it Cervicaria, because it is appropriated to the neck; and we Throatwort, because it is appropriated to the throat. Venus owns the herb, and the Celestial Bull will not deny it; therefore; a better remedy cannot be for the king's evil, because the Moon that rules the disease is exalted there. 'The decoction of the herb taken inwardly, and the bruised herb applied outwardly, dissolves clotted and congealed blood within the body, coming by any wounds, bruise, or fall; and is no less effectual for the king's evil, or any other knobs, kernels, bunches, or wens growing in the flesh wheresoever ; and for the hæmorrhoids, or piles. An ointment made hereof may be used at all times when the fresh herb is not to be had. 'The distilled water of the whole plant, roots and all, is used for the same purposes, and dries up the superfluous, virulent moisture of hollow and corroding, ulcers; it takes away
all reslness, spots, and freckles in the face, as also the scurf, and any foul deformity therein, and the leprosy likewise.

FITIPENDULA, OR DROP-WORT.P.X

Descript.] Turs sends forth many leaves, some larger, some smaller, set on each side of a middle rib, and each of them dented about the edges, somewhat resembling wild Tansy, or rather Agrimony, but harder in handling; among which rise up one or more stalks, two or three feet high, with the leaves growing thereon, and sometimes also divided into other branches spreading at the top into many white, sweet-smelling flowers, consisting of five leaves a-piece, with some threads in the middle of them, standing $(7,8$. together in a pith or umbie, each upon a small foot stalk, which after they have been blown upon a good while, do fall away, and in their places appear sinall, round, chaffy heads like buttons, wherein are the chaffy seeds set and placed. The root consists of many small, black, tuberous pieces, fastened together by many sinall, long, blackish strings, which run from one to another.

Place.] It grows in many places of this land, in the corners of dry fields and meadows, and the hedge sides.

Time.] They flower in June and July, and their seed is ripe in August.

Government and virtues.] It is under the dominion of Venus. It effectually opens the passages of the urine, helps the stranguary ; the stone in the kidneys or bladder, the gravel, and all other pains of the bladder and reins, by taking the roots in powder, or a decoction of them in white wine, with a little honey. The roots made into powder, and mixed with honey in the form of an electuary, doth much help them whose stomachs are swollen, dissolving and breaking the wind which was the cause thereof; and is also very effectual for all the diseases of the lungs, as shortness of breath, wheezing, hoarseness of the throat, and the cough ; and to expectorate tough phlegm, or any other parts thereabout.

THE VIG-TREE。 $\boldsymbol{P}$ :

To give a description of a tree so well known to every body that keeps it in his garden, were needless. 'They prosper very well in our English gardens, yet are fitter for medicine than for any other profit which is gotten by the fruit of them.

Govermment and virtues.] The tree is under the dominion of Jupiter. 'The milk that issues out from the leaves or branches where they are broken off, being dropped upon warts, takes them away. The de- 
coction of the leveas is excellently good to cover their bottoms; but instead of the wash sore heads with: and there is scarcely three upright leaves, as the Flower-de-luce a better remedy for the leprosy than it is. has, this has only three short pieces It clear's the face also of morphew, and the standing in their places, after which succeed body of white rcurf, scabs, and running thick and long three square heads, consores. If it be dropped into old fretting taining in each part somewhat big and flat ulcers, it cleanses out the moisture, and seed, like those of the Flower-de-luce. The brings up the flesh; because you cannot root is long and slender, of a pale brownish have the leaves green all the year, you may colour on the outside, and of a horseflesh make an ointment of them whilst you can. colour on the inside, with many hard fibres A decoction of the leaves being drank in- thereat, and very harsh in taste.

wardly, or rather a syrup made of them, dissolves congealed blood caused by bruises or falls, and helps the bloody flux. The ashes of the wood made into an ointment with hog's grease, helps kibes and chilblains. The juice being put into an hollow tooth, eases pain; as also pain and noise in the ears, being dropped into them ; and deafness. An ointment made of the juice and hog's grease, is an excellent remedy for the
biting of mad dogs, or other venomous beasts, as most are. A syrup made of the leaves, or green fruit, is excellently good for coughs, hoarseness, or shortness of breath, and all diseases of the breast and lungs; it is also extremely good for the dropsy and falling sickness. They say that the Fig Tree, as well as the Bay 'Tree, is never hurt by lightning; as also, if you tie a bull, be he ever so mad, to a Fig 'Tree, he will quickly become tame and gentle. As for such figs as come from beyond sea, I
have little to say, because I write not of exoticks.

THE YELLOW WATER-FLAG, OR FLOWERDE-IUCE.P. IX

Descript.] Turs grows like the Flowerde-luce, but it has much longer and narrower sad green leaves, joined together in that fashion; the stalk also growing oftentimes as high, bearing small yellow flowers has divers stalks full fiaught with long and
shaped like the Flower-de-luce, with three narro:w ash-coloured leaves, and from the Place.] It usually grows in watery ditches, ponds, lakes, and moor sides, which are always overflowed with water.

Time.] It flowers in July, and the seed is ripe in August.

Government and virtues.] It is under the dominion of the Moon. The root of this Water-flag is very astringent, cooling, and drying; and thereby helps all lasks and fluxes, whether of blood or humours, as bleeding at the mouth, nose, or other parts, bloody flux, and the immoderate flux of women's courses. The distilled water of the whole herb, flowers and roots, is a sovereign good remedy for watering eyes, both to be dropped into them, and to have cloths or sponges wetted therein, and applied to the forehead: It also helps the spots and blemishes that happen in and about the eyes, or in any other parts: The said water fomented on swellings and hot inflammations of women's breasts, upon cancers also, and those spreading ulcers called Noli me tangere, do much good: It helps also foul ulcers in the privities of man. or woman; but an ointment made of the flowers is better for those external applications.

FLAX-WEID, OR TOAD-FLAX. I. 10.

Descript.] Ou R common Flax-weed falling leaves, and other three arched that middle of them almost upward, stored with 
a number of pale yellow flowers, of a strong or spots, applied of itself, or used with some unpleasant scent, with deeper yellow mouths, powder of Lupines.

and blackish flat seed in round heads, The root is somewhat woody and white, especially the main downright one, with many fibres, abiding many years, shooting forth roots every way round about, and new branches every year.

FLEA-WORT. P, 10

Descript.] Ordinary Flea-wort rises up with a stalk two feet high or more, full of joints and branches on every side up to the top, and at every joint two small, long,

Place.] This grows throughout this land, and narrow whitish green, leaves somewhat both by the way sides and in meadows, as hairy; At the top of every branch stand also by hedge-sides, and upon the sides of divers small, short scaly, or chaffy heads, banks, and borders of fields.

Time.] It flowers in Summer, and the yellow: threads, like to those of the Planseed is ripe usually before the end of tain herbs, which are the bloomings of August.

Government and virtues.] Mars owns the herb: In Sussex we call it Gallwort, and lay it in our chicken's water to cure them of the gall; it relieves them when they are drooping. This is frequently used to spend the abundance of those watery humours by urine, which cause the dropsy: The decoction of the herb, both leaves and flowers, in wine, taken and drank, doth somewhat rosin.

move the belly downwards, opens obstructions of the liver, and helps the yellow jaundice; expels poison, provokes women's courses, drives forth the dead child, and after-birtl. The distilled water of the herb and flowers is effectual for all the same pur- what greater, the heads somewhat less, the poses; being drank with a dram of the seed alike; and the root and leaves abide powder of the seeds of bark or the roots of all winter, and perish not as the former.

Wall-wort, and a little Cinnamon, far certain days together, it is held a singular remedy for the dropsy. The juice of the herb, or the distilled water, dropped into the eyes, is a certain remedy for all heat, inflammation, and redness in them. 'The juice or

Place.] The first grows only.in gardens, the seccond plentifully in fields that are near the sea.

T'ime.] They flower in July, or thereabouls"

Government and virtues.]. 'The herb is water put into foul ulcers, whether they be cold, and dry, and saturnine. I suppose cancerous or fistulons, with tents rolled it obtained the name of Flea-wort, because therein, or parts washed and injected there- the seeds are so like Fleas, The seeds fried, with, cleanses them thoroughly from the and taken, stays the flux or lask of the bottom, and heals them up safely. The belly, and the corrosions that come by reasame juice or water also cleanses the skin son of hot choleric, or sharp and malignant wonderfully of all sorts of deformity, as humours, or by too much purging of any leprosy, morphew, scurf, wheals, pimples, violent inedicine, as Scammony, or the 
like. The mucilage of the seed made with Rose-water, and a little sugar-candy put thereto, is very good in all hot agues and they have a strong evil savour, being burning fevers, and other inflammations, to smelled unto, and are of a drying taste.

cool the thirst, and lenify the dryness and roughness of the tongue and throat. It helps also hoarseness of the voice, and diseases of the breast and lungs, caused by heat, or sharp salt humours, and the pleurisy also. The mucilage of the seed made with Plantain water, whereunto the yolk of an egg or two, and a little Populeon are put, is a most safe and sure remedy to ease the sharpness, pricking, and pains of the hæemorrhoids or piles, if it be laid on a cloth, and bound thereto. It helps all inflammations in any part of the body, and the pains that come thereby, as the headache and megrims, and all hot imposthumes, swellings, or breaking out of the skin, as blains, wheals, pushes, purples, and the like ; as also the joints of those that are out of joint, the pains of the gout and sciatica, the burstings of young children, and the swellings of the navel, applied with oil of roses and vinegar. It is also good to heal the nipples and sore breasts of women, being often applied thereunto. The juice of the herb with a little honey put into the ears helps the running of them, and the worms breeding in them: The same also mixed with hog's grease, and applied to corrupt and filthy ulcers, cleanses them and heals them.

\section{F C UX-WEED.}

Descript.] Ir rises up with a round upright hard stalk, four or five feet high, spread into sundry branches, whereon grow many greyish green leaves, very finely cut and severed into a number of short and almost round parts. The flowers are very small and yellow, growing spike fashion, after which come small long pods, will small yellowish seed in them. The root is lotig and woody, perishing every.year.
There is another sort, differing in nothing, Place.] They flower wild in the fields by hedge-sides and highways, and among rubbish and other places.

Time.] They flower and seed quickly after, namely in June and July.

Government and virtues.] This herb is saturnine also. Both the herb and seed of Flux-weed is of excellent use to stay the flux or lask of the belly, being drank in water wherein gads of steel heated have been often quenched; and is no less effectual for the same purpose than Plantain or Comfrey, and to restrain any other flux of blood in man or woman, as also to consolidate bones broken or out of joint. The juice thereof drank in wine, or the decoction of the herb drank, doth kill the worms in the stomach or belly, or the worms that grow in putrid and filthy ulcers; and made into a salve doth quickly heal all old sores, how foul or malignant soever they be: The distilled water of the herb works the same effects, although somewhat weaker, yet it is a fair medicine, and more acceptable to be taken. It is called Flux-weed because it cures the flux, and for its uniting broken bones, \&c. Paracelsus extols it to the skies. It is fitting that syrup, ointment, and plaisters of it were kept in your houses.

FLOWER-DE-LUCE. P. 10.

It is so well known, being nourished up in most gardens, that $I$ shall not need to spend time in writing a description thereof. Time.] The flaggy kinds thereof have the most physical uses; the dwarf kinds thereof flower in April, the greater sorts in May.

Government and virtues.] The herb is Lunar. The juice or decoction of the green root of the flaggy kind of Flower-de-luce; with a little honey drank; doth purge and 
cleanse the stomach of gross and tough also the pains and noise in the ears, and phlegm, and choler therein; it helps the the stench of the nostrils. 'The root itself, jaundice and the dropsy, evacuating those either green or in powder, helps to cleanse, humours both upwards and downwards; heal, and incarnate wounds, and to cover and because it somewhat hurts the stomach, the naked bones with flesh again, that is not to be taken without honey and spike- ulcers have made bare; and is also very nard. The same being drank, doth ease good to cleanse and, heal up fistulas and the pains and torments of the belly and cankers that are hard to be cured. sides, the shaking of agues, the diseases of ? the liver and spleen, the worms of the belly, the stone in the reins, convulsions and cramps that come of old humours; it also helps those whose seed passes from them unawares: It is a remedy against the bitings and stingings of venomous crea-s sometimes more long than round, without tures, being boiled in water and vinegar order thereon, somewhat hairy, and of an and drank. Boiled in water and drank, it evil greenish white colour; at the joints all provokes urine, helps the cholic, brings along the stalks, and with the leaves come down women's courses; and made up into forth small flowers, one at a place, upon a pessary with honey, and put up into the a very small short foot-stalk, gaping somebody, draws forth the dead child. It is what like Snap-dragons, or rather like Toadmuch commended against the cough, to flax, with the upper jaw of a yellow colour, expectorate rough phlegm ; it much eases and the lower of a purplish, with a small heel pains in the head, and procures sleep; or spur behind; after which come forth being put into the nostrils it procures small round heads, containing small black sueezing, and thereby purges the head of sced. The root is small and thready, dying phlegm. The juice of the root applied to every year, and rises itself again of its the piles or hæmorrhoids, gives much ease. own sowing.

The decortion of the roots gargled in the There is another sort of Lluellin which mouth, eases the tooth-ache, and helps the has longer branches wholly trailing upon stinking breath. Oil called Oleum Irinum, the ground, two or three feet long, and if it be rightly made of the great broad somewhat more thin, set with leaves thereflag Flower-de-luce and not of the great on, upon small foot stalks. 'The leaves are bulbous blue Flower-de-luce, (as is used by a little larger, and somewhat round, and some apothecaries) and roots of the same, of cornered sometimes in some places on the the flaggy kinds, is very effectual to warm edges; but the lower part of them being and comfort all cold joints and sinews, as the broadest, hath on each side a small also the gout and sciatica, and mollifies, point, making it seem as if they were ears, dissolves and consumes tumours and swell- sometimes hairy, but not hoary, and of a ings in any part of the body, as also of the better green colour than the former. The matrix ; it helps the cramp, or convulsions flowers come forth like the former, but the of the sinews. The head and temples colours therein are more white than yellow, anointed therewith, helps the catarrh or and the purple not so far. It is a large thin rheum distilled from thence; and used flower, and so are the seed and seed-vesupon the breast or stomach, helps to ex-ssels. The root is like the other, and tenuate the cold tough phlegm; it helps perishes every year.

$(7,8$. 
Place.] They grow in divers corn fields, and hanging downwards, having some and in borders about them, and in other threads also in the middle, from whence fertile grounds about Southfleet in Kent rise round heads, pointed sharp at the ends, abundantly; at Buchrite, Hamerton, and wherein small brown seed lies. The roots Richmanworth in Huntingdonshire, and in are so many small fibres, and some greater divers other places.

Time.] They are in flower about June and July, and the whole plant is dry and withered before August be done.

Government and virtues.] It is a Lunar for the most part, and as well on the higher herb. The leaves bruised and applied with as the lower places under hedge-sides in barley meal to watering eyes that are hot almost every county of this land.

and inflamed by defluxions from the head, do very much help them, as also the fluxes of blood or humours, as the lask, bloody flux, women's courses, and stays all manner of bleeding at the nose, mouth, or any other place, or that comes by any bruise or hurt, or bursting a vein; it wonderfully lielps all those inward parts that need consolidating or strengthening, and is no less effectual both to heal and close green wounds, than to cleanse and heal all foul or old ulcers, fretting or spreading cankers or the like. This herb is of a fine cooling, drying quality, and an ointment or plaister of it miglit do a man a courtesy that hath any hot virulent sores: "Tis admirable for the ulcers of the French pox; if taken inwardly, may cure the disease.

FOX-GLOVE. P.10.

Time.] It seldom flowers before July, and the seed is ripe in August.

Government and virtues.] The plant is under the dominion of Venus, being of a gentle cleansing nature, and withal very friendly to nature. The herb is familiarly and frequently used by the Italians to heal any fresh or green wound, the leaves being but bruised and bound thereon; and the juice thereof is also used in old sores, to cleanse, dry, and heal them. The decoction hereof made up with some sugar or honey, is available to cleanse and purge the body both upwards and downwards, sometimes of tough phlegm and clammy. humours, and to open obstructions of the liver and spleen. It has been found by cxperience to be available for the king's evil, the herb bruised and applied, or an ointment made with the juice thereof, and so used; and a decoction of two handfuls

Descript.] IT has many long and broad leaves lying upon the ground dented upon the edges, a little soft or woolly, and of a hoary green colour, among which rise up sometimes sundry stalks, but one very oftcn, bearing such leaves thereon from the bottom to the middle, from whence to the top it is stored with large and long hollow reddish purple flowers, a little more long and eminent at the lower edge, with some white spots within them, one above another thereof, with four ounces of Polipody in ale, has been found by late experience to cure divers of the falling sickness, that have been troubled with it above twenty years. I am confident that an ointment of it is one of the best remedies for a scabby lead that is. with small green leaves at cvery one, but square, a slender wcak stalk, and leaning all of them turning their heads one way, downwards on all sides, many branches 
two or three feet long, with finely cut and tilled water of the herb is also of good jagged leaves of a whitish or rather blueish effect in the former diseases, and conduces sea green colour; At the tops of the much against the plague and pestilence, branches stand many small flowers, as it being taken with good treacls. The diswere in a long spike one above another, tilled water also, with a little water and made like little birds, of a reddish purple honey of roses, helps all sores of the mouth colour, with whitish bellies, after which or throat, being gargled often therewith. come sinall round husks, containing small 'The juice dropped into the eyes, clears the black seeds. The root is yellow, sinall, and sight and takes away redness and other not very long, full of juice while it is green, defects in them, although it procure some but quickly perishes with the ripe seed. In pain for the present, and cause tears. the corn fields in Cornwall, it bears white Dioscorides saith it hinders any fiesh flowers.

springing of hairs on the eye-lids (after they

Place.] It grows in corn fields almost are pulled away) if the eye-lids be anointed every where, as well as in gardens.

with the juice hereof, 'with Gum Arabic

Time.] It flowers in May, for the most dissolved therein. The juice of the Fumipart, and the seed ripens shortly after.

Government and virtues.] Saturn owns the herb, and presents it to the world as a all sorts of scabs, pimples, blotches, wheals, cure for his own disease, and a strengthener and pushes which arise on the face or of the parts of the body he rules. If by hands, or any other parts of the body.

my astrological judgment of diseases, from the decumbiture, you find Saturn author of the disease, or if by direction from a nativity you fear a saturnine disease approaching, you may by this herb prevent in some counties by the name of Gorz or it in the one, and cure it in the other, and Whins, that I shall not need to write any therefore it is fit you kecp a syrup of it description thereof, nuy intent being to always by you. 'The juice or syrup made teach my countrymen what they know not, thereof, or the decoction made in whey by rather than to tell them again of that which itself, with some other purging or opening is generally known before.

herbs and roots to cause it to work the Place.] They are known to grow on dry better (itself being but weak) is very effec- barren heaths, and other waste, gravelly tual for the liver and spleen, opening the or sandy grounds, in all counties of this obstructions thereof, and clarifying the land.

blood from saltish, choleric, and adust 'Time.] They also flower in the Summer humours, which cause leprosy, scabs, tet- months.

ters, and itches, and such like breakings- Government and virtues.]' Mars owns the out of the skin, and after the purgings doth herb. They are hot and dry, and open strengthen all the inward parts. It is also obstructions of the liver and spleen. A degood against the yellow-jaundice, and coction made with the flowers thereof hath spends it by urine, which it procures in been found effectual against the jaundice, abundance. The powder of the dried herb as olso to provoke urine, and cleanse the given for some time together, cures melan- lidneys from gravel or stone ingendered choly, but the seed is strongest in opera- in them. Mars doth also this by symtion for all the former diseases. The dis-palhy. 


\section{GA R LIC K， T. . 12,}

Trie offensiveness of the breath of him that hath eaten Garlick, will lead you by the nose to the knowledge hereof, and (instead of a description) direct you to the place where it grows in gardens, which kinds are the best, and most physical.

Goverment and virtues.] Mars owns this herb. This was anciently accounted the poor man's treacle, it being a remedy for comes from beyond sea, therefore be pleased all diseases and hurts (except those which to take the description of them as follows. itself breed.) It provokes urine and women's courses, helps the biting of mad dogs, and other venomous creatures; kills worms in children, cuts and voids tough phlegm, purges the head, helps the lethargy, is a good preservative against, and a remedy for any plague, sore, or foul ulcers; takes away spots and blemishes in the skin, eases pains in the ears, ripens and breaks imposthumes, or other swellings. And for all those diseases the onions are as effectual. But the Garlick hath some more peculiar virtues besides the former, viz. it hath a special quality to discuss inconveniences coming by corrupt agues or mineral vapours, or by drinking corrupt and stinking waters; as also by taking wolf-bane, hen-bane, hemlock, or other poisonous and dangerous herbs. It is also held good in hydropick diseases, the jaundice, falling sickness, cramps, convulsions, the piles or hæmorrhoids, or other cold diseases. Many authors quote many diseases this is good for; but conceal its vices. Its heat is very vehement, and all vehement hot things send up but ill-favoured vapours to the brain. In choleric men it will add fuel to the fire; in men oppressed by nielancholy, it will attenuate the humour, and send up strong fancies, and as many strange visions to the head; therefore let it be taken inwardly with great moderation; outwardly you may niake more bold with it:
GENTIAN, FELWORT, OR BALDMONY.

IT is confessed that Gentian, which is most used amongst us, is brought over from beyond sea, yet we have two sorts of it growing frequently in our nation, which besides the reasons so frequently alledged why English herbs should be fittest for English bodies, has been proved by the experience of divers physicians, to be not a whit inferior in virtue to that which Descript.] 'The greater of the two hath many small long roots thrust down deep into the ground, and abiding all the Winter. The stalks are sometimes more, sometimes fewer, of a brownish green colour, which is sometimes two feet high, if the ground be fruitful, having many long, narrow, dark green leaves, set by couples up to the top; the flowers are long and hollow, of a purple colour, ending in fine corners. The smaller sort which is to be found in our land, grows up with sundry stalks, not a foot high, parted into several small branches, whereon grow divers small leaves together, very like those of the lesser Centaury, of a whitish green colour; on the tops of these stalks grow divers perfect blue flowers; standing in long husks, but not so big as the other; the root is very small, and full of threads.

Place.] The first grows in divers places of both the East and West counties, and as well in wet as in dry grounds; as near Long-field by Gravesin l, near Cobham in Kent; near Lillinstone in Kent; also in a chalk pit hard by a paper-mill not far from Dartford in Kent. 'The second grows also in divers places in Kent, as about Southfleet and Longfield ; upon Barton's hills in Bedfordshire; also not far from St. Albans; upon a piece of waste chalky ground, as you go out by Dunstable way towards Gorhambury. 
Time.] They flower in August.

Government and virtues.] They are under the dominion of Mars, and one of the principal herbs he is ruler of. They resist putrefactions, poison, and a more sure? remedy cannot be found to prevent the pestilence than it is; it strengthens the stonrach exceedingly, helps digestion, comforts the heart, and preserves it against faintings and swoonings: The powder of the dry roots helps the biting of mad dogs and venomous beasts, open obstructions of the liver, and restores an appetite for thcir meat to such as have lost it. 'The horb steeped in wine, and the winc drank, refreshes such as be over-weary with traveling, and grow lame in their joints, either by cold or evil lodgings; it helps stitches, and griping pains in the sides; is an excellent remedy for such as are bruised by falls; it provokes urine and the terms exceedingly, therefore let it not be given to women with child: The same is very profitable for such as are troubled with cramps and convulsions, to drink the decoction: Also they say it breaks the stone, and holps ruptures most certainly: it is excellent in all cold cliseases, and such as are troubled with tough phlegm, scabs, itch, or any frètting sores and ulcers; it is an admirable remedy to kill the worms, by taking half a dram of the powder in a morning in any convenient liquor; the same is excellently good to be taken inwardly for the king's evil. It helps agues of all sorts, and the yellow jaundice, as also the bots in cattle; when kine are bitten on the udder by any venomous beast, do- but stroke the place with the decoction of any? of these, and it will instantly heal them.

\section{CLOVE GILLIFLOWERS. P. $"$.}

It is vain to describe an herb so well known.

Gocernment and virtues.] They are gallant, ine, temperatc flowers, of the nature and inder the dominion of Jupiter; yea, so $(9,10$. tempcrate, that no excess, neither in heat, cold, dryness, nor moisturc, can be perceived in then ; they are great strengtheners both of the brain and heart, and will therefore serve either for cordials or cephalics, as your occasion will serve. There is both a syrup and a conserve made of them alone, commonly to be had at every apothecary's. To take now and then a little of either, strengthens nature much, in such as are in consumptions. They are also excellently good in hot pestilent fevers, and expel poison.

\section{GERMANDER. I . /}

Descript.] Common Germander shoots fortl sundry stalks, with small and somewhat round leaves, dented about the edges. The flowers stand at the tops, of a deep purple colour. The root is composed of divers sprigs, which shoots forth a great way round about, quickly overspreading a garden.

Place.] It grows usually with us in gardens,

Time.] And flowers in June and July.

Govermment and virtues.] It is a most prevalent herb of Mercury, and stréngthens the brain and apprehension exceedingly when wcak, and relieves them when drooping. This taken with honey (saith Dioscorides) is a remedy for coughs, hardness of the spleen and difficulty of urine, and helps those that are fallen into a dropsy, especially at the beginning of the discase, a decoction being made thereof when it is green, and drank. It also brings down women's courses, and expels the dead child. It is most effectual against the poison of all serpents, being drank in wine, and the bruised herb outwardly applied; used with honey, it cleanses old and foul ulcers; and made into an oil, and the eycs anointed therewith, takes away the dimness and moistness. I I is likewise good for the pains in the sides and cramps. The a 
decoction thereof taken for four days places of this land, and is usually nursed together, drives away and cures both ter- up in gardens.

tain and quartan agues. It is also good. Time.] It flowers not until July, and against all diseases of the brain, as con- the seed is ripe in August or September, tinual head-ache, falling-sickness, nuelan- yet the husks after they are ripe, opening choly, drowsiness and dullness of the spirits, themselves, will hold their seed with them convulsions and palsies. A dram of the for two or thrce months, and not shed them. seed taken in powder purges by urine, and is good against the yellow jaundice. 'The juice of the leaves dropped into the ears? used by many country people to purge kills the worms in them. The tops thereof, corrupt phlegm and choler, which they do when they are in flowers, stceped twenty- by drinking the decostion of the roots; four hour's in a draught of white wine, and and some to make it more gentle, do but drank, kills the worms in the belly.

\section{STINKING GLADWIN.}

\section{P. 11 .}

Descript.] 'THIs is one of the kinds of Flower-de-luce, having divers leaves arising from the roots, very like a Flower-de-luce, but that they are sharp-edged on both sides, and thicker in the middle, of a deeper green colour, narrower and sharper pointed, and a strong ill-scent, if they be bruised be-? tween the fingers. In the middle rises up a reasonably strong stalk, a yard high at least, bearing three or four flowers at the top, made somewhat like the flowers of the Flower-de-luce, with three upright leaves, of a dead purplish ash-colour, with some veins discoloured in then! the other three
do not fall down, nor are the three other small ones so arched, nor cover the lower leaves as the Flower-de-luce doth, but stand loose or asunder from them. After they are past, there come up three square hard husks, opening wide into three parts when they are ripe, wherein lie reddish turns black when it hath abiden long. 'The of the spleen. root is like that of the Flower-de-luce, but in all wounds, especially of the head; as reddish on the outside, and whitish within, also to draw forth any splinters, thorns, or very sharp and hot in the taste, of as evil broken bones, or any other thing sticking

a scent as the leaves.
Place.] This grows as well in upland used with a little verdigrease and honey, grounds, as in moist places, woods, and shadowy places by the sea-side in many boiled in vincgar, and laid upon any tumour weaker stomach: 'The juice hereof put up, or snuffed up the nose, causes sneezing, and draws from the head much corruption; and the powder thereof doth the same. The powder thereof drank in wine, helps those that are troubled with the cramps and convulsions, or with the gout and sciatica, and gives ease to those that lave griping pains in their body and belly, and lielps those that have the stranguary. It is given with much profit to those that have had long fluxes by the sharp and evil quality of humours, which it stays, having first cleansed and purged them by the drying and binding property therein. The root boiled in wine and drank, doth effectually procure women's courses, and used as a pessary, works the same effect, but causes abortion in women with child. Half a dram of the seed beaten to powder, and taken in wine, doth speedily cause one to make water abundantly. The same taken with vinegar, dissolves the hardness and swellings The root is very effectual

infuse the sliced roots in ale; and some take the leaves, which serve well for the 
or swelling, doth very effectually dissolve inferior to none, both for the inward and and consume them; yea, even the swell- outward hurts; green wounds, old sores ings of the throat called the king's evil; and ulcers, are quickly cured therewith. the juice of the leaves or roots heals the It also is of especial use in all lotions for itch, and all running or spreading scabs, sores or ulcers in the mouth, throat, or privy sores, blemishes, or scars in the skin, where- parts of man or woman. The decoction soever they be.

\section{GOLDEN ROD. 0.32}

Descript.] 'Turs rises up with brownish small round stalks, two feet high, and sometimes more, having thereon many narrow and long dark green leaves, very seldom with any dents about the edges, or any stalks or white spots therein, yet they are sometimes so found divided at the tops into many small branches, with divers small yellow flowers on every one of them; all which are turned one way, and being ripe, do turn into down, and are carried away by the wind. The root consists of many small fibres, which grows not deep in the ground, but abides all the winter therein, shooting forth new branches every year, the old one lying down to the ground.

Place.]. It grows in the open places of woods and copses, on both moist and dry grounds, in many places of this land.

Time.] It - flowers about the month of July.

Government and virtucs.] Venus claims the herb, and therefore to be sure it respects beauty lost. Arnoldus de Villa Nova commends it much against the stone in the reins and kidncys, and to provoke urine in abundance, whereby also the gravel and stone may be voided. The decoction of the herb, green or dry, or the distilled water thereof, is very effectual for inward bruises, as also to be outwardly applied, it stays bleeding in any part of the body, and of wounds; also the fluxes of humours, the bloody-flux, and women's courses; and is no less prevalent in all ruptures or burst- on. At the joints, with the leaves, come ings, being drank in wardly, and outwardly fortli very small blue flowers, and after them applied. It is a sovereign wound harb, hard stony roundish secd. The root is long

\section{GOUT-WORT, OR HERB GERRARD.}

Descript.] IT is a low herb, seldom rising half a yard high, having sundry lcaves standing on brownish green stalks by three, snipped about, and of a strong unpleasant savour: The umbels of the flowers are white, and the seed blackish, the root runs in the ground, quickly taking a great deal of roon.

Place.] It grows by hedge and wallsides, and often in the border and corner of fields, and in gardens also.

Time.] It flowers and seeds about the end of July.

Gorernment and virtucs.] Saturn rules it. Neither is it to be supposed Gout-wort hath its name for nothing but upon experiment to heal the gout and sciatica; as also jointaclies, and other cold griefs. The very bearing of it about one eases the pains of the goout, and defends him that bears it fron the discase.

\section{GROMEL. I . $19 \ldots 1.2$.}

OF this I shall brietly describe their kinds, which are principally used in physic, the virtucs whereof are alike, though somewhat different in their nlanner and form of growing.

Descript.] The greater Gromel grows up with slender hard and hairy stalks, trailing and taking root in the ground, as it lies thereon, and parted into many other small branches with hairy dark grcen leaves therein the gunıs. 
and woody, abiding the Winter, and shoots forth fresh stalks in the spring.

The smaller wild Gromel divers upright hard branched stalks, two or three feet high, jull of joints, at every one of which grow snall, long, hard, and rough leaves like the former, but less; among? which leaves conie forth small white flowers, and after them greyish round seed like the former; the root is not very big, but with many strings thereat.

The garden Gromel has divers upright, slender, woody, hairy stalks, blown and cressed, very little branched, with leaves like the former, and white flowers; after which, in rough brown husks, is contained a white, hard, round seed, shining like pearls, and greater than either the former ; the root is like the first described, with divers branches and sprigs thereat, which continues (as the first doth) all the Winter.

Place.] 'The two first grow wild in barren or untilled places, and by the way side in many places of this land. The last is a nursling in the gardens of the curious.

Time.] They all flower from Midsummer until September sometimes, and in the mean time the seed ripens.

Govermment and virtues.] The herb belongs to Dame Veniss; and therefore if Mars cause the cholic or stone, as usually he? doth, if in Virgo, this is your cure. These are accounted to be of as singular force as any herb or seed whatsoever, to break the stone and to void it, and the gravel either in the reins or bladder, as also to provolie urine being stopped, and to help stranguary. The seed is of greatest use, being bruised Pear-tree; from whence arises a slender and boiled in white wine or in broth, or the weak stalk, yet standing upright, bearing like, or the powder of the seed taken there- at the top many small white sweet-smelling in. Two drams of the seed in powder flowers, laid open like a star, consisting of taken with women's breast milk, is very five round pointed leaves, with many yellow effectual to procure a very speedy delivery threads standing in the middle about a green to such women as have sore pains in their head, and a long stalk with them, which in travail, and cannot be delivered: 'The herb time grows to be the seed-vessel, which itself, (when the seed is not to be had) either being ripe is found five square, with a small

boiled, or the juice thereof drank; is "effectual to all the purposes aforesaid, but not so powerful or speedy in operation.

\section{GOOSERERRY BUSH. I. 12.}

CALLED also Feapberry, and in Sussex Dewberry-Bush, and in some Counties Wineberry.•

Government and virtues.] They are under the donninion of Venus. The berries, while they are unripe, being scalded or baked, are good to stir up a fainting or decayed appetite, especially such whose stomachs are afflicted by choleric humours: They are excellently good to stay longings of woinen with child. You may keep them preserved with sugar all the year long. The decoction of the leaves of the tree cools hot swellings and inflammations; as also St. Anthony's fire. The ripe Gooseberries being eaten, are an excellent remedy to allay the violent heat both of the stomach and liver. The young and tender leaves break the stone, and expel gravel both from the kidneys and bladder. All the evil they do to the body of man is, they are supposed to breed crudities, and by crudities, worms.

Descript.] Tris sends forth seven, eight or nime leaves from a small brown creeping root, every one standing upon a long foot stalk, which are almost as broad as long, round pointed, of a sad green colour, and hard in handling; and like the leaf of a Yorms.

\section{W INTER-GR EEN.}


point at it, wherein is contained seed as small as dust.

Place.] It grows seldom in fields, but frequent in the woods northwards, viz. in Yorkshire, Lancashire, and Scotland.

Time.] It flowers about June and July.

Government and virtues.7 Winter-green is under the dominion of Saturn, and is a singularly good wound herb, and an especial remedy for healing green wounds speedily, the green leaves being bruised and applied, or the juice of them. A salve made of the green herb stamped, or the juice boiled with hog's lard, or with salad oil and wax, and some turpentine added to it, is a sovereign salve, and highly extolled by the Germans, who use it to heal all manner of wounds and sores. 'The herb boiled in wine and water, and given to drink to them that have any inward ulcers in their kidneys, or neck of the bladder, doth wonderfully help them. It stays all fluxes, as the lask, bloody fluxes, women's courses, and bleeding of wounds, and takes away any inflammations rising upon pains of the heart; it is no less helpful for foul ulcers hard to be cured; as also for cankers or fistulas. The distilled water of the herb effectually performs the same things.

\section{GROUNDSEL. I. 12 .}

Descript.] Ou common Groundsel has a round green-and somewhat brownish stalk, spreading toward the top into branches, set with long and somewhat narrow green leaves, cut in on the edges, somewhat like the oak-leaves, but less, and round at the end. At the tops of the branches stand many small green heads, out of which grow several small, yellow threads or thumbs, which are the flowers, and continue many days blown in that manner, before it pass away into down, and with', the sced is away into down, and with, the sced is a in the wind. The root is small and thready, and soon perishes, and as soon rises again of its own sowing, so that $(9,10$. it may be seen many months in the year, both green and in flower, and seed ; for it will spring and seed twice in a year at least, if $i t$ be suffered in a garden.

Place.] They grow almost every where, as well on tops of walls, as at the foot, amongst rubbish and untilled grounds, but especially in gardens.

Time.] It flowers, as was said before, almost every month throughout the year.

Government and virtues.] This herb is Venus's mistress-piece, and is as gallant and universal a medicine for all diseases coming of heat, in what part of the body soever they be, as the sun shines upon; it is very safe and friendly to the body of man: yet causes vomiting if the stomach be afHicted; if not, purging: and it doth it with more gentleness than can be expected; it is moist, and something cold withal, thereby causing expulsion, and repressing the heat caused by the motion of the internal parts in purges and romits. Lay by our learned receipts; take so much Sena, so much Scammony, so much Colocynthis, so much infusion of Crocus Metallorum, \&c. this herb alone preserved in a syrup, in a dislilled water, or in an ointment, shall do the deed for you in all hot diseases, and, shall do it, 1, Safely ; 2, Speedily.

The decoction of this herb (saith Dioscorides) made with wine, and drank, lielps the pains of the stomach; proceeding of clioler, (which it may well do by a vomit) as daily experience shews. The juice thereof taken in drink, or the decoction of it in ale, gently performs the same. It is good against the iaundice and falling sickness, being taken in wine; as also against difficulty of making water. It provokes urine, expels gravel in the reins or kidneys; a dram thereof given in oxymcl, after some walking or stirring of the body. It helps also the sciatica, griping of the belly, the cholic, defects of the liver, and provokes women's courses. 'The fresh herb boiled, $: \mathbf{A} \mathbf{A}$ 
and made into a poultice, applied to the and a gallant remedy for the inflammation breasts of women that are swollen with pain of the lungs and breasts, pleurisy, scabs, and heat, as also the privy parts of man or itch, \&c. It is under the celestial sign woman, the seat or fundament, or the ar- Cancer.

teries, joints, and sinews, when they are inflamed and swollen, doth inuch ease them; and used with some salt, helps to dissolve knots or kernels in any part of the body. The juice of the herb, or as (Dioscorides saith) the leaves and flowers, with some fine Frankincense in powder, used in wounds of the body, nerves or sinews, doth singularly help to heal them. The distilled water of the herb performs well all the aforesaid cures, but espccially for inflammations or watering of the eyes, by reason of the defluxion of rheum unto them.

\section{HEART'S-EASE. P. 12}

Tirs is that herb which such physicians as are licensed to blaspheme by authority, without danger of having their tongues burned through with an hot iron, called an herb of the 'Trinity. It is also called by those that are more moderate, Three Faces in a Hood, Live in Idleness, Cull me to you; and in Sussex we call them Pancies.

Place.] Besides those which are brought up in gardens, they grow commonly wild in the fields, especially in such as are very barren: sometimes you may find it on the tops of the high hills.

Time.] They flower all the Spring and Summer long.

Govermment and virtues.] The herb is really saturnine, something cold, viscous, and slimy. A strong decoction of the herbs and flowers (if you will, you may make it intosyrup) is an excellent cure for the French pox, the herb being a gallant antivenereal: and that antivencreals are the best cure for that disease, far better and safer than to torment them with the flux, divers foreign physicians have confessed. The spirit of it is excellently good for the convulsions in children, ạs also for the falling sickness, 
dominion over this herb, therefore it is a was the father of slander; Or are men's singular remedy for the liver, both to tongues so given to slander one another, strengthen it when weak, and ease it when that they must slander Nuts too, to keep afflicted, you shall do well to keep it in a their tongues in use? If any part of the syrup all the year; For though authors say Hazel Nut be stopping, it is the husks and it is green all the year, I scarcely believe it. shells, and no one is so mad as to eat them, Hart's Tongue is much commended against unless physically; and the red skin which the hardness and stoppings of the spleen ? covers the kernel, you may easily pull off: and liver, and against the heat of the liver And so thus have I made an apology for and stomach, and against lasks, and the Nuts, which cannot speak for themselves. bloody-flux. The distilled water thereof is? also very good against the passions of the heart, and to stay the hiccough, to help the falling of the palate, and to stay the bleeding of the gums, being gargled in the mouth. Dioscorides saith, it is good against the stinging or biting of serpents. As for the use of it, my direction at the latter end will be sufficient, and enough for those that are studious in physic, to whet their brains upon for one year or two.

\section{HAZEL-NUT. P. 40}

HAZEL Nuts are so well known to every body, that they need no description.

Government and virtues.] They are under the dominion of Mercury. The parted kernels made into an electuary, or the milk drawn from the kernels with mead or honeyed water, is very good to help an old cough; and being parched, and a little pepper put to them and drank, digests the distillations of rheum from the head. The dried husks and shells, to the weight of two drams, taken in red wine, stays lasks and women's courses, and so doth the red skin that covers the kernels, which is more effectual to stay women's courses.

And if this be true, as it is, then why should the vulgar so familiarly affirm, that eating nuts causes shortness of breath, than which nothing is falser? For, how can that which strengthens the lungs, cause shortness of breath? I confess, the opinion is far therefore good for the heat of the stomach, older than I am; I knew tradition was a and gnawings therein; for inflammations, friend to error before, but never that he sand the hot fits of agues. The juice thereof

There are several sorts of Hawk-weed, but they are similar in virtues.

Descript.] It has many large leaves lying upon the ground, much rent or torn on the sides into gashes like Dandelion, but with greater parts, more like the smooth Sow 'Thistle, from among which rises a hollow, rough stalk, two or three feet higl, branched from the middle upward, whereon are set at every joint longer leaves, little or nothing rent or cut, bearing on them sundry pale, yellow flowers, consisting of many small, narrow leaves, broad pointed, and nicked in at the ends, set in a double row or more, the outermost being larger than the inner, which form most of the Hawk-weeds (for there are many kinds of them) do hold, which turn into down, and with the small brownish seed is blown away with the wind. The root is long, and somewhat great, with many small fibres thereat. The whole plant is full of bitter-milk.

Place.] It grows in divers places about the field sides, and the path-ways in dry grounds.

Time.] It flowers and flies away in the Suminer months.

Government and virtues.] Saturn owns it. Hawk-weed (saith Dioscorides) is cooling, somewhat drying and binding, and friend to error before, but never that he? and the hot fits of agues. 'The juice thercof 
in wine, helps digestion, discusses wind, day, it rather shews the superstition of hinders crudities abiding in the stomach, those that observe it for the time of its and helps the difficulty of making water, flowering, than any great wonder, since the biting of venomous serpents, and sting- the like may be found in divers other places ing of the scorpion, if the herb be also of this land; as in Whey-street in Romney outwardly applied to the place, and is very Marsh, and near unto Nantwich in Chegood against all other poisons. A scruple shire, by a place called White Green, where of the dried root given in wine and vine- it flowers about Christmas and May. If gar, is profitable for those that have the the weather be frosty, it flowers not until dropsy. The decoction of the herb taken January, or that the hard weather be over. in honey, digests the phlegm in the chest or lungs, and with Hyssop helps the cough. The decoction thereof, and of wild Succory, made with wine, and taken, helps the wind cholic and hardness of the spleen ; it procures "rest and sleep, hinders venery and venerous dreams, cooling heats, purges the stomach, increases blood, and helps the diseases of the reins and bladder. Outwardly applied, it is singularly good for all the defects and diseases of the eyes, used with some women's milk; and used with good success in fretting or creeping ulcers, especially in the beginning. The green leaves bruised, and with a little salt applied to any place burnt with fire, before blisters do rise, helps them; as also inflammations, St. Anthony's fire, and all pushes and eruptions, hot and salt phlegm. The same applied with incal and fair water in manner of a ponltice, to any place affected with convulsions, the cramp, and such as are out of joint, doth gire help and ease. The distilled water cleanses the skin, and takes away freckles, spots, morphew, or wrinkles in the face.

\section{HA WTHORN.}

It is not my intention to trouble you with a description of this tree, which is so well known that it needs none. It is ordinarily but a hedge bush, although being pruned and dressed, it grows to a tree of a reasonable height.

As for the Hawthorn Tree at Glastonbury, land, by walls and hedge-sides, in waste which is said to flower yearly on Christmas- grounds and untilled places. Mars. The seeds in the berries beaten to porvder being drank in wine, are held singularly good against the stone, and are good for the dropsy. The distilled water of the flowers stay the lask. The seed cleared from the down, bruised and boiled in wine, and drank, is good for inward tormenting pains. If cloths or sponges be wet in the distilled water, and applied to any place wherein thorns and splinters, or the like, do abide in the flesh, it will notably draw them forth.

And thus you see the thorn gives a medicine for its own pricking, and so doth almost every thing else.

\section{HEMT,OCK: P, 13 .}

\section{Descript.] THE common great Hémlock} grows up with a green stalk, four or five feet high, or more, full of red spots sometimes, and at the joints very large winged leaves set at them, which are divided into many other winged leaves, one set against the other, dented abont the edges, of a sad green colour, branched towards the top, where it is full of umbels of white flowers; and afterwards with whitish flat seed: The root is long, white, and sometimes crooked; and hollow within. The whole plant; and every part, has a strong, heady, and illsavoured scent, much offending the senses.

Place.] It grows in all counties of this 
Time.] It flowers and seeds in July, or ? thereabouts.

Government and virtues.] Saturn claims dominion over this herb, yet $I$ wonder why it may not be applied to the privities in a Priapism, or continual standing of the yard, it being very bencficial to that disease ; I suppose, my author's judgment was first upon the opposite disposition of Saturn to Venus in those facultics, and therefore he forbade the applying of it to those parts, that it might not cause barrenness, or spoil the spirit proereative; which if it do, yet applied to the privities, it stops its lustful thoughts. Hemlock is exeeedingly cold, and very dangerous, espeeially to be taken in wardly. It may safely be applied to inthammations, tumours, and swellings in any part of the body (save the privy parts) as also to St. Anthony's fire, wheals, pushes, and creeping ulecrs that arise of lot sharp humours, by cooling and repelling the heat; the lcaves bruised and laid to llie brow or forehead are good for their eyes that are red and swollen; as also to take away a pin and web growing in the eye; this is a tried medicine: Take a small handful of this herb, and half so mueh bay salt, beaten together, and applied to the contrary wrist of the hand, for 24 hours, doth remove it in thriec dressing. If the root thereof be roasted under the embers, wrapped in double wet paper, until it be soft and tender, and then applied to the gout in the hands or fingers, it will quiekly help this evil. If any through mistake eat the herb Hemlock instead of Parsley, or the roots instead of a Parsnip (both of which it is very like) whereby happens a kind of fienzy, or perturbation of the senses, as if they were stupid and drunk, the remedy is (as Pliny saith) to drink of the best and strongest pure wine, before it strikes to the heart, or Gentian put in wine, or' a draught of vine-? gar, wherewith 'Tragus doth affirm, that he cured a woman that had eaten the root.

$(9,10$.
II EMP. P. 13.

'Tins is so well known to every good housewife in the country, that I shall not need to write any deseription of it.

Time.] It is sown in the vary end of Mareh, or beginning of April, and is ripe in August or September.

Government and rivtues.] It is a plant of Saturn, and good for something else, you see, than to make halters only. The seed of Hemp eonsumes wind, and by too mueh use thereof disperses it so much that it drics up the natural seed for proereation; yet, being boiled in milk and taken, helps such as have a hot dry cough. The Duteh make an emulsion out of the seed, and give it with good success to those that have the jaundice, especially in the beginning of the disease, if there be no ague accompanying it, for it opens obstructions of the gall, and causes digestion of eholer. The emulsion or decoetion of the seed stays lasks and continual fluxes, eases the cholie, and allays the troublesome humours in the bowels, and stays blceding at the mouth, nose, or other places, some of the leaves being fried with the blood of them that bleed, and so given them to eat. It is held very good to kill the worms in men or beasts; and the juiee dropped into the ears kills worms in them; and draws forth earwigs, or other living creatures gotten into them. 'The decoction "of the root allays inflammations of the head, or any other parts : the herb itself, or the distilled water thercof doth the like. 'The deeoetion of the root eases the pains of the gout, the hard humours of knots in the joints, the pains and shrinking of the sinews, and the pains of the hips. The fresh juice mixed with a little oil and butter, is good for any plaee that hath been burnt with fire, being thereto applied.

II F N B A NE.

Descript.] Oun common IIenbane has B $\mathrm{B}$ 
very large, thick, soft, woolly leaves, lying found without it growing by it. Ergo, it on the ground, much cut in, or torn on the is an herb of Saturn. The leaves of Henedges, of a dark, ill greyish green colour ; bane do cool all hot inflammations in the among which arise up divers thick and eyes, or any other part of the body; and short stalks, two or three feet high, spread are good to assuage all manner of swellings into divers small branches, with lesser leaves of the privities, or women's breast, or elseon them, and many hollow flowers, scarce where, if they be boiled in wine, and either appearing above the husk, and usually torn applied themselves, or the fomientation on one side, ending in five round points, warm; it also assuages the pain of the gout, growing one above another, of a deadish the sciatica, and other pains in the joints yellowish colour, somewhat paler towards? which arise from a hot cause. And applied the edges, with many purplish veins with vinegar to the forehead and temples, therein, and of a dark, yellowish purple helps the head-aclie and want of sleep in in the bottom of the flower, with a small hot fevers. The juice of the herb or seed, point of the same colour in the middle, or the oil drawn from the seed, does the each of them standing in a hard close husk, like. The oil of the seed is helpful for which after the flowers are past, grow very deafness, noise, and worms in the ears, like the husk of Asarabacca, and some- being dropped therein; the juice of the what sharp at the top points, wherein is herb or root doth the same. The decoction contained much small seed, very like Poppy of the herb or seed, or both, kills lice in seed, but of a dusky, greyish colour. The man or beast. 'The fume of the dried herb, root is great, white, and thick, branching stalks and seed, burned, quickly heals forth divers ways under ground, so like a swellings, chilblains or kibes in the hands Parsnip root (but that it is not so white) or feet, by holding them in the fume therethat it has deceived others. The whole plant of. The remedy to help those that have more than the root, has a very heavy, ill, taken Henbane is to drink goat's milk, soporiferous smell, somewhat offensive.

Place.] It commonly grows by the waysides, and under hedge-sides and walls.

Time.] It flowers in July, and springs again yearly of its own seed. I doubt my authors mistook July for June, if not for May.

Government and virtues.] I wonder how astrologers could take on them to make this an herb of Jupiter; and y'et Mizaldus, a ment, or plaister of it, is most admirable man of a penetrating brain, was of that for the gout, to cool the veneral heat of the opinion as well as the rest; the herb is in-reins in the French pox ; to stop the toothdeed under the dominion of Saturn, and aclie, being applied to the aching side; to I prove it by this argument: All the herbs allay all inflammations, and to help the which delight most to grow in saturnine diseases before premised. places, are saturnine herbs. Both Henbàne delights most to grow in saturuine? places, and whole cart loads of it may be? found near the places where they empty the the first of which is an Italian by birth, and common Jakes, and scarce a ditch to be only nursed up here in the gardens of the 
curious. Two or three sorts are found com- Bear's-foot, Christmas-herb, and Christmasmonly growing wild here, the description flowers. of two of which I shall give you.

Descript.] The first is a smooth, low plant, not a foot high, very bitter in taste, with many square stalks, diversly branched from the bottom to the top, with divers joints, and two small leaves at each joint, broader at the bottom than they are at the end, a little dented about the edges, of a sad green colour, and full of veins. The flowers stand at the joints, being of a fair purple colour, with some white spots in them, in fashion like those of dead nettles. The seed is small and yellow, and the roots spread nuch under ground.

The second seldom grows half a foot high, sending up many small branches, whereon grow many small leaves, set one against the other, somewhat broad, but very short. The flowers are like the flowers of the other fashion, but of a pale reddish colour. The seeds are small and yellowish. The root spreads like the other, neither will it yield to its fellow one ace of bitterness.

Place.] They grow in wet low grounds, and by the water-sides; the last may be found among the bogs on Hampstead Heath.

Time.] 'They flower in June or July, and the seed is ripe presently after.

Government and virtues.] They are herbs : of Mars, and as choleric and churlish as he is, being most violent purges, especially Saturn, and therefore no marvel if it has of choler and phlegm. It is not safe taking some sullen conditions with it, and would them inwardly, unless they be well rectified be far safer, being purified by the art of the by the art of the alchynist, and only the alchymist than given raw. If any have purity of them given; so used they may be taken any harm by taking it, the common: very helpful both for the dropsy, gout, cure is to take goat's milk: If you cannot and sciatica; outwardly used in ointments get goat's milk, you must make a shift with they kill worms, the belly anointed with it, such as you can get. 'The roots are very and are excellently good to cleanse old and effectual against all melancholy diseases, filthy ulcers.

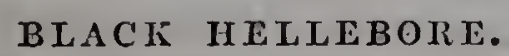

IT is also called Setter-wort, Setter-grass,

especially such as are of long standing, as quartan agues and madness; it helps the falling sickness, the leprosy, both the yellow and black jaundice, the gout, sciatica, 
by experience, that the root of that which stay blood, where or howsoever ftowing; it grows wild in our country, works not so speedily heals all green wounds, and is churlishly as those do which are brought effectual in old ulcers in the privy parts, or from beyond sea, as being. maintained by elsewhere. You may persuadê yourself a more temperate air. The root used as this is true, and also conceive a good reason a pessary, provokes the terms exceedingly; for it, do but consider it is an herb of also being beaten into powder, and strewed Venus, for all it hath a man's name.

upon fout ulcers, it consumes the dead flesh, and instantly heals them; nay, it will help gangrenes in the beginning. 'Twenty grains taken inwardly is a sufficient dose for one time, and let that be corrected with half so much cinnamon ; country people used to rowel their cattle with it. If a shooting forth stalks with leaves, some beast be troubled with a cough, or have whereof carry no berries, the others do; taken any poison, they bore a hole through every stalk smooth without joints, and the ear, and put a piece of the root in it, blackish green, rising about half a foot high, this will help him in 24 hours time. Many if it bear berries, otherwise seldom so high, other uses farriers put it to which I shall bearing at the top four leaves set directly forbear.

HERB TRUE-LOVE, OR ONE-BERRY.

Descript] OR 13 .

Descript.] Ondin has a small creeping root running under the uppermost crust of the ground, somewhat one against another, in manner of a cross or ribband tied (as it is called in a trucloves knot,) which are each of thein apart HERB ROBERT. P. A,

Tre Herb ${ }^{-}$Robert is held in great estimation by farmers, who use it in diseases of their cattle.

Descript.] It rises up with a reddish stalk two feet high, having divers leaves thereon, upon very long and reddish footstalks, divided at the ends into three or five divisions, each of them cut in on the open like a star, consisting of four small edges, which sometimes turn reddish. At and long narrow pointed leaves of a yellowthe tops of the stalks come forth divers ish green colour, and four others lying flowers made of five leaves, much larger between them lesser than they; in the midthan the Dove's-foot, and of a more reddish dle whereof stands a round dark purplish colour; after which come black heads, as in button or head, compassed about with others. The root is small and thready, eight small yellow mealy threads with three and smells, as the whole plant, very strong, colours, making it the more conspicuous, almost stinking.

Place.] This grows frequently every in the middle, when the other leaves are where by the way-sides, upon ditch banks withered, becomes a blackish purple berry, and waste grounds wheresoever one goes.

Time.] It flowers in June and July chiefly, and the seed is ripe shortly after.

Govemment and virtues.] It is under the taste. dominion of Venus. IIerb Robert is com- Place.] It grows in woods and copses, mended not only against the stone, but to and sometimes in the corners or borders of 
fields, and waste grounds in very many Hyssop boiled with rue and honey, and places of this land, and abundantly in the drank, helps those that arc troubled with woods, copses, and other places about coughs, shortness of breath, wheezing and Chislehurst and Maidstone in Kent. rheumatic distillation upon the lungs; taken Time.] They spring up in the middle of also with oxymel, it purges gross humours April or May, and are in flower soon after. by stool; and with honcy, kills worms in The berries are ripc in the end of May, the belly; and with fresh and new figs and in some places in June.

bruised, helps to loosen the belly, and more

Government and virtues.] Venus owns it; forcibly if the root of Flower-de-lucc and the leaves or berries hereof arc effectual to cresscs be added thercto. It amends and expel poison of all sorts, especially that of cherishes the native colour of the body, the aconites; as also, the plague, and other spoiled by the yellow jaundice; and being pestilential disorders; Matthiolus saith, that taken with figs and nitrc, helps the dropsy some that have lain long in a lingcring sick- and spleen ; being boiled with wine, it is ness, and others that by witchcraft (as it good to wash inflammations, and takes was thought) were become half foolish, by away the black and blue spots and marks taking a dram of the seeds or berries hereof that come by strokes, bruises, or falls, being in powder every day for 20 days together, applied with warm watcr. It is an excellent were restored to their former health. The medicine for the quinsy, or sivellings in roots in porder taken in wine eases the the throat, to wash and gargle it, being pains of the cholic speedily. The leaves boiled in figs; it helps the tooth-ache, bcirg are very effectual as wcll for green wounds, boiled in vinegar and gargled therewith. as to cleanse and heal up filthy old sores The hot vapours of the dccoction taken by and ulccrs; and is very powerful to discuss a funnel in at the ears, eases the inflammaall tumours and swellings in the privy tions and singing noise of them. Bcing parts, the groin, or in any part of the body, bruised, and salt, honcy, and cummin seed and specdily to allay all inflammations. put to it, helps thosc that are stung by The juice of the leaves applied to felons, or serpents. The oil thereof (the head being those nails of the hands or toes that have anointed) kills lice, and takcs away itching imposthumes or sores gathered together at of the head. It helps those that have the the roots of thcm, heals them in a short falling sickness, which way socver it be space. The herb is not to be described for applied. It hclps to expectorate tough the premises, but is fit to be nourished in phlegm, and is cffcctual in all cold griefs every good woman's garden.

\section{Y SSOP.}

Hyssop is so well known to be an inhabitant in every garden, that it will save me labour in writing a description thereof. 'The virtues are as follow.

Government and virtues.] T'he herb is Jupiter's, and the sign Cancer. Itstrcugthens ali the parts of the body under Cancer and no description ; I mean the manured kind, Jupitcr; which what they may be, is found which every good husband or housewife is amply described in my astrological judg- acquainted with. ment of diseases. Dioscorides saitl, that $(9,10$. or discases of the chests or lungs, being taken either in syrup or licking medicine. The grcen herb bruised and a little sugar put thercto, doth quickly lieal any cut or grcen wounds, bcing thereunto applied.

IIOPS.

These are so well known that they need Descript.] 'The wild hop grows up as the C C 
other doth, ramping upon trees or hedges, that stand next to them, with rough branches and leaves like the former, but it gives smaller heads, and in far less plenty than it, so that there is scarcely a head or two seen in a year on divers of this wild kind, wherein consists the chief difference.

Place.] They delight to grow in low up with square hairy stalks, half a yard or moist grounds, and are found in all parts of this land.

Time.] They spring not until April, and flower not until the latter end of June; the heads arc not gathered until the middle or latter end of September.

Government and virtues.] It is under the dominion of Mars. This, in physical operations, is to open obstructions of the liver and spleen, to cleanse the blood, to loosen the belly, to cleanse the reins from gravel, and provoke urine. The decoc-s tion of the tops of Hops, as well of the tame as the wild, works the same effects. In cleansing the blood they help to cure the French diseases, and all manner of scabs, itch, and other breakings-out of the body; as also all tetters, ringworms, and spreading sores, the morphew and all discolouring of the skin. The decoction of the flowers and hops, do help to expel poison that any one hath drank. Half a dram of the seed in powder taken in drink, kills worms in the body, brings down women's courses, and expels urine. A syrup made of the juice and sugar, cures the yellow jaundice, eases the head-ache that comes of heat, and tempers the heat of the liver and stomach, and is profitably given in long and hot agues that rise in choler and blood. Both the wild and the manured are of one property, and alike effectual in all the aforesaid diseases. By all these testimonies beer appears to be better than ale.

Mars owns the plant, and then Dr. Reason will tell you how it performs these actions.

\section{IIOREHOUND.}

THERE are two kinds of Horehound, the white and the black. 'The black sort is likewise called Hen-bit; but the white one is here spoken of.

Descript.] Common Horehound grows two feet high, set at the joints with two round crumpled rough leaves of a sullen hoary green colour, of a reasonable good scent, but a very bitter taste. The flowers are small, white, and gaping, set in a rough, hard prickly husk round about the joints, with the leaves from the middle of the stalk upward, wherein afterward is found sniall round blackish seed. The root is blackish, hard and woody, with many strings, and abides many years.

Place.] It is found in many parts of this land, in dry grounds, and waste green places.

Time.] It flowers in July, and the seed is ripe in August.

Government and virtues.] $\mathrm{It}$ is an herb. of Mercury. A decoction of the dried herb, with the seed, or the juice of the green herb taken with honey, is a remedy for those that are short-winded, have a cough, or are fallen into a consumption, either through long sickness, or thin distillations of rheum upon the lungs. It lielps to expectorate tough phlegm from the chest, being taken from the roots of Iris or Orris. It is given to women to bring down their courses, to expel the after-birth, and to them that have taken poison, or are stung or bitten by venemous serpents. 'The leaves used with honey, purge foul ulcers, stay running or creeping sores, and the growing of the flesh over the nails. It also helps pains of the sides. 'The juice thereof with wine and honey, helps to clear the eycsight, and snuffed up into the nostrils, purges away the yellow-jaundice, and with a little oil of roses dropped into the ears, 
eases the pains of them. Galen saith; it opens obstructions both of the liver and spleen, and purges the breast and lungs of phlegm : and used outwardly it both cleanses and digests. A decoction of Horehound (saith Matthiolus) is available for those that have hard livers, and for such as have itches and running tetters.' The powder hereof taken, or the decoction, kills worms. The green leaves bruised, and boiled in old hog's grease into an ointment, heals the biting of dogs, abates the swellings and pains that come by any pricking of thorns, or such like means; and used with vinegar, cleanses and heals tetters. 'There is a syrup made of Horehound to be had at the apothecaries, very good for old to void cold rheums from the lungs of old folks, and for those that are asthinatic or short-winded.

\section{HORSETIL。}

OF that there are many kinds, but $\mathbf{I}$ shall not trouble you nor myself with any large description of them, which to do, were but, as the proverb is, To find a knot in a rush, all the kinds thereof being nothing else but knotted rushes, some with leaves, and some without. Take the description of the most eminent sort as follows.

Descript.] The great Horsetail at the first springing has heads somewhat like those of asparagus, and afterwards grow to be hard, rough, hollow stalks, jointed at sundry places up to the top, a foot high, so made? as if the lower parts were put into the upper, where grow on each side a bush of small long rush-like hard leaves, each part resembling a horsetail, from whence it is so called. At the tops of the stalks come forth small scatkins, like those of trees. The root creeps under ground, having joints at sundry places.

Place.] This (as most of the other sorts hereof) grows in wet grounds.
Time.] They spring up in April, and their blooming catkins in July, seeding for the most part in August, and then perish down to the ground, rising afresh in the Spring.

Government and virtues.] The herb belongs to Saturn, yet is very harmless, and excellently good for the things following: Horsetail, the smoother rather than the rough, and the leaves rather than the bare, is most physical. It is very powerful to staunch bleeding either inward or outward, the juice or the decoction thereof being drank, or the juice, decoction, or distilled water applied outwardly. It also stays all sorts of lasks and fluxes in man or woman, and bloody urine; and heals also not only the inward ulcers, and the excoriation of the entrails, bladder, \&c. but all other sorts of foul, moist and running ulcers, and soon solders together the tops of green wounds. It cures all ruptures in children. The decoction thereof in wine being drank, provokes urine, and helps the stone and stranguary; and the distilled water thereof drank two or three times in a day, and a small quantity at a time, also eases the bowels, and is effectual against a cough that comes by distillations firom the head. The juice or distilled water being warmed, and hot inflammations, pustules or red wheals, and other breakings-out in the skin, being bathed therewith, doth help them, and doth no less the swelling heat and inflammation of the lower parts in men and women.

\section{HOUSELEEK OR SENGIEEN.}

Botr these are so well known to my countrymen, that I shall not need to write any description of them.

Place.] It grows commonly upon walls and house-sides, and flowers in July.

Government and virtues.] It is an herb of Jupiter, and it is reported by Mezaldus, to preserve what it grows upon from fire and lightning. Our ardinary Houseleek is 
good for all inward heats as wcll as out- which consist of small purplish red leaves ward, and in the cyes or other parts of the of a dead colour, rising out of the husks body; a possct made with the juice of wherein they stand with some threads in Houseleek, is singularly good in all hot the middle. It has sometimes a white agues, for it cools and tempers the blood flower. After the flowers are past, there and spirits, and quenches the thirst; and comes rough flat seed, with a small pointle also good to stay all hot defluctions or sharp in the middle, easily cleaving to any garand salt rhcums in the cyes, the juice being ment that it touches, and not so easily dropped into them, or into the ears. pulled off again. The root is black, thick, It helps also other fluxes of humours in the and long, hard to break, and full of clammy bowels, and the immoderate courses of juice, smelling somcivhat strong, of an evil women. It cools and restrains all other hot scent, as the leaves also do. inflammations, St. Anthony's fire, scaldings and burnings, the shingles, fretting ulcers, cankers, tettors, ringworms, and the like; and much eases the pains of the gout proceeding from any hot cause. The juice also takes away worts and corns in the hands or feet, being often bathed therewith, and the skin and leaves bcing laid on them afterwards. It eases also the head-ache, and distempered heat of the brain in frenzies, or through want of sleep, bcing applied to the temples and forehead. The leaves bruised and laid upon the crown or seam of the head, stays blecding at the nose very quickly. The distilled water of the herb is profitable for all the purposes aforesaid. 'The leaves being gently rubbed on any place stung with nettles or bees, doth quickly take away the pain.

\section{HOUND'S TONGUE. 卢.}

Descript.] The great ordinary Hound's Tongue has many long and somewhat narrow, soft, hairy, darkish green leaves, lying on the ground, somewhat like unto grcen wound doth heal it up quickly: the Bugloss leaves, from among which rises root baked under the embers, wrapped in up a rough hairy stalk about two fect ligh, paste or wet paper, or in a wet double cloth, with some smaller leaves thereon, and and thereof a suppository made, and put branched at the tops into divers parts, with up into or applied to the fundament, doth a small leaf at the foot of every branch, very effectually help the painful piles or which is somewhat long, with many flowers hruorrhoids. The distilled water of the set along the same, which branch is crooked herbs and roots is very good to all the puror turned inwards before it flowers, and poses aforcsaid, to be used as well inwardly opens by degrees as the flowers blow, to drink, as outwardly to wash any sore 
place, for it heals all manner of wounds and a-piece, with many yellow threads in the punctures, and those foul ulcers that arise middle, which being bruised do yield a redby the French pox. Mizaldus adds that dish juice like blood; after which come the leaves laid under the feet, will keep the small round heads, wherein is contained dogs from barking at you. It is called small blackish seed smelling like rosin. Hound's-tongue, because it ties the tongues The root is hard and woody, with divers of hounds; whether true, or not, I never strings and fibres at it, of a brownish colour, tried, yet I cured the biting of a mad $\operatorname{dog}$ ? which abides in the ground many years, with this only medicine.

IOLLY, IIOLM, OR IIULVER BUSH.

For to describe a tree so well known is necdless.

Government and virtues.] The tiee is Saturnine. The berries expel wind, and therefore are held to be profitable in the cholic. The berries have a strong faculty with them; for if you eat a dozen of them in the morning fasting when they are ripe and not dried, they purge the body of gross and clammy phlegm: but if you dry the berries, and beat them into powder, they bind the body, and stop fluxes, bloodyfluxes, and the terms in women. The bark of the tree, and also the leaves, are excellently good, being used in fomentations for broken bones, and such members as are out of joint. Pliny saith, the branches of the tree defend houses from lightning, and men froin witclicraft.

\section{ST. JOHN'S WORT. 卫. 13}

TIIIs is a very beautiful shrub, and is a great ornament to our meadows.

Descript.] Common St. John's Wort shoots fortl brownish, upright, hard, round stalks, two feet high, spreading many branches from the sides up to the tops of them, with two small leaves set one against another at every place, which are of a deep green colour, somewhat like the leaves of the lesser Centaury, but narrow, and full of small holes in every lcaf, which cannot be so well perceived, as when they are held up to the light; at the tops of the stalks and branches stand yellow flowers of five leaves? $(9,10$. shooting ancw every Spring.

Place.] 'This grows in woods and copses, as well those that are shady, as open to the sun:

Time.] They flower about Midsummer and $J u l y$, and their seed is ripe in the latter end of July or August.

Governient and virtues.] It is under the celestial sign Lco, and the dominion of the Sun. It may be, if you meet a Papist, he will tell you, especially if he be a lawyer, that St. John made it over to him by a leiter of attorney. It is a singular wound herb; boiled in wine and drank, it heals inward hurts or bruises ; made into an ointment, it open obstructions, dissolves swellings, and closes up the lips of wounds. The decoction of the herb and flowers, especi. ally of the seed, being drank in wine, with the juice of knot-grass, helps all manner of vomiting and spitting of blood, is good for those that are bitten or stung by any venomous creature, and for those that cannot make water. Two drams of the seed of St. John's Wort made into powder, and drank in a little broth, doth gently cxpel choler or congealed blood in the stomach. The decoction of the leaves and seeds drank somewhat warm before the fits of agues, whether they be tertains or quartans, alters the fits, and, by often using, doth take them quite away. The seed is much commended, being drank for forty days together, to help the sciatica, the fallingsickucss, and the palsy.

IVI.

Ir is so rell known in every chilet

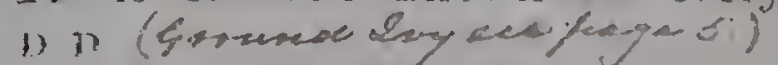


almost, to grow in woods upon the trees, and nose, and curing the ulcers and stench and upon the stone walls of churches, therein; the same dropped into the ears, houses, \&c. and sometines to grow alone of helps the old and running sores of them; itsclf, though but seldom.

Time.] It flower's not until July, and
the berries are not ripe till Christmas, when
they have feit i inter frosts. those that are troubled with the spleen, shall find much ease by continual drinking out of a cup made of Ivy, so as the drink may stand some small time therein before : Government and rirtues.] "It is under the it be drank. Cato saith, That wine put dominion of Saturn. A pugil of the Howers, into such a cup, will soak through it, by which may be about a drau, (saith Dioscn- reason of the antipathy that is between corides) drank twice a day in red wine, them.

helps the lasi, and bloody flux. It is an cleiny to the nerves and sinews, being? much taken inwardly, but very helpful to them, being outwardly applied. Pliny saith, the yellow berries are good against the joundice; and taken before one be set to dink hard, preserves from drunkenness, and helps those that spit blood; and that the white berries being taken inwardly, or applied outwarlly, kills the worms in the belly. The berries are a singular remedy to prevent the plague, as also to free then from it that have got it, by drinking the berries thereof made into a powder, for two mon without Highgate; lard by the Newor three days together. They being taken found Wells near Dulwich, upon a Common in wine, do certainly help to break the between Mitcham and Croydon, in the stone, provoke urine, and women's courses. The fresh leaves of Ivy, boiled in vinegar, and applied warm to the sides of those that are troubled with the splecn, ache, or stitch in the sides, do give much ease: The same applied with some Rosewater, and oil of Roses, to the temples and forehead, eases the head-ache, though it he of long continuance. The fresh leaves boiled in wine, and old filthy ulcers hard to be cured washed therewith, do wonderfully help to solar shrub is scarce to be paralleled for its cleanse them. It also yuickly lieals green virtues. The berries are hot in the third wounds, and is effectual to heal all burnings degree, and dry but in the first, being a and scaldings, and all kinds of exulccra- most admirable counter-poison, and as great tions coming thereby, or by salt phlegm or a resister of the pestilence, as any growing; humours in other parts of the body. The they are excellent good against the bitings juice of the berries or leaves snuffed up into of venomous beasts, they provoke urine the nose, purges the head and brain of thin exceedingly, and therefore are rery availrheum that makes defluxions into the eyes able to dysuries and stranguaries. It is so 
powerful a remedy against the dropsy, that on, usually not round as those below, but the very lye made of the ashes of the herb somewhat long, and divided at the edges: bcing drank, cures the clisease. It provokes the tops are somewhlat divided into long the terms, helps the fits of the mother, branches, bearing a number of flowers, set strengthens the stomach exceedingly, and round about a long spike onc above another, expels the wind. Indeed there is scarce a which are hollow and like a little bell of a bettcr remedy for wind in any part of the whitish green colour, after which come body, or the cholic, than the chymical oil small heads, containing very small brownish drawn from the berries; such country seed, which falling on the ground, will people as know not how to draw the chy- plentifully spring up before Winter, if it nical oil, may content themselves by eating have moisture: The root is round and most ten or a duzen of the ripe berries every usually smooth, grevish without, and white morning fasting. They are adnirably good within, having small fibres at the head of for a cough, shortness of brcath, and con- the root, and bottom of the stalk. sumption, pains in the belly, ruptures, cramps, and convulsions. They give safe and speedy delivery to women with child, they strengthen the brain exceedingly, help the meinory, and fortity the sight by strengthening the optic nerves; are excellently good in all sortis of agnes; help the gout and sciatica, and strengthen the limbs of the body. 'The ashes of the wood is a speedy renedy to such as have the scurvy, to rub their gums with. 'I'he berries stay all fluxes, help the hamorrhoids or piles, and kill worms in chikisen. A lye made of the ashes of the wood, and the body bathed with it, cures the itch, scabs and leprosy. 'The berries break the stone, procure appctite when it is lost, and are sickness.

KIDNEYWORT, OR WALL PENNYROYAL, OR WALL PENNYWORT. I

Descript.] It lias many thick, flat, and round leaves growing from the root, crery helps to hcal sore kidneys, torn or fretted
one having a long footstalk, fastened un- by the stone, or exulcerated within; it also one having a long about the middle of it, and a provokes urine, is available for the drous littlc unevenly weaved sometimes abount the and helps to break the stone. Bcing uscd edges, of a palc green colour, and some- as a bath, or made into an ointment, it what yellow on the upper side like a sau-cools the painful piles or hamorrhoidal cer; from among which arise one or more veins. It is no less effectual to give case tender, smootil, hollow stalks half a foot to the pains of the gout, the sciatica, and high, with two or three small leaves there- helps the kernels or knots in the neck ar. 
throat, called the king's evil : healing kibes of the herb and roots in wine, and applying and chilblains if they be bathed with the the same outwardiy to the place. It is juice, or anointed with ointment made singularly gond in ail running sores, canthereof, and some of the skin of the leaf cerous and fistulous, drying up of the moisupon them: it is also used in green wounds ture, and healing them up so gently, withto stay the blood, and to heal them quickly. out sliarpness; it doth the like to ruming

KN A PWEED. P. 13.

sores or scais of the head or other parts. It is of special use tor the soreness of the throat, swelling of the uvula and jaws, and

Descript.] THE common sort hereof excellently good to stay bleeding, and heal has many long and somewhat dark green up all.green wounds.

leaves, rising from the root, dented about the edges, and sometimes a little rent or torn on both sides in two or three places, and somewhat hairy, withal; amongst which arises a long round stalk, four or five feet high, divided into many branches, at the tops whereof stand great scaly green heads, and from the middle of them thrust forth a number of dark purplish red thrumbs or threads, which after they are withered and past, there are found divers black seeds, lying in a great deal of down, somewhat like unto 'Thistle seed, but smaller; the root is white, hard and woody, and divers fibres annexed thereunto, which perishes not, but abides with leaves therson all the Winter, shooting out fresh every spring.

KNOTGRASS. P. 14

$I_{T}$ is generally known so well that it needs no description.

Place.] It grows in every county of this land by the highway sides, and by foot-paths in fields; as also by the sides of old walls.

Time.] It springs up late in the Spring, and abides until the Winter, when all the branches perish.

Govermment and virtues.] Saturn seems to me to own the herb, and yet some hold the Sun; out of doubt 'tis Saturn. 'The, juice of the common kind of Knotgrass is most effectual to stay bleeding of the mouth, being drank in steeled or red wine; Place.] It grows in most fields and mea- and the bleeding at the nose, to be applied dows, and about their borders and hedges, to the forehead or temples, or to be squirted and in many waste grounds also every up into the nostrils. It is no less effectual where.

Time.] It usually flowers in June and July, and the seed is ripe shortly after.

Government and virtues.] Saturn challenges the herb for his own. This Knap- It is singularly good to provoke urine, help weed helps to stay fiuxes, both of blood at the stranguary, and allays the heat that the mouth or nosc, or other outward parts, comes thereby; and is porverful by urine and those veins that are inwardly broken, to expel the gravel or stone in the kidneys or inward wounds, as also the fluxes of the and bladder, a dram of the porder of belly; it stays distillation of thin and sharp the herb being taken in wine for many humours from the head upon the stomach days together. Buing boiled in wine and and lungs; it is good for those that are drank, it is profitable to those that are stung bruised by any fall, blows or otherwise, and or bitten by venemous creatures, and very is profitable for those that are bursten, and effectual to stay all defluxions of rheumatic have ruptures, by drinking the decoction humours upon the stomach, and kills worms 
in the belly or stomach, quiets inward pains that arise from the heat, sharpness and corruption of blood and choler. The distilled water hereof taken by itself or with the the herb as her own. Ladies' Mantle is powder of the herb or seed, is, very effectual sery proper for those wounds that have into all the purposes aforesaid, and is ac- flammations, and is very effectual to stay counted one of the most sovereign remedies bleeding, vomitings, fluxes of all sorts, to cool all manner of inflammations, breaking out through heat, lot swellings and imposthumes, gangrene and fistulous cankers, or foul filthy ulcers, being applied or put into them; but especially for all sorts of ulcers and sores happening in the privy parts of men and women. It helps all fresh and green wounds, and speedily heals them. The juice dropped into the ears, cleanses them being foul, and having running matter in them.

It is very prevalent for the premises; as also for broken joints and ruptures.

\section{IADIES' MANTLE. P.14:}

Descript.] IT has many leaves rising from the root standing upon long hairy foot-stalks, being almost round, and a little cut on the edges, into eight or ten parts, making it seem like a star, with so many corners and points, and dented round about, of a light green colour, somewhat hard in handling, and as it were folded or plaited at first, and then crumpled in divers places, gar and a little hairy, as the stalk is also, which rises up among them to the height of two or three feet; and being weak, is not able to stand upright, but bended to the ground, divided at the top into two or three small branches, with small yellowish green heads, and flowers of a whitish colour breaking out of them; which being past, there comes a small yellowish seed like a poppy seed : The root is somewhat long and black, with wany strings and fibres thereat.

Place.] It grows naturally in many pasPlace.] It grows naturaly indes in Hertfordshire, from obstructions, provokes women's courses,
tures and wood sides and other places of and expels the dead child and after-birth. this land.
Time.] It flowers in May and June; abides after seedtime green all the Winter. Government and virtues.] Venus claims bruises by falls or otherwise, and helps ruptures; and such women as have large breasts, causing them to grow less and hard, being both drank and outwardly applied; the distilled water drank for 20 days together helps conception, and to retain the birth; if the women do sometimes also sit in a bath made of the decoction of the herb. It is one of the most singular wound herbs that is, and therefore highly prized and praised by the Germans, who use it in all wounds inward and outward, to drink a decoction thereof, and wash the wounds therewith, or dip tents therein, and put them up all humidity of the sores, and abates inflammations therein. It quickly heals all green wounds, not suffering any corruption to remain behind, and cures all old sores, though fistulous and hollow.

\section{LAVENDER.}

BEING an inhabitant almost in every garden, it is so well known, that it needs no description.

Time.] It flowers about the end of June, and beginning of July.

Goternment and virtues.] Mercury owns the herb; and it carries his effects very potently. Lavender is of a special good use for all the griefs and pains of the head and brain that proceed of a cold cause, as the apoplexy, falling-sickness, the dropsy, or sluggish malady, cramps, convulsions, palsies, and often faintings. It strengthens the stomach, and frees the liver and spleen from obstructions, provokes women's courses, The flowers of Lavender steeped in wine E b into the wounds, which wonderfully dries 
helps them to make water that are stopped, or are troubled with the wind or cholic, if the place be bathed therewith. A decoction made with the flowers of Lavender, Hore-hound, Fennel and Asparagus root, and a little Cinnamon, is very profitably used to help the falling-sickness, and the which arise up divers tender, weak, round,
giddiness or turning of the brain: to gar- green stalks, somewhat streaked, with longle the mouth with the decoction thereof ger and smaller leares upon them; on the is good against the tooth-ache. 'Two tops of which stand flowers, almost like the spoonfuls of the distilled water of the Stock Gilliflowers, but rounder, and not so flowers taken, helps them that liave lost long, of a blushing white colour; the seed their voice, as also the tremblings and pas- is reddish, and grows "to small branches, sions of the heart, and faintings and swoon- being of a sharp biting taste, and so has the ing, not only being drank, but applied to herb.

the temples, or nostrils to be smelled unto ; but it is not safe to use it where the body is replete with blood and lumours, because of the hot and subtile spirits wherewith it is possessed. The chymical oil drawn from Lavender, usually called Oil of Spike, is of so fierce and piercing a quality, that it is cautiously to be used, some few drops being sufficient, to be given with other things, either for inward or outward griefs.

\section{LAVENDER-COTTON.P. XV.}

I $\mathrm{T}$ being a common garden herb, I shall forbear the description, only take notice, that it flowers in June and July.

Government and virtues.] It is under the dominion of Mercury. It resists poison, putrefaction, and heals the biting of venomous beasts: A dram of the powder of the dried leaves taken every morning fasting, stops the running of the reins in men, and whites in romen. The seed beaten into powder, and taken as worm-seed, kills the worms, not ouly in children, but also in people of riper years; the like doth the herb itself, being steeped in milk, and the milk drank; the body batlied with the decoction of it, helps scabs and itcl.

IADIES-SHOCK, OR CUCKOW-FLOWER.

This is a very pretty ornament to the sides of most meadows.
Descript.] The root is composed of many small white threads from whence spring up divers long stalks of winged leaves, consisting of round, tender, dark, green leares, set one against another upon a middle rib, the greatest being at the end, amongst Place.] They grow in moist places, and near to brooksides.

Time.] They Hower in April and May, and the lower leaves continuc green all the Winter.

Government and virtues.] They are under the dominion of the Moon, and very little inferior to Water Cresses in all their operations; they are excellently good for the scurvy, they provoke urine, and break the stone, and excellently warm a cold and weak stomach, restoring lost appetite, and help digestion.

\section{LETTUCE. P. 14.}

$I_{T}$ is so well known, being generally used as a Sallad-herb, that it is altogether needless to write any description thereof.

Govermment and virtues.] 'The Moon owns them, and that is the reason they cool and moisten what heat and dryness Mars causeth, because Mars has his fall in Cancer; and they cool the heat because the Sun rules it, between whom and the Moon is a reception in the generation of men, as you may see in my Guide for Women. The juice of Lettuce mixed or boiled with Oil of Roses, applied to the forehead and temples procures sleep, and eases the headache proceeding of an hot cause: Being ,eaten boiled, it helps to loosen the belly. 
It helps digestion, quenches thirst, in- great pools, and standing waters, and somecreases milk in nurses, eases griping pains in times in slow running rivers, and lesser

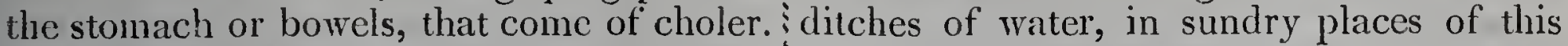
Applied ontwardly to the region of the land.

heart, liver or reins, or by bathing the said T Time.] They flower most commonly places with the juice of distilled water, , about the end of May, and their secd is wherein some white Sanders, or red Roses ripe in August.

are put; not only represses the heat and Government and virtues.] The herb is inflammations therein, but comforts and under the dominion of the Moon, and therestrengthens those parts, and also tempers fore cools and moistens like the former. the heat of urinc. Galen advises old men The leaves and flowers of the Water Lilies to use it with spice; and where spices are are cold and moist, but the roots and seeds wanting, to add Mints, Rochet, and such are cold and dry; the leaves do cool all like hot herbs, or else Citron Lemon, or inflammations, both outward and inward Orange seeds, to abate the cold of one and heat of agues; and so doth the flowers heat of the other. 'The seed and distilled also, either by the syrup or conserve; water of the Lettuce work the same effects the syrup helps much to procure rest, in all things; but the use of Lettuce is and to settle the brain of frantic perchiefly forbidden to those that are short-s sons, by cooling the hot distemperature winded, or have any imperfection in the of the head. 'The seed as well as the ront lungs, or spit blood.

WATER IILY. P. 14

OF these there are two principally noted kinds, viz. the White and the Yellow.

Descript.] The. White Lily has very large and thick dark green leaves lying on sharp, to be boiled in wine and water, and the water, sustained by long and thick the decoction drank. The distilled water foot-stalks, that arise from a great, thick, of the flowers is very effectual for all the round, and long tuberous black root diseases aforesaid, both inwardly taken, spongy or loose, with many knobs thereon, and outwardly applied; and is much com. green on the outside, but as white as snow mended to take away freckles, spots, sunwithin, consisting of divers rows of long burn, and morphew from the face, 'or other and somewhat thick and narrow leaves, parts of the body. The oil made of the smaller and thinner the more inward they flowers, as oil of Roses is made, is profitably be, encompassing a head with many yet- used to cool hot tumours, and to case the low threads or thrums in the middle; where, pains, and help the sores. ; after they are past, stand round Poppy-like heads, full of broad oily and bitter seed.

The yellow kind is little different from the former, save only that it las fewer leaves on the flowers, greater and more shining seed, and a whitish root, both within and without. 'The root of both is somewhat sweet in taste.

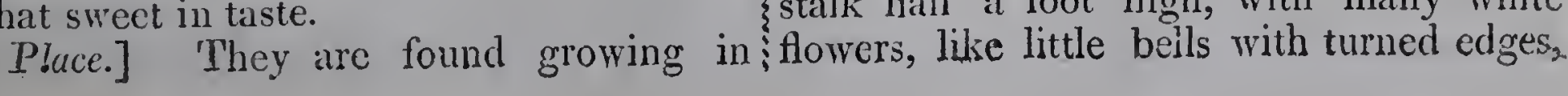

\section{LILY OF TIIE VALLEY. P. 14 .}

Catled also Conval Lily, Male Lily, and Lily Confancy.

Descript.] The root is small, and creeps far in the ground, as grass roots do. The leaves are many, against which rises up at stalk lalf a foot high, with many white 
of a strong, though pleasing smell ; the $\vdots$ birth. The root roasted, and mixed with a berries are red, not much unlike those of little hog's grease, makes a gallant poultice Asparagus.

Place.] They grow plentifully upon Hampstead-Heath, and many other places in this nation.

Time.] They flower in May, and the seed is ripe in September.

Government and virtues.] It is under the dominion of Mercury, and therefore it strengthens the brain, recruits a weak memory, and makes it strong again: The distilled water dropped into the eyes, helps intlammations therc; as also that infirmity which they call a pin and web. The spirit of the flowers distilled in wine, restores lost speech, helps the palsy, and is exccllently good in the apoplexy, comforts the heart and vital spirits. Gerrard saith, that the flowers being close stopped up in a glass, put into an ant-hill, and taken away again a month after, ye shall find a liquor in the glass, which, being outwardly applied, helps the gout.

\section{WHITE LILIES. P. F}

IT were in vain to describe a plant so commonly known in every one's garden; therefore I shall not tcll you what they are, but what they are good for.

Government and virtues.] They are under the dominion of the Moon, and by antipatly to Mars expel poison ; they are excellently good in pestilential fevers, the roots being bruised and boiled in wine, and the decoction drank; for it expels the in fair water, with some Maiden-hair and venom to the exterior parts of the body: figs, makes a good drink for those that have The juice of it being tempered with barley a dry cough or hoarseness, wheezing or meal, baked, and so eaten for ordinary shortness of breath, and for all the griefs of bread, is an excellent cure for the dropsy: An ointment made of the root, and hog's tions caused by the distillation of salt grease, is excellently good for scald heads, humours on them. It is also good in all unites the sinews when they are cut, and pains of the reins, the stranguary, and heat cleanses ulcers. The root boiled in any of urine: The fine powder of Licuorice conrenient decoction, gives speedy delivery blown through a quill into the eyes that to women in travail, and expels the after- have a pin and web (as they call it) or 
rheumatic distillations in them, doth cleanse to the tops into great and long branches, and help them. The juice of Liquorice is on all which, at the joints, there grow long as effectual in all the diseases of the breast and narrow leaves, but broader below, and and lungs, the reins and bladder, as the usually two at a joint, yet sometimes thrce decoction. The juice distilled in Rose- or four, somewhat like willow leaves, smooth watcr, with some Gum Tragacantl, is a fine on the edges, and of a fair green colour licking medicine for hoarseness, whecz- from the upper joints of the branclies, and ing, \&c.

\section{LIVER WORT. P. 32 .}

There are, according to some botanists, into small round heads, containing small upwards of three hundred different kinds cornered seeds : the root crecps under of Liverwort.

Descript.] Common Liverwort grows close, and spreads much upon the ground in moist and shady places, with many small green leaves, or rather (as it wcre) sticking that to one another, rcry unevenly cut in on the cdges, and crumpled; from among which arise small slender stalks, an inch or two high at most, bearing small star-like flowers at the top; the roots are very fine and small.

Government and virtues.] It is under thic dominion of Jupiter, and under the sign Cancer. It is a singularly good herb for all the diseases of the liver, both to cool and cleanse it, and hclps the inflammations in any part, and the yellow jaundice likcwise. Being bruised and boiled in small beer, and drank, it cools the heat of the liver and kidneys, and helps the running of the reins in men, and the whites in women; it is a singular remedy to stay the sprcading of tetters, ringworms, and other fretting and running sores and scabs, and is an excellent remedy for such whose livers are corrupted by surfeits, which cause their bodies to break out, for it fortifies the liver exceedingly, and makes it impregnable.

LOOSESTRIFE OR WILLOW-HERB.

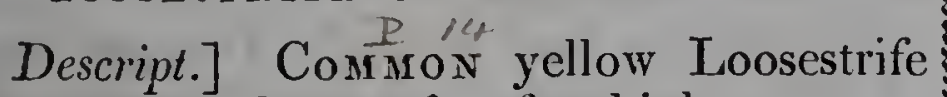
grows to be four or five feet high, or morc, and a greener colour than the former, with great round stalks, a little crested, and some brownish. The stalks are branched diversly branched from the middle of them into many long stems of spiked flowers half

(11 12.) at the tops of them also stand many yellow flowcrs of five leaves a-piecc, with divers yellow threads in the middle, which turn ground, almost like coughgrass, but greater, and shoots up every Spring brownish heads, which afterwards grow up into stalks. It has no scent or taste, and is only astringent. Place.] It grows in many places of this land in moist meadows, and by water sides. Time.] It flowers from Junc to August. Government and virtues.] This herb is good for all manner of bleeding at the mouth, nose, or wounds, and all tluxes of the belly, and the bloody-flux, given either to drink or taken by clysters; it stays also the abundance of women's courses; it is a singular good wound-herb for green wounds, to stay the blecding, and quickly close together the lips of the wound, if the herb be bruised, and the juice only applied. It is often used in gargles for sore mouths, as also for the secret parts. The smoak hereof being bruised, drives away flies and gnats, which in the night time molest people inhabiting near marshes, and in the fenny countries.

LOOSESTRIFE, WITH SPIKED HEADS OF FLOWERS. P. 14

IT is likcwise called Grass-polly.

Descript.] This grows with many woody square stalks, full of joints, about threc feet high at least; at every onc whereof stand two long lcaves, shorter, narrower, F $F$ 
a foot long, growing in bundles one above the place covered with a linen cloth doubled another, out of snall husks, very like the and anointed with the ointment; and this spiked heads of Lavender, eaeh of which is also an approved medicine. It likewise flowers have five round-pointed leaves of a cleanses and heals all foul ulcers, and sores purple violet eolour, or somewliat inclining whatsoever, and stays their inflammations to redness; in which husks stand small by washing them with the water, and layround leads after the flowers are fallen, ing on them a green leaf or two in the wherein is contained small seed. The root Summer, or dry leaves in the Winter. This ereeps under ground like unto the yellow, water, gargled warm in the mouth, and but is greater than it, and so are the heads sometimes drank also, doth cure the quinsy, of the leares when they first appear out of or king's evil . in the throat. 'The said the ground, and more brown than the water applied warm, takes away all spots, other.

marks, and seabs in the skin; and a little

Place.] It grows usually by rivers, and of it drank, quenches thirst when it is exditeh-sides in wet ground, as about the treme.

ditehes at and near Lambeth, and in many places of this land.

Time.] It flowers in the months of June
and July.

Govermment and virtues.] It is an herb of the Moon, and under the sign Cancer; neither do I know a better preserver of the sight when it is well, nor a better eure for sore eyes than Eyebright, taken inwardly, and this used outwardly; it is cold in quality. This herb is nothing inferior to the former, it having not only all the virtues which jounes seren or eight feet high, full of the former joints, but lesser leaves set on them than the former hath, but more peeuliar virtues grow below; and with them towards the of its own, found out by experience; as, tops eome forth large branches, bearing at namely, 'The distilled water is a present their tops large umbels of yellow flowers, remedy for hurts and blows on the eyes, and after them flat brownish seed. The and for blindness, so as the Christalline roots grow thick, great and deep, spreading humours be not perished or hurt; and this much, and enduring long, of a brownish hath been sufficiently proved true by the colour on the outside, and whitish within. experience of a nlan of jurlgment, who kept The whole plant and every part of it smellit long to himself as a great secret. It clears the eyes of dust, or any thing gotten into them, and preserves the sight. It is also very available against wounds and thrusts, being made into an ointment in this manner: 'T'o every ounce of the water, add two drams of May butter without salt, and of sugar and wax, of caeh as much also; let them boil gently together. Let of the Sun, under the sign Tamus. If ing strong, and aromatically, and is of a hot, sharp, biting taste.

Place.] It is usually planted in gardens, where, if it be suffered, it grows huge and great.

Time.] It flowers in the end of July, and seeds in August.

Goverment and virtues.] It is an herb after it is cold, be put into the wounds, and Saturn offend the throat (as he always doth 
Taurus is the Genesis) this is your cure is an excellent remedy boiled in beer for It opens, cures and digests humours, and broken-winded horses. mightily 'provokes women's courses and urine. Half a dram at a time of the dried root in powder taken in wine, doth wonderfully warm a cold stomach, helps digestion, forth many very long, weak, four-square, and consumes all raw and superfuous reddish stalks, trailing on the ground a moisture therein; eases all inward gripings great way, very rough or hairy, and full of and pains, dissolves wind, and resists poison joints: At every one of these joints come and infection. It is a known and much forth divers long and narrow leaves, standpraised remedy to drink the decoction of ing like a star about the stalks, round also the herb for any sort of ague, and to help and hairy, towards the tops whereof come the pains and torments of the body and forth many small pale yellow flowers, after bowels coming of cold. The seed is effec- which come small round heads, green at tual to all the purposes aforesaid (except first, and reddish afterwards, but black the last) and works more powerfully. The when they are ripe, wherein is contained distilled water of the herb helps the quinsy the seed. The root is not very great, but in the throat, if the mouth and throat be exceeding long, running down half a man's gargled and washed therewith, and helps length into the ground, red and very clear, the pleurisy, being drank three or four while it is fresh, spreading divers ways. times. Being dropped. into the eyes, it takes away the redness or dimness of them; it likewisc takes away spots or freckles in the face. 'The leaves bruised, and fried with a litite hog's lard, and put hot to any blotch or boil, will quickly break it.

\section{IUNGWORT. P. 15 .}

Descript.] Tris is a kind of moss, that grows on sundry sorts of trees, especially oaks and beeches, with broad, greyish, tough leaves diversly folded, crumpled, and gashed in on the edges, and some spotted also with many small spots on the upper- available for the palsy and sciatica, and side. It was never seen to bear any stalk effectual for bruises inward and outward, or flower at any time.

Place.] It is only manured in gardens, or larger fields, for the profit that is made thereof.

Time.] It flowers towards the end of Sunmer, and the seed is ripe quickly after. Government and virtues.]. It is an herb of Mars. It hath an opening quality, and afterwards to bind and strengthen. It is a sure remedy for the yellow jaundice, by opening the obstructions of the liver and gall, and cleansing those parts; it opens also the obstructions of the spleen, and dininishes the melancholy humour. It is and is therefore much used in vulnerary Goremment and virtues.] Jupitcr scems drinks. 'The root for all those aforesaid to own this herb. It is of great use to purposes, is to be boiled in wine or water, physicians to lielp the diseases of the lungs, as the cause recuires, and some honey and and for coughs, wheezings, and shortness of sugar put therento afterwards. 'The seed breath, which it cures both in man and hercof taken in vinegar and honey, helps beast. It is rery profitable to put into the swelling and hardness of the spleeu. lotions that are taken to stay the moist? The decoction of the leaves and brancines lumours that flow to ulcers, and hinder is a good fomentation for women that have their healing, as also to wash all other ulcers not their courses. 'The leares and roots in the privy parts of a man or woman. It beaten and applied to any part that is dis- 
coloured with freckles, morphew, the white with the cough, shortness of breath, the scurf, or any such deformity of the skin, yellow jaundice, diseases of the spleen, cleanses thoroughly, and takes them away. stopping of urine, and helps exceedingly to MAIDEN HAIR. $P$

break the stone in the kidneys, (in all which diseases the Wall Rue is also very effectual.)

Descript.] OuR common Maiden-Hair It provokes women's courses, and stays doth, from a number of hard black fibres, both bleedings and fluxes of the stomach send forth a great many blackish shining and belly, especially when the herb is dry ; brittle stalks, hardly a span long, in many for being green, it loosens the belly, and not half so long, on each side set very thick voids choler and phlegm from the stomach with small, round, dark green leaves, and spitted on the back of them like a fern.

Place.] It grows upon old stone walls in the West parts in Kent, and divers other places of this land; it delights likewise to grow by springs, wells, and rocky moist and shady places, and is always green.

WALL RUE, OR, WHITE MAIDEN-HAIR.

Descript.] ThIs has very fine, pale green stalks, almost as fine as hairs, set confusedly with divers pale green leaves on every short foot stalk, somewhat near unto the colour of garden Rue, and not much differing in form but more diversly cut in on the edges, and thicker, smooth on the upper part, and spotted finely underneath.

Place.] It grows in many places of this land, at Dartford, and the bridge at Ashford in Kent, at Beaconsfield in Buckinghamshire, at Wolly in Huntingtonshire, on Framlingham Castle in Suffolk, on the church walls at Mayfield in Sussex, in and I shall say no more but only describe Somersetshire, and divers other places of it to you, and for the virtues refer you to this land; and is green in Winter as well as Summer.

Government and virtues.] Both this and? the former are under the dominion of Mercury, and so is that also which follows after, and the virtue of both are so near alike, that though I have described them and their places of growing severally, yet I shall in writing the virtues of them, join them both together as follows.

The decoction of the herb Maiden-Hair being drank, helps those that are troubled the former, since whittever is said of them, may be also said of this.

Descript.] It has many small, brownish, red hairs, to make up the form of leaves growing about the ground from the root; and in the middle of them, in Summer, rise small stalks of the same colour, set with very fine yellowish green hairs on them, and bearing a small gold, yellow head, less than a wheat com, standing in a great husk. The root is very small and thready. Place.] It grows in bogs and moorish 
places, and also on dry shady places, as to those purposes. The same used by Hampstead Healh, and elsewhere. nurses procures them store of milk. The MALLOWS AND MARSHMALLOWS.

decoction of the seed of any of the common Mallows made in milk or wine, doth marCommon Mallows are generally so well vellously help excoriations, the phthisic, known that they need no description.

Our common Marshmallows have divers soft hairy white stalks, rising to be three or four feet ligh, spreading forth many branches, the leaves whereof are soft and hairy, somewhat less than the other Mallow leaves, but longer pointed, cut (for the most part) into some few divisions, but 'leep. The flowers are maty, but smaller also than of them therein, do help women to a speedy the other Mallows, and white, or tending to and easy delivery. "Pliny saith, that whoa bluish colour. After which come such soever takes a spoonful of any of the Mallong, round cases and sceds, as in the other lows, shall that day be free from all diseases Mallows. The roots are many and long, that may come unto him; and that it is slooting from one head, of the bigness of a especially good for the falling-sickness. The thumb or finger, very pliant, tough, and syrup also and conserve made of the flowers, being like liquorice, of a whitish yellow are very effectual for the same diseases, colour on the outside, and more whitish and to open the body, being costive. The within, full of a slimy juice, which being leaves bruised, and laid to the eyes with laid in water, will thicken, as if it were a litlle honey, take away the imposthumajelly.

Place.] The common Mallows grow in tions of them. The leaves bruised or rubbed every county of this land. The common the like, presently take away the pain, Marsh-mallows in most of the salt marshes, redness, and swelling that rise thereupon. from Woolwich down to the sea, both on And Dioscorides saith, The decoction of the Kentish and Essex shores, and in divers the roots and leaves helps all sorts of poison, other places of this land.

Time.] They flower all the Summer montlis, even until the Winter do pull them down. Gorernment and virtues.] Venus owns especial remedy against all hard tumours
them both. Tlie leaves of either of the and inflammations, or imposthumes, or so as the poison be presently voided by vomit. A poultice made of the leaves boiled and bruised, with some bean or bar. ley flower, and oil of Roses added, is an sorts, both specified, and the roots also swellings of the privitics, and other parts, boilcel in wine or water, or in broth with and eases the pains of them; as also against Parsley or Fennel roots, do help to open the the hardness of the liver or splcen, being body, and are very convenient in hot agues, applied to the places. The juice of Malor other distempers of the body, to apply lows boiled in old oil and applied, takes the leares so boiled warm to the belly. It away all roughness of the skin, as also the not only voids hot, choleric, and other scurf, dandriff, or dry scabs in the head, or offerisive humours, but eases the pains and other parts, if they be anointed therewith, torments of the belly coming thereby ; and or washed with the decoction, and preserves are therefore used in all clysters conducing the hair from falling off. It is also effec-
$(11,12$.
G $\mathrm{G}$ 
tual against scaldings and burnings, St. drink, to those that are wounded, and ready Anthony's fire, and all other hot, red, and to faint through loss of blood, and applied painful swellings in any part of the body. the same, mixed with honey and rosill, to The flowers boiled in oil or: water (as every the wounds. As also, the roots boiled in one is disposed) whereunto a little honey wine to those that have received any liurt and allum is put, is an excellent gargle to by bruises, falls, or blows, or had any bone wash, clcanse or heal any sore mouth or or meniber out of joint, or any swellingthroat in a short space. If the feet bespain, or ache in the muscles, sinews or bathed or washed with the decoction of the? arteries. . The muscilage of the roots, and of leaves, roots, and flowers, it helps much Linseedand Fénugreck put together, ismuch the defluxions of rheum from the head; used in poultices, ointments, and plaisters, if the head be washed therewith, it stays to molify and digest all hard swellings, and the falling and shedding of the liair. The the intlammation of them, and to case pains - green leaves (sailh Pliny) beaten with in any part of the body. The seed either nitre, and applied, draw out thorns or green or dry, mixed with vinegar, cleanses prickles in the Hesh.

The Marshmallows are more effectual in all the diseases before mentioned: The leaves are likewise used to loosen the belly gently, and in decoctions or clysters to ease all pains of the body, opening the strait passages, and making them slippery, whereby the stone may descend the more easily and without pain, out of the reins, kidneys, and bladder, and to ease the torturing pains thereof. But the roots are of more special use for those purposes, as well for coughs, country, was sent for up; the only thing hoarseness, shortness of breath and wheez-I gave him, was Mallows bruised and ings, being boiled in wine, or honeyed boiled both in milk and drink, in two days' water, and drank. The roots and seeds (the blessing of God being upon it) it cured hercof boiled in wine or water, are with him. And I here, to shew my thankfulgood success used by them that have ex- ness to God, in comnunicating it to his coriations in the bowels, or the bloody flux, creatures, leave it to posterity.

by qualifying the violence of sharp fretting huncurs, easing the pains, and healing the soreness. It is profitably taken by them that are troubled with ruptures, cramps, or convulsions of the sinews; and boiled in white wine, for the imposthumes by the throat, conmonly called the ling's evil, and of those kernels that rise behind the ears, and inflammations or swellings in women's breasts. 'The dried roots boiled in milk and drank, is especially good for the chincough. Hippocrates used to give the decoction of the rosts, or the juice thereof, to

You may remember that not long since there was a raging disease called the bloodyflux; the college of physicians not knowing what to make of it, called it the inside plague, for their wits were at $N e$ plus ultra about it: My son was taken with the sanse disease, and the excoriation of his bowels was exceeding great; mysclf being in the creatures, leave it to posterity.

$$
\text { MAPLE TREE. . E. } 1 \%
$$

Government and virtues.] $I_{\mathrm{T}}$ is under the dominion of Jupiter. The decortion either of the leaves'or bark, must needs strengthen the liver much, and so you shall find it to do, if you use it. It is excellently good to open obstructions botl of the liver and spleen, and eases pains of the sides thence proceeding.

WIND MARJORAM. P. 16 ,

CALLED also Origanum, Eastward Mar- 

joram; Wild Marjoram, and Grove Mar- dry places of this land; but it is not my
joram.

purpose to insist upon them. The garden Descript.] Wild or field Marjoram hath kinds being most used and useful.

a root which creeps much under ground, which continues a long time, sending up sundry-brownish, hard, square stalks, with small dark green leaves, very like those of an cxcellent remedy for the brain and other Sweet Marjoran, but harder, and some- parts of the body and mind, under the dowhat broader: at the top of the stalks stand minion of the same planet. Our common tufts of flowers, of a deep purplish red Sweet Marjoram is warming and comforcolour. The seed is small and something table in cold diseases of the head, stomach, blacker than that of Sweet Marjoram.

Place.] It grows plentifully in the borders of corn fields, and in some copses.

Time.] It flowers towards the latter end

of the Summer.
Government and virtues.] This is also liver and spleen. It helps the cold griefs under the dominion of Mercury. It of the womb, and the windiness thereof, and strengthens the stomach and head much, the loss of speech, by resolution of the there being scarce a better remedy growing tongue. The decoction thereof made with for such as are troubled with a sour humour some Pellitory of Spain, and long Pepper, in the stomach; it restores the appetite or with a little Acorns or Origanum, being being lost; helps the cough, and consump-? drank, is good for those that cannot make tion of the lungs; it cleanses the body of water, and against pains and torments in choler, expels poison, and remedies the in - the belly; it provokes women's courses, if firmities of the spleen; helps the bitings of it be used as a pessary. Being made into venomous beasts, and helps such as have powder, and mixed with honcy, it takes poisoned themselves by eating Hemlock, away the black marks of blows, and bruises, Henbane, or Opium. It provokes urine and heing thereunto applied; it is good for the the terms in women, helps the dropsy, and inflammations and watering of the eyes, the scurvy, scabs, itch, and yellow jaun- heing mixed with fine flour, and laid unto dice. The juice being clropped into the them. The juice dropped into the ears, ears, helps deafness, pain and noise in the cases the pains and singing noise in them. ears. And thus much for this herb, be- It is profitably put into those ointments tween which and adders, there is a deadly and salves that are warm, and comfort the antipathy.

SWEET MARJORAM: P. $\%$

outward parts, as the joints and sinews; for swellings also, and places out of joint. The powder thereof snuffed up into the nose SWEET Marjoram is so well known, provokes sneezing, and thereby purges. the being an inhabitant in every garden, that it brain; and chewed in the mouth, draws is needless to write any description thereof, forth much phlegm. The oil made thereof, neither of the Winter Sweet Marjoram, or is very warm and comfortable to the joints Pot Marjoram.

Place. I They grow commonly in garthat are stiff, and the sinews that are hard, to molify and supple them. Marjoram is dens; some sorts grow wild in the bor-much used in all odoriferous water, pówders of corn flelds and pastures, in sun- ders, \&c. thatare for ornament or delight. 
MARIGOLDS. P. $\%$.

These being so plentiful in every garden, and so well known that they need no description.

Time.] They flower all the Summer long, and sometimes in Winter, if it be mild.

Government and virtues.] It is an herb of the Sur, and under Leo. They strengthen the heart exceedingly, and are very expulsive, and a little less effectual in the smallpox and measles than saffron. The juice of Marigold leaves mixed with vinegar, and any hot swelling bathed with it, instantly gives ease, and assuages it. . The flowers, either green or dried, are much used in possets, broths, and drink, as a comforter of the heart and spirits, and to expel any malignant or pestilential quality which might annoy them. A plaister made with the dry flowers in powder, hog's-grease, turpentine, and rosin, applied to the breast, strengthens and succours the heart infinitely in fevers, whether pestilential or not.

\section{MASTERWORT.P.Ib}

\section{Descript.] Common Masterwort has} divers stalks of winged leaves divided into sundry parts, three for the most part standing together at a small foot-stalk on both sides of the greater, and three likewise at the end of the stalk, somewhat broad, and cut in on the edges into three or more divisions, all of them dented about the brims, of a dark green colour, somewhat resembling the leaves of Angclica, but that these grow lower to the ground, and on lesser stalks; among which rise up two or three short stalks about two feet higl, and slender, with such like learcs at the joints which grow below, but with lesser and fewer divisions, bearing unbels of white flowers, and after them thin, flat blackish sceds, bigger than Dill sceds. The root is somewhat greater and growing rather side-ways than down deep in the ground, shooting forth sundry heads, which taste sharp, biting on the tongue, and is the hottest and sharpest part of the plant, and the seed next unto it being somewhat blackish on the outside, and smelling well.

Place.] It is usually kept in gardens with us in England.

Time.] It flowers and seeds about the end of August.

Government and virtues.] It is an herb of Mars. The root of Masterwort is hotter than pepper, and very available in cold griefs and diseases both of the stomach and body, dissolving very powerfully upwards and downwards. It is also used in a decoction with wine against all cold rheums, distillations upon the lungs, or shortness of breath, to be taken morning and evening. It also provokes urine, and helps to break the stone, and expel the gravel from the kidneys; provokes women's courses, and expels the dead birth. It is singularly good for strangling of the mother, and other such like fcminine diseases. It is effectual also against the dropsy, cramps, and falling sickness; for the decoction in wine being gargled in the mouth, draws down much water and phlegm, from the brain, purging and easing it of what oppresses it. It is of a rare quality against all sorts of cold poison, to be taken as there is cause ; it provokes sweat. But lest the taste hereof, or of the seed (which works to the like effect, though not so powerfully) should be too offensive, the best way is to take the water distilled both from the herb and root. The juice hereof dropped, or tents dipped therein, and applied either to green wounds or filthy rotten ulcers, and those that come by envenomed weapons, doth soon cleanse and heal them. 'The same is also very good to help the gout coming of a cold cause.

SWEET MAUDLIN.

Descript.] Common Maudlin hath some- 
what long and narrow leaves, snipped about women's minds should run a gadding the edges. The stalks are two feet high, Also a plaister made of the fruit dried bebearing at the tops many yellow flowers set? fore they are rotten, and other conrenient round together and all of an equal height, things, and applied to the reins of the back, in umbels or tufts like unto tansy; after stops miscarriage in women with child, which follow small whitish seed, almost as "They are powerful to stay any Huxes of big as wormseed.

Place and Time.] It grows in gardens, and Howers in June and July.

Government and virtues.] The Virtues hereof being the same with Costmary or defluxions of blood to stay it, or of humours, Alecost, I shall not make any repetition which causes the pains and swellings. It thereof, lest my book grow too big; but is a good bath for women, that have their rather refer you to Costmary for satis- courses flow too abundant: or for the piles faction.

THE MEDIAR. P. $1 \%$

Descript.] Tine Tree grows near the bigness of the Quince Tree, spreading branches reasonably large, with longer and narrower leaves than either the apple or quince, and not dented about the edges. At the end of the sprigs stand the flowers, made of five white, great, broad-pointed leaves, nicked in the middle with some white threads also; after which comes the ? fruit, of a brownish green colour, being ripe, bearing a crown as it were on the top, which were the five grecn leaves; and being rubbed off, or fallen away; the head of the fruit is seen to be somewhat hollow. The fruit is very harsh before it is mellowed, and has usually five hard kernels within it. There is another" kind hercof notling differing from the former, but that it hath some thorns on it in several places, which the other hath not; and usually the "fruit is small, and not so pieasant.

Time and Place.] 'They grow in this land, and flower in May for the most part, and bear fruit in September and October.

Gocernment and virtues.] The fruit is old Saturn's, and sure a better medicine he hardly hath to strengthen the retentivé faculty; therefore it stays women's longings: 'The good old man cannot endure $(11,12$. blood or humours in men or women; the leaves also have this quality. The decoction of them is good to gargle and wash the mouth, throat and teeth, when there is any when they bleed too much. If a poultice or plaister be made with dried medlars, beaten and mixed with the juice of red roses, whercunto a ferw cloves and nutmegs may be added, and a little red coral also, and applied to the stomach that is given to casting or loathing of meat, it effectually helps. The dried leaves in powder strewed on fiesh bleeding wounds restrains the blood, and heals up the wound quickly. The medlar-stones made into powder, and drank in wine, wherein some Parsley-roots have Tain infused all night, or a little boiled, do break the stone in the kidneys, helping to expel it.

\section{MELLILOT, OR KING'S CLAVEK.}

Descript.] Tris Thath many grecn stalls, two or three feet high, rising from a tough, long, white root, which dies not every year, set round about at the joints with small and somewhat long, well-smelling leaves, set three iogether, unevently dented about the edges. The flowers are yellow, and well-smelling also, made like other trefoil, but small, standing in long spikes one above another, for an hand breath long or better, which afterwards turn into long crooked pods, wherein is contained flat seed, somewhat brown.

Place.] It grows plentifully in many places of this land, as in the cdge of Suftolls. II II 
and in Essex, as also in Huntingdonshire, male Mercury two small, round green and in other places, but most usually in heads, standing together upon a short corn fields, in corners of meadows.

Time.] It flowers in June and July, and is ripe quickly after.

Government and virtues.] Melilot, boiled in wine, and applied, mollifies all hard tumours and inflammations that happen in the eyes, or other parts of the body, and sometimes the yolk of a roasted egg, or fine flour, or poppy seed, or endive, is added unto it. It helps the spreading ulcers in the head, it being washed with a lye made thereof. It helps the pains of the stomach, being applied fresh; or boiled with any of of it.

the aforenamed things; also, the pains of the ears, bcing dropped into them; and steeped in vinegar, or rose water, it mitigates the head-ache. The flowers of Mellilot or Camomile are much used to be put together in clysters to expel wind, and ease pains; and also in poultices for the same purpose, and to assuage swelling tumours in the spleen or other parts, and helps inflammations in any part of the body. The juice dropped into the eyes, is a singularly good medicine to take away the film or skin that clouds or dimns the eye-sight. The head often washed with the distilled water of the herb and flower, or a lye made therewith, is effectual for those that suddenly lose their senses; as also to strengthen the memory, to comfort the head and brain, and to preserve them from pain, and the apoplexy.

FRENCH AND DOG MERCURT. P. 16.

Descript.] Tirs rises up with a square green stalk full of joints, two feet high, or thereabouts, with two leaves at every joint, and the branches likewise from both sides of the stalk, set with fresh green leaves, somewhat broad and long, about the bigness of the leaves of Bazil, fincly dented about the ter, and shoot forth new branches every edges; towards the tops of the stalk and year, for the old lie down to the ground. branches, come forth at every joint in the Place.] The male and female French

HAviNG described unto you that which is called French Mercury, I come now to shew you a description of this kind also.

Descript.] This is likewise of two kinds, maleand Female, having many stalks slender and lower than Mercury, without any branches at all upon them, the root is set with two leaves at every joint, somewhat greater than the female, but more pointed and full of veins, and somewhat harder in handling: of a dark green colour, and less dented or snipped about the edges. At the joints with the leaves come forth longer stalks than the former, with two lairy round seeds upon them, twice as big as those of the former Mercury. The taste hereof is herby, and the smell somewhat strong and virulent. The female has much harder lcaves standing upon longer footstalks, and the stalks are also longer ; from the joints come forth spikes of flowers like the French Female Mercury. The roots of them both are many, and full of small fibres which run under ground, and mat themselves very much, not perishing as the former Mercuries do, but abicle the Win- 
Mercury are found wild in divers places of or the juice rubbed upon warts, takes them this land, as by a village called Brookland a away. The juice mingled with some vinein Rumney Marsh in Kent.

The Dog Mercury in sundry places of worms, and the itch. Galen saitl, that Kent also, and elsewhere; but the female being applied in manner of a poultice to more seldom than the malc.

Time.] They flower in the Summer months, and therein give their seed.

Government and virtues.] Mercury, they say, owns the herb, but I rather think it is Mercury, although it be less used, yet may Venus's, and I am partly confident of it sserve in the same manner, to the same purtoo, for I never heard that Mercury ever pose, to purge waterish and melancholy minded women's business so much: I liumours.

believe he minds his study more. 'The decoction of the leaves of Mercury, or the juice thereof in broth, or drank with a little sugar put to it, purges choleric and waterish humours. Hippocrates commended it Mint, or Heart Mint, being most usual, wonderfully for women's diseases, and ap- I shall only describe as follows :

plied to the secret parls, to ease the pains of Descript.] Spear. Mint has divers round the mother; and used the decoction of it, stalks, and long but narrowish leaves set both to procure women's courses, and to thereon, of a dark green colour. The expel the after-birth; and gave the de-flowers stand in spiked heads at the tops coction thereof with myrrh or pepper, or of the branches, being of a pale blue used to apply the leaves outwardly against colour. The smell or scent thereof is somethe stranguary and diseases of the reins and what near unto Bazil; it encreases by the bladder. He used it also for sore and root under ground as all the others do. watering eyes, and for the deafness and Place.] It is an usual inhabitant in garpains in the ears, by dropping the juice dens; and because it seldom gives any thereof into them, and bathing them after- good seed, the seed is recompensed by wards in white wine. The decoction there- the plentiful increase of the root, which of made with water and a cock chicken, is being once planted in a garden, will hardly a most safe medicine against the hot fits of be rid out again.

agues. It also cleanses the breast and lungs Time.] It flowers not until the beginning of phlegm, but a little offends the stomach. : of August, for the most part.

The juice or distilled water snuffed up into Government and virtues.] It is an herb the nostrils, purges the head and eyes of of Venus. Dioscorides saith it hath a catarrhs and rheums. Some use to drink healing, binding and drying quality, and two or three ounces of the distilled water, therefore the juice taken in vinegar, stays with a little sugar put to it, in the morning bleeding: It stirs up venery, or bodily lust; fasting, to open and purge the body of two or three branches thereof taken in the gross, viscous, and melancholy humours. juice of four pomegranates, stays the hicMatthiolus saith, that both the seed of the cough, vomiting, and allays the choler. It male and female Mercury boiled with dissolves imposthumes being laid to with Wormwood and drank, cures the yellow barley-meal. It is good to repress the jaundice in a speédy manner. 'The leaves " milk in women's breasts, and for such as 
have swollen, nams...; Applied with salt, it helps the biting if a sled and held in the mouth.

mad $\operatorname{dog}$; with mead and honeyed water, The virtues of the Wild or Horse Mint, it eases the pains of the ears, and takes such as grow in ditches ? whose description away the roughness of the tongue, being? I purposely omitted, in regard they airo rubbed thereupon. It suffers not milk to well known) are serviceable to dissolve wind curdle in the stomach, if the leaves thereof in the stomach, to help the cholic, and those be steeped or boiled in it before you drink that are short-winded, and are an especial it. Briefly it is very profitable to the remedy for those that have veneral dreams stomach. The often use hereof is a very and pollutions in the night, being outwardly powerful medicine to stay women's courses applicd. The juice dropped in to the ears and the whites. Applied to the forehead eases the pains of then, and destroys the and temples, it eases the pains in the head, worms that breed therein. They are good and is good to wash the heads of young against the venemous biting of serpents. children therewith, against all manner of ?he juice laid on warm, helps the king's breakings-out, sores or scabs, therein. It evil, or kernels in the throat. 'The decoction is also profitable against the poison of ve- or distilled water helps a stinking breath, nomous creatures. The distilled water of proceeding from corruption of the tectl, Mint is available to all the purposes afore- and snuffed up the nose, purges the head. said, yet more weakly. But if a spirit Pliny saith, that eating of the leaves hath thereof be rightly and chymically drawn, been found by experience to cure the it is much more powerful than the herb leprosy, applying some of them to the face, itself. Simeon Sethi saith, it helps a cold liver, strengthens the belly, causes digestion, head used with vinegar. They are extremely stays vomits and hiccough.; it is good bad for wounded people; and they say a against the gnawing of the heart, provokes wounded man that eats Mint, his wound appetite, takes away obstructions of the will never be cured, and that is a long day. liver, and stirs up bodily lust; but therefore? too much must not be taken, because it makes the blood thin and wheyish, and turns it into choler, and therefore choleric persons must abstain from it. It is a safe medicine for the biting of a mad dog, being bruised with salt and laid thereon. 'The powder of it being dried and taken after micat, helps digestion, and those that are splenctic. 'Taken with wine, it helps women? with a greyish green bark, having two leaves in their sore travail in child-bearing. It is set at every joint, and at the end likewise, good against the gravel and stone in the which are somewhat long and narrow, small kidneys, and the stranguary. Being at the botton, but broader towards the end. smelled unto, it is comfortable for the head At the knots or joints of the boughs and and memory. The decoction hereof gar- branches grow sinall yellow flower's, which gled in the nouth, cures the gums and run into small, round, white, transparent mouth that are sore, and mends an ill-berries, three or four together, full of a savoured breath ; as also the Rue and Cori-glutinous moisture, with a blackish seed in ander, causes the palate of the mouth to each of them, which was never yet known 
to spring, being put into the ground, or any where else to grow.

Place.] It grows very rarely on oaks with us; but upon sundry other, as well timber as fruit trees, plentifully in woody groves, and the like, through all this land.

Time.] It flower's in the Spring-time, days together. Some have so highly esbut the berries are not ripe until October, teemed it for the virtues thereof, that they and abides on the branches all the Winter, liave called it Ligmum Sanctice Crucis, Wood unless the blackbirds, and other birds, do of the Holy Cross, believing it helps the devour them.

falling sickness, apoplexy and palsy very

Government and virtues.] This is under speedily, not only to be inwardly taken, but the dominion of the Sun, I do not question; to be hung at their neck. Tragus saith, and can also take for granted, that which that the fresh wood of any Misselto bruised, grows upnn oaks, participates something of the nature of Jupiter, because an oak is one of his trees; as also that which giows upon pear trees, and apple trees, participates something of his nature, because he rules the tree it grows upon, having no root of its own. But why that should have most virtues that grows upon oaks I know not, unless because it is rarest and hardest to conie by ; and our college's opinion is in this contrary to scripture, which saith, God's tender mercies are over all his works; and so it is, let the college of physicians walk as contrary to him as they please, and that is as contrary assthe east to the west. Clusius affirms that which grows upon pear trees to be as prevalent, and gives order, that it should not touch the ground after it is gathered; and also saith, that, being hung about the ncck, it remedies witchcraft. Both the leaves and berries of Misselto do heat and dry, and are of subtle parts ; the birdlime doih molify hard knots, tumours, and imposthumes; ripens and discusses them, and draws forth thick as well as thin hunours from the remote parts of the body, digesting and separating them. And being mixed with equal parts of rozin and wax, doth molify the hardness of the spleen, and helps old ulcers and sores. Being mixed with Sandaric and Orpiment, it helps to draw off foul nails; and if quick-lime and $(11,12$.

wine lees be added thereunto, it works the stronger. The Misselto itself of the oak (as the best) made into powder, and given in drink to those that have the falling sickness, does assuredly heal them, as Matthiolus saith : but it is fit to use it for forty and the juice drawn forth and dropped in the ears that have imposthumes in them, doth help and ease them within a few days.
MONFYWORT, OR HERB TWOPENCE

Descript.] ThE common Moneywort sends forth from a small thready root divers long, weak, and slender branches, lying and running upon the ground two or three feet long or more, set with leaves two at a joint one against another at equal distances, which are almost round, but pointed at the ends, smooth, and of a good green colour. At the joints with the leaves from the middle forward come forth at every point sometimes one ycllow flower, and sometimes two, standing each on a small foot-stalk, and made of five leaves, narrow-pointed at the end, with some yellow threads in the middle, which being past, there stand in their places small round heads of seed.

Place.] It grows plentifully in almost all places of this land, commonly in moist grounds by hedge-sides, and in the middle of grassy fields.

Time.] 'They flower in June and July, and their seed is ripe quickly after.

Government and virtues.] Venus owns it. Moneywort is singularly good to stay all fiuxes in man or woman, whether they be laslis, bluody-fluxes, bleeding inwardly or outwardly, or the weakness of the stomach I I 
that is given to casting. It is very good and May; for in June, when any hot also for the ulcers or excoriations of the weather comes, for the most part it is lungs, or other inward parts. It is exceed-withered and gone.

ingly good for all wounds, either fresh or

Govermment and virtues.] The Moon owns green, to heal them speedily, and for all the herb. Moonwort is cold and drying old ulcers that are of spreading natures. more than Adder's Tongue, and is therefore For all which.purposes the juice of the held to bc more available for all wounds herb, or the powder drank in water where- both inward and outward. The leaves in hot steel hath been often quenched; or boiled in red wine, and drank, stay the the decoction of the green herb in wine or immoderate flux of women's courses, and water drank, or used to the outward place, the whites. It also stays bleeding, vomitto wash or bathe them, or to have tents ing, and other fluxes. It helps all blows dipped therein and put into them, are ef- and bruises, and to consolidate all fracfectual.

\section{MOONWORT. P. $1 \%$}

Descript.] IT rises up usually but with one dark green, thick and tlat leaf, standing upon a short foot-stalk not above two fingers breadth; but when it flowers it may be said to bear a small slender stalk about four or five inches high, having but one leaf in the middle thereof, which is much divided on both sides into sometimes five or seven parts on a side, sometimes more; each of which parts is small like the middle rib, but broad forwards, pointed and round, resembling therein a half-moon, from whence it took the name; the uppermost parts or divisions being bigger than the lowest. The stalks rise above this leaf two or three inches, bearing many branches of small long tongues, erery onc like the spiky head of the adder's tongue, of a brownish colour, (which, whether I shall call them flowers, or the seed, I well know not) which, after they have continued awhile, resolve into a mealy dust. 'The root is small and fibrous. 'This hath sometimes divers such like leaves as are before described, with so many branches or tops rising from one stalk, cach divided from the other.

Place.] It grows on hills and heaths, yet where there is much grass, for therein it delights to grow.

Time.] It is to be found only in April? tures and dislocations. It is good for ruptures, but is chiefly used, by most with other herbs, to make oils or balsams to heal fresh or green wounds (as I said before) either inward or outward, for which it is excellently good.

Moonwort is an herb which (they say) will open locks, and unshoe such horses as tread upon it: This some laugh to scorn, and those no small fools neither; but country people, that I know, call it Unshoe the Horse. Besides I have heard commanders say, that on White Down in Dcvonshire, near 'Tiverton, there were found thirty horse shoes, pulled off from the feet of the Earl of Essex's horscs, being there drawn up in a body, many of them being but newly shod, and no reason known, which caused much admiration: the herb described usually grows upon heaths.

$$
\text { MOSSES. 卫. } 17
$$

I sin LI not trouble the reader with a description of these, since my intent is to speak only of two kinds, as the most principal, viz. Ground Moss and Tree Moss, both which arc very well known.

Place.] The Ground Moss grows in our moist woods, and at the bottom of hills, in boggy grounds, and in shadowy ditches, and many other such like places. The Treo Moss grows only on trees.

Government and virtues.] 
Mosses are under the dominion of Saturn. every joint, which are somewhat broad and The Ground Moss is held to be singularly long, as if it wcre rough or crumpled, with good to break the stone, and to expel and many great veins therein of a sad grcen drive it forth by urinc, being boiled in wine colour, and deeply dented about the edges, and drank. The herb being bruised and and ahmost divided. From the middle of boiled in water, and applied, eases all in- the branches up to the tops of them (which flammations and pains coming from an hot are long and small) grow the flowers round causc; and is therefore used to ease the them at distances, in sharp pointed, rough, pains of the gout.

The'Trec ATosses are cooling and binding, than Balm or Horehound, but in the same and partake of a digesting and molifying; quality withal, as Galen saith. But each which come small, round, blackish seeds in Moss partakes of the nature of the tree great plenty. 'The root sends forth a numfrom whence it is taken; therefore that of her of long strings and small fibres, taking the oak is more binding, and is of good effect strong hold in the ground, of a dark yellowto stay fluxes in man or woman; as also ish or brownish colour, and abides as the volniting or bleeding, the powder thereot Horehound docs: the smell of the one not being taken in wine. The decoction there- much differs from the other.

of in wine is very good for women to be bathed in, that are troubled with the over- in flowing of their courses. The same being drank, stays the stomach that is troubled with casting, or hiccough; ; and, as Avicena saith, it comforts the licart. T'he powder thereof taken in drink for some time together, is thought available for the dropsy. The oil that hąs had fresh Moss steeped therein for a time, and afterwards boilcd and applied to the temples and forehead, marvellously eases the head-ache coming of a hot cause; as also the distillations of liot rheums or humours in the eyes, or other parts. The ancients much used it in their ointments and other medicines against the lassitude, and to strengthen and comfort the sinews: For which, if it was good wi then, I know no reason but it may be found so still.

\section{MOTIERWORT.P̉. 15.}

Descript.] Tus hath a hard, square, brownish, rough, strong stalk, rising three or four feet high at least, spreading into many branches, whereon grow leaves on each side, with long foot-stalks, two at and to help cramps and convulsions. in England.

Government and virtues.] Venus owns the herb, and it is under Leo. There is no better herb to take melancholy vapours from the heart, to strengthen it, and make a merry, chearful, blithe soul than this herb. It may be kept in a syrup or conserve; therefore the Latins called it Cardiaca. Besides, it makes women joyful mothers of children, and settles their wombs as they should be, therefore we call it Motherwort. It is held to be of much use for the trembling of the heart, and faintings and swoonings; from whence it took the name Cardiaca. The powder thereof, to the quantity of a spoonful, drank in wine, is a wonderful help to women in their sore travail, as also for the suffocating or risings of the mother, and for these effects, it is likely it took the name of Motherwort with us. It also provokes urine and women's courses, cleanses the chest of cold phlegm, oppressing it, kills worms in the belly. It is of good use to warm and dry up the cold humours, to digest and disperse them that are settled in the veins, joints, and sinews of the body,

Place.] It grows only in gardens with us 
MOUSE-EAR. P. 16.19 .

Descript.] Mouse-ear is a low herb, creeping upon the ground by small strings, like the Strawberry plant, whereby it shoots forth small roots, whereat grow, upon the ground, many small and somewhat short leaves, set in a round form together, and very hairy, which, being broken, do give a whitish milk: From among these leaves spring up two or three small hoary stalks about a span high, with a few smaller leaves thereon; at the tops whereof stands usually but one flower, consisting of many pale yellow leaves, broad at the point, and a little dented in, set in three or four rows (the greater uppermost) very like a Dandelion flower, and a little reddish underneath about the edges, especially if it grow in a dry ground; which after they hare stood long in flower do turn into down, which with the seed is carried away with the wind.

Place.] It grows on ditch banks, and sometimes in ditches, if they be dry, and in sandy grounds.

Time.] It flowers about June or July, and abides green all the Winter.

Government and virtues.] The Moon owns this herb also; and though authors cry out upon Alchymists, for attempting to fix quicksilver by this herb and Moonwort, a Roman would not have judged a thing by the success; if it be to be fixed at all, it is by lunar influence. The juice thercof taken in wine, or the decoction thereof drank, doth help the jaundice, although of long continuance, to drink thereof norning and evening, and abstain from other drink two or three lours after. It is a special remedy against the stone, and the tormenting pains thereof: as also other tortures and griping pains of the bowels. The root shoots anew in the Spring. The whole decoction thereof with Succory and Cen- plant is of a reasonable scent, and is more taury is held very effectual to help the easily propagated by the slips than the dropsy, and thom that are inclining there- seed.

unto, and the diseases of the spleen. It ? Place.] It grows plentifully in many stays the fluxes of blood, either at the mouth or nose, and inward bleeding also, for it is a singular wound herb for wounds botle inward and outward: It helps the bloody flux, and helps the abundance of women's courses. There is a syrup made of the juice hereof and sugar, by the apothecaries of Italy, and other places, which is of numch account with them, to be given to those that are troubled with the cough or phthisic. The same àlso is singularly good for ruptures or burstings. The green hierb bruised and presently bound to any cut or wound, doth quickly solder the lips thereof. And the juice, decoction, or powder of the dried herb is most singular to stay the malignity of spreading and fretting cankers and ulcers whatsoever, yea in the mouth and secret parts. The distilled water of the plant is available in all the diseases aforesaid, and to wash outward wounds and sores, by applying tents of cloths wet therein.

\section{Descript.] Common Mugwort hath} divers leaves lying upon the ground, very much divided, or cut deeply in about the brims, some what like Worm wood, but much larger, of a dark green colour on the upper side, and very hoary white underneath. The stalks rise to be four or five feet high, having on it such like leaves as those below, but somerwhat smaller, branching forth very much towards the top, whereon are set very small, pale, yellowish flowers like buttons, which fall away, and after them come small seeds inclosed in round heads. The root is long and hard, with many sniall fibres growing tirom it, whereby it takes strong hold on the ground; but both stalks and leaves do lie down every year, and the . Place] 
places of this land, by the water-sides ; as also by small water courses, and in divers other places.

Time.] It flowers and secds in the end of Summer.

Government and virtues.] 'This is an herb there of Venus, thercfore maintains the parts of the berries, helps all inflammations or sores the body she rules, remedies the diseases of in the mouth, or throat, and palate of the parts that are under her signs, Taurus the mouth when it is fallen down. The and Libra. Mugwort is with good success juice of the leaves is a remedy against the put among other herbs that are boiled for biting of serpents, and for those that have women to apply the hot decoction to draw taken aconitc. 'The leaves beatcn with down their courses, to help the delivery of vinegar, are good to lay on any place that the birth, and expel the after-birth. As is burnt with fire. A decoction made of also for the obstructions and inflammations the bark and leaves is good to wash the of the mother. It breaks the stonc, and opens the urinary passages where they are stopped. The juice thereof made up with Myrrh, and put under as a pessary, works the same effects, and so docs the root also. Being made up with hog's grease into an ointment, it takes away wens and hard knots and kernels that grow about the neck and throat, and cases the pains about the neck more cffectually, if some Field Daisies be put with it. 'The herb itself the places. A branch of the tree taken being fresh, or the juice thereof taken, is a when the moon is at the full, and bound to special remedy upon the overmuch taking : the wrists of a woman's arm, whose courses of opium. Thince drams of the powder of come down too much, doth stay them in a the dried leaves taken in wine, is a speedy short space.

and the best ccrtain help for the sciatica. A decoction thereof made with Camomile and Agrimony, and the place bathed therewitl while it is warm, takes away the pains of the sinews, and the cramp.

THE MULBERRYTREF. P. 18.

Tirs is so well known where it grows, that it needs no description.

Time.] It bcars fruit in the montlis of July and August.

Gozermment and rirtues.] Mércury rulés the tree, therefore are its effects variable? for the most part, and are many set together as his are. The Mulberry is of different in a long spike, in some of a ycllow colour, parts; the ripe berrics, by reason of their in others more pale, consisting of five round sweetncss and slippery moisturc, opening "pointed leares, which afterwards have small $(13,14$. mouth and teeth when they ache. If the root be a little slit or cut, and a small hole made in the ground next thcreunto, in the Harvest-time, it will give out a certain juice, which being hardened the next day, is of good use to help the tooth-ache, to dissolve knots, and purge the belly. The leaves of Mulberries are said to stay bleeding at the mouth or nose, or the bleeding of the piles, or of a wound, being bound unto .

\section{Descript.] Common White Mullein has} many fair, large, woolly white leaves, lying next the ground, somcwhat larger than broad, pointed at the cud, and as it were up to be four or five feet high, covered over with such like leares, but less, so that no stalk can be seen for the multitude of leaves thereon up to the flowers, which come forth on all sides of the stalk, without any branches $\mathrm{K} \mathrm{K}$ dented about the edges. 'The stalk rises 
round heads, wherein is small brownish and leal then also. The leaves bruised seed contained. The rool is long, white, and wrapped in double papers, and covered and woody, perishing after it hath borne with hot ashes and embers to bake a while, seed.

Place.] It grows by way-sides and lanes, in many places of this land.

Time.] It flowers in July or thereabouts.

Government and virtues.] It is under the dominion of Saturn. A small quantity of and newly set again, takes away all swelthe root given in wine, is commended by ling and pain thereof.

Dioscorides, against lasks and fluxes of the belly. The decoction hereof drank, is profitable for those that are bursten, and for cramps and convulsions, and for those that are troubled with an old cough. The decoction thereof gargled, eases the pains of the tooth-ache. And the oil made by the often infusion of the flowers, is of very good effect for the piles. The decoction of the root in red ivine or in water, (if there be an ague) iwherein red hot steel hath been often quenched, doth stay the bloody-flux. The same also opens obstructions of the bladder and reins. A decoction of the pods, with small, lank, flat ends, wherein leaves hereof, and of Sage, Marjoram, and is contained round yellowish seed, sharp, Camomile flowers, and the places bathed lot, and biting upon the tongue. The root therewith, that have sinews stiff with cold is small, long, and woody when it bears or cramps, doth bring them much ease and stalks, and perishes every year.

comfort. Three ounces of the distilled water of the flowers drank morning and evening for some days together, is said to be the most excellent remedy for the gout. The juice of the leaves and flowers being Government and virtues.] It is an excel-
laid upon rough warts, as also the powder? lent sauce for such whose blood wants clariPlace.] 'This grows with us in gardens Descript.] Our common Mustard hath large and broad rough leaves, very much jagged with uneven and unorderly gashes, somewhat like turnip leaves, but less and rougher. The stalk rises to be more than a foot high, and sometimes two feet high, being round, rough, and branched at the top, bearing such like leaves thereon as grow below, but lesser, and less divided, and divers yellow flowers one above another at the tops, after which come small rough of the dried roots rubbed on, doth easily fying, and for weak stomach, being an take them away, but doth no good to herb of Mars, but naught for choleric smooth warts. The powder of the dried pcople, though as good for such as are flowers is an especial remedy for those that aged, or troubled with cold diseases. Aries are troubled with the belly-ache, or the claims something to do with it, therefore it pains of the cholic. The decoction of the strengthens the heart, and resists poison. root, and so likewise of the leaves, is of Let such whose stomachs are so weak they great effect to dissolve the tumours, swel- cannot digest their meat, or appetite it, take lings, or inflammations of the throat. The of Mustard-seed a dram, Cinnamon as much, seed and leaves boiled in wine, and ap-and having beaten them to powder, and plied, draw forth speedily thorns or splin- half as much Mastich in powder, and with ters gotten into the flesh, ease the pains, gum Arabic dissolved in rose-water, make 
it up into troches, of which they may take The seed bruised mixed with honey, and one of about half a dram weight an hour or applied, or made up with wax, takes away two before meals; let old men and women the marks and black and blue spots of make much of this medicine, and they will bruises, or the like, the roughness or scab-. either give me thanks, or shew manifest biness of the skin, as also the leprosy, and ingratitude. Mustard seed hath the virtue lousy evil. It helps also the crick in the of heat, discussing, ratifying, and drawing neck. 'T'he distilled water of the herb, when out splinters of bones, and other things of it is in the flower, is much used to drink the flesh. It is of good effect to bring inwardly to help in any of the diseases down women's courses, for the falling-sick-aforesaid, or to wash the mouth when the ness or lethargy, drowsy forgetful evil, to palate is down, and for the disease of the use it both inivardly and outwardly, to rub throat to gargle, but outwardly also for the nostrils, forehead and temples, to warm scabs, itch, or other the like infirmities, and and quicken the spirits; for by the fierce cleanses the face from morpliew, spots, sharpness it purges the brain by sncezing, freckles, and other deformities.

and drawing down rbeum and other viscous humours, which by their distillations upon the lungs and chest, procure coughing, and therefore, with some, lioney added thereto, doth much good therein. The decoction of the seed made in wine, and drank, provokes urine, resists the force of poison, the malignity of mushrooms, and venom of scorpions, or other venomous creatures, if it be taken in time; and taken before the cold fits of agues, alters, lessens, parts, some bigger, and some less, of a
and cures them. The seed taken either by dirty green colour. The flowers are small itself, or with other things, either in an elec- and yellow, that grow on the tops of the tuary or drink, doth mightily stir up bodily branches in long spikes, flowering by delust, and helps the spleen and pains in the sides, and gnawings in the bowels; and the stalk will have small round cods at the used as a gargle draws up the palate of the bottom, growing upright and close to the mouth, being fallen down; and also it dis- stalk, while the top flowers yet shew themsolves the swellings about the throat, if it selves, in which are contained small yellow be outwardly applied. Being chewed in seed, sharp and strong, as the herb is also. the mouth it oftentimes helps the tooth-ache. 'T'he root grows down slender and woody, The outward application hereof upon the yet abiding and springing again every pained place of the sciatica, discusses the year.

humours, and cases the pains, as also the gout, and other joint aches; and is much and often used to ease pains in the sides or loins, the shoulder, or other parts of the body, upon the plying thereof to raise July. blisters, and cures the disease by drawing Government and virtues.] Mars owns this. it to the outward parts of the body. It is herb also. "It is singularly good in all the. also used to help the falling off the hair. 
of voice: and by the use of the decoction thereof for a little space, those have been recovered who had utterly lost their voice, and almost their spirits also. The juice thereof made into a syrup, or licking medicine, with honey or sugar, is no less effectual for the same purpose, and for all other coughs, wheezing, and shortness of breath. The same is also profitable for those that have the jaundice, pleurisy, pains in the hack and loins, and for torments in the belly, or cholic, being also used in clysters. The seed is held to be a special remedy against poison and venom. It is singularly good for the sciatica, and in joint-aches, ulcers, and cankers in the mouth, throat, or behind the ears, and no less for the hard-
ness and swelling of the testicles, or of 'women's breasts.

\section{NAILWORT, OR WHITLOW-GRASS. P. 18}

Descript.] Tir rs very small and common herb hath no roots, save only a few strings: neither doth it ever grow to be above a hand's breadth high, the leaves are very small, and something long, not much unlike those of Chickweed, among which rise up divers slender stalks, bearing many white flowers one above another, which are exceeding small; after which come small Hat pouches containing the seed, which is very small, but of a sharp taste.

Place.] It grows commonly upon old stone and brick walls, and sometimes in gravelly grounds, especially if there be grass or moss near to shadow it.

Time.] They flower very early in the year, sometimes in January, and in in wine, is profitable for those that are February; for before the end of $A$ pril they bruised by an accident. The green herb are not to be found.

Government and virtues.] It is held to be lying there two or three hours, eases the cxccedingly good for those imposthumes in pains of the piles; the juice also being made the joints, and under the nails, which they up into an ointment, is effectual for the call Whitlows, Felons, Andicorns and Nail-s same purpose. The head washed with a whicals. bruised and applied to the fundament, and

\section{NEP, OR CATMINT.}

Plates. 17. 18 .

\section{Descript.] Comno n Garden Nep shoots} forth hard four-square stalks, with a hoariness on them, a yard high or more, full of branches, bearing at every joint two broad leaves like balm, but longer pointed, softer, white, and more hoary, nicked about the edges, and of a strong sweet scent. The flowers grow in large tufts at the tops of the branches, and underneath them likewise on the stalks many together, of a whitish purple colour. The roots are composed of many long strings or fibres, fastening themselves stronger in the ground, and abide with green leaves thereon all the winter. gardens.

Time.] And it flowers in July, or thereabouts.

Governmenit and virtues.] It is an herb of Venus. Nep is generally used for women to procure their courses, being taken inwardly or outwardly, either alone, or with other convenient herbs in a decoction to bathe them, or sit over the hot fumes thereof; and by the frequent use thereof, it takes away barrenness, and the wind, and pains of the mother. It is also used in pains of the head coming of any cold cause, catarrhs, rheums, and for swimming and giddiness thereof, and is of special use for the windiness of the stomach and belly. It is effectual for any cramp, or cold aches, to dissolve cold and wind that afflict the place, and is used for colds, coughs, and shortdecoction thereof, it takes away scabs, 
and may be effectual for other parts of the together, stays bleeding at the mouth. 'The body also.

NETTLES. E, 18

Nettes are so well known, that they need no description; they may be found by feeling, in the darkest night.

Government and virtues.] 'This is also temples in the lethargy, and the places an herb Mars clains dominion over. You stung or bitten with beasts, with a little salt. know Mars is lot and dry, and you know The distilled water of the herb is also effecas well that Winter is cold and moist; then tual (though not so powerful) for the disyou may know as well the reason why eases aforesaid; as for outward wounds Nettle-tops eaten in the Spring consume and sores to wash them, and to clcanse the the phlegmatic superfluities in the body of skin from morphew, leprosy, and other man, that the coldness and moistness of discolourings thereof. The seed or leaves Winter hath left behind. The. roots or bruised, and put into the nostrils, stays the leaves boiled, or the juice of either of them, bleeding of them, and takes away the flesh or both made into an electuary with honey growing in them called poly pus. The juice and sugar, is a safe and sure medicine to of the leaves, or the decoction of them, or open the pipes and passages of the lungs, of the root, is singularly good to wash cither which is the cause of wheezing and short- old, rotten, or stinking sores or fistulous, ness of breath, and helps to expectorate and gangrenes, and such as fretting, cating, tough phlegm, as also to raise the impost- or corroding seabs, manginess, and itch, humed pleurisy; and spend it by spitting; in any part of the body, as also green the same helps the swelling of the almonds wounds, by washing them therewith, or apof the throat, the mouth and throat being plying the grcen herb bruised thereunto, gargled therewitl. 'The juice is also effec- yea, although the flesh werc separated from tral to settle the palate of the mouth in its the bones; the same applicd to our wearied place, and to heal and temper the inflam- members, refresh them, or to place those mations and soreness of the mouth and that have been out of joint, being first set throat. The decoction of the leaves in up agaim, strengthens, dries; and comforts wine, being drank, is singularly good to pro- them, as also those places troubled with voke women's courses, and settle the suf- aches and gouts, and the defluxion of focation, strangling of the mother, and all humours upon the joints or sinews; it eases other diseases thereof; it is also applied out- the pains, and dries or dissolves the deflucwardly with a little myrrh. The same also, tions. An ointment made of the juice, or the seed provokes urine, and expels the oil, and a little wax, is singularly good to gravel and stone in the reins or bladder, rub cold and benumbed members. Au often proved to be effectual in many that handful of the leaves of green Nettles, and have taken it. 'The same' kills the worms another of Wallwort, or Deanwort, bruised $\mathrm{i}_{1}$ children, eases pains in the sides, and and applied simply themselves to the gout, dissolves the windiness in the spleen, as sciatica, or joint aches in any part, hath also in the body, although others think it been found to be an adminable help thereonly powerful to provoke venery. The uuto.

juice of the leaves taken two or threc days :

$(13,14$.

I. I. 


\section{NIGITSIIADE.卫, 18 ,}

Descript.] Comnor Nightshade hath an upright, round green, hollow stalk, about a foot or half a yard high, bushing forth in many branches, whereon grow many green leaves, somewhat broad, and pointed at the ends, soft and full of juice, somewhat like unto Bazil, but longer and a lille unevenly dented about the edges: At the tops of the stalks and branches come forth three or 3 four more white flowers made of five small pointed leaves a-piece, standing on a stalk together, one above another, with yellow pointels in the middle, composed of four or five yellow threads set together, which afterwards run into so many pendulous green berries, of the bigness of small pease, full of green juice, and small whitish round flat seed lying within it. 'The root is white, and a little woody when it hath given flower and fruit, with many small fibres at it; The whole plant is of a waterish insipid taste, but the juice within the berries is soniewhat viscous, and of a cooling and binding quality.

Place.]. It grows wild with us under our walls, and in rubbish, the common paths, and sides of hedges and fields, as also in our gardens here in England, without any planting.

Time.] It lies down every year, and rises up again of its own sowing, but springs not until the latter end of April at the soonest.

Govermment and rimules.] It is a cold Saturnine plant. The common Nightshade is wholly used to cool hot inflammations either inwardly or outwardly, being no ways dangerous to any that use it, virulency of cantharides, when one by cating as most of the rest of the Nightshades are; them hath his bladder exulcerated, and yet it must be used moderately. The dis- voids bloody urine. Hippocrates saith, he tilled water only of the whole herb is fittest used the fumes of Oak leaves to women that and safest to be taken inwardly: The juice were troubled with the strangling of the also clarified and taken, being mingled nother ; and Galen applied them, bring with a little vinegar, is good to wash the bruised, to cure grcen wounds. 'I'he dis:

mouth and throat that is inflaned: But outwardly the juice of the herb or berries, with oil of roses and a little vinegar and ceruse laboured together in a leaden mortar, is very good to anoint all hot inflammations in the eyes. It also doth much good for the shingles, ringworms, and in all running, fretting and corroding ulcers, applied thereunto. The juice dropped into the ear's, eases pains thereof that arise of heat or inflammations. And Pliny saith, it is good for hot swellings under the throat. Have a care you mistake not the deadly Nightshade for this; if you know it not, you may let them both alone, and take no harm, having other medicines sufficient in the book.

$I_{\mathrm{T}}$ is so well known (the timber thereof being the glory and safety of this nation by sea) that it needs no description.

Gorernment and virtues.] - Jupiter owns the tree. 'The leaves and bark of the Oak, and the acorn cups, do bind and dry very much. The inner bark of the tree, and the thin slin that covers the acorn, are most used to stay the spitting of blood, and the bloody-flux. The decoction of that bark, and the powder of the cups, do stay vomitings, spitting of bleod, bleeding at the mouth, or other fluxes of blood, in men or wonen; lasks also, and the nocturnal involuntary flux of men. The acorn in powder taken in wine, provokes urine, and resists the poison of venomous creatures: The decoction of acorns and the bark made in milk and taken, resists the force of poisonous herbs and medicines, as also the 
tilled water of the Oaken bud, before they the upper crust of the earth, shooting forth break out into leaves is good to be used in divers places.

either inwardly or outwaidly, to assuage Place.] It grows in moist, shadowy, inflammations, and to stop all manner of and grassy places of woods, in many parts fluxes in man or woman. The same is of this land.

singularly good in pestilential and hot Time.] It flowers about May, and the burning fevers; for it resists the force of berries are ripe in June, and then quickly the infection, and allays the leat: It cools perishes, until the next year it springs from the heat of the liver, breaking the stone in the same root again.

the kidneys, and stays women's courses. Government and virtues.] It is a precious The decoction of the leaves works the same herb of the Sun. Half a dram, or a dram effects. The water that is found in the at most, in powder of the roots hereof taken hollow places of old Oaks, is very effectual in winc and vinegar, of each equal parts, against any foul or spreading scabs. 'The and the party laid presently to sweat theredistilled water (or concoction, which is upon, is held to be a sovereign remedy for better) of the leaves, is one of the best those that are infected with the plague, and remedies that I know of for the whites in have a sore upon them, by expelling the women.

\section{oATs, P. 20.}

ARE so well known that they need no description.

Government and virtues.] Oats fried with bay salt, and applied to the sides, take away the pains of stitches and wind in the sides or the belly. A poultice made of meal of Oats, and some oil of Bays put thereunto, helps the itch and the leprosy, as also the fistulas of the fundament, and dissolves hard imposthumes. 'The meal of Oats boiled with vinegar, and applied, takes away freckles and spots in the face, and other parts of the body.

ONE BLADE.

Descript.] Tuis small plant never bears more than one leaf, but only when it rises up with his stalk, which thercon bears another, and seldom more, which are of a blueish green colour, pointed, with many ribs or veins querein, like Plantain. At the top of the stalk grow many small white flowers, star fashion, smelling somewhat sweet; after which come small red berries, when they are ripe. The root is sunall, of the bigness of a rush, lying and creeping under poison and infection, and defending the heart and spirits from danger. It is a singularly good wound herb, and is thereupon used, with other the like effects in many compound balms for curing of wounds, be they fresh and green, or old and malignant, and especially if the sinews be burnt.

\section{oRCIIIS.}

$\mathrm{I}_{\mathrm{T}}$ has almost as many several names attributed to the several sorts of it, as would almost fill a sheet of paper; as dog-stones, goat-stones, föol-stones, fox-stones, satiricon, cullians, together with many others too tedious to rehearse.

Descript.] 'T' describe all the several sorts of it were an endless piece of work; therefore I shall only describe the roots, because they are to be used with some discretion. 'They have each of them a double root within, some of them are round, in others like a hand ; these roots alter every year by course, when the one rises and waxes full, theother waxes lank, and porishes. Now, it is that which is fill which is 10 be used in medicines, the other being cither of no use at all, or clse, according to the humoun of some, it destroys and disannuls 
the virtues of the other, quite undoing what the place with good treacle, and after to that doth.

Time.] One or other of them may be taking away the outermost skin thereof; found in flower from the beginning of April being beaten together, is a sovereign salve to the latter end of August.

Government and virtues.] They are hot and moist in opcration, under the dominion of Dame Venus, and provoke lust exceedingly, which, they say, the dried and withered roots do restrain. They are held to kill worms in children; as also, being bruised and applied to the place, to heal the king's evil.

$$
\text { ONIONS. P. } 20 \text {. }
$$

for either plague or sore, or any other:

putrefied ulcer. The juice of Onions is good for either scalding or burning by fire, water, or gunpowder, and used with vinegar, takes away all blemishes, spots and marks in the skin: and dropped in the ears, eases the pains and noise of thens. Applied also with figs beaten together, helps to ripen and break imposthumes, and other sores.

Leeks are as like them in quality, as the

THeY are so well known, that I need not pome-water is like an apple: They are a spend time about writing a description of them.

Government and virtues.] Mars owns them, and they have gotten this quality, to help the piles. In other things they have draw any corruption to them, for if you the same property as the Onions, although peel one, and lay it upon a dunghill, you not so effectual.

shall find it rotten in half a day, by drawing putrefaction to it; then, being bruised and applied to a plague sore, it is very probable it will do the like. Onions are flatulent, or windy; yet they do somewhat provoke appetite, increase thirst, ease the belly and bowels, provoke women's courses, help the biting of a mad dog, and of other venomous creatures, to be used with honey and rue, increase sperm, especially the seed of them. They also kill worms in children if they thick, round, white tuberous clogs; and the drink the water fasting wherein they have plant grows not so big in some places as in been steeped all night. Being roasted others where it is found.

under the embers, and eaten with honey or sugar and oil, they much conduce to help? an inveterate cough, and expectorate the tough phlegm. The juice being snuffed up into the nostrils, purges the head, and helps the lethargy, (yet the often ealing them is said to procure pains in the head.) It hath been held by divers country people

Place.] It is frequent in almost erery Descript.] Common Orpine rises up. with divers rough brittle stalks, thick set with. fat and tleshy leaves, without any order, and little or nothing dented about the edges, of a green colour: 'The flowers are white, or whitish, growing in tufts, after which come small chaffy husks, with seeds like dust in them. 'The roots are divers county of this land, and is cherished in gardens with us, where it grows greater than that which is wild, and grows in shadowy sides of fields and woods.

Time.] It flowers about. July, and the seed is ripe in August.

Gorermment and rixtues.] The Moon a great prescrvative against infection, to eat owns the herb, and he that knows but her Onions fasting with bread and salt: As exaltaration, knows what I say is true. also to make a great Onion hollow, filling Orpine is seldom used in inward medicines 
with us, although Tragus saith from expe- is also effectual against the venom of any rience in Germany, that the distilled water poisonous creature, and the danger that thereof is profitable for gnawings or excori- comes to them that have the lethargy, and ations in the stomach or bowels, or for ul- is as good against the cough. The distilled cers in the lungs, liver, or other inward water of Parsley is a familiar medicine with parts, as also in the matrix, and helps all nurses to give their children when they are those diseases, being drank for certain days troubled with wind in the stomach or belly, together. It stays the sharpness of humours which they call the frets; and is also much in the blondy-flux, and other fluxes in the available to them that are of great years. body, or in wounds. The root thereof The leaves of Parsley laid to the eyes that also performs the like effect. It is used are inflamed with heat, or swollen, doth outwardly to cool any heat or inflammation much help them, if it be used with bread or upon any hurt or wound, and eases the meal ; and being fried with butter, and appains of them; as, also, to heal scaldings or burnings, the juice thereof being beaten with some green sallad oil, and anointed. The leaf bruised, and laid to any green wound in the hand or legs, doth heal them quickly; and being bound to the throat, much helps the quinsy; it helps also ruptures and burstenness. If you please to make the juice thereof into a syrup with honey or sugar, you may safely take a spoonful or two at a time, (let my author say what he will) for a quinsy, and you shall find the medicine pleasant, and the cure speedy.

PARSLEY.I.23.

TH Is is so well known, that it needs no description.

Government and virtues.] It is under the dominion of Mercury ; is very comfortable to the stomach; helps to provoke urine and to the stomach ; the to break wind both in the drink after it for three hours. This opens
stomach and bowels, and doth a little open obstructions of the liver and splcen, and the body, but the root much more. It expels the dropsy and jaundice by urine. opens obstructions both of liver and spleen, and is thercfore accounted one of the five ? opening roots. Galen commended it Descript.] Tue root, although it be very
arine mightily; ; especially if the roots be small and thready, yet it continues many boiled, and eaten like Parsnips. The seed years, from which arise many leaves lying is effectual to provoke urine and women's along on the ground, each standing upon courses, to expel wind, to break the stone, a long small foot-stalk, the lacaves as broad and ease the pains and torments thereof; it $;$ as a man's nail, very deeply dented on the 
edges, somewhat like a parsley-leaf, but of the seed being ripe about the beginning of a very dusky green colour. T'lie stalks are August, the second year after its sowing; very weak and slender, about three or four for if they do flower the first year, the counfingcrs in lcngth, set so full of leaves that try people call them Madneps.

they can hardly be seen, either having no Government and virtues.] The garden foot-stalk at all, or but very short; the Parsnips are under Venus. The garden flowers are so small they can hardly be Parsnip nourishes much, and is good and seen, and the seed as small as may bc.

wholesome nourishment, but a little windy,

Place.] It is a common herb throughout whereby it is thought to procure bodily the nation, and rejoices in barren, sandy, lust; but it fastens the body much, if much moist places. It may be found plentifully need. It is conducible to the stomach and about Hampstead Heath, Hyde Park, and reins, and provokes urine. But the wild in Tothill-fields.

Time.] It may be found all the Summer-time, even from the beginning of April to the end of October.

Government and virtues.] Its operation solves wind both in the stomach and bowels, is vcry prevalent to provoke urine, and to which is the cholic, and provokes urine. break the stone. It is a very good sallad The root is often used, but the seed much herb. "It were good the gentry would pickle more. The wild being better than the tamc, it up as they pickle up Samphire for their shews Dame Nature to be the best phyuse all the Winter. I cannot teach them sician.

how to do it; yet this I can tell them, it is a very wholesome herb. They may also liecp the herb dry, or in a syrup, if they please. You may take a dram of the powder of it in white wine; it would bring away lying often on the ground, or else raised a gravel from the kidneys insensibly, and little from it, with long, round, hairy footwithout pain. It also helps the stranguary.

\section{PARSNIPS.}

THE garden kind thereof is so well known each leaf, being almost round, yet some(the root being commonly eatell) that I what decply cut in on the colges in some shall not trouble you with any description leaves, and not so deep in others, of a whitish of it. But the wild kind being of more green colour, smelling somewhat strongly; physical use, I shall in this place describe among which rises up a round, crusted, it unto you.

liairy stalk, two or three fect high, with a Descript.] The wild Parsnip differs few joints and leaves thereon, and branched little from the garden, but grows not so fair at the top, where stand large umbels of and large, nor hath so many leaves, and the white, and sometimes redish flowcrs, and root is shorter, more woody, and not so fit after them flat, whitish, thin, winged sced, to be eaten, and therefore more medicinal. two always joined together. 'The root is

Place.]. The name of the first shews the long and white, with two or thice lnng place of its growth. The other grows wild strings growilg down into the ground, in divers places, as in the marshes in Roches- smclling likewise strongly and unpleasant. ter, and elsewhere, and flowers in July; Place.] It grows in moist mradows, and 
the borders and eorners of fields, and near within the stone. It sooner waxes old, ditches; through this land.

Time.] It flowers in July, and seeds in August.

Government and virtues.] Mereury hath the dominion over them. The seed thereof, and decays, than the A prieot, by mueh.

as Galen saith, is of a sharp and eutting Place.] They are nursed in gardens and orchards through this land.

Time:] They flower in the Spring, and fruetify in Autumn.

Government and virtues.]. Lady Venus quality, and therefore is a fit medieine for owns this tree, and by it opposes the ill a eough and shortness of breath, the falling effeets of Mars, and indeed for children and siekness and jaundice. 'The root is avail-y young people, nothing is better to purge able to all the purposes aforesaid, and is eholer and the jaundice, than the leaves or also of great use to take away the hard fllowers of this tree being made into a syrup skin that grows on a fistula, if it be but or conserve. Let such as delight to please scraped upon it. The seed hereof being their lust regard the fruit; but sueh as have drank, cleanses the belly from tough phleg- lost their liealth, and their ehildren's, let matie matter therein, eases them that are them regard what I say, they may safely liver-grown, women's passions of the mother, give two spoonfuls of the syrup at a time; as well being drank as the smoke thereof it is as gentle as Venus herself. The leaves reeeived, and likewise raises suel as are of peaehes bruised and laid on the belly, fallen into a deep sleep, or have the lethargy, kill wornis, and so they do also being by burning it under their nose. The seed boiled in ale and drank, and open the belly and root boiled in oil, and the head rubbed likewise; and, being dried, is a far safer therewith, helps not only those that are minedieine to discuss humours. The powfallen into a frenzy, but also the lethargy or drowsy evil, and those that have been-long wounds stays their bleeding, and eloses troubled with the head-ache, if it be like- them up. The flowers steeped all night in wise used with Rue. It helps also the runn- a little wine standing warm, strained forth ing seab and shingles. The juiee of the in the morning, and drank fasting, doth flowers dropped into the ears that run and gently open the belly, and move it downare full of matter, eleanses and heals them. ward. A syrup marde of them, as the syrup THE PEACH TREE.

of roses is made, works more forcibly than that of roses, for it provokes vomiting, and

Descript.] A релси Tree grows not so spends waterish and hydropie humours by great as the Aprieot tree, yet spreads the continuance thereof. 'The flowers made branches reasonable well, from whenee into a eonserve, work the same effeet. spring smaller reddish twigs, whereon are 3 The liquor that dropped from the tree, being set long and narrow grcen leaves dented wounded, is given in the decoction of Colisabout the edges. The blossoms are greater foot, to those that are troubled with it eough than the plumb, and of a light purple or shortness of breath, by adding thereunto colour; the fruit round, and somctimes as some sweet wine, and putting some saffron big as a reasonable Pippin, others smaller, also therein. It is good for those that are as also differing in eolour and taste, as rus- hoarse, or have lost their voiec; helps all set, red, or yellow, watcrish or firm, with a defects of the lungs, and those that vonit frize or cotton all over, with a cleft therein and spit blood. Two drams hereof given like an Apricot, and a rugged, furrowed, in the juice of kmons, or of radish, is good great stone within it, and a bitler kernel for them that are troubled with the stone, 
the kernels of the stones do wonderfully much more, and are very good in repelling ease the pains and wringings of the belly medicines; and if the wild sort be boiled through wind or sharp humours, and help with mushrooms, it makes them less danto make an excellent inedicine for the stone? gerous. The said Pears boiled with a little upon all occasions, in this manner: I take honey, help much the oppressed stomach, fifty kernels of peach-stones, and one hundred as all sorts of them do, sone more, some of the kernels of cherry-stones, a handful of lless: but the harsher sorts do more cool elder flowers fresh or dried, and three pints and bind, serving well to be bound to green of Muscadel; set them in a close pot into it bed s. wounds, to cool and stay the blood, and heal of horse-dung for ten days, after which distil : up the green wound without farther trouble, in a glass with a gentle fire, and keep it for or inflammation, as Galen saith he hath found your use: You may drink upon occasion by experience. The wild Pears do sooner three or four ounces at a time. The milk or close up the lips of green wounds than others. cream of these kernels being drawn forth with some Vervain water and applied to the forehead and temples, doth much help to procure rest and sleep to sick persons wanting it. The oil drawn from the kernels, the temples being therewith anointed, doth? working hard, and it will do as well as the like. The said oil put into clysters, drinking wine.

eases the pains of the wind cholic : and anointed on the lower part of the belly, doth the like, and dropped into the ears, eases pains in them; the juice of the leaves? doth the like. Being also anointed on the forehead and temples, it helps the megrim, and all other pains in the head. If the kernels be bruised and boiled in vinegar, until they become thick, and applied to the head, it marvellously procures the hair to grow again upon bald places, or where it is too thin.

THE PEAR TREE. Rathz:

Pear Trees are so well known, that they need no description.

Governmentand virtues.] The Tree belongs place, having a border of many leaves, to Venus, and so doth the Apple tree. For white on the upper side, and reddish undertheir physical use they are best discerned neath, with a yellow thrum in the middle, by their taste. All the sweet and luscious sorts, whether manured or wild, do help to move the belly downwards, more or less. Those that are hard and sour, do, on the contrary, bind the belly as much, and the that before described, from whence arise leaves do so also: Those that are moist do divers brittle stalks, a yard high and more, in some sort cool, but harsh or wild sorts with narrow leaves finely dented about the 
edges, standing one above another up to black, rough seed, which will stick to any the tops. 'The flowers are many and white, cloth or garment that shall touch' it. The standing in tufts like those of Yarrow, with root is soméwhat long, with small fibres a small yellowish thrum in the middle. thereat, of a dark reddish colour, which The seed is very small.

abides the Winter, although the stalks and

Place.] The last grows in fields by the leaves perish and spring every year. hedge sides and paths, almost every where. Place.] It grows wild generally through Time.] It flowers at the latter end of the land, about the borders of fields, and by June and July.

Government and virtues.] It is under the government of Mercury, and I am persuaded it is one of the best purgers of the brain that grows. An ounce of the juice taken in a draught of Muskadel an hour before the fit of the ague comes, it will assuredly drive away the ague at the second or third time taken at the farthest. Either the herb or root dried and chewed in the mouth, purges the brain of plegmatic humours; thereby not only easing pains in honey, is a singular remedy for an old' or the head and teeth, but also hinders the dry cough; the shortness of breath, and distilling of the orain upon the lungs and wheezing in the throat. Three ounces of eyes, thereby preventing coughs, phthisicks the juice thereof taken at a time, doth wonand consumption, the apoplexy and falling derfully help stopping of the urine, and to sickness. It is an excellently approved expel the stone or gravel in the kidneys or remedy in the lethargy. The powder of the bladder, and is therefore usually put among herb or root being snuffed up the nostrils, Oother herbs used in clysters to nitigate procures sneezing, and eases the head-ache; pains in the back, sides, or bowels, probeing made into an ointment with hog's cceding of wind, stopping of urine, the grease, it takes away black and blue'spots gravel or stone, as aforesaid. If the bruised occasioned by blows or falls, and helps both herb, sprinkled with some Muskadel, be the gout and sciatica.

PELLITORY OF THE WALL.D.21. warmed upon a tile, or in a dish upon a few quick coals in a chafing-dish, and applied to the belly, it works the same effect.

Descript.] It rises with brownish, red, The decoction of the herb being drank, tender, weak, clear, and almost transparent eases pains of the mother, and brings down stalks, about two feet high, upon which women's courses: It also eases those griefs grow at the joints two leaves somewhat that arise from obstructions of the liver, broad and long, of a dark green colour, spleen, and reins. The same decoction, which afterwards turn brownish, smootl on with a little honey added thereto, is good the edges, but rough and hairy, as the to gargle a sore throat. The juice held a stalks are also. At the joints with the while in the mouth, eases pains in the tceth. leaves from the middle of the stalk upwards, ? The distilled water of the herb drank with where it spreads into branches, stand many some sugar, works the same effects, and small, pale, purplish flowers in hairy, rough clcanses the skin from spots, freckles, purheads, or husks, after which come small, iples, wheals, sun-burn, morphew, \&c. 'The

$(13,14$. 
guice dropped into the ears, eases the noise in them, and takes away the pricking and shooting pains therein: The same, or the distilled water, assuages hot and sivelling imposthumes, burnirigs and scaldings by fire or water; as also all other hot tumours and inflammations, "or breakings-out, of heat, being bathed often witl wet cloths dipped therein: The said juice made into a liniment with ceruss, and oil of roses, and anointed therewith, cleanses foul rotten ulcers, and stays spreading or creeping? ulcers, and running scabs or sores in children's heads; and helps to stay the hair from falling off the head. The said ointment, or the herb applied to the fundament, opens the piles, and eases their pains ; and being mixed with goats' tallow, helps the gout. The juice is very effectual to cleanse fistulas, and to heal them up safely; or the herb itself bruised and applied with a little salt. It is likewise also effectual to heal any green wound; if it be bruised and bound thereto for three days, you shall need no other medicine to heal it further. A poultice made hereof with Mallows, and boiled in wine and wheat bran and bean flour, and some oil put thereto, and applied warm to any bruised sinews, tendon, or muscle, doth in a very short time restore them to their strength, taking arvay the pains of the bruises, and dissolves the con-? gealed blood coming of blows, or falls from high places.

The juice of Pellitory of the Wall clarified and boiled in a syrup with honey, and a spoonful of it drank every morning by such as are subject to the dropsy; if continuing that course, though but once a weak, they ever have the dropsy, let them but come to me, and I will cure them gratis.

PEN NYROYAL.

Pennyroyal is so well known unto all, I mean the common lind, that it needs no description.
There is a greater kind than the ordinary sort found wild with us, which so abides, being broughe into gardens, and differs not from it, but only in the largeness of the leaves and stalks, in rising higher, and not creeping upon the ground so much. The Howers whereof are purple, growing in rundles about the stalks like the other.

Place.] The first, which is common in gardens, grows also in many moist and watery places of this land.

'The second is found wild in effect in divers places by the highways fiom London to Colchester, and thereabouts, more abundantly than in any other counties, and is also planted in their gardens in Essex:

Time.] They flower in the latter end of Summer, about August.

Government, and virtues.] The herb is under Venus. Dioscorides saith, that Pennyroyal makes thin tough phlegm, warms the coldness of any part whereto it is applied, and digests raw or corrupt matter; Being boiled and drank, it provokes women's courses, and expels the dead child and after-birth, and stays the disposition to romit, being taken in water and vinegar mingled togetlier. And being mingled with honey and salt, it voids phlegm out of the lungs, and purges melancholy by the stool. Drank with wine, it helps such as are bitten and stung with venomous beasts, and applied to the nostrils with vinegar, revives those that are fainting and swooning. Being dried and burnt, it strengthens the gums. It is helpful to those that are troubled with the gout, being applied of itself to the place until it was red; and applied in a plaister, it takes away spots or marks in the face; applied with salt, it profits those that are splenetic, or livergrown. The decoction doth help the itch, if washed therewith. The green herb bruised and put into vinegar, cleanses foul ulcers, and takes awa the marks of bruises and blows about the eyes, and all discolourings of the face 
by fire, yea, and the leprosy, being drank The ordinary Female Peony hath as and outwardly applied: Boiled in wine many stalks, and more leaves on them than with honey and salt, it helps the tooth-ache. the Male; the leaves not so large, but nicked It helps the cold griefs by the joints, taking? on the edges, some with great and deep, away the pains, and warms the cold part, others with small cuts and divisions, of a being fust bound to the place, after a dead green colour. The flowers are of a bathing or sweating in a hot house. Pliny strong heady scent, usually smaller, and of adds, that Pennyroyal and Mints together, a more purple colour than the Male; with help faintings, being put into rinegar, and yellow thrums about the head, as the Male smelled unto, or put into the nostrils or hath. The seed vesscls are like horns, as in mouth. It eases head-aches, pains of the the Male, but smaller, the seed is black, breast and belly, and gnawings of the but less shining. The root consists of many stomach; applied with honey, salt, and short tuberous clogs, fastened at the end of vinegar, it helps cramps or convulsions of long strings, and all from the heads of the the sinews: Boiled in milk, and drank, it roots, which is thick and short, and of the is effectual for the cough, and for ulcers like scent with the Male.

and sores in the mouth; drank in wine it Place and Time.] They grow in gardens, provokes women's courses, and expels the and flower usually about May.

dead child, and after-birth. Matthiolus saith, 'The decoction thereof being drank, helps the jaundice and dropsy, all pains of the head and sinews that come of a cold cause, and clears the eye-sight. It helps the lethargy, and applied with barley-meal, helps burnings ; and put into the ears, eases the pains of them.

Government and virtues.] It is an herb of the Sun, and under the Lion. Physicians say, Male Peony roots are best; but $\mathrm{Dr}$. Reason told me Male Peony was best for men, and Female Peony for women, and he desires to be judged by his brother Dr. Experience. The roots are held to be of more virtue than the seed; next the flowers; and, last of all, the leaves. The roots of MALE AND FEMALE PEONY.I. 2/, the Male Peony, fiesh gathered, having Descript.] MaLe Peony rises up with been found by experience to cure the fallbrownish stalks, whereon grow green and ing sickness; but the surest way is, besides reddish leaves, upon a stalk without any hanging it about the neck, by which children particular division in the leaf at all. The lave been cured, to take the root of the flowers stand at the top of the stalks, con- Male Peony washed clean, and stamped sisting of five or six broad leaves, of a fair somewhat small, and laid to infuse in sack purplish red colour, with many yeilow for 24 hours at the least, afterwards strain threads in the middle stantling about the it, and take it first and last, morning and head, which after rises up to be the seed erening, a good draught for sundry days vessels, divided into two, three, or four together, before and after a full moon: and crooked pods like horns, which being full this will also cure old persons, if the disripe, open and turn themselves down back- ease be not grown too old, and past cure, wards, slewing with them divers round, especially if there be a due and orderly black, shining seeds, having also many preparation of the body with posset-drink crimson grains, intermixed with black, made of Betony, \&c. The root is also whereby it makes a very pretty shew. The effectual for women that are not sufficiently roots are great, thick and long, spreading cleansed after child-birth, and such as are and running down deep in the ground. troubled with the mother; for which like- 
wise the black seed beaten to powder, and hereof to be bruised, and mixed with old given in wine, is also available. The black hog's grease, and applied to the place, and seed also taken before bed-time, and in to continue thereon four hours in men, and the morning, is very effectual for such as in two hours in women, the place being aftertheir sleep are troubled with the disease wards bathed with wine and oil mixed called Ephialtes, or Incubus, bul we do together, and then wrapped up with wool commonly call it the Night-mare: a disease or skins, after they have sweat a little. It which melancholy persons are subject unto: also amends the deformities or discolourIt is also good against melancholy dreams. ings of the skin, and helps to take away The distilled water or syrup made of the marks, scars, and scabs, or the foul marks flowers, works the same effects that the root of burning with fire or iron. The juice and seed do, although more weakly. The hereof is by some used to be given in ale to Females is often used for the purpose afore-? drink, to women with child, to procure said, by reason the Male is so scarce a them a speedy delivery in travail. plant, that it is possessed by few, and those great lovers of rarities in this kind.

\section{PEPPERWORT, OR DITTANDER.}

Descript.] OU common Pepperwort sends forth somewhat long and broad leaves, of a light blueish green colour, finely in the ground, and rooteth in divers places. dented about the edges, and pointed at tlie At the joints of these branches sland two ends, standing upon round hard stalks, three or four feet high, spreading many branches on all sides, and having many small white flowers at the tops of them, after which follow small seeds in small heads. The root is slender, running much under ground, and shooting up again in many places, and both leaves and roots are very hot and sharp of taste, like pepper, for which cause it took the name.

Place.] It grows naturally in many places of this land, as at Clare in Essex places of this land, as at Clare in Essex ; far about, whereby it quickly possesses a also hear unto Exeter in Devonshire; upon? great compass, and is therefore most Rochester common in Kent; in Lanca- usually planted under hedges where it may shire, and divers other places; but usually have room to run.

kept in gardens.

Place.] Those with the pale blue, and

Time.] It flowers in the end of June, those with the white flowers, grow in woods and in July.

and orchards, by the hedge-sides, in divers

Govenment and virtues.] Here is another places of this land ; but those with the purmartial herb for you, make much of it. ple flowers, in gardens only.

Pliny and Paulıs Agineta say, that Pepperwort is very successful for the sciatica, Time.] They flower in March and April. Govermment and virtues.] Venus owns or any other gout or pain in the joints, or this herb, and saith, 'That the leaves eaten any other invetcrate grief: 'The leaves by man and wife together, cause love be- 
tween them. The Periwinkle is a great and Northamptonshire; as also near waterbinder, stays bleeding both at mouth and courses in other places. nose, if some of the leaves be chewed. The French used it to stay women's courses. Dioscorides, Galen, and Ægineta, commend it against the lasks and fluxes of the belly to be drank in wine.

\section{ST. PETER'S WORT.P. $2 /$,}

Ir Superstition had not been the father of 'Tradition, as well as Ignorance the Mother of Devotion, this herb, (as well as St. John's Wort) hath found some other name to be known by ; but we may say of our forefithers, as St. Paul of the Athenians, I perceive in many things you are too superstitious. Yet seeing it is come to pass,
that custom having got in possession, pleads prescription for the name, I shall ? let it pass, and come to the description of the herb, which take as follows.

Descript.] It rises up with square upright stalks for the most part, some greater and higher than St. John's Wort (and good reason, too, St. Peter being the greater apostle, (ask the Pope else;) for though pase the stalk. The flowers stand singly God would have the saints equal, the Pope each by themselves at them and the stalk, is of another opinion,) but brown in the consisting of fivesmall round-pointed leaves, same manner, having two leaves at every of a pale red colour, tending to an orange, joint, somewhat like, but larger, than St. s with so many threads in the middle, in whose John's Wort, and a little rounder pointed, places succeed smooth round héads, wherewith few or no holes to be seen thereon, and in is contained small seed. 'The root is having sometimes some smaller leaves rising small and fibrous, perishing every year.

from the bosom of the greater, and sometimes a little hairy also. At the tops of two stalks stand many star-like flowers, with as by the way-sides, and in gardens, arising yellow threads in the middle, very like of itself.

those of St. John's Wort, insomuch that Time.] It flowers from May until April, this is hardly discerned from it, but only by and the seed ripens in the mean time, and the largeness and height, the seed being falls.

alike also in both. 'The root abides long, Government and virtues.] It is a gallant

sending forth new shoots every year.
Place.] It grows in many groves, and whereby it draws forth thorns or splinters, small low woods, in divers places of this or other such like things gotten into the land, as in Kent, Huntingdon, Cambridge, flesh; and put up into the nóstrils, purgo

$(13,14$.
00 
the head; and Galen saith also, they have along among the leaves; after which cone a drying faculty, whereby they are good to small and round husks. The root is small solder the lips of wounds, and to cleanse and woody, perishing every year.

foul ulcers. The distilled water or juice is Place.] It grows more plentifully in much esteemed by French dames to cleanse Kent than any other county of this land, as the skin from any roughness and defor- namely, in many places on this side Dartmity, or discolouring thereof; being boiled ford, along to Southflect, Chatham, and in wine and given to drink, it is a good Rochester, and upon Chatham down, hard remedy against the plague, and other pes- by the Beacon, and half a mile from tilential fevers, if the party after taking it Rochester, in a field near a house called be warm in his bed, and sweat for two Selesys.

hours after, and use the same for twice at least. It helps also all stingings and
bitings of venomous beasts, or mad doos, being used inwardly, and applied outwardly. The same also opens obstructions of the liver, and is very available against the infirmities of the reins : It provoles urine, and helps to expel the stone and gravel out of the kidneys and bladder, and helps much in all inward pains and ulcers. 'The decoction, or distilled water, is no less ef- wont in former times to make pills with the fectual to be applied to all wounds that are powder thereof, and the pulp of figs. It fresh and green, or old, filthy, fretting, and marvellously helps all the diseases of the running ulcers, which it very effectually mother, inwardly or outwardly applied, cures in a sliort space. A little mixed procuring women's courses, and expelling with the juice, and dropped into the eyes, the dead clild and after-birth; yea, it is so cleanses them from cloudy mists, or thick powerful upon those feninine parts, that it films which grow over them, and hinder is utterly forbidden for women witl child, the sight. It helps the tooth-ache, being for it will cause aborion or delivery before dropped into the ear on a contrary side of the time. The decoction of the herb in the pain. It is also effectual to ease the wine taken inwardly, or applicd outwardly, pains of the hæmorrhoids or piles.

GROUND PINE, OR CHAMEPITYS.

Descript.] OuR common Ground Pine or both, for some time together, is also effectual in all pains and diseases of the joints, as gouts, cramps, palsies, sciatica, and aches; for which purpose the pills made grows low, seldom rising above a hand's with powder of Ground Pine, and of Herbreadth high, shooting forth divers small modactyls witl Venice Turpentine are very brariches, set with slender, small, long, nar- effectual. The pills also, continued for row, greyish, or whitish leaves, somewhat some time, are special good for those that hairy, and divided into three parts, many have the dropsy, jaundice, and for griping bushing together at a joint, some growing pains of the juints, belly, or inward parts. scatteringly upon the stalks, smelling some- It helps also all diseases of the brain, prowhat strong; like unto rozin: The flowers ceeding of coid and phlcgmatic humours are small, and of a pale yellow colour, and distillations, as also for the falling sickgrowing from the joint of the stalk all ness. It is a special remedy for the poison 
of the aconites, and other poisonous herbs, from the head, and stays all manner of as also against the stinging of any veno- fluxes, even women's courses, when they mous creature. It is a goor remedy for a flow too abundantly. It is good to stay cold cough, especially in the beginning. spitting of blood and other blcedings at the For all the purposes aforesaid, the herb mouth, or the making of foul and bloody being tunned up in new drink and drank, water, by reason of any ulcer in the reins is almost as effectual, but far more accepta- or bladder, and also stays the too free ble to weak and dainty stomachs. The bleeding of wounds. It is held an especial distilled water of the herb hath the same remedy for those that are troubled with the effects, but more weakly. The conserve phthisic, or consumption of the lungs, or of the flowers doth the like, which Mat- ulcers of the lungs, or coughs that come of thiolus much commends against the palsy. heat. The decoction or powder of the The green herb, or the decoction thereof, roots or seeds, is much more binding for all being applied, dissolves the harhness of the purposes aforesaid than the leaves. wonen's breasts, and all other hard swell- Dioscorides saith, that threc roots boiled in ings in any other part of the body. The wine and taken, helps the tertain agues, and green herb also applied, or the juice thereof for the quartan agues, (but letting the numwith some honey, not only clcanses putrid, her pass as fabulous) I conceive the decocstinking, foul, and malignant ulcers and tion of divers roots may be effectual. The sores of all sorts, but lieals and solders up herb (but especially the sced) is held to be the lips of green wounds in any part also. profitable against the dropsy, the fallingLet pregnant women forbear, for it works sickness, the yellow jaundice, and stoppings violently upon the feminine part.

$$
\text { PLA NTAIN.T. } 23 .
$$

This grows usually in meadows and away the pains of then. The clarified fields, and by path sides, and is so well juice, or distilled water, dropped into the known, that it needs no description.

Time.] It is in its beatuly about June, and the seed ripens shortly after.

Government and virtues.] It is true, Mis- helps and removes the heat. The same aldus and others, yea, almost all astrology-also with the juice of Houseleck is profitable physicians, hold this to be an herb of Mars, against all inflammations and breakings out because it cures the diseases of the head of the skin, and against burnings and scaldand privities, which are under the houses ings by fire and water. The juice or deof Mars, Aries, and Scorpio: 'The truth is, coction made either of itself, or other things it is under the command of Tenus, and of the like nature, is of much use and good curcs the head by antipathy to Mars, and effect for old and hollow ulcers that are hard the privities by sympatiy to Venus; neither to be cured, and for cankers and sores in is there hardly a martial disease but it the mouth or privy parts of man or woman; cures. and helps also the pains of the piles in the The juice of Plantain clarified and drank fundament. The juice mixed with oil of for divers days together, either of itself, or roses, and the temples and forehead anointin other drink, prevails wondertully against ed therewith, eases the pains of the liead all torments or excoriations in the intestines proceeding from heat, and helps lunatic or bowels, helps the distillations of rheurs and frantic persons very much; as also the 
biting of serpents, or a mad dog. The stone. The gum or leaves boiled in vine-
same also is profitably applied to all hot gar, and applied, kills tetters and ring-
gouts in the feet or hands, especially in the worms. Matthiolus saith, 'The oil pre-
beginning. It is also good to be applied served out of the kernels of the stones, as
where any bone is out of joint, to hinder oil of almonds is made, is good against the
inflammations, swellings, and pains that inflamed piles, the tumours or swellings of
presently rise thereupon. The powder of ulcers, hoarseness of the voice, rougliness
the dried leaves taken in drink, kills worms of the tongue and throat, and likewise the
of the belly; and boiled in wine, kills pains in the ears. And that five ounces
worms that breed in old and foul ulcers. of the said oil taken with one ounce of mus-
One part of Plantain water, and two parts kadel, drives forth the stone, and helps the
of the brine of powdered beef, boiled cholic.

biting of serpents, or a mad dog. The stone. The gum or leaves boiled in vine-
same also is profitably applied to all hot gar, and applied, kills tetters and ring-
gouts in the feet or hands, especially in the worms. Matthiolus saith, 'The oil pre-
beginning. It is also good to be applied served out of the kernels of the stones, as
where any bone is out of joint, to hinder oil of almonds is made, is good against the
inflammations, swellings, and pains that inflamed piles, the tumours or swellings of
presently rise thereupon. The powder of ulcers, hoarseness of the voice, rougliness
the dried leaves taken in drink, kills worms of the tongue and throat, and likewise the
of the belly; and boiled in wine, kills pains in the ears. And that five ounces
worms that breed in old and foul ulcers. of the said oil taken with one ounce of mus-
One part of Plantain water, and two parts kadel, drives forth the stone, and helps the
of the brine of powdered beef, boiled cholic.

biting of serpents, or a mad dog. The stone. The gum or leaves boiled in vine-
same also is profitably applied to all hot gar, and applied, kills tetters and ring-
gouts in the feet or hands, especially in the worms. Matthiolus saith, 'The oil pre-
beginning. It is also good to be applied served out of the kernels of the stones, as
where any bone is out of joint, to hinder oil of almonds is made, is good against the
inflammations, swellings, and pains that inflamed piles, the tumours or swellings of
presently rise thereupon. The powder of ulcers, hoarseness of the voice, rougliness
the dried leaves taken in drink, kills worms of the tongue and throat, and likewise the
of the belly; and boiled in wine, kills pains in the ears. And that five ounces
worms that breed in old and foul ulcers. of the said oil taken with one ounce of mus-
One part of Plantain water, and two parts kadel, drives forth the stone, and helps the
of the brine of powdered beef, boiled cholic.

biting of serpents, or a mad dog. The stone. The gum or leaves boiled in vine-
same also is profitably applied to all hot gar, and applied, kills tetters and ring-
gouts in the feet or hands, especially in the worms. Matthiolus saith, 'The oil pre-
beginning. It is also good to be applied served out of the kernels of the stones, as
where any bone is out of joint, to hinder oil of almonds is made, is good against the
inflammations, swellings, and pains that inflamed piles, the tumours or swellings of
presently rise thereupon. The powder of ulcers, hoarseness of the voice, rougliness
the dried leaves taken in drink, kills worms of the tongue and throat, and likewise the
of the belly; and boiled in wine, kills pains in the ears. And that five ounces
worms that breed in old and foul ulcers. of the said oil taken with one ounce of mus-
One part of Plantain water, and two parts kadel, drives forth the stone, and helps the
of the brine of powdered beef, boiled cholic.

biting of serpents, or a mad dog. The stone. The gum or leaves boiled in vine-
same also is profitably applied to all hot gar, and applied, kills tetters and ring-
gouts in the feet or hands, especially in the worms. Matthiolus saith, 'The oil pre-
beginning. It is also good to be applied served out of the kernels of the stones, as
where any bone is out of joint, to hinder oil of almonds is made, is good against the
inflammations, swellings, and pains that inflamed piles, the tumours or swellings of
presently rise thereupon. The powder of ulcers, hoarseness of the voice, rougliness
the dried leaves taken in drink, kills worms of the tongue and throat, and likewise the
of the belly; and boiled in wine, kills pains in the ears. And that five ounces
worms that breed in old and foul ulcers. of the said oil taken with one ounce of mus-
One part of Plantain water, and two parts kadel, drives forth the stone, and helps the
of the brine of powdered beef, boiled cholic.

biting of serpents, or a mad dog. The stone. The gum or leaves boiled in vine-
same also is profitably applied to all hot gar, and applied, kills tetters and ring-
gouts in the feet or hands, especially in the worms. Matthiolus saith, 'The oil pre-
beginning. It is also good to be applied served out of the kernels of the stones, as
where any bone is out of joint, to hinder oil of almonds is made, is good against the
inflammations, swellings, and pains that inflamed piles, the tumours or swellings of
presently rise thereupon. The powder of ulcers, hoarseness of the voice, rougliness
the dried leaves taken in drink, kills worms of the tongue and throat, and likewise the
of the belly; and boiled in wine, kills pains in the ears. And that five ounces
worms that breed in old and foul ulcers. of the said oil taken with one ounce of mus-
One part of Plantain water, and two parts kadel, drives forth the stone, and helps the
of the brine of powdered beef, boiled cholic.

biting of serpents, or a mad dog. The stone. The gum or leaves boiled in vine-
same also is profitably applied to all hot gar, and applied, kills tetters and ring-
gouts in the feet or hands, especially in the worms. Matthiolus saith, 'The oil pre-
beginning. It is also good to be applied served out of the kernels of the stones, as
where any bone is out of joint, to hinder oil of almonds is made, is good against the
inflammations, swellings, and pains that inflamed piles, the tumours or swellings of
presently rise thereupon. The powder of ulcers, hoarseness of the voice, rougliness
the dried leaves taken in drink, kills worms of the tongue and throat, and likewise the
of the belly; and boiled in wine, kills pains in the ears. And that five ounces
worms that breed in old and foul ulcers. of the said oil taken with one ounce of mus-
One part of Plantain water, and two parts kadel, drives forth the stone, and helps the
of the brine of powdered beef, boiled cholic.

biting of serpents, or a mad dog. The stone. The gum or leaves boiled in vine-
same also is profitably applied to all hot gar, and applied, kills tetters and ring-
gouts in the feet or hands, especially in the worms. Matthiolus saith, 'The oil pre-
beginning. It is also good to be applied served out of the kernels of the stones, as
where any bone is out of joint, to hinder oil of almonds is made, is good against the
inflammations, swellings, and pains that inflamed piles, the tumours or swellings of
presently rise thereupon. The powder of ulcers, hoarseness of the voice, rougliness
the dried leaves taken in drink, kills worms of the tongue and throat, and likewise the
of the belly; and boiled in wine, kills pains in the ears. And that five ounces
worms that breed in old and foul ulcers. of the said oil taken with one ounce of mus-
One part of Plantain water, and two parts kadel, drives forth the stone, and helps the
of the brine of powdered beef, boiled cholic.

biting of serpents, or a mad dog. The stone. The gum or leaves boiled in vine-
same also is profitably applied to all hot gar, and applied, kills tetters and ring-
gouts in the feet or hands, especially in the worms. Matthiolus saith, 'The oil pre-
beginning. It is also good to be applied served out of the kernels of the stones, as
where any bone is out of joint, to hinder oil of almonds is made, is good against the
inflammations, swellings, and pains that inflamed piles, the tumours or swellings of
presently rise thereupon. The powder of ulcers, hoarseness of the voice, rougliness
the dried leaves taken in drink, kills worms of the tongue and throat, and likewise the
of the belly; and boiled in wine, kills pains in the ears. And that five ounces
worms that breed in old and foul ulcers. of the said oil taken with one ounce of mus-
One part of Plantain water, and two parts kadel, drives forth the stone, and helps the
of the brine of powdered beef, boiled cholic.

biting of serpents, or a mad dog. The stone. The gum or leaves boiled in vine-
same also is profitably applied to all hot gar, and applied, kills tetters and ring-
gouts in the feet or hands, especially in the worms. Matthiolus saith, 'The oil pre-
beginning. It is also good to be applied served out of the kernels of the stones, as
where any bone is out of joint, to hinder oil of almonds is made, is good against the
inflammations, swellings, and pains that inflamed piles, the tumours or swellings of
presently rise thereupon. The powder of ulcers, hoarseness of the voice, rougliness
the dried leaves taken in drink, kills worms of the tongue and throat, and likewise the
of the belly; and boiled in wine, kills pains in the ears. And that five ounces
worms that breed in old and foul ulcers. of the said oil taken with one ounce of mus-
One part of Plantain water, and two parts kadel, drives forth the stone, and helps the
of the brine of powdered beef, boiled cholic.

biting of serpents, or a mad dog. The stone. The gum or leaves boiled in vine-
same also is profitably applied to all hot gar, and applied, kills tetters and ring-
gouts in the feet or hands, especially in the worms. Matthiolus saith, 'The oil pre-
beginning. It is also good to be applied served out of the kernels of the stones, as
where any bone is out of joint, to hinder oil of almonds is made, is good against the
inflammations, swellings, and pains that inflamed piles, the tumours or swellings of
presently rise thereupon. The powder of ulcers, hoarseness of the voice, rougliness
the dried leaves taken in drink, kills worms of the tongue and throat, and likewise the
of the belly; and boiled in wine, kills pains in the ears. And that five ounces
worms that breed in old and foul ulcers. of the said oil taken with one ounce of mus-
One part of Plantain water, and two parts kadel, drives forth the stone, and helps the
of the brine of powdered beef, boiled cholic.

biting of serpents, or a mad dog. The stone. The gum or leaves boiled in vine-
same also is profitably applied to all hot gar, and applied, kills tetters and ring-
gouts in the feet or hands, especially in the worms. Mitthiolus saith, The oil pre-
beginning. It is also good to be applied served out of the kernels of the stones, as
where any bone is out of joint, to hinder oil of almonds is made, is good against the
inflammations, swellings, and pains that inflamed piles, the tumours or swellings of
presently rise thereupon. The powder of ulcers, hoarseness of the voice, rougliness
the dried leaves taken in drink, kills worms of the tongue and throat, and likewise the
of the belly; and boiled in wine, kills pains in the ears. And that five ounces
worms that breed in old and foul ulcers. of the said oil taken with one ounce of mus-
One part of Plantain water, and two parts kadel, drives forth the stone, and helps the
of the brine of powdered beef, boiled cholic. together and clarified, is a most sure remedy to heal all spreading scabs or itch in the head and body, all manner of tetters, ringworms, the shingles, and all other running? and fretting sores. Briefly, the Plantains are singularly good wound herbs, to heal fresh or old wounds or sores, either inward or outward

PLUMS.

ArE so well known that they need no description.

Govermment and virtues.] All Plums are under Venus, and are like women, some sad grcen colour, and smooth on the upper better, and some worse. As there is great diversity of kinds, so there is in the operation of. Plums, for some that are sweet moistens the stomach, and make the belly little finger, lying aslope, or creeping along soluble; those that are sour quench thirst under the upper crust of the earth, brownmore, and bind the belly; the moist and ish on the outside and greenish within, of waterish do sooner corrupt in the stomach, a sweetish harshness in taste, set with cerbut the firn do nourish more, and offend tain rough knags on each side thereof, less. The dried fruit sold by the grocers having also much mossiness or yellow hairunder the names of Damask Prunes, do iness upon it, and some fibres underneath it, somewhat loosen the belly, and being? whereby it is nourished.

stewed, are often used, both in health and sickness, to relish the mouth and stomach, to procure appetite, and a little to open the body, allay choler, and cool the under them, and upon old mud walls, as stomach. Plum-trce leaves boiled in wine, also in mossy, stony, and gravelly places are good to wash and gargle the moutl and near unto wood. That which grows upon throat, to dry the flux of rheum coming to oak is accounted the best; but the quantity the palate, gums, or almonds of the ear. thereof is scarce sufficient for the common The gum of the, tree is good to break the use.

Place.] It grows as well upon old rotten stumps, or trunks of trees, as oak, beech, hazel, willow, or any other, as in the woods

POLY PODY OF THE OAK.
Descript.] THIs is a small herb consisting of nothing but roots and leaves, bearing neither stalk, flower, nor seed, as it is rising from the root, every one single by itself, of about a hand length, are winged, consisting of many small narrow leaves cut into the middle rib, standing on each side of the stalk, large below, and smaller up to the top, not dented nor notched at side, but on the other side somewhat rough by reason of some ycllowish flowers set thereon. The root is smaller than one's 
Time.] It being always green, may be doth much help it ; and applied also to the gathered for use at any time.

nose, cures the disease called Polypus, which

Goternment and virtues.] Polypodium of is a piece of flesh growing therein, which the Oak, that which grows upon the earth in time stops the passage of breath through is best; it is an herb of Saturn, to purge that nostril ; and it helps those clefts or melancholy ; if the humour be otherwise, ? chops that come between the fingers or chuse you Polv podium accordingly. Meuse toes.

(who is called the physician's Evangelist for the certainty of his medicines, and the truth of his opinion) saith, That it dries up

THE POPLAR TREE. P. thin humours, digests thick and tough, and are most familiar with us, viz. the Black purges burnt choler, and especially tough and White, both which I shall here desand thick phlegm, and thin phlegm also, cribe unto you.

even from the joints, and therefore good for Descript.] The White Poplar grows those that are troñbled with melancholy, or great, and reasonably high, covered with quartan agues, especially if it be taken in thick, smooth, white bark, especially the whey or honied water, or in barley-water, branches; having long leaves cut into or the brotlı of a chiclien with Epithymum, several divisions almost like a vine leaf, but or with Bects and Mallows. It is good for not of so deep a green on the upper side, the hardness of the spleen, and for pricking and hoary white underneath, of a reasonor stitches in the sides, as also for the cholic: able good scent, the whole form representSome use to put to it some Fenncl secds, or ing the form of Coltsfoot. The cathins Annise seeds, or Ginger, to correct that which it brings forth before the leaves, are loathing it brings to the stomach, which is long, and of a faint reddish colour, which more than needs, it being a safe and gentlc fall away, bearing seldom good seed with medicine, fit for all persons, which daily them. The wood hereof is smooth, soft, experience confirms; and an ounce of it and white, very finely waved, whereby it is may be given at a time in a decoction, if much esteened.

there be not Sena, or some other strong 'The Black Poplar grows higher and purger put with it. A dram or two of the straighter than the White, with a greyish powder of the dried roots, taken fasting in bark, bearing broad grcen leaves, somewhat a cup of honied water, works gently, and like ivy leaves, not cut in on the edges like for the purposes aforesaid. The distilled the White, but whole and dented, ending water, hoth of roots and leaves, is much in a point, and not white underneath, hangcommended for the quartan ague, to be ing by slender long foot stalks, which with takeit for many days together, as also the air are continually shaken, like as the against melancholy, or fearful and trouble- Aspen leaves are. The catkins hereof are some sleeps or dreams; and with some greater than those of the White, composed. sugar-candy dissolved thorein, is good of many round green borries, as if they against the cough, shortness of breath, and were set togecher in a long cluster, containwheezings, and those distillations of thin ing much downy matter, which being ripe, rheum upon the lungs, which cause phthi- is blown away with the wind. The clammy sicks, and oftentimes consumptions. The buds licreof, before they spread into leaves, fresh roots beaten small, or the powrler of are gathered to make Unguentum and the dried roots mixed with honey, and ap- Populneum, and are of a yellowish green plied to the member that is out of joint, colour, and somewhat small, sweet, but

$$
(15,16 .)
$$


strong. The wood is smooth, tough, and the White and Black of the Garden, and white, and easy to be cloven. On both the Erratic Wild Poppy, or Corn Rose. these trees grows a sweet kind of musk, Descript.] The Whitc Poppy hath at which in former times was used to put into first four or five whitish green lcaves lying sweet ointments.

Place.] They grow in moist woods, and compassing it at the bottom of them, and by water-sides in sundry places of this land; are very large, much cut or torn on the yet the White is not so frequent as the other. edges, and dented also besides : The stalk,

Time.] 'Their time is likewise expressed which is usually four or five feet high, hath before: The catkins coming forth before sometimes no branches at the top, and the leaves in the end of Summer.

susually but two or three at most, bearing hath every one but one head wrapped up in a dominion over both. White Poplar, saith thin skin, which bows down before it is Galen, is of a cleansing property: 'The ready to blow, and then rising, and being weight of an ounce in powder, of the bark broken, the flowers within it spreading itself thereof, being drank, saith Dioscorides, is open, and consisting of four very large, a remedy for those that are troubled with white, round lcaves, with many whitish the sciatica, or the stranguary. The juice round threads in the middle, set about a of the leaves dropped warm into the ears, small, round, green head, having a crown, eases the pains in them. The young or star-like cover at the head thereof, which clammy buds or eyes, before they break growing ripe, becomes as large as a great out into leaves, bruised, and a little honey apple, wherein are contained a great numput to them, is a good medicine for a dull ber of small round seeds, in several parsight. The Black Poplar is held to be tions or divisions next unto the shcll, the more cooling than the White, and therefore middle thercof remaining hollow, and the leaves bruised with vinegar and applicd, empty. 'The whole plant, both leares, help the gout. The seed drank in vinegar, stalks, and heads, while they are fresh, is held good against the falling-sickness. young, and green, yield a milk when they The water that drops from the hollow places are broken, of an unpleasant bitter taste, of this tree, takes away warts, pushes, almost ready to provoke casting, and of a wheals, and other the like breakings-out of strong heady smcll, which being condensed, the body. The young Black Poplar buds, is called Opium. The root is white and saith Matthiolus, are much used by women woody, perishing as soon as it hath given to beautify their hair, bruising them with ripe seed.

fresh butter, straining them after they have The Black Poppy little differs from the been kept for some time in the sun. The former, until it bears its Hower, which is ointment called Populneon, which is made somewhat less, and of a black purplish of this Poplar, is singularly good for all colour, but without any purple spots in the heat and inflammations in any part of the bottom of the leaf. The head of the seed body, and tempers the heat of wounds. It is much less than the former, and opens is much used to dry up the milk of women's itsclf a little round about the top, under breasts when they have weaned their the crown, so that the seed, which is very children. black, will fall out, if one turn the head thercof downward.

POPPY. P. 23.

The wild Poppy, or Corn Rose, hath long OE this I shall describe three kinds, riw. and narrow leaves, very much cut in on the 
edgcs into many divisions, of a light green the Moon. 'The garden Poppy lieads with colour, sometimes hairy withal. The stalk seeds made into a syrup, is frequently, and is blackisl and lairy also, but not so tall as to good effect used to procure rest, and the garden kind, having some such like sleep, in the sick and weak, and to stay leaves thereon to grow below, parted into catarrhs and defluxions of thin rheums three or four branchcis sometimes, whereon from the head into the stomach and lungs, grow small hairy licads bowing down be- causing a continual cougl, the fore-runner fore the skin break, wherein the flower is of a consumption; it helps also hoarseness inclosed, which when it is fully blown open, of the throat, and when one hath lost their is of a fair yellowish red or crimson colour, voice, which the oil of the sced doth likeand in some much paler, without any spot wise. The black seed boiled in wine, and in the bottom of the leaves, having many drank, is said also to stay the flux of the black soft threads in the middle, compass- belly, and women's courses. 'The empty ing a small green head, which when it is shells, or poppy heads, are usually boiled ripe, is not bigger than onc's little finger's in water, and giren to procure rest and end, wherein is contained much black seed, sleep : so doth the leaves in the same mansmaller than that of the garden. The root ner; as also if the head and temples be perishes every year, and springs again of bathed with the decoction warm, or with its own sowing. Of this kind there is one the oil of Poppies, the green leaves or the lesser in all the parts thereof, and differs in heads bruised, and applied with a little nothing else.

Place.] The garden kinds do not naturally grow wild in any place, but all are sown in gardens where they grow.

The Wild Poppy or Corn Rose, is plentifully enoughl, and many times too much so in the corn fields of all counties through this land, and also upon ditch banks, and by hedge sides. 'The smaller wild kind is also found in corn fields, and also in some other places, but not so plentifully as the former.

Time.] The garden kinds are usually sown in the spring, which then flower about the end of May, and somewhat earlicr, if they spring of their own sowing.

T'he wild kind flower usually from May until July, and the seed of then is ripe soon after the flowering.

Government and zirtues.] The herb is ? vinegar, or made inlo a poultice with barleymeal or hog's grease, cools and tempers all inflammations, as also the disease called St. Anthony's fire. It is generally used in treacle and mitlridate, and in all other medicines that are made to procure rest and sleep, and to ease pains in the head as well as in other parts. It is also used to cool inflammations, agues, or frenzies, or to stay defluxions which cause a cough, or consumption, and also other fluxes of the belly or women's courses; it is also put into hollow teeth, to ease the pain, and hath been found by experience to case the pains. of the gout.

The Wild Poppy, or Corn Rose (as Matthiolus saith) is good to prevent the fallingsickness. 'T'he syrup made with the flower, is with good effect given to those that have Lumar, and of the juice of it is made opium; only for lucre of money they chcat you, and drank, cither in the distilled water of and tell you it is a hind of tear, or some them, or some other drink, works the like such like thing, that drops from Poppies seffect. 'The distilled water of the flowers when they weep, and that is somewhere is leeld to be of much good use against beyond the süas, I kriow not where beyoud surfeits, being drank evening and morning: 
It is also more cooling than any of the parts where pushes, wheals, pimples, St. other Poppies, and therefore cannot but Anthony's fire and the like, break forth; be as effectual in hot agues, frenzies, and if a little vinegar be put to it, and laid to other inflammations either inward or out- the neck, with as much of galls and linseed ward. Galen saith, The seed is dangerous together, it takes away the pains therein, to be used inwardly.

\section{PURSLAIN.P.2N}

Garden Purslain (being used as a sallad herb) is so well known that it needs no and to allay the heat in all other sores or description; I shall therefore only speak of hurts; applied also to the navels of chilits virtues as follow.

Government and virtues.] 'Tis an herb of the Moon. It is good to cool any heat in the liver, blood, reins, and stomach, and in hot agues nothing better: It stays hot and choleric fluxes of the belly, women's courses; the whites, and gonorrhæa, or running of the reins, the distillation from the head, and pains therein proceeding from heat, want of sleep, or the frenzy. The seed is more effectual than the herb, and is of singularly good use to cool the heat and sharpness of urine, venereous dreams, and the like: insomuch that the over frequent use hereof extinguishes the heat and virtue- of natural procreation. The seed bruised and boiled in wine, and given to children, expels the worms. 'The juice of the herb is held as effectual to all the purposes aforesaid; as also to stay vomitings, and taken with some sugar or honey, helps an old and the book: make this as you are taught dry cough, shortness of breath, and the there, and do not (you that have any inphthisick, and stays immoderate thirst. genuity in you) sec your poor neighbours The distilled water of the herb is used by go with wounded limbs when an halfpenny many (as the more pleasing) with a little cost will heal them. sugar to work the same effects. The juice? also is singularly good in the infiammations and ulcers in the secret parts of man or woman, as also the bowels and hremorrhoids, when ti:ey are ulccrons, or excoriations in reasonable height and breadth, to cover them. The herb bruised and applied to the arbours, bower's and banquetting houses, forehead and temples, allays excessive heat and brought, wrought, and cut into so many thereiu, that hinders rest and sleep; and forms, of men, horses, birds, \&c. which, applied to the eyes, takes away the redness though at first supported, grows afterwards and inflammation in them, and those other strong of itself. It bears long and narrow 
green leaves by the couples, and sweet (as Agrimony hath) somewhat deeplydented smelling white flowers in tufts at the end of about the edges, of a sad green colour on the branches, which turn into small black the upper side, and greyish underneath, berries that have a purplish juice with them, of a pretty sharp scent and taste, somewhat and some seeds that are flat on the one like unto the Burnet, and a leaf hereof put side, with a hole or dent therein.

Place.] It grows in this land, in divers woods.

into a cup of claret wine, gives also a fine

Time.] Our Privet flowers in June and flowers thrust thick together, which smell July, the berries are ripe in August and much sweeter than the leaves; and in their Scptember.

Government and virtues.] The Moon is cornered seed. The root is somewhat lady of this. It is little used in physic with woody, and blackish on the outside, and us in these times, more than in lotions, to brownish within, with diver's great strings, wash sores and sore mouths, and to cool and lesser fibres set thereat, of a strong scent, inflammations, and dry up fluxes. Yet but nothing so pleasant as the flowers and Matthiolus saith, it serves all the uses for leaves, and perishes not, but abides many which Cypress, or the East Privet, is ap- years, shooting forth a-new every Spring. pointed by Dioscorides and Galen. He Place.] It grows in moist meadows further saitl, That the oil that is made of that lie mostly wet, or near the courses of the flowers of Privet infused therein, and water.

set in the Sun; is singularly good for the inflammations of wounds, and for the head- other all the three Summer months, that is, ache, coming of a hot cause. There is a June, July, and August, and the seed is sweet water also distilled from the flowers, ripe soon after.

that is good for all those diseases that need Government and virtues.] Venus claims cocling and drying, and therefore helps all dominion over the herb. It is used to stay fluxes of the bclly or stomach, bloody-fluxes, all manner of bleedings, fluxes, romitings, and women's courses, being either drank or applied; as all those that void blood at the It is said to alter and take away the fits of mouth, or any other place; and for distilla- the quartan agues, and to make a merry tions of rheum in the eyes, especially if it heart, for which purpose some use the be used with thens.

flowers, and some the leaves. It helps spcedily those that are troubled with the QUEEN OF TIE MEADOWS, MEADOW SWEET, OR MEAD SWEET.

\section{23 .}

Descript.] The stalks of these are redcholic; being boiled in wine, and with a little honey, taken warm, it opens the belly; but boiled in red wine, and drank, it stays the flux of the belly. Outwardly applied, dish, lising to be three feet high, sometimes it helps old ulcers that are cankerous, or four or five feet, having at the joints thereof? hollow fistulous, for which it is by many large winged lcaves, standling one above much commended, as also for the sores in another at distances, consisting of many the mouth or secret parts. The laves when and somewhat broad leaves, set on each they are full grown, being laid on the skin, side of a middle rib, being hard, rough, or will, in a short time, raise blisters thereon, rugged, crumpled much like unto elm leaves, as Tragus saitl. The water thereof helps having also some smaller leaves with them the heat and imflamnlation in the eyes.

$(15,16$.

Q $Q$ 
THE QUINCE TREE.P.23.

Descript.] The ordinary Quince Tree grows often to the height and bigness of a reasonable apple tree, but more usually lower, and crooked, with a rough bark, spreading arms, and branches far abroad. The leaves are somewhat like those of the apple tree, but thicker, broader, and full of reins, and whiter on the under side, not bore. If there be need of any outwardly dented at all about the edges. The flowers are large and white, sometimes dashed over with a blush. The fruit that follows is yellow, being near ripe, and covered with a white freeze, or cotton; thick set on the younger, and growing less as they grow to be thorough ripe, bunched out oftentimes in some places, some being like an apple, and some a pear, of a strong heady scent, from and and of an unpleasant taste to eat fresh; and heal the sore breasts of women. The but being scalded, roasted, baked, or pre- same, with a little sugar, is good to lenify served, becomes more pleasant.

Place and Time.] It best likes to grow near ponds and water sides, and is frequent through this land: and flowers not until pror the leaves be come forth. The fruit is ripe in September or October.

\section{Government and virtues.]} owns the 'Tree. Quinces when they are green, help all sorts of fluxes in men or women, and clioleric lasks, casting, and whatever needs astriction, more than any way prepared by fire; yet the syrup of the juice, or the conserve, are much conducible, much of the binding quality being consumed by the fire; if a little vinegar be added, it stirs up the languishing appetite, and the stomach given to casting; sone spices being added, comforts and strengthens the decaying and fainting spirits, and helps the liver oppressed, that it cannot perfect the digestion, , corrects clioler and phlegm. If you would have them purging, put honey t) them instead of sugar: and if more laxative, for choler, Rhubarb; for phlegm, Tur- bith; for watery humours, Scammony; but if more forcible to bind, use the unripe Quinces, with roses and acacia, hypocistis, and some torrified rhubarb. To take the crude juice of Quinces, is held a preservative against the force of deadly poison; for it hath been found most certainly true, tliat the very smell of a Quince hath taken away all the strength of the poison of white Helleof Quinces, or otlier medicines that may be made thereof, are very available to anoint the belly or other parts therewith; it likewise strengthens the stomach and belly, and the sinerrs that are loosened by sharp humours falling on them, and restrains immoderate sweatings. Themuscilage taken from the seeds of Quinces, and boiled in a the harshness and lioarseness of the throat, and rougliness of the tongue. 'The cotton or down of Quinces boiled and applied to plague sores, lieals them up: and laid as a plaister, made up with wax, it brings hair to them that are bald, and lieeps it from falling, if it be ready to shed.

\section{RADDISII, OR IIOHSF-RADDISH.}

THe garden Raddish is so well known, that it needs no description.

Descript.] 'The Horse-Raddish hath its first leaves, that rise before Winter, about a foot and a half long, very much cut in or torn on the edges into many parts, of a dark green colour, with a great rib in the middle; after these have been up a while, others follow, which are greater, rougher, broader and longer, whole and not divided at first, but only somewhat rougher dented about the edges; the stalks when it hears flowers (which is scliloni) is great, rising up with some few lesser leaves thercon, to three or four feet high, spreading at the top many binding and cooling of hot fluxes, the oil 
sinall branches of whitish flowers, made of leaves lying on the ground, very much four leaves a-piece; after which come small rent and torn on the sides in many places: pods, like those of Shepherd's Purse, but from among which rise up sometimes but seldom with any seed in them. The root is one, and sometimes two or three square or great, long, white and rugged, shooting up crested blackish or brownish stalks, three divers heads of lcaves, which may be parted or four fect high, sometimes branched, for increase, but it doth not creep in the bearing divers such-like leaves upon them, ground, nor run above ground, and is of a several distances upon the top, where it strong, sharp, and bitler tastc almost like branches forth into many stalks bearing mustard.

Place.] It is found wild in some places, but is chictly planted in gardens, and joys in moist and shadowy places.

Time.] It seldom flowers, but when it doth, it is in July.

Gorernment and rivtues.] They are both under Mars. The juice of Horse-raddish given to drink, is held to be very effectual for the scurvy. It kills the worms in children, being drank, and also laid upon the belly. The root bruised and laid to the place gricved with the sciatica, joint-ache, or the hard swellings of the liver and splecn, doth wonderfully help them all. The distilled water of the herb and root is morc familiar to be taken with a littlc sugar for all the purposes aforesaid.

Garden Raddishes are in wantonness by the gentry eaten as a sallad, but they breed but scurvy humours in the stomach, and corrupt the blood, and then send for a physician as fast as you can; this is one cause which nakes thc owner's of such nice palates so unhcalthful; yet for such as are troubled with the gravel, stonc, or stoppage of urine, they are good physic, if the borly be strong that takes thcm; you may niake the juice of the roots into a syrup if you please, for that use: they purge by urine exceedingly.

$$
\text { RAGWORT. P. } 24 \text {. }
$$

It is called also St. James'-wort, and Stagger-wort, and Stammer-wort, and Segrum.

Descript.] 'I'he greater common Ragwort wounds and ulccrs; stays the malignity of hath many large and long, dark green freiting and running cankers, and hollow

Thcre is another sort thereof differs from the former only in this, that it rises not so high; the leaves are not so finely jagged, nor of so dark a green colour, but rather sonicwhat whitish, soft and woolly, and the flowers usually paler.

Place.] They grow, both of them, wild in pastures, and untilled grounds in many places, and oftentimes both in one field.

Time.] They flower in June and July, and the seed is ripe in August.

Government and virtues.] Ragwort is under the command of Dame Venus, and clcanses, digests, and discusscs. The decoction of the herb is good to wash the mouth or throat that hath ulcers or sores therein: and for swellings, hardness, or imposthumes, for it thoroughly clcanses and heals them; as also thic quinsy, and the ling's cvil. It helps to stay catarrhs, thin rheums, and defluxions from the head into the cyes, nose, or lungs. The juice is found by expericnce to be singularly good to heal green wounds, and to cleanse and heai all old and filthy ulcers in the privities, and in other parts of the body, as also inward 
fistulas, not suffering them to spread farther. It is also much commended to help aches and pains either in the fleshy part, or in the nerves and sinews, as also the sciatica, or pain of the hips or knuckle-bone, to bathe the places with the decoction of the herb, or to anoint them with an ointment made of the herb bruised and boiled in old hog's suet, with some Mastick and Olibanum in powder added unto it after it is strained forth. In Sussex rve call it Ragweed.

\section{RATTLE GRASS.I.24}

OF this there are two kinds which I shall speak of, viz. the red and yellow.

Descript.] The common Red Rattle hath sundry reddish, hollow stalks, and sometimes green, rising from the root, lying for the most part on the ground, some growing more upright, with many small reddish or green leaves set on both sides of a middle rib, finely dented about the edges: The Howers stand at the tops of the stalks and branches, of a fine purplish red colour, like small gaping hooks; after which come blackish seed in small husks, which lying loose therein, will rattle with shaking. The root consists of two or three snall whitish strings with some fibres thereat.

The common Yellow Rattle bath seldom above one round great stalk, lising from the foot, about half a yard, or two feet high, and but few branches thereon, having two long and somewhat broad leaves set at a joint, deeply cut in on the edges, resembling the comb of a cock, broadest next to the stalk, and smaller to the end. 'T'he flower's grow at the tops of the stalks, with some shorter leaves with them, hooded after the same manner that the others are, but of a fair yellow colour, or in some paler, and in some more white. The seed is contained in large husks, and being ripe, will rattle or make a noise with lying loose in them. The root is small and slender, perishing every yeạr.
Place.] They grow in meadows and woods generally through this land.

Time.] They are in flower from Midsummer until August be past, sonctinies.

Government and virtues.] They are both of them under the dominion of the Moon. The Red Rattle is accounted profitable to heal up fistulas and hollow ulcers, and to stay the flux of humours in them, as also the abundance of women's courses, or any other fluxes of blood, being boiled in red wine, and drank.

'The yellow Rattle, or Cock's Comb, is held to be good for those that are troubled with a cough, or dimuess of sight, if the herb, being boiled with beans, and some honey put thereto, be drank or dropped into the eyes. The whole seed being put into the eyes, draws forth any skin, dimness or film, from the sight, without trouble, or pain.

\section{REST HARROW, OR CAMMOCK.}

Descript.] Common Rest Harrow rises up with divers rough woody twigs half a yard or a yard high, set at the joints without order, with little roundish leaves, sometines more than two or three at a place, of a dark green colour, without thorus while they are young; but afterwards armed in sundry places, with short and sharp thorns. The flowers come forth at the tops of the twigs and branches, whereof it is full fashioned like pease or broom blossoms, but lesser, flatter, and somewhat closer, of a faint purplish colour; after which come small pods containing small, flat, round seed: The root is blackish on the outside, and whitish within, very rough, and hard to break when it is fresh and green, and as hard as an horn when it is dried, thrusting down deep into the ground, and spreading likewise, every piece being apt to grow again if it be left in the ground.

Place.] It grows in many places of this land, as well in the arable as waste ground. 
Time.] It flowers about the beginning have; of a sad grcen colour, from among or middle of July, and the seed is ripe in which rise up divers stalks two or thrce August.

feet high, sometimes set with the like lcaves,

Government and virtues.] It is under the but smaller and smaller upwards, branched dominion of Mars. It is singularly good from the middle into divers stiff stalks, to provoke urine when it is stopped, and to bearing sundry yellow flowers on them, break and drive forth the stone, which the made of four leaves a-piece, as the others powder of the bark of the root taken in wine are, which afterwards yield them small redperforms effectually. Matthiolus . saith, dish seed, in small long pods, of a more The sansc helps the disease called Herma bitter and hot biting taste than the garden Carnosa, the Heshy rupture, by taking the kinds, as the leavcs are also.

said powder for three months together constantly, and that it hath cured some which seemed incurable by any other means than by cutting or burning. The dccoction thereof made with some vinegar, gargled in the mouth, eases the touth-ache, especially when it comes of rheum; and the said decoction is very powerful to open obstructions of the liver and spleen, and other parts. A distilled water in Balneo Maric, fear of inflaming their blood, and thereforc with four poutrls of the root hereof first for such we may say a little doth but a sliced small, and afterwards stceped in a little harm, for angry Mars rules them, and gallon of Canary wine, is singularly good he sometimes will be restive when he mects for all the purposes aforesaid, and to cleanse with fools. 'The wild Rockct is more strong the urinary passages. The powder of the and effectual to increase sperm and vencsaid root madeintoan electuary, or lozenges, rous qualitics, whereunto all the secd is with sugar, as also the bark of the fresh more effectual than the garden kind. It roots boiled tender, and afterwards beaten serves also to help digestion, and provokes to at conserve with sugar, works the like urine cxceedingly. The sced is used to cure efiect. The powder of the roots strewed the biting of serpents, the scorpion, and the upon the brims of ulcers, or mixed with shrew mouse, and other poisons, and expels any other convenicnt thing, and applicd, worms, and other noisome creatures that consumes the hardness, and causes them to breed in the belly. 'The herb boiled or heal the better.

$$
\text { ROCKT. }
$$

stewed, and some sugar put thereto, lielps the cough in children, being taken often. The seed also taken in drink, takes away

Is regard the Garden Rocket is rather the ill scent of the aim-pits, increases milk wed as a sallad herb than to any physical in nurses, and wastes the spleen. 'The seed purposes, I shall omit it, and only speak mixed with honey, and used on the face, of the common wild Rocket. 'The descriplion whereof take as follows.

Descripi.] 'The common wild Rocket? bas longer and narrower leaves, much more the gall of an ox, it mends foul scars, black divided into slender cuts and jags on both and blue spots, and the marlis of the smallsidcs the middle rib than the garden kinds pox. 
WINTER-ROCKET, OR CRESSES.P. 24 ?

have authors made with Roses! What a Descript.] WINTER-Rocket, or Winter- Roses are under Jupiter, Damask under Cresses, hath divers somewhat, large sad? Venus, White under the Moon, and Progreen leaves lying upon the ground, torn or velice under the King of France. This cut in divers parts, somewhat like unto white and red Roses are cooling and dryRocket or turnip leaves, with smaller pieces? ing, and yet the white is taken to exceed next the bottom, and broad at the ends, the red in both the properties, but is seldom which so abide all the Winter (if it spring? used inwardly in any medicine: The bitup in Autumn, when it is used to be eaten) from among which rise up divers small round stalks, full of branches, bearing many small yellow flower's of fou leaves a-piece, heat which caused the bitterness being conafter which come small pods, with reddish sumed, they have then a binding and asseed in them. The root is somewhat stringy, tringent quality: Those also hat are not and perishes every year after the seed is full blown, do both cool and bind nore ripe.

Place.] It grows of its own accord in gardens and fields, by the way-sides, in divers places, and particularly in the next pasture to the Conduit-head behind Gray's Inn, that brings water to Mr. Lamb's conduit in Holborn.

Time.] It flowers in May, secds in June, and then perishes.

Government and virtues.] This is profitable to provoke urine, to help stranguary, and expel gravel and stone. It is good for the scurvy, and found by experience to be a singularly good wound herb to cleanse inward wounds; the juice or decoction being drank, or outwardly applied to wash foul ulcers and sores, cleansing them by sharpness, and lindering or abating the dead flesh from growing therein, and healing them by their drying quality.

\section{ROSES.}

I I HoLD it altogether necdless to trouble the reader with a description of any of these, since both the garden Roses, and the Roses of the briars are well enough known: take therefore the virtues of them as follows; And first I shall begin with the garden kinds.

Government and virtues.]

What a pother than those that are full blown, and the white Rose more than the Red. The decoction of red Roses made with wine and used, is very good for the head-ache, and pains in the eyes, ears, throat, and gums; as also for the fundament, the lower part of the belly and the matrix, being bathed or put into them. The same decoction with the Roses remaining in it, is profitably applied. to the region of the heart to ease the inflammation therein; as also St. Anthony's fire, and other diseases of the stomach. Being dried and beaten to powder, and taken in steeled wine or water, it helps to stay women's courses. The yellow threads in the middle of the Roses (which are erroneously called the Rose Seed) being powdered and drank in the distilled water of Quinces, stays the overflowing of women's courses, and doth wonderfully stay the defluctions of rheum upon the gums and teetl, preserving them from corruption, and fastening them if they be loose, being washed and gargled therewith, and some vinegar of Squills added thereto. The heads with the seed being used in powder, or in a decoction, stays the lask and spilting of blood. Red Roses do strengthen the hcart, the stomach and the liver, and the retcn. tive faculty: They nitigate the pains that 
arise from heat, assuage inflammations, of mastich, is very good for the gonorrhea, procure rest and sleep, stay both whites and for the looseness of the humours in the and reds in women, the gonorrhea, or runn- body. The old Couserve mixed with Aroing of the reins, and fluxes of the belly: the maticum Rosarum, is a very good cordial juice of thein doth purge and cleanse the against faintings, swoonings, weaknèss, body from choler and phlegm: 'The husks and tremblings of the heart, strengthens, of the Roses, with the beards and nails of both it and a weak stomach, helps digesthe Roses, are binding and cooling, and the tion, stays casting, and is a very good predistilled water of either of them is good for servative in the time of infection. The dry the heat and redness in the eyes, and to Conserve, which is called the Sugar of Roses, stay and dry up the rheums and watering is a very good:cordial to strengthen the of them. Of the Red Roses are usuallymade heart and spirits ; as also to stay deflucmany compositions, all serving to sundry tions. The syrup of dried red Roses good uses, viz. Electuary of Roses, Con- strengtliens a stomach given to casting, serve, both moist and dry, which is more cools an over-heated liver, and the blood in usually called Sugar of roses, Syrup of dry agues, comforts the heart, and resists putreRoses, and Honey of Roses.. The cordial faction and infection, and helps to: say powder called: Diarrhoden: Abbatis, and lasks and fluxes. Honey of Roses is much Aromatica Rosurum. The distilled Water of used in gargles and lotions to wash sores, Roses, Vinegar of Roses, Ointment, and Oil either in the mouth, throat, or other parts, of Roses, and the Rose leaves dried, are of both to cleanse and heal them, and to stay great use and effect. 'Towriteat large of every the fluxes of humours falling upon them. one of these, would make my book: smell It is also uscd in clysters both to cool and too big, it being sufficient for a volume of cleanse. 'The cordial powders, called itself, to speak fully of them. But briefly, Diarrhoden Abbatis and Aromaticum Ro. the Electuary is purging, whereof two or sarum, do comfort and strengthen the heart three drams taken by itself in some con- and stomach, procure an appetite, help venient liquor, is a purge sufficient for a digestion, stay vomiting, and are very good weak constitution, but may be increased to for those that have slippery bowels, to six drams, according to the strength of the strengthen them, and to dry up their moispatient. It purges choler without trouble, ture. Red Rose-water is well known, and it is good in hot fevers, and pains of the of familiar use on all occasions, and better head arising from hot cholcric bumours, than Damask Rose-water, being cooling and and heat in the eyes, the jaundice also, and cordial, refreshing, quickening the weak joint-aches proceeding of hot humours. and faint spirits, used either in meats or The moist Conserve is of much use, both broths, to wash the temples, to smell at the binding and cordial; for until it be about nose, or to smell the swcet vapours thereof two years old, it is more binding than out of a perfuming pot, or cast into a hot cordial, and after that, more cordial than fire shovel. It is also of much good use binding. Some of the younger Conserve against the redncss and inflammations of the taken with mitluridate mixed together; is eyes to bathe them therewith, and the temgood for those that are troubled with dis- ples of the head; as also against pain and illations of rheum from the brain to the ache, for which purpose also Vinegar of nose, and defluctions of rhem into the Roses is of much gond use, and to procure eycs; as also for tiuxes and lasks of the rest and slecp, if some thereot, and Rosebelly; and being mixed with the powder? water together, be used to smill unto, or the 
nose and temples moistened therewith, but more usually to moisten a piece of a red melancholichunıurs; and available against Rose-cake, cut for the purpose, and heated the leprosy, itch, tetters, \&c. and the French between a double folded cloth, with a little disease: Also honey of Roses solutive is beaten nutmeg, and poppy-seed strewed on made of the same infusions that the syrup) the side that must lie next to the forehead is made of, and therefore works the same and temples, and bound so thereto all night. ? effect, both opening and purging, but is The ointment of Roses is much used against oftener given to phlegmatic than choleric heat and infammations in the head, to anoint persons, and is more used in clysters than the forehead and temples, and being mixt in potions, as the syrup made with sugar is. with Unguentum Populneum, to procure rest: The conserve and preserved leaves of those: it is also used for the heat of the liver, the Roses are also opcrative in gently opening back and reins, and to cool and heal pushes, the belly.

wheals, and other red pimples rising in the face or other parts. Oil of Roses is not only used by itself to cool any hot swellings or inflammations, and to bind and stay fluxes of humours unto sores, but is also put into ointments and plaisters that are cooling and binding, and restraining the flux of humours. The dried leaves of the red Roses are used both inwardly and outwardly, both cooling, binding, and cordial, for with them are made both Aromaticum, Rosarum, Diarrhoden Abbatis, and Saccharum Rosarum, each of whose properties are before declared. Rose leares and mint, heated and applied outwardly to the strengthen a weak stomach; and applied as a fomentation to the region of the liver and heart, do much cool and temper them, and also serve instead of a Rose-cake (as is said before) to quiet the over-hot spirits, and cause rest and sleep. The syrup of Damask Roses is both simple and compound, and made with Agaric. The simple solutive syrup is a familiar, safe, gentle and casy medicine, purging choler, taken from one herein, that the distilled water of this syrup should notably bind tlie belly. 'The syrup is found by experience of many to kill and with Agaric is more strong and effectual, for drive forth the worms of the belly.

one ounce thereof by itself will open the body more than the other, and works as much on phlegm as choler. The com-?

The simple water of Damask Roses is chiefly used for fumes to sweeten things, as. the dried leaves thereof to make sweet powders, and fill sweet bags; and little use they are put to in physic, although they have some purging quality; the wild Roses also are few or none of them used in physic, but are generally held to come near the nature of the manured Roses. The fruit of the wild briar, which are called Hips, bcing. thoroughly ripe, and made into a conserve with sugar, besides the pleasantness of the taste, doth gently bind the belly, and stay defluctions from the bead uppon the stomach, drying up the moisture thereof, and helps digestion. The pulp of the hips clried into a hard consistence, like to the juice of the liquorice, or so dried that it may be made into powder and taken into drink, stays speedily the whites in women. The briar ball is often used, being made into powder and drank, to break the stone, to provoke urine when it is stopped, and to ease and help the cholic; some appoint it to be burnt, and then taken for the same purpose: In the middle of the balls are often found certain white worms, which being dried and made into powder, and some of it drank, (a) 


\section{ROSA SOLIS, OR SUN DEW.}

IT is likewise called Red-rot, and. Youthwort.

Descript.] It hath, divers small, round, hollow leaves somewhat greenish, but full of certain red hairs, which make them secm red, every onc standing upon his own footstalk, reddish, hairy likewise. T'he leaves are continually moist in the hottest day, in these days. as any whatsoever, not only yea, the hotter the sun shines on them, the for physical but civil purposes. The phymoister they are, with a sliminess that will ssical use of it (being my present task) is rope (as we say), the small hairs always very much used both for inward and outward holding the moisture., Ansong these leaves diseases, for by the warming and comfortrise up slender stalks, reddish also, three or ing heat thereof it helps all cold: diseases, four fingers high, bearing divers sinall white both of the head, stomach, liver, and belly. knobs one above another, which are flowers; The decoction thereof in. wine, liclps the after which in the hoads are contained cold distillations of rheum into the eyes, small seeds. 'The root is a, few small hairs. and all other cold diseases of the head and

Place.] It grows usually. in bogs and brain, as the giddiness or swimmings wet places, and sometimes in moist woods. therein, drowsiness or dulness of the mind

Time.] It flowers in June, and the leaves and senses like a stupilness, the dumb palsy, are then fittest to be gathered

Gorennment and rintues.]: The Sun rules it, and it is under the sign Cancer. Rose Solis is accomited good to help those that have a salt rheuin distilling on the lungs, which breeds a consumption, and therefore the distilled water thereof in wine is held fit and profitable for such to drink, which water will be of a gnod yellow colour. The same water is held to be good for all other diseases of the lungs, as phthisics, wheezings, shortness of breath, or the cough; as also to heal the ulcers that happen in the lungs; and it coinforts the licart and fainting spirits. 'The leaves, outwardly applica. to the skin, will raise blisters, whicli has caused some to think it dangerous to be taken inwardly; but there are other things which will also clraw blisters, yet nothing dangerous to be taken inwardly. 'I'here is an usual drink made thereof with aqua vita and spices frequently, and without any offence or dinger, but to good purpose used in qualms and passions of the heart. $(15,16$. or loss of speech, the lethary, and fallingbathed therewith. It helps the pains in the gums and teeth, by rlseum falling into thon, not by putrefaction, causing an evil smell from them, or a stinking breath. It helps a weak momory, and quickens the senses. It is very comfortable to the stomach in all the cold griefs thereof, helps both retention of meat, and digestion, the decoction or powder being taken in wine. It is a remedy for the windiness in the stomach, bowels, and spleen, and expels it powerfilly. It ${ }^{3}$. liclps those that are liver-grow, by opening the obstructions thiceof. It helps dim eyes, and procures a ciear sight, the flowers thercof being taken all the while it is flowcring, every morning fasting, with bread and salt. Both Diosrorides and Galen say, I'hat if a decoction be made thercof witl' water, and they that have the yellow jamdice excrcise their bodies presently after thic taking thereof, it will certainly cure thom. 'The flowers and conserve made of 1 liem, $\mathrm{s}$ s sickness, to be botls drank, and tinc temples 
are singularly good to comfort the heart, pursued its virtues, you will conclude it and to expel the contagion of the pestilence; nothing inferior to that which is brought to burn the herb in houses and cliambers, out of China, and by that time this hath corrects the air in them. Both the flowers been as much used as that hath been, the and leares are very profitable for women name which the other hath gotten will be that are troubled with the whites, if they be eclipsed by the fame of this ; take theredaily taken. The dried leaves shred small, fore a description at large of it as follows : and taken in a pipe, as tobacco is taken, helps those that have any cough, phthisic, or consumption, by warming and drying the thin distillations which cause those diseases. The leaves are very much used in bathings; and made into ointments or oil, are singularly good to help cold benumbed joints, sinews, or members. The chymical oil drawn from the leaves and flowers, is a sovereign help for all the diseases aforesaid, to touch the temples and nostrils with two or three drops for all the diseases of the head and brain spoken of before; as also to take one drop, two, or three, as the case requires, for the inward griefs: Yet must it be done with discretion, for it is very quick and piercing, and therefore but a little must be taken at a time. There is also another oil made by insolation in this manner: Take what quantity you will of the flowers, and put them into a strong glass close slopped, tie a fine linen cloth over the mouth, and turn the mouth down into anotlier strong glass, which being set in the sun, an oil will distil down into the lower glass, to be preserved as precious for divers uses, both invard and outward, as a sovereign balm to heal the diseases beforc-mentioned, to clear dim sights, and take away spots, marks, and scar's in the skin.

RIUBARB, ÓR REPIIONTIC.

Do not start, and say, This grows you larger, whereby it may be plainly known to know not how far off: and then ask me, be a Dock. 'The root grows in time to be How it comes to pass that I bring it among very great, with divers and sundry great our English simples? For though the name spreading branches from it, of a dark may speak it foreign, yet it grows with us in brownish or reddish colour on the outside, Kingland, and that frequent enough in our having a pale yellow skin under it, which gardens; and when you have thoroughly :covers the inner substance or root; which 
rind and skin being pared away, the root of the stalks being divided into many small appears of so fresh and lively a colour, branches, bear reddish or purplish flowers, with fresh coloured veins running through and three-square seed, like unto other it, that the choicest of that Rhubarb that is Docks. The root is long, great and yellow, brcught us from beyond the seas cannot like unto the wild Docks, but a little redder; excel it, which root, if it be dried carefully, and as it ought (which must be in our discoloured. veins than the other does when country by the gentle heat of a fire, in re- it is dry.

gard the sun is not lot enough here to do it, and every piece kept from toucling one another) will hold its colour almost as well as when it is fresh, and has been approved of, and commended by those who have oftentimes used them.

Place.] It grows in gardens, and flowcrs about the beginning or middle of June, and the seed is ripe in July.

Time.] The roots that are to be dried and kept all the year foilowing, are nol to thereon, but smaller; at the top whereof be taken up before the stalk and leaves be stand in a long spike many small brownish quite turned red and gone, and that is not flowers, which turn into a hard three square until the middle or cnd of Octoher, and if slining brown seed, likethe garden Patience they be taken a little before the leaves dó spring, or when they are sprung up, the ronts will not have half so good a colour in them.

GREAT ROUND-LEAVED DOCK, OR BAS-

TARD RIIUBARB. Plate 24 .

Descript.] This has divers large, round, thin, yellowish green leaves rising from the root, a little waved about the edges, every one standing upon a reasonably thick and long brownish footstalk, from among which rises up a pretty big stalk, ahout two fcet high, with some such high leaves growing before described. The root grows greater than that, with many branches or great fibres thereat, yellow on the outside, and somewhat pale; yellow within, with some I have given the precedence unto this, discoloured veins like to the Rhubarb which because in virtues also it hath the pre-emi- is first described, but much less than it, nence. I come now to describe unto you especially when it is dry.

that which is called Patience, or Monk's

Place and Time.] These also grow in Rhubarb; and the next unto that, the great gardens, and flower and seed at or near the round-leaved Dock, or Bastard Rhubarb, same time that our true Rhubarb doth, viz. for the one of these may happily supply in they flower in June, and the seed is ripc in the absence of the other, being not much July.

unlike in their virtues, only one more

Government and virtues.] Mars clains powerful and cfficacious than the other. predominancy over all these wholesome And lastly, shall shew you the virtues of all herbs: You cry out upon him for an inthe three sorts.

GARDEN-PATIEXCE, OR MONK'S RHUBA R B. Plate z4. fortunate, when God created him for rour good (only he is angry with fools.) What dishonour is this, not to Mar's, bitt to God himself. A dram of the dried root of Descript.] This is a Dock bearing the Monk's Rhubarb, with a scruple of Ginger name of Rhubarb for some purging quality made into powder, and taken fasting in a therein, and grows up with large tall stalks, draught or mess of warm broth, purges set with somewhat broad and long, fair, choler and phlegm downwards very gently green leaves, not dented at all. The tops s, and safely without danger. The secr 
thereof contrary doth bind the belly, and blood, opening obstructions, and helping helps to stay any sort of lasks or bloody- those griefs that come thereof, as the jaumflux. The distilled water thereof is very dice, dropsy, swelling of the spleen, tertain profitably used to heal scabs; also foul and daily agues, and pricking pains of the ulccrous sores, and to allay the inflamma- sides; and also stays spitting of blood. tion of them: the juice of the leaves or The powder taken with cassia dissolved, roots or the decoction of them in vinegar, and washed Venice turpentine, cleanses the is used as a most effectual remedy to heal reins and strengthens them afterwards, and scabs and running sores.

The Bastard Rhubarb hath all the pro- is also given for the pains and swellings in perties of the Monk's Rhubarb, but more the head, for those that are troubled with effectual for both inward and outwaîd dis-melancholy, and helps the sciatica, gout, eases. The decoction thereof without vine- and the cramp. 'The powder of the Rhugar dropped into the ears, lakes away the barb taken with a litlle mummia and madpains; gargled in the mouth, takes away der roots in some red wine, dissolves clotted the tooth-ache; and being drank, heals the blood in the body, happening by any fall jaundice. The seed thereof taken, eases or bruise, and helps burstings and broken the gnawing and griping pains of the parts, as well inward as outward. The oil stomach, and takes away the loathing there- likewise wherein it hath been boiled, works of unto meat. The root thereof helps the the like effects, being anointed. It is used ruggedness of the nails, and being boiled in to heal those ulcers that happen in the eyes wine helps the swelling of the throat, com- or eyelids, being steeped and strainer ; as monly called the king's evil, as also the also to assuage the swellings and inflamswellings of the kernels of the ears. It mations; and applied with honcy, boiled helps them that are troubled with the stone, in wine, it takes away all blue spots or provoles urine, and helps the dimness of marks that happen therein. Whey or the sight. 'The roots of this Bastard Rhu- white wine are the best liquors to steep it barb are used in opening and purging diet-in, and thereby it works more effectual in drinks, with other things, to open the liver, opening obstructions, and purging the and to clcanse and cool the blood.

stomach and liver. Many do use a little

'The properties of that which is called the Indian Spikenard as the best corrector English Rhubarb are the same with the thercof.

former, but much more effectual, and hath all the properties of the true Italian Rhubarbs, except the force in purging, wherein? it is but of half the strength thereof, and a yellow stringy root, nuch spreacling in therefore a double quantity must be used: the ground, shooting forth new sprouts it likewise hath not that bittcrness and as- round about, with many herby green stalks, triction ; in other things it works almost in two feet high, crested all the length of them, an equal quantity, which are these: It set with joints here and there, and many purges the body of choler and phlegm, being large leares on them, above as well as beeither taken of itself, made into powder, and low, being divided into smaller learies, drank in a dranght of white wine, or steeped nicked o: dented in the fore part of them, therein all night, and taken fasting, or put of a red green colour on the upper-side, anong other purges, as shall be thought and pale green underneath; 'Toward the convenient, cleansing the siomach, liver, and top of the stalli there shoots forth divers 
short branches, on every one whereof stand often taken in meat and drink, it abates two, three or four small heads, or buttons, venery. 1 decoction thereof with some which breaking the skin that incloses them, dried dill Jeaves and Howers, eases all pains shoots forth a tuft of pale grcenish yellow and torments, inwardly to be drank, and threads, which falling away, there come in outwardly to be applied warm to the place thcir places small thrce-cornered cods, grieved. 'Ihe same being drank, helps the whercin is contained smiall, long and round pains both of the cliest and sides, as also seed. The whole plant has a strong un- coughs and hardness of breathing, the inplcasant scent.

Place.] It grows in many places of this ing pains of the sciatica and the joints, being land, in the border's of moist meadows, and a anointed, or laid to the places; as also the ditch-sides.

Time.] It flowers about July, or the beginning of August.

Government and virhues.] Dioscorides saith, That this herb bruised and applied, and frees women from the strangling or perfectly heals old sores, and the distilled suffocation thereof, if the share and the water of the herb and flowers doth the like, parts thereabouts be anointed therewith. It It is used by some among other pot-herbs kills and drives forth the worms of the to open the body, and make it soluble; but belly, if it be drank after it is boiled in wine the roots washicd clean, and boiled in ale to the half, with a little honey; it helps the and drank, provokes to stool more than the leaves, but yct very gently. The root knees, applied thereunto; and with figs it boiled in water, and the places of the body hiclps the dropsy, being bathed thercwith: most troubled with rermin and lice washed Bcing bruised and put into tho nostrils, it therewith while it is warm, destroys them slays the bleeding thereof. It takes away utterly. In Italy it is good against the wheals and pimples, if being bruised with a plague, and in Saxony against the jaundice, f few myrtlc leaves, it be made up with wax, as Camerarius saith.

GA R D N $\mathrm{R} U \mathrm{E}$.

and applied. It cures the morpliew, and takes away all sorts of warts, if boiled in wine with some pepper and nitre, and the

GARDEN-RUE is so well known by this place rubbed therewith, and with almond name, and the name Herb of Grace, that $I$ and honey helps the dry scabs, or any shall not need to write any farther descrip- tetter or ringworm. The juice thereof tion of it, but shall shew you the virtue of it, warmed in a pomegranate shell or rind, and as follows.

Government and virlues.] It is an lierb of the Sun, and under Leo. It provokes urine dropped into the ears, helps the pains of them. The juice of it and fennel, with a little honey, and the gall of a cock put thereand women's courses, being taken either in unto, helps the dimness of the eye-sight. meat or drink. 'T'le seed thereof taken in An ointment made of the juice thereof with wine, is, an antidote against all dangerous oil of roses, ceruse, and a little vinegar, and medicincs or dcadly poisons. 'The leaves anointed, cures St. Anthony's fire, and all takcn either by themselves, or with figs and running sores in the head: and the stinking walnuts, is called Mithridate's counter-poi- ulcers of the nose, or other parts. 'The son against the plaguc, and causes all veno- antidote used by Mithridates, every mornmows things to become harmless; being ing fasting, 10 sccure himself from any $(15,16$.$) .$ 
poison or infection, was this: 'Take twenty ground, about a span long, divided into leaves of rue, a little salt, a couple of wal- many other smaller parts full of small joints nuts, and a couple of tigs, beaten together set very thick together, whereat come forth into a mess, with twenty juniper berries, two very small leaves of a French yellow, which is the quantity appointed for every green coloured branches and all, where day. Another electuary is made thus : grows forth also a number of exceedingly Take of nitre, pepper, and cummin seed, small yellowish flowers, scarce to be disof each equal parts; of the leaves of Rue cerned from the stalks and leaves, which clean picked, as much in weight as all the turn into seeds as smail as the very dust. other three weighed; beat them well toge- The root is very long and small, thrusting ther, and put as much honey as will make down deep into the ground. This lias it up into an electuary (but you must first neither smell nor taste at first, but aftersteep your cummin seed in vinegar twenty wards has a little astringent taste, without four hours, and then dry it, or rather roast any manifest heat; yet a little bitter and it in a hot fire-shovel, or in an oven) and is sharp withal.

a remedy for the pains or griefs in the chest or stomach, of the spleen, belly, or sides, by wind or stitches; of the liver by obstructions; of the reins and bladder by the stopping of urine; and helps also to extenuate fat corpulent bodies. What an infamy is cast upon the ashes of Mithridates, or Methridates (as the Augustines read his name) by unworthy people. They that is Saturn's own, and is a noble antivenedeserve no good report themselves, love to rean. Rupture-wort hath not its name in give none to others, viz. That renowned vain: for it is found by experience to cure King of Pontus fortified his body by poison the rupture, not only in children but also in against poison. (He cast out devils by Beel- elder persons, if the disease be not too inzebub, Prince of the devils.) What a sot is veterate, by taking a dram of the powder he that knows not if he had accustomed his of the dried herb every day in wine, or a body to cold poisons, but poisons would decoction made and drank for certain days have dispatched him? on the contrary, if together. 'The juice or distilled water of not, corrosions would have done it. The the green herb, taken in the same manner, whole world is at this present time beholden helps all other fluxes either: of man or to him for his studies in plysic, and he that woman; vomitings also, and the gonorrhea, uses the quantity but of an hazel-nut of that being taken any of the ways aforesaid. It receipt every morning, to which his nanc doth also most assuredly help those that is adjoined, shall to adniration preserve have the stranguary, or are troubled with his body in health, if he do but consider the stone or gravel in the reins or bladder. that Rue is an herb of the Sun, and under The same also helps stitches in the sides, Leo, and gather it and the rest accord. ingly.

RUPTURE-WORT. $\mathbb{P}_{\text {F }} 24$.

Descript.] Turs spreads very many griping pains of the stomach or belly, the obstructions of the liver, and cures the yellow jaundice; likewise it kills also the worms in children. Being outwardly apthready branches round about upon the plied, it conglutinates wounds notably, and 
fiom the head to the eyes, nose, and teetl, id did describe it, they would presently say, being bruised green and bound thereto; or I might as well have spared that labour. the forehead, temples, or the nape of the Its virtue follows.

neck behind, bathed with the decoction of Goternment and virtues.] Rye is more the dried herb. It also dries up the mois- digesting than wheat; the bread and the ture of fistulous ulcers, or any other that are leaven thereof ripens and breaks imposfoul and spreading.

RUSHES. P. 32 .

A LThougir there are many kinds Rushes, yet I shall only here insist upon those which are best known, and most medicinal; as the bulrushes, and other of the soft and smooth kinds, which grow so commonly in almost every part of this land, and are so generally noted, that I suppose it needless to trouble you with any description of them: Briefly. then take the virtues of them as follows:

Government and virtues.] The seed of the soft Rushes, (saith Dioscorides and Galen, toasted, saith Pliny) being drank in wine and water, stays the lask and women's courses, when they come down too abundaily: but it causes head-ache; it provokes sleep likewise, but must be given with caution. 'The root boiled in water; to the consumption of onc third, helps the cough.

Thius you see that conveniences have their inconveniences, and virtue is seldom unaccompanied with some vices. What I have written concerning Rushes, is to satisfy my countrymen's questions: Are our Rushes good for nothing? Yes, and as good let them alone as taken. There are remedies enough without them for any disease, and therefore as the proverb is, I care not a rush for them; or, rather they will do you as much good as if one had given you a Rush.

$$
\text { RIE. P. } 23 .
$$

TIIIs is so well known in all the counties crocus, both. white and yellow, purge of this land, and especially to the country- phlegm as hermodactyls do ; and if you people, who feed much thereon, that if $I$ please to dry the roots of any crocus, neither meal of Rye put between a double cloth, and moistened with a little vinegar, and dish of coals, and bound fast to the head while it is hot, doth much case the continual pains of the head. Matthiolus saith, that the ashes of Rye straw put into water, and steeped therein a day and a night, and the chops of the hands or feet washed therewith, doth heal them.

\section{SAFFRON. $\theta \cdot 27 \%$}

T'us herb needs no description, it being known generally where it grows.

Place.] It grows frequently at Walden in Essex, and in Cambridgeshire.

Government and vivtues.] It is an herb of the Sun, ind under the Lion, and therefore you need not demand a reason why it strengtliens the heart so exccedingly. Let not above ten grains be given at one time, for the Sun, which is the fountain of light, way dazzlc the eyes, and make them blind; a cordial being taken in an immoderate quantity, hurts the heart instead of helping it. It quickens the brain, for the Sun is exalted in Aries, as he hath liis house in Leo. It lielps consumptions of the lung's, and difficulty of breathing. It is excellent in epidemical diseases, as pestilence, smallpox, and measles. It is a notable expulsive medicinc, and a notable remedy for the yellow jaundice. My opinion is, (but I have no author for it) that hermodactyls are nothing else but the roots of Saffion dried; and my reason is, that the roots of all

thumes, boils, and other swellings: The 
your eyes nor your taste shall distinguish with wormwood is good for the bloody-flux. them from hermodactyls.

$$
\text { SAGE. P. } 26 \text {. }
$$

Ou ordinary garden Sage needs no description.

\section{Time.] It flowers in or about July.}

Government and virtues.] Jupiter claims this, and bids me tell you, it is good for the liver, and to breed blood. A decoction of the leaves and branches of Sage made and drank, saith Dioscorides, provokes urine, brings down women's courses, helps to expel the dead child, and causes the hair to become black. It stays the bleeding of wounds, and cleanses foul ulcers. Thrce spoonfuls of the juice of Sage taken fasting, with a little honey, doth presently stay the spitting or casting of blood of them that are in a consumption. These pills are much commended; Take of spikenard, ginger, of each two drams; of the seed of Sage toasted at the fire, eight drams; of long pepper, twelvedrams; all these being brought into powder, put thereto so much juice of Sage as may make them into a mass of pills, taking a dram of them every moining fasting, and so likewise at night, drinking a little pure water after them. Matthiolus saith, it is very profitable for all manner of pains in the head coming of cold and rheumatic humours : as also for all pains of the joints, whether inwardly or outwardly, and therefore helps the falling-sickness, the lethargy such as are dull and heavy of least, wilh two leaves set at every joint; spirit, the palsy; and is of much use in all somewhat like other Sage leaves, but defluctions of rheum from the head, and for smaller, softer, whiter, and rounder, and a the diseases of the chest or breast. The little dented about the edges, and smelling leaves of Sagc and nettles bruised together, somewhat stronger. At the tops of the and laid upon the imposthume that lises stalks and branches stand the flowers, on a behind the ears, doth assuage it much. slcnder like spike, turning thenselves all 'The juice of Sage taken in warm water, one way when they blow, and are of a pale helps a hoarseness and a cough. The and whitish colour, shaller than Sage, but leaves sodden in wine, and laid upon the hooded and gaping like unto them. The place affected with the palsy, helps much, seed is blackish and round; four usually if the decoction bedrank: Also Sage taken secm in a husk together : the root is long 
and stringy, with divers fibres thereat, and abides many years.

Place.] It grows in woods, and by wood-sides; as also in divers fields and bye-lanes in the land.

Time.] It flowers in June, July, and August.

Government and virtues.] The herb is under Vellus. The decoction of the Wood Sage provokes urine and women's courses: It also provokes sweat, digests humours, round circle representing a Seal, whereof it and discusses swellings and nodes in the took the name, lying along under the upper flesh, and is therefore thought to be good crust of the earth, and not growing downagainst the French pox. The decoction ward, but with many fibres underneath. of the green herb, made with wine, is a safe and sure remedy for those who by falls, bruises, or blows, suspect some vein to be inwardly broken, to disperse and void the congealed blood, and to consolidate the veins. The drink used inwardly, and the herb used outwardly, is good for such as are inwardly or outwardly bursten, and is found to be a sure remedy for the palsy. The juice of the herb, or the powder thereof dried, is good for moist ulcers and sores in the legs, and other parts, to dry them, and cause them to heal more speedily. It
is no less effectual also in green wounds, to be used upon any occasion.

$$
\text { SOLOMON'S SEAL. } 27 \%
$$

Descript.] THe common Solomon's Seal rises up with a round stalk half a yard high, bowing or bending down to the ground, set with single leaves one above another, somewliat large, and like the leaves of the lily-convally, or May-lily, with an eye of bluish upon the green, with some ribs therein, and more yellowish underfroth. At the for the from the bottom up to the top of the stalk, places; yea, it hath been found by expecome forth small, long, white and hollow rience, and the decoction of the root in pendulous flowers, somewhat like the wine, or the bruised root put into wine or flowers of May-lily, but ending in five long other drink, and after a night's infusion, points, for the most part two together, at the strained forth hard and drank, hath helped end of a long foot-stalks, and sometimes but both man and beast, whose bones hath been $(17,18$.

Place.] It is frequent in divers places of this land; as, namely in a wood two miles from Canterbury, by Fish-Pool Hill, as also in Bushy Close belonging to the parsonage of Alderbury, near Clarendon, two miles from Salisbury : in Cheffon wood, on Chesson Hill, between Newington and Sittingbourn in Kent, and divers other places in Tine.] It flowers about May: The
Tinex, and other counties. Government and virtues.] Saturn owns the plant, for he loves his bones well. The to be available in wounds, hurts, and outward sores, to lieal and close up the lips of those that are green, and to dry up and restrain the flux of humours to those that are old. It is singularly good to stay vomilings and bleeding wheresoever, as also all fluxes in man or woman; also, to knit any joint, which by weakness uses to be often out of place, or will not stay in long when it is set; also to knit and join broken bones in any part of the body, the flowers at the foot of a leaf, which are withwhen they are ripe, wherein lie small, 
broken by any occasion, which is the most are the cause of most of the diseases which assured refuge of help to people of divers the frail nature of man is subject to ; both counties of the land that they can have. It which might be remedied by a more freis no less effectual to help ruptures and quent use of this herb. If people would burstings, the decoction in wine, or the have sauce to their meat, they may take powder in broth or drink, being inwardly some for profit as well as for pleasure. It taken, and outwardly applied to the place. is a safe herb, very pleasant both to taste The same is also available for inward or out- and stomach, helps digestion, and in some ward bruises, falls or blows, both to dispel sort opening obstructions of the liver and the congealed blood, and to take away spleen: provokes urine, and helps thereby both the pains and the black and blue to wash away the gravel and stone engenmarks that abide after the hurt. The same dered in the kidneys or bladder. also, or the distilled water of the whole plant, used to the face, or other parts of the skin, cleanses it from morphew, freckles, spots, or marks whatsoever, leaving the place fresh, fair, and lovely; for which purpose it is much used by the Italian Dames.

SA M PH IRE. P. 25.26.27,

\section{SAN ICLE. 0.26 .}

Tis Is herb is by many called Butterwort.

Descript.] Ordinary Sanicle sends forth many great round leaves, standing upon long brownish stalks, erery one somewhat deeply cut or divided into five or six parts, and some of these also cut in somewhat like 'Descript.] Rock Samphire grows up the leaf of crow's-foot, or dove's-foot, and with a tender green stalk about half a yard, finely dented about the edges, smootl, and or two feet high at the most, branching of a dark shining colour, and somewhat forth almost from the very bottom, and reddish about the brims; from amung stored with sundry thick and almost round which arise up small, round green stalks, (somewhat long) leaves of a deep green without any joint or leaf thereon, saving at colour, sometimes two together, and some- the top, where it branches forth into flowers, times more on a stalk, and sappy, and of liaving a leaf divided into three or four a pleasant, hot, and spicy taste. At the parts at that joint with the flowers, which top of the stalks and branches stand umbels are small and white, starting out of small of white flowers, and after them come large round greenish yellow heads, many standseed, bigger than fennel seed, yet some- ing together in a tuft, in which afterwards what like it. The root is great, white, and are the seeds contained, which are small long, continuing many years, and is of an hot and spicy taste likewise.

Place.] It grows on the rocks that are often moistened at the least, if not over-flowed with the sea water.

Time.] And it flowers and seeds in the end of July and August.

Government and virtues.] It is an herb of Jupiter, and was in former times wont to be used more than now it is; the more is the pity. It is well known almost to every! body, that ill digestions and obstructions? round burs, somewhat like the leaves of clevers, and stick in the same manner upon any thing that they touch. 'The root is composed of many blackish strings or fibres, set together at a little long head, which abides with green leaves all the Winter, and perishes not.

Place.] It is found in many shadowy woods, and other places of this land.

Time.] It flowers in June, and the seed is ripe shortly after.

Government and virtues.]. This is one of 
Venus's herbs, to cure the wounds or mis- no leaf appears in the Winter. The taste chiefs Mars inflicts upon the body of man. hereof is strong and unpleasant; and so is It heals green wounds speedily, or any the smell also. ulcers, imposthumes, or bleedings inward, also tumours in any part of the body; for the decoction or powder in drink taken, and the juice used outwardly, dissipates the humours: and there is not found any herb that can give such present help either to man or beast, when the disease falleth upon the lungs or throat, and to lieal up putrid malignant ulcers in the moutl, throat, and privities, by gargling or washing with the decoction of the leaves and roots made in water, and a little honey put thereto. It helps to stay women's courses, and all other fluxes of blood, either by the mouth, urine, or stool, and lasks of the belly; the ulcerations of the kidneys also, and the pains in the bowels, and gonorrihea, being boiled in wine or water, and drank. The same also is no less powerful to help any ruptures or burstings, used both in-
wardly and outwardly: And briefly, it is as effectual in binding, restraining, consolidating, heating, drying and healing, as
comfrey, bugle, self-heal, or any other of the vulnerary lierbs whatsoever.

SARACEN'S CONFOUND, OR SARACEN'S WOUNDWORT. 2.6 .

Descript.] TH Is grows sometimes, with brownish stalks, and other whiles with green, to a man's height, having narrow green leaves snipped about the edges, somewhat like those of the peach-tree, or willow leaves, but not of such a white green colour. The tops of the stalks are furnished with many yellow star-like flowers, standing in green heads, which when they are fallen, and the seed ripe, which is somewhat long, small and of a brown colour, wrapped in down, is therefore carried away, with the joint being somewhat round and broad, wind. The root is composed of fibres set pointed at the ends, dented also about the together at a head, which perishes not in edges, somewhat resembling nettle learcs Winter, although the stalks dry away and for the form, but of. a fresher green colour.

Place.] It grows in moist and wet grounds, by wood-sides, and sometimes in moist places of shadowy groves, as also by the water side.

Time.] It flowers in July, and the seed is soon ripe, and carried away with the wind.

Government and virtues.] Saturn owns the herb, and it is of a sober condition, like him. Among the Germans, this wound herb is preferred before all others of the same quality. Being boiled in wine, and drank, it helps the indisposition of the liver, and freeth the gall from obstructions ; whereby it is good for the yellow jaundice, and for the dropsy in the beginning of it; for all inward ulcers of the reins, mouth or throat, and inward wounds and bruises, likewise for such sores as happen in the privy parts of men and women; being steeped in wine, and then distilled, the water thereof drank, is singularly good to ease all gnawings in the stomach, or other pains of the body, as also the pains of the mother: and being boiled in water, it lielps continual agues; and the said water, or the simple water of the herb distilled, or the juice or decoction, are very effectual to heal any green wound, or old sore or ulcer whatsoever, cleansing them from corruption, and quickly healing them up: Briefly, whatsoever hath been said of bugle or sanicle, may be found herein.

\section{SAUCE-ALONE, OR JACK-BY-TIIE-IHEDGE- SIDE. P.a $\Leftarrow 26$.}

Descript:] The lower leaves of this are rounder than those that grow towards the top of the stalks, and are set singly on a
joint being somewhat round and broad, 
not rough or pricking: The flowers are you all the year, if you love yourself and white, growing at the top of the stalks one your ease, and it is a hundred pounds to a above another, which being past, follow penny if you do not; keep it dry, make small round pods, wherein are contained conserves and syrups of it for your use, and round seed somewhat blackish. The root withal, take notice that the Summer kind stringy and thready, perishes every year is the best. They are both of them hot after it hath given seed, and raises itself and dry, especially the Summer kind, again of its own sowing. The plant, or which is both sharp and quick in taste, any part thereof, being bruised, smells of expelling wind in the stomach and bowels, garlic, but more pleasantly, and tastes and is a present help for the rising of the somewhat hot and sharp, almost like unto mother procured by wind; provokes urine rocket.

Place.] It grows under walls, and by hedge-sides, and path-ways in fields in many places.

Time.] It flowers in June, July, and August.

Government and virtues.] It is an herb of Mercury. This is eaten by many country people as sauce to their salt fish, and helps well to digest the crudities and other corrupt humours engendlered thereby. It warms also the stomach, and causes digestion. The juice thereof boiled with honey is accounted to be as good as hedge mustard for the cough, to cut and expectorate the tough phlegm. The seed bruised and boiled in wine, is a singularly good remedy for the wind colic, or the stone, being drank warm: It is also given to women troubled with the mother, both to drink, and the sced put into a cloth, and applied while it is warm, is of singularly good use. 'The leaves also, or the seed boiled, is good to be used in clysters to ease the pains of the stone. 'The green leaves are held to be good to heal the ulcers in the legs.

WINTER AND SUMMER SAVOURY.

Boty these are so well known and women's courses, and is much commended for women with child to take inwardly, and to smell often unto. It cures tough phlegm in the chest and lungs, and helps to expectorate it the more casily; quickens the dull spirits in the lethargy, the juice thereof being snuffed up into the nostrils. 'T'he juice dropped into the eyes, clears a dull sight, if it proceed of thin cold humours distilled from the brain. 'The juice heated with the oil of Roses, and dropped into the ears, eases them of the noise and singing in them, and of deafness also. Outwardly applied with wheat flour, in manner of a poultice, it gives ease to the sciatica and palsied members, heating and warming them, and takes away their pains. It also takes away the pain that comes by stinging of bees, wasps, \&c.

\section{SAVINE.}

To describe a plant so well known is needless, it being nursed up almost in every garden, and abides green all the Winter.

Government and virtues.] It is under the dominion of Mars, being hot and dry in the third degree, and being of exceeding clean parts, is of a very digesting quality. If you dry the herb into powder, and mix entertained as constant inhabitants in our it with honey, it is an excellent remedy to gardens) that they need no description.

Govermment and virtues.] Mercury claims cleanse old filthy ulcers and fistulas; but it hinders them from healing. 'The same is dominion over this herb, neither is there excellently good to break carbuncles and a better remedy against the colic and iliac plague-sores; also helps the king's evil, passion, than this herb; keep it, dry by : being applied to the place. Being spread 
over a piece of leather, and applied to the them, and to expel it and the gravel by navel, kills the worms in the belly, helps urine; to help the stranguary; for which scabs and itch, running sores, cankers, tet- purpose the decoction of the herb or roots ters, and ringworms ; and being applied to in white wine, is most usual, or the powder the place, may haply cure venereal sores. of the small kernelly root, which is called This I thought good to speak of, as it may the seed, taken in white wine, or in the be safely used outwardly, for inwardly it same decoction made with white wine, is cannot be taken without manifest danger.

THE COMMON WHITE SAXIFRAGE."

Descript.] Thrs hath a few small redmost usual. 'The distilled water of the whole herb, root and flowers, is most familiar to be taken. It provokes also women's courses, and frees and cleanses the stomach and lungs from thick and tough phlegm that trouble them. There are not many better medicines to break the stone than this.

dish kernels of roots covered with some
skins, lying among divers small blackish fibres, which send forth divers round, faint or yellow green leaves, and greyish under-
neath, lying above the grounds, unevenly dented about the edges, and somewhat? hairy, every one upon a little foot-stalk, from whence rises up round, brownish, hairy, green stalks, two or three feet high, with a few such like round leaves as grow below, but smaller, and somewhat branched at the top, whereon stand pretty large white flowers of five leaves a-piece, with some yellow threads in the middle, standing in a long crested, brownish green husk. After the Howers are past, there arises sometimes a round hard head, forked at the top, wherein is contained small black seed, but usually they fall away without any seed, and it is the kernels or grains of the root which are usually called the White Saxifrage-seed, and so used.

Place.] It grows in many places of our land, as well in the lower-most, as in the upper dry corners of meadows, and grassy sandy places. It used to grow near Lamb's conduit, on the backside of Gray's Inn.

Time.] It flowers in May, and then gathered, as well for that which is called the seed, as to distil, for it quickly perishes down to the ground when any hot weather cones.

Gocernment and virtues.] It is very ef- of them herbs of the Moon. The Saxifectual to cleanse the reins and bladder, frages are hot as pepper; and Tragus saith, and to dissolve the stone engendered in by his experience, that they are wholesome $(17,18$. 
They have the same properties the pars- great, white and thick, growing down deep leys have, but in provoking urine, and into the ground, and abides many years. causing the pains thereof, and of the wind and colic, are much more effectual, the roots or seed being used either in powder, or in decoctions, or any other way; and likewise helps the windy pains of the to break and void the stone in the kidneys, to digest cold, viscous, and tough phlegm in the stomach, and is an especial remedy against all kind of venom. Castoreum being boiled in the distilled water thereof, is singularly good to be given to those that are troubled with cramps and convulsions. Some do use to make the seeds into conifits (as they do carraway seeds) which is effectual to all the purposes aforesaid. The juice of the herb dropped into the most grievous wounds of the head, dries up their moisture, and heals them quickly. Some women use the distilled water to take away freckles or spots in the skin or face; and to drink the same sweetened with sugar for all the purposes aforesaid.

\section{SCABIOUS, THREE SOR'TS.}

\section{Descript.] Cоммом field Scabious grows} up with many hairy, soft, whitish green leaves, some whereof are very little, if at all jagged on the edges, others very much rent and torn on the sides, and have threads in them, which upon breaking may be plainly seen; from among which rise up divers hairy green stalks, three or four feet high, with such like hairy green leaves on them, but more deeply and finely divided and branched forth a little: At the tops thereof, which are naked and bare of leaves for a good space, stand round heads of flowers, of a pale blueish colour, set together in a head, the outermost whereof are larger than infection of pestilence, if after the taking the inward, with many threads also in the of it the party sweat two hours in bed, and middle, somewhat flat at the top, as the this medicine be again and again repeated, head with the seed is likewise; the root is if need require. The green herb bruised much alike, take them as follow.

Government and virtues.] Mercury owns the plaint. Scabious is very effectual for all sorts of coughs, shortness of breath, and all other disceases of the breast and lungs, ripening and digesting cold phlegm, and other tough humours, voids them forth by coughing and spitting: It ripens also all sorts of inward ulcers and imposthumes; pleurisy also, if the decoction of the herb dry or green be made in wine, and drank for some time together. Four ounces of the clarified juice of Scabious taken in the morning fasting, with a dram of mithridate, or Venice treacle, frees the heart from any

There are many other sorts of Scabious, but I take these which I have here des-
cribed to be most familiar with us. The
virtues of both these and the rest, being but I take these which I have here des-
cribed to be most familiar with us. The
virtues of both these and the rest, being but I take these which I have here des-
cribed to be most familiar with us. The
virtues of both these and the rest, being

There is another sort of Field Scabious different in nothing from the former, but The Corn Scabious differs little from the first, but that it is greater in all respects, and the flowers more inclining to purple, and the root creeps under the upper crust Place.] The first grows more usually in meadows, especially about London every 'The second in some of the dry fields about this city, but not so plentifully as the ormer.

The third in standing corn, or fallow Time.] They flower in June and July,
The and the borders of such like fields. and some abide flowering until it be late in August, and the seed is ripe in the mean time. 
and applied to any carbuncle or plague sore, is found by certain experience to dissolve and break it in three hours spáce. The same decoction also drank, helps the pains and stitches in the side. The decoction of the roots taken for forty days together, or a dram of the powder of them taken at a tinue in whey, doth (as Matthiolus saith) wonderfully lielp those that are troubled with runving of spreading scabs, tetters, ringworms, yea, although they proceed from the French pox, which, he saith he hath tried by experience. The juice or decoction drank, helps also scabs and breakings-out of the itch, and the like. The juice also made up into an ointment and used, is effectual for the same purpose. 'The same also heals all inward wounds by the drying, cleansing, and healing quality therein: And a syrup made of the juice and sugar, is very effectual to all the purposes aforesaid, and so is the distilled water of the herb and flowers made in due season, especially to be used when the green herb is not in force to be taken. The decoction of the herb and roots outwardly applied, doth wonderfully help all sorts of hard or cold swellings in any part of the body, is
effectual for shrunk sinews or veins, and lieals green wounds, old sores, and ulcers. The juice of Scabious, made up with the powder of Borax and Samphire, cleanses the' skin of the face, or other parts of the body, not only from freckles and pimples, but also from morphew and leprosy ; the head washed with the decoction, cleanses it from dandriff, scurf, sores, itch, and the like, used warm: 'The herb bruised and applied, doth in a short time loosen, and draw forth any splinter, broken bone, arrow head, or other such like thing lying in the flesh.

SCURVYGRAss. 26

Descript.] Tue ordinary English Scurvy- grass hath many thick flat leaves; more long than broad, and sometimes longer and narrower; sométimes also sniooth on the edges, and sometimes a hittle waved; sometimes plain, smooth and pointed, of a sad green; and sometimes a blueish colour, every one standing by itself upón a long foot-stalk, which is brownish or greenish also, from among which arise many slender stalks, bearing few leaves thereon like the other, but longer and less for the most part: At the tops whereof grow many whitish flowers, with yellow threads in the middle, standing about à green head, which becomes the seed vessel, which will be somewhat flat when it is ripe, wherein is contained reddish seed, tasting somewhat hot. The root is made of many white strings, which stick deeply' into the mud, wherein it chiefly delights, yet it will weH abide in the more upland and drièr grounnd, and tastes a little brackish and salt even there, but not so much as where it hath the salt water to feed upon.

Place.] It grows all along the Thames sides, both on the Essex and Kentish shores, from Woolwich round about the sea costs to Dover, Portsmouth, and even to Bristol, where it is had in plenty; the other with round leaves grows in the marshes in Hoiland, in Lincolnshire, and other places of Lincolnshire by the scä side.

Descript.] There is also another sort called Dutch Scurvygrass, which is most known, and frequent in gardens, which has fresh, green, and alinost round leaves rising from the root, not so thick as the former, yet in some rich ground, very large, even twice as big as in otkers, not dented about the hedges, or hollow in the middle, standing on a long foot-stalk; from among these rise long, slender stalks, higher than the former, with more white flowers at the tops of them, which turn into small pods, and smaller brownish seed than the former. The root is white, small and thready. 'The 
taste is nothing salt at all; it hath a hot, the ground, whereby it is made a great tuft aromatical spicy taste.

Time.] It flowers in April and May, and gives seed ripe quickly after.

Government and virtues.] It is an herb of Jupiter. The English Scurvy grass is more used for the salt taste it bears, which doth in a short time. somewhat open and cleanse; but the Dutch herb of Venus, Self-heal, whereby when Scurvygrass is of better effect, and chiefly you are hurt you may heal yourself: It is used (if it may be had) by those that have a special herb for inward and outward the scurvy, and is of singular good effect to $;$ wounds. Take it inwardly in syrups for cleanse the blood, liver, and spleen, taking inward wounds: outwardly in unguents, the juice in the Spring every morning fast- and plaisters for outward. As Self-heal is ing in a cup of drink. The decoction is like Bugle in form, so also in the qualities good for the same purpose, and opens and virtues, serving for all the purposes obstructions, evacuating cold, clammy and wherete Bugle is applied to with good success', phlegmatic humours both from the liver either inwardly or outwardly, for inward and the spleen, and bringing the body to a wounds or ulcers whatsoever within the more lively colour. The juice also helps body, for bruises or falls, and such like all foul ulcers and sores in the mouth, hurts. If it be accompanied with Bugle, gargled therewith; and used outwardly, Sanicle, and other the like wound herbs, cleanses the skin from spots, marks, or scars it will be more effectual to wash or inject that happen therein.

SELF-HEA L. $0.26,2 \%$.

* Descript.] Tux common Self-heal which is called also Prunel, Carpenter's Herb, Hook-heal, and Sickle-wort, is a small, low, creeping herb, having many small, roundish pointed leaves, like leaves of wild mints, of a dark green colour, without dents on the edges; from among which rise square hairy stalks, scarce a foot high, which spread somelimes into branches with small leaves set thereon, up to the top, where stand brown spiked heads of small brownish leaves like scales and flowers set together, ans all ulcers, in the mouth, and throat, almost like the heads of Cassidony, which and those also in the secret parts. And the flowers are gaping, and of a blucish purple, proverb of the Germans, French, and or more pale blue, in some-places sweet, others, is verified in this, That he needs but not so in others. The root consists of neither physician nor surgeon that hath Selfmany fibres, downward, and spreading heal and Sanicle to help himself. strings also whereby it increases. The small stallis, with the leaves creeping on the ground, shoot forth fibres taking hold on 


\section{THE SERVICE-TREE. $\$ .27$.}

IT is so well known in the place where it grows, that it needs no description.

Time.] It flowers before the end of May, and the fruit is ripe in October.

Government and virtues.] Services, when they are mellow, are fit to be taken to stay fluxes, scouring, and casting, yet less than medlers. If they be dried before they be mellow, and kept all the year, they may be used in decoctions for the said purpose, either to drink, or to bathe the parts re-
quiring it; and are profitably used in that
manner to stay the bleeding of wounds,
and of the mouth or nose, to be applied to
the forehead and nape of the neck; and are
under the dominion of Saturn. either to drink, or to bathe the parts re-
quiring it ; and are profitably used in that
manner to stay the bleeding of wounds,
and of the mouth or nose, to be applied to
the forehead and nape of the neck; and are
under the dominion of Saturn. either to drink, or to bathe the parts re-
quiring it ; and are profitably used in that
manner to stay the bleeding of wounds,
and of the mouth or nose, to be applied to
the forehead and nape of the neck; and are
under the dominion of Saturn. either to drink, or to bathe the parts re-
quiring it ; and are profitably used in that
manner to stay the bleeding of wounds,
and of the mouth or nose, to be applied to
the forehead and nape of the neck; and are
under the dominion of Saturn. either to drink, or to bathe the parts re-
quiring it; and are profitably used in that
manner to stay the bleeding of wounds,
and of the mouth or nose, to be applied to
the forehead and nape of the neck; and are
under the dominion of Saturn. either to drink, or to bathe the parts re-
quiring it; and are profitably used in that
manner to stay the bleeding of wounds,
and of the mouth or nose, to be applied to
the forehead and nape of the neck; and are
under the dominion of Saturn.

\section{SHEPHERD'S PURSE.}

IT is called Whoreman's Permacety, She pherd's Scrip, Shepherd's Pounce, Toywort, Pickpurse, and Casewort.

Descript.] 'The root is small, white, and and spleen, rarefies thick phlegm, and perishes every year. The leaves are small cleanses it and the blood withal. It proand long, of a pale green colour, and deeply vokes urine and women's courses, and is cut in on both sides, among which spring up a stalk which is small and round, containing small leaves upon it even to the top. The flowers are white and very small; after which come the little cases which hold the seed, which are flat, almost in the form of a heart.

Place.] 'They are frequent in this nation, almost by every path-side.

Time.] They flower all the Summer long; nay some of them are so fiuitful, that they flower twice a year.

Govermment and virtues.] It is under the dominion of Saturn, and of a cold, dry, all the purposes aforesaid, and is held to and binding nature, like to him. It helps be stronger in operation than the herb, but all fluxes of blood, either caused by inward especially to open obstructions, and to rid or outward wounds; as also flux of the away any ague, if the juice thereof be taken belly, and bloody flux, spitting blood, and in wine, or the decoction thereof in wine bloodly urine, stops the terms in women; used.

$(17,18$. the soles of the feet, it helps the yellow jaundice. The herb being made into a poultice, helps inflammations and St. Anthony's fire. The juice being dropped into the ears, heals the pains, noise, and mutterings thereof. A good ointment may be made of it for all wounds, especially wounds in the head.

Tris is also very well known, and therefore I shall not trouble the reader with any description thereof.

Place.] It grows naturally in dry and marshy ground; but if it be sown in gardens. it there prospers very well.

Time.] It abides green all the Winter, and seeds in August.

Government and virtues.] $\mathrm{It}$ is an herb of Mercury. Smallage is hotter, drier, and much more medicinal than parsley, for it much more opens obstructions of the liver singularly good against the yellow jaundice, tertian and quartan agues, if the juice thereof be taken, but especially made up into a syrup. The juice also put to honey of roses, and barley-water, is very good to gargle the mouth and throat of those that have sores and ulcers in them, and will quickly heal them. 'I'he same lotion also cleanses and lieals all other foul ulcers and cankers elsewhere, if they be washed therewith. The seed is especially used to break and expel wind, to kill worms, and to lielp a stinking breath. The root is effectual to either to drink, or to bathe the parts re-
quiring it ; and are profitably used in that
manner to stay the bleeding of wounds,
and of the mouth or nose, to be applied to
the forehead and nape of the neck; and are
under the dominion of Saturn.

being bound to the wrists of the hands, and 
SOPEWORT, OR BRUISEWORT. Phat 2:-
Government and virtues.] It is under the dominion of Venus. Sorrel is prevalent in all hot diseases, to cool any inflammation Descript.] The roots creep under and heat of blood in agues pestilential or
ound far and near, with many joints choleric, or sickness and fainting, arising ground far and near, with many joints choleric, or sickness and fainting, arising
therein, of a brown colour on the outside from heat, and to refresh the overspent and yellowish within, shooting forth in spirits with the violence of furious or fiery divers places weak round stalks, full of fits of agues; to quench thirst, and procure joints, set with two leaves a-piece at every one of them on a contrary side, which are For it resists the putrefaction of the blood, ribbed somewhat like to plantain, and kills worms, and is a cordial to the heart, fashioned like the common field white cam- which the seed doth more effectually, being pion leaves, seldom having any branches more drying and binding, and thereby stays from the sides of the stalks, but set with the hot fluxes of women's courses, or of flowers at the top, standing in long husks humours in the bloody flux, or flux of the like the wild campions, made of five leaves stomach. The root also in a decoction, or a-piece, round at the ends, and dented in in powder, is effectual for all the said purthe middle, of a rose colour, almost white, ? poses. Both roots and seeds, as well as the sometimes deeper, sometimes paler; of a herb, are held powerful to resist the poison reasonable scent.

Place.] It grows wild in many low and wet grounds of this land, by brooks and the sides of running waters.

Time.] It flowers usually in July, and so continues all August, and part of September, before they be quite spent.

Government and virtues.] Venus owns it. The country people in divers places do use to kill those sharp humours that cause the to bruise the leaves of Sopewort, and lay it itch. The juice thereof, with a little vineto their fingers, hands or legs, when they gar, serves well to be used outwardly for are cut, to heal them up again. Some the same cause, and is also profitable for make great boast thereof, that it is diureti-: tetters, ringworms, \&c. It helps also to cal to provoke urine, aud thereby to expel discuss the kernels in the throat; and the gravel and the stone in the reins or kidneys, juice gargled in the mouth, helps the sores and do also account it singularly good to therein. 'The leaves wrapt in a colewort void hydropical waters : and they no less leaf and roasted in the embers, and applied extol it to perform an absolute cure in the to a hard imposthume, botch, boil, or plague French pox, more than either sarsaparilla, sore, doth both ripen and break it. 'The guiacum, or China can do; which, how distilled water of the herb is of much good true it is, I leave others to judge.

SORREL. S1ate 27.

OU R ordinary Sorrel, which grows in gardens, and also wild in the fields, is so well known, that it needs no description. of the scorpion. The decoction of the roots is taken to help the jaundice, and to expel the gravel and the stone in the reins or kidneys. The decoction of the flowers made with wine and drank, helps the black jaundice, as also the inward ulcers of the body and bowels. A syrup made with the juice of Sorrel and fumitory, is a sovereign help use for all the purposes aforesaid.

\section{WOOD SORREL.}

$$
\text { vinte } 26 \text {. }
$$

Descript.] Tur grows upon the ground, having a number of leaves coming from the root made of three leaves, like a trefoil, 
but broad at the ends, and cut in the middle, of a yellowish green colour, every one standing on a long foot-stalk, which at their first coming up are close folded together to the stalk, but opening themselves afterwards, and are of a fine sour relish, and yielding a juice which will turn red when it is clarified, and makes a most dainty clear syrup. Among these leaves rise up divers slender, weak foot-sialks, with every one of them a flower at the top, consisting of five small pointed leaves, starfashion, of a white colour, in most places, and in some dashed over with a small show of blueish, on the back side only. After the flowers are past, follow small round heads, with small yellowish seed in them. The roots are nothing but small strings fastened to the end of a small long piece; all of them being of a yellowish colour.

Place.] It grows in many places of our land, in wootis and wood-sides, where they be moist and sliadowed, and in other places not too much upon the Sun.

Time.] It flowers in April and May.

Government and virtues.] Venus owns it. Wood Sorrel serves to all the purposes that hoids. The juice boiled or thoroughly the other Sorrels do, and is more effectual heated in a little oil of bitter almonds in the in hindering putrefaction of blood, and peel of a poinegranate, and dropped into ulcers in the mouth and body, and to the ears, is a sure remedy for deafness, singquench thirst, to strengthen a weak stomach, ings, \&c. Tluree spoonfuls of the juice to procure an appetite, to stay vomiting, taken, warmed in white wine, and some and very excellent in any contagious sick- wine put thereto, causes women in travail ness or pestilential fevers. The syrup made? to have so easy and speedy a delivery, that of the juice, is effectual in all the cases they may be able to walk presently after. aforesaid, and so is the distilled water of: It is wonderful good for women to wash the herb. Sponges or linen cloths wet in their faces with, to clear the skin, and give swelling or intlammations, doth much cool and help them. 'The same juice taken and gargled in the mouth, and after it is spit forth, taken afresh, doth wonderfully help a foul stinking canker or ulcer therein. It is singularly good to heal wounds, or to stay the bleeding of thrusts or scabs in the body.
Sow 'Thistles are generally so well known, hat they need no description.

Place.] 'They grow in gardens and pathsides of fields, and high ways.

former are under the influence of Venus. Sow Thistles are cooling, and somewhat binding, and are very fit to cool a hot stomach, and ease the pains thereof. The the dissolution of the stomach, and the milk broken, given in drink, is beneficial to those that are short winded, and have a wheezthat the eating thereof helps a stinking breath. The decoction of the leaves and stalks causes abundance of milk in nurses, juice or distilled water is good for all hot inflammations, wheals, and erputions or heat in the skin, itching of the hremorr-

\section{SOUTHERN WOOD. P, 32 .}

Soutuern Wood is so well known to be an ordinary inhabitant in our gardens, that I shall not need to tronble you with any description thereof.

Time.] It Howers for the most part in July and August. manured grounds, sometimes by old walls, herb boiled in wine, is very helpful to stay ing. Pliny saith, That it hath caused the 
Government and virtues. It is a gallant mercurial plant, worthy of more esteem than it hath. Dioscorides saith, That the seed bruised, heated in warm water, and drank, helps those that are bursten, or troubled with cramps or convulsions of the sinews, the sciatica, or difficulty in making water, and bringing down women's courses. The same taken in wine is an antidote, or counter-poison against all deadly poison, and drives away serpents and other venomous creatures; as also the smell of the herb, being burnt, doth the same. The oil thereof anointed on the back-bone before the fits of joints and leaves on them, and at the tops agues come, takes them away: It takes an umbel of pure white flowers; at the away inflammations in the eyes, if it be put edges whereof sometimes will be seen a with some part of a roasted quince, and shew of the reddish blueish colour, especiboiled with a few crumbs of bread, and ap- ally before they be full blown, and are plied. Boiled with barley-meal it takes succeeded by small, somewhat round seeds, away pimpels, pushes or wheals that arise in the face, or other parts of the body. The seed as well as the dried herb, is often given to kill the worms in children: The herb bruised and laid to, helps to draw forth splinters and thorns out of the flesh. The ashes thereof dries up and heals old ulcers, that are without inflammation, although by the sharpness thereof it bites sore, and puts them to sore pains; as also the sores in the privy parts of man or woman. The ashes mingled with old sallad oil, helps those that have hair fallen, and are bald, causing the hair to grow again either on the head or beard. Daranters saith, That the oil made of Southern-wood, and put among the ointments that are used against the French disease, is very effectual, and likewise kills lice in the head. The distilled water of the herb is said to help them much that are troubled with the stone, as also for the diseases of the spleen and mother. The Germans commend it for a singular wound herb, and therefore call it Stabwort. It is held by all writers, ancient and modern, to be more offensive to the stomach than worm-wood.
SPIGNEL, OR SPIKENARD.

Descript.] THE roots of common Spignel do spread much and deep in the ground, many strings or branches growing from one head, which is hairy at the top, of a blackish brown colour on the outside, and white within, from whence rise sundry long stalks taste, from whece rise sundry long stallis of most fine cut leaves like hair, smaller than dill, set thick on both sides of the stalks, and of a good scent. Among these leaves rise up round stiff stalks, with a few bigger than the ordinary fennel, and of a brown colour, divided into two parts, and crusted on the back, as most of the umbelliferous seeds are.

Place.] It grows wild in Lancashire, Yorkshire, and other northern counties, and is also planted in gardens.

Government and virtues.] It is an herb of Venus. Galen saith, The roots of Spignel are available to provoke urine, and women's courses; but if too much thereof be taken, it causes head-ache. The roots boiled in wine or water, and drank, helps the stranguary and stoppings of the urine, the wind, swellings and pains in the stomacl, pains of the mother, and all joint-aches. If the powder of the root be mixed with honey, and the same taken as a licking medicine, it breaks tough phlegm, and diries up the rheum that falls on the lungs. The roots are accounted very effectual against the stinging or biting of any venomous creature. SPLEENWORT, CETERACII, OR HEART'S TONGUE. P. Z?

Descript.] THe snooth Spleenwort, from a black, thready and bushy root, sends forth 
many long single leaves, cut in on both down to the ground, that it seems a pretty. sides into round dents almost to the middle, bush, set with divers the like divided leaves which is not so hard as that of polypody, up to the tops, where severally do stand each division being not always set opposite small whitish green heads, set with sharp unto the other, cut between each, smooth, white pricks (no part of the plant else being and of a light green on the upper side, and prickly) which are somewhat yellowish; a dark yellowish roughness on the back, out of the middle whercof rises the flowers, folding or rolling itself inward at the first springing up.

Place.] It grows as well upon stone walls, as moist and shadowy places, about Bristol, and other the west parts plentifully; long and woody, perishing every year, and as also on Framlingham Castle, on Bea- rising again of its own sowing.

consfield church in Berkshirc, at Stroud in Place.]. It grows wild in the fields about Kent, and elsewhere, and abides green all London in many places; as at Mile-End the Winter.

Government and virtues.] 'Saturn owns it. green, and many other places.

Time.] It flowers early, and seeds in It is generally used against infirmities of July, and sometimes in August.

the Spleen: It helps the stranguary, and Government and virtues.] This, as almost wasteth the stone in the bladder, and is all Thistles are, is under Mars. The seed good against the yellow jaundice and the of this Star Thistle made into powder, and hiccough; but the juice of it in women drank in wine, provokes urine, and helps hinders conception. Matthiolus saith, That to break the stone, and drives it forth. The if a dram of the dust that is on the back- root in powder, and given in wine and side of the leaves be mixed with half a dram drank, is good against the plague and pes:of amber in powder, and taken with the tilence; and drank in the morning fasting juice of purslain or plantain, it helps the for some time together, it is very profitable gonorrhea speedily, and that the herb and for fistulas in any part of the body. root being boiled and taken, lielps all Baptista Sardas doth much commend the melancholy diseases, and those especially distilled water thereof, being drank, to help that arise from the French diseases. the French disease, to open the obstructions Camerarius saith, That the distilled water of the liver, and cleanse the blood from thereof being drank, is very effectual against corrupted humours, and is profitable against the stone in the reins and bladder; and the quotidian or tertian ague.

that the lye that is made of the ashes thereof being drank for some time together, helps splenetic persons. It is used in outward remedies for the same purpose.

STAR TIIISTLE. $\theta_{.}^{\prime} \&$

Descript.] A common Star Thistle has divers narrow leaves lying next the ground, cut on the edges somewhat deeply into herb. Strawberries, when they are green, many parts, soft or a little woolly, all over are cool and dry ; but when they are ripe, green, ainong which rise up divers weak they arc cool and moist: 'The berries are stalks, parted into many branches, all lying excellently good to cool the liver, the blood, 
and the spleen, or an lot choleric stomach; and for such other defects in them as may to refresh and comfort the fainting spirits, be helped by any outward medicine.

and quench thirst: They are good also for other inflammations; yet it is not amiss to refrain from them in a fever, lest by their putrifying in the stomach they increase the fits. The leaves and roots boiled? in wine and water, and drank, do likewise cool the liver and blood, and assuage all inflammations in the reins and bladder, provoke urine, and allay the heat and sharpness thereof. The same also being drank stays the blocdy flux and women's courses, and helps the swelling of the spleen. The water of the Berries carefully distilled, is a sovereign remedy and cordial in the panting and beating of the heart, leaves, from among which rises up a hard, and is good for the yellow jaundice. The round, woody stalk, spreading into many juice dropped into foul ulcers, or they branches, set with smaller and less divided washed therewith, or the decoction of the leaves on them up to the tops, where stand herb and root, doth wonderfully cleanse the flowers, which are like the garden kind, and help to cure them. Lotions and gar- and the seed is also (only take notice that gles for sore mouths, or ulcers therein, or in the flowers of the garden kind are gone in the privy parts or elsewhere, are made with on a sunny day, they being so cold, that the leaves and roots thereof; which is also they are not able to endure the beams of the good to fasten loose teeth, and to heal sun, and therefore more delight in the shade) spungy foul gums. It helps also to stay the root is white, but more hard and woody catarrhs, or defluctions of rheum in the than the garden kind. 'The whole plant is mouth, throat, teeth, or eyes. The juice or exceedingly bitter.

water is singularly good for hot and red inflamed eyes, if dropped into them, or they bathed therewith. It is also of excellent property for all pushes, wheals and other breakings forth of hot and sharp humours in the face and hands, and other parts of the body, to bathe them therewith, and to take away any redness in the face, or spots, or other deformities in the skin, and to make it clear and smooth. Some use this medicine, Take so many 'Strawberries as you shall think fitting, and put them into a distillatory, or body of glass fit for them, which being well closed, set it in a bed of horse dung for your use. It is an excellent water for hot intlamed eyes, and to take away a film or skin that kegins to grow over them,
Place.] This grows in many places of our land in waste untilled and barren fields. The other only in gardens.

Government and virtues.] It is an herb of Jupiter. Garden Succory, as it is more dry and less cold than Endive, so it opens more. An handful of the leaves, or roots boiled in wine or water, and a draught thereof drank fasting, drives forth choleric and plilegmatic humours, opens obstructions of the liver, gall and spleen; helps the yellow jaundice, the heat of the reins, and of the urine; the dropsy also; and those that have an evil disposition in their bodies, by reason of long sickness, evil diet, \&c. which the Greeks call Cachexia. A decoction thereof made with wine, and drank, is 
very effectual against long lingering agues, of of choler, thercby preventing diseases and a dram of the seed in powder, drank in arising from choleric humours. It expels winc, before the fit of the aguc, helps to poisonmuch, resists pestilential fcvers, being drive it away. The distilled water of the exceeding good also for tertian agues: You herb and flowers (if you can take them in may drink the decoction of it, if you please, time) hath the like properties, and is es- for all the foregoing infirmities. It is so pecially good for hot stomachs, and in harmless all herb, you can scarce use it agues, either pestilcntial or of long con- amiss: Being bruised and applied to the tinuance; for swoonings and passions of place, it helps the king's evil, and any other the heart, for the heat and head-ache in knots or kernels in the flesh; as also the children, and for the blood and liver. The piles.

said water, or the juice, or the bruised leaves applied outwardly, allay swellings, inflammations, St. Anthony's fire, pushes, wheals, and pimples, especially used with a little vinegar; as also to wash pestiferous sores. The said water is very effectual for sore eyes that are inflamed with redness, for nurses' breasts that are pained by the abundance of milk.

The wild Succory, as it is more bitter, so it is more strengthening to the stomach and liver.

STONE-CROP, PRICK-MADAM, OR SMALLHOUSELEEK.

Descript.] IT grows with divers trailing branches upon the ground, set with many. thick, flat, roundish, whitish green leaves, pointed at the ends. The flowers stand many of them togetlier, somerwhat loosely. The roots are small, and run creeping under ground.

Place.] It grows upon the stone walls and mud walls, upon the tiles of houses and pent-houses, and amongst rubbish, and in other gravelly places.

Time.] It flowers in June and July, and the leaves are green all the Winter.

Government and rirtues.] It is under the Government and rirtues.] It is under the be available to cxpectorate tough phlegm
dominion of the Moon, cold in quality, from the stomach, chest, and lungs. The and something binding, and therefore very juice thereof made into a syrup, or the disgood to stay defluctions, especially such as tilled water of the herb drank with some fall upon the cyes. It stops bleeding, both sugar, or without, if you will, or the smoak inward and outward, helps cankers, and all taken by a pipe, as is usual, but fainting, fretting sores and ulcers; it abates the heat helps to expel worms in the stomach and

Descripi.] Turs rises up with a round thick stalk, about two feet high, whereon do grow thick, flat green leaves, nothing so large as the other Indian kind, somewhat round pointed also, and nothing dented about the edges. The stalk branches forth, and bears at the tops divers flowers set on great husks like the other, but nothing so large : scarce standing above the brims of the husks, round pointed also, and of a greenish yellow colour. The seed "that follows is not so bright, but larger, contained in the like great heads. The roots are neither so great nor woody; it perishes every year with the hard frosts in Winter, but rises generally from its own sowing.

Place.] This came from some parts of Brazil, as it is thought, and is more familiar in our country than any of the other sorts; carly. giving ripe sced, which the others seldom do.

Time.] It flowcrs from June, sometimes to the end of August, or later, and the seed ripens in the mean cime.

Government and virtues.] It is a martial plant. It is found by good experience to be available to cxpectorate tough phlegm 
belly, and to ease the pains in the head, or or June, and the seed is ripe and blown megrim, and the griping pains in the bowels. away in the beginning of September.

It is profitable for those that are troubled Government and virtues.] A gallant Sawith the stone in the kidneys, both to ease turnine herb it is. The root, leaves, young the pains by provoking urine, and also to branches, or bark boiled in wine, and drank, expel gravel and the stone engendered stays the bleeding of the hrmorrhodical therein, and hath been found very effectual veins, the spitting of blood, the too aboundto expel windiness, and other humours, ing of women's courses, the jaundice, the which cause the strangling of the mother. cholic, and the biting of all venomous serThe seed hereof is very effectual to expel pents, except the asp; and outwardly apthe tooth ache, and the ashes of the burnt plied, is very powerful against the hardness herb to cleanse the gums, and make the of the spleen, and the tooth-ache, pains in teeth white. The herb bruised and ap- the ears, red and watering eyes. The deplied to the place grieved with the king's coction, with some honey put thereto, is evil, helps it in nine or ten days effectually. good to stay gangrenes and fretting ulcers, Monardus saith, it is a counter poison and to wash those that are subject to nits against the biting of any venomous crea- and lice. Alpinus and Veslingius affirm, ture, the herb also being outwardly applied That the Egyptians do with good success to the hurt place. The distilled water is use the wood of it to cure the French disoften given with some sugar before the fit ease, as others do with lignum vitæ or of an ague, to lessen it, and take it away in guiacum; and give it also to those who three or four times using. If the distilled have the leprosy, scabs, ulcers, or the like. freces of the herb, having been bruised be- Its ashes doth quickly heal blisters raised fore the distillation, and not distilled dry, by burnings or scaldings. It helps the be set in warm dung for fourteen days, and dropsy, arising from the hardness of the afterwards be hung in a bag in a wine spleen, and therefore to drink out of cups cellar, the liquor that distills therefrom is made of the wood is good for splenetic singularly good to use in cramps, aches, persons. It is also helpfiul for melancholy, the gout and sciatica, and to heal itches, and the black jaundice that arise thereof. scabs, and running ulcers, cankers, and all foul sores whatsoever. The juice is also good for all the said griefs, and likewise to kill lice in children's heads. The green herb bruised and applied to any green wounds, cures any fresh wound or cut? whatsoever: and the juice put into old sores, both cleanses and heals them. There is also made hereof a singularly good salve to help imposthumes, hard tumours, and other swellings by blows and falls.

THE TAMARISK TREE.

GARDEN TANSY.

alence 28.

Garden Tansy is so well known, that it needs no description.

Time.] It flowers in June and July.

Government and virtues.] Dame Venus was minded to pleasure women with child by this herb, for there grows not an herb, fitter for their use than this is; it is just as though it were cut out for the purpose. This herb bruised and applied to the naval, stays miscarriages; I know no lierb like it for that use : Boiled in ordinary beer, and the decoction drank, It is so well known in the place where it doth the like; and if her womb be not as grows, 'bat it needs no description.

Time.] It Howers about the end of May, she would have it, this decoction will make it so. Let those women that desire chil- 
dren love this herb, it is their best com- and it is true enough, that it will stop the panion, their husbands excepted. Also it terms, if worn so, and the whites too, for consumes the phlegmatic humours, the cold ought I know. It stays also spitting or and moist constitution of Winter most vomiting of blood. The powder of the usually affects the hody of man with, and herb taken in some of the distillcd water, that was the first reason of eating tansies in helps the whites in women, put more esthe Spring. The decoction of the common pecially if a little coral and ivory in powTansy, or the juice drank in wine, is a sin- der be put to it. It is also recommerrued gular remedy for all the griefs that come to help children that are bursten, and lave by stopping of the urine, helps the stran- a rupture, being boiled in water and salt. guary and those that have weak reins and Being boiled in water and drank, it eases kidneys. It is also very profitable to dis- the griping pains of the bowels, and is good solve and expel wind in the stomach, belly, for the sciatica' and joint-aches. The same or bowels, to procure women's courses, and boiled in vinegar, with honey and allum, expel windiness in the matrix, if it be bruis- and gargled in the mouth, eases the pains ed and often smelled unto, as also applied of the tooth-ache, fastens loose teeth, helps to the lower part of the belly. It is also the gums that are sore, and settles the very profitable for such women as are given palate of the mouth in its place, when it is to miscarry. It is used also against the fallen down. It cleanses and heals ulcers stone in the reins, especially to men. The in the mouth, or secret parts, and is very herb fried with eggs (as it is the custom in good for inward wounds, and to close the the Spring-time) which is called a Tansy, (lips of green wounds, and to heal old, moist, helps to digest and carry downward those and corrupt running sores in the legs or bad humours that trouble the stomach. elsewhere. Being bruised and applied to The seed is very profitably given to chil- the soles of the feet and hand wrists, it dren for the worms, and the juice in drink wonderfully cools the hot fits of agues, be is as effectual. Being boiled in oil, it is they never so violent. The distilled water good for the sinews shrunk by cramps, or cleanses the skin of all discolourings therepained with colds, if thereto applied.

WILD TANSY, OR SILVER WEED.

THIs is also so well known, that it needs no description.

Place.] It grows in every place.

Time.] It flowers in June and July

Government and virtues.] Now Dame in, as morphew, sun-burnings, \&c. as also pimples, freckles, and the like; and dropped into the eyes, or cloths wet therein and applied, takes away the heat and inflammations in them.

\section{TII I S T LES.}

OF these are many kinds growing here in England which are so well known, that they need no description: 'Their difference is casily known on the places where they grow, viz.

Place.] Some grow in fields, some in mcadows, and some among the corn; others on lieatlss, greens, and waste grounds in many places.

Time.] They flower in June and August, one name, the one to help conccption, and the other to maintain beauty, and what nore can be expected of her? What now remains for you, but to love your husbands, and not to be wanting to your poor neighbours? Wild Tansy stays the lask, and all the fluxes of blood in men and women, which some say it will do, if the grcen herb be worn in the shoes, so it be next the skin : and their seed is ripe quickly after. 
Government and virtues.] Surely Mars being drank, expels superfluous melancholy rules it, it is such a prickly business. All out of the body, and makes a man as merry these thistles are good to provoke urine, as a cricket; superfluous melancholy causes and to mend the stinking smell thereof; as care, fear, sadness, despair, envy, and many also the rank smell of the arm-pits, or the evils more besides; but religion teaches to whole body; being boiled in wine and wait upon God's providence, and cast our drank, and are said to help a stinking care upon him who cares for us. What a breith, and to strengthen the stomach. fine thing were it if men and women could Pliny saith, That the juice bathed on the live so? And yet seven years' care and fear place that wants hair, it being fallen off, makes a man never the wiser, nor a farthing will cause it to grow speedily.

THE MELANCHOLY THISTLE.

Descript.] IT rises up with tender single hoary green stalks, bearing thereon four or five green leaves, dented about the edges
the points thereof are little or nothing prickly, and at the top usually but one head, yet sometimes from the bosom of the uppermost leaves there shoots forth another small head, scaly and prickly, with many reddish thrumbs or threads in the middle, which being gathered fresh, will keep the colour a loug time, and fades not from the stalk a long time, while it perfects the seed, which is of a mean bigness, lying in the down. The root hath many strings fastened to the head, or upper part, which is blackish, and perishes not.

There is another so:t little differing from the former, but that the leaves are more green above, and more hoary underneath, and the stalk being about two feet high, bears but one scaly head, with threads and seeds as the former.

Place.] 'They grow in many moist meadows of this land, as well in the southern, as in the northern parts.

Time.] They flower about July or August, and their seed ripens quickly after.

Government and virtues.] It is under Capricorn, and therefore under both Saturn and Mars, one rids melancholy by sympathy, the other by antipathy. Their virtues Thistle is under Jupiter, and thought to be are but few, but those not to be despised; as effectual as Carduus Benedictus for for the decoction of the thistle in wine agues, and to prevent and cure the infection 
of the plague: as also to open the obstruccions of the liver and spleen, and thereby is good against the jaundice. It provokes urine, breaks and expels the stone, and is good for the dropsy. It is effectual also for the pains in the sides, and many other inward pains and gripings. The seed and distilled water is held powerful to all the purposes aforesaid, and besides, it is often applied both outwardly with cloths or spunges to the region of the liver, to cool the distemper thereof, and to the region of the heart, against swoonings and the passions of it. It cleanses the blood exceedingly: and in Spring, if you please to boil the tender plant (but cut off the prickles, unless you have a mind to choak yourself) it will change your blood as the season changes, and that is the way to be safe.

THE WOOLLEN, OR, COTTON THISTLE.

Descript.] TuIs has many large leaves lying upon the ground, somewhat cut in, and as it were crumpled on the edges, of a green colour on the upper side, but covered over with a long hairy wool or cotton down, set with most sharp and cruel pricks ; from the middle of whose heads of flowers come forth many purplish crimson threads, and sometimes white, although but seldom. 'The seed that follow in those white downy heads, "is somewhat large and round, re-" doth heal the cleft thereof, cankers and sembling the seed of Lady's 'Thistle, but fistulas therein, also takes away warts and paler. The root is great and thick, spread - wens. The juice of the leaves dropped into ing much, yet usually dies after seed time. the ears, kills worms in them. The dis-

Place.] It grows on divers ditch-banks, tilled water of the leaves dropped into the and in the com-fields, and highways, gene- eyes, takes away redness and mists in them rally throughout the land, and is of ten that hinder the sight, and is often used by growing in gardens.

Government and virtues.] $I t$ is a plant of Mars. Dioscorides and Pliny write, That the leaves and roots hercof taken in drink, help those that have a crick in their neck, that they cannot turn it, unless they turn their whole body. Galen saith, 'Iliat the roots and leaves hereof are good for such persons that have their bodies drawn together by some spasm or convulsion, or other infirmities; as the rickets (or as the college of physicians would have it, Rachites, about which name they have quarrelled sufficiently) in children, being a disease that hinders their growth, by binding their nerves, ligaments, and whole structure of their body.

THE FULLER'S THISTLE, OR TEASLE.

IT is so well known, that it needs no description, being used with the clothworkers.

'The wild Teasle is in all things like the former, but that the prickles are small, soft, and upright, not hooked or stiff, and the flowers of this are of a fine blueish, or pale carnation colour, but of the manured kind, whitish.

Place.] The first grows, being sown in The other near ditches and rills of water in many places of this land.

Time.] They flower in July, and are ripe in the end of August.

Government and virtues.] It is an herb of Venus. Dioscorides saith, That the root bruised and boiled in wine, till it be thick, and kept in a brazen vessel, and after spread as a salve, and applied to the fundament, women to preserve their beauty, and to take away redness and inflammations, and all other lieat or discolourings.

TREACLE MUSTARD.

Descript.] It rises up with a hard round stalk, about a foot high, parted into some branches, having divers soft green leaves. gardens or fields for the use of clothworkers: 
long and narrow, set thereon, waved, but resisting poison, venom and putrefaction. not cut into the edges, broadest towards the It is also available in many cases for which ends, somewhat round pointed; the flowers the common Mustard is used, but somewhat. are white that grow at the tops of the weaker.

branches, spike-fashion, one above another; after which come round pouches, parted in the middle with a furrow, having one blackish brown seed on either side, somewhat sharp in taste, and smelling of garlick, especially in the fields where it is natural, but not so much in gardens: The roots are small and thready, perishing every year. Give me leave here to add Mithridate Mustard, although it may seem more properly by the name to belong to $\mathrm{M}$, in the alphabet."

MITHRIDATE MUSTARD. P. 38 .

Descript.] Thus grows higher than the former, spreading more and higher branches, whose leaves are smaller and narrower, sometimes unevenly dented about the edges. The flowers are small and white, growing on long branches, with much smaller and rounder vessels after them, and parted in the same manner, having smaller brown' seeds than the former, and much sharper in taste. The root perishes after : seed time, but abides the first Winter after springing.

Place.] They grow in sundry places in this land, as half a mile from Hatfield, by the river side, under a hedge as you go to Hatfield, and in the street of Peckham on Surrey side.

Time.] They flower and seed from May to August.

Government and virtues.] Both of them are herbs of Mars. The Mustards are said to purge the body both upwards and downwards, and procure women's courses so abundantly, that it abundantly, that it suffocates the birth. defluctions of rhcum to the eyes, or other It breaks inward imposthumes, being taken parts; as also to cool the heat and inflaminwardly; and used in clysters, helps the mations of then, and ease hot pains of the sciatica. The seed applied, eloth the same. head, to bathe the forehead and temples It is an especial ingredient in mithridate therewith. The simple distilled water of and treacle, being of itself an antidote the flowers is very effectual for the said

$\mathrm{I}_{\mathrm{T}}$ is so well known, that it needs no description.

Place.] It grows in every county in the hedges and borders of fields.

Time.] It flowers in April, and sometimes in March, but the fruit ripens after all other plums whatsoever, and is not fit to be eaten until the Autumn frost mellow them.

Government ana virtues.] All the parts of the Sloe-Bush are binding, cooling, and dry, and all effectual to stay bleeding at the nose and mouth, or any other place; the lask of the belly or stomach, or the bloody flux, the too much abounding of women's courses, and helps to ease the pains of the sides, and bowels, that come by overmuch scouring, to drink the decoction of the bark of the roots, or more usually the decoction of the berries, either fresh or dried: 'The conserve also is of very much use, and more familiarly taken for the purposes aforesaid. But the distilled water of the flower first steeped in sack for a night, and drawn therefrom by the heat of Balneum and Anglico, a bath, is a most certain remedy, tried and approved, to ease all manner of gnawings in the stomach, the sides and bowels, or any griping pains in any of them, to drink a small quantity when the extremity of pain is upon them. The leaves also are good to make lotions to gargle and wash the mouth and throat, wherein are swellings, sores, or kernels; and to stay the

THE BLACK TIORN, OR SLOE-BUSH. 
purposes, and the condensate juice of the applied with a little flour and wax to chilSloes. The distilled water of the green dren's navels that stick forth, it helps them. berries is used also for the said effects.

THOROUGH WAX, OR TIIOROUGII LEAF. Plate 28

Descript.] Common Thorough-Wax sends forth a strait round stalk, two feet high, or better, whose lower teaves being of a bluish colour, are smaller and narrower than those up higher, and stand close thereto, not compassing it; but as they grow higher, they do not, encompass the stalks, until it wholly pass through them, branching toward the top into miany parts, where? the leaves grow smaller again, every one standing singly, and never two at a joint. 'The flowers are small and yellow, standing in tufts at the heads of the branches, where afterwards grow the seed, being blackish, many thick thrust together. The root is small, long and woody, perishing every year, after secd-time, and rising again plen-? tifully of its own sowing.

Place.] It is found growing in many corn-fields and pasture grounds in this land.

Time.] It flowers in July, and the secd is ripe in August.

Goremment and virtues.] Both this and the former are under the influence of Saturn. 'Thorough-Wax is of singular good use for all sorts of bruises and wounds either inward or outward; and old ulcers and sores likewise, if the decoction of the herb with water and wine be cliank, and the place washed therewith, or tlic juice of the green herb bruised, or boiled, either by itself, or with other herbs, in oil or hog's grease, to be marle into an ointment to serve all the year. 'Ihe decoction of the herb, or powder of the dried herb, taken intwardly, and tue same, or the leaves bruised, and applied outwardy, is singularly good for London Dispensatory) and anoint the head all ruptures and burstings, especially in with it, it presently stops the pains thereof.
childiven before they be too old. Being It is excellently good to be given either in $(19,20$.
TIY YIE. P. 32 .

It is in vain to describe an herb so commonly known.

Govermment and virtues.] $\mathrm{It}$ is a noble strengthener of the lungs, as notable a one as grows; neither is there scarce a better remedy growing for that disease in children which they commonly call the Chin-cough, than it is. It purges the body of phlegin, and is an excellent remedy for shortness of breath. It kills worms in the belly, and being a notable herb of Venus, provokes the terms, gives safe and speedy delivery to women in travail, and brings away the after birth. It is so harmless you need not fear the use of it. An ointment made of it takes away hot swellings and warts, helps the sciatica and dullness of sight, and takes away pains and hardness of the spleen. Tis excellent for those that are troubled with the gout. It eases pains in the loins and hips. The herb taken any way inwardly, comforts the stomach much, and expels wind.

WILD THYME, OR MOTHER OF THYME.

WiLD Thyme also is so well known, that it needs no description.

Place.] It may be found commonly in commons, and otlier barren places throughout the nation.

Gorernment and rirtues.] It is under the dominion of Venus, and under the sign to the head. It provokes urine and the terms, and eases the griping pain of the belly, cramps, ruptures, and inflamation of the liver. If yon make a rinegar of the herb, as vinegar of roses is marle (you may find out the way in my translation of the London Dispensatory) and anoint the head $3 \mathrm{~B}$ Aries, and therefore chiefly appropriated 
phrenzy or lethargy, although they are two is an ingredient in all antidotes or counter contrary diseases: It helps spitting and poisons. Andreas Urlesius is of opinion voiding of blood, coughing, and vomiting; that the decoction of this root is no less it comforts and strengthens the head, effectual to cure the French pox than Guistomach, reins, and womb, expels wind, acum or China; and it is not unlikely, and breaks the stone.

because it so mightily resists putrefaction.

TORMENTIL, OR SEPTFOIL. Paters.

'The root taken inwardly is most effectual to help any flux of the belly, stomach,

Descript.] THis hath reddish, stender, spleen, or blood; and the juice wonderweak branches rising from the root, lying fully opens obstructions of the liver and on the ground, rather leaning than standing lungs, and thereby helps the yellow jaunupright, with many short leaves that stand dice. The powder or decoction drank, or closer to the stalk than cinquefoil (to which to sit thereon as a bath, is an assured remedy this is very like) with the root-stalk com-against abortion, if it proceed from the passing the branches in several places; but over flexibility or weakness of the inward those that grow to the ground are set upon retentive faculty; as also a plaster made long foot stalks, each whereof are like the therewith, and vinegar applied to the reins leaves of cinquefoil, but somerwhat long and of the back, doth much help not only this, lesser dented about the edges, many of but also those that cannot hold their water, them divided into five leaves, but most the powder being taken in the juice of of them into seven, whence it is also called plaintain, and is also commended against Septfoil; yet some may have six, and some the worms in children. It is very powerful eight, according to the fertility of the soil. in ruptures and burstings, as also for bruises At the tops of the branches stand divers and falls, to be used as well outwardly as small yellow flowers, consisting of five inwardly. The root hereof made up with leaves, like those of cinquefoil, but smaller. sellitory of Spain and allum, and put into The root is smaller than bistort, somewhat a hollow tooth, not only assuages the pain, thick, but blacker without, and not so red but stays the flux of humours which causes within, yet sometimes a little crooked, it. 'I'ormentil is no less effectual and having blackish fibres thereat.

Place.] It grows as well in woods and sores and hurts, than for inward, and is shadowy places, as in the open champain therefore a special ingredient to be used in country, about the borders of fields in many wound drinks, lotions and injections, for places of this land, and almost in every foul corrupt rotten sores and ulcers of the broom field in Essex.

Time.] It flowers all the Summer long. mouth, secrets, or other parts of the body. The juice or powder of the root put in Government and virtues.] This is a gallant ointnients, plaisters, and such things that herb of the Sun. Tormentil is most ex- are to be applied to wounds or sores, is very cellent to stay all kind of fluxes of blood or effectual, as the juice of the leaves and the humours in man or woman, whether at root' bruised and applied to the throat or nose, mouth, or belly. 'The juice of the jaws, heals the king's evil, and eases the herb of the root, or the decoction thereof, pain of the sciatica; the same used with a taken with some Venice treacle, and the little vinegar, is a special remedy against person laid to sweat, expels any venom or the running sores of the head or other poison, or the plague, fever, or other contagious diseases, as pox, measles, \&c. for it eruptions in the skin, proceeding of salt and 
sharp humours. The same is also effectual of joint and newly set, and full of pain, do for the piles or hrmorrhoids, if they be give much ease; the seed and juice of the washed or bathed therewith, or with the leaves also being rubbed with a little salt distilled water of the herb and roots. It is upon warts and wens, and other kernels in found also helpful to dry up any sharp the face, eye-lids, or any other part of the rheum that distills from the head into the body, will, by often using, take them away. eyes, causing redncss, pain, watterings, itching, or the like, if a little prepared tutia, or white amber, be used with the distilled water thereof. And here is enough, only remember the Sun challengeth this herb.

TURNSOLE, OR HELIOTROPIUM.

Descript.] Tre greater Turnsole rises in this land. with one upright stalk, about a foot high, or Government and virtues.] Mercury hath more, dividing itself almost from the bottom, dominion over the common sort. Dodointo divers small branches, of a hoary colour ; neus saith, The leaves and flowers are good at each joint of the stalk and branches grow to ease the griping pains of the gout, the small broad lcaves, somewhat white and herb being boiled and used in a clyster. hairy. At the tops of the stalks and branches If the herb be made into a poultice, and stand small white flowers, consisting of four, applied to inflammations, it will ease them. and sometimes five small leaves, set in 'The juice dropped in the eyes, is a familiar order one above another, upon a small medicine, with many country people, to crooked spike, which turns inwards like a take away the pin and web (as they call it) bowed finger, opening by degrees as the in the eyes; it also allays the heat and flowers blow open; after which in their blood shooting of them. Country people place come forth cornered seed, four for the do also in many places drink the juice most part standing together; the root is thereof against the biting of an adder; and small and thready, perishing every year, having boiled the bcrb in water, they first and the seed slicdding every year, raises it wash the place with the decoction, and then again the next spring.

Place.] It grows in gardens, and flowers lay some of the herb also to the liurt place. The herb also boiled in swine's grease, and and seeds with us, notwitlsstanding it is not so made into an ointment, is good to apply natural to this land, but to Italy, Spain, and to the biting of any venomous creature. France, where it grows plentifully.

The lierl also bruised and lieated between

Government and virtues.] It is an licrb of tiles, and applied hot to the share, causes the Sun, and al good one too. Dioscorides them to malse water who had it stopt besaith, 'Ihat a good handful of this, which is fore. It is hold likewise to be good for called the Great Turnsole, boiled in water, wounds, and to take away sced. The deand chank, purges both choler and phlegm, coction of the herb and flowers. with the and boiled with cummin, helps the stone in seed and root, taken for some time, helps the reins, kidncys, or bladder, provolics women that are troubled with the whites. urine and women's courses, and causes an? The seed and flowers boiled in water, and casy and speedy delivery in child-birtlo afterwards made into a poultice with some The laves bruised and applied to places oil, and applied, helps hard swellings and pained with the gout, or that have been out imposthumes. 


\section{HEART TREFOIL.} BESIDEs the ordinary sort of Trefoil, taste, as the leaves also and the flowers be, here are two more remarkable, and one of although much less, but do not yield such which may be properly called Heart 'Tre- a clear claret wine colour, as some say it foil, not only because the leaf is triangular, , doth, the root is brownish, somewhat great, like the heart of a man, but also because hard and woody, spreading well in the each leaf contains the perfection of a heart, ground.

and that in its proper colour, viz. a flesh colour.

Place.] It grows between Longford and Bow, and beyond Southwark, by the highway and parts adjacent.

Government and virtues. dominion of the Sun, and if it were used, it would be found as great a strengthener of the heart, and cherisher of the vital spirits as grows, relieving the body against fainting and swoonings, fortifying it against poison and pestilence, defending the lieart against the noisome vapours of the spleen.

\section{PEARL TREFOIL.}

It differs not from the common sort, save only in this particular, it luath a white spot in the leaf like a pearl. It is particularly under the dominion of the Moon, and its icon shews that it is of a singular virtue against the pearl, or pin and web in the eyes.

TUSTAN, OR PARK LEAVES. P. 28.

Descript.] IT hath brownish round stalks, crested the length therenf, rising two by two, and sometimes three feet high, branching forth even from the bottom, having divers joints, and at cach of them two fair large leaves standing, of a dark blueish green colour on the upper side, and of a yellowish green underneath, turning reddish toward Antumn. At the top of the stalks stand large yellow flowers, and heads with seed, which being greenish at the first such like small pieces of roots, which have and afterwards reddish, turn to be of a all of them many long green strings and blackish purple colour when they are ripe, fibres under them in the ground, whereby with small brownish seed within them, and it draws nourishment. From the liead of
Place.] It grows in many woods, groves, and woody grounds, as parks and forests, and by hedge-sides in many places in this land, as in Hampstead wood, by Ratley in Essex, in the wilds of Kent, and in many other places needless to recite.

Timc.] It flowers later than St. John's or St. Peter's-wort.

Gorcrnment and virtues.] It is an herb of Saturn, and a most noble anti-vencrean. T'ustan purges choleric humours, as St. Peler's-wort is said to do, for therein it works the same effects, both to help the sciatica and gout, and to heal burning by fire; it stays all the bleedings of wounds, if either the green herb be bruised, or the powder of the dry be applied thereto. It hath been accounted, and certainly it is, a sovereign herb to heai either wound or sore, either outwardly or inwardly, and therefore always used in drinks, lotions, green wounds, ulcers, or old sores, in all balms, oils, ointments, or any other sorts of which the continual experience of former ages hath confirmed the use thereof to be admirably good, though it be not so much in use now, as when physicians and sur. geons were so wise as to use herbs more than now they do.

\section{GARDEN VALERIAN. P. 3D}

Descript.] Tins hath a thick short greyish root, lying for the most part above ground, shooting forth on all other sides 
these roots spring up many green leaves, virtue against the plague, the decoction which at first are somewhat broad and long, : thereof being drank, and the root bring without any divisions atall in them, or dent-, used to smell 'to. It helps to expel the ing on the edges; but those that rise up wind in the belly. The green herb with after are more and more divided on each the root taken fresh, being bruised and apside, some to the middle rib, bcing winged, plied to the bead, takcs away the pains and as nrade of many leaves together on a stalk, prickings there, stays rheum and thin disand those upon a stalk, in like manner more tillation, and being boiled in white wine, divided, but smaller wowards the top than and a drop thereof put into the eyes, takes below; the stalk rises to be a yard ligh or away the dimness of the sight, or any pin more, somclinics branched at the top, with or web therein. It is of excellent property many small whitish flowers, sometimes to heal any inward sores or wounds, and dashed orer at the edge's with a pale pur- also for outward hurts or wounds, and plish colour, of a little scent, which passing drawing away splinters or thorns out of the away, there follows small browinsh white flesh.

seed, that is easily carried away with the wind. The root smells more strong than either leaf or thower, and is of more use in medicines.

Place.] It is gencrally kept with us in gardens.

Timie.] It flowers in June and July, and continues flowering until the frost pull it down.

Government and rimtues.] 'This is under the influence of Mercury. Dioscorides saith, 'That the Garden Valerian hath a of them, which are set on all sides one above warming ficulty, and that being dried and another, and sometimes two or three togegiven to drink it provokes urine, and helps ther, being small and gaping, of a blue the stranguary. The decoction thereof taken, doth the like also, and takes away come small round sced, in small and somepains of the sides, provokes women's courses, what long heads. The root is small and and is used in antidotes. Pliny saith, 'That long. the powder of the rool given in drmk, or: the decoction thereof taken, helps all stoppings and stranglings in any part of the body, whether they proceed of pains in the chest or sides, and takes them away. The

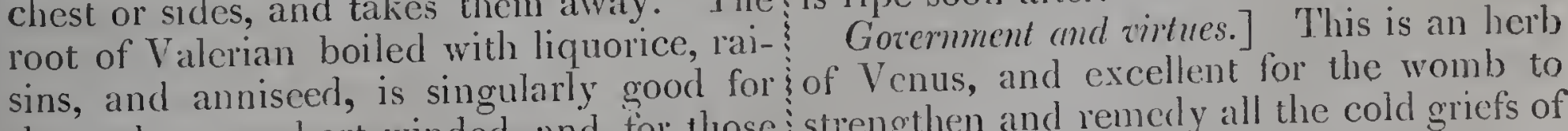
those that are short-winded, and for those strengthen and remedy all the cold griefs of that are troubled with the cough, and helps it, as Plantain doth the hot. Vervain is to open the passages, and to expectoratc hot and dry, opening obstructions, cleansphlegin casily. It is given to those that are ing and healing. It helps the yellow jaunbitten or stung by any renomous creature, dice, the dropsy and the gout; it kills and being boiled in wine. It is of a special expels worms in the belly, and causes a $(19,20$. B $\mathrm{C}$ 
good colour in the face and body, strengthens meal into a poultice, it cools inflammations as well as corrects the diseases of the stomach, of wounds; the dropping of the vine, when liver, and spleen ; helps the cough, wheez- it is cut in the Spring, which country people ings, and shortness of breath, and all the call Tears, being boiled in a syrup, with defects of the reins and bladder, expelling sugar, and taken inwardly, is excellent to the gravel and stone. It is held to be good stay women's longings after every thing against the biting of sepents, and other they see, which is a disease many women venomous beasts, against the plague, and with child are subject to. The decoction of both tertian and quartan agues. It con- Vine leaves in white wine doth the like. solidates and heals also all wounds, both Also the tears of the Vine, drank two or inward and outward, stays bleedings, and used with some honey, heals all old ulcers in and fistulas in the legs or other parts of the body; as also those ulcers that happen in the mouth ; or used with hog's grease, it helps the swellings and pains of the secret parts in man or woman, also for the piles as a coal, to be as white as snow, if you but or hæmorrhoids; applied with some oil of every morning rub them with it. It is a roses and vinegar unto the forehead and most gallant Tree of the Sun, very sympatemples, it eases the inveterate pains and thetical with the body of men, and that is ache of the head, and is good for those that the reason spirit of wine is the greatest corare frantic. The leaves bruised, or the dial among all vegetables. juice of them mixed with some vinegar, doth wonderfully cleanse the skin, and takes away morphew, freckles, fistulas, and other such like inflamations and deformities of the skin in any parts of the body. The distilled water of the herb when it is in full strength, dropped into the eyes, cleanses them from filns, clouds, or mists, that darken the sight, and wonderfully strengthens the optic nerves. The said water is very powerful in all the diseases aforesaid, either inward or outward, whether they be old and are used to cool any heat, or distemcorroding sores, or green wounds. The perature of the body, either inwardly or dried root, and peeled, is known to be ex- outwardly, as inflammations in the eyes, in cellently good against all scrophulous and the matrix or fundament, in imposthumes scorbutic habits of body, by being tied to also, and hot swellings, to drink the decocthe pit of the stomacli, by a piece of white ribband round the neck.

\section{TIYE VINE. $7.29 \cdot 30$}

The leaves of the English vine ( $\mathrm{I}$ do not or any other pains arising of heat, being mean to send you to the Canaries for a applicd in the same manner, or with oil of medicine (being boiled, inakes a good lotion roses. A dram weight of the dried leaves for sore mouths; being boiled wilh barley or flower of Violets, but the leaves more 
strongly, doth purge the body of choleric rough, hairy, or prickly sad grcen leaves, humours, and assuages the heat, being? somewhat narrow; the middle rib for the taken in a draught of wine, or any other most part being white. The flowers stand drink; the powder of the purple leares of at the top of the stalk, branched forth in the flowers, only picked and dried and many long spiked leaves of flowers bowing drank in water, is said to help the quinsy, or turning like the turnsole, all opening for and the falling-sickness in clildren, espe- the most part on the one side, which are cially in the beginning of the disease. 'I'he long and hollow, turning up the brims a flowers of the white Violets ripen and dis- little, of a purplish violet colour in them solve swellings. The herb or flowers, white that are fully blown, but more reddish while they are fresh, or the flowers when they are they are in the bud, as also upon their dedry, are effectual in the pleurisy, and all cay and withering; but in some places of diseases of the lungs, to lenify the sharp- a paler purplish colour, with a long pointel ness of hot rheums, and the hoarseness of in the iniddle, feathered or parted at the the throat, the heat also and sharpness of top. After the flowers are fallen, the seeds urine, and all the pains of the back or reins, 乡growing to be ripe, are blackish, cornered and bladder. It is good also for the liver and pointed somewhat like the head of a and the jaundice, and all hot agucs, to cool viper. The root is somewhat great and the heat, and quench the thirst; but the blackish, and woolly, when it grows toward syrup of Violets is of most use, and of better seed-time, and perishes in the Winter:

effect, being taken in some convenient There is another sort, little differing from liquor: and if a little of the juice or syrup the former, only in this, that it bears white of lcmons be put to it, or a few drops of the oil of vitriol, it is made thercby the more powerful to cool the heat, and quench the thirst, and gives to the drink a claret wine colour, and a fine tart relish, pleasing to the taste. Violets taken, or made up with honey, do more cleanse and cool, and with sugar contrary-wise. The dried flower of Violets are accounted amongst the cordial drinks, powder's, and oller medicines, es- remedy agrainst the biting of the Viper, and pecially where cooling cordials are neces- all other venomous beasts, or serpents; as sary. 'Thle green leaves are used with other also against poison, or poisonous herbs. herbs to make plaisters and poultices to Dioscorides and others say, That whosoever inflammations and swellings, and to case all shall take of the herb or root befere they be pains whatsoever, arising of heat, and for bitten, shall not be hurt by the poison of any the piles also, being fried with yolks of eggs, serpent. The root or seed is thought to be and applied thereto.

VIPER'S BUGLOSS.

Descript.] 'T'urs hath many long rough leaves lying on the ground, from among which rises up divers hard round stalks, very rough, as if they were thick set with prickles or hairs, whereon are set such like
Place.] The first grows wild almost every where. That with white flowers about the castlc-walls at Lewis in Sussex.

Time.] 'They flower in Summer, and their seed is ripe quickly after.

Govermment and virtues.] It is a most gallant herb of the Sun ; it is a pity it is no more in use than it is. It is an especial most effectual to comfort the heart, and expel sadness, or causeless mclancholy ; it compers the blood, and allays hot fits of agues. The seed drank in wine, procures abundance of nilk in women's breasts. The same also being taken, eases the pains in the loins, back, and kidneys. 'The distilled water of the herb when it is in flower, 
or its chief strength, is excellent to be ap-ings, comforts and strengthens any weak plied either inwardly or outwardly, for all part, or out of joint; helps to cleanse the the griefs aforesaid. There is a syrup made eyes from mistiness or films upon them, nereof very effectual for the comforting and to cleanse the filthy ulcers in the mouth, the heart, and expelling sadness and melan- or any other part, and is a singular remedy choly.

WALL FLOWERS, OR WINTER GILLIFLOWERS.

THE garden kind are so well known that they need no description.

Descript.] The common single Wallflowers, which grow wild abroad, have sundry small, long, narrow, dark green leaves, set without order upon small round, whitish, woody stalks, which bear at the tops divers single yellow flowers one above another, every one bearing four leaves a-piece, and of a very sweet scent: after which come gathered accordingly, which you shall find long pods, containing a reddish seed. The roots are white, hard and thready.

for the gout, and all aches and pains in the joints and sinews. A conserve made of the flowers, is used for a remedy both for the apoplexy and palsy.

\section{THE WALLNUT TREE.}

D. $x \times x$ ill.

$I_{T}$ is so well known, that it needs no description.

Time.] It blossoms early before the leaves come forth, and the fruit is ripe in September.

Government and virtues.] 'This is also a plant of the Sun. Let the fruit of it be to be of most virtues while they are green, before they have shells. The bark of the

Place.] It grows upon church walls, and Tree doth bind and dry very much, and the old walls of many houses, and other stone leaves are much of the same temperature: walls in divers places; The other sort in but the leaves when they are older, are heatgardens only.

Time.] All the single kinds do flower harder of digestion than when they are many times in the end of Autumn; and if fresh, which, by reason of their sweetness, the Winter be mild, all the Winter long, are more pleasing, and better digesting in but especially in the months of February, the stomach; and taken with sweet wine, March, and April, and until the heat of the they move the belly downwards, but being spring do spend them. But the double old, they grieve the stomach; and in hot kinds continue not flowering in that manner bodies cause the choler to abound and the all the year long, although they flower very head-ach, and are an enemy to those that early sometimes, and in some places very llave the cough ; but are less hurtful to those late.

Govermment and virtues.] The Moon rules them. Galen, in his seventh book of simple medicines, saith, 'That the yellow Wall- honey, they help the biting of a mad dog, flowers work more powerfully than any or the venons or infectious poison of any' of the other kinds, and are therefore of more beast, \&c. Caiats Pompeius found in the use in physic. It cleanses the blood, and treasury of Mithridates, king of Pontus, fretteth the liver and reins from obstruc- when he was overthrown, a scroll of his own tions, provokes women's courses, expels the hand witing, contirining a medicine against secundine, and the dead child; helps the any poison or infection; which is this; liardness and pain of the mother, and of Take two dry walnuts, and as many good spleen also; stays intlammations and swell- figs, and twenty leaves of rue, bruised and 
beaten together with two or three corns of the green husks being ripe, when they are salt and twenty juniper berries, which take shelled from the nuts, and drank with a every morning fasting, preserves from dan- little vinegar, is good for the place, so as ger of poison, and infection that day it is before the taking thereof a vein be opened. taken. The juice of the other green husks The said water is very good against the boiled with honey is an excellent gargle for quinsy, being gargled and bathed theresore mouths, or the heat and inflammations with, and wonderfully helps deafness, the in the throat and stomach. The kernels, noise, and other pains in the ears. The when they grow old, are more oily, and distilled water of the young green leaves in therefore not fit to be eaten, but are then the end of May, performs a singular cure used to heal the wounds of the sinews, on foul running ulcers and sores, to be gangrenes, and carbuncles. The said ker- bathed, with wet cloths or spunges applied nels being burned, are very astringent, to them every morning.

and will stay lasks and women's courses, being taken in red wirie, and stay the falling of the hair, and make it fair, being anointed with oil and wine. The green husks will do the like, being used in the same manner. The kernels beaten with rue and wine, being applied, help the quinsy ; and bruised with some honey, and applied to the ears, ease the pains and inflammation of them. A piece of the green husks put into a hollow tooth, eases the pain. The catkins hereof, taken before they fall off, dried, and given a dram thereof in powder with white wine, wonderfully helps those that are troubled with the rising of the mother. The oil that is pressed out of the kernels, is very profitable, taken inwardly like oil of almonds, to help the cholic, and to expel wind rery effectually; an ounce or two thereof may be taken at whole herb changes to be yellow, after it any time. The young green nuts taken hath been in flower awhile.

before they be half ripe, and preserved with Place.] It grows every where by the sugar, are of good use for those that have way sides, in moist grounds, as well as dry, weak stomachs, or defluctions thereon. The in corners of fields and bye lanes, and somedistilled water of the green husks, before times all over the field. In Sussex and they be half ripe, is of excellent use to cool Kent they call it Green Weed.

the heat of agues, being drank an ounce or two at a time: as also to resist the infection of the plague, if some of the same be also applied to the sores thereof. The digests raw phlegm, thins gross humours, same also cools the heat of green wounds dissolves hard tumours, and opens obstrucand old ulcers, and heals them, being tions. Some do highly commend it against nathed therewith. The distilled water of the biting of venomous creatures, to be take

Time.] It flowers in June.

Government and virtues.] Matthiolus saith, that the root hereof cures tongh phlegn, $3 \mathrm{D}$ 
inwardly and applied outwardly to the bran of Wheat meal steeped in sharp vinehurt place; as also for the plague or pes- gar, and then bound in a linen cloth, and tilence. The people in some countries of rubbed on those places that have the scurf, this land, do usc to bruise the herb, and lay morphew, scabs or leprosy, will take them it to cuts or wounds in the hands or legs, to away, the body being first well purged and heal them.

WH Е А . 8.34 ,

prepared. 'The decoction of the bran of Wheat or barley, is of good use to bathe those places that are bursten by a rupture;

ALL the several kinds thereof are so well and the said bran boiled in good vinegar, known unto almost all people, that it is all and applied to swollen breasts, helps them. together needless to write a description thereof:

Government and virtues.] It is under the biting of vipers (which I take to be no Venus. Dioscorides saith, That to eat the venomous creatures. The leaves of Wheat corn of green Wheat is hurtful to the meal applied with some salt, take away stomach, and breeds worms. Pliny saith, hardness of the skin, warts, and hard knots That the corn of Wheat, roasted upon an in the flesh. Wafers put in water, and iron pan, and eaten, are a present remedy drank, stays the lask and bloody flux, and for those that are chilled with cold. 'The are profitably used both inwardly and outoil pressed from wheat, between two thick wardly for the ruptures in children. Boiled plates of iron, or copper heated, heals all in water unto a thick jelly, and taken, it tetters and ring-worms, being used warm; stays spitting of blood; and boiled with and hereby Galen saith, he hath known mint and butter, it helps the hoarseness of many to be cured. Mitthiolus commends the throat.

the same to berput into hollow ulcers to heal? them up, and it is good for chops in the hands and feet, and to make rugged skin smooth. The green corns of Wheat being? no description. I shall therefore only shew chewed, and applied to the place bitten by you the virtues therof.

a mad dog, heals it; slices of Wheat bread Government and virtues.] The Moon soaked in red rose water, and applied to owns it. Both the leaves, bark, and the the eyes that are lot, red, and inflamed, or seed, are used to stanch bleeding of wounds, blood-shotten, helps them. Hot bread ap- and at mouth and nose, spitting of blood, plied for an hour, at times, for three days and other fluxes of blood in man or woman, together, perfectly heals the kernels in the and to stay vomiting, and provocation therethroat, commonly called the king's evil. The flour of Wheat mixed with the juice of drank. It helps also to stay thin, hot, sharp, henbane, stays the flux of humours to the salt distillations from the head upon the joints, being laid thereon. The said meal lungs, causing a consumption. The leaves boiled in vinegar, helps the shrinking of the bruised with some pepper, and drank in sinews, saith Pliny; and mixed with vine- wine, helps much the wind cholic. 'The gar, and boiled together, heals all freckles, leaves bruised and boiled in wine, and spots and pimples on the face. Wheat drank, stays the heat of lust in man or flour, mixed with the yolk of an egg, honey, woman, and quite extinguishes it, if it be and turpentine, doth draw, cleanse and heal long used: The seed also is of the same any boil, plague, sore, or foul ulcer. The effect. Water that is gathered from the 
Willow, when it flowers, the bark being fit of it, where those that sow it, cut it three slit, and a ressel fitting to receive it, is very times a year.

good for redness and climness of sight, or Time.]. It flowers in June, but it is long films that grow over the eyes, and stay the after before the seed is ripe.

rheums that fall into them; to provoke Government and virtues.]. It is a eold and urine, being stopped, if it be drank; to elear dry plant of Saturn. Some people affirm the face and skin from spots and diseolour- the plant to be destruetive to bees, and ings. Galen saith, The flowers have an fluxes them, which, if it be, I cannot help admirable faculty in drying up humours, it. I should rather think, unless bees be being a medieine without any sharpness or contrary to other creatures, it possesses them corrosion; you may boil them in white with the contrary disease, the herb being wine, and drink as much as you will, so exceeding dry and binding. However, if you drink not yourself drunk. The bark any bees be diseased thereby, the cure is, works the same effect, if used in the same to set urine by them, but set it in at ressel, manner, and the Tree hath always a bark that they cannot drown themselves, which upon it, though not always flowers; the may be remedied, if you put pieces of eork burnt ashes of the bark heing mixed with in it. The herb is so drying and binding, vinegar, takes away warts, corns, and that it is not fit to be given inwardly. An superfluous flesh, being applied to the place. ointment made thereof stanches bleeding. The decoetion of the leaves or bark in wine, A plaister matle thereof, and applied to the takes away scurff and dandriff by washing ? region of the spleen which lies on the left the place with it. It is a fine cool tree, the side, takes away the hardness and pains boughs of which are very convenient to therenf. The ointment is excellently good be placed in the chamber of one sick of a in such uleers as abound with moisture, and ferer.

WOAD. P.33 35

Descript.] It hath divers large leaves, long, and somewhat broad withal, like those of the greater plntain, but larger, thieker, of a greenish colour, somewhat blue withal. From among which leaves rises up a lusty stalk, three or four feet high, with divers leaves set thereon; the higher the stalk rises, the smaller are the leaves; at the top it spreads divers branches, at the end of which appear rery pretty, little yellow flowers, and after they pass away like other flowers of the field, eome husks, long and somewhat flat withal; in form they resemble a tongue, in colour they are black, and they hang bobbing downwards. 'The seed contained within these husks (if it be a little chewed) gives an azure colour. The root is white and long.

Place.] It is sored in fields for the bene- takes away the corroding and fretting humours: It eools inflammations, quenches St. Anthony's fire, and stays defluxion of the blood to any part of the body.

WOODBINE, OR HONEY-SUCKLES:

IT is a plant so common, that every one that hath eyes knows it, and he that hath none, eannot read a description, if I should write it.

Time. They flower in June, and the fruit is ripe in August.

Gocernment and riptues.] Doctor Tradition, that grand introdueer of errors, that hater of truth, lover of folly, and the mortal foe to Dr. Reason, hath taught the common people to nse the leaves or flowers of this plant in mouth-water, and by long eontinuanee of time, hath so grounded it in the brains of the vulgar, that you eannot beat it out with a beetle: All mouth-waters onght to be cooling and drying, but. Honey 

Suckles are cleansing, consuming and di-
gesting, and therefore fit for inflammations thus Dr. Reason. Again if you please, we will leave Dr. Reason a wlile, and come to Dr. Experience, a learned gentleman, and his brother. Take a leaf and chew it in your mouth, and you will quickly find it likelier to cause a sore mouth and throat than to cure it. Well then, if it be not good? for this, What is it good for? It is good for something, for God and nature made nothing in vain. It is an herb of Mercury, and appropriated to the lungs; neither is it Crab claims dominion over it; neither is it a foe to the Lion; if the lungs be afflicted by Jupiter, this is your cure: It is fitting a conserve made of the flowers of it were kept in every gentlewoman's house ; I know no better cure for an asthma than this : besides, it takes away the evil of the spleen, provokes urine, procures speedy delivery of women in travail, helps cramps, convulsions, and palsies, and whatsoever griefs come of cold or stopping; if you please to make use of it as an ointment, it will clear your skin of morphew, freckles, and sun-burnings, or whatsoever else discolours it, and then the maids will love it. Authors say, The flowers are of nore effect than the leaves, and that is true; but they say the seeds are least effectual of all. But Dr. Reason told me, That there. was a vital spirit in every seed to beget its like; and Dr. Experience told me, That there was a greater heat in the seed than there was in any other part of the plant: and withal, 'That heat was the mother? of action, and then judge if old Dr. Tradition (who may well be honoured for his age, but not for his goodness) hath not so poisoned the world with errors before I was born, that it was never well in its wits since, and there is a great fear it will die mad.

$$
\text { WOR M WOOD. P. } 31 \text {, }
$$

THREe Wormwoods are familiar with ter, I do not know the father of it, unless us; one I shall not describe, another I shall the devil, but she christened it, and called describe, and the third be critical at; and I care not greatly if I begin with the last first.

Sea Wormwood hath gotten as many names as virtues, (and perhaps one more) Scriphian, Santomeon, Belchion, Narbinense, Hantonicon, Misneule, and a matter of twenty more which I shall not blot paper withal. A papist got the toy by the end, and he called it Holy Wormwood; and in truth I am opinion, their giving so much holiness to herbs, is the reason there remains so little in themselves. The seed of this Wormwood is that which women usually give their children for the worms. Of all Wormwood's that grow here, this is the weakest, but Doctors commend it, and apothecaries sell it; the one must keep his credit, and the other get money, and that is the liey of the work. The herb is good for something, because God made nothing in vain: Will you give me leave to weigh things in the balance of reason; Then thus: The seeds of the common Wormwood are far more prevalent than the seed of this, to expel worms in children, or people of ripe age; of both some are weak, some are strong. The Seriphian Wormwood is the weakest, and haply may prove to be fittest for the weak bodies, (for it is weak enough of all conscience.) Let such as are strong take the common Wormwood, for the others will do but little good. Again, near the sea many people live, and Seriphian grows near them, and therefore is more fitting for their bodies, because nourished by the same air ; and this I had from Dr. Reason. In whose body Dr. Reason dwells not, dwells Dr. Madness, and he brings in lis brethren, Dr. Ignorance, Dr. Folly, and Dr. Sickness, and these together make way for Death, and the latter end of that man is worse than the beginning. Pride was the cause of Adam's fall ; pride begat a daugh- 
it Appetite, and sent her daughter to taste with many round, woody, hairy stalks from these wormwoods, who finding this the one root. Its height. is four feet, or three at least bitter, made the squeamish wench least. 'The leaves in longitude are long, in extol it to the skies, though the virtues of it latitude narro:w, in colour white, in form. never reached the middle region of the air. hoary, in similitude likeSouthernwoorl, only Its due praise is this; It is weakest, there- broader and longer; in taste rather salt than fore fittest for weak bodies, and fitter for bitter, because it grows so near the saltthose bodies that dwell near it, than those water; at the joints, with the leaves toward that divell far from it; my reason is, the sea the tops it bears little yellow flowers; the (those that live far from it, know when they root lies deep, and is woods.

come near it) casts not such a smell as the land doth. The tender mercies of God being over all his works, hath by his eternal Providence, planted Seriphian by the seaside, as a fit medicine for the bodies of those that live near it. Lastly, It is known to all that know any thing in the course of nature, that the liver delights in sweet things, if so, it abhors bitter ; then if your liver be weak, it is none of the wisest courses to plague it with an enemy. If the liver be weak, a? consumption follows; would you know the reason? It is this, A man's flesh is repaired by blood, by a third concoction, which transmutes the blood into flesh, it is well I said, (concoction) say I, if I had said me. The liver makes blood, and if it be weakened that if it makes not enough, the flesh wastes; and why must flesh always be renewed? Because the eternal God, when he made the creation, made one part of it in continual dependency upon another; and why did he so? Because himself only is permanent; to teach us, That we should not fix our affections upon what is transitory, but what endures for ever. The result of this is, if the liver be weak, and cannot make blood enough, I would have said, Sanguify, if I lad written only to scholars, the Seriphian, which is the weakest of Wormwoods, is better than the best. I have been critical enough, if not too much.

Place.] It grows familiarly in England, by the sea-side.

Descript.] It starts up out of the earth, $(19,20$.
Common Wormwood I shall not describe, for every boy that can eat an egg knows it. Roman Iformwood; and why Roman, seeing it grows familiarly in England? It may be so called, because it is good for a stinking breath, which the Romans cannot. be very free from, maintaining so mâny bad houses by authority, of his Holiness.

Descript.] 'The stalks are slender, and shorter than the conimon Wormwood by one foot at least; the leaves are more finely cut and divided than they are, but something smaller; both leaves and stalks are hoary, the flowers of a pale yellow. colour; it is altogether like the common Wormwood, save only in bigness, for it is smaller; in taste, for it is not so bitter; in smell, for it is spicy.

Place.] It grows upon the tops of the mountains (it seems 'tis aspiring) there 'tis natura!, but usually nursed up in gardens for the use of the apothecaries in London. Time.] All Wormwoods usually flower in August, a little sooner or later.

Government and virtues.] Will you give me leave to be critical a little? I must take leave. Wormwood is an herb of Mars, and if Pontanus say otherwise, he is beside the bridge; I prove it thus: What delights in martial places, is a martial herb; but Wormwood delights in martial places (for about forges and iron works you may gather a cart-load of it, ) ergo, it is a martial herb. It is hot and dry in the first degree, viz. just as hot as your blood, and no hotter. It remedies the evils choler can inflict on the $3 \mathbf{E}$ 
body of man by sympathy. It helps the herb of Mars, is a present remedy for the evils Venus and the wanton Boy produce, biting of rats and mice. Mushrooms (I by antipathy; and it doth something else cannot give them the title of Herba, Frubesides. It cleanses the body of choler tex, or Arbor) are under the dominion of (who dares say Mars doth no good?) It Saturn, ( and take one time with another, provokes urine, helps surfeits, or swellings they do as much harm as good;) if any have in the belly; it causes appetite to meat, poisoned himself by eating them, Wormbecause Mars rules the attractive faculty in wood, an herb of Mars, cures him, becausc man: 'The sun never shone upon a better Mars is exalted in Capricorn, the house of herb for the yellow jaundice than this; Why Saturn, and this it doth by sympathy, as it should men cry out so much upon Mars for did the other by antipathy. Wheals, pushes, an infortunate, (or Saturn either?) Did black and blue spots, coming either by God make creatures to do the creation a bruises or beatings. Wormwool, an herb mischief? This herb testifies, that Mars is of Mars, helps, because Mars, (as bad you willing to cure all diseases he causes; the love him, and as you hate him) will not truth is, Mars loves no cowards, nor Saturn break your head, but he will give you a fools, nor I neither. Take of the tlowers of plaister. If he do but teach you to know Wormwood, Rosemary, and Black Thorn, yourselves, his courtesy is greater than is of each a like quantity, half that quantity discourtesy. The greatest antipathy beof saffron; boil this in Rhenish wine, but tween the planets, is between Mars and put it not in saffron till it is almost boiled; Venus: one is hot, the other cold; one This is the way to keep a man's body in diurnal, the other noclurnal; one dry, the health, appointed by Camerarius, in his other moist; their houses are opposite, one book intitled Hortus Medicus, and it is a masculine, the other feminine; one public. good one too. Besides all this, Wormwood the other private; one is valiant, the other provokes the terms. I would willingly effeminate: one loves the light, the other teach astrologers, and make them physi- hates it; one loves the field, the other sheets; cians (if I knew how) for they are most then the throat is under Venus, the quinsy fitting for the calling; if y ou will not believe lies in the throat, and is an inflammation me, ask Dr. Hippocrates, and Dr. Galen, there; Venus rules the throat, (it being a couple of gentlemen that our college of under Tamıs her sign.) Mars eradicates physicians keep to vapour with, not to all diseases in the throat by his herbs (for follow. In this our herb, I shall give the pat- wormwood is one) and sends them to Egypt tern of a ruler, the sons of art rough cast, on an errand never to return more, this yet as near the truth as the men of Benja- done by antipathy. The eyes are under min could throw a stone: Whereby, my the Luminaries; the right eye of a man, brethren, the astrologers may know by a and the left eye of a woman the Sun claims penny how a shilling is coined : As for the dominion over: the left eye of a man, and college of physicians, they are too stately to the right eye of a woman, are privileges of learn, and too proud to continue. They the Moon, Wormwood, an herb of Mars say a mouse is under the dominion of the cures both; what belongs to the Sun by Moon, and that is the reason they feed in the sympathy, because he is exalted in his night; the house of the Moon is Cancer; house; but what belongs to the Moon by rats are of the same nature with mice, but antipathy, because he hath his fall in her's. they are a little bigger; Mars receives his Suppose a man be bitten or stung by a fall in Cancer, ergo, Wormwood being an martial creature, imagine a wasp, a hornet, 
a scorpion, Wormwood, an herb of Mars, either linen or woolen draper) yet as brave as gives you a present cure; that Mars, cho- they looked, my opinion was that the moths leric as he is, hath learned that patience, to might consume them; moths are under the pass by your evil speeches of him, and tells dominion of Mars; this herb Wormwood beyou by my pen, That he gives you no af- ing laid among cloaths, will make a moth fliction, but he gives you a cure; you need scorn to meddle with the cloaths, as much not run to A pollo, nor Esculapius; and if as a lion scorns to meddle with a mouse, or he was so choleric as you make him to be, an eugle with a fly. You say Mars is anhe would have drawn his sword for anger, gry, and it is true enough he is angry with to see the ill conditions of these people many countrymen, for being such fools to that can spy his vices, and not his virtues, be led by the noses by the college of phyThe eternal God, when he made Mars, sicians, as they lead bears to Paris garden. made him for public good, and the sons of Melancholy men cannot endure to be men shall know it it in the latter end of the wronged in point of good fame, and that world. Et calum Mars solus babet. . You doth sorely trouble old Saturn, because they say Mars is a destrnyer; mix a little Worm- call him the greatest infortunate; in the wood, an herb of Mars, with your ink, body of man he rules the spleen, (and that neither rats nor mice touch the paper writ- makes covetous man so splenetic) the poor ten with it, and then Mars is a preserver. old man lies crying out of his left side. Astrologers think Mars causes scabs and Father Saturn's angry, Mars comes to him; itch, and the virgins are angry with him, Come, brother, I confess thou art evilspoken because wanton Venus told them he de- of, and so am I ; thou kuowest I have my forms their skins; but, quoth Mars, my ; exaltation in thy house, I give him an herb only desire is, they should know themselves; of mine, Wormwood, to cure the old man : my herb Wormwood will restore them to Saturn consented, but spoke little, and so the beauty they formerly had, and in that Mars cured him by sympatiy. When I will not come an inch behind my opposite, Mars was free from war, (for he loves to be Venus: for which doth the greatest evil, he fighting, and is the best friend a soldier that takes away an innate beauty, and when hath) I say, when Mars was free from war, he has done, knows how to restore it again? he called a council of war in his own brain, or she that teaches a company of wanton to know how he should do poor sinful mian lasses to paint their faces? If Mars be in good, desiring to forget his abuses in being a Virgin, in the nativity, they say he causes called an infortunate. He mustcrs up his the cholic (it is well God hath set some own forces, and places them in battalia. body to pull down the pride of man.) He Oh! quoth he, why do I hurt a poor in the Virgin troubles none with the cholic, silly man or woman? His angel answers but them that know not themselves (for who him, It is because they have offended their knows himself, may easily know all the God, (Look back to Adam:) Well, says world.) Wurmwood, an herb of Mars, is a Mars, though they speak evil of me, I will prcsent cure for it ; and whether it be most do good to them; Death's cold, my herb like a C'hristian to love him for his good, or shall heat them: they are full of ill humours hate him for his evil, judge ye. I had al- (else they would never bave spoken ill of most forgotten, that charity thinks no evil. me;) my herb shall cleanse thenn, and dry I wasonce in the 'lower and viewed the ward- them; they are poor weak creatures, my robe, and there wasagreat many fine clothes: herb shall strengthen theni ; they are dull (I can give them no other title, for I was never witted, my herb shall fortify their appre- 
hensions; and yet among astrologers all night, the one from Aries, and the other this does not deserve a good word: Oh the from Scorpio; give 111 e thy leave by sympatience of Mars!

Felix qui potuit rerum cognoscere caucas,

Inque domus superum scandere cura facit.

O happy he that can the knowledge gain,

To know the eternal God made nought in vain.

To this 1 add,

I know the reason causeth such a dearth

Of knowledge; 'tis because men love the earth.

The other day Mars told me he met with Venus, and he asked her, What was the reason that she accused him for abusing women? He never gave them the pox. In quarrel; Mars (not delighting nuch in the dispute they fell out, and in anger women's tongues) went away, and did it parted, and Mars told me that his brother? whether she would or no.

Saturn told him, that an antivenerean He that reads this, and understands what medicine was the best against the pox. he reads, hath a jewel of more worth than Orice a month he meets with the Moon. a diamond; he that understands it not, is Mars is quick enough of speech, and the as little fit to give physick. There lies a Moon not much behind hand, (neither are key in these words which will unlock, (if it most women.) The Moon looks much be turned by a wise hand) the cabinet of after children, and children are much trou- physick : I have delivered it as plain as I bled with the worms; she desired a medi- durst; it is not only upon Wormwood as cine of him, he bid her take his own herb, I wrote, but upon all plants, trees, and Wormwood. He had no sooner parted herbs; he that understands it not, is unfit with the Moon, but he met with Venus, and (in my opinion) to give physic. 'This shall she was as drunk as a hog; Alas! poor live when I am dead. And thus I leave it Venus, quoth he; What! thou a fortune, to the world, not caring a farthing whether and be drunk? I'll give thee antipathetical they like it or dislike it. The grave equals cure; Take my herb Wormwood, and thou all men, and therefore shall equal me with shall never get a surfeit by drinking. A all princes; until which time the eternal poor silly countryman hath got an ague, Providence is over me: Then the ill tongue and cannot go about his business: he of a prattng fellow, or one that hath more wishes he had it not, and so do I; but I tongue than wit, or more proud than will tell him a remedy, whereby he shall honest, shall never trouble me. Wisdom is prevent it; Take the herb of Mars, Worm- justified by her children. And so much for wood, and if infortunes will do good, what Wormwood.

will fortunes do? Some think the lungs are under Jupiter; and if the lungs then the breath; and though sometimes a man gets a stinking breath, and yet Jupiter is a fortune, forsooth; up comes Mars to him spread upon the ground, finely cut, and Come brother Jupiter, thou knowest I sent divided into many small parts: It flowers thee a couple of trines to thy house last are white, but not all of a whiteness, and 
stayed in knots, upon divers green stalks It stays the shedding of hair, the head being which rise from among the leaves.

Place.] It is frequent in all pastures. taken it helps the retentive faculty of the Time.] It flowers late, even in the latter stomach: it helps the gonorrhea in men, end of August.

Government and virtues.] It is under the cannot hold their water; and the leaves influence of Venus. An ointment of them schewed in the mouth eases the tooth-ache; cures wounds, and is most fit for such as and these virtues being put together, shew have inflammations, it being an herb of the herb to be drying and binding. Achilles Dame Venus; it stops the terms in women, is supposed to be the first that left the virbeing boiled in white wine, and the decoc- tues of this herb to posterity, having learned tion drank; as also the bloody flux; the them of this master Chiron, the Centaur; ointment of it is not only good for green and certainly a very profitable herb it is in wounds, but also for -ulcers and fistulas, cramps, and therefore called Militaris. especially such as abound with moisture.

\section{DIRECTIONS FỌ MAKING SYRUPS, CONSERVES,}

$$
\text { \&.c. \&.c. }
$$

Having in divers places of this 'Treatise promised you the way of making Syrups, Conserves, Oils, Ointments, \&c, of herbs, roots, flowers, \&c. whereby you may have them ready for your use at such times when they cannot be had otherwise; I come now to perform what I promised, and you shall find me rather better than worse than iny word.

That this may be done methodically, I shall divide my directions into two grand sections, and cach section into several chapters, and then you shall see it look with such a countenance as this is.

\section{S ECTION I.}

Of gathering, drying, and keeping. Simples, and their juices.

CII P. I Of leates of Herbs, \& c.

CIIA P. II. Of Flower's.

Снар. II I. Of Seeds.

CIlAP. Iv. Of Roots.

$(19,20$.
CII P. v. Of Barks.

c II A P. VI. Of Juices.

$\mathrm{S}$ E C T I O N I

Of making and keeping Compounds.

снар. I. Of distilled waters.

снар. II. Of Syrups.

снар. II . Of Juleps.

CHA P. IV. Óf Decoctions.

CuAp.v. Of Oils.

CIA P. VI Of Electuaries.

CII AP. viI. Of Conserves.

c In a P. viri. Of Preserves.

CII A P. IX. Of Lohochs.

CHAP. X. Of Ointments.

CII A P. х I. Of Plaisters.

снат. хіт. Of Poultices.

снар. х I I. Of Troches.

CHAP. xiv. Of Pills.

CII A. xv. The way of fitting Medicines to Compound Diseases.

Of all these in order. 


\section{H A P T E R I. \\ Of Leaves of Herbs, or Trees.}

1. Of leaves, choose only such as are green, and full of juice; pick them carefully, and cast away such as are any way declining, for they will putrify the rest: So shall one handful be worth ten of those you buy at the physic herb shops.

2. Note what places they most delight to grow in, and gather them there ; for Betony that grows in the shade, is far better than that which grows in the Sun, because it delights in the shade; so also such herbs as delight to grow near the water, shall be gathered near it, though happily you may find some of them upon dry ground: The Treatise will inform you where every herb delights to grow.

3. The leaves of such herbs as run up to seed, are not so good when they are in flower as before (some few excepted, the leaves of which are seldom or never used) in such cases, if through ignorance they were not known, or through negligence forgotten, you had better take the top and the flowers, then the leaf:

4. Dry them well in the Sun, and not in the shade, as the saying of physicians is ; for if the sun draw away the virtues of the herb, it must need do the like by hay, by the same rule, which the experience of every country farmer will explode for a notable piece of nonsense.

5. Such as are artists in astrology, (and indeed none else are fit to make physicians) such I advise; let the planet that governs the herb be angular, and the stronger the better ; if they can, in herbs of Saturn, let Saturn be in the ascendant; in the herbs of Mars, let Mars be in the mid heaven, for in those houses they delight; let the Moon apply to them by good aspect, and let her not be in the houses of her encmies; if you smell, they are good; eit
cannot well stay till she apply to them, let gone, so is the virtue also. fire. thicir pleasure ; for, planet that governs them. when it is in its prime. et or dewy, they will not keep. you in the foregoing chapter. gone, so is the virtue also.

her apply to a planet of the same triplicity; if you cannot wait that time neither, let her be with a fixed star of their nature.

6. Having well dried them, put them up in brown paper, sewing the paper up like a sack, and press them not too hard together, and keep them in a dry place near the

7. As for the duration of dried herbs, a just time cannot be given, let authors prate

1st. Such as grow upon dry grounds will kecp better than such as grow on moist.

$2 \mathrm{dly}$, Such herbs as are fill of juice, will not keep so long as such as are drier.

$3 \mathrm{dly}$. Such herbs as are well dried, will keep longer than such as are slack dried. Yet you may know when they are corrupted, by their loss of colour, or smell, or both; and if they be corrupted, reason will tell you that they must needs corrupt the bodies of those people that take them.

4. Gather all leaves in the hour of that

\section{I A P T E R I I. \\ Of Flowers.}

1. THe flower, which is the beauty of the plant, and of none of the least use in physick, grows yearly, and is to be gathered

2. As for the time of gathering them, let the planetary hour, and the planet they come of, be observed, as we shewed you in the foregoing chapter : as for the time of the day, let it be when the sun shine upon them, that so they may be dry; for, if you gather either flowers or herbs when they are

3. Dry them well in the sun, and keep them in papers near the fire, as I shewed

4. So long as they retain the colour and smell, they are good; either of them being 


\section{II A P T E R I I I \\ Of Seeds.}

1. TuE seed is that part of the plant which is endowed with a vital faculty to bring forth its like, and it contains potentially the whole plant in it.

2. Is for place, let them be gathered from the place where they delight to grow.

3. Let them be full ripe when they are gathered; and forget not the celestial harmony before mentioned, for I have found by experience that their virtues are w wice as great at such times as other's: "There is the sun."

4. When you have gathered them, dry them a little, and but a little in the sun, before you lay them up.

5. You need not be so careful of keeping them so near the fire, as the other beforementioned, because they are fuller of spirit, and therefore not so subject to corrupt.

6. As for the time of their auration, it is palpable they will keep a good many years; yet, they are best the first year, and this I make appear by a good argument. They is to dry in the sun, or else hang them in will grow sooner the first year lhey be set, the chimney corner upon a string; as for therefore then they are in their prime; such as are hard, you may dry them any and it is an easy matter to renew them yearly.

\section{II $\Lambda$ P TER I V. \\ Of Roots.}

1. Or roots, chuse such as are neither rotten nor worm-eaten, but proper in their taste, colour, and smell; such as exceed neither in softness nor hardness.

2. Give me leave to be a little critical th against the vulgar received opinion, which gentle fire; or, if you can with convenience is, That the sap falls down into the roots in keep them near the fire, you may save yourthe Autumn, and rises again in the Spring, self the labour. as men go to bed at night, and rise in the morning; and this idle talk of untruth is so grounded in the heads, not onily of the vulgar, but also of the learned, that a man cannot drive it out by reason. I pray let such sapmongers answer me this argument; If the sap falls into the roots in the fall of the leaf, and lies there all the Winter, then must the root grow only in the Winter. But the root grows not at all in the Winter, as experience teaches, but only in the kernel in the Spring, you shall find the root to grow to a pretty bigness in the Summer, and be not a whit bigger next Spring. What doth the sap do in the rool all that while? Pick straws? "Tis as rotten as a rotten post.

The truth is, when the sun declines from the tropic of Cancer, the sap bugins to congeal both in root and branch; when he touches the tropic of Capricorn, and ascends to us-ward, it begins to wax thin again, and by degrees, as it congealed. But to proceed.

3. The drier time you gather the roots in, the better they are; for they have the less excrementitious moisture in them.

4. Such roots as are soft, your best way
to dry in the sun, or else hang them in
he chimney corner upon a string; as for
ch as are hard, you may dry them any

5. Such roots as arc great, will keep longer than such as are small; yet most of them will keep a year.

8. Such roots as are soft, it is your best way to keep them always near the fire, and ter-time you find any of your roots, herbs or flowers begin to be moist, as many times you shall (for it is your best way to look to them once a month) dry then by a very 7. It is in vain to dry roots that may Summer: Therefore, If you set an appleto take this general rule for it: If in Win- 
commonly be had, as Parsley, Fennel, Plantain, \&c. but gather them only for pre- Put it into a pipkin or skillet, or some such sent need.

\section{H A P T E R V \\ Of Barks.}

1. BARKs, which physicians use in medicine, are of these sorts : Of fruits, of roots, of boughs.

2. The barks of fruits are to be taken when the fruit is full ripe, as Oranges, Lemons, \&c. but because I have nothing to do with exotics here, I pass them without any more words.

3. The barks of trees are best gathered in the Spring, if of oaks, or such great trees; because then they come easier off, and so you may dry them if you please; but indeed the best way is to gather all barks only for present use.

4. As for the barks of roots, 'tis thus' to be gotten. Take the roots of such herbs as have a pith in them, as parsley, fennel, \&c. slit them in the middle, and when you have taken out the pith (which you may easily do) that which remains is called (tho' improperly) the bark, and indeed is only to be used.

\section{II A P T E R V I. \\ Of Juices.}

1. JUICES are to be pressed out of herbs when they are young and tender, out of some stalks and tender tops of herbs and plants, and also out of some flowers.

2. Having gathered the herb, would you preserve the juice of it, when it is very dry (for otherwise the juice will not be worth times improperly; for in truth, nothing is a button) bruise it very well in a stone mor-simple but pure elements; all things else tar with a wooden pestle, then having put are compounded of them. We come now it into a canvas bag, the herb I mean, not to treat of the artificial medicines, in the the mortar, for that will give but little juice, form of which (because we must begin press it hard in a press, then take the juice somewhere) we shall place distilled waters ; and clarify it. thing, and set it over the fire; and when the fire till no more scum arise; when you have your juice clarified, cast away the scum as a thing of no use.

4. When you have thus clarified it, you have two ways to preserve it all the year.

(1.) When it is cold, put it into a glass, and put so much oil on it as will cover it to the thickness of two fingers; the oil will swim at the top, and so keep the air from coming to purtify it: When you! intend to use it, pour it into a porringer, and if any oil come out with it, you may easily scum it off with a spoon, and put the juice you use not into the glass again, it will quickly sink under the oil. This is the first way. ficult, and the juice of fruits is usually preserved this way. When you have clarified it, boil it over the fire, till (being cold) it be of the thickness of honey; This is most commonly used for diseases of the mouth, and is called Roba and Saba. And thus much for the first section, the second follows

$$
\text { SECTION II. }
$$

The way of making and keeping all necessary Compounds.

C H A P T E R V.

\section{Of distilled Waters.}

Hitmer to we have spoken of medicines which consist in their own nature, which authors vulgarly call Simples, though somein which consider, scum arises, take it off; let it stand over the

(2.) The second way is a little more dif 
1. Waters are distilled of herbs, flowers, fruits, and roots.

2. We trent not of strong waters, but of cold, as being to act Galen's part, and not viz. Paracelsus's.

3. 'I'he herbs ought to be distilled when they are in the greatest vigour, and so ought the flowers also.

4. The vulgar way of distillations which people use, because they know no better, a is in a pewter still; and although distilled waters are the weakest of artificial medicines, and good for little but mixtures of other medicines, yet they are weaker by many degreés, than they would be were they distille.d in sand. .If I thought it not impossible, to teach you the way of distilling in sand, 1 would attempt it.

5. When you have distilled your water, put it into a glass, covered over with a paper pricked full of holes, so that the excrementitious and fiery vapours may exhale, which cause that settling in distilled waters called the Mother:, which corrupt them, then cover it close, and keep it for your use.

6. Stopping distilled waters with a cork, makes them musty, and so does paper, if it but touch the water : it is best to stop them with a bladder, being first put in water, and bound over the top of the glass.

Such cold waters as are distilled in a pewter still (if well kept) will endure a year; such as are distilled in sand, as they are twice as strong, so they endure twice as long.

$$
\begin{gathered}
\text { C II A P T E R I } 1 . \\
\text { Of Syrups. }
\end{gathered}
$$

1. A Srrup is a medicine of a liquid form, composed of infusion, decoction and juice. And, 1. For the morc grateful taste. 2. For the better keeping of it: with a certain quantity of honey or sugar, hereafter mentioned, boiled to the thickness of new honey.

$(21,22$.
2. You see at the first view, That this aphorism divides itself into 7 hree branches, which deserve severally to be treated of,

1. Syrups made by infusion.

2. Syrups made by decoction.

3. Syrups made by juice.

Of each of these, (for your instructionsake, kind countrymen and women) I speak a word or two apart.

1st, Syrups made by infusion, are usually made of flowers, and of such flowers as soon lose their colour and strength by boiling, as roses, violets, peach flowers, \&c. They are thus made: Having picked your flowers clean, to every pound of them add three pounds or three pints, which you will (for it is all one) of spring water, made boiling hot; first put your flowers into a pewter-pot, with a cover, and pour the water on them; then shutting the pot, let it stand by the fire, to keep hot twelve hours, and strain it out: (in such syrups as purge) as damask roses, peach flowers, \&c. the usual, and indeed the best way, is to repeat this infusion, adding fresh fiowers to the same liquor divers times, that so it may be the stronger) having strained it out, put the infusion into a pewter bason, of an earthen one well glazed, and to every pint of it add two pounds of sugar, which bcing only melted over the fire, without boiling, and scummed, will produce you the syrup you desire.

2 dly, Syrups made by decoction are usually made of compounds, yet may any simple herb be thus converted into syrup: 'Take the herb, root, or flowers you would make into a syrup, and bruise it a little; then boil it in a convenient quantity of spring water; the more water you boil it in, the weaker it will be; a handful of the herb or root is a convenient quantity for a pint of water, boil it till half the water be consumed, then let it stand till it be almost cold, and strain it through a woollen cloth, $3 \mathrm{G}$ 
letting it run out at leisure : without press- want help, or such as are in health, and want ing. lo every pint of this decoction add no money to quench thirst.

one pound of sugar, and boil it over the fire till it come to a syrup, which you may know, if you now and then cool a little of it with a spoon: Scum it all the while it boils, and when it is sufficiently boiled, whilst it is hot, strain it again through a woollen cloth, but press it not. Thus you have the syrup perfected.

$3 \mathrm{dlly}$, Syrups made of juice, are usually made of such herbs as are full of juice, and indeed they are better made into a syrup this way than any other; the operation is thus: Having beaten the herb in a stone mortar, with a wooden pestle, press out the juice, and clarify it, as you are taught before in the juices; then let the juice boil away till about a quarter of it be consumed; to a pint of this add a pound of sugar, and when it is boiled, strain it throughta woollen cloth, as we taught you before, and keep it for your use.

3. If you make a syrup of roots that are any thing hard, as parsley, fennel, and grass roots, \&c. when you have bruised them, lay them in steep some time in that water which you intend to boil them in hot, so will the virtue the better come out.

4. Keep your syrups either in glasses or stone pots, and stop them not with cork nor bladder, unless you would have the glass break, and the syrup lost, only bind paper about the mouth.

5. All syrups, if well made, continue a year with some arivantige; yet such as are made by infusion, keep shortest.

C II A P T E R I I I.

Of Juleps.

1. JULEPs were first invented, as I suppose, in Arabia ; and my reason is, because the word Julep is an Arabic word.

2. It signifies only a pleasant potion, as is vulgarly used by such as are sick, and

3. Now-a-day it is commonly used-

1. To prepare the body for purgation.

2. To open obstructions and the pores.

3. 'To digest tough humours.

4. 'To qualify hot distempers, \&c.

4. Simple Juleps, (for I have nothing to say to compounds here) are thus made; Take a pint of such distilled water, as conduces to the curc of your distemper, which this treatise will plentifully furnish you with, to which add two ounces of syrup, conducing to the same effect; (I shall give you rules for it in the next chapter) mix them together, and drink a draught of it at your pleasure. If you love tart things, add ten drops of oil of vitriol to your pint, and shake it together, and it will have a fine grateful taste.

5. All juleps are made for present use; and therefore it is in vain to speak of their duration.

\section{H A P T E R I V.}

\section{Of Decoctions.}

1. AlL the difference between decoctions, and syrups made by decoction, is this; Syrups are made to keep, decoctions only for present use; for you can hardly keep a decoction a week at any time; if the weather be hot, not half so long.

2. Decoctions are made of leaves, roots, flowers, seeds, fruits or barks, conducing to the cure of the disease you make them for ; are made in the same nanner as we sherved you in syrups.

3. Decoctions made with wine last longer than such as are made with water; and if you take your decoction to cleanse the passages of the urine, or open obstructions, your best way is to make it with white wine instead of water, because this is penetrating.

4. Decoctions are of most use in such 
diseases as lie in the passages of the body, fruits or seeds by expression, as oil of sweet as the stomach, bowels, kidneys, passages and bitter almonds, linseed and rape-seed of urine and bladder, because decoctions oil, \&c. of which see in my Dispensatory. pass quicker to those places than any other form of medicines.

5. If you will sweeten your decoction with sugar, or any syrup fit for the occasion you take it for, which is better, you may, and no harm.

6 . If in a decoction, you boil both roots, herbs, flowers, and seed together, let the roots boil a good while first, because they retain their virtue longest ; then the next in order by the same rule, viz. 1. Barks. 2. The herbs. 3. 'The seeds. 4. The flowers. 5 . The spices, if you put any in, because their virtues come soonest out.

7. Such things as by boiling cause slininess to a decoction, as figs, quinceseed, linseed, \&c. your best way is, after you have bruised them, to tie them up in a linen rag, as you tie up calf's brains, and so boil them.

8. Keep all decoctions in a glass close stopped, and in the cooler place you keep them, the longer they will last ere they be sour.

Lastly, The usual dose to be given at one time, is usually two, three, four, or five ounces, according to the age and strength of the patient, the season of the year, the strength of the medicine, and the quality of the disease.

$$
\begin{gathered}
\text { C H A P T E R V. } \\
\text { Of Oils. }
\end{gathered}
$$

1. Oxu Olive, which is commonly known by the name of Sallad Oil, I suppose, because it is usually eaten with sallads by them that love it, if it be pressed out of ripe olives, according to Galen, is temperate, and exceeds in no one quality.

2. Of oils, some are simple, and some are compound.

3. Simple oils, are such as are made of

4. Compound oils, are made of oil of olives, and other simples, imagine herbs, flowers, roots; \&c.

5. The way of making them is this: Having bruised the herbs or flowers you would make your oil of, put them into an earthen pot, and to two or three handfuls of them pour a pint of oil, cover the pot with a paper, set it in the sun about a fortnight or so, according as the sun is in hotness; then having warmed it very well by the fire, press out the herb, \&c. very hard in a press, and add as many more herbs to the same oil; bruise the herbs (I mean not the oil) in like manner, set them in the sun as before; the oftener you repeat this, the stronger your oil will be; At last when you conceive it strong enough, boil both herbs and oil together, till the juice be consumed, which you may know by its bubbling, and the herbs will be crisp; then strain it while it is hot, and keep it in a stone or glass vessel for your use.

6. As for chymical oils, I have nothing to say here.

7. The general use of these oils, is for pains in the limbs, roughness of the skin, the itch, \&c. as also for ointments and plaisters.

8. If you have occasion to use it for wounds or ulcers, in two ounces of oil, dissolve half an ounce of turpentine, the heat of the fire will quickly do it; for oil itself is offensive to wounds, and the turpentine qualifies it.

$$
\text { C II } \Lambda \mathrm{P} T \mathrm{TE} \text { VI. }
$$

\section{Of Electuaries.}

Puysicinns make more a quoil than needs by lialf, about clectuaries. I shall prescribe but one general way of making them up; as for ingredients, you may vary 
them as you please, and as you find occa-? sion, by the last chapter.

1. That you may make electuaries when herbs, as of scurvy-grass, wormwood, rue, you need them, it is requisite that you keep and the like, take only the leaves and tenalways herbs, roots, flowers, seeds, \&c. der tops (for you may beat your heart out ready dried in your house, that so you may before you can beat the stalks small) and be in a readiness to beat them into powder when you need them.

2. It is better to keep them whole than beaten; for being beaten, they are more subject to lose their strength; because the air soon penetrates them.

3. If they be not dry enough to beat into powder wlien you need them, dry them by a gentle fire till they are so.

4. Having beaten them, sift them through a fine tiffany searce, that no great pieces may be found in you electuary.

5 . To one ounce of your powder add three ounces of clarified honey ; this quantity I hold to be sufficient. If you would make more or less electuary, vary your proportion accordingly.

6. Mix them well together in a mortar, and take this for a truth, you cannot mix them too much.

7. The way to clarify honey, is to set it over the fire in a convenient vessel, till the scum rise, and when the scum is taken off, it is clarified.

8. The usual dose of cordial electuaries, is from half a dram to two drams; of purging electuaries, from half an ounce to an ounce.

9. The manner of keeping them is in a pot.

10. The time of taking them, is either in a morning fasting, and fasting an hour after them ; or at night going to bed, three or four hours after supper.

$$
\begin{gathered}
\text { CH A P T E R V́ I. } \\
\text { Of Conserves. }
\end{gathered}
$$

1. 'The way of making conserves is twofold, one of herbs and flowers, and the other of fruits.
2. Conserves of herbs and flowers, are thus made: if you make your conserves of having beaten them, weigh them, and to every pound of them add three pounds of sugar, you cannot beat them too much.

3. Conserves of fruits, as of barberries, sloes and the like, is thus made: First, Scald the fruit, then rub the pulp through a thick hair sieve made for the purpose, called a pulping sieve; you may do it for a need with the back of a spoon: then take this pulp thus drawn, and add to it its weight of sugar, and no more ; put it into a pewter vessel, and over a charcoal fire; stir it up and down till the sugar be melted, and your conserve is made.

4. Thus you have the way of making conserves; the way of keeping them is in earthen pots.

5. The dose is usually the quantity of a nutmeg at a time morning and evening, or (unless they are purging) when you please.

6. Of conserves, some keep many years, as conserves of roses: other but a year, as conserves of Borage, Bugloss, Corwslips and the like.

7. Have a care of the working of some conserves presently after they are made; look to them once a day, and stir them about: conserves of Borage, Bugluss, Wormwood, have got an excullent faculty at that sport.

8. You may know when your conserves are almost spoiled by this; you shall find a hard crust at top with little holes in it, as though worms had been eating there.

$$
\text { C.H A P TE R VIII. }
$$

$$
\text { Of Preserves. }
$$

OF Preserves are sundry sorts, and the 
operation of all being somewhat different, you cut it into it, and let it remain until we will handle them all apart. These are you have occasion to use it.

preserved with sugar;
1. Flowers.
3. Roots.
2. Fruits.

3. Roots are thus preserved; First, Scrape them very clean, and cleanse them from the pith, if they have any, for some roots have 1. Flowers are very seldom preserved; not, as Eringo and the like; Boil them in I never saw any that I remember, save water till they be soft, as we shewed you only cowslip flowers, and that was a great before in the fruits; then boil the water you fashion in Sussex when I was a boy. It is boiled the root in into a syrup, as we shewed thus done, Take a flat glass, we call them you before; then keep the root whole in the jat glasses; strew on a laying of fine sugar, syrup till you use them.

on that a laying of flowers, and on that another laying of sugar, on that another laying of flowers, so do till your glass be few that I can remember, are, oranges, full ; then tie it over with a paper, and in lemons, citrons, and the outer bark of wala little time, you shall have very excellent nuts, which grow without side the shell, and pleasant preserves.

There is another way of preserving scurvy preserves; these be they I can reflowers; namely, with vinegar and salt, member, if there be any more put them as they pickle capers and broom-buds; but into the number.

as I have little skill in it myself, I cannot teach you.

2 Fruits, as quinces, and the like, are preserved two ways ;

(1.) Boil them well in water, and then pulp them through a sieve, as we shewed mot this way and my reason is this; Because you before; then with the like quantity of I doubt when their bitterness is gone, so is sugar, boil the water they were boiled in their virtue also; I shall then prescribe one into a syrup, viz. a pound of sugar to a pint common way, namely, the same with the of liquor; to every pound of this syrup, former, viz. First, boil them whole till they add four ounces of the pulp ; then boil it be soft, then make a syrup with sugar and with a very gentle fire to their right con-s the liquor you boil them in, and keep the sistence, which you may easily know if you barks in the syrup.

drop a drop of it upon a trencher; if it be enough, it will not stick to your fingers when it is cold.

(2.) Another way to preserve fruits is this; First, Pare off the rind; then cut them in halves, and take out the core: then boil them in water till they are soft; if you know when beef is boiled enough, you may easily know when they are; Then boil the water with its; like weight of sugar into a syrup; put the syrup into a pot, and put the boiled fruit as whole as you left it when

$(21,22$.
5. They are kept in glasses or in glaz'd pots.

6. The preserved flowers will keep a year, if you can forbear eating of them; the roots and barks much longer.

7. This art was plainly and first invented for delicacy, yet came afterwards to be of excellent use in physic; For,

(1.) Hereby medicines are made pleasant for sick and squeamish stomachs, which else would loath them.

(2.) Hereby they are preserved from de: caying a long time.

$3 \mathrm{H}$ 
C H A P T E R I X.

\section{Of Lohocks.}

1: That which the Arabians call Lohocks, and the Greeks Eclegma, the Latins call Linctus, and in plain English signifies nothing else but a thing to be licked up.

2. They are in body thicker than syrup, and not so thick as an electuary.

3. The manner of taking them is, often to take a little with a liquorice stick, and let it go down at leisure.

4. They are casily thus made; Make a decoction of pectoral herbs, and the treatise will furnish you with enough, and when you have strained it, with twice its weight of honey or sugar, boil it to a lohock; if you are molested with much phlegm, honey is better than sugar; and if you add a little vinegar to it, you will do well ; if not, I hold sugar to be better than honey.

5. It is kept in pots, and may be kept a year and longer.

6. It is excellent for roughness of the ? wind-pipe, inflammations and ulcers of the lungs, difficulty of breathing, asthmas,coughs, and distillation of humours.

\section{I A A P T E R $R$ X.}

\section{Of Ointments.}

7. VARIous are the ways of making ointments, which authors have left to posterity, which I shall omit, and quote one which is easiest to be made, and therefore most beneficial to people that are ignorant in physic, for whose sake I write this. thus done.

Bruise those herbs, flowers, or roots, you will make an ointment of, and to two handfuls of your bruised herbs add a pound of hog's grease dried, or cleansed from the skins, beat them very well together in a stone mortar with a wooden pestle, then put roots, herbs,
it into a stone pot, (the herb and grease I rosin, gums. mean, not the mortar,) cover it with a paper and set it either in the sun, or some other warm place; three, four, or five days, that it may melt ; then take it out and boil it a little; then whilst it is hot, strain it out, pressing it out very hard in a press : to this grease add as many more herbs bruised as before; let them stand in like manner as long, then boil them as you did the former; If you think your ointment is not strong enough, you may do it the third and fourth time: yet this I will tell you, the fuller of juice the herbs are, the snoner will your ointment be strong; the last time you boil it, boil it so long till your herbs be crisp, and the juice consumed, then strain it pressing it hard in a press, and to every pound of ointment add two ounces of turpentine, and as much wax, because grease is offensive to wounds, as well as oil.

2. Ointments are vulgarly known to be kept in pots, and will last above a year, some above two years.

C II A P T E R X I.

\section{Of Plaisters.}

1. Tin E Greeks made their plaisters of divers simples, and put metals into the most of them, if not all ; for having reduced their metals into powder, they mixed them with that fatty substance whereof the rest of the plaister consisted, whilst it was thus hot, continually stirring it up and down, lest it should sink to the bottom; so they continually stirred it till it was stiff; then they made it up in rolls, which when they needed for use, they could melt by the fire again.

2. The Arabians made up theirs with oil and fat, which needed not so long boiling.

3. 'The Greeks emplaisters consisted of these ingredients, metals, stones, divers sorts of earth, feces, juices, liquors, seeds, roots, herbs, excrements of creatures, wax, 
C II A P T E R XII.

\section{Of Poultices.}

1. Poultices are those kind of things which the Latins call Cataplasmata, and our learned fellows, that if they can read English, that's all, call them Cataplasms, because 'tis a crabbed word few understand; it is indeed a very fine kind of medicine to ripen sores.

2 , They are made of herbs and roots, fitted for the disease, and menbers afflicted, being chopped small, and boiled in water almost to a jelly; then by adding a little barleymeal, or nieal of lupins, and a little oil, or rough sweet suet, which I hold to be better, spread upon a cloth and apply to the grieved places.

3. Their use is to ease pain, to break sores, to cool inflammations, to dissolve hardness, to ease the spileen, to concoct humours, and dissipate swellings.

4. I beseech you take this caution along with you; Use no poultices (if you can help it) that are of an healing nature, before you have first cleansed the body, because they are subject to draw the humours to them from every part of the body.

C II A P TER XIII.

\section{Of Troches.}

1. Trie Latins call them Placentula, or little cakes, and the Greeks Prochiliois, Kukliscoi, and Artiscoi; they are usually little ruund that cakes, or you may make them square if you will.

2. Their first invention was, that powders grounded upon reason too, not upon fancy, being so kept might resist the intermission or hearsay. The first invention of pills was of air, and so endure pure the longer.

3. Besides, they are easier carried in the before, such infirmities as lie near the paspockets of such as travel; as many a man sages were best removed by decoctions, (for examplc) is forced to travel whose because they pass to the grieved part stomach is too cold, or at least not so hot as soonest; so here, if the infirmity lies in the it should be, which is most proper, for the head, or any other remote part, the best way in such $\boldsymbol{u}$ case, it is better to carry troches of wormwood, or galangal, in a paper in his pocket, than to lay a gallipot along with him.

4. 'They are made thus; At night when you go to bed, take two drams of fine gum tragacanth ; put it into a gallipot, and put half a quarter of a pint of any distilled water fitting for the purpose you would make your troches for to cover it, and the next moruing you shall find it in such a jelly as the physicians call mucilage; With this you may (with a little pains taken) make a powder into a paste, and that paste into cakes called troches.

5. Having made them, dry them in the shade, and keep them in a pot.for your use.

\section{II A P T E R X I V.}

of Pills.

1. 'Tн е y are called Pilula, because they resemble little balls; the Greeks call them Catapotia.

2. It is the opinion of modern physicians, that this way of making medicines, was invented only to deceive the palate, that so by swallowing them down whole, the bitterness of the medicine might not be perceived, or at least it might not be unsufferable: and indeed most of their pills, though not all, are very bitter.

3. I am of a clean contrary opinion to this. I rather think they were done up in this hard form, that so they might be the longer in digesting; and my opinion is to purge the head, now, as I told you

stomach is never cold till a man be dead; 
is to use pills, because they are longer in| digestion, and therefore the better able to call the offending humour to them.

4. If I should tell you here a long tale of medicine working by sympathy and antipathy, you would not understand a word of it: They that are set to make physicians may find it in the treatisc. All modern physicians know not what belongs to a sympathetical cure, no more than a cuckow what belongs to flats and sliarps in music, but follow the vulgar road, and call it a hidden quality, because tis hidden from the eyes of dunces, and indeed none but astrologers can give a reason for it; and physic without reason is like a pudding without fat.

5. The way to make pills is very easy, for with the help of a pestle and mortar, and a little diligence, you may make any powder into pills, either with syrup, or the jelly I told you before.

$$
\text { C H A P T E R X V : }
$$

The way of miaing Medicines according to the Cause of the Disease, and Parts of the Body afflicted.

THIs being indeed the key of the work, I shall be somewhat the more diligent in it. I shall deliver myself thus ;

1. To the Vulgar.

2. To such as study Astrology; or such as study physic astrologically.

1st, To the Vulgar. Kind souls, I am sorry it hath been your hard mishap to have been so long trained in such Egyptian darkness, which to your sorrow may be felt; The vulgar road of physic is not my prac-
tice, and $I$ am therefore the more unfit to give you advice. I have now published a little book, (Galen's Art of Physic,) which will fully instruct you, not only in the knowledge of your own bodies, but also in fit medicines to remedy each part of it when afflicted; in the mean season take these few rules to stay your stomachs.
1. With the disease, rogard the cause, and the part of the body afflicted; for example, suppose a woman be subject to miscarry, through wind, thus do ;

(1.) Look Abortion in the table of diseases, and you shall be directed by that, how many herbs prevent miscarriage.

(2.) Look Wind in the same table, and you shall see how many of these herbs expel wind.

These are the herbs medicinal for your grief.

2. In all diseases strengthen the part of the body afflicted.

3. In mix'd diseases there lies some difficulty, for sometimes two parts of the body are afflicted with contrary humour's, as sometimes the liver is afflicted with choler and water, as when a man hath both the dropsy and the yellow-jaundice; and this is usually mortal.

In the former, Suppose the brain be too cool and moist, and the liver be too hot and dry ; thus do ;

1. Keep your head outwardly warm.

2. Accustom yourself to the smell of hot herbs.

3. Take a pill'that heats the head at night going to bed.

4. In the morning take a decoction that cools the liver, for that quickly passes the stomach, and is at the liver immediately.

You must not think, courteous people, that I can spend time to give you examples of all diseases; These are enough to let you see so much light as you without art are able to receive; If I should set you to look at the sun, I should dazzle your eyes, and make you blind.

2dly, T'o such as study Astrology, (who are the only men I know that are fit to study physic, physic without astrology being. like a lamp without oil : you are the men I exceedingly respect, and sucl documents. as my brain can give you at present (being absent from my study) I shall give you. 
1. Fortify the body with herbs of the 5 . If this cannot well be, make use of the nature of the Lord the Ascendant, 'tis no: medicines of the Light of 'Time.

matter whether he be a Fortune or Infortune! 6 . Be sure always to fortify the grieved in this case.

2. Let your medicine be something antipathetical to the lord of the sixlh. s part of the body by sympathetical remedies.

7. Regard the heart, keep that upon the wheels, because the Sun is the foumdation of

3. Let your medicine be something of life, and therefore those universal remedic's, the nature of the sign ascending. Alirum Potabile, and the Plilosopher's

4. If the Lord of the 'l'enth be strong, Stone, cure all diseases by fortifying the make use of his medicines. heart.

\section{ENGIISH PHYSICIAN}

\section{FAMILY DIPENSATORY.}

\section{AN ASTROLOGO-PHYSICAL DISCOURSE OF THE HUMAN VIRTUES IN 'THE BODY OF MAN; BO'IH PRINCIPAL ANU ADMINIS'TERING.}

HunAN virtues are cither PRINCIPAL The scope of this discourse is, To prefor procreation, and conservation; or $A D-$ serve in soundness and vigour, the mind Ministring, for Attraction, Digestion, and underslanding of man; to strengthen Retention, or Expulsion.

Virtues conservative, are Vital, Natural, and Animal.

By the natural are bred Blood, Choler, Flegm, and Melancholy.

The animal virtue is Intellective, and Sensitive.

'T'he Intellective is Imagination, Judgment, and Memory.

The sensitice is Common, and Particular.

The particular is Secing, Hearing, Suelling, Tasting, and Feeling.

$(21,22$. the brain, preserve the body in healih, to teach a man to be an able co-artificer, or lielper of nature, to withstand and expel Diseases.

I shall touch only the principal faculties both of body and mind; which being kept in a due decorum, preserve the body in health, and the mind in vigour.

I shall in this place speak of them only in the general, as they are laid down to your view in the Synopsis, in the former pagres, and in the same order.

Virtue Procreative.] The first in order, is $3 \mathrm{r}$ 
the Virtue Procreative ; for natural regards nol only the conservation of itself, but to beget its like, and conserve in Species.

"The seat of this is the Member' of Generation, and is governed principally by the influence of Venus.

It is augmented and encreased by the strength of Venus, by her Herbs, Roots, Trees, Minerals, \&c.

It is diminished and purged by those of Mars, and quite extinguished by those of Saturn.

Observe the hour and Medicines of Venus, to fortify; of Mars, to cleanse this virtue; of Saturn, to extinguish it.

Consertative.] 'The conservative virtue is Vital, Natural, Animal.

Vital.] The Vital spirit hath its residence in the heart, and is dispersed from it by the Arteries; and is governed by the intluence of the Sun. And it is to the body, as the Sun is to the Creation; as the lieart is in the Microcosm, so is the Sun in the Megacosm: for as the Sun gives life, light, and motion to the Creation, so doth the heart to the body; therefore it is called Sol Corporis, as the Sun is called Cor Coeli, because their operations are similar.

Inimical and destructive to this virtue, are Saturn and Mars.

'The Hcrbs and Plants of Sol, wonderfully fortify it.

Natural.] The natural faculty or virtue resicles in the liver, and is generally governed by Jupiter, Quasi Juvans Pater; its office is to nourish the body, and is dispersed through the body by the veins.

From this are bred four particular humours, Blood, Choler, Flegm, and Melancholy.

Blood is made of meat perfectly concocted, in quality hot and moist, governed by Jlipiter: It is by a third concoction transmuted into flesh, the superfluity of it into seed, and its receptacle is the veins, by which it is dispersed through the body.
Choler is made of meat more than perfectly concocted; and it is the spume or frotl of blood : it clarifies all the humours, heats the body, nourishes the apprehension, as blood dotlı the judgment: It is in quality hot and dry; fortifies the attractive faculty, as blood doth the digestive; moves man to activity and valour: its receptacle is the gall, and it is under the influence of Mars. Flegm is made of meat not perfectly digested; it fortifies the virtue expulsive, makes the body slippery, fit for ejection; it fortifies the brain by its consimilitude with it; yet it spoils apprelicnsion by its antipathy to it: It qualifies choler, cools and moistens the heart, thereby sustaining it, and the whole body, from the fiery effects, which continual motion, would produce. Its receptacle is the lungs, and is governed by Vemus, some say by the Moon, perliaps it may be governed by them both, it is cold and moist in quality.

Melancholy is the sediment of blood, cold and dry in quality, fortifying the retentive faculty, and memory; makes men sober, solid, and staid, fit for study ; stays the unbridled toys of lustful blood, slays the wandering thoughts, and reduces them home to the centre: its receptacle is in the spleen, and it is governed by Saturn.

Of all these humours blood is the chief, all the rest are superthuities of blood; yet are they nccessary superfluities, for without any of them, man cannot live.

Namely; Choler is the fiery superfluities; Flegm, the Watery; Melancholy, the Earthly.

Animal.] The third principal virtue remains, which is Animal; its residence is in the brain, and Morcury is the general significator of it. Ptolomiy held the Moon signified the Animal virtue; and I am of opinion, both Mercury and the Moon dispose it; and my reason is, 1 , Because both of them in nativities, either fortify, or impedite it. 2, Ill directions to either, or from either, 
afflict it, as gond onc's help it. Indeed the So that it is one of the surest rulcs to Moon rules the bulk of it, as also the sensi- know a man's own complexion, by his tive part of it : Mercury the rational part: dreams, I mean a nan roid of distractions, and that's the reason, if in a nativity the or deep studies: (this most assuredly shows' Moon be stronger thin MLercury, sense many Mercury to dispose of the Imagination, as times over-powers reason; but if Mercury also because it is mulable, applying itself to be strong, and the Moon weak, reason will any object, as Mercury's nature is to do;) for be master ordinarily in despite of sense. then the imagination will follow its old bent; It is divided into Intellective, and Sen- tor if a man be bent upon a business, his sitive.

apprehension will work as mucl, when he is

1. Intellective.] The Intellectual resides asleep, and find out as many truth by study, in the brain, within the Pia mater, is govern- as when the man is awake; and perhaps ed gencrally by Mercury.

more too, because then it is not hindered

It is divided into Imagination, Judgment, hy ocular objects.

and Memory.

And thus much for imagination, which is

Imagination is seated in the forepart of governed by Mercury, and fortified by his the brain; it is hot and dry in quality, influence; and is also strong or weak in quick, active, always working; it receives man, according as Mercury is strong or vapours firm the heart, and coins them into weak in the nativity.

thoughts: it never sleeps, but always is Judgment is seated in the midst of the working, both when the man is sleeping and brain, to shew that it ought to bear rule over waking; only when Judgment is awake it all the other faculties: it is the judge of the regulates the Imagination, which runs at little world, to approve of what is good, random when Judgment is asleep, and forms and reject what is bad; it is the seat of any thought according to the nature of the reason, and the guide of actions; so that all vapour sent up to it. Mercury is out of failings are committed through its infirmity, question the disposer of it.

A man may easily perceive his Judgment asleep before himself many times, and then he shall perceive his thoughts run at Jupiter. random.

Judgment always sleeps when men do, Imagination nerer sleeps; Memory sometimes sleeps when men sleep, and sometimes it doth not: so then when memory is awake, and the man aslecip, then memory remembers what apprehension coins, and that is a

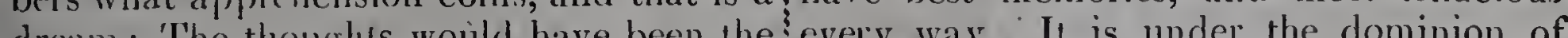
same, if memory had not been awake to remember it.

'These thoughts are commonly (I mean in sleep, when they are purely nal ural, framed according to the nature of the humour, called complexion, which is predominate in the body; and if the humour be peccant it is always so.

Memory is seated in the hinder cell of the brain, it is the great register to the little world ; and its office is to record things either done and past, or to be done.

It is in quality cold and dry, melancholic, and therefore generally melancholic men have best memories, and most tenacious Suturn, and is fortified by his influence, but purged by the luminaries.

2. Sensitive.] 'The second part of the animal virtue, is sensitive, and it is dirided into two parts, eommon and particular.

Common seuse is an imaginary term, and that which gives virtue to all the particular senses, and knits and unites them 
together within the Pia Mater. - It is regu- It is under the dominion of Vemus, some say, lated by Mercury, (perhaps this is one rea-Mercury: A thousand to one, but it is son why men are su fickle-headed) and its under Mercury.

office is to preserve a harmony among the senses.

Particular senses are five, viz. seeing, hearing, smelling, tasting, and feeling.

These senses are united in one, in the brain, by the common sense, but are operatively distinguished into their several seats, and places of residence.

The sight resides in the eyes, and particularly in the christaline humour. It is in quality cold and moist, and governed by the luminaries. They who have them weak in their genesis, have always wcak sights; if one of them be so, the weakness possesses but one eye.

The hearing resides in the ears; is in quality, cold and dry, melancholy, and under the dominion of Saturn.

The smelling resides in the nose, is in quality hot and dry, choleric, and that is the reason choleric creatures have so good smells, as dogs. It is under the influence of Mars.

The taste resides in the palate, which is placed at the root of the tongue on purpose to discern what food is congruous for the stomach, and what not; as the meseraik veins are placed to discern what nourishment is proper for the liver to convert into blood. In some very few men, and but a few, and in those few, but in few instances these two tasters agree not, and that is the reason some inen covet meats that make them sick, viz. the taste craves them, and the meseraik vcins reject them: In quality hot and moist, and is ruled by Jupiter.

The four ADMINISTERING VIRTUES are, attractive, digestive, retentive, and expulsive.

The attractive virtue is hot and dry, hot by quality, active, or principal, and that appears because the fountain of all heat is attractive, viz. the sun. Dry by a quality passive, or an effect of its heat; its office is to remain in the body, and call for what nature wants.

It is under the influence of the Sum, say authors, and not under Mars, because he is of a corrupting nature, yet if we cast an impartial eye upon experience, we shall find, that martial men call for meat none of the least, and for drink the most of all other men, although many times they corrupt the body by it, and therefore I see no reason why Mars being of the same quality with the Sun, should not liave a share in the dominion. It is in vain to object, that the influence of Mars is evil, and therefore he should have no dominion over this virtue; for then,

1. By the same rule, he should have no dominion at all in the body of inan.

2. All the virtues in man are naturally evil, and corrupted by Adam's fall.

'This attractive virtue ought to be fortified when the Moon is in fiery signs, viz. Aries and Sagitary, but not in Leo, for the sign is so violent, that no physic ought to be-given when the Moon is there: (and why not Leo, seeing that is the most attractive sign of all; and that's the reason such as have it ascending in their genesis, are The feeling is deputed to no particular such greedy caters.) If you connot stay till organ, but is spread abroad, over the whole the Moon be in one of then, let one of them body ; is of all qualities, hol, cold, dry, and ascend when you administer the medicine. moist, and is the index of all tangible The digestive virtue is lot and moist, and things; for if it were only hot alone, it is the principal of them all, the other like could not feel a quality contrary, viz. cold, handmaids attend it. and this might be spoken of other qualities.

The attractive virtue draws that which it 
should digest, and serves continually to feed Although $I$ did what I could throughout
and supply it. The retentive virtue, retains the substance langnage as might be understood by all, and with it, till it be perfectly digested.

therefore avoided terms of art as imuch as The expulsive virtuc casteth out, expels might be, Yet, 1. Some rcords of necessity fall what is supertluous by digestion. It is in which need explanation. 2. It romild be under the intluence of Jupiter, and fortified very tedions at the end of every reccipt to repeat by his herbs and plants, \& c. In fortifying over and over again, the zay of administration it, let your Moon be in Gemini, Aquary, or of the receipt, or ordering your bodies after it, the first half of Libra, or if matters be come or to instruct you in the miature of medicines, to that extremity, that you cannot stay till \{ and indeed would do nothing else but stuff the that time, let one of them ascend, but both book fill of tautolog\%.

of then together would do better, always To answer to both these is my task at this provided that the Moon be not in the as- time.

cendent. I cannot believe the Moon afflicts To the first: The words which need exthe ascendent so much as they talk of, if plaining, such as are obvious to my eye, are she be well dignified, and in a sign she these that follawe.

delights in.

1. To distil in Balno Mariæ, is the usual

'I'he retentive virtue is in quality cold and way of distilling in water. It is no more than $\mathrm{dry}$; cold, because the nature of cold is to to place your glass body which holds the matter compress, witness the ice; dry, because the to be distilled in a covenient vessel of water, nature of dryness, is to keep and hold what when the water is cold (for fear of breaking) is compressed. It is under the influcnce of put a wisp of straw, or the like under is, to keep Saturn, and that is the reason why usually it from the bottom, then make the water loil, Saturnine men are so coretous and tenaci- that so the spirit may be distilled forth; take ous. In fortifying of it, make use of the not the glass out till the reater be cold again, heris and plants, \&c. of Saturn, and let the for fear of breaking: It is impossible for a Moon be in Tanrus or Virgo, Capricorn is not man to learn how to do it, unless he saw it so good, say authors, (I can give no reason done. for that neither;) let not Saturn nor his ill aspect molest the ascendent.

'The expulsive faculty is cold and moist; cold because that compasses the superfluitics; moist, because that makes the body slippery and fit for ejcction, and disposes it to it. It is under the dominion of Luna, with whom you may join Yerus, because she is of the same nature.

Also in whatsoever is before written, of the nature of the plancts, take notice, that fixed stars of the same nature, work the same effect.

In fortifying this, (which ought "to "be done in all purgations,) let the Moon be in Cancer, Scorpio, or Pisces, or let one of these signı ascend.

$(21,22$.
2. Manica Hippocrates. Hippocrates's sleeve, is a piece of woolen cloth, new and white, sewed together in form of a sugar-loaf. Its use is, to strain any syrup or decoction through, by pouring it into it, and suffering it to run through without pressing or crushing it.

3. Calcination, is a burning of a thing in a crucible or other such convenient ressel that will endure the fire. A crucible is such a thing as goldsmiths melt silver in, and founders metals; you may place it in the midst of the fire, with coals above, below, and on every side of it.

4. Filtrition, is straining of a liquid body through a brown paper: makc up the paper in form of a funnel, the which having placed $3 \mathrm{~K}$ 
in a funnel, and the fumel and the paper? so medicines might be more delightful, or at in it in an empty glass, pour.in the liquor? last less burdensome. You may make the you would filter, and let it run through at its mixtures of them in what form you please; leisure. only for your better instruction at present,

5. Coagulation, is curdling or hardening: accept of these few lines. it is used in physic for reducing a ligund body to hardness by the heat of the fire.

6. Whereas you find vital, natural, and animal spirits often montioned in the virtues or receipts, I shall explain what they be, cine appropriated to it; if wind, sce how and what their operation. is in the body of manymcelicines appropriated to that disease man.

The actions or operations of the animal virtues, are, 1. sensitive, 2. motize.

The sensitive is, 1. enternal, 2. internal.

The external senses are, 1, seeing, 2. hearing, 3. tasting, 4. smelling, 5. feeling.

The internal senses are, 1. the Imagination, to apprehend a thing. 2. Judgment, to judge of it. 3. Memory, to remember it.

The seat of all these is in the brain.

The vital spirits proceed from the heart, and cause in man mirth, joy, hope, trust, humanity, mildness, courage, \&c. and their opposite: viz. sadness, fear, care, sorrow, despair, envy, hatred, stubbommess, revenge, fc. by heat natural or not natural.

'I'he natural spirit nourishes the body throughout (as the vital quickens it, and the animal gives it sense and motion) its office is to alter or concoct food into chile, chile into blood, blood into flesh, to form, engender, nourish, and increase the body.

7. Infusion, is to steep a gross body into one more liquid.

8. Decoction, is the liquor in which any thing is boiled.

As for the manner of using or ordering the body after any s'veating, or purging medicines, or pills, or the like, they will be
found in different parts of the work, as also in the next page.

The different forms of making up medicines, as some into syrups, other's into electuaries, pills, troches, \&c. Was partly to please the different palates of people, that? expel wind, and use them.

2. Have a care you use not such medicines to one part of your body which are appropriated to another, for if your brain be orer heated, and you use such medicines as conl the heart or liver, you may make bad work.

3. The distilled water of any herb you would take for a disease, is a fit mixture for the syrup of the same herb, or to make any electuary into a drink, if you affect such liquid medicines best; if you hare not the distilled water, make use of the decoction.

4. Diseases that lie in the parts of the body remote from the stomach and bowels, it is in vain to think to carry away the cause at once, and therefore you had best lo it by degrees; pills, and such like medicines which are hard in the body, are fittest for such a business, because they are longest before they digest.

5. Use no strong medicines, if weak will serve the turn, you had better take one too weak by half, than too strong in the least.

6. Consider the natural temper of the part of the body afflicted, and maintain it in that, clse you extinguish nature, as the heart is hot, the brain cold, or at least the coldest part of the body.

7. Observe this general rule; 'That such medicines as are hot in the first degree are most habitual to our bodic's, becaluse they are just of the heat of our blood.

8. All opening medicines, and such as 
provoke urine or the menses, or break the electuaries, as also all pills that have neither stone, may most conveniently be given in Diagrydium nor Colocynthus, in them. But white wine, hecause white wine of itself is all violent purges require a due ordering of of an opening nature, and cleanses the the body; such ought to be taken in the reins.

morning after you are up, and not to sleep

9. Let all such medicines as are taken after them before they are done working, at to stop fluxes or looseness, be taken before leant before night: two hours after you have meat, alsout an hour before, more or less, taken them, drink a draught of warm possetthat so thry may strengthen the digestion and retentive fatilty, before the food come into the stomach, but such as are subject to vomit.11) their meat, let them take such medicines as stay vomiting presently after meat, at the conclusion of their meals, that so they may close up the mouth of the stomach; and that is the reason why usually men eat a bit of cheese after meat, because by its sourness and binding it closes the month of the stomach, thereby staying belching and vomiting.

10. In taking purges be very careful, and that you may be so, observe these rules.

(1.) Consider what the liumour offending is, and let the medicine be such as purges that liumour, else you will weaken nature, not the disease.

(2.) 'Take notice, if the humour you would purge out be thin, then gentle medicines will serve the turn, but if it be tough and viscous, then such medicines as are cutting and opening, the night before you would take the purge.

(3.) In purging tough humours, forbear as much as may be such medicines as leave a binding quality behind them.

(4.) Have a care of taking purges when your body is astringent; your best way, is first to open it by a clyster.

(5.) In taking opening medicines, you may safely take them at night, eating hut or brankursine, it is meanly hot and dry,
a little supper three or four hours before, helps aches and mumness of the joints, and is and the next morning drinking a draught of of a binding quality, good for wounds and warm posset-drink, and you need not fear broken hones. Dioscorides saith, they are to go about your business. In this manner piofitable for ruptures, or such as are you mity take Lenitize Electury, Diacatho- bursten, or burnt with fire, a dram of the licon, Pulp of Cassia, and the like gentle root in powder being taken in the morning repel the vapours back.
I confess these, or many of these directions may be found in one place of the book or other, and I delight as little to write tautology as another, but considering it might make for the public good, I inserted them in this place: if, notwithstanding, any will be so mad as to do themselves a mischief, the fault is not mine.

\section{R O O' 'S.}

Acanths, Brance Ursince. Of bearsbreech, or brankursine, it is meanly hot and dry, helps aches and mumness of the joints, and is bit of mutton, often walking about the chamber; let there be a good fire in the chamber, and stir not out of the chamber next day.

you are in bed, covered warm, and in the time of your sweating drink posset-drink as
hot as you can. If you sweat for a fever, boil sorrel and red sage in your posset-drink, permit, then (the chamber being kept very warm) shift yourself all but your head, about which (the cap which you sweat in being still kept on) wrap a napkin very hot, to 
fasting, in a decection made with the sane root alld water.

Acriri, Veri, Perigrini, rulgaris, \&s. See Calamus Aromaicus. I shall wot speati concerving the sererit sorts of it, one of which is Walcr-thag, or Flower-dic-luce, which is hot and diy in the second degree, binds, strengthens, stops fluxes of the belly, and immolerate fowing of the mosses, a dram being latien in red wine every morning.

slikum. Liarlic. It is hot and dry in the fourth degree, breeds corrupt blood, yet is an encmy to all poisons, and such as ale bitt'n by cold venomous beasts, viz. Adders, Toads, Spiders, \&c. it provokes uine, and expels wind.

Alcanne. Of privet. See the leaves.

Althcee. Of Marsh mallows, are meanly hot, of a digesting, sofiening nature, ease pains, help bloudy fluxes, the stone, and gr.ıvel; being bruised and boiled in milk, and the milk drank, is a good remed y for gripings of the belly, and the bloody tlux. It a ferer accompriny the disease, boil a handful of common mallow leaves with a handful of these roots.

Angelice. Of Angolica ; is hot and dry in the third degree, strengthens the heart, and is good against pestilence and poison, half a dram taken in the morning fasting. Anchusce. Ot Alkantet; cold and diy, binding, good tor old ulcers.

Anthora. A foreign root, the counterpoison for Monkshood, it is an adnirable remedy for the wind cholic, and resists poisolli.

Apii. Of surallage. See the barks.

Aristolochice. Of birthwort ; of which are three sorts, long, romol, and climing: All hot and dry in the third degree. 'The long, being rlask in wine, brillgs away boll birth and after-birth, and whatsoever a careleis midwife hath lat' behind. Dioscorides, Galen. 'The round, being rlank with wine, helps (besiles the former) stuffings of the
lungs, hardness of the spleen, ruptures, con- vulsions; both of them resist poison. I never read any use of the climing birthwort.

Artanita, Cyclaminis, $\& c$. Or Sowbread ; hot and dry in the third degree, a niost violent purge, dangerous; outwardly applied to the place, it profits much in the bitings of venomous beasts, also being hung about women in labour, it causes speedy dieliverance. See the Herb.

Arundinis, Vallanorice, and Saccharino. Of common recds and sugar reeds. The roots of common reeds applied to the place draw out thorns, and ease sprains; the ashes of them mixed with vinegar, take scurf, or dandrif off from the head, and prevent the falling off of the hair, they are hot and dry in the second degree, according to Galen. I never read any virtue of the root of sugar cane.

Ari, \&.c. Of Cuckow-points, or WakeRobin, hot and dry in the third degree, I know no great good they do inwardly taken, unless to play the rogue withal, or make sporl: outwardly applicd, they take off scurf, morphew, or treckles fiom the face, clear the skiu, and ease the pains of the gout.

Asclepiadis, vincetoxici. Of Swallow-wort, hot and dry, good against poison, and gripings of the belly, as also against the bitings of mad dogs, taken inwardly.

Asari. Of Asarabacca: the roots are a safer purge than the leaves, and not so violent, they purge by vomit, stool, and urine; they are profitable for such as have agues, dropsies, stoppings of the liver, or spleen, green sichness.

Asparagi. Of Asparagus, or sperage: they are lcmperate in quality, opening, they provoke urine, and cleanse the reins and bladder, being boilcd in white wine, and the wine drank.

Asphodeli, Hastce Reige fam. Of Kings Spear, or Female Asphodel. I know no physical use of the roots; probably there 
is, for I do not believe God created any thing of no use.

Asphodeli, Albuci, muris. Of male Asphodel. Hot and dry in the second degree, black: they are both hot and dry, some inwardly taken, they provoke vomit, urine, say in the tihird degree, and some say but and the menses: outwardly used in oint- 3 in the first; they purge flegm and watery ments, they cause hair to grow, cleanse humours, but they trouble the stomach ulcers, and take away morphew and freckles much, they are very good for dropsies; from the face.

Bardano, \&c. Of Bur, Clot-bur, or Bur-" dock, temperately hot and dry. Helps
such as spit blood and matter; bruised and such as spit blood and matter; bruised and
mixed with salt and applied to the place, helps the bitings of mad dogs. It expels wind, "eases pains of the teeth, strengthens the back, helps the running of the reins,
and the whites, heing talken invardly. Dioscorides, Apuleius.

Behen. alb. $r u b$. Of Valerian, white and red. Mesue, Serapio, and other Arabians, name.

say they are hot and moist in the latter end of thie first, or beginning of the second degree, and confort the heart, stir up lust. strengthens the lungs, helps bruises, resists The Grecians held them to be dry in the poison, \&c. being taken inwardly in powsecond degree, that they stop fluxes, and der, the quantity of half a dram at a time. provolie urine.

Bellidis. Of Dasies. See the Leaves.

Bete, nigre, alloe, rubre. Of Beets, black, white, and red; as for black Beets and dry in the second degree, cutting and I have nothing to say, I doubt they are as cleansing: they provoke menses, help ralre as black swans. The red Bcet root malignant ulcers, ease the toothache, assuage boiled and preserved in vinegar, makes a sstrelling, and help the rickets. See Oil of fine, cool, pleasing, cleansing, digesting Cappers. sauce. See the leaves.

Bistorte, \&c. Of Bistort, or snakeweed, Bennet. 'The roots are dry, and something cold and dry in the third degree, binding : hot, of a cleansing quality, they keep garhalf a dram at a time taken inwardly, resists pestilence and poison, helps ruptures ? leaves.

and bruises, stays tluxes, voniting, and immoderate flowing of the menses, helps inflammations and soreness of the mouth, and fastens loose teeth, being bruised and boiled in white wine, and the mouth washed with it.
Borraginis. Of Borrage, hot and moist stortness of wind, coughs, convulsions, $3 \mathrm{~L}$

Caniium. Of Colewort. I know nothing the roots are good for, but only to bear the herbs and flowers.

Centrurii majoris. Of Centaury the Greater. 'The roots help such as are bursten, such as spitblood, shrinking of sinews, 
cramps: half a dram in powder be- it opens obstructions of the liver, being ing taken inwardly, either in muskadel, boiled in white wine, and the decoctions or in a decoction of the same rools. drank; and if chewed in the moutl it helps They are either not at all, or very scarce the tooth-ache. Celandine the lesser is in England, our centaury is the small cen- that which usually we call Pilewort, which tuary.

Cepæ. Of Onions. Are hot and dry of the root mixed with honey and snuffed (according to Galen) in the fourth degrec: up in the nose, purges the head, helps the they cause dryness, and are extremely hurt- hemorrboids or piles being bathed with it, ful for choleric people, they breed but little as also dotl the root only carried about one: nourishment, and that little is naught: they being made into an ointment, it helps the are bad meat, yet good physic for phleg- king's evil or Scrophula.

matic people, they are opening, and pruvoke urine and the menses, if cold be the cause obstructing: bruised and outwardly applied, they cure the bitings of mad dogs, roasted and applied, they help boils, and aposthumes: raw, they take the fire out of burnings, but ordinarily eaten, they cause headlache, spoil the sight, dull the senses, and fill the body full of wind.

Chameleontis albi nigri, \&c.

of Chame-

Chima, wonderfully extenuates and dries, provokes sweat, resists putrefaction; it strengthens the liver, helps the dropsy and malignant ulcers, lcprosy, itch, and venereal, and is profitable in diseases coming of fasting. It is commonly used in diet drinks for the premises.

Cichorii. Of Succory; cool and dry in the second degree, strengthens the liver and leon, leon, white and black. Tragus calls the liver and spleen, being boiled in white wine carline thistle by the name of white chame- and the decoction drank.

leon, the root whereof is hot in the second degree, and dry in the third, it provokes sweat, kills worms, resists pestilence and poison; it is given with success in pestilential fevers, helps the toothache by being chewed in the mouth, opens the stoppings of the liver and spleen, provokes urine, and the menses: give but little of it at a time, Colchici. Of Meadow Saffron. The roots are held to be hurtful to the stomach, therefore I let them alone.

Consolide, majoris, minoris. Consolida Major, is that which we ordinarily call Comfry, il is of a cold quality, yet pretty temperate, so glutinous, that, according to by reason of by reason of its heat. As for the black that is cut in sunder, if they he boiled with chameleon, all physicians hold it to have it; it is excellent for all wounds, both ina kind of venomous quality, and unfit to be ternal and external, for spitting of blood, used invardly, Galen, Clusius, Nicander, ruptures or burstness, pains in the back, it Dioscorides, and AEgineta. Outwardly in strengthens the reins, it siops the menses, ointments, it is profitable for scabs, mor- and helps hemorrhoids. The way to use pliew, tetters, \&c. and all things that need them is to boil them in water and driuk the cleansing.

Chelidonij majoris, minoris. Of celandine, the greater and lesser: The greater is that which we usually call Celandine: the root is hot and dry, cleansing and scouring, being roots coming from beyond sea, hot
proper for such as have the yellow jaundice, and dry, break wind, being boiled in oil, decoction. Consolida minor', is that we call Self-heal, and the latins Prunella. See the herb.

Costi utriusque. Of Costus both sorts, 
it is held to help the gout by anointing the the pestilence: it helps the vertigo or grieved place with it.

swimming of the head, is admirable against

Cncumeris a grestis. Of wild Cueumber the bitings of venomous beasts, and such as roots; they purge flegn, and that with such have taken too much opium, as 'also for violence, that I would advise the country lethargies, the juice helps hot rheums in the man that knows not how to correct them, to eyes; a scruple of the root in powder is let them alone.

Cinarce, \&c. Of Artichokes. The roots enough to take at one time.

Dracontii, Dracunculi. Divers authors purge by urine, whereby the rank savour of attribute divers herbs to this name. It is the body is much amended. Cynnoglosste, \&s.. Of Hounds-tongue, the roots of which cleanse mightily, and Cold and dry : being roasted and laid to take away proud, or dead flesh, the very the fundament, helps the hemorrhoids, is smell of them is hurtful for pregnant wonien: also good for burnings and scaldings.

outwardly in ointments, they take away

Curcumce. Of Turmerick, hot in the scurf, morplew, and sun-burning; I would third degree, opens obstructions, is profita- not wish any, unless very well read in physic, ble against the yellow jaundice, and cold to take them inwardly. Matthiolus, Diosdistemper of the liver and spleen, half a corides. dram being taken at night going to bed in the pulp of a roasted apple, and if you add a little saffiron to it, it will be the better by far.

Cyperiutriusque, longi, rotundi. Of Cyprus Grass, or English Galanga, both sorts, long and round : is of a warm nature, provokes urine, breaks the stone, provokes the menses; the ashes of them (being burnt) are used for ulcers in the mouth, cankers, \&c.

Dauci. Of Carrots. Are moderately hot and noist, breed but little nourishment, and are windy.

Dentaria majoris, \&s. Of Tootliwort, toothed violets, or corralivort: they are bore, or sncezewort, being grated and snuffed drying, binding, and strengthening; are up the nose, canseth sneezing; lills rats and good to ease pains in the sides and bowels; mice being mixed with their meat.

also being boiled, the decoction is said to be good to wash green wounds and ulcers witl.

Dictiammi. Of Dittany: is hot and dry? in the third degree, hastens travail in women, provokes the menses. (See the leaves.)

Black Hellebore, Bears-foot or Christmas flower: both this and the former are hot and dry in the third degree. This is neither so violent nor dangerous as the former.

Enulæ Campanæ Helenij. Of Elecampane. It is hot and dry in the third degnee, wholesome for the stomach, resists poison, heips

Doronici. Of Doronicum, a supposed old conghs, and sortness of breath, helps rupkind of 'Wolf's bane: It is hot and dry in tures, and provokes lust; in ointments, it is good the third degree, strengthens the heart, is a against scabs and itch.

sorereign cordial, and preservative against Endjvae, \&c. Of Endize, Garden Endize, 
which is the root here specified, is held to be? amorous disenses. You may lake half a drarn somerchat colder, though not so dry and cleans- at a time. Maithiolus.

ing as that which is wild ; it coolshot stomachs, Gentiana. Of Gentian; some call it Felhot livers, amends the blood commpted by heat, wort, and Baldimoney. It is hot, clearsing, and therefore is good in fevers, it cools the and scouring, a notable counterpoison, it opens reins, and therefore prevents the slone, it opens obstructions, helps the biting of venemous beasts, obstructions, and provokes urine: youmay bruise and mad dogs, helps digestion, and cleanseth the root, and boil it in wehite wine, 'tis very the body of raw humours; the root is profitable harmless.

Eringij. Of Eringo or Sea-holly: the for ruptures, or such as are bursten.

roots are moderately hot, something drying and that is grows in England: it is hot and moist cleansing, bruised and applied to the polace; in temperature, helps the roughness of the they help the Scrophula, or disease in the windpipe, hoarsness, diseases in the lirdneys and throat called the King's Evil, they break the bladder, and ulcers in the bludder, it concocts stone, encrease seed, stiv up lust, provoke the raw humour's in the stomach, helps difficulty of terms, \&c.

Esulæ, majoris, minoris. Of Spurge the the root dried and beaten into powder, and the greater and lesser, they are both (taken in-sowder put into the eye, is a special remedy zardly) too violent for common use; outwardly for a pin and web.

in ointments they cleanse the skin, take away Gramminis: Of Grass, such as in London sunburning.

Filicis, \&c. Fearn, of which are two grand they call couch grass, and Squitch-grass; in distinctions, viz. male and female. Both are urine, and easeth the kidneys oppressed with hot and dry, and good for the rickets in chil-sravel, gripings of the belly, and difficulty of dren, and diseases of the spleen, but dangerous urine. Let such as are troubled with these for pregnant women.

Filipendula. Of Dropwort. The roots these roots (being bruised) have been boiled, are hot and dry in the third degree, opening, for their morning's dranght, bruised and apcleansing, yet somezhat binding; they provoke plied to the place, they speedily help green urine, ease pains in the bladder, and are a good? woumds. Galen, Dioscorides. preservative against the falling-sickness.

Hermodactyli. Of Hernodactils. They Fœniculi. Of Fermél. The root is hot are hot and dry, purge flegm, especially from and dry, some say in the third degree, opening; the joints, therefore are good for gouts, and it provokes urine, and menses, strengthens the other diseases in the joints. Their vices are liver, and is good against the dropsy.

Fraxini. Of Ash-tree. I know no great virtues in physic of the roots.

Galanga, majoris, minoris. Galanga, corrected with long pepper, ginger, cimamon, or mastich. I would not have unstilfiul people

too busy with purges.
Hyacinthi. Of Jacinths. The roots are commonly called Galingal, the greater and dry in the first degree, and cold in the second; lesser: They are hot and dry in the third they stop looseness, bind the belly. degree, and the lesser are accomted the hotter, Iridis, vulgaris, and Florentine, \&c. it strengthens the stomach exceedingly, and Orris, or Flower-de-luce, both that which takes away the pains thereof coming of cold grows with us, and that which comes from or wind; the smell of it strengthens the brain, Florence. 'They are hot and dry in the it relieves faint hearts, takes away windiness third degree, resist poison, help shortness of the womb, heats the reins, and provokes of the breath, provoke the menses; the 
Root being green and bruised, takes away applied, it helps ulcers in the head, and blackness and blueness of a stroke, being amends the ill colour of the face. applied thereto.

Malvo. Of Mallows. They are cool, Imperitorice, \&c. Of Master-wort. The and digesting, resist poison, and help corroot is hot and dry in the third degree; rosions, or gnawing of the bowels, or any. mitigates the rigour of agues, helps dropsies, other part; as also ulcers in the bladder. provokes sweat, breaks carbuncles, and See Marsh-mallows.

plague-sores, being applied to them; it is Mandragorce. Of Mandrakes. A root very profitable being given inwardly in dangerous for its coldness, being cold in the bruises.

fourth degree: the root is dangerous.

Isotidis, Glasti. Of Woad. I know no Mechoachance. Of Mechoacalı. It is great physical virtue in the root. See the corrected with Cinnamon, is temperate yet Herb.

drying, purges flegm chiefly from the head

Labri Veneris, Dipsaci. Fullers-Thistle, and joints, it is good for old diseases in the Teazle. The root being boiled in wine till head, and may safely be given even to feit be thick (quoth Dioscorides) helps by verish bodies, because of its temperature: it unction the clefts of the fundament, as also is also profitable against coughs and pains in takes away warts and wens. Galen saitl, the reins; asalso against venereal complaints; they are dry in the second degree: and the strong may take a dram at a time.

I take it all Authors hold them to be cold Mci, \&c. Spignel. The roots are hot and dry. Unslacked lime beaten into pow- and dry in the second or third degree, and der, and mixed with black soap, takes send up unwholesome vapours to the head. away a wen being anointed with it.

Lactuce. Of Lettice. I know no physical virtue residing in the roots.

Lauri. Of the Bay-tree. The Bark of the root drunk with wine, provokes urine, breaks the stone, opens obstructions of the liver and spleen. But according to Dioscorides is naught for pregnant women. Galen.

Lapathi acuti, Oxylapathi. Sorrel, according to Galen; but Sharp-pointed Dock, according to Dioscorides. The roots of Sorrel are held to be profitable against the jaundice. Of Sharp-pointed Dock; cleanse, and help scabs and itch.

Levistici. Of Lovage. They are hot and dry, and good for any diseases coming of wind.

Lillij albi. Of white Lillies. The root is something lot and dry, lielps burnings, softens the womb, provokes the menses, if boiled in wine, is given with good success They are cold and dry, and stop lust: in rotten Fevers, Pestilences, and all dis- I never dived so deep to find what virtuc eases that require suppuration: outwardly ? the roots have.

$(23,24$.

Mezerei, \&c. Of Spurge, Olive, or Widowwail. See the Herb, if you think it worth the seeing.

Merorum Celci. Of Mulberry Tree. The bark of the root is bitter, hot and dry, opens stoppings of the liver and spleen, purges the belly, and kills worms, boiled in vinegar, helps the tooth-ache.

Morsus Diaboli, Succisa, \&c. Devil's-bit. See the herb.

Norpi Spica, Indice, Celtica, \&c. Of Spikenard, Indian, and Cheltic. Cheltic Nard wonderfully provokes urine. They are both hot and dry. The Indian, also provokes urine, and stops fluxes, helps windiness of the stomach, resists the pestilence, helps gnawing pains of the stomach; and dries up rheums that molest the head. The Celtic Spikenard performs the same offices, though in a weaker measure.

Nemupharis, Nympha. Of Water-lilics.
They are cold and dry, and stop lust:
never dived so deep to find what virtue
the roots have.

3 II 
Ononidis, Arreste Bovis, \&c. Of Cam- speedy deliverance to women in travail, and mock, or Rest-harrow, so called because it brings away the placenta. makes oxen stand still when they are ploughing. The roots are hot and dry in the third degree; it breaks the stone (viz. the bark of it.) The root itself, according to Plimy, helps the falling-sickness; according to Matthiolus, helps ruptures: you may take half a dram at a time.

Ostrutij. Masterwort, given once before under the name of Imperitoria. But I have something else to do than to write one thing twice as they did.

Pastinate, Sative, and silvestris. Garden and Wild Parsnips. They are of a temperate quality, inclining something to heat : The Garden Parsnips provoke lust, and nourish as much and more too, than any root ordinarily eaten: the wild are more physical, being cutting, cleansing, and opening: they resist the bitings of venomous beasts, ease pains and stitches in the sides, and are a sovereign remedy against the wind cholic.

Pentafylli. Of Cinqfyl, commonly called Five-leaved, or Five-finger'd grass : the root is very drying, but moderately hot: It is admirable against all fluxes, and stops blood flowing from any part of the body : it helps infirmities of the liver and lungs, helps putrified ulcers of the mouth, the root boiled in vinegar is good against the shingles, and appeases the rage of any fretting sores. You may safely take half a dram at a time in any convenient liquor.

Petacite. Of Butter-bur. The roots are hot and dry in the second degree, they are exceeding good in violent and pestilential fevers, they provoke the menses, expel poison, and kill worms.

Pencedani, Faniculi porcini. Of Sulphurwort, Hogs-fennel, or Hore-strange. It is very good applied to the navels of children that stick out, and ruptures: held in the mouth, it is a present rcmedy for the fits of according to the strength of the discased, it the mother : being taken inwardly, it gives? works verv safely.

Pooniø, maris, fomella. Of Peomy male and female. They are meanly hot, but more drying. The root helps women not sufficiently purged after travail, it provokes the menses, and helps pains in the belly, as also in the reins and bladder, falling sickness, and convulsions in children, being either taken invardly, or hmmg abont their necks. You may take half a dram at a time, and less for children.

Phu, Valcrina, majoris, minoris. Valerian, or Setwal, greater and lesser. They are temperately hot, the greater provolies urine and the menses, helps the stranguary, stays rheums in the head, and takes away the pricking pains thereof: The lesser resist poison, assuages the swelling of the testicles, coming either through wind or cold, helps cold taken after sweating or labour, wind cholic: outwardly it draws out thorns, and cures both wounds and ulcers.

Pimpinellæ, \&c. Of Burnet. It doth this good, to bring forth a gallant physical herb.

Plantaginis. Of Plantane. The root is something dryer than the leaf, but not so cold, it opens stoppages of the liver, helps the jamdice, and ulcers of the reins and bladder. A little bit of the root being eaten, instantly stays pains in the hear, even to admiration.

Polypodij. Of Polypodium, or Fern of the Oak. It is a gallant though gentle purger of melancholy; Also in the opinion of Mesue (as famous a physician as ever I read for a Galenist,) it dries up superfluous humours, takes away swellings fron the hands, feet, knees, and joints, stitches and pains in the sides, infirmities of the spleen, rickets; correct it with a few Annis seeds, or Fennel seeds, or a little ginger, and then the stomach will not loath it. Your best way of taking it, is to bruise it well, and boil it in white wine till half be consumed, you may put in much, or little, 
Poligonati, sigilli Solomonis, \&c. Of Solo- ing takes away the virtue of it, and theremon's Seal. Stamped and boiled in wine it fore it is best given by infusion only; If speedily helps(beingdrank)all broken bones, your body be any thing strong, you may and is of incredible virtue that way; as also take two drams of it at a time being sliced being stamped and applied to the place, it thin and steeped all night in white wine, in soon healsall wounds, and quickly talsesaway the black and blue marks of blows, being? white wine; it purges but gently, it leaves bruised and applied to the place, and for a binding quality behind it, therefore dried these, I am persuaded there is not a better a little by the fire and beaten into powder, medicine under the sun.

Porri. Of Leeks. They say they are hot and dry in the fourtli degree; they breed ill-favoured nourishment at the best, they spoil the eyes, heat the body, cause troublesome sleep, and are noisome to the stomach : yet are they good for something else, for the juice of them dropped into the ears takes away the noise of them, mixed with a little vinegar and snuffed up the nose, it stays the bleeding of it, they are better of the two boiled than raw, but both ways exceedingly hurtful for ulcers in the bladder: and so are onions and garlic.

Prunellorum Silvestrium. Of Sloe-bush, or Sloe-tree. I think the college set this amongst the roots only for fashion sake, and I did it because they did.

Pyrethri Salivaris, \&.c. Pelitory of Spain. It is hot and dry in the fourth degree, chewed in the mouth, it draws away rheum in the tooth-ache; bruised and boiled in oil, it provokes sweat by unction; inwardly taken, théy say it helps palsies and other cold effects in the brain and norves.

Rhapontici. Rhupontick, or Rlubarb of Pontus. It takes away windiness and weakness of the stomach, sighings, sobbings, spittings of blood, diseases of the liver and spleen, rickets, \&c. if you take a dram at a time it will purge a little, but bind much, and therefore fit for foul bodies that have fluxes.

Rhabarbari. Of Rhubarb. It gently is usually given in fluxes.

Rhaphani, Domestica and Sylvestris. Of Raddishes, garden and wild. Garden Raddishes provoke urine, break the stone, and purge by urine exceedingly, yet breed very bad blood, are offensive to the stomach, and hard of digestion, hot and dry in quality. Wild, or Horse Raddislies, such as grow in ditches, are hotter and drier than the former, and more effectual.

Rhodie Rad. Rose Root. Stamped and applied to the head it mitigates the pains thereof, being somewhat cool in quality.

Rhabarbari Monachorum. Monks Rhubarb, or Bastard-Rhubarb, it also purges, and cleanses the blood, and opens obstructions of the liver.

Rubice tinctorum. Of Madder. It is both drying and hinding, yet not without some opening quality, for it helps the ycllow jaundice, and therefore opens obstructions of the liver and gall ; it is given with good success, to such as have had bruises by falls, stops loosencss, the hemorrhoids, and the menses.

Rusci. Of Knec-holly or Butchersbroom, or Brtiscus. 'They are meanly hot and dry, provoke urine, break the stone, and help-such as camnot evacuate urine freely. Use them like grass ronts.

Sambuci. Of Elder. I know no wonders the root will do.

Sarsce-Pariglice. Of Sarsa-Parilla, or Bind-weed ; somcwhat hot and dry, helpful purges choler from the stomach and liver, against pains in the head, and joints; they opens stoppings, withstands the dropsy, provoke sweat, and are used familiarly in Hypocondriac Melancholly; a little boil 
Satyrij utriusque. Of Satyrion, each sort. called our Ladies-thistles by that name; They are hot and moist in temper, provoke the roots of which are drying and bindvenery, and increase seed; each branch ing, stop fluxes, bleeding, take away bears two roots, both spongy, yet the one cold swellings, and ease the pains of the more solid than the other, which is of most teeth.

virtue, and indeed only to be used, for some say the most spongy root is quite contrary in opcration to the other, as the one increaseth, the other decreaseth.

Saxifragice albce. Of white Saxifrage, in Sussex we call them Lady-smocks. The roots powerfully break the stune, expcl wind, provoke urine, and cleanse the reins.

Sanguisorbe. A kind of Burnet.

Scabiosa. Of Scabious. The roots either boiled, or beaten into powder, and so taken, help such as are extremely troubled with scabs and itch, are medicinal in the french disease, hard swellings, inward wounds, being of a drying, cleansing, and healing faculty.

Scordij. Of Scordium, or Water-Germander. See the herb.

Scillce. Of Squills. See vinegar, and wine of Squills, in the compound.

Scropularice, \&c. Of Figwort. The roots being of the same virtue with the herb, I refer you thither.

Scorzonerce. Of Vipers grass. The root cheers the heart, and strengthens the vital spirits, resists poison, helps passions and tremblings of the heart, faintness, sadness, and melancholy, opens stoppings of the liver and spleen, provokes the menses, ease women of the fits of the mother, and helps swimmings in the head.

Seseleos. Of Seseli, or Hartwort. The roots provoke urine, and help the fallingsickness.

Sisari, secacul. Of Scirrets. They are hot and moist, of good nourishment, something windy, as all roots are; by reason of which, they provoke venery, they stir up appetite, and provoke urine.

Sconchi. Of Sow-thistles. See the herb. Spince albe, Bedeguar. The Arabians

Spatula fatida. Stinking Gladon, a kind of Flower-de-luce, called so for its unsavory smell. It is hot and dry in the third degree; outwardly they help the king's evil, soften hard swellings, draw out broken bones: inwardly taken, they help convulsions, ruptures, bruises, infirmities of the lungs.

Tamarisci. Of Tamaris. See the herbs, and barks.

Tanaceti. Of Tansie. The root eaten, is a singular remedy for the gout: the rich may bestow the cost to preserve it.

Thapsi, \&c. A venomous foreign root: therefore no more of it.

Toimentilla. Of Tormentil. A kind of Sinqfoil; dry in the third degree, but moderately hot; good in pestilences, provokes sweat, stays vomiting, cheers the heart, expels poison.

Trifolij. Of Trefoil. See the herb.

Tribuli Aquatici. Of Water Caltrops. The roots lie ton far under water for me to reach to.

Trachellij. Of Throat-wort: by some called Canterbury Bells: by some Coventry Bells. They help diseases and ulcers in the throat.

Trinitatis herba. Hearts-ease, or Pansies. I know no great virtue they have.

Tunicis. I shall tell you the virtue when I know what it is.

Tripolij. The root purges flegm, expels poison.

Turbith. The root purges flegm, (being hot in the third degree) chiefly from the exterior parts of the body : it is corrected with ginger, or Mastich. Let not the vulgar be too busy with it.

Tuburnum. Or Toad-stools. Whether these be roots or no, it matters not much. 
for iny part I know but little need of them, either in food or physic.

Victorialis. A foreign kind of Garlick. They say, being hung about the neck of cattle that are blind suddenly, it helps them; and defends those that bear it, from evil spirits.

Swallow-wort, and teazles were handled before.

Ulmarice, Reginc, prati, \&.c. Mead-sweet. Cold and dry, binding, stops fluxes, and the immoderate flowing of the menses: you may take a dram at a time.

Urtice. Of Nettles. See the leaves.

Zedoarice. Of Zedoary, or Setwall. 'This and Zurumbet, according to Rhasis, and Mesue, are all one; Avicenna thinks them different: I hold with Mesue; indeed they differ in form, for the one is long, the other round; they are both hot and dry in the second degree, expel wind, resist poison, stop fluxes, and the menses, stay vomiting, help the cholic, and kill worms; you may take half a dram at a time.

Zingiberis. Of Ginger. Helps digestion, warms the stomach, clears the sight, and is profitable for old men: heats the joints, and therefore is profitable against the gout, expels wind; it is hot and dry in the second degree.

\section{B A R K S.}

A Pil Rad. Of the roots of Sinallage. Take notice here, that the Barks both of this root, as also of Parsley, Fennel, \&c. is all of the root which is in use, neither can it properly be called bark, for it is all the root, the hard pith in the middle excepted, which is always thrown away, when the roots are used. It is something hotter and drier than Parsley, and more medicinal; it opens stoppings, provokes urine, helps digestion, expels wind, and warms a cold stomach: use them like grass roots.

$(83,24$.
Avellanarum. Of Hazel The rind of the tree provokes urine, breaks the stone; the husks and shells of the nuts, dried and given in powder, stay the immoderate flux of the menses.

Aurantiorum. Of Oranges. Both these, and also Lemons and Citrons, are of different qualities: the outward bark, viz. what looks red, is hot and dry, the white is cold and moist, the juice colder than it, the seeds hot and dry; the outward bark is that which here $I$ am to spcak to, it is somewhat hotter than either that of Lemons or Citrons, therefore it warms a cold stomach more, and expels wind better, but strengthens not the heart so much.

Berber, \&c. Barberries. The Rind of the tree according to Closius, being stecped in wine, and the wine drank, purges choler, and is a singular remedy for the yellow jaundice. Boil it in white wine and drink it. See the directions at the beginning.

Cassia Lignea, \&c. It is something more oily than Cinnamon, yet the virtues being not much different, I refer you thither.

Capparis Rad. Of Caper roots. See the roots.

Castanearum. Of Chesnuts. The bark of the Chesnut tree is dry and binding, and stops tluxes.

Cinnamonum. Cinnamon, and Cassia Lignea, are hot and dry in the second degree, strengthens the stomach, help digestion, cause a sweet breath, resist poison, provoke urine, and the menses, cause speedy delivery in women to travail, help coughs and defluxions of humours upon the lungs, dropsy, and difficulty of urine. In ointments it takes away red pimples, and the like deformities from the face. There is scarce a better remedy for women in labour, than a dram of Cinnamon newly beaten into powder, and taken in white wine.

Citrij. Of Pome Citrons. The outward pill, which I suppose is that which is meant $3 \mathrm{~N}$ 
here: It strengthens the heart, resists poison, amends a stinking breath, helps digestion, comforts a cold stomach.

Ebuli Rad. Of the roots of Dwarf-Elder, or Walwort. See the herbs.

Enulce. Of Elecampane. See the roots. Esulce Rad. See the roots.

Fabarum. Of Beans. Bean Cods (or Pods, as we in Sussex call them) being bruised, the ashes are a sovereign ren edy for aches in the joints, old bruises, gouts, and sciaticas.

Freniculi Rad. Of Fennel roots. See the roots, and remember the observation given in Smallage at the beginning of the barks.

Fraxini Rad. Of the bark of Ash-tree roots. The bark of the tree, helps the rickets, is moderately hot and dry, stays vomiting; being burnt, the ashes made into an ointment, helps leprosy and other deformity of the skin, eases pains of the spleen. You may lay the bark to steep in white wine for the rickets, and when it hath stood so for two or three days, let the diseased child drink now and then a spoonful of it.

Granatorum. Of Pomegranates. rind cools, and fercibly binds, stays fluxes, and the menses, helps digestion, strengthens weak stomachs, fastens the teeth, and are good for such whose gums waste. You may take a dram of it at a time inwardly. Pomegranate flowers are of the same virtue.

Gatrujaci. See the wood.

Juglandium Virid. Of green Walnuts. As for the outward green bark of Walnuts, I suppose the best time to take them is before the Walnuts be shelled at all, and then you may take nuts and all (if they may properly be called nuts at such a time) you shall find them exceeding comfortable to $P$ the stomach, they resist poison, and are a most excellent preservative against the plague, inferior to none: they are admirable for such as are troubled with consumptions of the lungs. :
Lauri. Of the Bay-tree. See the root.

Limonum. Of Lemons. The outward peel is of the nature of Citron, but helps not so effectually; however, let the poor country man that cannot get the other, use this.

Mandragora Rud. Be pleased to look back to the root.

Myrobalanorum. Of Myrobalans. See the fruits.

Macis. Of Mace. It is hot in the third degree, strengthens the stomach and heart exceedingly, and helps concoction.

Maceris, $\& c$. It is held to be the inner bark of Nutmeg-tree, helps fluxes and spitting of blond.

Petroselini Rad. Of Parsley root: opens obstructions, provokes urineand themenses, warms a cold stomach, expels wind, and breaks the stone. Use them as grass roots, and take out the inner pith as you were taught in smallage roots.

Prunelli Silvestris. Of Sloe-tree. I know no use of it.

Pinearum putaminae. Pine shucks, or husks. I suppose they mean of the cones that hold the seeds; both those and also the bark of the tree, stop fluxes, and help the lungs.

Querci. Of Oak-tree. Both the bark of the oak, and Acorn Cups are drying and cold, binding, stop fluxes and the menses, as also the running of the reins; have a care how you use them before due purging.

Rhaphani. Of Radishes. I could never see any bark they had.

Suberis. Of Cork. It is good for something else besides to stop bottles : being dry and binding, stanches blood, helps fluxes, especeially the ashes of it being burnt. Paulus.

Sambuci, \&c. Of Elder roots and branches; purges water, helps the dropsy.

Cort. Medius Tamaricis. The middle Bark of Tameris, eases the spleen, helps the rickets. Use them as Ash-tree bark. 
Tillio. Of Line-tree. Boiled, the water helps burnings.

Thuris. Of Frankinsenses. I must plead Ignor amus.

Ulmi. Of Elm. Moderately hot and cleansing, good for wounds, burns, and broken bones, riz. boiled in water and the grieved place bathed with it.

\section{WOODS AND THEIR CHIPS, OR RASPINGS.}

A Gallochus, Lignum Aloes. Wood of Aloes; is moderatcly hot and dry: a good cordial: a rich perfuine, a great strengthener to the stomach.

Aspalathus. Rose-wood. It is moderately hot and dry, stops looseness, provokes urine, and is excellent to cleanse filthy ulcers.

Bresilium. Brasil. All the use I know of it is, to die cloth, and leather, and make red ink.

Buxus. Box. Many Physicians have written of it, but no physical virtue of it.

Cypressus. Cypress. The Wond laid amongst cloaths, secures them from moths. See the leaves.

Ebenum. Ebony. It is held to clear the sight, being either boiled in wine, or burnt to ashes.

Guajacum, Lignum vita Dries, attenuates, causes sweat, resists putrefaction, is good for the French disease, as also for ulcers, scabs, and leprosy: it is used in diet drinks.

Juniperus. Juniper. The smoak of the wood, drives away serpents; the ashes of it made into lie, cures itch, and scabs.

Nephriticum. It is a light wood and comes from Hispaniola; being steeped in water, will soon turn it blue, it is hot and dry in the first degree, and so used as before, is an admirable remedy for the stone, and for obstructions of the liver and spleen. ?
Rhodium. Encreases milk in nurses.

Santalum, album, Rubrum, citrinum. White, red, and yellow Sanders: They are all cold and dry in the second or third degree: the red stops defluxions from any part, and helps inflanimations: the white and yellow (of which the yellow is best) cool the heat of fevers, strengthen the heart, and cause cheerfulness.

Sassafras. Is hot and dry in the second degree, it opens obstructions or stoppings, it strengthens the breast exceedingly; if it be weakened through cold, it breaks the stone, stays vomiting, provokes urine, and is very profitable in the venereal, used in diet drinks.

Tamaris. Is profitable for the rickets, and burnings.

Xylobalsamum. Wood of the Balsam tree, it is hot and dry in the second degree, according to Galen. I never read any great virtues of it.

\section{HERBS AND THEIR LEAVES.}

A Brotanum, mas, famina. Southernwood, male and fenale. It is hot and dry in the third degree, resists poison, kills worms ; outwardly in plaisters, it dissolves cold swellings, and helps the bitings of venomous beasts, makes hair grow: take not above half a dram at a time in powder. Absinthium, \&\&. Wormwood. Its several sorts, are all hot and dry in the second or third degrees, the common Wormwoor is thought to be hottest, they all help weakness of the stomach, cleanse choler, kill worms, open stoppings, help surfeits, clear the sight, resist poison, cleanse the blood, and secure cloaths from moths.

Abugilissa, \&c. Alkanet. The leaves are something drying and binding, but inferior in virtue to the roots, to which $I$ refer you.

Acetosa. Sorrel. Is moderately cold 
dry and binding, cuts tough humours, cools held to be more cordial; cools the blood, the brain, liver and stomach, cools the helps ulcers in the mouth; hot defluxions blood in fevers, and provokes appetite. Acanthus. Bear's-breech, or Branks ursine, is temperate, something moist. See the root.

Adiantum, Album, mgrum. Maiden hair, white and black. They are temperate, yet drying. White Maiden hair is that we usually call Wall-rue; they both open obstructions, cleanse the breast and lungs of gross slimy humours, provoke urine, help ruptures and shortness of wind.

Adiantum Aurcum Politrycum. Golden. Maiden-hair. Its temperature and virtues are the same with the former; helps the spleen; burned, and lye made with the ashes, keeps the hair from falling off the head.

Agrimonia. Agrimony. Galen's Eupatorium. It is hot and dry in the first degree, binding, it amends the infirmities of the liver, helps such as evacuate blood instead of water, helps inward wounds, opens obstructions. Outwardly applied it helps old sores, ulcers, \&c. Inwardly, it helps the jauinice and the spleen. Take a dram of this or that following, inwardly in white wine, or boil the herb in white wine, and drink the decoction. Galen, Pliny, Dioscorides, Serapio.

Ageretum. Hot and dry in the second degree, provokes urine and the menses, dries the brain, opens stoppings, helps the green sickness, and profits such as have a cold, weak liver; outwardly applied, it takes away the hardness of the matrix, and fills hollow ulcers with flesh.

A gnu Castus, \&c. Chast-tree. The leaves are hot and dry in the third degree; expel wind, consume the seed, cause chastity being only borne about one; it dissolves swellings of the testicles, being applied to them, head-ache, and lethargy.

sllajula. Lujula, \&c. Wood Sorrel. is of the temperature of other Sorrel, and etses in the brain, being only smelled to upon the lungs, wounds, ulcers, \&c.

Alcea. Vervain Mallow. The root helps fluxes and burstness. Atius, Dioscorides. Allium. Garlick. Hot and dry in the fourth degree, troublesome to the stomach; it dulls the sight, spoils a clear skin, resists poison, eases the pains of the teeth, helps the bitings of mad $\operatorname{dog} s$, and venomous beasts, helps ulcers, leprosies, provokes urine, is exceedingly opening, and profitable for dropsies.

Althea, \&c. Marsh-Mallows. Are moderately hotand drier than other Mallows; they help digestion, and mitigate pain, case the pains of the stone, and in the sides. Use them as you were taught in the roots, whose virtues they have, and both together will do better.

Alsine. Chickweed. Is cold and moist without any binding, assuages swelling, and comforts the sinews much; therefore it is good for such as are shrunk up ; it dissolves aposthumes, hard swellings, and helps mange in the hands and legs, outwardly applied in a pultis. Galen.

Alchymilla. Ladies-Mantle. Is hot and dry, some say in the second degree, some say in the third: outwardly it helps wounds, reduces women's breasts that hang down: inwardly, helps bruises, and ruptures, stays vomiting, and the Fluor Albus, and is very profitable for such women as are subject to miscarry through cold and moisture.

Alkanna. Privet hath a binding quality, helps ulcers in the moutl, is good against burnings and scaldings, cherishes the nerves and sinews; boil it in white wine to wash the mouth, and in hog's grease for burnings and scaldings.

Amaracus, Majorana. Marjoram. Some say 'tis hot and dry in the second degree, some advance it to the third. Sweet MarIt $\mathrm{j}$ ram, is an excellent eemedy for cold dis- 
helps such as are given to much sighing, easeth pains in the belly, provokes urine, being taken inwardly: you may take a dram of it at a time in powder. Outwardly in oils or salves, it helps sinews that are shrunk; limbs out of joint, all aches and swellings coming of a cold cause.

Angelica. Is hot and dry in the third degree; opens, digests, makes thin, strengthens the heart, helps fluxes, and loathsomeness of meat. It is an enemy to poison and pestilence, provokes menses, and brings away the placanta. You may take a dram of it at a time in powder.

Anagallis, mas, femina. Pimpernel, male and female. 'They are something hot and dry, and of such a drying quality that they draw thorns and splinters out of the flesh, amend the sight, clcanse ulcers, help infirmities of the liver and reins. Galen.

Anethum. Dill. Is hot and dry in the second degree. It stays vomiting, eases hiccoughs, assuages swellings, provokes urine, helps such as are troubled with fits of the mother, and digests raw humours.

Apium. Smallage; So it is commonly used; but indeed all Parsley is called by the name of Apium, of which this is one kind. It is something hotter and dryer than Parsley, and more efficacious; it opens stoppings of the liver, and spleen, cleanses the blood, provokes the menses, helps a cold stomach to digest its meat, and is good against the yellow jaundice. Both Smallageand Clevers, may be well used in pottage in the morning instcad of herbs.

Aparine. Goose-grass, or Clevers : They are meanly hot and dry, cleansing, help the bitings of venomous beasts, keep men's bodies from growing too fat, help the yellow jaundice, stay bleeding, fluxes, and help green wounds. Dioscorides, Plimy, Galen, Tragus.

Aspergula odorata. Wood-roof: Cheers the heart, malies men merry, helps melancholy, and opens the stoppings of the liver. the lungs.

$(23,24$.
Aquilegia. Columbines: help sorethroats, are of a drying, binding quality.

Argentina. Silver-weed, or Wild Tansy ; cold and dry almost in the third degree; stops lasks, fluxes, and the menses, good against ulcers, the stone, and inward wounds: easeth gripings in the belly, fastens loose teeth: outwardly it takes away freckles, morphew, and sunburning, it takes away inflammations, and bound to the wrists stops the violence of the fits of the ague.

Artanita. Sow-bread: hot and dry in the third degree, it is a dangerous purge: outwardly in ointments ittakes awa y freckles, sunburning, and the marks which the small pox leaves behind them: dangerous for pregnant women.

Aristolochia, longa, rotunda. Birth-wort long and round. See the roots.

Artemisia. Mugwort: is hot and dry in the second degree: binding: an lierb appropriated to the female sex; it brings down the menses, brings away both birth and placenta, eases pains in the niatrix. You may take a dram at a time.

\section{Asparagus. See the roots.}

Asarum, \&.c. Asarabacca : hot and dry ; provokes romiting and urine, and are good for dropsies. They are corrected with mace or cinnamon.

Atriplex, fc. Orach, or Arrach. It is cold in the first degree, and moist in the second, saith Galen, and makes the belly soluble. It is an admirable remedy for the fits of the mother, and other infirmities of the matrix, and therefore the Latins called it Vulvaria.

Aricula muris, major. Mouse-ear: lot and dry, of a binding quality, it is adnirable to heal wounds, inward or outward, is also ruptures or burstness: Eilge-tools quenched in the juice of it, will cut -iron without turning the edge, as easy as they will lead: And, lastly, it helps the swelling of the spleen, coughs and consumptions, of 
Attractivis hirsuta. Wild Bastard-saffron, Betony: hot and dry in the second degree, Distaff-thistle, or Spindle-thistle. Is dry and helps the falling sickness and all head-aches moderately digesting, helps the biting of coming of cold, cleanses the breast and venomous beasts: Mesue saith, it is hot in lungs, opens stoppings of the liver and the first degree, and dry in the second, and spleen, as the rickets, \&c. procures appetite, cleanseth the breast and lungs of tough helps sour bclchings, provokes urine, breaks flegm.

Balsamita, \&c. Costmary, Alecost: See Maudlin.

Barbajovis, sedum majus. Houseleck or Sengreen: cold in the third degree, profitable against the Shingles, and other hot? creeping ulcers, inflammations, St. Anthony's fire, frenzics; it cools and takes away corns from the toes, being bathed with the juice of it, and a skin of the leaf laid over the place; stops fluxes, helps scalding and burning.

Bardana. Clot-bur, or Bur-dock: temperately dry and wasting, something cooling; it is held to be good against the shrinking of the sinews; eases pains in the bladder, and provokes urine. Also Mizaldus saith, that a leaf applied to the top of the head of a woman draws the niatrix upwards, but applied to the soles of the feet draws it downwards, and is therefore an admirable remedy for suffocations, precipitations, and dislocations of the matrix, if a wise man have but the using of it.

Beta, alba, nigra, rubra. Beets, white, black, and red; black Bects I have no knowledge of. The white are something colder and moister than the red, both of them loosen the belly, but have little or no nourishment. The white provoke to stool, and are more clcansing, open stoppings of the liver and spleen, help the vertigo or swimming in the head: The red stay fluxes,
help the immoderate flowing of the menses, and are good in the yellow jaundice.

Beuedicta Cariphyllwa. Avens: hot and dry, help the cholic and rawness of the stomach, stitches in the sides, and take away clotted blood in any part of the body.

the stone, mitigates the pains of the reins and bladder, helps cramps, and convulsions, resists poison, helps the gout, such as evacuate blood, madness and head-ache, kills worms, helps bruises, and cleanseth women after labour: You may take a dram of it at a time in white wine, or any other convenient liquor proper against the disease you are afflicted with.

Betonica Pauli, \&c. Paul's Betony, or Male Lluellin, to which add Elative, or Female Lluellin, which comes afterwards; they are pretty temperate, stop defluxions of humours that fall from the head into the eyes, are profitable in wounds, help filthy foul eating cankers.

Betonica Coronaria, \&.c. Is Clove Gilliflowers. Sce the flowers.

Bellis. Dasies : are cold and moist in the second degree, they ease all pains and swellings coming of heat, in clysters they loose the belly; are profitable in fevers and inflammations of the lesticles, they take away bruises, and blackness and blueness; they are admirable in wounds and inflammations of the lungs or blood

Blitum. Blites. Some say they are cold and moist, others cold and dry: none niention any great virtues of them.

Borrago. Borrage: hot and moist, comforts the heart, checrs the spirits, drives away sadness and melancholy, they are rather laxative than binding; help swooning and heart-qualms, brecd good blood, help consumptions, madness, and such as are much weakened by sickness.

Bomus Henricus. Good Henry, or all good; hot and dry, cleansing and scouring; i Betonica vulgaris. Common or .Wood wardly it clcanseth old sores and ulcers. 
Botrys. Oak of Jerusalem : hot and dry in the second degree, helps such as are shortwinded, cuts and wastes gross and tough flegn, laid annong cloaths they preserve them from moths, and give them a sweet sinell.

\section{Branca ursina. Bears-breech.}

Brionia, \&s. Briony, white and black; oth are hot and dry in the third degree, purge violently, thet are held to be wholesome physic for such as have dropsies, vertigo, or swimining in the head, fallingsickness, \&c. Certainly it is a strong, troublesome purge, therefore not to be tampered with by the unskilful, outwardly in ointments it takes away freckles, wrinkles, morphew, scars, spots, \&c. from the face.

Bur'sa pastoris. Shepherd's Purse, is manifestly cold and dry, though Lobel and Pena thought the contrary; it is binding? and stops blood, the menses; and cools inflammations.

Buglossom. Buglosse. Its virtues are the same with Borrage.

Bugula. Bugle, or Middle Comfrey; is temperate for heat, but rery drying, excellent for falls or inward bruises, for it dissolvescongealed blood, profitable for inward wounds, helps the rickets and other stoppings of the liver; outwardly it is of wonderful force in curing wounds and ulcers, though festered, as also gangreens and fistulas, it helps broken borics, and dislocations. Inwardly you may take it in powder a dram at a time, or drink the decoction of it in white-wine: being made into an ointment with hog's grease, you slrall find it admirable in green wounds.

Buphthalmum, \&c. Ox eye. Matthiolus saith they are commonly used for black Hellehore, to the ritues of . which I refer.

Buxus. Boxtree: the leaves are hot, dry, and binding, they are profitable against the biting of mad dogs; both taken inwardly boiled and applied to the place: besides they are good to cure horses of the bots.
Calamintha, Montana, Palustris. Mountain and Water Calamint: For the Water Calamint: see mints, than which it is accounted stronger. Mountain Calamint, is hot and dry in the third degree, provokes urine and the menses, hastens the birth in women, brings away the placenta, helps cramps, convulsions, difficulty of breathing, kills worris, helps the dropsy: outwardly used, it helps such as hold their necks on one side: half a dram is enough at one time. Galen, Dioscorides, Apuleius.

Calendula. \&ic. Marigolds. The leaves arc hot in the second degree, and something moist, loosen the belly: the juice held in the mouth, helps the toothache, and takes away any infiammation or hot swelling being bathed with it, mixed with a little vinegar.

Callitricum. Maiden-hair. SecAdianthum. Caprisolium. Honey-suckles: The leaves are liot, and therefore naught for inflammations of the mouth and throat, for which the ignorant people oftentime give them: and Galen was true in this, let modern writers write their pleasure. If you chew but a leaf of it in your mouth, experience will tell you that it is likelier to cause, than to cure a sore throat, they provoke urine, and purge by urine, bring speedy delivery to women in travail, yet procure barrenness and hinder conception, outwardly they dry up foul ulcers, and cleanse the face from morphew, sun-burning and freckles.

Carduncellus, \&c. Groundsell. Cold and moist according to Tragus, helps the cholic, and gripings in the belly, helps such as cannot nllake water, cleanses the reins, purges choler and sharp humours: the usual way of taking it is to boil it in water with currants; and so cat it. I hold it to be a wholesomeand harnless purgc. Outwardly it easeth women's breasts that are swollen and inflamed; as also inflammations of the joints, nerves, or sinews. Agineta. 
Carduus B. Marice. Our Ladies Thistles. are drying and binding, help dimness of the They are far more temperate than Carduus sight: help the spleen, preserve from Benedictus, open obstructions of the liver, drunkenness, and help the evil effects of it: help the jaundice and dropsy, provoke provoke the menses.

urine, break the stone.

Carduus Benedictus. better known by the Blinsed Thistle, but and dry in the second degree, cleansing and opening, helps swimming and giddiness in the head, deafness, strengthens the memory, helps griping pains in the belly, kills worms, provokes sweat, expels poison, helps in-? flammation of the liver, is very good in pestilence and venereal: outwardly applied, it ripens plague-sores, and helps hot swellings, the bitings of mad dogs and venomous beasts, and foul filthy ulcers. Every one that can but make a Carduus posset, knows how to use it. Camerarius, Armuldus vel anovamus.

Chalina. See the roots, under the name of white Chameleon.

Corallina. A kind of Sea Moss : cold, binding, drying, good for hot gouts, inflammations : also they say it kills worms, and therefore by some is called Maw-wormseed.

Cussutha, cascuta, potagralini. Dodder. See Epithimum.

Caryophylluta. Avens, or Herb Bennet, hot and dry: they help the cholic, rawness of the stomach, stitches in the sides, stoppings of the liver, and bruises.

Cataputia minor. A kind of Spurge. See Tythymalus.

Cattaria, Nepeta. Nep, or Catmints. The virtues are the same with Calaminth.

Cauda Equina. Horse-tail; is of a binding drying quality, cures wounds, and is an Water cresses; they help mangy horses: admirable remed y for sinews that are shrunk: see Water cresses.

it is a sure remedy for bleeding at the nose, or by wound, stops the mienses, fluxes, ulcers in the reins and bladder, coughs, ulcers in the lungs, difficulty of breathing.

Caulis, Brassica hortensis, silvestris. wort, or Cabbages, garden and wild.
Centaurium, majus, minus. Centaury the greater and less. They say the greater will do wonders in curing wounds: see the root. The less is a present remedy for the yellow jaundice, opens stoppings of the liver, gall, and spleen : purges choler, helps gout, clears the sight, purgeth the stomach, helps the dropsy and green sickness. It is only the tops and flowers which are useful, powder, or half a handful boiled in possetdrink at a time.

Centinodium, \&c. Knotgrass : cold in the second degree, helps spitting and other evacuations of blood, stops the menses and all other fluxes of blood, vomiting of blood, gonorrhae, or running of the reins, weakness of the back and joints, inflammations of the privities, and such as make water by drops, and it is an excellent remedy for hogs that will not eat their meat. Your only way is to boil it, it is in its prime about the latter end of July, or beginning of August: at which time being gathered it may be kept dry all the year. Brassavolus, Camerarius.

Caryfolium vulgare et Myrrhis. Common and great chervil: Take them both together, and they are temperately hot and dry, proheart, and are good for old people; help pleurises and pricking in the sides.

Capea, Anagallis aquatica. Brooklime, hot and dry, but not so hot and dry as

Ceterach, \&c. Spleenwort: moderately hot, waste and consumes the spleen, insomuch that Vitruevius affirms he hath known hog's that have fed upon it, that have had Cole- (when they were killed) no spleens at all. They It is excellently good for melancholy people, of which you may take a drain inwardly in voke urine, stir up venery, comfort the 
helps the stranguary, provokes urine, and breaks the stone in the bladder, boil it and drink the decoction; but because a little boiling will carry away the strength of it in vapours, let it boil but very little, and let it stand close stopped till it be cold before you strain it out; this is the general rule for all simples of this nature.

Chanapitys. Ground-pine; hot in the second degree, and dry in the third, helps the jaundice, sciatica, stopping of the liver, and spleen, provokes the menses, cleanses the entrails, dissolves congealed blood, resists poison, cures wounds and ulcers. Strong bodies may take a dram, and weak bodies half a dram of it in powder at a time.

Chamæemelum, sativum, sylvestre. Garden and Wild Chamomel. Garden Chamomel, is hot and dry in the first degree, and as gallant a medicine against the stone in the bladder as grows upon the earth, you may take it inwardly, I mean the decoction of it, being boiled in white wine, or inject the juice of it into the bladder with a syringe. It expels wind, helps belchings, and potently provokes the menses: used in baths, it helps pains in the sides, gripings and gnawings in the belly.

Chancedris, fc. Germander: hot and dry in the third degree; cuts and brings away tough humours, opens stoppings of the liver and spleen, helps coughs and shortness of breath, stranguary and stopping of urine, and provokes the menses; half a dram is enough to take at a time.

Chelidonium utrumque. Celandine both sorts. Small Celandine is usually called Pilewort; it is something hotter and dryer than the former, it helps the hemorrhoids or piles, bruised and applied to the grief. Celandine the greater is hot and dry (they say in the third degree) any way used; either the juice or made into an oil or ointment, it is a great preserver of the sight, and an excellent help for the eyes.

$(23,24$.
Cinara, \&.c. Artichokes. They provoke venery, and purge by mine.

Cichorium. Succory, to which add Endive which comes after. 'They are cold and dry in the second degree, cleansing and opening; they cool the heats of the liver, and are profitable in the yellow jaundice, and burning fevers; help excoriations in the privities, hot stomachs; and outwardly applied, help hot rheuns in the eyes.

cicuta. Henlock: cold in the fourth degree, poisonous: outwardly applied, it helps Priapismus, the shingles, St. Anthony's fire, or any eating ulcers.

Clematis Daphnoides, Vinca provinca. Periwinkle. Hot in the second degree, something dry and binding; stops lasks, spitting of blood, and the menses.

Consolida major. Comfrey, I do not conccive the leaves to be so virtuous as the roots.

Consolida media. Bugles, of which before. Consolida minima. Daises.

Consolida rubra. Golden Rod: hot and dry in the second degree, cleanses the reins, provokes urine, brings away the gravel: an admirable herb for wounded people to take inwardly, stops blood, \&c.

Consolida Regalis, Delphinium. Lark heels: resist poison, help the bitings of renomous beasts.

Saracenica Solidago. Saracens Confound Helps inward wounds, sore mouths, sore throats, wasting of the lungs, and liver.

Coronepus. Buchorn Plantane, or Seaplantain: cold and dry, helps the bitings of venomous beasts, either taken invardly, or applied to the wound: helps the cholic, breaks the stone. Agineta.

Coronaria. Hath got many English names. Cottonweed, Cudweed, Chaffweed, and Petty Cotton. Of a drying and binding nature; boiled in lye, it keeps tho liead from nits and lice; being laid among clothes, it keeps them safe from moths, kills worms, helps the bitings of venomous $3 \mathrm{P}$ 
beasts; taken in a tobacco-pipe, it helps it is to poison; it is an admirable remedy coughs of the lungs, and vehement head- against wounds and gunshot, wounds made aches.

Cruciata. Crosswort: (there is a kind of Gentian called also by this name, which I pass by) is drying and binding, exceeding good for inward or outward wounds, either inwardly taken, or outwardly applied: and an excellent remedy for such as are bursten.

Crassula. Orpine. Very good: outwardly used with vinegar, it clears the skin; inwardly taken, it helps gnawings of the stomach and bowels, ulcers in the lungs, bloody-flux, and quinsy in the throat, for which lasi disease it is inferior to none, take not too much of it at a time, because of its coolness.

Crithamus, \&c. Sampire. Hot and dry, helps difficulty of urint, the yellow jaundice, provokes the menses, helps digestion, opens stoppings of the liver and spleen. Galen.

Cucumis Asinimus. Wild Cucumbers. See Elaterium.

Cyanus major, minor. Blue bottle, great and small, a fine cooling herb, helps, bruises, wounds, broken veins; the juice dropped into the eye, helps the inflanmations thereof.

Cygnoglossam. Hound's-Tongue, cold and dry: applied to the fundament helps the hemorrhoids, heals wounds and ulcers, and is a present remedy against the bitings of dog's, burnings and scaldings.

C'ypressus, Chame Cyparissus. Cypresstree. The leaves are hot and binding, help ruptures, and Polypus or flesh growing on the nose.

Chamce cyparissus. Is Lavender Cotton. Resists poison, and kills worms.

Disetımnus Cretensis. Dictamny, or Dittany of Creet, hot and dry, brings away dead children, hastens delivery, brings away the placenta, the verv smell of it druves away venomous peasts, so deadly an enemy with poisoned weapons, it draws out splinters, broken bones, \&c. 'The dose from half a dram to a dram.

Dipsacus, sativ. sylv. Teazles, garden and wild, the leaves bruised and applied to the temples, allay the heat in fevers, qualify the rage in frenzies; the juice dropped into the ears, kills worms in them, dropped into the eyes, clears the sight, helps redness and pimples in the face, being anointed with it. Ebulus. Dwarf Elder, or Walwort. Hot and dry in the third degree; waste hard swellings, being applied in form of a poultice; the hair of the head anointed with the juice of it turns it black; the leaves being applied to the place, help inflammations, burnings, scaldings, the bitings of mad dogs; mingled with bulls suet is a present remedy for the gout; inwardly taken, is a singular purge for the dropsy and gout.

Echium. Viper's-bugloss, Viper's-herb, Snake bugloss, Wal-bugloss; Wild-bugloss, several counties give it these several names: It is a singular remedy being eaten, for the biting of venomous beasts: continually eating of it makes the body invincible against the poison of serpents, toads, spiders, \&c. however it be administered; it comforts the heart, expels sadness and melancholy. The rich may make the flowers into a conserve, and the herb into a syrup, the poor may keep it dry; both may keep it as a jewel.

Empetron, Calcufragra, Herniaria, \&c. Rupture-wort, or Burst-wort. The English name tells you it is good against ruptures, and so such as are bursten shall find it, if they please to make trial of it, either inwardly taken, or outwardly applied to the place, or both. Also the Latin names liold it forth to be good against the stone, which whoso tries shall find true.

Enula Campana. Elicampane. Provokes urine. See the root.

Epthimum. Dodder of 'Time, to which 
add common Dodder, which is usually that which grows upon flax : indeed every Dodder retains a virtue of that herb or plant it grows upon, as Dodder that grows upon Broom, provokes urine forcibly, and loosens the belly, and is moister than that which grows upon flax: that which grows upon time, is hotter and dryer than that wich grows upon flax, even in the third degree, opens obstructions, helps infirmities of the spleen, purgeth miancholy, relieves drooping spirits, helps the rickets: 'That which grows on tlax, is excellent for agnes in young children, strengthens weak stomachs, purgeth choler, provolscs urine, opens stoppings in the reins and bladder. That which grows upon nettles, provokes urine exccedingly. "The way of using it is to boil it in white wine, or other convenient decoction, and boil it very little. Ritias, Mesue, Actuarius, Serapio, Avincena.

Eruch. Rucket, hot and dry third degree, being eaten alone, causeth head-ache, by its heat procures urine. Galen.

Eupatorium. Sce Ageratum.

Euphragia. Fyebright is something hot and dry, the very sight of it refresheth the eyes; inwardly taken, it restores the sight, and makes old men's eyes young, a dram of it taken in the morning is worth a pair of spcctacles, it comforts and strengthens the niemory, outwardly applied to the place, it helps the eyes.

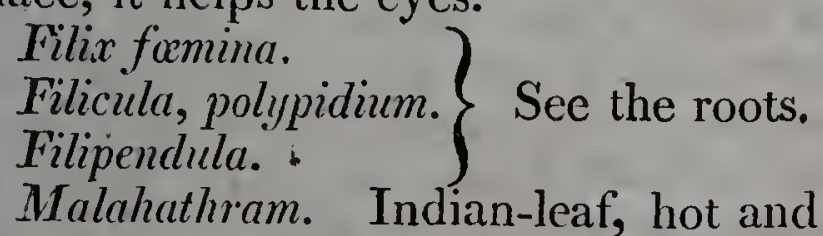
in the second degree, comforts the stomach exceedingly, helps digestion, provokes urine, helps inflammations of the eyes, secures cloaths fiom moths.

Ficriculım. Fennel, encreaseth milk in nurses, provokes urine, breaks the stone, easeth pains in the reins, opens stoppings, breaks wind, provokes the menses; you may boil it in white wine.
Fragaria. Strawberry leaves; are cold, dry, and binding, a singular remedy for inflammations and wounds, hot discases in the throat; they stop fluxes and the terms, cool the heat of the stomach, and the inflammations of the liver. The best way is to boil them in barley water.

Frainus, \&s. Ashli-trees, the leaves are: moderately lot and dry, curc the bitings of Adders, and Serpents; they stop looseness, and stay vomiting, help the rickets, open stoppages of the liver and spleen.

Fumaria. Fumitory: cold and dry, it opens and cleanses by urine, helps such as are itchy, and scabbed, clears the skin, opens stoppings of the liver and spleen, helps rickets, hypochondriac melancholy, madness, fienzies, quartan agues, loosens the belly, gently purgeth melancholy, and addust choler: boil it in white wine, and take this one general rulc. All things of $a$ cleansing or opening nature may be most commodionsly boiled in white wine. Runicmber but this, and then I need not repeat it.

Galega. Goat's-1'ue : 'Temperate in quality, resists poison, kills worms, helps the falling-sickness, resists the pestilence. You may take a dram of it at a time in powder.

Galion. Ladies-bed straw : dry and binding, stanches blood, boiled in oil, the oil is good to anoint a weary traveller; inwardly it provokes venery.

Gentiana. See the root.

Gerista. Brooms: hot and dry in the second degree, cleanse and open the stomach, break the stone in the reins and bladiler, help the green sickness. Let such as are troubled with heart-qualms or faintings, forbear it, for it weakens the heart and spirit vital. Sec the flowers.

Geranium. Cranebill, the divers sorts or it, one of which is that which is called Muscata ; it is thought to be cool and dry, helps hot swellings, and by its smell amends a hot orain. 
Geranium Columbinum. Doves-foot; helps milk in nurses, and outwardly by ointment the wind cholic, pains in the belly, stone in helps leprosies.

the reins and bladder, and is good in ruptures, and inward wounds. I suppose these are the general virtues of them all.

\section{Gramen. Grass : See the root.}

Gratiola. Hedge-Hyssop, purges water and flegm, but works very churlishly. Gesner commends it in dropsies.

Asphodelus form. See the root.

Hepatica, Lichen. Liverwort, cold and dry, good for inflammations of the liver, or any other inflammations, yellow jaundice.

Hedera Arborea, Terrostris. 'I'rce and Ground-Ivy. Tree-Ivy helps ulccrs, burnings, scaldings, the bad effects of the spleen; the juice snuffed up the nose, purges the head, it is admirable for surfcits or headache, or any other ill effects coming of drunkenness. Ground-Ivy is that which usually is called Alehoof, hot and dry, the juice helps noise in the ears, fistula's, gouts, stoppings of the liver, it strengthens the reins and stops the menses, helps the yellow jaundice, and other diseases coming of stoppings of the liver, and is excellent for wounded people.

Herba Camphorata. Stinking Groundpine, is of a drying quality, and therefore stops defluxions either in the eyes or upon the lungs, the gout, cramps, palsies, aches : strengthens the nerves.

Herbu Paralysis, Primula veris. Primroses, or Cowslips, which you will. The leaves help pains in the head and joints; see the flowers which are most in use.

Herba Paris. Heri True-love, or Oneberry. It is good for wounds, falls, bruises, aposthumes, inflammations, ulcers in the privities. Herb 'True-love, is very cold in temperature. You may take half a dram of it at a time in powder.

Herba Roberti. A kind of Cranebill.

Herba venti, Anemone. Wind-rlower. The juice snuffed up in the nose purgeth the head, it cleanses filthy ulcers, encreases for the gout, being roasted between two

Helxine. Pellitory of the wall. Cold,
Hellone moist, cleansing, helps the stonc and grarcl in the kidnics, difficulty of urine, sore throats, pains in the ears, the juice being dropped in them; outwardly it helps the shingles and St. Anthony's fire.

Hyppoglossum. Horse-tongue, Tongueblade or Double-Tongue. 'The roots help the stranguary, provoke urine, case the hard labour of womcn, provoke the menses, the herb helps ruptures and the fits of the mother : it is liot in the second degree, dry in the first: boil it in white wine.

Hyppolapathum. Patience, or Monk's Rhubarb: see the Root.

Hypposclimum. Alexanders, or Alisanders: provoke urine, cxpel the placenta, help the stranguary, expel wind.

Sage either taken inwardly or beaten and applied plaister-wise to the matrix, draws forth both menses and placenta.

Hormimm. Clary: liot and clry in the third degree; helps the weakness in the back, stops the running of the reins, and the Fluor Albus, provokes the menses, and helps women that are barren through coldness or moisture, or both: causes fruitfulness, but is hurtful for the memory. The usual way of taking it is to fry it with butter, or make a tansy with it.

Hydropiper. Arsmart. Hot and dry, consumes all cold swellings and blood congealed by bruises, and stripes ; applied to the placc, it helps that aposthume in the joints, commonly called a felon: strewed in a chamber, kills all the fleas there: this is hottest Arsmart, and is unfit to be given inwardly: there is a milder sort, called Persicaria, which is of a cooler and milder quality, drying, excellently good for putrified ulcers, kills worms : I had almost forgot that the former is an admirable remedy for the gout, being roasted between two 
tiles and applied to the grieved place, and yet I had it from Dr. Butler too.

Hysopus. Hysop. Helps coughs, shortness of breath, wheezing, distillations upon the lungs: it is of a cleansing quality: kills worms in the body, amends the whole colour of the body, helps the dropsy and spleen, sore throats, and noise in the ears. See Syrup of Hysop.

Hyosciamus, fic. Henbane. The white Henbane is held to be cold in the third degree, the black or common Henbane and the yellow, in the fourth. 'They stupify the senses, and therefore not to be taken inwardly, outwardly applied, they help inflammations, hot gouts: applied to the temples they provoke sleep.

Hypericon. St. John's Wort. gallant a wound-herb as any is, either given inwardly, or outwardly applied to the wound: it is hot and dry, opens stoppings, helps spitting and vomiting of blood, it cleanses the reins, provokes the menses, helps congealed blood in the stomach and meseraic veins, the falling-sickness, palsy, cramps and aches in the joints; you may give it in powder or any convenient decoction.

Hypoglottis, Laurus, Alexandrina. Laurel of Alesandria, provokes urine and the inenses, and is held to be a singular help to women in travail.

Hypoglossum, the same with Hypoglossum before, only different names given by different authors, the one deriving his name from the tongue of a horse, of which form the leaf is; the other the form of the little leat, because small leaves like small tongues grow upon the greater.

Iheris Cardamantice.

Sciatica - cresses. I suppose so called because they help the Sciatica, or Huckle-bone Gout.

In guvialis, Asther. Setwort or Shartwort: being bruised and applied, they help
swellings, botches, and venerous swellings in the groin, whence they took their name,

$(23,24$. as also inflammation and falling out of the fundament.

Iris. See the roots.

Isatis, Glastum. Woad. Drying and binding; the side being bathed with it, it easeth pains in the spleen, cleanseth filthy corroding gnawing ulcers.

Iva Arthritica. The same with Camapytis.

Iuncus oderatus. 'The same with Schananthus.

Labrum veneris. The same with Dipsacus.

Lactuca. Lettice. Cold and moist, cools the inflammation of the stomach, commonly called heart-burning: provokes sleep, resists drunkenness, and takes away the ill effects, of it; cools the blood, quenches thirst, breeds milk, and is good for choleric bodies, and such as have a frenzy, or are frantic. It is more wholesome eaten boiled than raw.

Logabus, Herba Leporina. A kind of Trefoil growing in France and Spain. Iet them that live there look after the virtues of it.

Lavendula. Lavender: Hot and dry in the third degree: the temples and foreliead bathed with the juice of it; as also the smell of the herb helps swoonings, catalepsis, falling-sickness, provided it be not accompanied with a fever. See the flowers.

Laureola. Laurel. The leaves purge upwarr and downward: they are good for rheumatic people to chew in their mouths, for they draw forth much water.

Laurus. Bay-tree. The leaves are hot and dry, resist drunkenness, they gently bind and help diseases in the bladder, help the stinging of bees and wasps, mitigate the pain of the stomach, dry and lieal, open obstructions of the liver and spleen, resist the pestilence.

Lappa Minor. The lesser Burdock.

Lentiscus. Mastich-tree. Botll the leaves and bark of it stop fluxes (being hot and dry in the second degree) spitting and evacuations of blood, and the falling out of the fundament.

3 a 
Lens palustris. Duckmeat: Cold and provoke urine; the young sprouts open moist in the second degree, helps inflamma- stoppings of the liver and spleen, clcanse tions, hol swellings, and the falling out of the blood, clear the skin, help scabs and the fundament, being warmed and applied itch, help agues, purge choler: they are to the place.

Lepidium Piperites. Dittander, Pepperwort, or Scar-wort: A hot fiery sharp herb, admirable for the gout being applied to the place: being only held in the hand, it helps the tooth-ache, and withall leaves a. wan colour in the hand that holds it.

Livisticum. Lovage. Clears the sight, takes away redness and freckles from the face.

\section{Libanotis Coronaria. See Rosemary.}

Linaria. Toad-flax, or Wild-flax: usually boiled and taken as they eatasparagus, but if you would kecp them, for they are excellent for these diseases, you may make them into a conserve, or into a syrup.

Lychnitis Coronaria: or as others write it, Lychnis. Rose Campion. I know no great physical virtue it hath.

Macis. See the barks.

Magistrantia. \&.c. Masterwort. Hot and dry in the third degree: it is good against poison, pestilence, corrupt and unwholesome air, helps windiness in the stomach, and dry, cleanses the reins and bladder, causeth an appetite to one's victuals, very provolies urine, opens the stoppings of the profitable in falls and bruises, congealed liver and spleen, and helps diseases coming and clotted blood, the bitings of mad-dogs; thereof: outwardly it takes away yellow- the leaves chewed in the mouth, cleanse the ness and deformity of the skin.

Lillium convallium. Lilly of the Valley. See the Howers.

Lingua Cervina. Hart's-tongue: drying and binding, stops blood, the menses and fluxes, opens stopping of the liver and spleen, and cliseases thence arising. 'The like quantity of Hart's-tongue, Knotgrass and Comfrey Roots, being boiled in water, and a draught of the decoction drunk every morning, and the materials which have boiled applied to the place, is a notable remedy for such as are bursten.

Limorinu. Sea.bugloss, or Marsh-bugloss, or Sea-Lavender; the seeds being very drying and binding, stop fluxes and the menses, help the cholic and siranguary.

Lotus urbana. Authors make some tlutter about this lierb, I conceive the best take it ointments. to be Trisolium Odoratum, Sivcet Trefoyl, which is of a temperate nature, cleanses the eycs gently of such things as hinder the sight, cures green wounds, ruptures, or burstness, helps such as urine blond or are the opens the liver and spleen, cleanses burstness, helps such as urine blond or are the breast and lungs, helps old coughs, Lupulus. Hops. Opening, cleansing, the lungs, it provolics the menses, eases
the ling the sides, ptisicks, or ulceration of

Marrubium, album, nigrum, fotidum.

Marribium album, is commion Horehound. Hot in the second degree, and dry in the third, opens the liver and spleen, cleanses 
hard labour in child-bearing, brings away the placenta. See the syrups. Murrubium, nigrum, et fietidum. Black
and stinking Horeliouud, I take to be all one. Hot and dry in the third degree; cures the bitings of mad dogs, wastes and consumes hard knots in the fundament and matrix, cloanses filthy ulcers.

Marum. Herb Mastich. Hot and dry in the third degree, good against cramps and convulsions.

Matricaria. Feverfew. Hot in the third degree, dry in the second; opens, purges ; a singular remedy for diseases incident to the matrix, and other diseases incident to women, eases their travail, and infirmities coming after it ; it helps the vertigo or dissiness of the hicad, melancholy sad thoughts : you may boil it eitler alone, or with other herbs fit for the sanie purpose, with which this treatise will furnish you: applied to the wrists, it helps the ague.

Matrisylia. 'I'he same with Caprifolium. Meliotus. Melilot. Inwardly taken, provokes urine, breaks the Stone, cleanses the reins and bladder, cutteth and cleanses the lungs of tough flegm; the juice dropped into the eyes, clears the sight, into the cars, mitigates pain and noise there; the liead bathed with the juice mixed with vinegar, takes away the pains thereof: outwardly in pultisses, it assuages swellings in the privities and elsewhere.

Mellissa. Balm. Hot and dry: outwardly mixed with salt and applied to the neck, helps the King's-evil, bitings of mad dogs, venomous beasts, and such as cannot hold their neck as they should do; inwardly it is an excellent remedy for a cold and moist stomach, cheers the heart, refieshes the mind, takes away griefs, sorrow, and care, instead of which it produces joy and mirth. Sce the syrup. Galen, Avicenmu.

Mentha sativa. Garden Mints, Spear Mints. Are hot and dry in the third degree, provoke hunger, are wholesome for the stomach, stay vomiting, stop the menses, help sore heads in children, strengthen the stornach, cause digestion; outwardly applied, they help the bitings of mad-dogs: Yet they hinder conception.

Memba aquatica. Watcr Mints: Ease pains of the belly, head-aclie, and vomiting, gravel in the kidnies and stone.

Methastrum. Horse-mint. I know no difference between them and watcr mints.

Mercurialis, mas, fcemina. Mercury male and fimale, they are both hot and dry in the second degree, cleansing, digesting, they purge watery humours, and further conception.

Mezerem. Spruge-Olive, or Widdowwail. A dangerous purge, better let alone than meddled wilh.

Millefolium. Yarrow. Mcanly cold and binding, an lealing herb for wounds, stanches bleeding; and some say the juice snuffed up the nose, causetlr it to blced, whence it was called, Nose-bleed; it stops lasks, and the menses, helps the running of the reins, holps inflammations and excoriations of the priapus, as also inflammations of wounds. Galen.

Muscus. Mosse. Is something cold and binding, yet usually retains a smatch of the property of the tree it grows on; therefore that which grows upon oaks is very dry and binding. Serapio saith that it being infused in wine, and the wine drank, it stays vomiting and fluxes, as also the Fluor Albus.

Myrtus. Myrtle-tree. The leaves are of a cold earthly quality, drying and binding, good for fluxes, spitting and vomiting of blood; stop the Flior Albus and menses.

Nerrlus. Sce the root.

Nasturtium, Aquaticum, Hortense. Water crrsses, and Garden-cresses. Gardencresses are hot and dry in the fourth degree, good for thes curvy, sciatica, hard swellings, yct io they trouble the belly, ease pains of thesplecn, provokelust. Dioscorides. Watercresses are hot and dry, eleanse the blood, 
help the scurvy, provoke urine and the menses, break the stone, help the green-sickness, cause a fresh lively colour.

Nasturtium Alhum, Thlaspie. mustard. Hot and dry in the third degree, purges violently, dangerous for pregnant women. Outwardly it is applied with profit to the gout.

Nicorimi. Tobacco. It is hot and dry in the second degree, and of a cleansing nature: the leaves warmed and applied to the head, are excellently good in inveterate head-aches and megrims, if the diseases come through cold or wind, change them often till the diseases be gone, help such whose necks be stiff: it eases the faults of the breast: Asthma's or head-flegm in the lappets of the lungs: eases the pains of the stomach and windiness thereof: being heated by the fire, and applied hot to the side, they loosen the belly, and kill worms being applied unto it in like manner: they break the stone being applied in like manner to the region of the bladder : help the rickets, being applied to the belly and sides: applied to the navel, they give present ease to the fits of the inother: they take away cold aches in the joints applied to them : boiled, the liquor absolutely and speedily cures scabs and itch: neither is there any better salve in the world for wounds than may be made of it: for it cleanses, fetches out the filth though it lie in the bones, brings up the flesh from the bottom, and all this it doth speedily: it cures wounds made with poisoned weapons, and for this Clusius brings many experiences too tedious here to relate. It is an admirable thing for carbuncles and plague-sores, inferior to none: green wounds 'twill cure in a trice: ulcers and gangreens very speedily, not only in men, but also in beasts, therefore the Indians dedicated it to their god. 'Taken in a pipe, it hath almost as many virtues; it easeth weariness, takes away the sense of hunger and thirst, provokes to stool: he saith, the Indians will travel four days without erther meat or drink, by only chewing a little of this in their mouths: It eases the body of superfluous humours, opens stoppings. See the ointment of Tobacco.

Nummularia. Money-wort, or Herb Two-pence; cold, dry, binding, helps fluxes, stops the menses, helps ulcers in the lungs; outwardly it is a special herb for wounds.

Nymphea. See the flowers.

Ocymum. Basil, hot and moist. The best use that I know of it, is, it gives specdy deliverance to women in travail. Let them not take above half a dram of it at a time in powder, and be sure also the birth be ripe, else it causes abortion.

Olece folia. Olive leaves: they are hard to come by here.

Ononis. Restharrow. See the roots.

Ophioglossum. Adder's-tongue. 'Theleaves are very drying: being boiled in oil they make a dainty green balsam for green wounds : taken inwardly, they help inward wounds.

Origanum. Origany: a kind of wild Marjoram ; hot and dry in the third degree, helps the bitings of venomous beasts, such as have taken Opium, Hemlock, or Poppy; provokes urine, brings down the menses, helps old coughs; in an ointment it helps scabs and itch.

\section{Oxylapathum. Sorrel. See Acetosa.}

Papaver, \&c. Poppies, white, black, or erratick. I refer you to the syrups of cach. Parietaria. Given once betore under the name of Helaine.

Pastincea. Parsnips. See the roots.

Persicaria. See Hydropiper. This is the milder sort of Arsmart I described there: If ever you find it amongst the compounds, take it under that notion.

Pentaphyllium. Cinquefoil: very drying, yet but meanly hot, if at all; helps ulcers in the mouth, roughness of the wind-pipe (whence comes hoarsness and coughs, \&c.) helps fluxes, creeping ulcers, and the yellow 
jaundice; they say one leaf cures a quotidian ague, three a tertain, and four a quartan. I know it will cure agues without this curiosity, if a wise man have the handling of it; otherwise a cart load will not do it.

Petroselimum. Parsley. See Smallage. Per Columbinus. See Geramium.

Persicarium folia. Peach Leaves: they are a gentle, yet a complete purger of choler, and disease coming from thence; fit for children because of their gentleness. You may boil them in white wine: a handfull is enough at a time.

Pilosella. Mouse-ear: once before and this is often enough.

Pithyusa. A new name for Spurge of the last Edition.

Plantago. Plantain. Cold and dry; an herb, though common, yet let none despise it, for the decoction of it prevails mightily against tormenting pains and excoriations of the entrails, bloody fluxes, it stops the menses, and spitting of blood, phthisicks, or consumptions of the lungs, the running of the reins, and the Fluor Albus, pains in the head, and frenzies: outwardly it clears the sight, takes away inflammations, scabs, itch, the shingles, and all spreading sores, and is as wholesome an herb as call grow about any an house. Tragus, Dioscorides.

Polium, f.c. Polley, or Pellamountain: All the sorts are hot in the second degree, and dry in the third: helps dropsics, the yellow jaundice, infirmities of the spleen, and provokes urine. Dioscorides.

Polygonum. Knotgrass.

Polytricum. Maidenhair.

Portulaca. Purslain: Cold and moist in the second or third degrce: cools hot stomachs, and it is admirable for one that hath his teeth on edge by eating sour apples, it cools the blood, liver, and is good for hot diseases, or inflammations in any of these places, stops fluxes, and the menses, and $(25,26$. helps all inward inflanmations whatsoever.

Porrum. Leeks. See the roots.

Primula Veris. See Cowslips, or the Flowers, which you will.

Prumella. Self-heal, Carpenter's-herb, and Sicklewort. Moderately hot and dry, binding. See Bugle, the virtues being the same.

Pulegium. Pennyroyal; hot and dry in the third degree; provokes urine, breaks the stone in the reins, strengthens women's backs, provokes the menses, easeth their labour in child-bed, brings away the placenta, stays vomiting, strengthens the brain, breaks wind, and helps the vertigo.

Pulmonaria, arborea, et Sympluytum maculosum. Lung-wort. It helps infirmities of the lungs, as hoarsness, coughs, wheezing, shortness of breath, \&c. You may boil it in Hyssop-water, or any other water that strengthens the lungs.

Pulicaria. Fleabane; hot and dry in the third dcgree, helps the biting of venomous beasts, wounds and swellings, the yellow jaundice, the falling sickness, and such as cannot make water ; being burnt, the smoak of it kills all the gnats and fleas in the chamber; it is dangerous for pregnant women.

Pyrus sylvestris. Wild Pear-tree. I know no virtue in the leaves.

Pyrola. Winter-green. Cold and dry, and very binding, stops fluxes, and the menses, and is admirably good in green wounds.

Quercus foliu. Oak Leaves: Are much of the nature of the former, stay the Fluor Albus. See the bark.

Ramenculns. Hatl got a sort of English Namcs : Crowfoot, King-kob, Gold-cups, Gold-knobs, Butter-flowers, \&c. they are of a notable hot quality, unfit to be taken inwardly: If you bruise the roots and apply them to a plaguc-sore, they are notable things to draw the venom to them.

Raparum folia. If they do mean Turnip -3.R 
leaves, when they are young and ténder, they are held to provoke urine.

Rosmarirum. Rosemary, hot and dry in the second degree, binting, stops fluxes, helps stuffings in the head, the yellow jaundice, helps the memory, expels wind. "See the flowers. Serapio, Dioscorides.

Rosa solis. See the water.

Rosa alba, rubra, Damascena. White, Red, and Damask Roses.

Rumer. Dock; All the ordinary sort of Docks are of a cool and drying substance, and therefore stop fluxes; and the leaves are seldom used in physic.

Rubus Idaus: Raspis, Raspberries, or Hind-berries: I know no great virtues in the leaves.

Ruta, Rue, or Herb of Grace; hot and dry in the third degree, consumes the seed, and is an enemy to generation, helps difficulty of breathing, and inflammations of the lungs, pains in the sides, inflammations of the priapus and matrix, naught for pregnant women: no herb resists poison more. It strengthens the heart exceedingly, and no herb better than this in pestilential times, take it what manner you will or can.

Ruta Muraria. Sce Adianthum.

Sabina. Savin: hot and dry in the third degree, potently provokes the inenses, expels both birth and afterbirth, they (boiled in oil and used in ointments) stay creeping ulcers, scour away spots, freckles and sunburning from the face; the belly anointed with it kills worms in children.

Salvia. Sage: hot and dry in the second or third degree, binding, it stays abortion in such women as are subject to come before their times, it causes fruitfulness, it is singularly good for the brain, strengthens the senses and memóry, helps spitting and vomiting of blood: outwardly, heat hot with a little vinegar and applied to the side, helps stitches and pains in the sides.

Salix. Willow leaves, are cold, dry, and binding, stop spitting of blood, and Huxes; the boughs stuck about a chamber, wonderfully cool the air, and refresh such as have. fevers; the leaves applied to the head, help hot diseases there, and frenzies.

\section{Sampsucum. Marjoram.}

Sunicula. Sanicle; hot and dry in the second degree, cleanses wounds and ulccrs.

Saponaria. Sope-wort, or Bruise-wort, vulgarly used in bruises and cut fingers, and is of notable use in the veneral disease

Satureia. Savory. Summer savory is hot and dry in the third degree, Winter. savory is not so hot, both of them expel wind.

Sazifragia atba. White Saxifrage, breaks wind, helps the cholic and stonc.

Scabiosa. Scabious: hot and dry in the second degrec, cleanses the breast and lungs, helps old rotten coughs, and difficulty of breathing, provokes urine, and cleanses the bladder of filthy stuff, breaks aposthumes, and cures scabs and itch. Boil it in white wine.

Scariola. An Italian name for Succory. Schonanthus. Schœnanth, Squinanth, or Chamel's hay; hot and binding. It digests and opens the passages of the veins: surely it is as great an expeller of wind as any is.

Scordium. Water-Germander, hot and dry, cleanses ulcers in the inward parts, it provokes urine and the menses," opens stopping of the liver, spleen, reins, bladder, and matrix, it is a great counter poison, and eases the breast oppressed with flegm: see Diascordium.

Scrophularia'. Figwort, so called of Scrophula, the King's Evil, which it cures they say, by being only hung about the neck. If not, bruise it, and apply it to the place, it helps the piles or hemorrhoids. Sedum. And all his sorts: see Barla Jovis.

Sema. It heats in the second degrec and dries in the first, cleinses, purges, and digests; it carries downward both choler, 
flegm, and melancholy; it cleanses the usually fornd about old ruinous buildings; brain, heart, liver, spleen; it cheers the it is so ealled beeause of its virtue in senses, opens obstructions, takes away dul- stopping fluxes.

ness of sight, helps deafness, helps melancholy and madness, resists resolution of the ncrves, pains of the head, seabs, iteh, falling-siekness, the windiness of it is eorreeted with a little ginger. You may boil half an ounce of it at a time, in water or white wine, but boil it not too muels; half an the herbs too; though I am of another ounce is a moderate dose to be boiled for opinion. any reasonable body.

Shinuchiu. Spinage. I never read any physieal virtues of it.

Spina Allia. See the root.

Spica. See Nardus.

Stcebe. Silver Knapweed: The virtues Scrpillum. Mother-of-Time, with Time; ; is a great counterpoison, opens obstructions it is hot and dry in the third degree, it pro- of the liver and spleen, cleanscs the matrix vokes the menses, and helps the stranguary and bladder, brings out eorrupt humours, or stoppage of urine, gripings in the belly, provokes urine.

ruptures, convulsions, inflanmation of the Succisa, Marsus Diaboli. Devil's-bit. liver, lethargy, and infirmities of the spleen, Hot and dry in the seeond degree: inwardly boil it in white wine. Atius, Galcn.

Sigillum Solomonis. Solomon's scal. the root.

Simyrnium. Alexander of Crete.

Solamum. Night-shade: very cold and dry, binding; it is somewhat danmerons the neck whieh the vulgar eall the almonds given inwardly, unless by a skillul hand, of the ears, than this herb bruised and outwardly it helps the Shingles, St. Anthony's applied to them. fire, and other hot inflammations.

Suchaha. An Egyptian Thorn. Very

Soldinclla. Bindweed, hot and dry in hard, if not impossible to come by here. the seeond degree, it opens obstruetions of : Tanacetum. Tansy : hot in the seeond the liver, and purges watery humours, and degree and dry in the third; the very smoll is therefore very profitable in dropsics, it is of it stays abortion, or miscarriages in very hurtful to the stomach, and therefore women; so it doth being bruised and apif taken inwardly it had need be well eor- plied to their navels, provokes urine, and rected with cinnamon, ginger, or annisseed, \&e.

Sonchus levis Asper. Sow-thistles smooth and rough, they are of a cold, watery, yet binding quality, good for frenzies, they inerease milk in nurses, and cause the ehildren which they nurse to have a good colour, help gnawings of the stomach coming of a liot cause; ontwardly they help inflammations, and hot swellings, eoo the heat of the fundament and privitics.

Sophi Chirurgoruin. Fluxweed: drying without any manifest heat or coldness; it is is a special help against the gont.

Taraxacon, Dandelion, or to write better Freneh, Dent-de-lion, for in plain English, it is called lyon's tooth ; it is a kind of Succory, and thither I refer you.

Tameriscus. Tamiris. It hath a dry eleansing quality, and hath a notable virtue against the riekets, and infirmities of the spleen; provokes the menses. Galen, Dioscorides.

Telcplium. A kind of Opine.

Thlaspi. Sec Naśturitum.

Thymbra. A wild Savory. 
Thymum. Thyme. Hot and dry in the a digesting, cleansing quality, stops fluxes third degree; helps coughs and sliortness of kreath, provokes the menses, brings away dead children and the after birth; purges Hegm, cleanses the breast and lungs, reins and natrix; lielps the sciatica, pains in the breast, expels wind in any part of the body, resists fearfulness and melancholy, continual pains in the head, and is profitable for such as have the falling-sickness to smell to.

Thymcelea. The Greek name for SpurgeOlive: Mezereon being the Arabick name.

Tithymallus, Esula, \&c. Spurge. Hot and dry in the fourth degree: a dogged purge, better let alone than taken inwardly: harr anointed with the juice of it will fall off: it kills fish, being mixed with any thing that they will eat: outwardly it cleanses ulcers, takes away freckles, sunburning and morphew from the face.

Tormentilla. See the root.

Trinitatis herba. Pansies, or Heart'sease: They are cold and moist, both herbs and flowers, excellent against inflammations of the breast or lungs, convulsions or falling-sickness, also they are held to be good for venereal complaints.

Trifolium. Trefoil: dry in the third degree, and cold: The ordinary Meadow Trefoil, cleanses the bowels of slimy humours that stick to them, being used either in drinks or clysters; outwardly they take away inflammations.

Tussilago. Colt's-foot : something cold and dry, and therefore good for inflammations, they are admirably good for coughs, and consumptions of the lungs, shortness of breath, \&c. It is often used and with good success taken in a tobacco-pipc, being cut and mixed with a little oil of annis seeds. See the Syrup of Colt's-foot.

Valeriana. V Valerian, or Setwall. See the roots.

Verbascum, Thapsus Barbatus. Mullin, they provoke lust, help difficulty of breathor Higtaper. It is something dry, and of ing, pleurisies, inflammations of the lungs, 
that troublesome cough that women call the Chincough; they exceedingly break the stone, provoke urine, and help such as cannot hold their necks upright. : Boil them in white wine.

Usnea. Moss; once before.

\section{FLOWERS.}

Bor AGE, and Bugloss flowers strengtnen the brain, and are profitable in fevers.

Chamomel flowers, heat and assuage swellings, inflammation of the bowels, dissolve wind, are profitably given in clysters or drink, to such as are troubled with the cholic, or stone.

Strechea, opens stoppings in the bowcls, and strengtheus the wliole body.

Saffron powerfully concocts, and sends out whatever humour offends the body, drives back inflammations; applied outwardly, encreases venery, and provokes urine.

Clove-Gilliflowers, resist the pestilence, strengthen theheart, liver, and stomach, and provoke venery.

Schenanth (which I touched slightly amongst the herbs) provokes urine potently, provokes the menses, breaks wind, helps such as spit or vomit blood, eases pains of the stomach, reins, and spleen, helps dropsies, convulsions, and inflammations of the womb.

Lazender-fozers, resist all cold afflictions of the brain, convulsions, falling-sickness, they strengthen cold stomachs, and open obstructions of the liver, they proroke urine and the menses, bring forth the birth and placenta.

Hops, open stoppings of the bowels, and for that cause beer is better than ale.

Bulm-flowers, clieer the heart and vital spirits, strengthen the stumach.

Rosemary-flowers, strengthen the brain exccedingly, and resist madness; clear the sight.

$(25,26$.
Winter-Gilliflowers, or Wall-flowers, help inflammation of the womb, provolie the menses, and help ulcers in the mouth.

Honey-suchles, provoke urine, ease the pains of the spleen, and such as can hardly fetch their breath.

Mallows, help coughs.

Red Roses, cool, bind, strengthen both vital and animal virtue, restore such as are in consumptions, strengthen. There are so many compositions of them which makes me more brief in the simples.

Violets, (to wit, the blue ones,) cool and moisten, provoke sleep, loosen the belly, resist ferers, help inflammations, correct the heat of choler, ease the pains in the head, help the roughness of the wind-pipe, diseases in the throat, inflammations in the breast and sicles, plurisies, open stoppings of the liver, and help the yellow.jaundice.

Chicory, (or Succory as the vulgar call it) cools and strengthens the liver, so doth Endive.

Watev lilies ease pains of the head coming of choler and heal, provoke sleep, cool intlammations, and the heat in fevers.

Pomegranatc-fluzers, dry and bind, stop fluxes, and the menses.

Cowslips, strengthen the brain, senses, and memory', excecdingly, resist all diseases there, as convulsions, falling-sickness, palsies, sec.

Contanry, purgescholer and gross humours helps the yellow jaundice, opens ohstructions of the liver, helps pains of the spleen, prorokes the menses, brings awaly birth and afterbirth.

Elder flowers, help dropsies, cleanse the blood, clear the skin, open stoppings of the liver and spleen, and diseases arising therefrom.

Bean-fozers, clear the skin, stop humours flowing into the eyes

Peach-tree flowers, purge choler gently.

Broom-fluzers, purge water, and are good in dropsies.

$3 \mathrm{~s}$ 
The temperature of all these differ either veriy little or not at all from the herbs.

'I'he way of using the flowers I did forbear, because most of them may, and are usually made into conserves, of which you cold, heats the stomach. may take the quantity of a nutmeg in the morning; all of them may be kept dry a ycar, and boiled with other herbs conducing to the cures they do.

\section{FRUITS AND THEIR BUDS.}

Green Figs, are held to be of ill juice, but the hest is, we are not much troubled with them in England; dry figs help coughs, cleanse the breast, and help infirmities of the lungs, shortness of wind, they loose the belly, purge the reins, help inflammations of the liver and splcen; outwardly they dissolve swellings.

Pine-nuts, restore such as are in consumptions, amend the failings of the lungs, concoct flegm, and yet are naught for such as are troubled with the head-ache.

Dates, are binding, stop eating ulcers being applied to them; they are rery good for weak stomachs, for they soon digest, and breed good nourishment, they help infirmities of the reins, bladder, and womb.

Sebestens, cool choler, violent heat of the stomach, help roughness of the tongue and wind-pipe, cool the reins and bladder.

Rusisins of the Sum, help infirmities of the breast and liver, restore consumptions, gently cleanse and move to stool.

Walnuts, kill worms, resist the pestilence, (I mran the green ones, not the dry.) ger.

Capers eaten before meals, provoke hun-

Nutmegs, slrengthen the brain, stomach, and liver, provoke urine, ease the pains of the splicn, stop looseness, ease pains of the liead, and pains in the joints, strergthen the body, rake away weakness coming of cold, and cause a sweet breath.
Cloves, help digestion, stop looseness, provoke lust, and quicken the sight.

Pepper, binds, expels wind, helrs the cholic, quickens digestion oppressed with Quinces. See the Compositions.

Pears are grateful to the stomach, drying, and therefore help fluxes.

All plums that are sharp -or sour, are binding, the sweet are loosening.

Cucumbers, cool the stomach, and are good against ulcers in the bladder.

Galls, are exceeding binding, help ulcers in the mouth, wasting of the gums, ease the pains of the teeth, help the falling out of the womb and fundament, make the hair black.

Pompions are a cold and moist fruit, of small nourishment, they provoke urine, outwardly applied; the flesh of them helps inflammations and burnings; applied to the forehead they help inflammations of the eyes. Melons, have few other virtues.

Apricots, are very grateful to the stomach, and dry up the humours thereof. Peaches are held to do the like.

Cubebs, are hot and dry in the third degree, they expel wind, and cleanse the stomach of tough and viscous humours, they case the pains of the spleen, and help cold diseases of the womb, they cleanse the head of flegm and strengthen the brain, they heat the stomach and provoke vencry.

Bitter. Almonds, are hot in the first degree and dry in the second, they cleanse and cut thick humours, cleanse the lungs; and eaten every morning, they are held to preserve from drunkenness.

Bay-berries, heat, expel wind, mitigate pain ; are excellent for cold infirmities of the womb, and dropsies.

Cherries, are of different qualities according to their different taste, the sweet are quickest of digestion, but the sour are more pleasing to a hot: stomach, and procure appetite to one's meat. 
Medlars, are strengthening tothe stomach, confident a clild of three years old, if you binding, and the green are more binding should give it Raisins of the sun or Cherries, than the rotten, and the dry than the green. would not ask how it should take them.

Olives, cool and bind.

English-currants, cool the stomach, and are profitable in acute fevers, they quench thirst, resist vomiting, cool the heat of choler, provoke appetite, and are good for hot complexions.

Services, or Chockers are of the nature of Medlars, but something weaker in operation.

Barberries, quench thirst, cool the heat of choler, resist the pestilence, stay vomiting and fluxes, stop the menses, kill worms, help spitting of blood, fasten the teeth, and strengthen the guns.

Stratsberries, cool the stomach, liver, and blood, but are very hurtful for such as have agues.

Winter-Cherries, potently provoke urine, and break the stone.

Cassia-fistula, is temperate in quality, gently purgeth choler and flegm, clarifies the blood, resists fevers, cleanses the breast and lungs, it cools the reins, and thereby resists the breeding of the stone, it provokes urine, and therefore is excecding good for the running of the reins in men, and the Fluor Albus in women.

All the sorts or Myrobalans, purge the stomach; the Indian Myrobalans, are held to purge melancholy most especially, the other flegm; yet take heed you use them not in stoppings of the bowels: they are cold and dry, they all strengthen the heart, brain, and sinews, strengthen the stomach, relieve the senses, take away tremblings and heart-qualms. They are seldom used alone.

Prunes, are cooling and loosening.

Tamarinds, are cold and dry in the second encreases seed, kills worms, eascs pains of degree, they purge choler, cool the blood, stay vomiting, help the yellow jaundice, Busil secut: If we may believe Diosco. quench thirst, cool hot stomachs, and hot rides and Crescentius, chcers the heart, and livers.

strengthens a noist stomach, drives away I omit the use of these also as resting 'melancholy, and provokes urine. 
Nettle secd, provokes venery, opens stoppages of the womb, helps inflamnations of of the sides and lungs; purgeth the breast: boil them (being bruised) in white wine also.

The seeds of Ammi, or Bishop's-zeed, heat and dry, help difficulty of urine, and the pains of the cholic, the bitings of venomous beasts; they provoke the menses, and purge the womb.

Ammis secds, heat and dry, ease pain, expel wind, cause a sweet breath, help the dropsy, resist poison, breed milk, and stop the Fluor Albus in women, provoke venery, and ease the head-ache.

Cardamoms, heat, kill worms, cleanse the reins, and provoke urine.

Femel sced, breaks wind, provokes urine and the menses, encreases milk in nurses.

Cummin sced, heat, bind, and dry, stop blood, expel wind, ease pain, help the bitngs of venomous beast: outwardly applied (viz. in Plaisters) they are of a discussing nature.

Carrot seeds, are windy, provoke lust exceedingly, and encrease seed, provoke urine and the menses, cause speedy delivery to wonen in travail, and bring away the placenta. All these also may be boiled in white wine.

Nigella seeds, boiled in oil, and the forenead anvinted with it, ease pains in the head, take away leprosy, itch, scurf, and holp scald heads: Inwardly taken they expel worms, they provolie urine, and the menses, help difficulty of breathing.

Staresncre, kills lice in the head, I hold it not fiting to be given inwardly.

Olibanum mixed with as much Barrow's Grease (beat the Olibanum first in powder) and boiled together, make an ointment which will kill the lice in children's heads, and such as are subject to breed them, will never breed them. A Medicine cheap, safe, and sure, which breeds no annoyance to the brain.
The seeds of Water-cresses, heat, yet trouble the stomach and belly; ease the pains of the spleen, are very dangerous for pregnant women, yet they provoke lust: outwardly applied, they help leprosies, scald heads, and the falling off of hair, as also carbuncles, and cold ulcers in the joints.

Mustard seed, heats, extenuates, and draws moisture from the brain: the head being shaved and anointed with Mustard, is a good renedy for the lethargy, it helps filthy ulcers, and hard swellings in the month, it hclps old aches coming of cold.

French Barley, is cooling, nourishing, and breeds milk

Sorrel sceds, potently resist poison, help fluxes, and such stomachs as loath their meat.

Succory seed, cools the heat of the blood, extinguishes lust, opens stoppings of the liver and bowcls, it allays the heat of the body, and produces a good colour, it strengthens the stomach, liver, and reins.

Poppy steds, ease pain, provoke sleep. Your best way is to make an emulsion of them with barley water.

Mallow seeds, ease pains in the bladder. Chich-pease, are windy, provoke lust, encrease milk in nurses, provoke the menses, outwardly, they help scabs, itch, and inflammations of the testicles, ulcers, \&c.

White Sarifrage sceds, provoke urine, expel wind, and break the stone. Boil them in white wine.

Rue seeds, helps such as cannot hold their water.

Lettice seed, cools the blood, restrains venery.

Also Gourds, Citruls, Cucumbers, Melons, Purslain, and Endive seeds, cool the blood, as also the stomach, spleen, and reins, and allay the heat of fevers. Use them as you werc taught to do poppy-seeds.

Wormseed, expels wind, kills worms.

Ash-tree Keys, ease pains in the siles, help the dropsy, relieve men weary with 
labour, provoke venery, and make the body name you please, is a desperate purge, hurtlean.

ful to the body by reason of its heat, windi-

Piony seeds, help the Ephialtes, or the ness, corroding, or gnawing, and vinlence disease the vulgar call the Mare, as also the of working. I would advise my countrymen fits of the mother, and other such like in- to let it alone; it will gnaw their bodies as firmities of the womb, stop the menses, and fast as doctors gnaw their purses.

help convulsions.

Broom seed, potently provoke urine, break the stone.

Citron seeds, strengthen the heart, chcer the vital spirit, resist pestilence and poison.

\section{TEARS, LIQUORS, AND ROZINS.}

Laudanum, is of a heating, mollifying nature, it opens the mouth of the veins, stays the hair from falling off, helps pains in the ears, and hardness of the womb. It is used only outwardly in plaisters.

Assafotida. Is commonly used to allay the fits of the mother by smelling to it; they say, inwardly taken, it provokes lust, and expels wind.

Benzoin, or Benjamin, makes a good perfume.

Sanguis Draconis, cools and binds exceedingly.

Aloes, purges choler and flegm, and with such deliberation that it is often given to withstand the violence of other purges, it preserves the senses and betters the apprehension, it strengthens the liver, and helps the yellow-jaundice. Yet is naught for such as are troubled with the hemorrhoids, or have agues. I do not like it taken raw. Sec Aloe Rosata, which is nothing but it washed with the juice of roses.

Manna, is temperately hot, of a mighty dilative quality, windy, cleanses choler gently, also it cleanses the throat and stomach. A child may take an ounce of it at a time melted in milk, and the dross strained out, it is good for them when they are scabby.

Scamony, or Diagridium, call it by which $(25,26$.
Opopanax, is of a heating, molifying, digesting quality.

Gum Elemi, is exceeding good for fractures of the skull, as also in wounds, and therefore is put in plaisters for that end. See Arceus his Liniment.

Tragacanthum, commonly called Gum Traganth, and Gum Dragon, helps coughs, hoarseness, and distillations on the lungs.

Bdellium, heats and softens, helps hard swellings, ruptures, pains in the sides, hardness of the sinews.

Galbanum. Hot and dry, discussing; applied to the womb, it hastens both birth and after-birth, applicd to the navel it stays the strangling of the womb, commonly called the fits of the mother, helps pains in the sides, and difficulty of breathing, being applied to it, and the smell of it helps the vertigo or diziness in the head.

Myrh, heats and dries, opens and softens the womb, provokes the birth and afterbirth ; inwardly taken, it helps old coughs and hoarsencss, painsin the sides, kills worms, and helps a stinking breath, helps the wasting of the gums, fastens the teeth: outwardly it helps wounds, and fills up ulcers with flesh. You may take half a dram at a time.

Mastich, strengthens the stomach exceedingly, helps such as vomit or spit blood, it fastens the teeth and strengthens the gums, being chewed in the mouth.

Frankinsense, and Olibanum, heat and bind, fill up old ulcers with flesh, stop bleeding, but is extremely bad for mad people. Turpentine, Purges, cleanses the reins, helps the running of them.

Styrax Calamitis, helps coughs, and distillations upon the lungs, hoarseness, want $3 \mathrm{~T}$ 
of voice, hardness of the womb, but it is bad for head-aches.

Ammonicaum, applied to the side, helps the hardness and pains of the spleen.

Camphire, eases pains of the head coming of heat, takes away inflammations, and cools any place to which it is applied.

\section{JUICES.}

Tuat all juices have the same virtues sight, help the vices of the nerves, resist poison with the herbs or fruits whereof they are exceedingly, neither is there any better remedy made, I suppose few or none will deny, under the sun for their bitings than the head therefore I shall only name a few of them, and that briefly.

Sugar is held to be hot in the first degree, strengthens the lungs, takes away the roughness of the throat, succours the reins and blardder.

The juice of Citrons cools the blood, strengthens the heart, mitigates the violent heat of fevers.

The juice of Lemons works the same eftect, but not so powerfully.

Juice of Liquorice, strengthens the lungs, helps coughs and colds.

\section{THINGS BRED FROM PLAN'IS.}

These have bcen treated of before, only täo excepted. The first of which is,

Agaricus. Agarick: It purges flegm, choler, and melancholy, from the brain, nerces, muscles, marrow, (or more properly brain) of the back, it cleanses the breast, lungs, liver, stomach, spleen, reins, romb, joints; it protokes urine, and the menses, liills worms, helps pains in the joints, and causes a good colour: it is very seldom or never taken alone. See Syrup of Roses with Agarick.

Lastly, Vicus Quircinus, or Misleto of the Oak, helps the falling-sickness being either taken inwardly, or hung about one's neck.

\section{LIVING CREATURES.}

Millepedes (so called from the multitude of their feet, though it camnot be supposed they have a thousand) sows, hog-lice, wood-lice, being bruised and mixed with wine, they provole urine, help the yellow jaundice: outwardly being boiled in oil, help pains in the ears, a drop being put into them.

The flesh of vipers being eaten, clear the
ceedingly, neither is there any better remedy
nder the sun for their bitings than the head
the viper that bit you, bruised and applied to the place, and the flesh eaten, you need not eat aboie a dram at a time, and make it up as you shall be taught in troches of vipers. Neither any comparable to the stinging of bees and wasps, 'flc. than the same that sting you, bruised and applied to the place.

Land Scorpions cure their oren stingings ly the same means; the ashes of them (being burnt) potently provolies urine, and breaks the stone.

Earth-worms, are an admirable remedy for cut nerces being applied to the place; they provole urine; see the oil of them, only let me not forget one notable thing quoted by Mizaldus, which is, That the pozider of them put into an hollow tooth, makes it drop out.

To draw a tooth without pain, fill an earth eggs and all, and when you have buned them, licep the ashes, with which if you touch a tooth it will fall out.

Ecls, being put into wine or beer, and suffered to die in it, he that drinks it will nerer. endure that sort of liquor again.

Oysters applied alive to a pestilential sacelling, draw the venom to them.

Crab-fish, burnt to ashes, and a dram of it taken every morning helps the biting's of mad dogs, and all other venomous beasts.

Swallows, being eaten, clear the sight, the ashes of them (being burnt), eaten, preserves en crucible fiull of Emmets, Ants, or Pismires, 
from drunkemness, helps sore throats being applied to them, and inflammations.

Grass-hoppers, being eaten, ease the cholic, and pains in the bladder.

Hedge Sparrows, being kept in salt, or dried and eaten raw, are an admirable remedy for the stone.

Young Pigeons being eaten, help pains in the reins, and the disease called Tenesmus.

\section{PARTS OF LIVING CREATURES, AND EXCREMENTS.}

THE brain of Sparrow's being eaten, provokes lust exceedingly.

The brain of an Hare being roasted, helps trembling, it makes children breed tcetis easily, their gums being rubbed with it, it also helps scald lieads, and falling off of hair, the head being anointed with it.

The head of a young Kite, being burnt to ashes and the quantity of a drachm of it taken every morning in a little water, is an admirable remedy against the gout.

Crab-eyes break the stone, and open stoppings of the bowels.

The lungs of a For, wcll dried, (but not burned) is an admirable strengthener to the lungs: see the Lohoch of Fox lungs.

The liver of a Duck, stops fluxes, and strengthens the liver exccedingly.

The liver of a Frog, being dried and eaten, helps quartan agues, or as the vulgar call them, third-day agues.

Castoreum resists poison, the bitings of venomous beasts; it provokes the menses, and brings forth birth and after-birth: it expels wind, eases pains and aches, convulsions, sighings, lethargies; the smell of it allays the fits of the mother ; inwardly given, it helps tremblings, falling-sickness, and other such ill effects of the brain and nerves: A scruple is enough to take at a time, and indeed spirit of Castorium is bet- ter than Castorium, raw, to which I refer you.

A Sheep's or Goat's bladder being burnt, and the ashes given inwardly, helps the Diabetes.

A flayed Mouse dricd and beaten into powder, and given at a time, helps such as cannot hold their water, or have a Diabetes, if you do the like three days together.

Ivory, or Elephant's tooth, binds, stops the Whites, it strengthens the heart and stomach, helps the yellow jaundice, and makkes women fruitful.

'Those sinall bones which are found in the fore-feet of an Hare, being beatcn into powder and drank in wine, powerfully provoke urine.

Goose grease, and Capons grease, are both softening, help gnawing sores, stiffiness of the womb, and mitigate pain.

I am of opinion that the suet of a Goat mixed with a little saffion, is as excellent an ointment for the gont, especially the gout in the knees, as any is.

Bears grease stays the falling off of the hair.

For grease helps pains in the ears.

Elk's Cluze's or hoof's are a sovereign remedy for the falling sickness, though it be but worn in a ring, much more being taken inwardly; but saith Mizaldus, it must be the hoof of the right foot behind.

Milk is an extreme windy meat; therefore $I$ am of the opinion of Dinscorides, viz. that it is not profitable in head-aches; yet this is for certain, that it is an admirable remerly for inward ulcers in any part of the body, or any corrosions, or excoriations, pains in the reins and bladder: but it is very bad in diseases of the liver, spleen, the falling-sickness, vertigo, or dissiness in the head, fevers and head-aches; Goat's milk is hild to be better than Cow's for Hectic fevers, phthisick, and consumptions, and so is Ass's also.

Whey, attenuates and cleanses both cho- 
ler and melancholy: wonderfully helps the Fluor Albus, helps such as spit blood, melancholy and madness coming of it; it is an approved remedy for the falling opens stoppings of the bowels; helps such sickness. Also if ten grains of red Coral as have the dropsy and are troubled with be given to a child in a little breast-milk so the stoppings of the spleen, rickets and soon as it is born, before it take any other hypochondriac melancholy: for such dis- food, it will never have the falling-sickness, eases you may make up your physic with nor convulsions. The common dose is whey. Outwardly it cleanses the skin of from ten grains to thirty.

such deformities as come through choler or Pearls, are a wonderful strengthener to melancholy, as scabs, itch, morphew, lepro- the heart, encrease milk in nurses, and sies, \&c.

Honey is of a gallant eleansing quality, exceeding profitable in all inward ulcers in what part of the body soever; it opens the resist fevers. The dose is ten grains or veins, cleanses the reins and bladder. I fewer; more, I suppose, because it is dear, know no vices belonging to it, but only it than because it would do harm. is soon converted into choler.

Wax, softens, heats, and meanly fills sores with flesh, it suffers not the milk to curdle in women's breasts ; inwardly it is given (ten grains at a time) against bloodyfluxes.

Amber, (viz. yellow Amber) heats and dries, therefore prevails against moist diseases of the head; it helps violent coughs, helps consumption of the lungs, spitting of blood, the Fluor Albus; it stops bleeding at the nose, helps difficulty of urine: You

Raw-silk, heats and dries, cheers the heart, drives away sadness, comforts all the spirits, both natural, vital and animal.

may take tcn or twenty grains at a time.

The Froth of the Sea, it is hot and dry, helps scabs, itch, and leprosy, scald heads, \&c. it cleanses the skin, helps difficulty of unine, makes the teeth white, bcing rubbed

BELONGING TO THE SEA.

Sperma Cati, is well applied outwardly to with hair. eating ulcers, the marks which the small ? pox leaves behind them; it clears the sight, provokes sweat; inwardly it troubles the stomach and belly, helps bruises, and stretching of the nerves, and therefore is good for women newly delivered.

Amber-grease, heats and dries, strengthens the brain and nerves exceedingly, if the infirmity of them come of cold, resists pestilence.

Sea-sand, a man that hath the dropsy, being set up to the middle in it, it draws out all the water.

Red Coral, is cold, dry and binding, Red Coral, is cold, dry and binding, fevers, falling-sickness, and all such like
stops the immoderate flowing of the menses, infirmities, incident either to the vital or stops the immoderate flowing of the menses, infirmities, incid
bloody-fluxes, the running of the reins, and animal spirit.

\section{METALS, MINERALS, AND S'TONES.}

GOLD is temperate in quality, it wonderfully strengtleens the heart and vital spirits, which one perceiving, very wittily inserted these verses :

For Gold is cordial : and that's the reason,

Your raking Misers live so long a seasnn.

However, this is certain, in cordials, it resists melancholy, faintings, swonnings, 
Alum. Heats, binds, and purges; scours filthy ulcers, and fastens loose teeth.

and consumptions, viz. taken inwardly; for this stone is not used to be worn as a jewel; Brimstone, or flower of brimstone, which the powder of it put upon wounds made is brimstone refined, and the better for by venomous beasts, draws out the poison. physical uses; helps coughs' and rotter ' Topaz (if Epiphanius spake trutlı)' if you Hlegm; outwardly in ointments it takes away put it into boiling water, it doth so cool it leprosies, scabs, and itch; inwardly it helps that you may presently put your hands into yellow jaundice, as also worms in the belly, it without harm; if so, then it cools inespecially being mixed with a little Salt- flammations of the body by touching them. petre: it helps lethargies being snuffed up Toadstone; Being applied to the place in the nose.

Litharge, both of gold and silver; binds and dries much, fills up ulcers with flesh, and heals them.

Lead is of a cold dry earthly quality, of an healing nature; applied to the place it helps any inflanmation, and dries up humours.

Pompholix, cools, dries and binds.

Jacynth, strengthens the heart being either beaten into powder, and taken inwardly, or only worn in a ring.

Sapphire, quickens the senses, helps such as are bitten by venomous beasts, ulcers in the bowels.

Emerald; called a chaste stone because it resists lust: being worn in a ring, it helps, or at least mitigates the falling sickness and vertigo; it strengthens the memory, and stops the unruly passions of men.

Ruby (or carbuncle, if there be such a stone) restrains lust; resists pestilence; takes away idle and foolish thoughts, makes men' cheerful. Cardanus.

Granite. Strengthens the heart, but hurts the brain, causes anger, takes away sleep.

Diamond, is reported to make him that bears it unfortunate.

Amethist, being worn, makes men sober and steady, keepsmen from drunkennessand too much sleep, it quickens the wit, is profitable in huntings and fightings, and repels vapours from the head.

Bezoar, is a notable restorer of nature, a great cordial, no way hurtful nor danger- I hope my fault is not much, or at a least. ous, is admirably good in fevers, pestilences,

$(25,26$. helps the bitings of venomous beasts, and quickly draws all the poison to it; it is known to be a true one by this; hold it near to any toad, and she will make proffer to take it away from you if it be right; else not. Lemmius.

Nephritichus lapis; helps pains in the stomach, and is of great force in breaking and bringing away the stone and gravel.

Jasper; being worn, stops bleeding, eases the labour in women, stops lust, resists fevers and dropsies. Mathiolus.

Atites, or the stone with child, because being hollow in the middle, it contains another little stone within it, is found in an Eagle's nest, and in many other places; this stone being bound to the left arm of women with child, stays their miscarriage or abortion, but when the time of their labour comes, remove it from their arm, and bind it to the inside of their thigh, and it brings forth the child, and that (almost) without any pain at all. Dioscorides, Plimy. Lapis Lazuli, purges melanclioly being taken inwardly; outwardly worn as a jewel, it makes men cheerful, fortunate and rich.

And thus I end the stones, the virtues of which if any think incredible, I answer; 1. I quoted the authors where I had them. 2. I know nothing to the contrary but why it may be as possible as the sound of a trumpet is to incite a man to valour; or a fiddle to dancing: and if $I$ have added a few simples which the Colledge left out, $3 \mathrm{v}$ 


\section{A CA'TALOGUE OF SIMPLES}

IN TII E

\section{NEW DISPENSATORY.}

ROO'TS.

Narew, Spikenard, Celtic ana Induan, Water lilies, Rest-harrow', sharp pointed Dock, College.] Sorrel, Calamus Aromaticus, Peony, male and female, Parsinps, garden and Water-Alug, Privet, Garlick, Marsh-mallows, wild, Cinquefoil, Butter-Bu", Parsley, Hog's Alcanet, Angelica, Anthora, Smallage, Aron, Fennel, Valerian, greater and lesser, Burnet, Birth-wort long and round, Sowbread, Reeds, Land and Water Plantaim, Polypodium of the Asarabacca, Virginian Snakezeed, Swall- Oak, Solomon's Seas, Leeks, Pellitory of Spain, wort, Asparagus, Asphodel, male and female. Cinquefoil, Turnips, Raddishes, gardenandwild, Burdocls great and small, Behen, or Bazil, Rhapontick, common Rhubarb, Monk's RhuValerian, white and red. Daisies, Beets, barl, Rose Root, Madder Bruscus. Soperort, vhite, red, and black. Marsh-mallows, Bis- Sarsaparilla, Satyprion, male and female, White iort, Borrage, Briony, white and black, Bugloss, Sarifrage, Squills, Figzort, Scorzonera, Engzarden and wild. Calamus Aromaticus, Our lish and Spanish, Virginian Snake zeed, SoloLady's thistles, Avens, Coleworts, Centaury the mon's Seal, Cicers, stinking Gladnn, Devil's bit, less. Onions, Chameleon, white and black. Dandelion, Thapsus, Tormentil, Turbith, Colt'sCelandine, Pilezort. China, Succory, Arti-foot, Vulerian, greater and lesser, Vervain, chokes. Virginian Snakcroot, Comfry greater Swallow-zwort, Nettles, Zedoary long and round, and lesser. Contra yerra, Costus, sweet and Ginger.

bitter. Turmerick, wild Cncumbers, Sowbread, Culpeper.] These be the roots the colHound's-tongue, Cypres, long and round. lege hath named, and but only named, and Toothwort, white Dittany, Doronicum, Dra- in this order I have set them down. It gons, Woody Niglatshade, Vipers Bugloss, seems the college holds a strange opinion, Smallage, Hellebore, white and black, Endive, viz. 'That it would do an Englishman a Elicampane, Eringo, Colt's-foot, Fearn, male mischief to know what the herbs in his and female, Filipendula or Drop-wort, Fennel, garden are good for.

white Dittany, Galanga, great and small, But my opinion is, that those herbs, Gentian, Liquorice, Dog-gress, Hermodactils. roots, plants, \&c. which grow near a man, Swallow wort, Jacinth, Herbune, Jallap, are far better and more congruous to his Master-wort, Orris or Flower-de-luce, both nature than any outlandish rubbish whatEnglish and Florentine, sharp pointed Dock, soever, and this I am able to give a reason Burdocli greater and lesser, Lotage, Privet, of to any that shall demand it of me, therewhite Lilies, Liquorice, Mallows, Mechoacan, fore I am so copious in handling of them, Jallap, Spignel, Mercury, Devil's bat, szect you shall observe them ranked in this order 
1. The temperature of the roots, herbs, flowers, \&c. viz. Hot, cold, dry, moist, Lecks, Pellitory of Spain. together with the degree of each quality.

2. What part of the body crch root, herb, flower, is appropriated to, viz. head, throat, breast, heart, stomach, liver, spleen, bowels, reins, bladder, womb, joints, and in those which heat those places, and which cool them.

3. The property of each simple, as they bind, open, molify, harden, extenuate, discuss, draw out, suppure, cleanse, glutinate, break wind, breed seed, provoke or stop the menses, resist poison, abate swellings, ease pain.

This I intend shall be my general method throughout the simples, which, having finished I shall give you a paraphrase explaining these terms, which rightly considered, will be the key of Galen's way of administering physic.

\section{Temperature of the Roots.}

Roots hot in the first degree. Marsl-mallows, Bazil, Valerian, Spattling, Poppy, Burdocks, Borrage, Bugloss, Calamus Aromaticus, Avens, Pilewprt, China, Selfheal, Liquorice, Dog-grass, white Lilies, Peony, male and female, wild Parsnips, Parsley, Valerian, great and small, Knecholly, Satyrion, Scorzonera, Skirrets.

Hot in the second degree. Water-flag, Reeds, Swallow-wort, Asphodel, male, Carline 'Ihistle, Cypress, long and round, Fennel, Lovage, Spignel, Mercury, Devil's bit, Butter Bur, Hog's Fennel, Sarsa parilla, Squils, Zedoary.

Hot in the third degree. Angelica, Aron, Birthwort long and round, Sowbread, Asarabacca, Briony, white and black, Sallendine, Virgianian snakeroot, Hemeric, White Dittany, Dorouicum, Hellebore, white and b'ack, Elicarpane, Fillipendula, Gialanga greater and lesser, Masterwort, Oris Eng!ish and Florentine, Restharrow, anking Gladen, Turbith, Gincer
Hot in the fourth degree. Garlick, Onions, Roots temperate in respect of heat, are Bear's breech, Sparagus, our Lady's Thistle, Eringo, Jallap, Mallows, Mechoacan, garden Parsnips, Cinquefoil, Tormentil.

Roots cold in the first degree. Sorrel, Beets, white and red, Comfrey the greater, Plantain, Rose Root, Madder.

Cold in the second degree. Alcanet, Daisies, Succory, Hound's tongue, Endive, Jacinth. Cold in the third degree. Bistort and Mandrakes are cold in the third degree, and Henbane in the fourth.

Roots dry in the first degree. Bears-breech, Burdocks, Redbeets, Calamus Aromaticus, Pilewort, Selt-heal, Endive, Eringo, Jacintl, Malder, Kneeholly.

Dry in the second degree. Watcrflag, Marshmallows, Alkanet, Smallage, Reeds, Sorrel, Swallow-wort, Asphodel male, Bazil, Valerian and Spatling Peppy, according to the opinion of the Greeks. Our Lady's 'Thistles, Avens, Succory, Hound's tongue. Cypress long and round, Fennel, Lovage, Spignel, Mercury, Devil's bit, Butter-bur, Parsley, Plantain, Zedoary.

Dry in the third degrce. Angelica, Aron, Birthwort, long and round, Sowbread, Bistort, Asarabacca, Briony white and black, Carline Thistle, China, Sallendine, Virginian Snake-root, white Dittany, Doronicum, Hellebore white and black, Elicampane, Fillipendula, Galanga greater and lesser, Masterwort, Orris, English and Florentine, Restharrow, Peony male and female, Cinquetoil, Iog's Fennel, Sarsaparilla, stinking Gladen, Tormentil, Ginger.

Dry in the fourth degree. Garlick, Onions, Costus, Leeks, Pellitory of Spain.

Roots moist are, Bazil, Valerian, and Spatling-poppy, according to the Arabian Physicians, Daisies, white Beets, Borrage, Bugloss, Liquorice, Dog grass, Mallows, Satyrion, Scorzonera, Parsnips, Skirrets. 
Roots appropriated to several parts of the body. simples may be found out by the ensuing explanation of the terms, and I suppose by Heat the head. Doronicum, Fennel, that means they were found out at first; Jallap, Mechoacan, Spikenard, Celtic and and although I hate a lazy student from Indian. Peony male and female.

Neck and throat. Pilewort, Devil's bit.

Breast and lungs. Birthwort long and round, Elicampane, Liquorice, Orris English and Florentine, Calamus Aromaticus, Cinquefoil, Squills.

Heart. Angelica, Borrage, Bugloss, Carline Thistle, Doronicum, Butter bur, Scorzonera, Tormentil, Zedoary, Bazil, Valerian white and red.

Stomach. Elicampane, Galanga greater and lesser, Spikenard, Celtic and Indian, Ginger, Fennel, Avens, Raddishes.

Bowels. Valerian great and small, Zedoary, Ginger.

Liver. Smallage, Carline Thistle, Sullendine, China, Turmerick, Fennel, Gentian, Dog-grass, Cinquefoil, Parsley, Smallage, Asparagus, Rhubarb, Rhapontic, Kneeholly.

Spleen. Smallage, Carline Thistle, Fern male and female, Parsley, Water-flag, Asparagus, round Birthwort, Fennel,Capers, Ash, Gentian.

Reins and Bladder. Marshmallows, Smallage, Asparagus, Burdock, Bazil, Valerian, Spatling Poppy, Carline Thistle, China, Cyprus long and round, Fillipendula, Dog grass, Spikenard, Celtic and Indian, Parsly, Knee-holly, white Saxifrage.

Womb. Birthwort long and round, Galanga greater and lesser, Peony male and female, Hog's Fennel.

Fundament. Pilewort.

Joints. Bear's-breech, Hermodactils, Jallap, Mecoacan, Ginger, Costus.

Roots cool the head. Rose root.

Stomach. Sow 'Thistles, Endive, Succory, Bistort.

Liver. Madder, Endive, Chicory.

Properties of the Roots. Although I confess the properties of the holly, \&c. my heart, yet to encourage young students in the art, I shall quote the chie of them: I desire all lovers of physic to compare them with the explanation of these rules, so shall they see how they agree, so may they be enabled to find out the properties of all simples to their own benefit in physic. Roots, bind. Cypress, Bistort, Tormentil, Cinquefoil, Bear's breech, Water-flag, Alkanet, Toothwort, \&c.

Discuss. Birthwort, Asphodel, Briuny, Capers, \&c.

Cleanse. Birthwort, Aron, Sparagus, Grass, Asphodel, Celandine, \&c.

Open. Asarabacca, Garlic, Leeks, Onions, Rhapontick, Turmerick, Carline Thistle, Succory, Endive, Fillipendula, Fennel, Parsly, Bruscus, Sparagus, Smallage, Gentian, \&c.

Extemuate. Orris Fnglish and Florentine, Capers, \&c.

Burn. Garlick, Onions, Pellitory of Spain, \&c.

Mollify. Mallows, Marshmallows \&c.

Suppur. Marshmallows, Briony, white Lillies, \&c.

Glutinate. Comfrey, Solomon's Seal, Gentian, Birthwort, Daisies, \&c.

Expel Wind. Smallage, Parsly, Fennel, Water-flag, Garlick, Costus, Galanga. Hog's Fennel, Zedoary, Spikenard Indian, and Celtic, \&c.

Breed Seed. Waterflag, Eringo, Satyrian, Galanga, \&c.

Provoke the menses. Birthwort Asarabacca, Aron, Waterflag, white Dittany, Asphodel, Garlick, Centaury the less, Cyperus long and round, Costus, Capers, Calamus Aromaticus, Dittany of Crete, Carrots, Eringo, Fennel, Parsly, Smallage, Grass, Elicampane, Peony, Valerian, Knee- 
Stop the menses. Comfrey, Tormentil, convenient liquor, is an excellent remedy Bistort, \&c.

for the scurvy; the powder of it being snufProvoke sweat. Carolina Thistle, China, fed up in the nose, cleanses the head of Sarsa parilla, \&c.

Résist poison. Angelica, Garlick, long rheum gallantly.

The bark of the black Alder tree purges Birthwort, Smallage, Doronicum, Costus, choler and flegm if you make a decoction Zedoary, Cyprus, Gentian, Carolina This- with it. Agrimony, Wornwood, Dodder, tle, Bistort, Tormentil, Swallow-wort, Viper's? Hops, Endive and Succory roots: Parsly Bugloss, Elicampane, \&c.

Help burnings. Asphodel, Jacinth, white Lilies, \&c.

Ease pains. Waterflag, Eringo, Orris, Restharrow, \&c.

Purge choler. Asarabacca, Rhubarb, Rhapontick, Fern, \&c.

Relieve melancholy. Hellebore, white and black, Polipodium.

Purge flegm and watery humours. Squills, Turbith, Hermodactils, Jallap, Mecoacan, wild Cucumbers, Sowbread, male A sphodel, Briony white and black, Elder, Spurge great and small.

I quoted some of these properties to reach you the way how to find the rest, which the explanation of these terms will give you ample instructions in: I quoted not all because I would fain have you studious : be diligent gentle reader.

How to use your bodies in, and after taking purges, you shall be taughi by and by.

Barks mentioned by the College are these.

College.] Hazel Nuts, Oranges, Barberries, Birch-tree, Caper roots, Cassia Lignea, Chestnuts, Cimnamon, Citron Pills, DwarfElder, Spurgeroots, Alder, Ash, Pomegranates, Guajaeum, Walnut tree, green IValmuts, Laurel, Bay, Lemon, Mace, Pomegranates, Mandrake roots, Mezereon. MTulbermy tree roots, Sloe tree roots, Pinemiuts, Fistiek-muts, Poplar tree, Oak, Elder, Sassafias, Cork, Tamerisk, Lime tree, Frankineense, Elm, Capt. Winter's Cimunamon.

Crlpeper.] Of these, Captain Winter's Cinnamon, being taken as ordinary spice, or half a dram taken in the morning in any $(2 \bar{j}, 26$. and Smallage roots, or you may bruise a handful of each of them, and put them in a gallon of ale, and let them work together: put the simples into a boulter-bag, and a draught, (half a pint, more or less, according to the age of him that drinks it,) being drunk every morning, helps the dropsy, jaundice, evil disposition of the body ; also helps the rickets, strengthens the liver and spleen; makes the digestion good, troubles not the stomach at all, causes appetite, and helps such as are scabby and itchy.

I'he rest of the barks that are worth the noting, and the virtues of them, are to be found in the former part of the book.

Barks are hot in the first degree. Guajacum, Tamarisk, Oranges, Lemons, Citrons.

In the second. Cinnamon, Cassia, Lignea, Captain Winter's Cinnamon, Frankincense, Capers.

In the third. Mace.

Cold in the first. Oak, Pomegranates.

In the third. Mandrakes.

Appropriated to parts of the body.

Heat the head. Captain Winter's Cinnamon.

The heart. Cinnamon, Cassia, Lignea, Citron Pills, Walnuts, Lemon pills, Mace.

The stomach. Orange pills, Cassia Lignea, Cinnamon, Citron pills, Lemon pills, Mace, Sassafias.

The lungs. Cassia Lignea, Cinnamon, Walnuts.

The liter. Barberry-tree, Bay-tree, Captain Winter's Cinnamon.

The splcen. Caper bark, Ash tree oark, Bay trce. 

fras.

The reins and bladder. Bay-tree, Sassa-

lime, or zater Pinipermel, Bects white, red, and

The zomb. Cassia Lignea, Cinnamon.

Cool the stomach. Ponegranate pills. tree.

Purge eholer. The bark of Barberry

Purse flesm and water. Elder, Dwarf- Purse, Ox-eye, Box leaves, Calaminth of the Elder, Spurge, Laurel.

black, Bctomy of the zood and zater. Daises greater and lesser, Blite, Siereury, Borrage, Oak of Jenusalem, Cubbages, Sodonella, Briomy white and black, Bugloss, Buglesse, Shepherd's Mountains and Fens, Ground Pine, Wood-bine, or Honey-suckles, Lady-smocks, Marygolds, Our Lady's Thistle, Carduus Beriedictus, W O O D S. Avens, small Spurge, Horse-tail, Colezcorts, Centaury the less, Knotgrass, Cervil, GerCollege.] Firr, Wood of Aloes, Rhodium, mander, Camomle, Chamepytis finale SouthernBrazil, Box, Willow, Cypress, Ebony, Guaja-wood, Chelene, Pilewort, Chicory, Hemiock, cum, Juniper, Lentisk, Nephriticum, Rhodium, garden and sea Seurvy-grass, Fleazeort, ComRosemary, Sanders, white, yellow, and red, firy great, middle, or burgle; least or Daisies, Sassafras, Tamarisk.

Sarasens, Confound, Buck-horn, Plantain,

Of these some are hot. Wood of Aloes, May weed, (or Margzeed, as we in Sussex Rhodium, Box, Ebony, Guajacum, Neph-s call it) Orpine, Sampeer, Crosezert, Dodder, riticum, Rosemary, Sassafras, Tamarisk. B Blue Bottle great and small, Artichokes,

Some cold. As Cypress, Willow, Sanders Houndstone, Cypress leates, Dandelion, Ditwhite, red, and yellow.

Rosemary is appropriated to the head, wood of Aloes to the heart and stomach, Rhodium to the bowels and bladder, Nephriticum to the liver. spleen, reins and bladder, Sassatiras to the breast, stomach and bladder, Tamarisk to the spleen, Sanders cools the heart and spirits in fevers.

For the particular virtues of each, see:Museatu, Herb Robert, Dores Foot, Cottonthat part of the book preceding.

\section{$\mathrm{H} \mathrm{E} \cdot \mathrm{R} \mathrm{B}$.}

College.] Southernzood male and female. Wormwood, common, Roman, and such as bear eresses, small Sengreen, Shareâtort, Woad, Wormseed, Sorrel, wood Sorrel, Maiden-hair Reeds, Schcenanth, Chamepitys, Glassarort, common, white or wall Rue, black and golden Lettice, Lagobus, Arch-angel, Burilock great Maudlin, Agremomy, Vervain, Mallow, Ladies and small, Lavender, Laurel, Bay leares, Mantle, Chickweed, Marshmallows, and Pim- English and Alexandrian, Duckaced, Dittanpernel both male and female, Water Pimpernel, der, or Pepper-wort, Locage, Prinet, Sea bugDill, Angelica, Smallage, Goose-grass, or loss, Toad flax, Harts-tongue, sweet Trefoil, Cleavers, Columbine, reild Tansie, or Silver Wood-sorrel, Hops, Willow-herl, Marjoram, Weed, Mugzert, Asarabacea, Woodroofe, sommon and tree Mallows, Manárake, HoreArach, Distaff Thistle, Mousear, Costmary, ?hound white and black, Herb Mastich, Fea- 
therfew, Woodbine, Melilot, Bawm garden and golden Maiden-hair, Woodroof, Bugle, zoater, Mints, Horse-mints, Mercury, Mezereon, Goat's Rue, Har's-tongue, swect Trefoil, Yarrow, Devil's-bit, Moss, sweet Chivil, Mir- Flixweed, Cinquefoil, Trefoil, Paul's Bettle leaves, Garden and water Cresses, Nep, tony, Lluellin.

Tobacco, Money-rort, Water Lilies, Bazil, Intemperate and hot in the first degree, are Olive Leaves, Rest-harrow, Adder's Tongue, Agrimony, Marsh-mallows, Goose-grass or Origanum, sharp-pointed Dock, Poppy, white, Cleavers, Distaff 'Thistle, Borrage, Bugloss, black, and red, or Erratick, Pellitory of the or Lady's Thistles, Avens, Cetrach, Chervil, Wall, Cinquefoil, Ars-smart spotted and not Chamomel, Eycbright, Cowslips, Melilot, spotted, Peach Leaves, Thoroughwax, Parsley, Bazil, Self-heal.

Hart's Tungue, Valcriak, Mouse-car, Burnet, In the second. Common and Roman small Spurge, Plantain common and narrow Wormwood, Maudlin, Lady's Mantle, leaved, Mountain and Cretick Poley, Knot- Pimpernel male and femalc, Dill, Smallage, grass, Golden Mnidenhair, Poplar leaves and Mugwort, Costmary, Betony, Oak of Jerubuds, Leeks, Purslain, Silverweed, or wild salem, Marigold, Cuckootiowers, Cardius Tansy, Horchound white and black, Primroses, Benedictus, $\mathbf{C}$ ntaury the less, Chamepitys, Sclf-heal, Field Pellitory, or Sneezereort, Penny- Scurvy-grass, Indian Leaf, Broom, Alcroyal, Fleabane, Lungroort, Winter-green, hoof,Alexanders, Double-tongue,or'TongueOak leaves and buds, Docks, common rue, blade, Archangel, or dead Nettles, Bay Wall Rue or white Maiclenhair, wild Rue, Leaves, Marjoram, Horehound, Bawm, Savin, Osier Leaves, Garden Sage the greater Mercury, Devil's-bit, Tobacco, Parsley, and lesser, IVild Sage, Elder leaves and buds, Poley mountain, Rosemary, Sage, Sanicle, Marjorum, Burnet, Sanicle, Soperert, Sarory, Scabious, Senna, Soldanella, Tansy, VcrWhite Saxifrage, Scabious, Chicory, Schonanth, vain, Percwinkle.

Clary, Scordium, Figwort, Houseleek, or In the third degree. Southernwood nale Sengreen the greater and lesser, Groundsel, and female, Biroklimc, Angelica, Briony Serina leaves and pods, Mother of Time, Solcmon's Seal, Alexanders, Nightshade, Soldanela, Sorc-thistles, smooth and rough, Flixweed, common Spilie, Spinach, Hawthom, Deril's-bit, Comfiry, Tamarisk leaves, Tansu, Dandelyon, Mullen or Higcaper, Tïme, Lime Origanum, biting Arsmart, called in Latin tree leaves, Spurge, Tormentil, common and Hydropipcr, (the college confounds this golden Trefoil, Wood-sorrel, sweet Trefoil, Coit's-foot, Valerian, Mullen, Vertain, Paul's cold (Sneezewort, Pennyroyal, Rue, Savin, Bettomy, Lluellin, Violets, Tansy, Perezinliles, summer and winter Savory, Mother of Swallow-zort, golden Rod, Vine l:aves, Mead-i'Time, Lavender, Spikc, Time, Nettles. saceet, Elm learcs, Naval-reort, Netties, comnon and homan, Archangel, or dead Nettles, white and red.

Culpeper. These be the herbs as the Spurge.

college set down to look upon, we will Herbs cold in the first degree. Sorrel, sec if we can translatc them in another Wood-sorrel, Arach, Burdock, Shepherd'sform to the benefit of the body of man. purse, Pellitory of the wall, Hawk-werd, Herbs temperate in respect of heat, are Mallows, Yarrow, mild Arsmart, called common Maiden-hair, Wall-rue, black and Persicaria, Burnet, Coltsfoot, Violets. 
Cold in the second degree. Chickweed, wild 'Tansy, or Silverweed, Daisies, Knotgrass, Succory, Buck-horn, Plantain, Dandelyon, Endive, Fumitory, Strawberry leaves, Lettice, Duck-meat, Plantain, Purslain, Willow leaves.

In the third degree. Sengreen, or HouseIcek, Nightshade.

In the fourth degree. Hemlock, Henbane, Mandrakes, Poppies.

Herbs dry in the first degree. Agrimony, Marsh-mallows, Cleavers, Burdoclis, Shepherds-purse, our Lady's Thistle, Chervil, Chamoniel, Eye-bright, Cowslips, Hawkweed, Tongue-blade, or double tongue, Melilot, mild Arsmart, Self-heal, Senna, Flixweed, Coltsfoot, Perewinkle.

Dry in the second degree. Common and Roman Wormwood, Sorrel, Wood-sorrel, Maudlin, Lady's mantle, Pimpernel male and female, Dill, Smallage, wild Tansy, or Silverweed, Mugwort, Distaff Thistle, Costmary, Betony, Bugle, Cuckooffowers, Carduus Benedictus, Avens, Centanry the less, Chicory, commonly called Succory, Scurvy-grass, Buckhorn, Plantain, Dandelyon, Endive, Indian Leaf, Strawberry leaves, Fumitory, Broom, Alehoof, Alexanders, Archangel, or Dead Nettles, white and red, Bay Leaves, Marjoran, Featherfew, Bawm, Mercury, Devil's-bit, Tóbacco, Parsley, Burnet, Plantain, Rosemary, Willow Leaves, Sagc, Santicle, Scabious, Soldanella, Vervain.

Dry in the third degree. Southernwood, male and female, Brooklime, Angelica; Briony, white and black, Calamint, Germander, Chamepitys, Selandine, Pilewort, Fleabane, Epithinum, Dwarf-Elder, Bank cresses, Clary, Glasswort, Lavender, Lovage Horehound, Herb Mastic, Mints, Watercresses, Origanuı., Cinquefoil, hot $\Lambda$ rsmart, Poley mountain, Sneezewort, Penny-royal, Rue, or herb of Grace, Savin winter and summer Savory, Mother of Time, Lavender, Silk, Tansy, Time, Trefoil.
In the fourth degree. Garden-cresses, wild Rue, Leeks, Onions, Crowfoot, Rosa Solis, Garlic, Spurge.

Herbs moist in the first degree. Borrage, Bugloss, Marigolds, Pellitory of the wall, Mallows, Bazil.

In the fourth degree. Chickweed, Arach, Daisies, Lettice, Duckmcat, Purslain, Sow 'Thistles, Violets, Water-lilies.

Herbs appropriated to certain parts of the body of man.

Heat the head. Maudlin, Costmary, Betony, Carduus Benedictus, Sullendine, Scurvy-grass, Eye-bright, Goat's Rue, Cowslips, Lavender, Laurel, Lovage, herb Mastich, Feather-few, Melilot, Sncezewort, Penny-royal, Senna, Mother of Time, Vervain, Rusemary.

Heat the throat. Archangel white and red, otherwise called dead Nettles, Deril'sbit.

Heat the breast. Maiden-hair, white, black, common and golden, Distaff Thistle, Time, Betony, Calaminth, Chamomel, Fennel, Indian-leaf, Bay leaves, Hyssop, Bawm, Horehound, Oak of Jerusalen, Germander, Melilot, Origanum, Rue, Sxbious, Periwinkles, Nettles.

Heat the heart. Southernwood male and female, Angelica, Wood-roof, Bugloss, Carduus Benedicius, Borrage, Goat's Rue, Senna, Bazil, Rosemary, Elecampane.

Heat the stomach. Wormwood common and Roman, Smallage, Avens, Indian leaf, Broom, Schenanth, Bay leaves, Bawm, Mints, Parsley, Fennel, Time, Mother of Time, Sage.

Heat the liver. Agrimony, Maudlin, Pimpernel, male and female, Smallage, Costmary, or Ale cost, our Lady's Thistles, Centaury the less, Germander, Chamepytis, Selandine, Sampier, Fox Gloves, Ash-tree leaves, Bay leaves, Toad-flax, Hops, Horehound, Water-cresses, Parsley, Polcy Mountain, Sage, Scordium, Senna, Mother 
of Time, Soldanella, Asarabacca, Fennel, Strawberry-leaves, and Water-Lilies.

Hyssop, Spikenard.

Heat the bowels. Chamomel, Alehoofe, Alexanders.

Heat the spleen.

Stomach. Sorrel, Wood sorrel, Succory, Orpine, Dandelyon, Endive, Strawberry leaves, Hawkweed, Lettice, Purslain, Sow Thistles, Violet leaves. Maiden-hair, Agrimony, Smallage, Centaury the less, Cetrach, Germander, Chamepitys, Samphire, Fox-glove, Epithimum, Ash-tree, Bay leaves, Toad-flax, Hops, Horehound, Parsley, Poley, Mountain Sage, Scordium, Senna, Mother of'Time,'Tamarisk, Wormwood, Water-cresses, Hart's-tongue.

Heat the reins and bladder. Agrimony, Maudlin, Marsh-mallows, Pimpernel male and female, Brooklime, Costmary, Bettony, Chervil, Germander, Chamomel, Samphire, Broom, Rupture-wort, Clary, Schenanth, Bay-leaves, Toad-flax, Hops, Melilot, Water-cresses, Origanum, Pennyroyal, Scordium, Vervain, Mother of Time, Rocket, Spikenard, Saxifrage, Nettles.

Heat the womb. Maudlin, Angelica, Mugwort, Costmary, Calaminth, Flea-bane, May-weed, Ormarg-weed, Dittany of Crete, Schenanth, Arch-angel or Dead Nettles, Melilot, Feather-fer, Mints, Devil's-bit, Origanum, Bazil, Pennyroyal, Savin, Sage, Scordium, Tansy, Time, Vervain, Periwinkles, Nettles.

Heat the joints. Cowslips, Sciatica-cresses, hot Arsmart, Garden-cresses, Costmary, Agrimony, Chamomel, Saint John's-wort, Melilot, Water-cresses, Rosemary, Rue, Sage Stechas.

Herbs cooling the head. Wood-sorrel, Teazles, Lettice, Plantain, Willow-lcaves, Sengreen or Houseleek, Strawberry-leaves, Violet-leaves, Fumitory, Water Lilies.

Cool the throat. Orpine, Strawberry leaves, Privet, Bramble leaves.

Breast. Mulberry leaves, Bramble leaves, Violet leaves, Strawberry leaves,
Sorrel, Wood-sorrel, Poppies, Orpine,

Moneywort, Plantain, Colt's-foot.
Heart. Sorrel, Wood sorrel, Viper's Pennyroyal,Stochas, Time, Mother of'lime, Bugloss, Lettice, Burnet, Violet leaves, Juniper, \&c.

$(27,28$. $3 \mathrm{r}$

Liver. Sorrel, Woodsorrel, Dandelyon, Endive, Succory, Strawberry leaves, Fumitory, Liverwort, Lettice, Purslain, Nightshade, Water Lilies.

Bowels. Fumitory, Mallows, Buckthorn, Plantain, Orpine, Plantain, Burnet. Lettice.

Reins and bladder. Knotgrass, Mallows, Yarrow, Moneywort, Plantain, Endive, Succory, Lettice, Purslain, Water Lilies, Houseleek or Sengreen.

The roomb. Wild Tansy, Arrach, Burdocks, Willow herb, Mirtle leaves, Moneywort, Purslain, Sow Thistles, Endive, Succory, Lettice, Water Lilies, Sengreen.

The joints. Willow leaves, Vine leaves, Lettice, Henbane, Nightshade, Sengreen or Houseleek.

Herbs altering according to property, in operation, some bind, as

Amomus, Agnus Castus, Shepherd's purse, Cypress, Horsetail, Ivy, Bay leaves, Melilot, Bawm,. Mirtles, Sorrel, Plantain, Knot-grass, Comfry, Cinquefoil, Fleawort, Purslain, Oak leaves, Willow leaves, Sengreen or Houseleek, \&c.

Open, as, Garlick, Onions, Wormwood, Mallows, Marsh-mallows, Pellitory of the Wall, Endive, Succory, \&c.

Soften. Mallows, Marsh-mallows, Beets, Pellitory of the Wall, Violet leaves, Strawberry leaves, Arrach, Cypress leaves, Bay leaves, Fleawort, \&c.

Harden. Purslain, Nightshade, Houseleek or Sengreen, Duckmeat, and most other herbs that are very cold.

Extenuate. Mugwort, Chamomel, Hysop, 
Discuss. Southernwood male and female, St. John's Wort, Marjoram, Horehound, all the four sorts of Maidenhair, Marsh-Bawm, Water-cresses, Origanum, Bazil, mallows, Dill, Mallows, Arrach, Beets, Pennyroyal,Poley mountain, Parsley, SmallChamomel, Mints, Melilot, Pelitory of the age, Rue, Rosemary, Sage, Savin, Hartwort, Wall, Chickweed, Rue, Stochas, Marjoram. Time, Mollier of 'Time, Scordium, Nettles.

Draw. Pimpernel, Birthworl, Dittany, Leeks, Onions, Garlick, and also take this general rule, as all cold things bind and harden, so all things very hot are drying.

Suppure. Mallows, Marsh-mallows, White Lily leaves, \&c.

Cleanse. Pimpernel, Southernwood, Sparagus, Cetrach, Arrach, Wormwood, Beet, Pellitory of the Wall, Chamepitis, Dodder, Liverwort, Horehound, Willow leaves, \&c.

Glutinate. Marsh-mallows, Pimpernel, Centaury, Chamepitis, Mallows, Germander, Horsetail, Agrimony, Maudlin, Strawberry leaves, Woad-chervil, Plantain, Cinquefoil, Comfiy, Bugle, Self-heal, Woundwort, Tormentil, Rupture-wort, Knot-grass, Tobacco.

Expel wind. Wormwood, Garlick, Dill, Smallage, Chamomel, Epithimum, Fennel, Juniper, Marjoram, Origanum, Savory both winter and summer. Tansy is good to cleanse the stomach and bowels of rough viscous flegm, and humours that stick to them, which the flegmatic constitution of the winter usually infects the body of man with, and occasions gouts and other diseases of like nature and lasting long. This was the original of that custom to eat Tansys in the spring; the herb may be made into a conserve with sugar, or boil it in wine and drink the decoction, or make the juice into a syrup with sugar, which you will.

Herbs breed seed. Clary, Rocket, and most herbs that are hot and moist, and breed wind.

Provoke the terms. Southernwood, Garlick, all the sorts of Maiden hair, Mugwort, Wormwood, Bishops-weed, Cabbages, Bettony, Centaury, Chamomel, Calaminth, Germander, Dodder, Dittany, Fennel,

Stop the terms. Shepherd's purse, Strawberries, Mirtles, Water Lilies, Plantain, Houseleek or Sengreen, Comfiy, Knotgrass.

Resist poison. Southernwood, Wornwood, Garlick, all sorts of Maiden hair, Snallage, Bettony, Carduus Benedictus, Germander, Calaminth, Alexanders, Carline Thistle, Agrimony, Fennel, Juniper, Horehound, Origanum, Pennyroyal, Poleymountain, Rue, Scordium, Plantain.

Discuss swellings. Maiden-hair, Cleavers, or Goosegrass, Mallows, Marsh-mallows, Docks, Bawm, Water-cresses, Cinquefoil, Scordium, \&c.

Ease pain. Dil, Wormwood, Arach, Chamomel, Calaminth, Chamepitis, Henbane, Hops, Hog's Fennel, Parsley, Rosemary, Rue, Marjoram, Mother of Time.

\section{Herbs Purging.}

Choler. Groundsel, Hops, Peach leaves, Wormwood, Centaury, Mallows, Senna.

Melancholy. Ox-eye, Epithimum, Fumitory, Senna, Dodder.

Flegm and water. Briony, white and black, Spurge, both work most violently and are not fit for a vulgar use, Dwarf Elder, Hedge Hyssop, Laurel leaves, Mercury, Mezereon also purges violently, and so doth Sneezewort, Elder leaves, Senna.

For the particular operations of these, as also how to order the body after purges, the quantity to be taken at a time, you have been in part instructed already, and shall be more fully hereafter.

\section{F L O W E R.}

College.] Wormwood, Agnus Castus, Amaranthus, Dill, Rosemary, Columbines, 
Orrenges, Balaustins, or Pomegranate Flowers, Epithimum, Winter-gilliflowers, or WallBettony, Borrage, Bugloss, Marigolds, Wood- flowers, Woodbine, or Honey-suckles. bime or Honey-suckles, Clove Gilliflowers, Centanty the less, Chamomel, Winter Gilliflowers, Succory, Comfry the greater, Saffron, Bluebottle great and small, (Synosbatus, Tragus, and Dedonæus hold our white thorn to be it, Cordus and Marcelus thimk it to be Bryars, Lugdunensis takes it for the sweet Bryar, but what our College takes it for, I know not) Cytimus, (Dioscorides calls the flowers of the Manured Pomegranates, Cytimus, but Pliny calls the flower's of the wild kind by that name,) Fox-glove, Viper's Bugloss, Rocket, Euyebright, Beuns, Fumitory, Broom, Cowslips, St. John's Wort, Hysop, Jessamine or Shrub, Trefoil, Archangel, or Dead Nettles white and red, Lavender, Wall-flowers, or WinterGilliflowers, Privet, Lilies white, and of the valley, Hops, Common and tree Mallows, Feather-fex", Woodbine, or Honey-suckles, Melilot, Bazom, Walnuts, Water-Lilies white and yellow, Origannm, Poppies white and red, or Erraticks, Poppies, or corn Roses, so called because they grow amongst Corn, Peomy, Honey-suckles, or Woodbine, Peach-flower's, Primroses, Self-heal, Sloebush, Rosemary flowers, Roses, white, damask and red, Sage, Elder, white Saxifrage, Scabions, Siligo, (I think they mean reheut by it, Authors are not agreed about it) Steches, Tamarisk, Tansy,
Mullen or Higtaper. Limetree, Clove Gilliflowers, Colt's-foot, Violets, Agmus Castus, Dead Nettles white and red.

Culpeper.] 'That these may be a little explained for the public good: be pleased to take notice.

Some are hot in the first degree, as Borrage, Bugloss, Bettony, Ox-eye, Melilot, Cliamomel, Stoechas.

Hot in the second degree. Amomus, Saffron, Clove-gilliflowers, Rocket, Bawm, Spikenard, Hops, Schenanth, Lavender, Jasmine, Rosentary.

In the third degree. Agnus Castus,
Cold in the first degree. Mallows, Roses, red, white, and damask Violets.

In the second. Anemom, or Wind-flower, Endive, Succory, Water-lilies, both white and yellow.

In the third. Balaustins, or Pomegranate flowers.

In the fourth. Henbane, and all the sorts of Poppies, only whereas authors say, field Poppies, which somecall red, others erratick and corn Roses, are the coldest of all the others; yet my opinion is, that they are not cold in the fourth degree.

Moist in the first degree. Borrage, Bugloss, Mallows, Succory, Endive.

In the second. Water-lilies, Violets.

Dry in the first degree. Ox-cye, Saffron, Chamomel, Melilot, Roses.

In the second. Wind-flower, Amomus, Clove-gilliflowers, Rocket, Lavender, Hops, Peony, Rosemary, Spikenard.

In the third. Woodbine, or Honeysuckles, Balaustines, Epithimum, Germander, Chamepitis.

The temperature of any other flowers not here mentioned are of the same temperature with the herbs, you may gain skill by searching there for them, you can loose none.

For the parts of the body, they are appropriated to, some heat

The head; as, Rosemary flowers, Selfheal, Chamomel, Bettony, Cowslips, Lavender, Melilot, Peony, Sage, Stoechas.

The breast. Bettony, Bawm, Scabious, Schœnanth.

The heart. Bawm, Rosemary flowers, Borrage, Bugloss, Saffiron, Spikenard.

The stomach. Rosemary-flowers, Spikenard, Schœnanth.

The liver. Centaury, Schænanth, Elder, Bettony, Chamomel, Spikenard. 
The spleen. Bettony, Wall-flowers.

SThe reins and bladder. Bettony, Marsh. mallows, Melilot, Schoenanth, Spikenard.

The zoomb. Bettony, Squinanth or Schenanth, Sage, Orris or Flower-dc-luce.

The joints. Rosemary-flowers, Cowslips, Chamomel, Melilot.

\section{Flowers, as they are cooling, so they cool}

The head. Violets, Roses, the three sorts of Poppies, and Water-lilies.

The breast and heart. Violets, Red Roses, Water-lilies.

The stomach. Red Roses, Violets.

The liter and spleen. Endive, and Succory.

Violets, Borrage, and Bugloss, moisten the heart, Rosemary-flowers, Bawm and Bettony, dry it.

\section{According to property, so they bind.}

Balaustins, Saffron, Succory, Endive, red-roses, Melilot, Bawm,Clove-gilliflowers, Agnus Castus.

Discuss. Dill, Chamomel, Marsh-mallows, Mallows, Melilot, Stoechas, \&c.

Cleanse. Damask-roses, Elder flowers, Bean flowers, \&c.

Eatenuate. Orris, or Flower-de-luce, Chamomel, Melilot, Stoechas, \&c.

Mollify. Saffron, white Lilies, Mallows, Marsh-mallows, \&c.

Suppure. Saffron, white Lilies, \&c.

Glutinate. Balaustines, Centaury, \&c.

Provole the terms. Bettony, Centaury, Chamoinel, Schøenanth, Wall-flowers, Bawm Peony, Rosemary, Sage.

Stop the terms. Balaustines, or Ponegranate flowers, Water Lilies.

Expel wind. Dill, Chamomel, Schœenanth, Spikenard.

Help burnings. White Lilies, Mallows, Marsh-mallows.

Resist poison. Bettony; Centaury.

Ease prin. Dill, Chamomel, Centaury, Melilot, Rosemary.
Flowers purge choler. Peach flowers ${ }^{6}$ Damask Roses, Violets.

Flegm. Broom flowers, Elder flowers.

If you compare but the quality of the flowers witl the herbs, and with the explanation of these terms at the latter end, you may easily find the temperature and property of the rest.

The tlowers of Ox-eye being boiled into a poultice with a little barley meal, take away swellings and hardness of the flesh, being applied warm to the place.

Chamomiel flowers heat, discuss, loosen and rarify, boiled in Clysters, they are excellent in the wind cholic, boiled in wine, and the decoction drunk, purges the reins, break the stone, opens the pores, cast out choleric humours, succours the heart, and cases pains and aches, or stiffness coming by travelling.

The flowers of Rocket used outwardly, discuss swellings, and dissolve hard tumors, you may boil them into a poultice, but inwardly taken they send but unwholesome vapours up to the head.

Hops open obstructions of the bowels, liver, and spleen, they cleanse, the body of choler and flegm, provoke urine.

Jasmine flowers boiled in oil, and the grieved place bathed with it, takes away cramps and stitches in the sides.

The flowers of Woodbine, or Honeysuckles, bcing dryed and beaten into powder, and a dram taken in white wine in the morning, helps the rickets, difficulty of breathing; provoke urine, and help the stranguary.

The flowers of Mallows being bruised and boiled in honey (two ounces of the flowers is sufficient for a pound of honey; and having first clarified the honey before you put them in) then strained out; this honey taken with a liquorice stick, is an excellent remedy for Coughs, Asthmas, and "consumptions of the lungs. 
Capers; Nutmegs, dry Walnuts, dry Hazel

\section{F R U I'T S.}

College.] Winter-cherries, Love Apples, Almonds strect and bitter, Anucardiu, Oranges, Hazel Nuts, the oily Nut Ben, Barberries, Capers, Guinmy Pepper, Figs, Carpobalsamum, Cloves, Cassia Fistula, Chestnuts, Cherries black and red, Cicers, white, black and red, Pome Citrons, Coculus Indi, Colocynthis, Cur-? rants, Cornets or Cornelian Cherries, Cubebs, Cucumbers garden and wild, Gourds, Cynosbatus, Cypress, Cones, Quinces, Dutes, DwarfElder, Green Figs, Strawberries, common and Turkey Galls, Acorns, Acom Cups, Pomegramates, Gooseberries, Ivy, Herb Tine-Love, Wulnuts, Jujubes, Juniper berries, Bayberries, Lemons, Oranges, Citrons, Quinces, Pomegranutes, Lemons, Mandralies, Peaches, Stramonium, Apples, garden and wild," or Crabs and Apples, Musk Melons, Medlars Mulberries, Myrobalans, Bellericks, Chebs, Emblicks, Citron and Indian, Mirtle, Berries, water Nuts, Husel Nuts, Chestmuts, Cypress Nuts, Walmuts, Nutmegs, Fistick Nuts, Voinitiug Nuts, Olizes pickled in brine, Heuds of zohite and black Poppies, Pompions, Peaches, French or Kidney Beans, Pine, Cones, white black, and long Pepper, Fistick Nuts, Apples and Crabs, Primes, French and Damasli, Sloes, Pears, English Curronts, Berries of Purging Thom, black Berries, Raspberries, Elder berries, Scbastens, Services, or Checkers, Hawthorn berries, Pine Nuts, Wuter Nuts, Grapes, Gooseberries, Raisins, Currunts.

Culpeper.] That you may reap benefit by these, be pleased to consider, that they are some of them

Temperale in respect of heat. Raisins of the sun, Currants, Figs, Pine Nuts, Dates, Selbastens.

Hot in the first degree. Sweet Almonds, Jujubes, Cypress Nuts, green Hazel Nuts, green Wallunts.

Hot in the second degree. . The Nut Ben, $(27,28$.
Nuts, Fistick Nuts.

In the third degree. Juniper Berries, Cloves, Carpobalsamum, Cubebs, Anacardium, bitter Almonds.

In the fourth degree. Pepper, white, black and long, Guinny Pepper.

Cold in the first degree. The flesh of Citrons, Quinces, Pears, Prunes, \&c.

In the second. Gourds, Cucumbers, Melons, Pompions, Oranges, Lemons, Citrons, Pomegranates, viz. the juice of them, Peaches, Prunes, Galls, Apples.

In the third. Mandrakes.

In the fourth. Stramonium.

Moist in the first degree. The Hesh of Citrons, Lemons, Oranges, viz. the inner rhind which is white, the outer rhind is hot. In the second. Gourds, Melons, Peaches, Prunes, \&c.

Dry in the first degree. Juniper Berries.

In the second. The Nut Ben, Capers, Pears, Fistick Nuts, Pine Nuts, Quinces, Nutmegs, Bay berries.

In the third. Cloves, Galls, \&c.

In the fourth. All sorts of pepper.

As appropriated to the body of Man, so they heat the head: as

Anacardia, Cubebs, Nutmegs.

The breast. Bitler Almonds, Dates, Cubebs, Hazel Nuts, Pine Nuts, Figs, Raisins of the sun, Jujubes.

The heart. Walnuts, Nutmegs, Juniper berries.

The stomach. Sweet Alnonds, Cloves, Ben, Juniper berries, Nutmegs, Pine Nuts, Olives.

The spleen. Capers.

The reins and bladder. Bitter Almonds, Juniper Berries, Cubebs, Pine Nuts, Raisins of the sun.

The romb. Walnuts, Nutmegs, Bayberries, Juniper berries.

Cool the breast. Sebastens, Prunes, Oranges, Lemons.

$3 \mathrm{Y}$ 
The heart. Oranges, Lemons, Citrons, Myrobalans of all sorts, especially Chebs, Pomegranates, Quinces, Pears.

The stomach. Quinces, Citruls, Cucumbers, gently, and without danger. Gourds, Musk Melons, Pompions, Cherries, Gooseberries, Cornelian Cherries, Lemons, Apples, Medlars, Oranges, Pears, English Currants, Cervices or Checkers.

The liver. Those that cool the stomach and Barberries.

The reins and womb. Those that cool the stomach, and Strawberries.

\section{By their several operations, some}

Bind. As the berries of Mirtles, Barberries, Chestnuts, Cornels, or Cornelian Cherries, Quinces, Galls, Acorns, Acorncups, Medlars, Checkers or Cervices, Pomegranates, Nutmegs, Olives, Pears, Peaches.

Discuss. Capers, all the sorts of Pepper.

Extemate. Sweet and bitter Almonds, Bayberries, Juniper berries.

Glutinate. Acorns, Acorn Cups, Dates, Raisins of the sun, Currants.

Expel Wind. Bay berries, Juniper berries, Nutmegs, all the sorts of Pepper.

Breed seed. Raisins of the sun, sweet Almonds, Pine Nuts, Figs, \&c.

Provoke urine. Winter Cherries.

Provole the terms. Iv y berries, Capers, \&c.

Stop the terms. Barberries, \&c.

Resist poison. Bay berries, Juniper berries, Walnuts, Citrons, commonly called Pome Citrons, all the sorts of Pepper.

Ease pain. Bay berries, Juniper berries, Ivy berries, Figs, Walnuts, Raisins, Currants, all the sorts of Pepper.

\section{Fruits purging.}

Choler. Cassia Fistula, Citron Myrobalans, Prunes, Tamarinds, Raisins.

Melancholy. Indian Myrobalans.

Flegm. Colocynthis and wild Cucumbers purge violently, and therefore not rashly to be meddled withal: I desire my book should Of all these give me leave to commend only one to you as of special concernment, which is Juniper berries.

\section{S E E D S.}

College.] Sorrel, Agmis Castus, Marshmallows, Bishop's weed true and common, Amomis, Dill, Angellica, Amis, Rose-seed, Smallage, Columbines, Sparaguz, Arach, Oats, Oranges, Burdocks, Bazil, Barberries, Cotton, Bruscus or Knee-holly, Hemp, Cardamoms greater and lesser, Carduus Benedictus, oner Ludy's Thistles, Bastard, Saffron, Caraway, Spurge greater and lesser, Coleworts, Orions, the Kermels of Cherry stones, Chervil, Succory, Hemlock, Citrons, Citmuls, Garden Scurvy-grass, Colocynthis, Coriander, Samphize, Cucumbers garden and wild, Gourds, Quinces, Cummin, Cynosbatus, Date-stones, Carrots English, and cretish, Divarf-Elder, Endive, Rocket, Hedge Mustard, Orobus, Beans, Femel, Femugreck, Ash-tree keys, Fumitory, Brooms, Grains of Paradise, Pomegranates, wild Rue, Aleacunders, Barley, white Henbane, St. Jolın's Wort, Hyssop, Lettice, Sharp-pointed-Dock, Spurge, Laurel, Lentils, Lovage, Lemons, Ash-tree-keys, Linseed, or Flaxweed, Gromwell, Darnel, Sweet Trefoil,Lupines, Masterwort, Marjoram, Mallows, Mandrakes, Melons, Medlars, Mezereon, Gromwell, sweet Navew Nigella, the kernels of Cherries, Apricots, and Peaches, Bazil, Orobus, Rice, Panick, Poppics white and black, Parsnips garden and zeild, Thorough Wax, Parsley, English and Macedonian, Bumet, Pease, Plantain, Peony, Leeks, Purslain, Fleazort, Turnips, Radishes, Sumach Spurge, Roses, Rue, garden and wild, Wormseed, Savifiage, Succory, Sesami, Hartwort, common and cretish, Mustard-sced, Alexanders, Nightshade, Steves Ager, Sumach, Treacle, be beneficial, not hurtiul to the vulgar, but Mustard, sweeet Trefoil, Wheat, both the fine 
flour and the bran, and that which starch is made of, Vetches or Tares, Violets, Nettles, common and Roman, the stones of Grapes, Greele Whent, or Spelt Wheat.

Culpeper.] 'That you may receive a little more benetit by these, than the bare reading of them, which doth at the most but tell you what they are; the following method may instruct you what they are good for.

\section{Seeds are hot in the first degree.}

Linseed, Fenugreek, Coriander, Rice, Gromwell, Lupines.

In the second. Dill, Smallage, Orobus, Rocket, Bazil, Nettles.

In the third. Bishop's Weed, Annis, Amomus, Carraway, Fennel, (and so I believe Smallage too, let authors say what they will, for if the herb of Smallage be somewhat hotter than Parsley; I know Iittle reason why the sced should not be so hot) Cardamonis, Parsley, Cummin, Carrots, Nigella, Navew, Hartwort, Staves Ager.

In the fourth. Water-cresses, Mustardseed.

Cold in the first degree. Barley, \&c.

In the second. Endive, Lettice, Purslain, Succory, Gourds, Cucumbers, Melons, Citruls, Pompions, Sorrel, Nightshade.

In the third. Henbane, Hemlock, Poppies white and black.

Moist in the first degree. Mallows, \&c.

Dry in the first degree. Beans, Fennel, Fcnugreek, Barley, Wheat, \&c.

In the second. Orobus, Lentils, Rice, Poppies, Nightshade, and the like.

In the third. Dill, Smallages, Bishop's Weed, Annis, Caraway, Cummin, Coriander, Nigella, Gromwell, Parsley.

Appropriated to the body of man, and so they \&c.

Heat the head. Fennel, Marjoram, Peony,

The breast. Nettles.

The heart. Bazil, Rue, \&c. Mustard seed, \&c.
The stomach. Annis, Bishop's weed, Amomus, Smallage, Cummin, Cardamoms, Cubebs, Grains of Paradise.

The liver. Annis, Fennel, Bishop's weed, Amomus, Smallage, Sparagus, Cummin, Caraway, Carrots.

The spleen. Annis, Caraway, Watercresses.

The reins and bladder. Cicers, Rocket, Saxifrage, Nettles, Gromwell.

The womb. Peony, Rue.

The joints. Water-cresses, Rue, Mustardseed.

Cool the head. Lettice, Purslain, white Poppies.

The breast. White Poppies, Violets.

The heart. Orange, Lemon, Citron and Sorrel seeds.

Lastly, the four greater and four lesser cold seeds, which you may find in the beginning of the compositions, as also the seed of white and black Poppies cool the liver and spleen, reins and bladder, womb and joints.

\section{According to operation some sceds ,}

Bind, as Rose-seeds, Barberries, Shepherd's purse, Purslain, \&c.

Discuss. Dill, Carrots, Linseeds, Fenugreek, Nigella, \&c.

Cleanse. Beans, Orobus, Barley, Lupines, Nettles, \&c.

Mollify. Linseed, or Flax seed, Fenugreek seed, Mallows, Nigella.

Harden. Purslain seed, \&c.

Suppure. Linseed, Fenugreek seed, Darnel, Barley husked, commonly called French Barlcy.

Glutinate. Orobus, Lupines, Darnel, \&c, Erpel wind. Annis, Dill, Sinallage, Caraway, Cummin, Carrots, Fennel, Nigclla, Parsley, Hartwort, Wormseed.

Breed seed. Rocket, Beans, Cicers, Ashtrce keys.

Provole the menses. Amomus, Sparagus, Annis, Fennel, Bishop's wced, Cicers, Carrots, Smallage, Parsley, Lovage, Hartwort, 
Break the stone. Mallows, Marsh-mallows, Gromwell, \&c.

Stop the terms. Rose sceds, Cummin, Burdock, \&c.

Resist poison. Bishop's weed, Annis, Smallage, Cardamoms, Oranges, Lemons, Citrons, Fcnnel, \&c.

Ease pain. Dill, Amomus, Cardamoms, Cummin, Carrots, Orobus, Fenugreek, Linseed, Gromwell, Parsley, Panick.

Assuage sweellings. Linseed, Fenugreek seeds, Marsh-mallows, Mallows, Coriander, Barley, Lupines, Darnel, \&c.

The College tells you a tale that there are such things in Rerum Natura, as these, Gums, Rozins, Balsams, and Juices made thick, viz.

College.] Juices of Wormwood and Maudlin, Acacia, Aloes, Lees of Oil, Assa-foelida, Balsam of Peru and India; Bdellium, Benzoin, Camphire, Carama, Colophonia, Juice of Maudlin, Euphorbium, Lees of Wine, Lees of Oil, Gums of Galbamum, Amoniacum, Anime, Arabick, Cherry Trees, Copal, Elemy, Juniper, Ivy, Plumb Trees, Cambuge, Hypocystis, Labdanum, Lacca, Liquid Amber, Mamna, Mastich, Myrrh, Olibanum, Opium, Opopanax, Pice-bitumen, Pitch of the Cedar of Greece, quencheth thirst, and strengthens the Liquid and dry Rozins of Fir-tree, Larch-tree, stomach exceedingly: It may easily be Pine tree, Pine-finit, Mastich. Venice and carried about in onc's pocket, and eat a little Cyprus Turpentine. Sugar, white, red, and now and thon.

Christaline, or Sugar Candy white and red, Sagapen, Jumiper, Gum, Sanguis Draconis, roughness of the tongue, it strengthens the Surcocolla, Scamomy, Styrax, Liquid and reins and bladder, bcing weakcned: being Calumitis, Tacha, Mahaccu, Tartar, Frankin- beaten into fine powder and put into the cense, Olibannm, Tragaganth, Birdlime.

Culpeper.] That my country may receive more bencfit than cver the college of Physicians intended them from these, I shall treat of them severally.

1. Of the Juices.

2. Of the Gums and Rosins.
Concrete Juices, or Juices made thick, are either.

Temperate, as, Juice of Liquoricc, white Hot in the first degree. Sugar.

In the second. Labdanum.

In the third. Benzoin, Assafoetida.

Cold in the third degree. Sanguis Draconis, Acacia.

In the third. Hypocistis.

In the fourth. Opium, and yet some authors think Opium is hot because of its bitter taste.

Aloes and Manna purge choler gently: and Scamony doth purge choler violently, that it is no ways fit for a vulgar man's use, for it corrodes the Bowels. Opopoanax purges flegm very gently.

White starch gently levigates or makes smooth such parts as are rough, syrup of Violets being made thick with it and so taken on the point of a knife, helps coughs, roughness of the throat, wheezing, excoriations of the bowels, the bloody-flux.

Juice of Liquorice helps roughness of the Trachea Aitera, which is in plain English causes coughs and hoarseness, difficulty of breathing, \&c. It allays the heat of the stomacly and livcr, eases pains, soreness and roughncss of the reins and bladder, it cyes, it takes away films that grow over the sight.

Labdanum is in operation, thickening, heating and mollifying, it opens thc passagc of the veins, and keeps the hair fiom falling off ; the usc of it is usually cxternal: being mixed with winc, myrrh, and oil of mirtles, called the windpipe, the roughness of which 
and applied like a plaister, it takes away flthy scars, and the deformity the small pox leaves behind them; being mixed with oil of Roses, and dropped into the ears, it helps pains there; being used as a pessary, it provokes the menses, and helps hardness or stiffiness of the womb. It is sometinies used inwardly in such medicines as ease pains and help the cough: if you mix a little of it with old white wine and drink it, it botll provokes urine and stops looseness or fluxes.

Dregons blood, cools, binds, and repels. Acasia, and Hyposistis, do the like.

The juice of Maudin, or, for want of it tormer, being spread upon a cloth is exCostmary, which is the same in effect, and cellently good for old aches coming of former better known to the vulgar, the juice is made thick for the lretter keeping of it; first clarify the juice before you boil it to its due thickness, which is something thicker than honey.

It is appropriated to the liver, and the quanticy of a dram taken every morning, helps the Cacluxia, or evil disposition of the body proceeding from coldness of the liver: it helps the rickets and worms in children, provokes urine, and gently (without purging) disburdens the body of choler and flegm; it succours the lungs, opens obstructions, and resists putrifaction of blood.

Gums are either temperate, as, Lacca, Elemi, Tragacanth, \&c.

Intemperate, and so are hot in the first degree, as Bdellium, Gum of Iry.

In the second, Galbanum, Myrrh, Mastich, Trankincense, Olibanum, Pitch, Rozin, Styrax.

In the third. Amoniacum.

In the fourth. Euphorbium.

Gum Arabick is cold.

Colophonia and Styrax soften. larack or Juniper Grum, and Sarcocolla jind.

Gum of Cherry trees, breaks the stone.

Styrax provokes the menses.

$(27,28$.
Opopanax gently purges flegm.

From the prickly Cedar when it is burned comes forth that which, with us, is usually known by the name of Tar, and is excellently good for unction either for scabs, itch, or manginess, either in men or beasts, as also against the leprosy, tetters, ringworms, and scald heads.

All sorts of Rozins fill up hollow ulcers, and relieve the body sore pressed with cold griets.

The Rozin of Pitch-tree, is that which is commonly called Burgundy pitch, and is something hotter and sharper than the bruises or dislocations.

Pitch mollifies hard swellings, and brings boils and sores to suppuration, it breaks carbuncles, disperses apostliumes, cleanses ulcers of corruption and fills them with Hesh.

Bdellium heats and mollifies, and that very tempcrately, being mixed with any conrenient ointment or plaister, it helps kernels in the neck and throat, Scrophula, or that disease which was called the King's Evil. Inwardly taken in any convenient medicine, it provokes the menses, and breaks the stone, it helps coughs and bitings of venomous beasts: it helps windiness of the spleen, and pains in the sides thence coming. Both outwardly applied to the place and inwardly taken, it helps ruptures or such as are burst, it softens the hardness of the womb, dries up the moisture thereof and expels the dead child.

Bitumen Jadaicum is a certain dry pitch which the dead sea, or lake of Sodom in India casts forth at certain timcs, the inhabitants thereabouts pitch their ships with it. It is of excellent use to mollify the hardness of swellings and discuss them, as also against inflanmmations; the smoke of it burnt is excellently good for the fits of the mother, $4 \mathrm{~A}$ and the falling-sickness: Inwardly taken in 
wine it provokes the menses, helps the bitings tures in the skull and head. See Arceus: of venomous beasts, and dissolves congealed liniment.

blood in the body.

Gum Lacca being well purified, and the Ambergreese is hot and dry in the second quantity of half a dram taken in any condegree, I will not dispute whether it be a venient liquor, strengthens the stomach and Gum or not: It strengthens nature much liver, opens obstructions, helps the yellow which way soever it be taken, there are but jaundice and dropsy; provokes urine, few grains usually given of it at a time: breaks the stone in the reins and bladder. mixed with a little ointment of Orange flowers, and the temples and forehead. anointed with it, it eases the pains of the head and strengthens the brain exceedingly; the same applied to the privities helps the fits of the mother; inwardly taken it strengthens the brain and memory, the heart and vital spirit, warms cold stomachs, and is an exceeding strengthener of nature to old people, adding viguur to decayed and worn-out spirits : it provokes venery, and makes barren women fruitful, if coldness and moisture or weakness be the cause inspediting.

Assafictida being smelled to, is vulgarly known to repress the fits of the mother; a little bit put into an aching tooth, presently eases the pain, ten grains of it taken before dinner, walking half an hour after it, provokes appetite, helps digestion, strengthens the stomach, and takes away loathing of meat, it provokes lust exceedingly and expels wind as much.

Borax, besides the virtues it has to solder Gold, Silver, Copper, \&c. inwardly given in small quantities, it stops fluxes, and the running of the reins : being in fine powder, and put into green wounds, it cures them at once dressing.

Gambuge, which the College calls Guttà Gamba. I know no good of it.

Liquid Amber is not much unlike liquid Styrax: by unction it warms and comforts a cold and moist brain, it eases all griefs coning of a cold cause, it mightily comforts and strengthens a weak stomach, being anointed with it, and helps digestion exceedingly, it dissolves swellings. It is hot in the third degree, and moist in the first.

I think it would do the commonwealth no liarm if I should speak a word or two of Manna here, although it be no Gum : I confess authors make some flutter about, it, what it is, some holding it to be the juice of a tree; I am confident it is the very same condensated that our honey-dews here are, only the contries whence it comes being far hotter, it falls in great abundance. Let him that desires reason for $\mathrm{it}$, be pleased to read Butler's book of Bees, a most excellent experimental work, there he shall find reason enough to satisfy any reasonable man. Choose the driest and whitest; it is a very gentle purger of choler, quenches thirst, provokes appetite, eases the roughness of the throat, helps bitterness in the throat, and often proneness to vomit, it is very good for such as are subject to be costive to put it into their drink instead of sugar, it hath no obnoxious quality at all in it, but may be taken by a pregnant woman without any danger ; a child of a year old may take an Caranna outwardly applied, is excellent ounce of it at a time dissolved in milk, it for aches and swellings in the nerves and joints: If you lay it behind the ears, it draws back humours from the eyes; applied to the temples as they usually do Mastich, it helps the tooth-ache.

will melt like sugar, neither will it be known from it by the taste.

Myrrh is hot and dry in the second degree, dangerous for pregnant women, it is bitter, and yet held to be good for the roughness Gum Elimi, authors anprovriate to frac-? of the throat and wind-pipe; half a dram of 
it taken at a time helps rheumatie distilla- not very easy. It stops defluctions from tions upon the lungs, pains in the sides; it the head, if you perfume your cap with the stops fluxes, provokes the menses, brings smoke- of it, it helps the headache and away both birth and after-birth, softens the megrim, strengthens the brain, and therefore hardness of the womb; being taken two the sinews.

hours before the fit eomes, it helps agues. Mathiolus saith he seldom used any other medicine for the quartan ague than a dram of myrrh given in Muskadel an hour before the fit usually came; if you make it up into pills with treacle, and take one of them every morning fasting, it is a sovereign pieservative against the pestilenee, against the poison of serpents, and other venomous beasts; a singular remed y for a stinking breath if it arise from putrefaetion of the stomach, it fastens loose teeth, and stays the shedding off of the hair, outwardly used il breeds flesh in deep wounds, and eovers the naked bones with flesh.

Olibanum is hot in the seeond degree, and dry in the first, you may take a dram of it at a time, it stops looseness and the running of the reins; it strengthens the memory exeeedingly, eomforts the heart, expels sadness and melancholy, strengthens the heart, helps coughs, rheums and pleurises; your best way (in my opinion,) to take it is to mix it with conserve of roses, and take it in the morning fasting.

Tachamacha is seldom taken inwardly, outwardly spread upon leather, and applied to the navel; it stays the fits of the mother, applied to the side, it mitigates speedily, and in little time quite takes away the pain and windiness of the spleen; the truth is, whatsoever ache or swelling proceeds of wind or eold raw humours, I know no better plaister eoming from beyond sea than this gum. It strengthens the brain and memory cxecedingly, and stops all such defluctions thence as trouble the eyes, ears, or teeth, it helps the gout and seiatiea.

Gum Tragaganth, which the vulgar call Gum Dragon, being mixed with peetoral Syrups, (which you shall find noted in their proper plaees) it helps eoughs and hoarseness, salt and sharp distillations upon the lungs, being taken with a liquoriee stiek, being dissolved in sweet wine, it helps (being drank) gnawing in the bowels, sharpness and freetings of the urine, whiel causes excoriations either in the reins or bladder, being dissolved in' milk and the eyes washed with it, it takes away weals and scabs that grow on the eyelids, it is exeellently good to be put in poultice to fodder wounds, especially if the nerves or sinews be hurt.

Sagapen, dissolved in juice of rue and taken, wonderfully breaks the stone in the bladder, expels the dead child and afterbirth, clears the sight; dissolved in wine and drank, it helps the eough, and distillation upon the lungs, and the fits of the mother; outwardly in oils or ointments, it helps sueh members as are out of joint or over-stretehed:

Galbanum is of the same operation, and also taken from the same plant, viz. Fennel, Giant.

Gum Arabic, thiekens and cools, and corrects choleric sharp humours in the body, being dissolved in the white of an egg, well beaten, it helps burnings, and keeps the place from blistering.

Masticlb stays fluxes, being taken inwardly any way. 'Three or four small grains of Mastieh, swallowed at night going to bed, is a remedy for pains in the stomach: being beaten into powder, and mixed with conserre of Roses, it strengthens the stomach,

Gum Coopul, and Gum Anime, are very stops distillations upon the lungs, stays like one another both in body and opera-s vomiting, and causes a sweet breath; being tion, the former is hard to come by, the last? mixed with white wine and the mouth 
washed with it. it cleanses the gums of corruption, and fastens loose teeth.

Frankincense being used outwardly in the way of a plaister, heats and binds; being applied to the temples, stops the rheums that flow to the eyes, helps green wounds, and fills hollow ulcers with flesh, stops the bleeding of wounds, though the arteries be cut; being made into an ointment with Vinegar and Hog's-grease, helps the itch, pains in the ears, inflammations in women's breasts commonly called agues in the breast; beware of taking it inwardly; lest it cause madness.

Turpentine is hot in the second degree, it heals, softens, it discusses and purges, cleanses the reins, provokes urine.

Styrax Calamitis is hot and dry in the second degree, it heals, mollifies, and concocts; being taken inwardly helps the cough, and distillations of the lungs, hoarseness and loss of voice, helps the hardness of the womb, and provokes the menses.

Ammoniacum, hot and dry in the third degree, softens, draws, and heats; being dissolved in vinegar, strained and applied plaister-wise, it takes away carbuncles and hardness in the flesh, it is one of the best remedies that I know for infirmities of the spleen, being applied to the left side; being made into an ointment with oil, it is good to, anoint the limbs of such as are weary: a scruple of it being taken in the form of a pill loosens the belly, gives speedy delivery to women in travail, helps diseases of the spleen, the sciatica and all pains in the joints, and have any humour afflicting their breast.

Camphire, it is held by all authority to be cold and dry in the third degree, it is of very thin subtile parts, insomuch that being beaten into very fine powder it will vanquish away into the air, being beaten into powder and mixed with oil, and the temples anointed therewith, eases lieadaches proceeding of ? heat, all inflammations whatsoever, the back being anointed with the same, cools the reins, and seminal vessels, stops the running of the reins and Fluor Albus, the moderate use of Venery, the like it doth if it be drank inwardly with Bettony-water, take but a small quantity of it at a time invardly, it resist poison and bitings by venomous beasts; outwardly, applied as before, and the eyes anointed with it, stops hot rheums that flow thither.

Opopanax purges thick flegm from the most remote parts of the body, vix. the brain, joints, liands, and feet, the nerves and breast, and strengthens all those parts when they are weak, if the weakness proceed of cold, as usually it doth; it helps weakness of the sight, old rotten coughs, and gouts of all sorts, dropsies, and swellings of the spleen, it helps the stranguary and difficulty of making urine, provokes the menses, and helps all cold afflictions of the womb; have a care you give it not to any pregnant women. The dose is one dram at most, corrected with a little Mastich, dissolved in Vinegar and outwardly applied helps the passions of the splecn.

In the next place the College tells you a tale concerning Liquid, Juices, and Tears, which are to be kept for present use, viz.

College.] Vinegar, Juice of Citrons, Juice of sour Grapes, Oranges, Barberries, Tear's of a Birch-tree, Juice of Chermes, Quinces, Pomegranutes, Lemons, Wood-sorrel, Oil of unripe Olives, and ripe Olives, both new and cold, Juice of red and Damask Roses, Wine Tears of a Vine.

Culpeper.] The virtues of the most of these may be found in the Syrups, and are few of them used alone. 
broken, and they boiled in spring water, but not scummed at all, for the scum will Then the College tells you there are things sink of itself, and the water drank for bred of PLANTS.

ordinary drink is a most admirable remedy

College.] Agarick, Jew's-ears, the berries for consumption; being bruised and apof Chermes, the Spungy substance of the plied to the place they help the gout, draw Briar, Moss, Viscus Quercimus, Oak, Apples. thorns out of the flesh, and held to the nose Culpeper.] As the College would have help the bleeding thereof. you know this, so would I know what the chief of them are good for.

Jew's-ears boiled in milk and drank, helps sore throats.

Moss is cold, dry, and binding, thierefore good for fluxes of all sorts.

Misleto of the Oak, it helps the falling sickness and the convulsions, being discreetly gathered and used.

Oak Apples are dry and binding; being boiled in milk and drank, they stop fluxes and the menses, and being boiled in vinegar, and the body anointed with the vinegar, cures tlie itch.

Therefore consider that the College gave the Apothecaries a catalogue of what Parts of Living creatures and Excrements they must keep in their shops.

College.] The fat, grease, or suet, of a Duck, Goose, Eel,. Boar, Herron, Thymallows (if you know where to get it) Dog, Capon, Beaver, wild Cat, Stork, Coney, Horse, Hedge-hog, Hen, Man, Lion, Hare, Pike, or Jack, (if they have any fat, I ain persuaded 'tis worth twelve-pence a grain) $W_{\text {olf }}$, Mouse of the mountains, (if you can catch them) Pardal, Hog, Serpent, Badger, Grey or brock Fox, Vulture, (if you can catch Then the College acquaints you, That there them) Album Gracum, Anglice, Dog's ilung, are certain living Creatures called

College.] Bees, Woodlice, Silkworms, the hucklebone of a Hare and a Hog, East and West Bezoar, Butter not salted and salted, Toads, Crabs of the River, little Puppy Dogs, stone taken out of a man's bladder, Vipers Grass-hoppers, Cantharides, Cothanel, Hedge- flesh, fresh Cheese, Castorium, white, yellow, hogs, Emmets or Ants, Larks, Swallows, and and Virgin's War, the brain of Hares and this young ones, Horse-leeches, Sinails, Earth- Sparrows, Crabs' Claws, the Rennet of a worms, Dishwashers or Wagtails, House Lamb, a Kid, a Hare, a Calf, and a Horse, Sparrozs and Hedge Sparrows, Frogs, Scineus, the heart of a Bullock, a Stag, Hog, and Land Scorpions, Moles, or Monts, Tortoise of a Wether, the horn of an Eilk, a Hart, the Woods, Tenches, Vipers and Foxes.

Culpeper.] That part of this crew of killed by a violent death, a Cockscomb, the Cattle and some others which they have not tooth of a Bore, an Elephont, and a Sea-horse, been pleased to learn, may be made bene- Ivory, or. Elephant's 'Tooth, the skin a Snake ficial to your sick bodies, be pleased to hath cast off, the gall of a Hawk, Bullock, a understand, that

she Goat, a Hare, a Kite, a Hog, a Bull,

Bees being burnt to ashes, and a lye made a Bear, the cases of Silk-zcorms, the liver of a with the ashes, trimly decks a bald head Wolf, an Otter, a Frog, Isinglass, the gints being washed with it.

of a Wolf and a Fox, the milli of a she Ass,

Snails with shells on their backs, being? a she Goat, a Woman, an Ewe, a Heifer, first washed from the dirt, then the shells East and West Bezoar, the stone in the head of

$(27,28$. 
a Crab, and a Perch, if there be any stone in an Ox Gall, stone in the bladder of a Man, the Jaw of a Pike or Jack, Pearls, the marrow of the Leg of a Sheep, Ox, Goat, Stag, Calf; common and virgin Honey, Musk, Mummy, a Sreallow's nest, Crabs Eyes, the Omentum or call of a Lamb, Ram, Wether, Calf, the Seisile and Roach Amethist, Amianth, Amphezwhites, yolks, and shells of. Hen's Eggs, Emmet's lites, Antimony, leaves and filings of Silver, Eggss,bone of a Stag'sheart, an Ox leg, Ossepix, Quick Silver, Lapis, Armenius, native Arsenic, the iminer skin of a Hen's Gizzard, the wool of : both white and red, artificial Arsenic, white Hares, the feathers of Partridges, that which : and realgar, Argilla, Asteria, leaves and filBees make at the entrance of the hive, the ings of Gold, Belemites, Berril, Bole-armenicl, pizzle of a Stag, of a Bull, Fox Limigs, Borrax, Toad-stone, Lapis Calaminatis, Cadfasting spittle, the blood of a Pigeon, of a mia, Lime quick and quenched, Vitriol, white, Cat, of a he Goat, of a Hare, of a Partridge, blue, and green, Steel, Borrar, Chrisolite, of a Sow, of a Bull, of a Badger, of a Snail, Chrisopus, Cynabris, native and artificial, Silk, Whicy, the suet of a Bullock, of a Stag, Whetstones, Chalk, white and green, Crystal, of a he Goat, of a Sheep, of a Heifer, Sperma- Diphriges, the rnst, dust, scales, and flakes of oeti, a Bullock's spleen, the skin a Snake hath Iron, Granite, Mortar, such as walls are cast off, the excrements of a Goose, of a Dog, daubed with, Hematitis, Heliotropium, Jacinth, of a Goat, of Pigeons, of a stone Horse, of a Hyber, Nicius, Jasper, Lapis Judacious, Hen, of Swallows, of a Hog, of a Heifer, the Tiles, Lapis Lazuly, Lapis Lincis, Lithanancle of a Hare, of a Sow, Cobwebs, Water, thrax, Litharge of Silier and Gold, Loadshells, as Blatta Bazantia, Buccince, Crabs, stone, Marchasite, or fire stone Marble, Red Cockles, Dentalis, Entalis, Mother of Pearl, Lead, native and artificial, Miss, Naptha, Mytuli Purpure, Os sepice, Umbilious Mari- Lapis Nephriticus, Nitre, Oaker yellow and mus, the testicles of a Horse, a Cock, the hoof? red, Onyx, Opalus, Ophytes, Ostcocolla, Lead of an Elk, of an Ass, a Bullock, of a Horse, of white and black, Plumbago, Pompholix, Mar'a Lyon, the urine of a Boar, of a she Goat.

Culpeper.] The liver of an Hedge-hog being dried and beaten into powder and drank in wine, strengthens the reins exceedingly, and helps the dropsy, convulsions, and the falling sickncss, together with all fluxes of the bowels.

The liver being in like manner brought into powder, strengthens the liver excoedingly, and helps the dropsy.

Then the College tells you these things may be taken from the SEA, as

College.] Amber-grease, Sea-water, Seachasite, Realgar, Rnby, red Oaker, Sal Armoniach, Sal Gem, and salt Nitre, Saphyr and Sardine, Selenitis, Ftints, Emerald, Smiris, Sori, Spodium, Pezeter, Brimstone, quick and common, Talth, Earth of Cimolia, Sanes, Lemnos, Sylesia, Topas, Alana, Terra, Thtty, Vitriol, white, blue, and greén.

Precious stones alter by a way manifest or liidden.

By a way manifest, they are hot, in the first degree. Hemetitis, Pyritis, Lopis Asius, Thyitis, Smyres, Lapis Schistus.

Precious stones cold, are in the first degree. sand, Bitumen, Amber white and yellow, Jet, Jacinth, Saphyr, Emerale, Cristal, Lapis Carline, Coral, white and reds. Foam of the Sannius, Lapis Phrigius.

Sea, Spunge, Stone Punice, Sea salt, Spunges, Amber.

In the second degree. Ruby, Carbuncle, Granite, Sardony. 
In the fourth degree. Diamond.

In respect of property, they bind, as Lapis Asius, Nectius, Geodes, Pumice-stone.

Emolient; as Alabaster, Jet, Lapis Thrasius.

Stupify: as Memphitis, Jasper, Ophites. Cleanse: as Lapis Arabicus.

Glutinate: as Galactitis, Melites.

Scarify: as Morochtus.

Break the stone: as Lapis Lyncis, Lapis Judaicus, Lapis Sponge.

Retcin the fruit in the romb: as Etitis, Jasper.

Provolie the menses. Ostracites.

Stones altering by a hidden property (as they call it,) are

Bezoar, Topaz, Lapis Colubrinus, Toadstone, Emerald, Alectorius, Calcidonius, Amethist, Saphyr, Jasper, Lapis Nephriticus, Lapis Tibernum, Lapis, Spongites, the stone found in the maw of a Swallow, Load-stone, Lapis Vulturis, Merucius, Coral, Lynturius, Jet, Etites, the stones of Crabs, Amber, Crystal, \&c.

The Load-stone purges gross humours.

Lapis Armenius and Lapis Lazuli, purge melancholy.

Pyrites heat and cleanse, take away dimness of sight. Dioscorides. Lapis Asius binds and moderately corrodes and cleanses filthy ulcers, and fills them up with flesh; being mixed with honey, and applied to the place, is an admirable remedy for the gout.

Chrystal being beaten into very fine pow der, and a dram of it taken at a time helps the bloody-flux, stops the Fluor Albus, and increases milk in Nurses. Mathiolus.

Lapis Samius is cooling and binding, it is very comfortable to the stomach, but it dulls the senses, helps fluxes of the eyes and ulcers.

Geodetes binds and drys, being beaten into powder and mixed with water, and applied to the place, takes away inflammations of the Testicles.
Pumice-stone being beaten into powder, and the teeth rubbed with it, cleanses them. Dioscorides.

Jet, it is of a softening and discussing nature, it resists the fits of the mother.

Lapis Arabicus being beaten into powder, and made into an ointment helps the hemorrhoids.

Ostracites, a dram of it taken in powder provokes the menses; beingtaken after that purgation, causes conception, also being made into an ointment, helps inflammations of the breast.

Myexis being borne about one takes away pains in the reins, and hinders the breeding of the stone.

Lapis Armenius purges melancholy, and also causes vomiting, I hold it not very safe for our English bodies, and therefore I will speak no more of it.

\section{Explanation of certain Vacuations.}

The five opening Roots.

Smallage, Sparagus, Fennel, Parsley, Kneeholly.

The two opening Roots.

Fennel, Parsley.

The five emolient Herbs.

Marsh-mallows, Mallows, Beets, Mercury, Pellitory of the Wall, Violet Leaves.

The five Capillary Herbs.

Maidenhair, Wall Rue, Cetrach, Hart'stongue, Politricum.

The four cordial Flowers.

Borrage, Bugloss, Roses, Violets.

The four greater hot Seeds, Carminative, or breaking wind.

Annis, Carraway, Cummin, Fennel.

The four lesser hot seeds.

Bishop's weed, Amomus, Smallage, Carrots. The four greater cold seeds.

Citrul, Cucumber, Gourds, Melon.

The four lesser cold seeds.

Succory, Endive, Lettice, Purslain.

Five fragments of precious stones. Granite, Jacinth, Sapphire,Sardine,Emerald. 
The right worshipful, the College of $\mathrm{Phy}_{-}$ sicians of London in their New Dispensatory give you free leave to distil these common waters that follow, but they never intend you should know what they are good for.

\section{SIMPLE DISTILLED WATERS.}

\section{Of fresh Roots of}

Briony, Onions, Elecampane, Orris, or Flower-de-luce, Tumips.

Of flowers and buds of

Southernwcod, both sorts of Wormwood, Wood Sorrel, Lady's-Mantle, Marsh-mallows, Angelica, Pimpernel with purple flowers, Smallage, Columbines, Sparagus, Mouse-ear, Borrage, Shepherd's Purse, Calaminth, Woodbine or Honey-suckles, Carduus Benedictus, our Lady's Thistles, Knotgrass, Succor', Dragons, Coit's-foot, Fennel, Goat's Rue, Grass, Hyssop, Lettice, Lovage, Toad-flax, Hops, Marjoram, Mallows, Horehound, Featherfew, Bawm, Mints, Horse-mints, Water Cresses, English Tobacco, white PoppiesPellatory of the Wall, Parsley, Plantain, Purslain, Self-heal, Pennyroyal, Oak leaves, Sage, Scabions, Figrwort or Throatwort, Honseleek, or Sengreen, the greater and lesser. Mother of Time, Nightshade, Tansy, Tormentil, Valerian.

\section{Of Flowers of}

Oranges, (if you can get them) Blue-bottle the greater, Beans, Water-Lilies, Luvender, Nut-tree, Coreslips, Sloes, Rosemary, Roses white, damask, und red, Satyrien, Lime-tree, Clove-gilliflowers, Violets.

\section{Of Fruits of}

Oranges, Black Cherries, Pome Citrons, Quinces, Cucumbers, Strawberries, Winter Cherries, Lemons, Rasberries, unripe Walnuts, Apples.

Of parts of living Creatures and their ex-

Lobsters, Cockles, or Snails, Hartshom, $\begin{aligned} & \text { Into the } \\ & \text { ascribed. }\end{aligned}$

Bullocks dung made in May, Srollows, Earthworms, Magpies, Spawn of Frogs.

\section{SIMPLE WA'TERS DISTILLED, being digested before-hand. \\ Of the fresh Roots of Nettles.}

Of the leaves of Agrimony, wild Tansy, or Silverweed, Mugwort, Bettony, Marigolds, Chamomel, Chamepitys, Celandine, Pilewort, Scurvy-grass, Comfry the greater, Dandelyon, Ash-tree leaves, Eyebright, Fumitory, Alehoof, or ground Ivy, Horse-tail, St. John's Wort, Yarrow, Moneywort, Restharrow, Solomon's Seal, Res solis, Rue, Savin, Sảxifrage, Hart's tongue, Scordium, Tamarisk, Mullin, Vervain, Paul's Bettony, Mead-sweet, Nettles.

Of the Flowers of Mayweed, Broom, Cowslips, Butter-bur, Peony, Elder.

Of the berries of Broom, Elder.

Culpeper.]. Then the College gives you an admonition concerning these, which being converted into your native language, is as follows.

We give you warning that these common waters be better prepared for time to come, either in common stills, putting good store of ashes underneath, the roots and herbs being dryer, \&c. or if they be full of Juice, by distilling the juice in a convenient batl, that so burning may be avoided, which hitherto hath seldom been. But let the other Herbs, Flowers, or Roots, be bruised, and by adding Tartar, common salt, or leven be digested, then putting spring water to them, distil them in an Alembick with its refrigeratory, or Worm, till the change of the taste shew the virtue to be drawn off; then let the oil (if any) be separated from the water according to art. Into the number of these waters may be ascribed. 
The Tears of Vines, the liquor of the Birch-trce, May dew.

Culpeper.] That my country may receive the benefit of these waters, 1 shall first shew the temperatures, secondly, the virtues of the most usual and most easy to come by: If any should take exceptions that I mention not all, I answer first, I mention enough. Sccondly, who ever makes this objection, they shew extreme ingratitude; for had I mentioned but only one, I had revealed more to them than ever the College intended they should know, or give me thanks for doing.

The qualities and appropriation of the simple Distilled Waters.

Simple distilled waters either cool or heat: such as cool, either cool the blood or choler.

Waters cooling the blood. Lettice, Purslain, Water Lilies, Violets, Sorrel Endive, Succory, Fumitory.

Waters cooling and repressing choleric humours, or vapours in the head.

Nightshade, Lettice, Water Lilies, Plantain, Poppies, viz. The flowers both of white black and red Poppies, black Cheries.

The breast and lungs. Violets, Poppies all three sorts, Colt's-foot.

In the heart. Sorrel, Quinces, Water Lilies, Roses, Violets, green or unripe Walnuts.

In the stomach. Quinces, Roses, Violets, Nightshade, Houseleeks, or Sengreen, Lettice, Purslain.

In the liver. Endive, Succory, Night= shade, Purslain, Water Lilies.

In the reins and bladder. Endive, Succory, Winter Cherries, Plantain, Water Lilies, Strawberries, Houseleek or Sengreen, black Cherries.

In the womb. Endive, Succory, Lettice, Water Lilies, Purslain, Roses.

Simple waters which are hot, concoct either flegm or melancholy.

$(27,28$.
Waters concocting flegm in the head, are Bettony, Sage, Marjoram, Chamomel, Fennel, Calaminth, Rosemary-flowers, Primroses, Eye-bright:

In the breast and lungs. Maiden-hair, Bettony, Hysop, Horehound, Carduus Benedictus, Scabious, Orris, or Flower-deluces, Bawm, Self-heal, \&c.

In the heurt. Bawm, Rosemary.

In the slomach. Wormwood, Mints, Fennel, Chervil, Time, Mother of Time, Marigolds.

In the liver. Wormwood, Centaury, Origanum, Marjoram, Maudlin, Costmary, Agrimony, Fennel.

In the spleen. Water-cresses, Wormwood, Calaminth.

In the reins and bladder. Rocket, Nettles, Saxifrage, Pellitory of the Wall, Alicampane, Burnet.

In the womb. Mugwort, Calaminth, Penny-royal, Savin, Mother of Time, Lovage.

Waters concocting Melancholy in the head, are

Hops, Fumitory.

The breast. Bawm, Carduus Benedictus.

The heart. Borrage, Bugloss, Bawm, Rosemary.

The liver. Endive, Chicory, Hops.

The spleen. Dodder, Hart's-tongue, Tamarisk, Time.

Having thus ended the appropriation, I shall speak briefly of the virtues of distilled waters.

Lettice water cools the blood when it is over-heated, for when it is not, it needs no cooling: it cools the head and liver, stays hot vapours ascending to the head, and hinders sleep; it quenches immoderate thirst, and breeds milk in nurses, distil it in May.

Purslain water cools the blood and liver, quenches thirst, helps such as spit blood, have hot coughs, or pestilences.

The distilled water of water Lily-flower's, $4 \mathrm{c}$ 
cools the blood and the bowels, and all internal parts of the body; helps such as have the yellow jaundice, hot coughs and pleurisies, the head-ache, coming of heat, fevers pestilential and not pestilential, as also hectic fevers.

The water of Violet flowers, cools the blood, the heart, liver and lungs, overheated, and quenches an insatiable desire of drinking; they are in their prime about the latter end of March, or beginning of April, according as the year falls out.

The water of Sorrel cools the blood, heart, liver, and spleen: If Venice Treacle be given with it, it is profitable in pestilential fevers, distil it in May.

Endive and Succory water are excellent against heat in the stomach; if you take an ounce of either (for their operation is the same) morning and evening, four days one after another, they cool the liver, and cleanse the blood: they are in their prime in May.

Fumitory water is usual with the city dames to wash their faces with, to take away morphey, freckles, and. sun-burning; inwardly taken, it helps the yellow jaundice and itch, cleanses the blood, provokes sweat, strengthens the stomach, and cleanses the body of adust humours: it is in its prime in May aud Jume.

The water of Nightshade helps pains in the head coming of heat. Take heed you distil not the deadly Nightshade instead of the common, if you do, you may make mad, work. Let such as have not wit enough to know them asunder, have wit enough to let them both alone till they do.

The water of white Poppies extinguishes all heat against nature, helps head-aches coming of lieat, and too long standing in the sun. Distil them in Jume or July.

Colt's-foot water is excellent for burns to wash the'place with it; inwardly taken it helps Phthisicks and other diseases incident to the lungs, distil them in May or June.
The water of Distilled Quinces strengthens. the heart and stomach exceedingly, stays vomiting and fluxes, and strengthens the retentive faculty in man.

Damask Rose water cools, comforts, and strengthens the lieart, so dotlı Red Rosewater, only with this difference, the one is binding, the other loosening ; if your body be costive, use Damask Rose water, because it is loosening: if loose, use red, because it is binding:

White Rose water is gencrally known to be excellent against hot rheums, and inflammations in the eyes, and for this it is better than the former.

The water of Red Poppy fozcers, called by many Corn-roses, because they grow so frequently amongst corn, cools the bloosl and spirits over-heated by drinking or labour, and is therefore excellent in surfets. Green Walnuts gathered about the latter end of June or July, and bruised, and so stilled, strengthen the heart, and resist the. pestilence.

Plantain water helps the headache ; being. dropped into the ear it helps the tooth-ache, helps the phthisicks, dropsy and fluses, and is an admirable remedy for ulcers in the reins and bladder, to be used as common drink: the herb is in its.prime in Mag. Straceberry water cools, quenches thirst. clarifies the blood, breaks the stone, helps all inward inflammations, especially those in the reins, bladder and passages of the urine; it strengthens the liver and helps the yellow jaundice.

The distilled water of Dog grass, or Couch grass, as some call it, cleanses the reins gallantly, and provokes urine, opens obstructions. of the liver and spleen, and kills worms.

Black Cherry water provokes urine, helps. the dropsy. It is usually given in diseases. of the brain, as convulsions, falling-sickness, palsy and apoplexy.

Betomy is in its prime in May, the dis- 
tilled water thereof is very good for such as are pained in their heads, it prevails against the dropsy and all sorts of fevers; it succours the liver and, spleen, and helps want of digestion and evil disposition of the body thence arising; it hastens travail in women with child, and is excellent against the bitings of venomous beasts.

Distil Sage whilst the nowers be on it, the water strengthens the brain, provokes the menses, helps nature much in all its actions.

Marjoram is in its prime in June, distilled water is excellent for such whose brains are tou cold, it provoles urine, heats the womb, provokes the menses, strengthens the memory and helps the judgment, causes an able brain.

Distil Camomel water about the beginning of June. It eases the cholick and pains in the belly; it breaks the stone in the reins and bladder, provokes the menses, expels the dead child, and takes away pains in the head.

Femel water strengthens the heart and brain; dilates the breast, the cough, provokes the menses, encreases milk in nurses, and if you wash your eyes with it, it clears the sight.

The Hooses of the fore fect of a Cow dried and taken any away, encrease milk in nurses, the smoke of them drives away nice. Mizaldus.

Calaminth water heats and cleanses the romb, provokes the menses. and eases the pains of the head, distil it in May.

The distilled water of Rosemary flowers, helps such as are troubled with the yellow Jaundice, Asthmas, it cleanses the blood, helps concoction, strengthens the brain and body excecdingly.

Water of the flowers of Lities of the valley, strengthens. the brain and all the senses.

The water of Cozslip fioners helps the palsey ; takes atray pains in the head, the vertigo and megrin, and is exceeding good for ptegnant romen.
The eyes being washed every morning with Eyebright water, most strangely clears and strengthens the sight.

Maidenhair distilled in May, the water cleanses both liver and lungs, clarifies the blood, and breaks the stone.

Hyssop water cleanses the lungs of flegm, helps coughs and Asthmas, distil it in August.

'The water of Hore-hound, helps the cough and straitness of the breast; it strengthens the breast, lungs and stomach, and liver, clistil it in June.

Carduus water succours the head, strengthens the memory, helps such as are troubled with vertigoes and quartan agues; it provokes sweat, strengthens the heart, and all other fevers of choler. It is in its prime in May and June.

Scabious water helps pleurises and pains, and pricking in the sides; Aposthumes, coughs, pestilences, and straitness of the breast.

Water of Flower-de-luce is very profitable in dropsies, an ounce being drank continually every norning and evening; as also pains and torments in the bowels.

Bazem water distillid in May, restores memory, it quickens all the senses, strengthens the brain, heart, and stomach, causes a merry mind and a sweet breath.

'The water of Comfiey solders broken bones, being drank, helps ruptures, outwardly it stops the bleeding. of wounds, they being washed with it.

Wormzenod water distilled cold, about the end of May, heats and strengthens the stomach, lielps concoction, stays vomiling, kills worms in the stomach and bowcls, it mitigates the pains in the tecth, and is profitahly given in tevers of choler.

. Wint water strengthens the stomach, helps concoction and statys romiling, distil il in. the litter and of Mav, or heginning of June, as the year is in arwarlness or backwadliess, observe llatin all the lest. 
Chervil water distilled about the end of and lungs, provokes urine, and cleanses May, helps ruptures, breaks the stone, dis- the passages of it from gravel.

solves congealed blood, strengthens the lieart and stomach.

The water of Mother of Time strengthens the brain and stomach, gets a man a good stomach to his victuals, provoke urime and the menses, heats the womb. It is in its prime about the end of June.

The water of Marigold flowers is appropriated to most cold diseases of the head, eyes, and stomach: they are in their vigour when the Sun is in the Lion.

The distilled water of Centaury comforts a cold stomach, helps in fever of choler, it kills worms, and provokes appetite.

Maudlin and Costmary water distilled in May or June, strengthens the liver, lielps the yellow jaundice, opens obstructions, and helps the dropsy.

Water-cresses distilled in March, the water cleanses the blood, and provokes urine exceedingly, kills worms, outwardly mixed with honey, it clears the skin of morphew and sunburning.

Distil Nettles when they are in flower, the water helps coughs and pains in the bowels, provokes urine, and breaks the stone.

Saxifrage water provokes urine, expels wind, breaks the stone, cleanses the reins and bladder of gravel, distil them when they are in flower.

The water of Pellitory of the Wall, opens obstructions of the liver and spleen, by drinking an ounce of it every morning; it cleanses the reins and bladder, and eases the gripings of the bowels coming of wind. Distil it in the end of May, or beginning of June.

Cinquefoil water breaks the stone, cleanses the reins, and is of excellent use in putrified fevers. Distil it in May.

The water of Radishes breaks the stone, cleanses the reins and bladder, provokes the menses, and helps the yellow jaundice.

Distil Burnet in May or June, the water breaks the stone, cleanses the passages of urine, and is exceeding profitable in pestilential times.

Mugwort water distilled in May, is excellent in coughs and diseases proceeding from stoppage of tlie menses, it warms the stomach, and helps the dropsy.

Distil Penny-royal when the flowers are upon it: the water heats the womb gallantly, provokes the menses, expels the afterbirth; cuts, and casts out thick and gross humours in the breast, eases pains in the bowels, and consumes flegm.

The water of Lovage distilled in May, eases pains in the head, and cures ulcers in the womb being washed with it; inwardly taken it expels wind, and breaks the stone.

The tops of Hops when they are young, being distilled, the water cleanses the blood of melancholy humours, and therefore helps scabs, itch, and leprosy, and such like diseases thence proceeding; it opens obstructions of the spleen, helps the rickets, and hypochondriac melancholy.

The water of Borrage and Bugloss distilled when their flowers are upon them, strengthens the heart and brain exceedingly, cleanses the blood, and takes away sadness, griefs and melancholy.

Dodder water cleanses the liver and spleen, helps the yellow jaundice.

Tamarisk water opens obstructions, and helps the hardness of the spleen, and strengthens it.

English Tobacco distilled, the water is excellently good for such as have dropsy, to drink an ounce or two every morning; it helps ulcers in the mouth, strengthens the lungs, and helps such as have asthmas.

The water of Dwarf Elder, hath the same effects.

Thus you have the virtues of enough of Elicampane water strengthens the stomach cold waters, the use of which is for mix- 
tures of other medieines, whose operation cold and flegm, 'chilliness of the spirits, is the same, for they are very seldom given \&c.

alone: If you delight most in liquid medi- But that my countrymen may not be cines, having regard to the disease, and part mistaken in this, I shall give them some of the body afflicted by it, these will fur- symptoms of each complexion how a man nish you with where withal to make them may know when it exceeds its due limits. so as will please your pallate best.

\section{COMPOUNDS. SPIRIT AND COM- POUND DISTILLED WATERS.}

Culpeper.] Before I begin these, I the thonght good to premise a few words: They are all lot in operation, and therefore not to be meddlled with by people of hot constitutions when they are in health, for fear of fevers and adustion of blood, but for people of cold constitutions, as melancholy and flegmatic people. If they drink of them moderately now and then for recreation, due consideration being lad to the part of the body which is weakest, they
may do them good: yet in diseases of melancholy, neither strong watersnor sack is to be drank, for they make the hunour thin, and then up to the hearl it flies, where it fills the brain with foolish and fearful imaginations.

2. Let all young people forbear them whilst they are in health, for their blood is usually hot enough without them.

3. Have regard to the senson of the year, so shall you find them more beneficial in Summer than in Winter, because in summer the body is always coldest within, and digestion weakest, and that is the reason why neen and women eat less in Summer than in Winter.

Thus much for people in lealth, which drink strong waters for recreation.

As for the medicinal use of them, it shall be shewed at the latter end of every receipt, is only in general they are (due respect had Spiritus et Aqua Absinthis mimus Composita. to the humours afflicting, and part of the body afflicted) medicinal for diseases of: $(29,30$.
Signs of choler abounding.

Leanness of body, costiveness, hollow eyes, anger without a cause, a testy disposition, yellowness of the skin, bitterness in the throat, pricking pains in the head, the pulse swifter and stronger than ordinary, the urineligher coloured, thinner and brighter, troublesome sleeps, much dreaming of fire, liglıtning, anger, and fighting.

Signs of blood abounding.

The veins are bigger (or at least they seem so) and fuller thien ordinary; the skin is red, and as it were swollen; pricking pains in the sides, and about the temples, sliortness of breath, head ache, the pulse great and full, urine high coloured and thick, dreams of blood, \&c.

Signs of melancholy abounding.

Fearfulness without a cause, fearful and foolish imaginations, the skin rough and swarthy, leanness, want of sleep, frightinl dreanis, sourness in the throat, the pulse very weak, solitariness, thin clear urine, often sighing, \&c.

\section{Signs of flegm alounding.}

Sleepiness, dulness, slowness, heariness, cowardliness, forgetfulness, much spitting, much superfluities at the nose, little appetite to meat and as bad digestion, the skin whiter, colder and smoother than it was want to be; the pulse slow and decp: the urine thick and low coloured: dreams of rain, floods, and water, \&c.

These things thus prenised, I come to the matter.

The first the College presents you with, Or, Spirit and water of Wormwood, the lesser composition. 
College.] Take of the leaves of dryed ? of each one drachm: let the things be cut Wormwood two pounds, Annis seeds, halt that are to be cut, and the things that are a pound: steep them in six gallons of small bruised that are to be bruised, all of them wine twenty four hours, then distill them infused in twenty four pints of Spanish in an Alembick, adding to every pound of Wine, for twenty four hours, then disthe distilled water two ounces of the best Sugar.

Let the two first pound you draw out be called Spirit of Wormwood, those which follow, Wormwood water the lesser composition.

Culpeper.] I like this distinction of the Collcge very well, because what is first stilled out, is far stronger then the rest, and therefore very fltting to be kept by itself: you may take which you please, according as the temperature of your body, either to heat or cold, and the season of the year requires.

It hith the same virtues Wormwood hath, only fitter to be used by such whose bodies are chilled by age, and whose natural heat abates. You may search the herbs for the virtues, it heats the stomach, and helps digestion.

The College.] Aftcr the same manner (only omitting the Annis seeds) is distilled spirit and water of Angelica, both Herb and Root; Bawm, Mints, Sage, \&c. the Tlowers of Rosemary, Clary, Clove-gilliflowers, \&c. the seeds of Caraway, \&c. Juniper-berries, Orange Pills, Lemons, Citrons, \&c. Cinnamon, Nutmegs, \&c.

Spiritus et Aqua Absynthii magis composita.

()) splirit and water of Wormwood, the greater composition.

The College.] 'Take of common and Roman Wormwood, of each a pound; Sage, Mints, Bawm, of each two handfuls; the Roots of Galanga, Ginger, Calamus Aromaticus, Elecampane, of each three drachims; Liquorice an ounce, Ratisins of the Sun stoned, three onnces, $\Lambda$ nnis seeds, and sweet Fennel seeds, of each three drachms ; Cimnamon, Cloves, Nutmegs, of cach two drachms; Cardamoms, Cubebs,

tilled in an Alembick, adding two nunces of white sugar to every pint of distilled water.

Let the first pint be called Spirit of Wormwood the greater composition.

Culpeper.] The opinion of Authors is, That it heats the stomach, and strengthens it and the lungs, expels wind, and helps digestion in ancient people.

Spiritus et Aqua Angelica Magis composita. Or Spirit and water of Angelica, the greater composition.

The College.] 'Take of the leaves of Angclica eight ounces, of Carduus Benedictus six ounces, of Bawm and Sage, of cach four ounces, Angelica seeds six ounces; sweet Fennel seeds nine ounces: Let the herbs, being dryed, and the seeds be grosly bruised, to which add of the species called Aromaticum Rosarum, and of the species called Diamoschu Dulce, of each an ounce and a half, infuse them two days in thirty two pints of Spanish Wiuse, then distil them with a gentle fire, and with every pound mix two ounces of sugar dissolved in Rose-water.

Let the thrce first pounds be called by the name of Spirit the rcst by the name of water.

Culpeper.] 'I'he chief end of composing this medicine, was to strengthen the heart and resist infection, and therefore is very wholesome in pestilential times, and for such as walk in stinking air.

I shall now quote you their former receipt in their former Dispensatory.

\section{Angelica water the greater composition.}

The College.] 'Iake of Angelica two pounds, Annis seed laalf a pound, Coriander and Carawa y seeds, of cach four ounces, Zedoary bruised, three ounces : steep them twenty four hours in six gallons of small 
wine, then draw out the spirit, and sweeten it with sugar.

Culpeper.] It comforts the heart, cherishes the vital spirits, resists the pestilence, and all corrupt airs, which indeed are the natural causes of epidemical diseases, the sick may take a spoonful of it in any convenient cordial, and such as are in health, and have bodies either cold by nature, or cooled by age, may take as much either in the morning fasting, or a little before meat.

\section{Spiritus Lavendula compositus Matthice.}

Or compound spirit of Lavender. Matthias. The College.] 'Take of Lavender flowers one gallon, to which pour three gallons of the best spirits of wine, let them stand together in the sun six days, then distil them with an Alembick with his refrigeratory.

Take of the flowers of Sage, Rosemary, and Bettony, of each one handful: the flowers of Borrage, Bugloss, Lilies of the Valley, Cowslips, of each two handfuls: let the flowers be newly and seasonably gathered, being infused in one gallon of the best spirits of wine, and mingled with the foregoing spirit of Lavender flowers, adding the leaves of Bawm, Feather-few, and Orange tree fresh gathered; the flowers of Stœchas and Orange tree, Bay berries, of each one ounce: After convenient digestion distil it again, after which add Citron pills the outward bark, Peony seed husked, of each six drachms, Cimnamon, Mace, Nutmegs, Cardamoms, Cubebs, yellow Sanders, of each half an ounce, Wood of Aloes one dram, the best Jujubes, the stones being taken out half a pound, digest them six weeks, then strain it and filter it, and add to it prepared Pearls two drams, Emeralds prepared a scruple, Ambergrease, Musk, Saffion, of each half a scruple, red Roses dryed, red Sanders, of each halt an ounce, yellow Sanders, Citron Pills dryed, of each one dram. Let the species being tyed up in a rag, be hung into the aforementioned spirit.

Culpeper.] I could wish the Apothecaris would desire to be certified by the College.

1. Whether the gallon of Lavender flowers must be filled by heap, or by strike. 2. Next, whether the flowers must be pressed down in the measure, or not. 9. How much must be drawn off in the first distillation. 4. Where they should get Orange leaves and flowers fiesh gathered. 5. What they mean by comvenient digestion. 6. Where you shall find Borrage, Bugloss and Cowslips flowering together, that so you may have them all fresh according to their prescript, the one flowering in the latter end of April, and beginning of Nay, the other in the end of Jume, and beginning of July. 7. If they can make a shift to make it, how, or which way the virtues of it will countervail the one halt of the charge and cost, to leave the pains and trouble out.
Spiritus Castorii.
Or Spirit of Castoreum.

The College.] Take of tresh Castoreum four ounces, Lavender flower an ounce, the tops of Sage and Rosemary, of each half an ounce, Cinnamon six drams, Mace. Cloves, of each two drachms, spirits of Wine rectified, six pounds, digest them in a phial filled only to the third part, close siopped with cork and bladder in warm ashes for two days, then distilled in Balneo Maria, and the distilled water kept close stopped.

Culpeper.] By reason of its heat it is no ways fit to be taken alone, but nixed with other convenient medicines appropriated to the diseases you would give it for, it resists poison, and helps such as are bitten by venomous beasts: it causes speedy delivery to women in travail, and casteth out the Placenta: it helps the fits of the mother, lethargies and convulsions, being mixed with white wine, and dropped into 
the ears, it helps deafness; if stopping be pound, Peony flowers four ounces : steep thie cause of it, the dose to be given in-them together fourtecn days, then distil wardly is between one dram, and half a them in Balneo Marice till they be dry: in dram, according to the strength and age of the distilled liquor infuse again male Peony the patient.

\section{Aqua Petasitidis composita.}

Or, compound water of Butter-bur.

The College.] 'Take of the fresh roots of Butter-bur bruised, one pound and a half, the roots of Angelica and Masterwort, of each half a pound, steep them in ten pints of strong Alc, then distil them till the cliange of the taste gives a testimony that the strength is drawn out.

Culpeper.] 'I'his water is very effectual being mixed with other convenient cordials, for such as have pestilential fevers: also a spoonful taken in the morning, may prove a good preservative in pestilential times:' it helps the fits of the mother, and? such as are short windel, and being taken inwardly, dries up the moisture of such ? sores as are hard to be cured.

\section{Aqua Raphani Composita.}

Or Compound water of Radishes.

The College.] Take of the leaves of both sorts of Scurvy-grass, of each six pound, having bruised them, press the juice out of them, with which mix of the juice of brooklime, and Water-cresses, of each nne pound and a half, of the best white wine, eight pounds, twclve whole Lemons, pills and all, fresh Briony roots four pound, theroots of wild Radishes two pound, Captain Winter's Cinnamon half a pound, Nutmegs four ounces, steep them altogetlier, and then distil them

Culpeper.] I fancy it not, and so I leave it; I suppose they intended it for purgation of women in child-bed.

Aquu Peonice Composita.

$\mathrm{Or}$ Compound water of Pcony.

The College.] Take of the flowers of Iilies of the Valley, one pound: infuse them in four gallons of Spanish wine so long till the following flowers may be had fresh. roots gathered in due time, two ounces and a lialf, whitc Dittany, long Birthwort, of each half an ounce, the leaves of Misselto of the Oak, and Rue, of each two handfuls, Peony seeds husked, ten drams, Rue seeds three drams and a half, Castoreum two scruples, Cubebs, Mace, of each two drachms, Cinnamon an ounce and a half, Squills prepared, three draclims, Rosemary flowers six - pugils, Arabian Stæchas, Lavender, of : each four pugils, the florvers of Betony, Clove-gilli-flowers, and Cowslips, of each eight pugils, then adding four pound of the juice of black Cherries, distil it in a glass till it be dry.

\section{Aqua Bezoartica.}

Or Bezoar Water.

College.] Take of the leaves of Celandine, roots and all, three handfuls and a half, Rue two handfuls, Scordium four handfuls, Dittany of Crete, Carduus, of each one handful and a half, Zedoary and Angelica roots, of cach three drams, Citrous and Lemon pills, of cach six drams, Clovegilliflowers one ounce and a half, Red Rose, Centaury the less, of each two drams, Cinnamon, Cloves, of each three drams, Venice Treacle three ounces, Mitlıridates one ounce and a half, Camphire two scruples, Troches of Vipers two ounces, Mace two drams, Wood of Aloes half an ounce, Yellow Sanders one dram and a half, Carduus scerls one ounce, Citron sceds six drams, let them be cut and infused in spirits of Wine, and Malaga Winc, of each threc pound and a half, Vinegar of Clovegilliffowers, Juice of Lemons, of each one pound, and distilled in a glass still in liulnco Marice, after it is half distilled off, the residuc may be slrained through a linen cloath, and he reduced to the thickness of
Honcy, and called the Bezoartic catract 
Culpeper.] Extracts have the same vir- one pound and a half, the leaves and tues with the waters they are made from, thowers of Centaury the less, of each four only the different form is to please the ounces, steep them eight days in twelve partates of such whose fancy loathes any one pounds of white Wine, then distil them in particular form.

'I'his Bezoar water strengthens the heart, ateries, and vital spirits: It provokessweat, and is exceeding good in pestilential fevers, in health it withstands melancholy and consumptions, and makes a merry, blithe, chearful creature. Of the extract you may take ten grains at a time, or sumewhat
more, if your body be not feverish, half a spoonful of water is sufticient at a time, and that mixed with other cordials or medicincs appropriated to the disease that troubles you.

Aqua et Spiritus Lambricorum, magistralis.

Or Water and Spirit of Earthworms.

College.] 'I'ake of Linthworms well cleansed, three pound, Snail with shells
on their backs cleansed, two gallons, bcat them in a mortar, and put them into a convenient vessel, addling stinging Nettles, roots and all, six handiuls, wild Angelica, four handfuls, brank Ursinc, seven handfuls, Agrimony, Bettorry, of catch three handfuls, Rue one handful, common Wormwood two handfuls, Roserary flowers six steeped in four gallons of Canary Wine,
ounces, Dock roots ten ounces, the roots of still off three gallons in an alembick, to Sorrel five ounces, 'I'urmeriek, the immer which add three ounces of each of the bark of Barberries, of each four ounces, cordial flowers, Clove-gillitlowers six Fenugreck seeds two ounces, Cloves three ounces, Saffron half an ounce, Turmerick ounces, Hart's-horn, Ivory in gross pow- two ounces, Galanga, Bazil seeds, of each der, of each four ounces, Satfron three one dram, Citron pills one ounce, the seed drams, small spirits of Wine four gallons of Citrons and Carduus, Cloves of each and a half, after twenty-four hours infusion, five ounces, Hart's-horn four ounces, steep distil them in an alembick. Let the four them twenty four hours and then distil first pounds be reserved for spirit, the rest them in Balneo Marice: to the distilled water for water.

Culpeper.] 'Tis a mess altogether, it red Coral, Crabs eyes, white Amber, of each may be they intended it for an universal two drams, Crabs claws, six drams, Bezoar, medicine.

Aqua Gentiance compositce.

Or Gentian Waler compound.

College.] 'T'ake of Gentain roots sliced, may keep the powders for Spicord. (emp.) Ambergrease, of each two scruples, steep them six weeks in the sun, in a vessel welt stopped, often shaking it, then filter it, (you A $\mathrm{E}$ 
by mixing twelve ounces of Sugar candy, with six ounces of red Rose-water, and four Venice Treacle, Mithridates, of each eight ounces of spirit of Cinnamon with it.

Culpeper.] I suppose this was invented Vinegar six pounds, juice of Lemons two for a cordial to strengthen the heart, to pounds, digest them two days, either in relieve languishing nature. It is exceed-Horse-dung, or in a bath, the vessel being ing dear: I forbear the dose, they that close shut, then distil them in sand; in the have money enough to make it themselves, distillation you may make a 'Theriacal excannot want time to study both the virtues traction.

and dose: I would have gentlemen to be studious.

\section{Aqua cordialis frigida Sarenice}

College.] Take of the juice of Borrage, Bugloss, Bawm, Bistort, Tormentil, Scordium, Vervain, sharp-pointed Dock, Sorrel, Goat's Rue, Mirrlis, Blue Bottle great and small, Roses, Marigolds, Lenon, Citrons, of each three ounces, white Wine Vinegar one pound, Purslain seeds two ounces, Citron and Carduus seeds, of each half an Treacle. The dose is from a spoonful to Citron and Carduus seeds, of each half an Treacle.

the flowers of Borrage, Bugloss, Violets, Clove-gilliflowers, of each one ounce, Diatrion Sentaloñ six arams: let all of them, being rightly prepared, be infused three days, then distilled in a glass still: to the distilled Liquor add earth of Lemnos, Siletia, and Samos, of each one ounce and an half, Pearls prepared with the juice of Citrons, three drams, mix them, and keep them together.

Culpeper.] It mightily cools the blood, and therefore profitable in fevers, and all liseases proceeding of heat of blood; it movokes sherp. You may take half an ounce at a tiuc, or two drams if the party be weak.

\section{Aqua Theriacalis.}

Or 'Treacle Water.

College.] Take of the juice of green in all fevers, especially pestilential; it expels venomous hunours by sweat; it strengthens the heart and vitals; it is an admirable counter-poison, special good for such as have the plague, or are poisoned, or bitten by venomous beasts, and expels virulent humours from such as have the venereal disease. If you desire to know more virtues of it, see the virtues of Venice

\section{Aqua Brionice composita.}

Or Briony Water compound.

College.] Take of the juice of Briony. roots, four pounds, the leaves of Rue and Mugwort, of each two pounds, dryed Savin three handfuls, Featherfer, Nep, Pennyroyal, of each tiru handfuls. Bazil, Dittany, of Crete, of each one handful and a half, Orange pills four ounces, Myrrh two ounces, Castoreum one ounce, Canary Wuie twelve pounds, digest them four days in a convenient vessel, then still them in Balneo Marice: About the middle of the distillation strain it out, and make an Hysterical extraction of the residue.

Culpeper.] A spoonfül of it taken, eases the fits of the mother in women that have them; it potently expels the after-

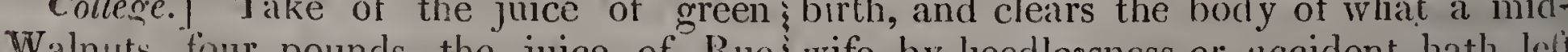
Walnuts, four pounds, the juice of Rue wife by lieedlessness or accident hath left three pounds, juice of Carduus, Marigolds, behind; it cleanses the womb exceedingly, and Bawm, of each two pounds, green and for tlat I fancy it much, take not above. Petasitis roots one pound and a half, the a tasterful at a time, and then in the morroots of Burs one pound, Angelica and ning fasting, for it is of a purging quality, Mastcr-wort, of ench half a pound, the and let pregnant women forbear it. 


\section{Aqua Imperialis. \\ Or Imperial Water.}

The College.] 'l'ake of dried Citron, and Orange pills, Nutnlegs, Clores, Cimnamon, of each two ounces, the roots of Cypress, Orris, Florentine, Calamus Aromaticus, of cach one ounce, Zedoary Galanga, Ginger, of cach hall an ounce, the tops of Livender and Rosemary, of each two nandfuls, the leaves of Bay, Marjoram, Bawm, Mints, Sage, Thyme, of each one handful, the flowers of white and Damask Roses fresh, of cach half a handful, Rosewater fonr pounds, white Winc eight pounds, let all of them be bruised and infused twenty four hours, then distil them according to art.

Culpeper.] Yon must distil it in a bath, and 1 ot in sand: It comforts and sirengthens the heart against faintings and swoonings, and is held to be a prescrvative against consumptions and apoplexies. You may take half a spoonful at a timc.

\section{Aqua Mirabilis.}

Cnllegc:] Take of Cloves, Galanga, Cubebs, Mace, Cardamoms, Nutmegs, Ginger, of cach one dram, Juice of Celanrime half a pound, spirits of Wine one pound, white. Wine three pounds, infuse thein twenty-four hours, and draw off two pounds with an alembick.

Culpeper.] The simples also of this, regard the stomach, and therefore the water heits cold stomachs, besides authors say it prescries from apoplexies, and restores lnst speech.

\section{Aqua Protheriacalis.}

College.] 'I'ake of Scordium, Scahius, Carduus, Goat's Rue, of each two handfuls, Citron and Orange pills, of ench two onnces, the seeds of Citrons, Carduus, Hartwort, Treacle, Mustard, of each one ounce, the flowers of Marigolds and Rosemary, of each one handful, cut them, and bruisc then grossly, then infuse them in four pounds of white Wine, and two pounds of
Carduus water, in a glass, close stopped, and set it in the sun or bath for a fortnight, often shaking it, then distilit in Balneo'Marice. Let the two first pounds be kept by themselves for use, and the remainder of the distillation by itself: Lastly, mix one ounce of Julep of A lexandria, and a spoonful of Cinnamon water with each pound.

Culpeper.] Aqua Protheriacalis, signifies a water for Treacle; so then if you put Diascoridum to it, it is a water for Diascoridum; well then, we will take it for a general water for all physick.

\section{Aqua Capomis. \\ Or Capon Water.}

College.] Take a Capon the guts bring pulled out, cut in pieces, the fat being taken away, boiled in a suficieint quantity of spring-water in a close vessel, take of this broth three pounds. Borrage and Violct-water, of each a pound and a half; white Wine one pound, red rose lcaves two drams and an half, the flowers of Borrage, Violets and Bugloss, of each one dram, pieces of bread, hot out of the oven, half a pound, Cinnamon bruised, half an ounce, distil it in a glass still according to art.

Culpeper.] The simples are niost of them appropriated to the heart, and 11 truth the composition greatly nourishes and strengthens such as are in consumptions, and restores lost strength, either by fevers or other sickness: It is a sovereign remedy for hectic fevers, and Marasmos, which is nothing else but a consumplion coining from them. Let silch as are subject to these diseases, hold it for a jewel.

$$
\begin{aligned}
& \text { Aqua Limacum Magistr. } \\
& \text { Or Water of Snails. }
\end{aligned}
$$

College.] Take of the juice of Ground Ivy, Coll's-foot, Scabious, Lungwort, of each one pound and a half, the juice of Purslain, Plantain, Aunbrosia, Paul's Bettony, of each a pound, Hog's blood, white Wine, of each four pounds, Garden Snails,
two pound, dried Tobacco leaves erghts 
powder of Liquerice two ounces, of Elecampane halt an ounce, of Orris an ounce, Cotton seeds an ounce and a half, the pound, green Asarabacca six ounces, Radish greater cold seeds, Annis seeds of each six seeds, six ounces. Let all of them, being drams, Satfron one dram, the flowers of bruised, be steeped in three pounds of white red Roses, six pugils, of Violets and Bor- Wine for three days, then distilled in a rage, of each four pugils, steep them three leaden still till they be dry.

days warm, and then distil them in a glass still, in sand.

Culpeper.] It purges the lungs of flegm and helps consumptions there. If you should happen to live where no better nor readier medicine can be gotten, you may use this.

\section{Aqua Scordii composita.}

Or Compound Water of Scordium.

College.] 'Take of the juice of Groat's Rue, Sorrel, Scordium, Citrons, of each one pound, London 'I'reacle, half a pound, steep it three days, and distil it in sand.

Culpeper.] A tasterful taken in the morning, preserves from ill airs.

\section{Aqua Marice.}

College.] Talie of Sugar Candy a pound, Canary Wine six ounces, Rose Water four ounces; boil it well into a Syrup, and add to it Imperial water two pounds, Ambergreese, Musk, of each eighteen grains, Saffron fifteen grains, yellow Sanders infused in Imperial water, two drams; make a clear water of it.

\section{Aqua Papaveries composita.}

Or Poppy Water compound.

College.] Take of red Poppies four pounds, sprinkle them with white Wine two pounds, then distil them in a common still, let the distilled water be poured upon fresh flowers and repeated three times; to which distilled water add two Nutmegs sliced, red Poppy flowers a pugil, Sugar two ounces, set it in the sun to give it a pleasing sharpness; if the sharpness be more than you would have it, put some of the same water to it which was not set in the sun. Aqua Juglandium composita:

Or Walnut Water compound

\section{College.] 'Take of green
ound and an half, liad
ound, green Asarabacca six
uised, be steeped in three por
ine for three days, then
aden still till they be dry.}

\section{T I N C T U R E.}

\section{Tenctura Croci.}

Or Tincture of Saffron.

College.] Take two drams of Saffron, eight ounces of Treacle water, digest them six days, then strain it.

Culpeper.] See the virtues of Treacle water, and then know that this strengthens the heart something more, and keeps melancholy vapours thence by drinking a spoonful of it every morning.

\section{Tinctura Castorii.}

\section{Or Tincture of Castoreum.}

College.] Take of Castoreum in powder half an ounce, spirit of Castoreum half a pound, digest them ten days cold, strain it, and keep the Liquor for Tincture.

Culpeper.] A learned invention! 'Tis omething more prevalent than the spirit.

\section{Tinctura Fragroram.}

Or Tincture of Strawberries.

College.] Take of ripe Wood-strawberries two pounds, put them in a phial, and put so much small spirits of Wine to them, that it may overtop them the thickness of four fingers, stop the vessel close, and set it in the sun two days, then strain it, and press it but gently ; pour this spirit to as many fresh Strawberries, repeat this six times, at last keep the clear liquor for your use.

Culpeper.] A fine thing for Gentlemen that have nothing else to do with their money, and it will have a lovely look to please their eyes. 


\section{Tinctura Scordii.}

Or Tincture of Scordium.

College.] Take of the leaves of Scordium gathered in a dry time, half a pound, digest them in six pounds of small spirits of Wine, in a vessel well stopped, for three days, press them out gently, and repeat the infusion three times, and keep the clarified liquor for use.

So is nlade 'Tincture of Celandine, Restharrow, and Rosa-solis.

Culpeper.] See the herbs for the virtues, and then take notice that these are better for cold stomachs, old bodies.

Tinctura Theriacalis vulgo Aqua Theriacalis Ludg - per infius.

Or Tincture of 'Treacle.

College.] Take of Canary Wine often times distilled, Vinegar in which half an ounce of Rue seeds have been boiled, two pounds choice treacle, the best Mithridate, of each half a pound; mix them and set them in the sun, or heat of a bath, digest them, and keep the water for use.

Tinctura Cimnamoni, vulgo, Aqua Clareta Cimnam.

Or Tincture of Cinnamon.

Culege.] Take of bruised Cinnamon wo ounces, rectified spirits of Wine two pounds, infuse them four days in a large glass stopped with cork and bladder, shake it twice a day, then dissolve half a pound of Sugar Candy by itself in two pounds of Rose water, mix both liquors, into which hang a nodule containing, Ambergris half a scruple, Musk four grains.

\section{Tinctura Viridis. \\ Or a green Tincture.}

College.] 'Takic of Verdigris, half an ounce, Auripigmentum six drams, Alum
three dranns, boil them in a pound of white Wine till half be consumed, adding, after it is cold, the water of red Roses, and Nightshade, of each six ounces.

Culpeper.] 'This was made to cleanse ulcers, but I fancy it not.

$(29,30$.
Aqua Aluminosa Magistralis.

College.] Take of Plantain and red Rose water, of each a pound, rocli Alum and Sublimatum, of each two drams; let the Alum and Sublimatum, being in powder, boil in the waters, in a vessel with a narrow mouth till half be consumed, when it has stood five days, strain it.

\section{PHYSICAL WINES.}

\section{Vimum Absynthitis.}

Or. Wormwood Wine.

College.] Take a handful of dried Wornuwood, for every gallon of Wine, stop it in a vessel close, and so let it remain in steep: so is prepared wine of Rosemary flowers, and Eye-bright.

Culpeper.] It helps cold stomachs, breaks wind, belps the wind cholic, strengthens the stomach, kills worms, and helps the green sickness.

Rosemary-flower Wine, is made after the same manner. It is good against all cold diseases of the head, consumes flegin, strengthens the gums and teeth.

Eye-bright Wine is made after the same manner. It wonderfully clears the sight being drank, and revives the sight of elderly men: A cup of it in the morning is worth a pair of spectacles.

All other Wines are prepared in the same mámner.

The best way of taking any of these Wines is, to drink a drauglit of them every morning. You may, if you find your body old or cold, make Wine of any other herb, the virtues of which you desire ; and make it and take it in the same manner.

Vimum Cerassorum Nigronum.

Or Winc of Black Cherries.

College.] Takic á gallon of Black Cherries, keep it in a vessel chose stopped till it begin to work, then filter it, and an ounce of Sugar being adrled to every pound, let $4 \mathrm{~F}$ 
it pass through Hippocrates' slecve, and and when it hath stood so four days, take keep in a vessel close stopped for use.

Vimum Helleboratum.

Or Helleborated Wine.

College.] 'Take of white Hellebore cut small, four ounces, Spanish Wine two pounds, steep it in the sun in a plial close stopped, in the dog days, or other hot weather.

\section{Vimum Rubellum.}

College.] Take of Stibium, in powder, one ounce, Cloves sliced two drams, Clarel Wine two pounds, keep it in a phial close shut.

\section{Vinum Benedictum.}

College.] Take of Crocus Metallorum, in powder, one ounce, Mace one dram, Spanish Wine one pound and an half, steep it.

\section{Vinum Antimoniale.}

\section{Or Antimonial Wine.}

College.] 'Take of Regulus of Antimony, in powcler, four ounces, stcep it in three pounds of white Wine in a glass well stopped, after the first shaking let the Regulus settle.

Culpeper.] These last mentioned are vomits, and vomits are fitting medicines for but a few, the mouth being ordained to take in nourishment, not to cast out excrements, and to regulate a man's borly in vomiting; and doses of vonits require a deeper study in physic, than I doubt the generality of people yet have; I omit it therefore at this time, not because I grudge it my country, but because I would not willingly lave them do thenselves a mischief, I shall shorly teach them in what diseases vomits may be used, and then, and not till then, the use of vomits.

\section{Vinum Scilliticum.}

Or Wine of Squills.

College.] 'Take of a white Squill of the mounlains, gathered about the rising of the dog star, cut it in thin pieces, and dried for a montl, one pound, put it in a glass bottle, bottom, cut in thin slices, and placed thirty and pour to it eight pounds of French Wine, or forty days in the sun or some reniss 
heat, then a pound of them (being cut dram, Saffron three drams, of these let the small with a knife made of jrory or some Saffion, Hart's-horn, Dittany, and Bole, white wood) being put in a vessel, and six be tied up in a rag, and stecped with the pounds of Vinegar put to them; set the ves-s things before mentioned, in five pints of sel, being close stopped, in the sun thirty or Vincgar, for certain diays by a temperate forty days, afterwards strain it, and kecp it heat in a glass woll stopped, strain it, and for use.

Culpeper.] A little of this medicine being taken in the morning fasting, and walking half an hour after, preserves the body in health, to extrome old age, (as Sanius tried, who using no other medicine but this, lived in perfect health till one hundred and seventeen years of agc) it makes the digestion good, a long wind, a clear voice, an acute sight, a good colour. it suffers no offensive thing to remain in the body, neither wind, rlegm, choler, melancholy, dung, nor urine, but brings them forth; it brings forth filth though it lie in the bones, it takcs away salt and sour belchings, though a man be ncver so licen-. tious in diet, lie shall feel ne harm: It hath cured such as have the phthisic, that have been given over by all Physicians: It cures such as have the falling sickness, gouts, and diseases and swellings of the joints: It takes away the hardness of the liver and splcen. add six drams of the best Treacle to it, slrake it together, and keep it for your use. Acetum Theriacale.

Or' Treacle Vinegar.

College.] Add to the description of Treacle water, Clove-gilliflowers two ounces, Lavender flowers an ounce and a half, Rose, and Elder flower Vinegar, of each four pounds, digcst it without boiling, three days, then strain it through Hippocrates sleeve.

Citlpeper.] See Trcacle Water for the virtues, only this is more cool, a little more fantastical.

We should never have done if we slould reckon up the particular benefits of this medicine: Therefore $w c$ conunend it as a wholesome medicine for soundness of body, prescrvation of health, and vigour of mind. 'Thus Galen.

Acetum Theriucule, Norimbers.

Or Trcacle Vinegar.

College.] Take of the roots of Celandine the greater, one ounce and a half: the roots of Angelica, Masterwort, Gentian, Bistort, Valerian, Burnet, white Dittany, Elecam pane, Zedoary, of cach one dram, of Plantain the greatcr one dram and a half, the leavcs of Mousear, Sage, Scabious, Scordium, Dittany of Crete, Carrluus, of rach half an handful, barks and seeds of Citrons, of each half a cram, Bole Amoniac one 3 an ounce, Mandlin fire drams, Polinodiun

\section{E C O C T. I O N S.}

Decoctum commune pro clystere.

Or a common Decoction for a Clyster. College.] Take of Mallows, Violets. Pellitory, Bects, and Mercury, Chamomel Howers, of each one handful, swect Fennel seeds half an ounce, Linsceds two drams, boil them in a sufficiont 'quantity of common water to a pound.

Culpeper:] This is the common decoction for all clysters, according to the quality of the humour abounding, so you may add what Simples, or Syrups, or Electuaries you plcase; only half a score Linsceds, and a handful of Chamomel flowers are adderl.

\section{Dccoclum Epythimi. \\ Or a Decoction of Epithimum.}

College.] Take of Myrobalans, Chebs, and Inds, of cach half an ounce, Stochas, Raisins of the sun stoned, Epithimum, Senua, of each one ounce, Fumitory half 
six drams, Turbith half an ounce, Whey made with Goat's milk, or Heifer's milk four pounis, let them all boil to two pounds, bount, Mousear, ithimum excepted, which boil but s of each two handfuls, Wormwood half a a second or iwo, then take it from the fire, handful, Southernwood, Bettony, Bugloss, and add black Hellebore one dram and Comfrey the greater and lesser, roots and an halt, A gerick lialf a dram, Sal. Gem. all, Avens, both sorts of Plantain, Sanicle, one dram and an half, steep them ten hours, Tormentil with the roots, the buds of Barthen press it strongly out.

Culpeper.] It purges melancholy, as these being gathered in May and June also choler, it resists madness, and all and diligently dried, let them be cut and diseases coming of melancholy, and there- put up in skins or papers against the time fore let melancholy people esteem it as a of use, then take of the forenamed herbs jewel.

Decoctum Sennce Gereonis.

Or a Decoction of Semna.

College.] Take of Senna two ounces, Pollipodium half an ounce, Ginger one dram, Raisins of the sun stoned two ounces, Sebestens, Prunes, of each twelve, do you good, this is as like to do it as any the flowers of Borrage, Violets, Roses, and I know.

Rosemary, of each two drams, boil them in four pounds of water till half be consumed.

Culpeper.] It is a common Decoction for any purge, by adding other simples or compounds to it, according to the quality of the humour you would lave purged, yet, in itself, it chiefly purges melancholy. Decoctum Pectorale.

Or a Pectoral Decoction.

College.] Take of Raisins of the sun stoned, an ounce, Sebestens, Jujubes, of advertise thee of these few things, which each fifteen, Dates six, Figs four, French concern the nature, making, and use ofSyrups Barley one ounce, Liquorice half an ounce, in general. 1. A Syrup is a medicine of Maiden-hair, Hyssop, Scabious, Colt's-foot, a liquid body, compounded of Decoction, of each one handful, boil them in three pounds of water till two remain.

Culpeper.] The medicine is chiefly appropriated to the lungs, and therefore causes a clear voice, a long wind, resists coughs, hoarseness, asthmas, \&c. You may drink a quarter of a pint of it every morning, without keeping to any diet, for it purges not.

I shall quote some Syrups fitting to be mixed with it, when I come to the Syrups. three handfuls, boil them in four pounds of conduit water and two pounds of white Wine gently till half be consumed, strain it, and a pound of Honey being added to it, let it be scummed and kept for use. Infusion, or Juice, with Sugar or Honey, and brought by the heat of the fire, into the thickness of Honey. 2. Because all Honey is not of a thickness, understand new Honey, which of all other is thinnest. 3. The reason why Decoctions, Infusions, Juices, are thus used, is, Because thereby, First, They will keep the longer. Secondly, They will taste the bettcr. 4. In boiling Syrups liave a great care of their just consistence, for if you boil them too much
Culpeper.] If sight of a medicine will 
they will candy, if too little, they will sour. 5. All simple Syrups liave the virtues of the simples they are made of, and are far more convenient for weak people, and delicate stomachs.

Syrupus de Absinthio simplex.

Or Syrup of Wornwwood simple.

The College.] Take of the clarified Juice of common Wormwood, clarified Sugar, of each four pounds, make it into a Syrup according to art. After the same manner, are prepared simple Syrups of Betony, Borrage, Bugloss, Carduus, Chamonel, Succory, Endive, Hedge-mustard, Strawberries, Fumitory, Ground Ivy, St. John's Wort, Hops, Mercury, Muusear, Plantain, Apples, Purslain, Rasberries, Sage, Scabious, Scordium, Houseleek, Colt's-foot, Paul's Bettony, and other Juices not sour. Exlpeper:] See the simples, "and then you may easily know both their virtues, and also that they are pleasanter and fitter for delicate stomachs when they are made into Syrups.

Syrupus de Absinthio Compositus.

Or Syrup of Wormwood compound.

College.] Take of common Wormwood meanly dry, half a pound, red Roses two ounces, Indian Spikenard three drams, old white Wine, juice of Quinces, of each two pounds and an half, steep them a whole day in an earthen vessel, then boil them gently, and strain it, and by adding two pounds of sugar, boil it into a Syrup according to art.

Culpeper.] Mesue is followed verbatim in this; and the receipt is appropriated to cold and flegmatic stomachs, and it is an admirable remedy for it; for it strengthens both stomach and liver, as also the instruments of concoction, a spoonful taken in the morning, is admirable for such as have a weak digestion, it prorokes an appetite to one's victuals, it prevails against the yellow jaundice, breaks wind, purges humours by urine.

$(29,30$. 
it cuts and brings away toingh flegm and four hours in three ounces of white Wine, choler, and is therefore a spccial remedy Radish and Fumitory water, of each two for such as have a stuffing at theirstomach. pounds, then boil it away to one pound Syrupus de Agno Casto.

Or Syrup of A gnus Castus.

College.] Take of the seeds of Rue and Gum Ammoniacum, first dissolved in white Hemp, of each half a dram, of Endive, Wine Vinegar, two ounces, boil the rest Lettice, Purslain, Gourds, Melons, of each with a pound and an half of white sugar two drams, of Fleawort half an ounce, of into a Syrup, adding the mixtures of the Agnus Castus four ounces, the flowers of Gum at the end.

Water Lilies, the leaves of Mints, of each half a handful, decoction of sceds of Lentils, and Coriander seeds, of each half an ounce, three pounds of the decoction, boil them all over a gentle fire till two pounds be con-? sumed, add to the residue, being strained, $t$ wo ounces of juice of Lemons, a pound and a half of white sugar, make it into a Syrup according to art. little.

Culpeper.] A pretty Syrup, and good for Syrupus de Althea.

Or Syrup of Marsh-mallows.

Culpeper.] It cools the liver, and opens obstructions both of it and the spleen, helps old surfeits, and such like diseases, as scabs, itch, leprosy, and what else proceed from the liver over heated. You may take an ounce at a time.

\section{Syrupus de Artemisia.}

Or Syrup of Mugwort.

College.] Take of Mugwort two handfuls, Pennyroyal, Calaminth, Origanum, Bawm, Arsmart, Dittany of Crete, Savin, Marjoram, Germander, St. John's -Wort, Camcpitis, Featherfer with the flowers, College.] 'Take of roots of Marsh-mal- Centaury the less, Rue, Bettony, Bugloss, lows, two ounces, the roots of Grass Aspara - of each a handful, the roots of Fennel, gus, Liquorice, Raisins of the Sun stoned, Smallage, Parsley, Sparagus, Bruscus, of each half an ounce, the tops of Mallows, Saxifrage, Elecampane, Cypress, Madder, Marsh-mallows, Pellitory of the Wall, Orris, Peony, of each an ounce, Juniper Burnet, Plantain, Maiden-hair white and Berries, the seeds of Lovage, Parsley, black, of each a handful, red Cicers an Snallage, Annis, Nigella, Carpobalsanum ounce, of the four greater and four lesser or Cubebs, Costus, Cassia Lignea, Cardacold seeds, of each three drams, boil them moms, Calamus Aromaticus, the roots. of in six pounds of clear Water till four remain, Asarabacca, Pellitory of Spain, Valcrian, which being strained, boil into a syrup with ? of each half an ounce, being cleansed, cut, four pounds of white sugar.

Culpeper.] It is a fine cooling, opening, slipery Syrup, and chietly commendable for the cholic, stone, or gravel, in the kidneys or bladder.

Syrupus de Ammoniaca.

Or Syrup of Aminoniacum.

College.] 'Take of Mitudlin and Cetrach, of cach four handfuls, common. Wormwood an ounce, the roots of Succory, Sparagus, and bruised, let them be infused twentyfour hours in fourteen pounds of clcar water, and boiled till half be consumed, bcing taken off from the fire, and rubbed betweer: your hands whilst it is warm, strain it, and witl honey and sugar, of each two pounds, sharp Vinegar fourounces, boil it to a Syrup, and perfume it with Cinnamon and Spikenard, of each three drams.

Culpeper.] It helps the passion of the bark of Caper roots, of each two ounces, matrix, and retains it in its place, it disafter due preparation steep them twenty- solves the coldness, wind, and pains thercof: 
it strengthens the nerres, opens the pores, corrects the blood, it corrects and provokes Liquorice stick, or take a spoonful in the the menses. You may take a spoonful of molning fasting.

it at a time.

Syrumus de Betonica compositus.

Or Syrup of Bettony compquind.

College.] Take of Bettony three handfuls, Marjoram four handfuls and a hals 'Thy rie, red Roses, of each a handful, Violets, Stachas, Sage, of each half a handful, the seeds of Fennel, Annis; and Ammi, of each half an ounce, the roots of Peons, Polypodium, and Fennel, of each five drams, boil thom in six pounds of river water, to three pounds, strain it, and add juice of Bettony two pounds, sugar three pounds and a half, make it into a Syrup.

Culpeper.] It helps diseases coming of cold, both in the head and stomel, as also such as come of wind, vertigos, nadness ; it concocts melancholy, it provokes the menses, and so doth the simple syrup more than the compound.

\section{Symupus Byzantimus, simple.}

Collegc.]. Take of the Juice of the leaves of Endive and Smallage, of each two pounds, of Hops and Bugloss, of each one pound, boil them togcther and scum them, and to the clarified liquor, add four pounds of white sugar, to as much of the juices, and with a gentle fire boil it to a Syrup.

Syprupus Byzantimus, compound.

College.] Take of the Juices so ordered as in the former, four pounds, in which boil red Roses, two ounces, Liquorice half an ounce, the seeds of Annis, Fennel, and Snlallage, of each three drams, Spikenard two drams, strain it, and to the three pounds remaining, add two pounds of 'Vinegar, four pounds of Sugar, make it into a syrup according to art.

Culpeper.] They both of them (viz. both Simple and Compound) open stopp-? ings of the stomach, liver, and spleen, help the rickets in children, cut and bring away trugh flegm, and help the yellow

\section{Symupus Botryos.}

Or Syrup of Oak of Jerusalem.

College.] 'Take of Oak of Jcrusalem, Hedge-mustard, Nettles, of each two handfils, Colt's-foot, one liandful and a lialf, boil them in a sufficient quantity of clear watcr till half be consumed; to two pounds of the Dccoction, add two pounds of the Juice of 'Turnips baked in an oven in a close pot, and with threc pounds of white sugar, boll it:into a Syrup.

Culpeper] This Syrup was composed against coughs, shortness of breath, and other the like infirnities of the breast proceeding of cold, for which (if you can get it) you mat take it with a Liquorice stick. Syrunus ciupilloram Veneris.

Or Syruip of Maiden-hair.

College. $]_{\text {inc }}$ ef Iquorice two ounces, Maiden-har five othes," stecp them " $a$ ' inatural day in four pounds of warm water, then after gentle boiling, and strong straining, with a pound and a half of fine sugar make it into a Syrup.

Culpeper.] It opens stoppings of the stomach, strengthens the lungs, and helps the infirmities of them.: This may be taken also either with a Jiquorice stick, or mixed with the Pectoral Decoction like Syrup of Coltsfoot.

Syrupus Cardiacus, rel Iulcpum Cardiacum. Or a Coidial Syrup.

College.] 'Jake of Rhenish Winc two pounds, Rose Watcr two ounces and a hall, Cloves two scruples, Cinnamon half a dram, Ginger two scruples, Sigar three ounces and a laalf, boil it to the cbisistence of a Julep, adding Ambergris three grains, Musk one grain.

Culpeper.] If you would have this Julep keep long, yon may put in "rub́c sugar, and yet if close stopiped, ielwill not easily corrupt because it is nade up buly of Nine; 
indeed the wisest way is to order the quan- a pound, Sugar two pounds, make it into tity of sugar according to the palate of a Syrup like Syrup of Clove-gilliflowers. him that takes it. It restores such as are in consumptions, comforts the heart, cherishes the drooping spirits, and is of an opening quality, thereby carrying away those vapours which might otherwise annoy the brain and heart: You may take an sunce at a time, or two if you please.

Syrupus infusionis florum Cariophillorum.

Or Syrup of Clove-gilliflowers.

College.] Take a pound of Clove-gilliflowers, the whites being cut off, infuse them a whole night in two pounds of water, then with four pounds of sugar melted in it, make it into a Syrup without boiling.

Culpeper.] This Syrup is a fine temperate Syrup: it strengthens the heart, liver, and stomach; it refreshes the vital spirits, and is a good cordial in fevers ; and usually mixed with other cordials, you can hardly err in taking it, it is so harmless a Syrup.

\section{Syrupus de Cinnamomo. \\ Or Syrup of Cinnamon.}

College.] Take of Cinnamon grossly bruised, four ounces, steep it in white Wine, and small Cinnamon Water, of each half a pound, three days, in a glass, by a gentle heat; strain it, and with a pound and a half of sugar, boil it gently to a Syrup.

Culpeper.] It refreshes the vital spirits exceedingly, and cheers both heart and stomach languishing through cold, it helps digestion exceedingly, and strengthens the whole body. You may take a spoonful at a time in a cordial.

College.] Thus also you may conveniently prepare Syrups (but only with white Wine,) of Annis seeds, sweet Fennel seeds; Cloves, Nutmegs, Ginger, \&c

Syrupus Acetositatis Citriorium.

Nir Syrup of Juice of Citrons.

College..] Take of the Juice of Citrons, and cork, a digestion being made three or strained without expression, and cleansed, four days, pour off what is dissolved; put 
in fresh clarified juice, and proceed as before, repeat this so often till all the coral be dissolved; lastly, to one pound of this juice add a pound and a half of sugar, and boil it to a Syrup gently.

Syrupus e Corulliis compositus.

Or Syrup of Coral compound.

College.] Take of red Coral six ounces, in very fine powder, and levigated upon a marble, add of clarified juice of Lemons, the flegm being drawn off in a bath, sixteen ounces, clarified juice of Barberries, eight ounces, sharp white Wine Vinegar, and juice of Wood-sorrel, of each six ounces, mix them together, and put them in a glass stopped with cork and bladder, shaking it every day till it have digested eight days in a bath, or horse dung, then filter it, of which take a pound and a half, juice of Quinces half a pound, sugar of Roses twelve ounces, nuke them into a Syrup in a bath, adding Syrup of Clove-gillitlowers sixteen ounces, keep it for use, omitting the half dram of Ambergris, and four grains of Musk till the physician command it.

Culpeper.] Syrup of Coral both simple and compound, restore such as are in consumptions, are of a gallant cooling nature, especially the last, and very cordial, good for hectic fevers, it stops fluxes, the running of the reins, and the Fluor Albus, helps such as spit blood, and such as have the falling-sickness, it stays the menses. Half a spoonful in the morning is enough.

\section{Symupus Cydomiorum. \\ Or. Syrup of Quinces.}

College.] Take of the Juice of Quinces clarified six pounds, boil it orer a gentle fire till half of it be consumed, scumming it, adding red Wine tlnce pounds, white sugar four pounds, boil it into a Syrup, to be perfumed with a dram and a half of Cinnamon, Cloves and Ginger, of cach two scruples.

Culneper.] It strengthens the heart and stomach, stays looseness and vomiting, relicres languishing nature: for looseness, $(29,30$. take a spoonful of it before meat, for vomiting after meat, for both, as also for the rest, in the morning.

\section{Syrupus de Erysimo.}

Or Syrup of Hedge-mustard.

College.] T'ake of Hedge-mustard, fresh, six handfuls, the roots of Elecampane, Colt's-foot, Liquorice, of each two ounces, Borrage, Succory, Maiden-hair, of cach a handful and a half, the cordial flowers, Rosemary and Bettony, of eacli half a handful, Annis seeds half an ounce, Raisins of the sun stoned, two ounces, let all of then, being prepared according to art, be boiled in a sufficient quantity of Barley Water and Hydromel, with six ounces of juice of Hedge-mustard to two pounds and a half, the which, witl three pounds of sugar, boil it into a Syrup according to art.

Culpeper.] It was invented against cold aftlictions of the breast and lungs, as asthmas, hoarseness, \&c. You may take it either with a Liquorice stick, or which is better, mix an ounce of it with three or four ounces of Pectoral Decoction, and drink it off warm in the morning.
Symupus de Fumaria.
Or Syrup of I'unitory.

College.] l'iake of Endive, common Wommood, Hops, Dodder, Hart's-tongue, of eacl a handful, Gpithinum an ounce and a half, boil them in four pounds of water till lialf be consumed, strain it, and add the juice of Fumitory a pound and a half, of Borrage and Bugloss, of each half a pound, white sugar four pounds, make then into a Syrup according to art.

Culpeper.] 'The reccipt is a pretty concoctcr of melancholy, and therefore a rational help for diseases arising thence, both internal and external, it helps diseases of the skin, as Leprosies, Cancers, Warts, Corns, Itch, 'Tetters, Ringworms, Scabs, \&c. and it is the better to be liked, because of its gentleness. It hiclps surfils cxceedingly, cleanses, cools, and strengthens the liver, 4 II 
and causes it to make goorl blood, and good the fruits the seeds, and the seeds the herbs, blood cannot make bad flesh. I commend about a quarter of an hour ; at last, five this receipt to those whose borlies are sub- pounds of water being consumed, boil the ject to scabs and itch. If you please you other three (being first strained and clarimay take two ounces by itself every morning.

\section{Syrupus de Glycyrrhiza.}

Or Syrup of Liquorice.

College.] Take of green Liquorice, scraped and bruised, two ounces, white Maiden-hair an ounce, diyed Hyssop half an ounce, steep these in four pounds of hot? water, after twenty-four hours, boil it till half be consumed, strain it, and clarify it, and with Honey, Penids, and Sugar, of each eight ounces, nuke it into a Syrup, adding, before it be perfectly boiled, red Rose Water six ounces.

Culpeper.] It cleanses the breast and and round, Briony, Dittany, Gentian, lungs, and helps continual coughs and Hog's Fenucl, Valerian, of each half an pleurisies. You may take it with a ounce, the roots of Smallage, Asparagus, Liçuorice stick, or add an ounce of it or Fennd, Parsley, Bruscus, of each an ounce, more to the Pectoral Decoction.

Syrupus Granatorum cum Aceto; rulgo,

O.rysuccharum simplex.

Pcllitory of Spain, an ounce and a half, Stoechas, the sceds of Annis, Ammi, Caraway, Fennel, Lovages, Harlwort, of each Or Syrup of Pomegranates with Vincgar. Llare drams, Raisins of the sun two ounces,

College.] 'T'ake of white sugar a pound boil them in ten pounds of water to tour, and a half, juice of Pomegranates eight to which add honey and sugar, of each two ounces, white Wine Vinegar four ounces, boil it gently into a Syrup.

Culpeper.] Look the virtues of Pome-: of each three drams. granates among the simples.

Syrumus de Hyssopo.

Or Syrup of IIyssop.

College.] 'lake eight pounds of Spring Watrr, half an ounce of Barley, boil it about half an hour, then add the Roots of Smallage, Parsiey, Fennel, Liquorice, of each ten drams, Jujubes, Sebestens, of each fiftecn, Raisins of the sun stoned, an ounce and a halt, Figs, Dates, of cach ten, the secds of Mallows and Quinces, Gum 'Tragacanth ticd up in a rag, of each three drans, Hyssop meanly dryed, ien drams, Maiden-hair six drams, boil them together, very available in coughs, hoarsoness, and yet so, that the roots may precede the firuits, pleurisies, ulcers of the lungs and bladder, 
as also in all inflammations whatsoever, You may take a spoontul of it once in three Devil's-bit, the flowers of both sorts of or four hours, or if you please take it with Bugloss, and Rosemary, of each a handful, a Liquorice sticks.

Syrupus de Weconio, size Diacodium.

Or Syrup of Meconiun, or Diacodium.

College.] T'ake of white Poppy lieads with their seeds, gathered a little after the flower's are fallen oft, and kept thrce days, cightounces, black Poppy heads (soordered) six ounces, rain Watcr cight pounds, steep them twenty-four hours, then boil and press them gently, boil it to three pounds, and with twenty-four ounces of sugar boil it into a Syrup according to art.

Syrupus de Meconio compositus.

Or Syrup of Meconium compound.

College.] 'Tike of white and black Poppy heads with their seeds, fitty drans, Maden-hair fifteen clrams, Jujubes thirly, the seeds of Lettice, forty drams, of Mal-? lows and Quinces tied up in a rag, a dram and a halt, Liquorice five drams, water eight pounds, boil it according to art, strain it, and to three pounds of Decoction add Sugar and Penids, of cach one pound, make it into a Syrup.

Culpeper.] Meconium is nothing else but the juice of English Poppies boiled till it be thich: It prevails agaunst dry coughs, phthisicks, hot and sharp gnawing rheums, and provolies sleep. It is an ustal fashion for nurses when they have heated their milk by exercise or strong liquor (no marvel then if their children be froward) then rum for Syrup of Poppies, to make their young ones sleep. I would fain have that fashion left, thercfore I forbear the close; let nurses kecp their own bodies lemperate, and their children will sleep well enough, never fear.

\section{Sylrupus Melissophylli.}

Or Sylup of Bawm.

College.] 'Tike of the Bark of Bugloss roots, an ounce, the roots of white Dittiny an ounce and an hall, with which, and
Cinquefoil, Scorzonera, of each half an three ounces of the aforesaid Decoction, the seeds of Sorrel, Citrons, Fennel, Carduus, Bazil, of each three drams, boil them in four ponnds of water till half be conwhite sugar, juice of Bawn and Rose Water, of each half a pound, boil them to a Syrup, the which perfume with Cinnamon and yellow Sanders, of each half an ounce. Culpeper.] It is an excellent cordial, and strengihens the heart, breast, and slomach, it resists melancholy, revives the spirits, is given with good success in fevers, it strengthens the memory, and relieves languishing nature. You may take a spoonfull of il at a time.

Syrupus de Mentha.
Or Syrup of Mints.

College.] 'Take of the juices of Quinces sweet and between sweet and sour, the juice of Pomegranates sweet, betwcen swect and sour, and sour, of cach a pound and a half, dried Mints half a pound, red Roses two ounces, let then lie in steep one day, then boil it half away, and with four pounds of sugar boil it into a Syrup according to art: perfume it not unless the Physicians command.

Culpeper.] The Syrup is in quality binding, yet it comforts the stonach much, helps digestion, stays vomiting, and is as excellent a remedy against sour or offensive belchings, as any is in the Dispensatory. 'Take a spoonful of it after meat. Sigrupus de Mureiluginibus.

Or Syrup of Mussilaeres.

College.] 'Take of the seeds of Marshmallows, Mallows, Quinces, of each an ounce, Gum 'lragacanth threr drams, let these intuse six hours in warm Decoction of Mallows, white Poppy seceds, and lliner Cherries, then press ont the Mussilage to an ounce and an hall, whth which, and
three ounces of the aforesaid Decoction, sumed, strain it, and add three pounds of 
and two ounces of sugar, make a Syrup according to art.

Culpeper.] $\Lambda$ spoonful taken by itsclf, or in any convenient liquor, is excellent for any sharp corroding hunours be they in what part of the body soever, phthisicks, bloody-flux, stone in the reins or bladder, or ulcers there: it is excellent good for such as have taken purges that are too strong for their bodies, for by its slippery nature it helps corrosions, and by its cooling helps inflammations.

\section{Syrupus Myrtimus.}

Or Syrup of Myrtles.

College.] Take of Myrtle Berries two ounces and an half, Sanders white and red, Sumach, Balaustines, Barberry stones, red Roses, of each an ounce and a half, Medlars half a pound, bruise them in eight pounds of water to four, strain it, and add juice of Quinces and sour Ponnegranates, of each six ounces, then with three pounds of sugar, boil it into a Syrup.

Culpeper.] 'The Syrup is of a very binding, yet comforting nature, it helps such as spit blood, all fluxes of the belly, or corrosions of the internal parts, it strengthens the retentive faculty, and stops immoderate flux of menses. A spoonful at a time is the dose.

Syrupus Florum Nymphe simplex. Or Syrup of Water-Lily flowers, simple.

College.] Take of the whitest of white Water-Lily flowers, a pound, steep them in three pounds of warm water six or scren hours, let them boil a little, and strain them out, put in the same weight of flowers again the second and third time, when you have strained it the last time, add its weight of sugar to it, and boil it to a Syrup.

Syrupus Florum Nymphce compositus. Syrup of Water-Lily flowers compound.

College.] Take of white Water-Lily flowers half a pound, Violets two ounces. Lettice two handfuls, the seeds of Lettice, Purslain, and Gourds, of each half an ounce, boil them in four pounds of clear water till one be consumed, strain it, and add half a pound of red liose water, white sugar four pounds, boil it into a Syrup according lo allt.

Culpeper.] 'They are both fine cooling Syrups, allay the heat of choler, and provoke sleep, they cool the body, both head, hearl, liver, reins, and matrix, and therefore are profitable for hot diseases in eirher, you mal take an ounce of it at a time when your stomach is cmpty.

\section{Syrupus de Papavere Erratico, size Rubro.}

Or Syrup of Erratic Poppies.

College.] Take of the fresh flowers of red Poppics two pounds, stecp them in four pounds of warm spring water, the next day strain it, and boil it into a Syrup with its equal weight in sugar.

Culpeper.] The Syrup cools the blood, helps surfeits, and may safely be given in freinzies, fevers, and hot agues.

\section{Syrupus de Pilosella. \\ Or Syrup of Mouscar.}

College.] 'Take of Mousear three handfuls, the roots of Lady's-mantle an ounce and an half, the roots of Comfrey the greater, Madder, white Dittany, Tormentil, Bistort, of cach an ounce, the leares of Wintergreen, Horsetail, Ground Ivy, Plantain, Adder's Tongue, Strawberries, St. John's Wort with the Howers, Golden Rod, Agrimony, Bettony, Burnet, A vens, Cinquefoil the greater, red Coleworts, Balaustines, red Roses, of each a handful, boil them gently in six pounds of Plantain Water to three, then strain it strongly, and when it is settled, add Gum Tragacanth, the secds of Fleawort, Marsh-mallows and Quinces, made into a Mussilage by themselves in Strawberry and Bettony Watcr, of each three ounces, white sugar two pounds, boil it to the thickness of honcy.

Culpeper.] It is drying and licaling, and therefore good fur ruptures 
Syrupus infusionis florum Pceonia.

Or Syrup of the infusion of Peony flowers. College.] It is prepared in the same manner as Syrup of Clove-gilliflowers.

Symupus de Pconia compositus.

Or Syrup of Pcony compound.

College.] 'lake of the Roots of both sorts of Peony taken up at the full Moon, cut in slices, and stecped in white Wine a whole day, of cach an ounce and an half, Contra Yerva halt an ounce, Siler Mountain six drams, Ell's Claws an ounce, Rosemary with the flowers on, one handful, Bettony, Hyssop, Origanum, Chamepitys, Rue, of cách three drams, Wood of Aloes, Cloves, Cardamoms the less, of each two drams, Ginger, Spikenard, of each a dram, Stoechas, Nutmegs, of cach two drams and an half, boil them after one day's warm. digestion, in a sufficient quantity of clistilled water of Pcony roots, to four pounds, in which (being strained through Hippocrates' slceve) put four pounds and an half of white sugar, and boil it to a Syrup.

Culpeper.] It holps the falling-sickness, and convulsions.

Syrupus de Pomis atterans.

$$
\text { Or Syrup of Apples. }
$$

College.] Take four pounds of the juice of sweet scented Apples, the juice of Bugloss, garden and wild, of Violet leaves, Rose Water, of each a pound, boil them together, and clarify them, and with six pounds of pure sugar, boil it into a Syrup according to art.

Culpeper.] It is a fine cooling Syrup for such whose stomachs are overpressed with heat, and may safely be given in fevers, for it rather loosens than hinds : it breeds good blood, and is profitable in hectic fevers, and for such as are troubled with palpitation of the lieart, it quenches thirst admirably in ferers, and stays hiccoughs. You may take an ounce of it at a time in the morning, or when you need. $(31,32$.
Syrupus de Prasio.

Or Syrup of Horehound.

College.] Take of white Horehound fresh, two ounces, Liquorice, Polipodium of the Oak, Fennel, and Smallage roots, of each half an ounce, white Maiden-hair, Origanum, Hyssop, Calaminth, Thyne, Savory, Scabious, Colt's-foot, of each six drams, the seeds of Annis and Cotton, of each three drams, Raisins of the sun stoned two ounces, fat Figs ten, boil them in eight pounds of Hydromel till half be consumed; boil the Decoction into a Syrup with honey and sugar, of each two pounds, and perfume it with an ounce of the roots of Orris Florentine.

Culpeper.] It is appropriated to the breast and lungs, and is a fine cleanser to purge them from thick and putrified flegm, it helps phthisicks and coughs, and diseases subject to old men, and cold natures. Take it with a Liqunrice stick.

Syrupus de quinq. Radicibus.

Or Syrup of the five opciing Roots.

College.] Take of the roots of Smallage, Fennel, Parsley, Bruscus Sparagus of each two ounces, spring Water, six pounds, hoil away the third part, and make a Syrup with the rest according to art, with three pounds of sugar, adding eight ounces of white Wine Vinegar, towards the latter end.

Culpeper.] It cleanses and opens very well, is profitable against obstructions, provokes urine, cleanses the body of flegm, and is safely and profitably given in the beginning of fevers. All ounce at a time upon an empty stomach is a good dose.

\section{Syrupus Raphani.}

\section{Or Syrup of Radishes.}

College.] Take of garden and wild Radish roots, of each an ounce, the roots of white Saxifrage, Lovage, Bruscus, Eringo, Rest-harrow, Rarsley, Fennel, of cach half an ounce, the leares of Betiony, Burnet, Pennyroyal, Nettles, Water-cresses, $4 \mathrm{I}$ 
Samphire, Maiden-hair, of each one hand- $\vdots$ and Cotton, of each three drams, boil them ful, Winter Cherries, Jujubes, of each ten, all (the roots being.infused in white Wine the secds of Bazil, Bur, Parsley of Mace- the day before) in a sufficient quantity of donia, Hartwort, Carraway, Carrots, Grom - Wine and Water to eight ounces, strain it, well, the bark of the root of Bay-tree, of and adding four ounces of the Juice of each two drams, Raisins of the sun stoned, Scabious, and ten ounces of sugar, boil it Liquorice, of each six drams, boil them in to a Syrup, adding to it twenty drops of oil twelve pounds of water to eight, strain it, of sulphur. and with four pounds of sugar, and two Culpeper.] It is a cleansing Syrup ap-
pounds of honey, make it into a Syrup, propriated to the breast and lungs, whe'n and perfume it with an ounce of Cinnamon, you perceive them oppressed by flegm, and half an ounce of Nutmegs.

crudites, or stoppings, your remedy is to

Culpeper.] A tedious long medicine for take now and then a spoonful of this Syrup, the stone.

it is taken also with good success by such Syrupus Regius, alias Julapium Alexandrinum.

Or Julep of Alexandria.

College.] Boil four pounds of Rosewater, and one pound of white Sugar into College.] 'I'ake of Hart's-tongue thrce a Julep. Julep of Roses is made with handfuls, Polypodium of the Oak, the Damask Rose water, in the very same roots of both sorts of Bugloss, bark of the manner.

Culpeper.] Two fine cooling drinks in the heat of summer.

Syrupus de Rosis siccis.

Or Syrup of dried Roses.

College.] Make four pounds of spring water hot, in which infuse a pound of driced Roses, by some at a time, press them ont and with two pounds of sugar, boil it into a Syrup according to art.

Culpeper.] Syrup of dricd Roses, strenethens the heart, conforts the spirits, binds the body; helps fluxes, and corrosions, or gnawings of the bowels, it strengthens the stomach, and says vomiting. Trou may take an ounce at a time, before meat, if for fluxes; after meal if tor romiting.

$$
\text { S:rupus Scabiose. }
$$

Or Syrup of Sca!rious.

College.] 'Take of the roots of Elecampanc, and Polypodium of the Oak, of each two onnces, Raisins of the sum sioned an ounce, Sebestens twenty, Coles's-font, Lumgroots of Capers and Tamcrisk, of each two ounces, Hops, Dodder, Maiden-hair, Bawin, of each two handluls, boil them in nine pounds of Spring water to five, and strain it, and with four pounds of white sugar, make it into a Syrup according to art.

Culpeper.] It leelps the stoppings of molancholy, opens obstructions of the liver and spleen, and is profitable anainst splenoric evils, and therefore is a choice remedy for the discass which the rulsar call the rickets, or liver-grown: A spontiul in a morming is a presious remedy for childeren troubled with that disease. Men that are troubled with the splecen, which is known by pain and hamelness in thrir latt side, may take lince or four spoonfuls, they shall find this one receipt worth the price of the whole book.

\section{Sumupus de Strchade. Syrup of Stoechas.}

College. 7 'Take of Stochas flowers forr

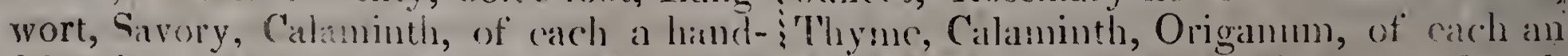
ful and an half, Liginorice, Spanish Tebacre, nunce and an half, Sage, Buttony, of cach of each half an ounce, the seeds of Nettles half an ounce, the seeds of Rue, Peony, and 
Femnel, of each three dranis, spring water the water of Violet flowers and sugar, like ten pounds, boil it till half be cousuned, Julej of Roses. and with honcy and sugar, of each two pounds, boil it into a Syrup, which perfume with Cinnamon, Ginger, and Calmus Aromaticus, of each two drams tied up in a rag.
Culpeper:]
It is cooling and pleasant.

\section{PURGING SYRUPS.}

\section{Syrupus de Cichorio cum Rhubarbaro. \\ Or Syrup of Succory with Rhubarb.}

College.] Take of whole Barley, the roots of Smallage, Fennel, and Sparagus, of each two ounces, Succory, Dandelyon, Endive, smooth Sow-thistles, of each two handfuls, Lettuce, Liverwort, Fumitcry, tops of Hops, of each one handful, Maidenhair, white and black, Cetrachs, Liquorice, winter Cherries, Dodder, of each six drams, to boil these take sixteen pouncis of spring water, strain the liquor, and boil in it six pounds of white sugar, adding towards the and six ounces of Rhubarb, six drams of spikenard, bound up in a thin slack rag, the which crush often in boiling, and so make it into a Syrup according to art.

Culpeper.] It cleanses the body of venemSylrupus Violurum. Or' Syrup of Violets.

College.] Take of Violet flowers fresh ous humours, as boils, carbuncles, and the like; it prevails against pestilential fevers, it strengthens the heart and nutritive virtue, purges by stool and urine, it makes a man have a good stomach to his meat, and provolies sleep. But by my author's leave, I never accounted purges to be proper physic in postilential fercrs; this I believe, the Syrup cleanses the liver well, and is exceeding goor for such as are troubled with hypocondriac melancholy. 'The strong may take: two ounces at a tinie, the weak, one, or you may mix an ounce of it with the Decoction of Senna.

\section{Sirrupus de Epthymo. \\ Or Syrup of Expithimum.}

Coliege.] 'iuke of Epithimum twenty drans, Mirobalans, Citron, and Indian of ,each fifteen drams, Emblicks, Bellorisess, Polyon!lum. Liguorice Agrick, Thyme, and resist the heat of the discase; it connforts hot stomachs excecdingly, cools the liver and leart, and resists putrefuction, pestilencere, and poison.

College.] Julep of Violets is made of 'CaTaminth. Bugloss, Stoechas of each six 
drams, Dodder, Fumitory, of each ten drams, red Roses, Annis-seeds and sweet Fennel seeds of each two drams and an half, sweet Prunes ten, Raisins of the sun stoned four ounces, Iamarinds two ounces and an half, after twenty-four hours infusion in ten pints of spring water, boil it away to six, then take it from the fire and strain it, and with five pounds of fine sugar boil it inty Syrup according to art.

Culpeper.] It is best to put in the Dodder, Stœchas and Agarick, towards the latter end of the Decoction. It purgesmelancholy, and other humours, it strengthens the stomach and liver, cleanses the body of addust clioler and addust blood, as also of salt humours, and helps diseases proceeding from these, as scabs, itch, tetters, ringworms, leprosy, \&c. A man may take two ounces at a time, or add one ounce to the Decoction of Epithimum.

\section{Syrupus e Floribus Persicorum.}

Or Syrup of Peach-flowers.

College.] Take of fresh Peach-flowers a pound, steep them a whole day in three pounds of warm water, then boil a little and strain it out, repeat this infusion five times in the same liquor, in three pounds of which dissolve two pounds and an half of sugar and boil it into a Syrup.

Culpeper.] It is a gentle purger of choler, and may be given even in fevers to draw away the sharp choleric humours.

\section{Syrupus de Pomis purgans. \\ Or Syrup of Apples purging.}

College.] Take of the juice of sweet smelling Apples two puunds, the juice of Borrage and Bugloss of each one pound and an half, Senna two ounces, Annis seeds? half an ounce, Saffron one dram, let the Senna be steeped in the juices twenty-four hours, and after a boil or two strain it, and with two pounds of white sugar boil it to a Syrup according to art, the saffron each one pound and an half, let them be being tied up in a rag, and often crushed mixed together warm all night, and in the in the boiling:
Culpeper.] The Syrup is a conling purge, and tends to reclify the distemper's of the blood, it purges choler and melancholy, and therefore inust needs be effectual both in yellow and black jaundice, madness, scurf, leprosy, and scabs, it is very gentle. The dose is from one ounce to three, according as the body is in age and strength. An ounce of it in the morning is excellent for such children as break out in scabs.

\section{Syrupus de Pomis magistralis}

Or Syrup of Apples magisterial.

College.] 'Take of the Juice and Water of Apples of each a pound and an half, the Juice and Water of Borrage and Bugloss of each nine ounces, Senna half a pound, Annis seeds, and sweet Fennel seeds, of each three drams, Epithimum of Crete, two ounces, Agarick, Rlubarb, of cach half an ounce, Ginger, Mace, of cach four scruples, Cinnamon two scruples, Saffron lialf a dram, infuse the Rhubarb and Cinnamon apart by itself, in white Wine and all the rest, the Saffron excepted, be steeper in the Waters above mentioned, and the next day put in the juices, which being boiled, scummed, and strained, then with four ounces of white sugar boil it into a Syrup, crushing the saffion in it being tied up in a linen rag, the infusion of the Rhubarb being added at the latter end.

Culpeper.] Out of doubt this is a gallant Syrup to purge choler and melancholy, and to resist madness.

\section{Syrupus de Rhubarbaro. Or Syrup of Rhubarb.}

College.] Take of the best Rhubarb and Senna of each two nunces and an half, Violet flowers a liandful, Cinnamon one Bettony, Succory and Bugloss Water of morning strained and boiled into a Syrup, Juice of Apples, of each two ounces, let dram and an half, Ginger half a dram, 
with two pounds of white slgar, adrling provokes the menses, purges the stomach towards the end four onnces of Sgam of and liver, and provokes urine.

Roscs.

Culpeper.] It cleanses choler andinelancholy very gently, and therefore fit for children, old people, and weak bodies. You may arld an ounce of it to the Decoction of Epithimum or to the Decoction of Senna.

Sijpupus Rosaceus solutivus.

Or Syrup of Roses solutive.

College.] 'Take of Spring Water boiling hot four pounds, Dianask Rose leaves fresh, as many as the water will contain, let them remain twelve hours in infusion, close stopped, then press them out and put in fresh Rose leaves, do so nine times in the same liquor, encreasing the quantity of the Roses as the liquor encreases, which will be almost hy the third part every time: Take six parts of this liquor, and with four parts of white sugar, boil it to a Syrup according to art.

Culpeper.] It loosens the belly, and gently brings out choler and flegm, but leaves a binding quality behind it.

Syrupus e succo Rosarum.

Or Syrup of the Juice of Roses.'

Collene.] It is prepared withrout steeping, only with the juice of Damask Roses pressed out, and clanified, and an equal proportion of suga! added to it.

Culpeser.] This is like the other.

Syrripas Rosaceus solutwus cum Agareco.

Or. Syrup of Roses solutive with Agarick.

College. $]$ Take of Agarick cut thin an ounce, Ginger two drams, Sal. Gem. one dram, Polipodium bruised two ounces, sprinkle them with white Wine and stcep? them two days over warm ashes, in a pound and an half of the infusion of Damask Roses prescribed before, and with one pound of sugar boil it into a Syrup according to art.

Culpeper.] It purges flegm from the head, relicres the senses oppressed by it,

$(31,32$.
Symupus Rosaceus solutivus cum Helleboro. Or Syrup of Roses solutive with Hellebore. College.] Take of the bark of all the Myrobalans, of each fourounces, bruise them grossly, and stcep them twenty four hours in twelve pounds of the infusion of Roses before spoken, Senna, Epithimum, Polypodium of the Oak, of each four ounces, Cloves an ounce, Citron seeds, Liquorice, of each four ounces, the bark of black Hellebore roots six drams, let the fourth part of the liquor gently exhale, strain it, and with five pounds of sugar, and sixteen drams of Rhubarb tied up in a linen rag, make it into a Syrup according to art.

Culpeper.] The Syrup rightly used, purges melancholy, resists madness.

Syrupus Rosaceus solutivus cum Senna. Or Syrup of Roses solutive will Semna.

College.] 'lake of Senna six ounces, Caraway, and sweet Fennel seeds, of each three drams, sprinkle them witl white Wine, and infuse them two days in three pounds of the infusion of Roses aforesaid, then strain it, and with two pounds of sugar boil it into a Syrup.

Culpeper.] It purges the body of choler and melancholy, and expels the relies a disease hath left behind it; the dose is fiom one ounce to two, you may take it in a Decoction of Senna, it lcaves a binding quality behind it.

\section{Syrupus de spina Cerina. \\ Or Syrup of Purging Thorn.}

College.] Take of the berries of Purging Thorn, gathered in September, as many as you will, bruise then in a stone mortar, and press out the juice, let the forth part of it evaporate, away in a bath, then to two pounds of it, ald sixteen ounces of white sugar, boil it into a Syrup, which perfume with Mastich, Cinnamon, Nutnegs, Annis seeds in fine powder, of each three drams. 


\section{SYRUPS MADE WITH VINEGAK AND HONEY.}

\section{Mel Anthosatum.}

Or Honey of Rosemary Flowers.

College.] 'Take of fresh Rosemary flowers a pound, clarified Honey three pounds, mix them in a glass with a narrow moull, set them in the surn, keep them for use.

Culpeper.] It hath the same virtues with Rosemary Howers, to which I refer you, only by reason of the Honey it may be somewhat cleansing.

\section{Mel Helleboratum.}

\section{Or Honey Helleborated.}

College.] 'Take of white Hellebore roots bruised a pound, clear Water fourteen pounds, after three days infusion, boil it till halt be consumed, then strain it diligently, and with three pounds of Honey, boil it to the thickness of Honey.

\section{Mel Mercuriale.}

Or Honey of Mercury.

College.] Buil three pounds of the juice of Mcrcury, with two pounds of Honey to the thickness of Honcy.

Culpeper.] It is used as an emollient in clysters.

\section{Ilel Mororum, vel Diamoron.}

Or Honey of Mulbcrries.

College.] Take of the juice of Mulberries and Blackberies, before they be ripe, gathered before the sun be up, of each a pound an a half, Honcy two pounds, boil them to their duc thickness.

Culpeper.] It is vulgarly known to be good for sore mouths, as also to cool inflammations there.

Mel Nuceum, alias, Diacarion et Dianucum. Or Honey of Nuts.

College.] 'Take of the juice of the outward bark of green Walnuts, gathered in the dog days tivo pounds, boil it gently till : it be thick, and with one pound of Honey, boil it to the thickness of Honey.

Culpeper.] It is a good preservative in pestilential times, a spoonful being taken as soon as you are up.

Mel Passalatum.

Or Honey of Raisins.

College.] Take of Raisins of the sun cleansed from the stones two pounds, steep them in six pounds of warm water, the next day boil it half away, and press it strongly, and with two pounds of Honey, let the expressed liquor boil to its thickness.

Culpeper.] It is a pretty pleasing medicine for such as are in consumptions, and are bound in body.

Mel Rosatum commune, sive Foliatum.

Or common Honey of Roses.

College.] Take of red Roses not quite open two pounds, Honey six pounds, set them in the sun according to art.

\section{Mel Rosatum Colatum.}

Or Honcy of Roses strained.

College.] 'Take of the best clarified Honey ten pounds, juice of fresh red Roses one pound, set it handsomely over the fire, and when it begins to boil, put in four pounds of fresh red Roses, the whites being cut off; the juice being consumed by boiling and stirring, strain it and keep it for use.

Culpeper.] 'They are both used for diseases in the niouth.

\section{Mel Rosutum solulivum.}

Or Honey of Roses solutive.

College.] 'Take of the often infusion of Damask Roses five poubds, Honey rightly clarified four pounds, boil it to the thickness of Honey.

Culpeper.] It is used as a laxative in clysters, and some use it to clcanse wounds.

College.] Afier the same manner is prepared Honey of the infusion of red Roses. Mel scilliticum.

Or Honey of Scpuils.

College.] Takie one Squil full of juice, 
cut in bits, and put it in a glass vessel, the Syuills prepared, Birthwort, long, round, mouth close stopped, and covered with a and climbing, Turbith, English Orris, Costus, skin, set in the sun forty days, to wit, twenty Polypodium, Lemon pills, of each an before and after the rising of the dog star, ounce, the strings of black Hellebore, then open the resscl, and take the juice Spurge, Agcrick, added at the end of the which lies at the bottom, and prescrve it with the best Honey.

College.] Honey of Violets is prepared th like as Honey of Roses.

$$
\text { Oxymel, simple. }
$$

College.] 'Take of the best Honey four Posca, made of equal parts of Water and pounds, clear Water and white Wine Vine- Vinegar, eight pounds, Sapa two ounces, gar, of each two pounds, boil them in an three days being expired, boil it little more earthen vessel, taking the scum off with than half away, strain it, pressing it gently, a wooden scummer, tit it be come to the and add the thequor a pound and a half consistence of a Syrup.

Culpeper.] It cuts Hegnn, and it is a good preparative against a vomit.

\section{Oxymel compound.}

College.] Take of the Bark of the Root of Fenncl, Smallage, Parsley, Bruscus, Asparagus, of cach two ounces, the seeds of Fennel, Smallage, Parslcy, Annis, of cach one ounce, stcep them all (the roots being first cleanser and the seeds bruised) in six pounds of clear Water and a pound and a half of Wine Vincgar, the ncxt day boil it of Honey Roses, whercin two ounces of Citron pills have been infused, boil it to the thickness of Honcy, and perfume it with Clores, Saffron, Ginger, Galanga, Mace, of each a dram. to the consumption of the third part, boil the rest being strained, with three pounds of Honey into a liquid Syrup according to art.

Culpeper.] First having bruised thie roots and sceds, bril then in the water till half be consunied, then strain it and add the Honey, and when it is almost boiled enorgh, add the Vincgar.

\section{Oxymel Helleboratum.}

Or Oxymel Helleborated.

College.] Talie of Rue, 'Tliyme, Dittany of Crete, Hyssop, Pennyroyal, Horehound, Carduus, the roots of Celtick, Spikenard without leaves, the inner bark of Ekders, of that are tough and viscous, and therefore cach a handiful, Mountain Calaninth two helps the stomach and bowels afticter by pugils, the seeds of Annis, Fenncl, Bazi:, such humours, and sour belchings. If you Roman Nettles, Dill, of each two drans, take but a spoonful in the morning, an able the roots of Angelica, Marsh-mallows, Aron, body will think enough. 
Or?mel Scilliticum compositus.

Or Oxymel of Squills compound. intimities, walincsses, or failings thereof, as want of voice, difficulty of breathing, College.] 'Take of Origanum, dried coughs, horseness, callarrs, \&c. 'The way
Hyssop, I'hyme, Lovage, Cirdanoms the of taling it is with a Liquorice-stick, or if less, Stoclias, of cach five drams, boil them you please, you may add an ounce of it to in three pounds of Water to one, strain it the Pectoral Drcoction before mentioned. and with two ponnds of Honey, Iloncy of Raisins half a pound, juice of Briony five ounces, Yinegar of Squills a pound and a inalf, boil it, and scum it according to art.

C'!peper.] This is good against the falling-sickness, Megrim, Head-ache, Vertigo, or swimming in the head, and if these be ? occanioned by the stomach as many times? they are, it helps the lungs obstructed by hmour, and is good for women not well cleansed after labour, it opens the passage of the womb.

Syrup of Purslain. Mesue.

Syrup of Poppies, the lesser composition.

College.] Take of the heads of white Poppies and black, when both of them arc green, of each six ounces, the seeds of Lettice, the flowers of Violets, of each one ounce, boil them in eight pints of water till the virtue is out of the heads; then strain them, and with four pounds of sugar boil the liquor to a Syrup.

Syrup of Poppies, the greater composition.

College.] Take of the heads of both white and black Poppies, seeds and ais, of each fifty drams, Maiden-hair, fiftecn drans,

College.] Take of the seeds of Purslain Liquorice, five drams, Jujubes, thirty by grossly bruised, half a pound, of the juice of number, Lettice seeds, forty drams, of the Endive, boiled and clarified, two pounds, sceds of Mallows and Quinces, (tied up in Sugar two pounds, Vinegar nine ounces, a thin linen cloth) of each one dram and infusc the sceds in the juice of Endive? an late, boil these in eight pints of water twenty-four hours, afterwards boil it half till five pints be consumed, when you have away with a gentle fire, then strain it, and strained out the three pints remaining, add boil it with the sugar to the consistence of to them, Penids and white sugar, of cach a Syrup, adding the Vinegar towards the a pound, boil them into a Syrup according latter end of the decoction.

(o) art.

Culpeper.] It is a pretty cooling Syrup, Culpeper.] All these former Syrups of fit for any hot risease incident to the Poppies provoke sleep, but in that, I desire stomach, reins, bladder, matrix, or liver ; they may be used with a great deal of cauit thickens flegm, cools the blood, and pro- tion and wariness: such as these are not vokes sleep. You may take an ounce of it fit to he given in the beginning of fevers, at a time when you have orcasion.

Compound Syrup of Colt's-foot. Renod. nor to such whose bodies are costive, yet to such as are troubled with hot, sharp rheums,

College.] 'Take six handfuls of green you may safely give them: The last is apColt's-foot, two handliuls of Maiden-hair, propriated to the lungs; It prevails against one handful of Hyssop, and two ounces of dry coughs, phthisicks, hot and sharp gnawLiquorice, boil them in four pints, either of ing rlieums, and provokes slecp. It is an rain or spring water till the fourth part be usual fashion for nurses when they have consumed, then strain it, and clarify it, to heated their milk by excrcise or strong liquor which and three pounds of white sugar, then run for Syrup of Poppies to make boil it to the perfect consistence of a Syrup. tleir young ones step. I would fain have Culpeper.] The composition is appro- that fashion left off, therefore I forbear the priated to the lungs, and therefore helps the dose: Let nurses keep their own bodies 
tempcrate, and their children will sleep the sun, or the fire, that it is capable of well chough.

Surup of Eupatorium (or Maudlin.) Mesue, use. was first invented for diseases in the College.] Take of the Roots of Smallage, mouth. 3. It is usually made, in respect Femmel, and Succory, of each two ounces, of body, somewhat thicker than new Honey. Liquorice, Schienanth, Dodder, Worn- 4t It may be kept about a yeur, little more wood, Rirses, of each six drams, Maiden- or less.

hair, Bcdeguar, or instead thereof, the roots of Carduus Marix, Suchaha or instead thereof the rools of Avens, the flowers or routs of Bugloss, Annis seeds, sweet Feunel ? sceds, Ageratum, or Maudlin, of each five drams, Rhubarb, Mastich, of each three? drams, Spikenard, Indian leaf, or instead of it put Roman spike, of each two drams, boil then in eight pints of Water till the third part be consumed, then strain the Decoction, and with four pounds of sugar, clarified juice of Smallage and Endive, of each half a pound, boil it into a Syrup.

Calpeper.] It anends infirmities of the liverconing of cold, opens obstructions, helps the dropsy, and evil state of the body ; it extenuates gross humours, strengthens tho liver, provokes urine, and is a present succour for hyporondriac melancholy. You may take an cunce at a time in the morning, it opens but purges not.

Hone? of Emblicks. Augustanus.

College.] T'iake fifty Einblick Myrobalans, stake a little on the point of a knife when bruise them and boil them in three pints of you need.

water till two be consumed, strain it, and with the like weight of. Honey, boil it into a Syrup.

Culpeper.] It is a fine gentle purger both of flegm and melancholy: it strengthens the brain and nerves, and senses both internal and external, helps tremblings of the hearl, stays romiting, provokes appetite. Yronl may take a spoonful at a time.

\section{ROB, OR SAPA: AND JUICES.} Rob de Erasis.
Or Rob of Cherries.
College.] Take of the juice of red
Cherries somewhat sowerish, as much as you will, and with half their weight in sugar boil them like the former.

Culpeper.] See the virtue of Cherries; and there you have a method to keep them all the year.

Rob sive Sava, simplex.
Or. Simple Rob, or Sapa.

College.] Take of Wine newly pressed from white and ripe Grapes, boil it over a gentle fire to the thickness of Honey

Culpeper.] Whenever you read the word Rob, or Sapa throughout the Dispenout any relation of what it should be made, this is that you ought to use.

Rob de Barberis.

Or Rob of Barberries.

College.] 'Take of the juice of Barberries strained as much as you will, boil it by itself (or else by adding half a pound of sugar Honey.

Culpeper.] It quenches thrst, closes the mouth of the stomach, thereby staying vomiting, and belching, it strengthens stomachs weakened by lieat, and procures appetite. Of any of these Robs you may , . two pounds, sugar a pound and an half, hoil it according to art.

Culpeper.] 1. Rob, or Sapa, is the juice of a fruit, made thick by the heat either of ? $(31,8.2$.
Culpeper:] Of these Cornel trees are $4 \mathrm{I}$ 
two sorts, male and female, the fruit of the Betony, only in the last, the sugar and juice male Cornel, or Cornelian Cherry is here to must be equal in weight.

be used. The fruit of male Cornel, binds exceedingly, and therefore good in fluxes, and the immoderate Howing of the menses. Rob Cydoniorum.

Or Rob of Quinces.

College.] Take of the clarified juice of Quinces, boil it till two parts be consumed and with its equal weight in sugar boil it into a Rob.

\section{Miva vel Gelatina Eorundem.}

()r Jelly of Quinces.

College.] Take of the juice of Quinces clarified twelve pounds, buil it half away, and add to the remainder, old white Wine five pounds, consume the third part orer a gentlc fire, taking. away the scum (all you oughi) let the rest settle, and strain it, and with three pounds of sugar boil it according to art.

Culpcper.] Both are good for weak and indisposed stomachs.

College.] Rob of snur Plums is made as Rob of Quinces, the use of sugar is indifferent in them both.

Rob of English Currants is made in the same manner, let the juice be clarified.

Culpeper.] The virtues are the same with Rob of Barberries.

\section{Rob Baccarum Sambuci}

Or Rob of Elder Berries.

College.] 'Take of the juice of Flder a gentle fire, cither by itself, or a quarter of its woight in sugar bring added.

Culpeper.] Both Roh of Elder Berries, and Dwarf-Elder, are excrllent for such whose hodies are inclining to dropsies, neitler let them néglect nor despise it. They may take the quantity of a nutmeg each morning, it will gently purgethe watery
humour.

College.] In the same manner is made Roh of Dwarf-Lider, Junipers, and Paul's

Succus Glycyrrthizce simpler.

Or Juice of Liquorice simple.

College.] Infuse Liquorice Roots cleansed and gently bruised, three days in Spring Water, so much that it may ovci-top the roots the brcadth of three fingers, then boil it a little, and press it hard out, and boil the liquor with a gentle fire to its due thickness.

Culpeper.] It is vulgarly known to be good against coughs, colds, \&c. and a stren gthner of the lungs.

Succus Glycyrrhize compnsitus.

Or Juice of Liquorice compound.

College.] Take of the water of tender Oak leaves, of Scabious, of each four pounds, English Liquorice scraped and bruised two pounds, boil them by degrees tiil they be soft, then press out the liquor strongly in a press, to which add three pounds of juice of Hyssop, and dry it away in the sun in a broad earthen vessel.

Culpeper.] 'The virtues are the same with the former.

\section{Succus Pronorum Sylvestrum. \\ Or Juice of Slocs, ralled Acacia.}

College.] 'I'ake of Sloes hardly ripe, press out the juice, and make it thick in a bath.

\section{Culpeper.] It stops fluxes, and procures} appetite.

Collegr.] So are the Juices of Wormwood, Maudlin, and Fumitory made thick, to wit, the horbs bruised while they be tender, and the juice pressed nut and after it be claritied, boil over the fire to its just thickness.

\section{LOHOCH, OR ECLEGMA'TA}

Culpeper.] Bccausc this word also is understood but by few, we will first explain what it is. 1. The word Lohoch is an Arabick word, called in Greek Eiciegma, in 
Latin Linctus, and signities a thing to be troubled with pleurises to take now and then licked up. 8. It is in respect of body, a little of it. something thicker than a Syrup, and not so thick as an electuary. 3. Its use was against the roughness of the windpipe, diseases, and intlammations of the lungs, difficulty of breathing, colds, coughs, \&c. 4. Its manner of reception is with a Liquorice stick, bruised at the end, to take up some and retain it in the mouth, till it melt of its own accord.

\section{Lohoch de Farfara.}

Or Lohoch of Coltsfoot.

College.] Take of Colts-foot roots cleansed eight ounces, Marsh-1nallow roots four ounces cleansed, boil them in a sufficient quantity of water, and press the pulp out through a sieve, dissolve this again in the Decoction, and let it boil once or twice, then take it from the fire, and add two pounds of white sugar, Honey of Raisins fourteen ounces, juice of Liquorice two drams and an half, stir them sloutly with a wooden pestle, mean scason sprinkle in Saffron and Cloves, of each a scruple, Cinnamon and Mace, of each two scruples, make them into a Lohoch according to art.

Culpeper.] It was invented for the cough. Lohoch de Papavers.

Or Lohoch of Poppies.

Collegc.] Take white Poppy seeds twenty four drams, swcet Almonds blanched. in Rose Water, Pine-nuts cleansed, Gum Arabick and 'Tragacanth, of each ten drams, juice of Liquorice an ounce, Starch three drams, the secds of Lettuce, Purslain, Quinces, of each half an ounce, Saffroil a breast. dram, Penids four ounces, Syrup of Meconium three pounds, make it into a Lohoch according to art.

Culpeper.] It helps salt, sharp and thin distillations upon the lungs, it allays the fury of such s arp humours, which occasion prith roughness of the throat, want of sleep, aud tevers; it is exccllent for such as arc
Lohoch e Passulis.

Or Lohoch of Raisins.

College.] Take of miale Peony roots, Liquorice, of each half an ounce, Hyssop, Bawm, Hart's-tongue, or Cetrach, of each half a handful, boil them in Spring Water, and press them strongly, and by adding a pound of Raisins bruised, boil it again, pressing it tìrough a linen cloth, then with a pound of white sugar, make it into a Lohoch according to art.

Culpeper.] It is very good against coughs, consumptions of the lungs, and other vices of the breast, and is usually given to children for such diseases, as also for convulsions, and falling-sickness.

\section{Lohoch e Pino.}

Or Lohoch of Pinenuts.

College.] Take of Pine-nuts, fifteen drams, sweet Almonds, Hazel Nuts gently roasted, Gum Arabick and Tragacanth, powder and juice of Liquorice, white Starch, Maidenhair, Orris roots, of each two drams, the pulp of Dates seventeen drams, hitter Almonds one dram and an half, Honey of Raisins, white Sugar-candy, fresh Butter, of each two ounces, Honey one pound and an half, dissolve the Gums in so much Decoction of Maiden-hair as is sufficient; let the rest be mixed over a gentle fire, and stirred, that so it may be made into a Lohoch.

Culpeper.] The medicine is excellent for continual coughs, and difficulty of breathing, it succours such as are asthmatic, for it cuts and atenuates tough humours in the

\section{Lohoch de Portulaca.}

Or Lohoch of Purslain.

College.] Take of the strained Juice of Purslain two pounds, 'Troches of Terra Lemniatwo drams, Troches of Amber, Gum, Arabic, Dragon's-blood of cach one dram, Lapis IIematitis, the wool of a Hare toasted, f each two seruples, white Sugar one 
pound, mix toth together, that so you may make a Lohoch of them.

Culpeper.] The medicine is so binding that it is better let alone than taken, unless in inward bruises when men spit blood, then you may safely take a little of it.

\section{Lohoch e Pulmone Vulpis.}

Or Lohoch of Fox Lungs.

College.] 'Take of Fox Lungs rightly prepared, juice of Liquorice, Maiden-hair, Annis-seeds, sweet Fennel seeds, of each equal parts, Sugar dissolved in Cclt's-foot, and Scabinus Water, and boiled into a Sylup, three times their weight; the rest being in fine powder, let them be put to it and strongly stirred together, that it may be made into a Lohoch according to art.

Culpeper.] It cleanses and unites ulcers in the lungs and breast, and is a present remedy in phthisicks.

\section{Lohoch samum et Expertum.}

Or a sound and well experienced Lohoch. College.] Take of dried Hyssop and Calaminth, of each half an ounce, Jujubes, Sebestens, the stones being taken out, fifteen Raisins of the Sun stoned, fat Figs, Dates, of each two ounces, Linseed, Fenugreek seed, of each five drams, Maiden-hair one handful, Annis-seeds, sweet Fennel seeds, Orris Roots cut, Liquorice, Cinnamon, of each an ounce, boil them according to art in four pounds of clear water till half be consumed, and with two pounds of Penids boil it into a Syrup, afterwards cut and bruise very small Pine-nuts five drams, sweet Almonds blanched, Liquorice, Gum Tragacanth and Arabick, white Starch of each three drains, let these be put into the Syrup when it is off the fire, and stir it about swiftly with a wonden pestle till it look white.

Culpeper.] It succors the breast, lungs, turoat; oppressed by cold, it restores the voice lost by teason of cold, and attenuates thick and gross humours in the breast and lungs:

\section{Tohoch Scilliticum.}

Or Lohoch of Squils.

College.] Take three drams of a Squil baked in paste, Orris Routs two drams, Hyssop, Hore-liound, of cach one dram, Saffron, Mylrh, of each half a dram, Honey two ounces and an half, bruise the Squil, after it is baked, in a stone mortar, and atter it hath boiled a walm or two with the Honey, put in the rest of the things in powder, diligently stirring it, and make it into a Lohoch according to art.

\section{Eclegma of Squils. Mesue.}

College.] Take of the juice of Squils and Honey, both of them clarified, of earh two pounds, boil then together according to art to the consistence of Honey.

Culpeper.] For the virtues of it see Vinegar of Squils, and Oximel of Squils, only this is more mild, and not so harsh to the throat, because it hath no Vinegar in it, and therefore is far more fitting for Asthmaes, and such as are troubled with difficulty of breathing, it cuts and carriesaway humours from the breast, be they thick or thin, and wonderfully helps indigestion of victuals, and eases pains in the breast, and for this, I quote the authority of Galen.

\section{Lohoch of Coleworts. Gordonius.}

College.] Take one pound of the juise of Coleworts, clarified Saffion three drams, clarified Honey, and Sugar, of each half a pound, make of them a Lohoch according to art.

Culpeper.] It helps hoarseness, and loss of voice, eases surfeits and head-achecoining of drunkenness, and opens obstructions of the liver and spleen, and therefore is gond for that disease in children called the rickets.

\section{PRESERVED ROOTS, STALKS,} BARKS, FLOWERS, FRUITS.

College.] Take of Eringo Roots as many as you will, clcanse them withnut and within, 
the pith bcing taken out, steep them two days in clear water, shifing the water some- that they are free from the stones, boil it times, then dry them with a cloth, then again 11 an earthen vessel over a gentle fire, take their equal weight in white Sugar, and often stirring them for fear of burning, till as much Rose-water as will make it into a the watery humour be consumed, then nix Syrup, which being almost boiled, prit in ten pounds of sugar with six pounds of this the ronts, and let them boil until the mois- pulp, boil it to its due thickness. Brom ture be consumed, and let it be brought to burls are also preserved, but with brine and the due body of a Syrup. Not mich un- vinegar, and so are Olives and Capers. like to this are preserved the roots of Acorus, Lastly, Anongst the Barks, Cimnamon; Angelica, Borrage, Bugloss, Succory, kle- amongst the flowers, Roses, and Marigolds, campane, Burnet, Satyrion, Sicers, Comfrey amongst the fruits, Almonds, Cloves, Pinethe greater, Ginger, Zedoary. Take of the muts, and Fistick-nuts, are said to be prestalks of Artichokes, not too ripe, as many served but with this difference, they anre as you will, and (contrary to the roots) take concrusted with dry sugar, and are more only the pith of these, and preserve thesn called confects than preserves.

with their equal weight in sugar, like the former. So is prepared the stalks of Angelica, Burs, Lettuce, \&c. before they be too ripe. Take of fresh Orange pillis as? many as you will, take away the cxterior ycllowness, and steep them in spring water Wormwood, Sorrel, Wood-sorrel, the flowers three days at the least, often renewing the of Oranges, Borrage, Bugloss, Bettony, water, then preserve them like the former. Marigolds, the'Jops of Carduus, the Flowas In like manner are Lemon and Citron pills of Centaury the less, Clove-gillitlowers, preserved. Preserve the flowers of Citrons, Gemande:, Succory, the Leaves of ScurvyOranges, Borrage, Priniroses, with Sugar, grass, the flowers of Confrey the greater; according to art. . Take of Apricots as Citratix, Cinosbati, the roots of Spurge, many as you will, take away the outer skin herbs and Howers of Eye-bright, the tops and the strnies, and mix them with their of Fumitory, Goat's-rue, the fiowers of like weight in sugar, after four hours take? Broom not quite open, Hyssop, Lavender, them out, and boil the Sugar without any other Liquor, then put, them in again, and Mallows, the tops of Bawm, the leaves of boil them a little. Other Fruits may be Mints, the flowers of Walce Lilics, red preserved in the same manner, or at Poppies, Peony, Peaches, Priniroses, Roses, least not much unlike to it, as whole Bar- the leaves of Rue, the flowers of Sage, Elder berries, Cherries, Corncls, Citrons, Quinces, iscabious, the leaves of Scordiun, the Peaches, common Apples, ihe five sorts of flowers of Limetree, Coltsfoet, Violets, with Myrobalans, Hazel Nuts, Walnuts, Nut- all these are conserves made with their treble megs, Raisins of the Sun, Pepper brought proportion of white sugur; yel note, that green from India, Plums, garden and wild all of them must not be mixed alike, some Pears, Grapes. Pulps are also preserved, of them must be cut, bealens and rently as Barberries, Cassia Fistula, Citrons, boiled, some neither cut, beaten nor bovilen, Cinosbatis, Quinces, and Slocs, \&e. T'alic and some admit but one of them, wheis or Barberries as many as you will, boil every artist in his trade may find out by shem in spring water till they are tender, this premonition and avoid crror.

$(31,33$.

$\Delta \mathrm{M}$ 


\section{SUGARS.}

\section{Diacodium Solidum, sive Tabulatum.}

College.] Take of white Poppy heads, meanly ripe, and newly gathered, twenty, steep them in three pounds of warm spring water, and the next day boil them until the virtue is out, then strain out the liquor, and with a sufficient quantity of good sugar, boil it according to art, that you may make it up into Lozenges.

Culpeper. The virtues are the same with the colmun Diacodium, viz. to provoke sleep, and help thin rheums in the liead, coughs, and roughness of the throat, and may easily be carried about in one's pocket.

Saccharum tabulatum simplex, et perlutum.

Or Lozenges of Sugar both simple and pearled.

College.] The first is made by pouring

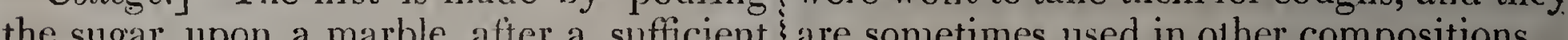
the sugar upon a marble, after a sufficient
boiling in half its weight in Damask Rose Water: And the latter by adding to every pound of the former towards the latter end of the decoction, Pearls, prepared and bruised, half an ounce, with eight or ten leares of gold.

Culpeper.] It is naturally cooling, appropriated to the heart, it restores lost strength, takes away burning fevers, and false imaginations, (I mean that with Pearls, for that without Pearls is ridiculous). it hath the same virtues Pearls have.

Saccharum Tabulatum compositum.

Or Lozenges of Sugar componnd.

College.] Tike of choice Rhubarb four scruples, Agarick Trochiscated, Corallins, burnt Hart's-horn, Dittany of Crete, Wormseed and Sorrel seed, of each a scruple, Cinnamon, Zedoary, Cloves, Saffiron, of each half a scruple, white Sugar a pound, dissolved in four ounces of.Wormwood Watter, Wormwood: Wine, an ounce, Cinnamon Water a spoonful, with the forenamed powders make.it into Lozenges according to art.
Culpeper.] The title shews you the virtues of it.

\section{Saccharum Penidium,}

Or Sugar Penids

College.] Are prepared of sugar dissolved in spring water by a gentle fire, and the whites of Rgggs diligently beaten, and clarified once, and again whilst it is boiling, then strain it and boil it gently again, till it rise up in great bubbles, and being chewed it stick not to your teeth, then pour it upon a marble, anointed witlı oil of Almonds, (let the bubbles first sink, after it is removed firom the fire) bring back the outsides of it to the middle till it look like Larch rosin. then, your hands being rubbed with white starch, you may draw it into threads eithe short or long, thick or thin, and let it cool in what form you please.

Culpeper:] I- remember country people were wont to take them for coughs, and they are sometimes used in other compositions. Confectio de Thure.

Or Confection of Frankincense.

College.] Take Coriander seeds prepared half an ounce, Nutmegs, white Frankin. cense, of each three drams, Liquorice, Mastich, of each two drams, Cubebs, Hart'sLorn prepared, of each one dram, couserve of Red roses an ounce, white Sugar as much as is sufficient to make it into mean bits.

Culpeper.] I cannot boast much of the rarity nor virtues of this receipt. Saccharum Rosatum. Or Sugar of Roses.

College.] Take of. red Rose eaves, the whites being cut off, and speedily dried in the sun an ounce, white Sugar a pound, Roses of each two ounces which being consumed by degrees, put in the Rose leav'es in powder, mix them, put it upon a marble, and make it into Lozenges according to art. Culpeper.]. As for the virtues of this, it strengthens weak stomachs, weak hearts, and. weak brains, restores, such as are in; melt the Sugar in Rose-water and juice o. 
consumptions, restores lost strength, stays fluxes, eases pains in the head, ear's and eyes, helps spitting, vomiting, and urining of blood; it is a fine commodity for a man in a consumption to carry about with him, and eat now and then a bit.

\section{SPLCIES, OR POWDERS.}

\section{Aromaticum Caryophyllatum.}

College.] Take of Cloves seven drams, Mace, Zedoary, Galanga the less, yellow Sander's, 'Troches, Diairhodon, Cinnamon, wood of Aloes, Indian Spikenard, long Pepper, Cardamoms the less, of each a dram, Red Roses four ounces, Gallia Moschata, Liquorice, of cacli two drams, of Indian leaf, Cubebs of cach two scruples, beat them all diligently into powder.

Culpeper.] 'This powder. strengthens the heart and stomach, helps digestion, expels wind, stays vomiting, and cleanses tho stomach of putrified lumors.

\section{Aromaticum Rosatum.}

College.] 'Take of Red Roses exungulated fifteen drams, Liquorice seven drams, wood of Aloes, yellow Sanders, of each three drams, Cinnamon five drams, Cloves, Mace, of each two drams and an lialf, Gum Arabic and Tragacantl, of each eight scruples, Nutmegs, Cardamons the less, Galanga of each one dram, Indian Spikenard two scruples, make it into a powder: to be kept in a glass for use.

Culpeper.] It strengthens the brain; heart aud stomach, and all such internal members as help towards decoction, it helps digestion, consumes this watery excrements of the bowels, strengthens such as are pined away by reason of the violence of a disease, and restores surle as are in consumption.

Pulvus ea chelus Cancrorum compositus.

Or Powder of Clab's claws compound.

College.] Take of Dearis prepared, Crab's eyes, red Coral, white Amber, Hart's-horn, oriential Bezoar, of each half an vunce, powder of the black tops of Crab's claws, the weight of them all, beat them into powder, which may be made into balls with jelly, and the skins which our vipers have cast off, warily dried and kept for use.

Culpeper.] 'I'his is that powder they ordinarily call Gascoigns powder, there are divers receipts of it, of which this is none of the worst, four, or five, or six grains is excellently good in a fever to betaken in any cordial, for it cheers the heart and vital spirits excedingly, and makes them impiegnable.

Species Cordiales Temperatre.

College.] 'lake of wood of Aloes, Spodium of each a dram, Cinnamon, Cloves, bone of a Stag's-heart, the roots of Angelica, Avens, and 'Torasentil, of each a dram and an half; Pearls prepared six drams, raw Silli toasted, both sorts of Coral of each two drams, Jacinth, Enerald, Samplire, of each half a dram, Satfiron a-scruple, the leaves of gold and silver, of cach ten, make them into powder according to art.

Culpeper.] It is a great cordial, a great strengtlener of the licart, and brain.

\section{Diacalamintlie Simple.}

College.]: 'Take of Mountain Calamintl, Penny royal, Origanum, the seeds of Macedonian Parsley, common Parsley, and Hartwort, of cach two drams, the seeds of Smallage, the tops of 'Tliyme of each half an ounce, the seeds of Lovage, black Pepper, of each an ounce, make them into powder according to art.

Culpeper.]. It heats and comforts cold bodies, cuts thick and gross flegm, provokes urine and the nucnses. I confess this differ's something from Galen, but is better for our bodies in my opinion than his. It cxpels wind excecdingly, you may take lialt a dram of the powder at a time. 'Tlere is nothing surce than that all their powders will keep better in Electuaries than they will in powders, and into such a body, you 
may make it with two pound and an half of ounce, Sugal-candy, Diatragacanthum white sugar dissolved in rose water.

\section{Diacalamintha compound.}

College.] 'Take of Diacalanin tha simple, half an ounce, the leaves of Horehound, Marjoram, Bawm, Mugwort, Savin dried, before. It comforts the breast, is good in of each a dram, Cypress roots, the secds of : colds, coughs, and hoarseness. You may Maddir and Pue, Macc, Cinnanon, of each mix it will any pectoral Syrups which are two scruples, beat them and mix them diligently into a powder according to art.

Culpeper.] This seems to be more appropriated to the feminine gender than the former, viz. to bring down the terms, to bring away the birth, and after-birth, to purge thent after labour, yet it is dangerous for pregnant women.

\section{Dianisum.}

College.] Take of Annis seeds two ounces and an half, Liquorice, Mastich, of eacli an ounce, the sceds of Caraway, Fennel, Galanga, Mace, Ginger, Cinnamon, of each five drams, the three sorts of $\mathrm{P}^{\mathrm{C}} \mathrm{epper}$, Pcpper, Ginger, of each a dram, make them Cassia Lignea, mountain Calaminth, Pel- into powder according to art.

litory of Spain, of each two drams, Cardamons the greater, Cloves, Cubebs, Indian Spikenard, Saffion, of cach a dram and an half, make them into powder.

Culpeper.] It is chictly appropriated to the stomach, and liclps the cold infirmitics thercof, raw, flegm, wind, continual coughs, and other such diseases coming of cold. You mat safely take a dram of the electuary at a tine. You may make an elcctuary of it with its treble weight of clarified Honey.

Pulvis Radicum Ari composicus.

Or Powder of Aron Roots compound. Ivory, Bole-amoniac, Furth of Germany,
College.] Take of Amn Roots two ounces, Samos and Lemnos, Elk's-claw, 'Tormentil of common Water Flag, and Burnet, of cach one ounce, Crab's eyes, half an ounce, Cinnamon thrce drams, salt of Wormwood, and Juniper, of each one dram, make them into powder.

Culpeper.] And when you have done tell me what it is good for.

Diareos simple.
College.] Take of Orris roots half an strengthener of the heart and vitals in fercrs. frigidum, of each two dram, make them into powder.

Culpeper.] I do not mean the Diatragacanthum frigidum, for that is in powder
before. It comforts the breast, is good in
colds, coughs, and hoarseness. You may
mix it will any pectoral Syrups which are
appropriated to the same, discases, and so lake it with a Liquorice stick.

\section{Dialacca.}

College. Take of $\bar{G}$ um-lacca, prepared Rhubarb, Schæenanth, of each three drams, Wormwood and Agrimony, made thick, the seeds of Smallage, Annis, Fennel, Amuni, Savin, bitter Almonds, Myrrh, Costus, or Zedoary, the roots of Maddir, Asarabacca, Birthwort long and round, Gentian, Saffron, Cinnamon, dried Hyssop, Cassia Lignea, Brlellium, of each a dram and an half, black Culpeper.] It strengthens the stomach and liver, opens obstructious, helps dropsies, yellow jaundice, provokes urine, breaks the stone in the reins and bladder. Half: a dram is a moderate close, if the patient be strong they may take a dram in white Wine. Let pregnant women forbear it.

\section{Pulvis Cardiacus Manistralis.}

College.] Take of East Bezoar, bone of a Stag's-heart, of each a dram and an half, Magisterium, of white and red Coral, white Amber, Magisterium of Pearl, Hart's-horn, Ivory, Bole-amoniac, Writh of Germany, Samos and Lemnos, Elk's-claw, 'Tormentil roots, of each a dram, Wood of Aloes, Citron peels, the roots of Angelica and Zedoary, of each two scruples, leaves of Gold twenty, Ambergris one scruple, Musk six grains, mix them and make them into powder. purse, yet a mighty cordial and gent strengthener of the heart and vitals in ferers. Indian Spikenard, Mastich, the juice of

Culpeper.] It is too dear for a vulgar 
Diumiurgariton firigidum.

College.] T'akie of the four greater cold seeds, the seeds of Purslain, white Poppies, Endive, Sorrel, Citrons, the three Sanders, Wood of Alues, Ginger, red Roses exungulated, the flowers of Water-lilies, Bugless, Viulets, the berries of Mirtles, bone in a Stag'sh eart, Ivory, Contra yerva, Cinnamon of each one dram, both sorts of Coral, of each half a dram, Pearls three drams, Camphire six grains, make them into powder according to art. Observe that the four liumours. greater cold seeds, and the Poppy seeds, are not to be added before the powder be required by physician for use. Do so by the other powder in the composition of which these powders are used.

Culpeper.] Authors hold it to be restorative in consumptions, to help such as are in hectic fevers, to restore strength lost, to help coughs, asthmaes, and consumiptions of the lungs, and restore such as have laboured long under languishing or pining diseases.

\section{Diamoschu Dulce.}

Take of Saffiron, Galanga, Zedoary, Wood of Aloes, Mace, of each two drans, Pearls, raw Silk toasted, white Amber, red Coral prepared, Gallia Moschata, Bazil, of each two drams and an half, Ginger, Cubebs, Long Pepper, of each a dram and an half, Nutmegs, Indian leaf or Cinnamon, Cloves, of each one dram, Musk two scruples, make them into powder according to art.

- Culpeper.] It wonderfully helps "cold afflictions of the brain, that come without a fever, melancholy and its attendants, viz. sadness without a cause, vertigo or diziness in the head, falling-sickness, palsies, resolution of the nerves, convulsions, heart-qualms, afflictions of the lungs, and difficulty of brcathing. 'The dose of the powder is half a dram, or two scruples, or less; according to the age or strength of him or her that takes it. Me'sue appoints it to be made into an electuary with clarified honey, and of (31, 32.) the electuary, two drams is the close: The time of taking it is, in the morning fasting: Diamoschu Amarum

College.] Is prepared by adding to the forenamed Wornwood, dried Roses, of each three drams, Aloes half an ounce, Cinnamon two drams and an half, Casturiun and Lovage, of each one dram, make them into powder.

Culpeper.] Besides the virtues of the former, it purges the stomach of putrificd

\section{Specia Dianthics.}

College.] 'Take of Rosemary flowers an ounce, red Boses, Violets, Liquorice, of each six drams, Cloves, Indian Spikenard, Nutmegs, Galanga, Cinnamon, Ginger, Zedoary, Mace, Wood of Aloes, Cardamoms the less, the sceds of. Dill and Anis, of each four scruples, ake them into powder according to art.

Culpeper.] It strengthens the heart and helps the passions thereof, it causes a joyful and cheerful mind, and strengthens such as have been : weakened by long sickness, it strengthens cold stomachs, and helps digestion notably. The dose is half a dram, you may make it into an electuary with honey, and take two drams of that at a time.

\section{Diapendion.}

College.] Take of Penides two ounces, Pinc-nuts, sweet Almonds blanched, white Poppy seeds, of each three drams and a scruple, (Cinnamon, Cloves, Ginger, which three being omitted, it is a Diapendion without spices) juice of Eiquorice, Gum Tragacanth and Arabic, white Starch, the four greater cold sceds husked, of each a dram and an half, Camphire seven grains, make them into powder.

Culpeper.] It helps the vices of the breast, coughs, colds, hoarseness, and consumptions of the lungs, as also such as spit matter. You may mix it witli any pectoral syrup, and take it with a Liquorice stick, if you fancy the powder best, but if the $4 \mathrm{~N}$ 
electuary, you may take a dram of it upon a knife's point at any time when the cough comes.

\section{Diamhodon:Abuatis.}

College.] 'Take of Sanders white and red, of each two drams and an half, Gum Tragacanth, Arabic, Irory of each two. scruples, Asarabacca roots, Mastich, Indian Spikenard, Cardamoms, Liquorice, Saffron, Wood of Aloes, Cloves, Gallia Moschata, Annis and sweet Fennel seeds, Cinnamon, Rlunbarh, Bazil seeds, Barherry sceds, the seeds of Succory, Purslain, the four greater cold seeds cleansed, white Poppy secds, of each a scruple, Pearls, bone of a Stag's-heart of each half a scruple, red Roses exungulated, one ounce and three drans, Cam phire seven grains, make them into powder according to art.

Culpeper.] It cools the violent heat of the heart and stomach, as also of the liver, lungs, and spleen, ease's pains in the body, and nost inlimities coming to the body by reason of heat. 'The dose of the powder is half a dram, and two ounces of the eleoturry, into which with sugar dissolved in Rose-water you may make it.

\section{Diospoliticum.}

College.] Take of Cummin seeds steeped in vinegar and dried, long Pepper, Ruc leaves, of each an ounce, Nitre half ounce, make them into powicler.

Culpeper.] It is an aduirable romedy for such whose ineat is putrified in the stomaclis, it helps cold stomachs, cold belchings and windy. You may take half a dram after meat, either in a spoonful of Muskadel, or in a Syrup of Mirtles or Quinces, or any Cordial IVater whose effects is the same.

\section{Species Diatragacanthi frigidi.}

College.] 'Take of Gum Tragacanth two ounces, Gum Arabic an ounce and two drams, whiteStarch half anounce, Licyorice, the seeds of Mclons and white Poppies, of each three drams, the seeds of Citruls,
Cucumbers and Gourds, of each two drams, Penids three ounces, Camphire half a scruple, make of then a powder according to art. Also you may make an clectuary of them with a sufficient quimtity of Syrup of Violets, but have a care of what was told you before of the seeds.

Culpeper.] Make up into an electuary. It holps the faults of the brcast and lumgs coming of heat and dryness, it helps consuwptions, leanness, inflammations of the sides, pleurises, \&c. hot and dry coughs, roughess of the tongue and jaws.

\section{Diatron Piperion.}

Coltcge.] 'lake of the three sorts of Peppers, of each six drams and fifteen grains, Annis seeds, 'I'lynue, Ginger, of each one dran, beat them into gross powder.

Culpeper.] It heats the stomach and cxpels wind. Half a dran in powder, or two drams in electuary (for so Galen who was author of it, appoints it to be marle with clarified honey, a sufficient quantity) if age and strength permit, if not, half so much, is a sufficient dose, to be taken before neat, if to lieat the stomach and help digestion; : after neat, if io expel wind.

\section{Diatrion Santalon.}

College.] Take of all the sorts of Sanders, red Roses, of each three drams, Rhubarb, I vory, Juice of Liquorice, Purslain seeds, of cach two dimus and lifteen grains, whrte Starch, Gum Arabic, Tragacanll, the sceds of Mrinns, Cucumbers, Citruls, Gourds, Endive, of each a diam and an half, Camphire a scruple, make them into powder according to art.

Culpeper.] It is very profitable against the heat of the stomach and liver, besides, it wondcrfully helps such as have the yellow jaundice, and consumptions of the lungs. You may sately take a dram of the powder, or two drans of the electuary in the norning fasting, for most of these powder will keep better by half in electuaries. 


\section{Pulvis Haly.}

College.] Take of white Poppy seeds ten drams, white Starch, Gum Arabic and Tragacanth, of each three drauns, the seeds of Purslain, Marsh-mallows, Mallows, of cach five drams, Cucumbers, Melons, Gourds, Citruls, Quinces of each seven drams, Ivory, Liquorice, of each three drams, Penids the weight of them all, make them into powder according to art.

Culpeper.] It is a gallant cool powder, fit for all hot inperfections of the breast and lungs, as consumptions, pleurisies, \&c. Your best way is to make it into a soft electuary witl Syrups of Violets, and take it as Diatragacanthum frigidum.

\section{Letificans.}

College.] Take the flowers of Clove-bazil, or the seads thereof, Saffron, Zedoary, Wood of Aloes, Cloves, Citron pills, Galanga, Mace, Nutmegs, Styrax Calamitis, of each two drams and an half, Ivory, Annis seeds, Thyme, Epithimum, of each one dram, bone of a Stag's heart, Pearls, Camphire, of each half a dram, leares of Gold and Silver, of each half a scruple, make it into powder according to art.

Culpeper.] It causes a merry hear', a good colour, helps digestion, and keeps back old age. You may mix half a dram of it to take at one time, or less if you please, in any cordial Syrup, or cordial clectuary appropriated to the same uses.

\section{Pulvis Saxonicus.}

College.] Take of the roots of both sorts of Angelica, Swallow-wort, garden.Valerian, Polipodiun of the Oak, Marsh-mallows, Nettles, of each half an ounce, the bark of German Nezereon, two drams, twenty grains of herb 'True-love, the leaves of the saine, roots and all, thirty six, the roots being? stecped in rinegar and dried, beat it all into powder.

Culpeper.] It seems to be as great an expeller of poisun, and as great a preserva- tive against it, and the pestilence, as one shall usually read of.

\section{Rosate Novelle.}

College.] Take of red Roses, Liquorice, of each one ounce, one dram, two scruples, and an half, Cinnamion two drams, two scruples, and two grains, Clores, Indian Spilienard, Ginger, Galanga, Nutmegs, Zedoary, Styrax, Calamitis, Cardamoms, Parsley' seeds, of each one scruple eight grains, beat them into powder.

Culpejer.] It quenches thirst, and stays vomiting, and the author saith it helps hot and dry stomachs, as also heat and dryness of the heart, liver, and lungs, (yet is the powder itself hot, it strengthens the vital spirits, takes away heart-qualms, it prorokes sweat, and strengthens such as have laboured under long chronical diseases. You may take a dram of the electuary: every morning, if with clarified Honey you please to make it into such a body.

\section{Pulvus Thuraloes.}

College.] 'Take of Frankinicense one dram, Aloes half a dram, beat them into powder.

Culpeper.] And when you have occasion to use it, mix so much of it with the white of an egg, (beat the white of the egg well first) as will make it of the thickness of Honey, then dip the wool of a Hare in it, and apply it to the sore or part that bleeds, binding it on.

\section{Pulvis Hermidactylorum composulus.}

Or Powder of Hermodactils compound.

College.] Take of men's hones burnt, Scammony, Hermodactils, 'Iurtith, Sena, Sugar, of each equal parts, beat them into powder.

\section{Putzis Sence compositus major.}

Or Powder of Sena the greater composition. College.] 'Take of the sceds of Amnis, Carraway, Fennel, Cummin, Spikenard, Cinnamon, Galanga, of each half an ounce, Liquorice, Gromwell, of each an ounce, 
Sena, the weight of them all, beat it into keep it, you materratse the cuantity powder.

Culpeper.] That this receipt is gallantly analogicali!y.

composed none can deny, and is an excellent purge for such whose bodies as are Ditany, Clove-gilliflowere, Scabious, the troubled with the wind cholic, or stoppage seed of Sorrel, Coriander prepared, Citron, either of guts or kidneys, two drams Carduus Benedictus, Ensive, Rue, of each taken in white Wine will work sufficiently one dram, of the three sorts of Sanders, with any ordinary body. Let weak men (white, red, and yellow, Been, white and and children take less, keeping within doors, red (or if you camnot get them, take the and warm.

\section{Pulvis Sence compositus minor.}

Or Powder of Sena, the lesser composition. College.] 'Take of Sena two ounces, Cremor 'Tartar half an ounce, Mace two scruples and an half, Ginger, Cinnamon, of each a dram and an half, Salgem one dram, beat it into porvder according to art.

Culpeper.] This powder purges melancholy, and cleanses the head.

\section{Diasence.}

College.] Take of Sena, Crcmor Tartar, of each two ounces, Cloves, Cinnamon, Galanga, Ammi, of each two drams, Diacridium half an ounce, beat it into powder according to art.

\section{Dinturbith with Rhubarb.}

College.] Take of Turbith, Hermodactils, of each an ounce, Rhubarb ten drams, Diacrydium half an ounce, Sanders red and white, Violets, Ginger, of each a dram and an half, Mastich, Annis seeds, Cinnamon, Saffron, of each half a dram, make it into powder.

Culpeper.] This also purges flegm and choler. Once more let me desire such as are unskilful in the rules of physic, not to meddle with purges of this nature (unless prescribed by a skilful Physician) lest they do themselves more mischief in half an hour, than they can rernove in half a year.

The lesser cordial Powder. Fernelius.

College.] 'Take of Hart's-horn, Unicorn's horn, Pearls, Ivory; of each six grains beat them into fine powder: If you mean to roots of Avens and Tormentil, in their stead) Roman Doronicum, (a kind of wolfbane) Cinnamon, Cardamoms, Saffron, the flowers of both surts of Bugloss, (viz. Borragc and Bugloss,) red Roses, and WaterLilies, Wood of Aloes, Mace, of each two scruples, Ivory, Spodium, bone of a Stay'sheart, red Coral, Pearls, Emerald, Jacinth, Granite of each one scruple, raw Silk torrified, (dried or roasted by the fire,) Bole-amoniac, Earth of Lemnos, of each half a dram, Camphire, Ambergris, Musk, of each six grains, beat them into powder according to art, and with eigh tinies their weight in white sugar, dissolved in Rosewater, you may make them into Lozenges, if you please.

Culpeper.] Both this and the former powder, are appropriated to the heart, (as the title shew) thefore they do strengthen that, and the vital spirit, and relieve languishing nature. All these are cordial Powders, and seldom above half a dram of ther. given at a time.

$A$ Powder for such as are bruised by a fall. The Augustan Physicians.

College.] Take of Terra sigillata, Sanguis Draconis, Mummy of each two drams, Spermaceti one dram, beat them into powder according to art.

Culpeper.] You must beat the rest into powder, and then add the Spermaceti to them afterwards, for if you put the Spermaceti and the rest all together and go to beat them in that fashion, you may as soon beat the mortar into powder, as the simples. 
Indeed your best way is to beat them severally, and then mix them altogether, which humours and cold afflictions of the stomach being done, makes you a gallant medicine and liver. You may take half a dram of the for the infirmities specified in the title, a powder at a time, or two of the electuary dram of it taken in Muskadel and sweating in the morning fasting, or an hour before after it.

Species Electuarii Dyacymini. Nicholaus.

College.] Take of Cummin seeds infused a natural day in Vinegar, one ounce and one scruple, Cinnamon, Cloves, of each two drams and an half, Galanga, Savory, Calaminth, of each one dram and two scruples, Ginger, black Pepper, of each two drams and five grains, the seeds of Lovage, and Ammi, (Bishop's-weed,) of each one dram and eighteen grains, long Pepper one dram, Spikenard, Nutmegs, Cardamoms, of each two scruples and an half, beat them and keep them diligently in powder for your use.

Culpeper.] It heats the stomach and bowels, expels wind exceedingly, helps the wind cholic, helps digestion hindered by cold or wind, is an admirable remedy for wind in the bowels, and helps quartan agues. The powder is very hot, half a dram is enough to take at one time, and too much if the patient be feverish, you may take it in white Wine. It is in my opinion a fine composed powder.

Species Electuarii Diagalanga. Mesue.

College.] Take of Galanga, wood of Aloes, of each six drans, Cloves, Mace, seeds of Lovage of each two drams, Ginger, long and white Pepper, Cinnamon, Calamus Aromaticus of each a dram and an half, Calaminth, and Mints dried, Cardamoms the greater, Indian Spikenard, the seeds of Smallage, Annis, Fennel, Caraway, of each one dram, beat them into powder according to art. Also it may be made into an electuary with white sugar dissolved in Malaga wine, or twelve times the weight of it of clarified Honey.

Culpeper.] Mesue quotes it only as an elecmeat. It helps digestion exceedingly, expels wind, and heats a cold stomach.

Species Electuarii Dianargariton Calidi. Avicenna.

College.] Take of Pearls and Pellitory of the Wall, of each one dram, Ginger, Mastich, of each half an ounce, Doronicum, Zedoary, Smallage seeds, both sorts of Cardamoms, Nutmegs, Mace, of each two drams, Been of both sorts, (if they cannot be procured take the roots of Avens and Tormentil) black and long Pepper of each three drams, beat them into powder and keep them for your use.

Culpeper.] This (quoth Avicenna) is appropriated to women, and in them to diseases incident to their matrix ; but his reasons I know not. It is cordial and heats the stomach.

\section{Lithontribon Nicholaus, according to Fernelius.}

College.] Take of Spikenard, Ginger, Cinnamon, black Pepper, Cardamoms, Cloves, Mace, of each half a dram, Costus, Liquorice, Cy press, Tragacanth,Germander, of each two scruples, the seeds of Bishop'sweed, (Anmi,) Smallage, Sparagus, ${ }^{\circ}$ Bazil, Nettles, Citrons, Saxifrage, Burnet, Caraway, Carrots, Fennel, Bruscus, Parsley of Macedonia, Burs, Seseli, (or Hartwort,) Asarabacca, of each one dram, Lapis Spongix, Lyncis, Cancri, Judaici, of each one dram and an half, Goat's blood prepared an ounce and half, beat them all into powder according to art.

Culpeper.] It heats the stomach, and helps want of digestion coming through cold, it eases pains in the belly and loins, the Illiac passion, powerfully breaks the stone in the reins and bladder, it speedily tuary, which he saith prevails against wind, ?helps the cholic, stranguary, and disury.

$(33,34$.

4 o 
The dose is from a dram to half a dram, Saphire, bone of a Stag's heart, of each take it either in white Wine, or decoction of one dram, beat them into powder accordherbs tending to the same purposes.

\section{Pleres Arconticon. Nicholaus.}

College.] Take of Cinnamon, Cloves, Galanga, Wood of Aloes, Indian Spikenard, Nutmegs, Ginger, Spodium, Schœenanthus, Cypress, Roses, Violets of each one dram, Indian Leaf or Mace, Liquorice, Mastich, Styrax Calamitis, Marjoram, Costmary, or Water-mints, Bazil, Cardamoms, long and white Pepper, Myrtle berries, and Citron pills, of each half a dram and six grains, Pearls, Been white and red, (or, if they be wanting, take the roots of Avens and Tormentil in their stead) red Coral, torrified Silk, of each eighteen grains, Musk six grains, Camphire four grains, beat them into powder according to art, and with ten times their weight in sugar dissolved in Bawm water, you may make them into an not. electuary.

Culpeper.] It is exceedingly good for sad, melancholy, lumpish, pensive, grieving, vexing, pining, sighing, sobbing, fearful, careful spirits, it strengthens weak siomachs exceedingly, and help such as are prone to faintings and swoonings, it strengthens such as are weakened by violence of sickness, it helps bad memories, quickens all the senses, strengthens the brain and animal spirits, helps the falling-sickness, and succours such as are troubled with asthmas, or other cold afflictions of the lungs. It will keep best in an electuary, of which you may take a dram in the morning, or more, as age and strength requires.

A Preservative Powder against the Pestilence. Montagnam.

College.] Take of all the Sanders, (white, red, and yellow, the seeds of Bazil, of each an ounce and an half, Bole Amoniac, Cinnamon, of each an ounce, the roots of each four scruples, juice of Liquorice, white Dittany, Gentian, and 'Tormentil, of each Starch, the seeds of white Poppies, Purslain, two drams and an half, the seeds of Citron Lettuce, and Endive, of each three drams, the and Sorrel, of each two drams, Pearls, four greater cold seeds husked, of Quinces, 
Mallows, Cotton, Violets, Pine-nuts, fistic Nuts, sweet Almonds, pulp of Sebestens, of each two drams, Cloves, Spodium, Cinnamon, of each one dram, Saffron five grains, Penids half an ounce, being beaten, make them all into a soft electuary with three times their weight in Syrup of Violets.

Culpeper.] It restores consumptions, and hectic fever's, lost strength, it nourishes much, and restores radical moisture, opens the pores, resists choler, takes away coughs, quenches thirst, and resists fevers. You may take an ounce in a day, by a dram at a time, if you please.

\section{Confectio Alkermes.}

College.] Take of the juice of Apples, Damask Rose-water, of each a pound and an half, in which infuse for twenty-four hours, raw' Silk four ounces, strain it strongly, and add Syrup of the berries of Cherms brought over to us, two pounds, Sugar one pound, boil it to the thickness of Honey; then removing it from the fire whilst it is warm, add Ambergris cut small, half an ounce, which being well mingled, put in these things following in powder, Cinnamon, Wood of Aloes, of each six drams, Pearls prepared, two drams, LeafGold a dram, Musk a scruple, make it up according to art.

Culpeper.] Questionless this is a great cordial, and a mighty strengthener of the heart, and vital spirits, a restorer of such as are in consumptions, a resister of pestilences and poison, a relief to languishing nature, it is given with good success in fevers, but give not too much of it at a a time, lest it prove too hot for the body, and too heavy for the purse. You may mix ten grains of it with other convenient cordials to children, twenty or thirty to men.

\section{Electuarium e Sassaphras.}

College.] Take of Sassafias two ounces, common Water three pounds, boil it to the consumption of the third part, adding,

towards the end, Cinnamon bruised half an ounce, strain it, and with two pounds of white sugar, boil it to the thickness of a Syrup, putting in, in powder, Cinnamon, a dram, Nutmegs, half a scruple, Musk three grains, Ambergris, two and thirty grains, ten leaves of Gold, Spirit of Vitriol four drops, and so make it into an electuary according to art.

Culpeper.] It opens obstruction of the liver and spleen, helps cold rheums or defluxions from the head to the lungs, or teeth, or eyes, it is excellent in coughs, and other cold afflictions of the lungs and breast, it helps digestion, expels wind and the gravel of the kidneys, it provokes the menses, warms and dries up the moisture of the womb, which is many times the cause of barrenness, and is generally a helper of all diseases coming of cold, raw thin humours, you may take half a dram at a time in the morning.

Electuarium de Baccis Lauri.

Or Electuary of Bay-berries.

College.] Take of theleaves of dried Rueten drams, the seeds of Amni, Cummin, Lovage, Origanum, Nigella, Caraway, Carrots, Parsley, bitter Almonds, Pepper black and long, wild Mints, Calamus Aromaticus, Bay-berries, Castorium of each two drams, Sagapenum half an ounce, Opopanax three drams, clarified Honey a pound and an half, the things to be beaten; being beaten, and the Gums dissolved in Wine, make it into an electuary according to art.

Culpeper.] It is exceeding good either in the cholic, or Iliac passion, or any other disease of the bowels coming of cold or wind, it generally eases pains in the bowels. You may give a dram in the morning fasting, or half an ounce in a clyster, according as the disease is.

Diacapparit.
College.] Take of Capers four ounces,
grimony Roots, Nigella seeds, Sqquils,
sarabacca, Centaury, black Pepper, Small- 

age, Thyme of each an ounce, Honey three
times their weight, make it into an electuary
according to art.

Culpeper.] They say it helps infirmities of the spleen, and indeed the name seems to promise so much, it may be good for cold bodies, if they have strength of nature in them.

College.] Take of Cinnamon fifteen drams, Cassia Lignea, Elecampane roots, of each half : an ounce, Galanga, seven drams, Cloves, long Pepper, both sorts of Cardainoms, Ginger, Mace, Nutmegs, Wood of Aloes, of each three drams, Saffron, one dram, Sugar five drams, Musk two scruples, adding according to the prescript of the Physician, and by adding three pounds eight ounces of clarified Honey, boil it and make it into an electuary according to art.

\section{Culpeper.] Diacimamomum, or in plain} English, $A$ composition of Cinnamon, heats the stomach, causes digestion, provokes the menses, strengthens the stomach and other parts that distribute the nourishment of the body, a dram of it taken in the morning fasting, is good for ancient people and cold bodies, such as are subject to dropsies and diseases of flegn, or wind, for it comforts and strengthens nature much. If you take it to help digestion, take it an hour before meat, do so in all things of like quality.

\section{Diacorallion.} College.] Take of Coral white and red,
Bole-amoniac, Dragon's-blood, of each one dram, Pearls half a dram, Wood of Aloes, red Roses, Gum 'Tragacanth, Cinnamon, of each two scruples, Sanders white and red, of each one scruple, with four times its weight in sugar dissolved in small Cinnamon Water, make it into an electuary, according to art.

Culpeper.] It comforts and strengthens the heart exceedingly, and restores such as are in consumptions, it is cooling, therefore good in hectic fevers, very binding, and therefore stops fluxes, neither do I know a better niedicine in all the dispensatory for such as liave a consumption accompanied with looseness. It stops the menses and Fluor Albus. Take but a dram at a time every morning, because of its binding quality, except you have a looseness, for then you may take so much two or three times a day.

\section{Diacorum.}

College.] Take of the roots of Cicers, Acorus, or Calamus Aromaticus, Pine-nuts, of each a pound and a half, let the Cicers roots, being cleansed, cut, boiled, and pulped, be added to ten pounds of clarified honey, and boiled, (stirring it) to its just thickness, then being removed from the fire, add the Acorus roots beaten, the Pinenuts cut, and these following in powder. Take of black Pepper an ounce, long Pepper, Cloves, Ginger, Mace; of eaeh half an ounce, Nutmegs, Galanga, Cardamons, of each three drams, mix them with the roots and Honey into an electuary according to art.

Culpeper.] The electuary provokes lust, heats the brain, strengthens the nerves, quickens the senses, causes an acute wit, eases pains in the head, helps the fallingsickness and convulsions, coughs, catharrs, and all diseases proceeding from coldness of the brain. Half a dram is enough to take at one time, because of its heat.

Peony is an herb of the sun, the roots of it cure the falling-sickness.

\section{Diacydonium simple.}

College.] Take of the flesh of Quinces cut and boiled in fair water to a thickness, eight pounds, white sugar six pounds, boil it to it just thickness.

\section{Diacydonium with Species.}

College.] Take of the juice of Quinces, Sugar, of each two pounds, white Wine Vinegar half a pound, added at the end of the decoction, it being gently boiled, and the scum taken away, add Ginger two ounces, white Pepper ten drams and two scruples, 
bruise them grossly, and boil it again to the thickness of Honey.

Diacydonium compound, Magisterial.

College.] Take of white Sugar six pounds, spring Water four pounds, clarify them well with the white of an egg, scumming them, then take of ripe Quinces cleansed from the rind and seeds, and cut in four quarters, eight pounds, boil them in the foregoing Syrup till they be tender, then strain the Syrup through a linen cloth, rocata Anglice, Boulter; boil them again to a jelly, adding four ounces of white wine Vinegar towards the end; remove it from the fire, and whilst it is warm put in these following species in powder, Ginger an ounce, white Peppér, Cinnamon, Nutmegs, of cach two drams, keep it for use.

Culpeper.] The virtues of all these three are, they comfort the stomach, help digestion, stays romiting, belchings, \&c. stop fluxes and the menses. They are all harmless, you may take the quantity of a nutnieg of them at a time, before meat to help digestion and fluxes, after meat to stay voniting, in the morning for the rest.

\section{Confectio de Hyacintho.}

College.] 'J'ake of Jacinth, red Coral, Bole-amoniac, Earth of Lemnos, of each half an ounce, the berries of Chermes, the Roots of 'Tormentil and Ditany, the seeds of Citrons, Sorrel, and Purslain, Saffron, Myrrh, red Roses exungulated, all the sorts of Sanders, bone of a Stag's heart, Hart's. horn, Ivory prepared, of each four scruples, Samphire, Emerald, Topaz, Pearls, raw Silk, leaves of Gold and Silver, of each two scruples, Camphire, Musk, Ambergris, of to two drams. each five grains, with Syrup of Lemons? make it into a confection according to art.

Culpeper] It is a great cordial and cool, excceding good in acute ferers and pestilences, it mightily strengthens and cherishes the heart. Never above half a dram is given at a time, very seldom so much.

$(33,34$.
Antidotum Hamagogum.

College.] Take of Lupines'husked two drams, black Pepper five scruples and six grains, Liquorice four scruples, long Birthwort, Mugwort, Cassia Lignea, Macedonian Parsley seed, Pellitory of Spain, Rue seed, Spikenard, Myrrh, Pennyroyal, of each two scruples and fourteen grains, the seeds of Smallage, Savin, of each two scruples and thirteen grains, Centaury the greater, Cretish Carrots, Nigella, Caraway, Annis, Cloves, Alum, of each two scruples, Bay leaves one scruple, one half scruple, and three grains, Schænanth one scruple and thirteen grains, Asarabacca, Calamus Aromaticus, Amomum, Centaury the less, the seed of Orrach, Peony, Fennel, of each one scruple and six grains, wood of Aloes, a scruple and fourteen grains, Cypress, Elecampane, Ginger, Cappar roots, Cummin, Orobus, of each one scruple, all of them being beaten into very fine powder, let them bé made into an electuary according to art, with four times their weight in sugar, let it stand one month before you use it.

Culpeper.] It provokes the menses, brings away both birth and after-birth, the dead child, purges such as are not sufficiently purged after travail, it provokes urine, breaks the stone in the bladder, helps the stranguary, disury, iskury, \&c. helps indigestion, the cholic, opens any stoppings in the body, it heats the stomach, purges the liver and splcen, consumes wind, stays romiting, but let it not be taken by pregnant women, nor such people as have the hemorrhoids. The dose is from one dram

\section{College.] Thasatyrion. \\ Diasatyrion.}

ounces, Dates, bitter Almonds, Indian Nuts, Pinc nuts, Festick nuts, green Ginger, Eringo roots preserved, of each one ounce, Ginger, Cloves, Galanga, Pepper long and black, of each three drauns, Ambergris one scruple, Musk two scruples, Penins a $\mathrm{p}$ 
four ounces, Cinnamon, Saffron, of each half an ounce, Malaga Wine three ounces, Nutmegs, Mace, Grains of Paradise, of each two drams, Ash-tree keys, the belly and loins and Scinks, Borax, Benjamin, of each threedrams, wood of Aloes, Cardamoms, of each two drams, the seeds of Nettles and Onions, the roots of Avens, of each a dram and and half, with two pounds and an half of Syrup of green Ginger, make them into an electuary according to art.

Electuarium Diaspermaton.

College.] Take of the four greater and lesser. cold seeds, the seeds of Asparagus, Burnet, Bazil, Parsley, Winter Cherries, of each two drains, Grom well, Juice of Liquorice, of each three drams, Cinnamon, Mace, of each one dram, with eight times their weight in white Sugar dissolved in Marshmallows water, make it into an electualy according to art.

Culpeper.] It breaks the stone, and provokes urine. Men may take half an ounce at a time, and children half 'so much, in water of any herb or roots, \&c. (or decoction of them) that break the stone.

\section{Micleta.}

College.] Take of the barks of all the Myrobalans torrified, of each two drams and an half, the seeds of Water-cresses, Cummin, Annis, Fennel, Ammi, Caraway, of each a dram and an half, bruise the seeds and sprinkle them with sharp white wine Vinegar, then beat them into powder, and add the Mirobalans, and these things that follow, Spodium, Balaustines, Sumach, Mastich, Gum Arabic, of each one dram and fifteen grains, mix them together, and with ten ounces of Syrup of Myrtles, make them into an electuary according to art.

Culpeper.] It gently eases the bowels of the wind cholic, wringing of the bowels, infirmities of the spleen, it stops fluxes, the hemorrhoids, as also the menses.

Electuarium Pectorale.

Or a Pectoral Electuary.'
College.] 'Take of the juice of Liquorice, sweet Almonds, Hazel-Nuts, of each half an ounce, Pine-nuts an ounce, Hysop, Maidenhair, Orris, Nettle seeds, round Birthwort, of each a dram and an half, black Pepper, the seeds of Water-cresses, the roots of Elecampane, of each. half a dram, Honey fourteen ounces, make them into an electuary according to art.

Culpeper.] It strengthens the stomach and lungs, and helps the vices thereof. Take it with a Liquorice stick.

\section{Theriaca Diatessaron.}

College.] Take of Gentain, Bay-berries, Myrrh, round Birthwort, of each two ounces, Honey two pounds, make them into an electuary according to art.

Culpeper.] : This is a gallant electuary. It wonderfully helps cold infirmities of the brain, as convulsions, falling-sickness, dead palsies, shaking palsies, \&c. As also the stomach, as pains there, wind, want of digestion, as also stoppings of the liver, dropsies, it resists the pestilence and poison, and helps the bitings of venomous beasts. The dose is from half a drain to two drams, according to the age and strength of the patient, as also the strength of the diseases: you may take it either in the morning, or when urgent occasion calls for it.

\section{Diascordium.}

College.] Take of Cinnamon, Cassia Lignea, of each lialf an ounce, Scordium, an ounce, Dittany of Crete, 'Tormentil, Bistort, Galbanum, Gum Arabic, of each half an ounce, Opium one dram and an half, Sorrel seeds one dram and a half, Gentain half an ounce, Bole-anıniac an ounce and an half, Earth of Lemnos half an ounce, long Pepper, Ginger, of each two drams, clarified Honey two pounds and an half, Sugar of Roses one pound, Canary Wine ten ounces, make them into an electuary according to art.

Culpeper.] It is a well composed electuary, something appropriated to the nature 
of women, for it provokes the menses, hastens labour, helps their usual sickness at the time of their lying in; I know nothing better, it stops fluxes, mightily strengthens the heart and stomaeh, neither is so hot but it may safely be given to weak people, and besides provokes sleep. It may sately be given to young children ten grains at a time, aneient people may take a dram or more. It is given as an excellent cordial in such fevers as are aecompanied with want of slcep.

\section{Mithridate.}

College.] Take of Myrrh, Saffron, Agariek, Ginger, Cinnamon, Spikenard, Frankincense, 'Treacle, Mustard seeds, of each ten drams, the seeds of Hartwort, Opobalsanum, or oil of Nutmegs by ex-? pression, Schenanth, Stochas, Costus, Galbanum, 'Iurpentine, long Pepper, Castorium, juice of Hypocistis, Styrax, Calamitis, Opopanax, Indian leaf, or for want of it Maee, of each an ounce, Cassia Lignea, Poley Mountain, white Pepper, Seordium, the seeds of Carrots of Crete, Carpobalsamum or Cubebs, 'Troeh, Cypheos, Bdelium, of cach seven drams, Celtie Spikenard, Gum A rabie, Maecdonian Parsley secds, Opium, Cardamoms the less, Fenncl seed, Gentian, red Rose leaves, Diltany of Crete, of caeh five drams, Annis seeds, Asarabacea, Orris Acorus, the greater Valcrian, Sagapen, of cach three drams, Meum Acacia, the bollies of Scinks, the tops of St. John's Wort, of each two drams and an half, Malaga Wine, so mueh as is sufficient to dissolve the juices and gums, elarified Honey the treble weight of all, the wine excepted, make them into an electuary according to art.

Culpeper.] It is good against poison and such as liave done themselres wrong by taking filthy medicines, it prorokes sweat, it helps eontinual waterings of the stomach, ulcer's in the body, consumptions, weakness of the limbs, rids the body of eold humours, and diseases coming of cold, it remedies and an half, Euphorbium prepared, Mymh, cold infirmities of the brain, and stopping of the passage of the senses, (viz. hearing, seeing, smelling, \&c.) by cold, it expels wind, helps the cholic, provokes appetite to one's victuals, it helps ulcers in the bladder, if Galen say true, as also diffieulty of urine, it casts out the dead child, and helps sueh women as cannot conceive by reason of cold, it is an admirable remedy for melancholy, and all discases of the body coming through cold, it would fill a whole sheet of paper to reckon them all up particularly. You may take a seruple or half a dram in the norning, and follow your business, two drams will make you sweat, yea one dram if your body be weak, for then two drams may be dangerous beeause of its heat.

\section{Phylonium Persicum.}

College.] 'Take of white Pepper, the seeds of white Henbanc, of each two drams, Opium, Earth of Lemnos, of each ten drams, Lap, Hematitus, Saffron, of each five drams, Castorium, Indian Spikenard, Euphorbium prepared, Pellitory of Spain, Pearl's, Amber, Zedoary, Elecampane, 'Troch, Ramach, of each a dram, Camphire a scruple, with their treble weight in Honey of Roses, make it into an eleetuary aecording to art.

Culpeper.] It stops blood flowing from any part of the body, the immoderate flowing of the menses, the hemorrhoids in mer, spitting of blood, bloody fluxes, and is profitable for such women as are subject to miscarry : See the next receipt.

\section{Phylonizm Romanim.}

College.] 'Take of white Pepper, white Henbane seeds, of each five drams, Opimn two drams and an half, Cassia lignea a dram and an half, the seeds of Snallage a dram, Parsley of Macedonia, Fennel, Carrots of Crete, of cach two scruples and fre grains, Saffion a scruple and an half, Indian Spikenard, Pellitory of Spain, Zedoary fifteen grains, Cinnamon a dram 
Castorium, of each a dram with their treble Rhapontic, Stoechas, Horehound, Macedo. weight in clarified Honey, make it into an nian Parsley seed, Calaminth, Cypress, electuary.

Electuarium de Oro.

Or electuary of Eggs.

College.] 'Take a Hen's Egg new laid, and the white being taken out by a small hole, fill up the void place with Saffron, leaving the yolk in, then the hole being? stopped, roast it in ashes till the shell begin to look black, take diligent heed the Saffron burn not, for then is the whole medicine spoiled, then the matter being taken out dry, if so that it may be beaten into powder and add to it as much powder of white Mustard seed as it weighs. Then take the roots of white Dittany and 'Tormentil, of each two drams, Myrrh, Hart's-hoin, Petasitis roots, of each one dram, the roots of Angelica and Burnet, Juniper Berries, Zedoary, Camphire of each half an ounce, mix them all together in a mortar, then add Venice Treacle the weigh of them all, stir them about with a pestle three hours together, putting in so much Syrup of Lemons, as is enough to make it into an electuary according to art.

Culpeper.] A dram of it given at a time, is as great a help in a pestilential fever as a man shall usually read of in a Galenist. It provokes sweat, and then you shall be taught how to use yourself. If years do not permit, give not so much.

Theriaca Andromachi.

Or Venice Treacle.

College.] 'Take of Troches of Squils forty-eight drams, Troches of Vipers, long Pepper, Opium of'Thebes, Magma,Hedycroi dried, of each twenty-four drams, red Roses exungulated, Orris, Illirick, juice of Liquorice, the seeds of sweet Navew, Scordium, Opobalsamum, Cinnamon; Agerick, of each twelve drams, Myrrh, Costus, or Zedoary, Saffron, Cassia Lignea, Indian Spikenard, Schenanth, Pepper white and black, Olibanum; Dittany of Crete;
Turpentine, the roots of Cinquefoyl and Ginger, of each six drams, Poley Mountain, Chamepitis, Celtic Spikenard, Amomus, Styrax Calamitis, the roots of Meum, the tops of Germander, the roots of Rhapontic Earth of Lemnos, Indian Leaf, Chalcitis burnt, or instead thereof Roman Vitriol burnt, Gentian roots, Gum Arabic, the juice of Hypositis, Carpobalsamum or Nutmegs, or Cubebs, the seeds of Annis, Cardamums, Fennel, Hartwort, Acacia, or instead thereof the juice of Sloes made thick, the seeds of Treacle Mustard, and Ammi, the tops of St. John's Wort, Sagapen, of each four drams, Castorium, the roots of long Birthwort, Bitumen, Judaicum, Carrot seed, Opopanax, Centaury the less, Galbanum, of each two drams, Canary Wine enough to dissolve what is to be dissolved, Honey the treble weight of the dry species, make them into an Electuary according to art.

Culpeper.] It resists poison, and the bitings of venomous beasts, inveterate headaches, vertigo, deafness, the falling-sickness, astonishment, apoplexies, dulness of sight, want of voice, asthmaes, old and new coughs, such as spit or vomit blood, such as can hardly spit or breathe, coldness of the stomach, wind, the cholic, and illiac passion, the yellow jaundice, hardness of the spleen, stone in the reins and bladder, difficulty of urine, ulcers in the bladder, fevers; dropsies, leprosies, it provokes the menses, brings forth birth and after-birth, helps pains in the joints, it helps not only the body, but also the mind, as vain fears, melancholy, \&c. and is a good remedy in pestilential fevers. You may take half a dram and go about your business, and it will do you good if you have occasion to go in ill airs, or in pestilent times, if you shall sweat under it, as your best way is, if your body be not in health, then take one dram, or between one and two, or less than one, according as 
age and strength is, if you cannot take this and clarified, make it into an electuany or any other sweating medicine by itself, according to art.

mix it with a little Carduus or Dragon's Culpeper.] It is exceeding good against water, or Angelica water, which in my cold diseases of the stomach, liver, or opinion is the best of the three.

\section{Theriacca Londinensis.}

Or London 'Treacle.

College.] 'T'ake of Hart's-horn two ounces, the seeds of Citrons, Sorrel, Peony, Bazil, of each one ounce, Scordium, Coralliana, of each six drams, the roots of Angelica, 'Tormentil, Peony, the leaves of 'Dittany, Bay-berries, Juniper-berries, of each half an ounce, the flowers of Rosemary, Marigolds, Clove Gilliflowers, the tops of Saint John's Wort, Nutmegs, Saffron, of each threedrams, the Roots of Gentian, Zedoary, Ginger, Mace, Myrrh, the leares of Scabious, Devil's-bit, Carduus, of each two drans, Cloves, Opium, of each a dram, Malaga Wine as much as is sufficient, with their treble weight in Honey, mix them according to art.

Culpeper.] The receipt is a pretty cordial, resists the pestilence, and is a good antidote in pestilential times, it resists poison, strengthens cold stomachs, helps digestion, crudities of the stomach. A man may safely take two drams of it in a morning, and let him fear no harm.

\section{Diacrocuma.}

College.] Take of Saffron, Asarabacca roots, the seeds of Parsley, Carrots, Annis, Sinallage, of each half an ounce, Rhubarb, the roots of Meum, Indian Spikenard, of eacl six drams, Cassia Lignea, Costus, Myrrh, Schenanth, Cubebs, Madder roots, the juices of Maudlin, and Wormwood made thick, Opobalsanuum, or oil of Nutmegs, of each two drams, Cinnamon, Calamus Aromaticus, of each a dram and an half, Scordium, Cetrach, juice of Liquorice, of each two drams and an half, Tragacanth a dram, with eight times their weight? in white sugar, dissolved in Endive water,

$(33,34$.$) ,$ spleen, corruption of humours and putrefaction of meat in the stomach, ill favoured colour of the body, dropsies, cold faults in the reins and bladder, provokes urine. Take a dram in the morning.

\section{PURGING ELECTUARIES.}

\section{Benedicta Laxativa.}

College.] Take of choice Turbith ten drams, Diacridium, bark of Spurge Roots prepared, Hermodactils, Red Roses, of.each five drans, Cloves, Spikenard, Ginger, Saffron, long Pepper, Amomus, or for want of it Calamus Aromaticus, Cardamons the less, the seeds of Smallage, Parsley, Fennel, Asparagus, Bruscus, Saxifrage, Gromwell, Caraway, sal. gem. Galanga, Mace, of each a dram, with their treble weight of clarified Honey: make them into an clectuary according to art. Also you may keep the species itself in your shops.

Culpeper.] It purges tlegm, chiefly from the joints, also it purges the reins and bladder.

College.] Take of Cloves, Costus, or Zedoary, Ginger, Cummin, of each two drams, Hermodactils, Diacridium, of each half an ounce: with their double weight of Honey clarified in white wine, make them into an electuary according to art.

Culpeper.] Authors say it purges hot rheuns, and takes away inflammations in wounds, I assure you the electuary works violcntly, and may safely be given in clysters, and so you may give two or three drams at a time, if the patient be strong. For taken otherwise it would kill a horse cum privilegio.

Cassia Extracta pro Clysteribus.

Or Cassia extracted for Clysters. $4 Q$ 
College.] 'Take of the leaves of Violets, Mallows, Beets, Mercury, Pellitory of the purges without advice of a physician.

Wall, Violet Hower's, of each a handful, boil them in a sufficient quantity of water, the benefit of which let the Cassia be ex-? tracted, and the canes washed; then take of this Cassia so drawn, and boil it to its consistence, a pound, Sugar a pound and a half, boil them to the form of an electuary according to art.

Culpeper.] You may take it in white Wine, it is good for gentle bodies, for if your body be hard to work upon, perhaps it will not work at all; it purges the reins gallantly, and cools them, thereby preventing the stone, and other diseases caused by their beat.

Electuarium Amarum Magistrale majus.

Or the greater bitter Electuary.

College.] Take of Agarick, Turbith, Species Hiera Simplex, Rhubarb, of each one dram, choice Aloes unwashed two drams, Ginger, Crystal of Tartar, of each two scruples, Orris, Florentine, sweet Fennel seeds, of each a scruple, Syrup of Roses solutive as much as is sufficient to make it into an electuary according to art.

Electuarium Amarum minus.

Or the lesser bitter Electuary.

College.] Take of Epithimum half an ounce, the roots of Angelica three drams, of Gentian, Zedoary, Acorus, of each two drains, Cinnamon one dram and an half, Cloves, Mace, Nulmegs, Saffron, of each one dram, Aloes six ounces, with Syrup of Fumitory, Scabious and Sugar so much as is sufficient to make it into a soft electuary.

Culpeper.] Both these purge choler, the former ftegm, and this melancholy, the former works strongest, and this strengthens most, and is good for such whose brains are annoyed. You may take half an ounce of the former, if your body be any thing strong, in white Wine, if very strong an ounce, a reasonable body may take an ounce of the latter, the weak less. I would

\section{Diacassia with Manna.}

College.] Take of Damask Prunes two ounces, Violet Howers a handful and an half, Spring Water a pound an an lialf, boil it according to art till half be consumed, strain it, and dissulve in the decoction six ounces of Cassia newly drawn, sugar of Violets, Syrup of Violets, of each four ounces, Pulp of T'amarinds an ounce, Sugar Candy. an ounce and an half, Manna two ounces, mix them, and make them into an electuary according to art.

Culpeper.] It is a fine cool purge for such as are bound in the body, for it works gently, and without trouble, it purges choler, and may safely be given in fevers coming of choler: but in such cases, if the body be much bound, the best way is first to administer a clyster, and then the next morning an ounce of this will cool the body, and keep it in due temper.
Cassia extracta sine soliis Sence.

Or Cassia extracted without the leaves of Sena.

College.] Take twelve Prunes, Violet flowers a handful, French Barley, the seed of Annis, and bastard Saffron, Polypodium of the Oak, of each five drams, Maidenhair, Thyme, Epithimum, of each half a handful, Raisins of the Sun stoned half an ounce, sweet Fennel seeds two drams, the seeds of Purslain, and Mallows, of each three drams, Liquorice half an ounce, boil them in a sufficient quantity of water, strain them and dissolve in the decoction, pulp of Cassia two pounds, of Tamarinds an ounce, Cinnamon three drams, Sugar a pound, boil it into the form of an electuary.

$$
\text { Cassia extracta cum soliis Sence. }
$$

Or Cassia extracted with the leaves of Sena. College.] Take of the former receipt two pounds, Sena in powder two ounces, mix them according to art.

Culpeper.] This is also a fine cool gentle 
purge, cleansing the bowels of choler and melancholy without any griping, very fit for fererish bodies, and yet the former is gentler than this. They both cleanse and
cool the reins; a reasonable body may take cool the reins; a reasonable body may take an ounce and an half of the former, and an
ounce of the latter in white Wine, if they keep the house, or their bodies be oppressed with melancholy, let them take half the quantity in four ounces of decoction of Epithimum.

\section{Diacarthamum.}

College.] Take of Diatragacanthum frigidum, half an ounce, pulp of preserved Quinces an ounce, the inside of the seeds of Bastard Saffron half an ounce, Ginger two drams, Diacrydium beaten by itself three drams, Turbith six drams, Manna two ounces, Honey of Roses solutive, Sugar Candy, of each an ounce, Hermodactils half an ounce, Sugar ten ounces and an half, make of them a liquid electuary according to art.

\section{Diaphenicon.}

College.] Take of the pulp of Dates boiled in Hydromel, Penids, of each laalf a pound, sweet Almonds blanched, three ounces and an half, to all of them, being bruised and mixed, add clarified Honey two pounds, boil them a little, and then strew in Ginger, long Pepper, Mace, Cinnamon, Rue leaves, the seeds of Fennel and Carrots, of each two drams, Turbith four ounces, Diacridium an ounce and an half, make of them an electuary according to art.

Culpeper.] I cannot believe this is so profitable in fevers taken downwards as authors say, for it is a very violent purge.

\section{Diaprumum Lenitive.}

College.] Take one hundred Damask Prunes, boil them in water till they be soft, then pulp them, and in the liquor they were boiled in, boil gently one of Violet flowers, strain it, and with two pounds of sugar boil it to a Syrup, then add half a pound of the aforesaid pulp, the pulp of Cassia, and Tamarinds, of each one ounce, then mix with it these powders following: Sanders white and red, Spodium, Rhubarb, of each three drams, red Roses, Violets, the seeds of Purslain, Succory, Barberries, Gum Tragacanth, Liquorice, Cinnamon, of each two drams, the four greater cold seeds, of each one dram, make it into an electuary according to art.

Culpeper.] It may safely, and is with good success, given in acute, burning, and all other fevers, for it cools much, and loosens the body gently: it is good in agues, hectic fevers, and Mirasmos. You may take an ounce of it at a time, at night when you go to bed, three hours after a light supper, neither need you keep your chamber next day, unless the weather be very cold, or your body very tender.

\section{Diaprunum solutive.}

College.] Take of Diaprunum Lenitive whilst it is warm, four pounds, Scammony prepared two ounce and five drams, mix them into an electuary according to art.

Seeing the dose of Scammony is increased according to the author in this medicine, you may use a less weight of Scammony if you please.

\section{Catholicon.}

College.] Take of the pulp of Cassia and Tamarinds, the leaves of Sena, of each two ounces, Polypodium, Violets, Rhubarb, of each one ounce, Annis seeds, Penids, Sugar Candy, Eiquorice, the seeds of Gourds, Citruls, Cucumbers, Melons, of each two drams, the things to be bruised being bruised, take of fresh Polypodium three ounces, sweet Fennel seeds six drams, boil tliem in four pounds of water till the third part be consumed, strain it, and with two pounds of sugar, boil the decoction to the thickness of a Syrup; then with the pulps and powder make it into an electuary according to art.

Culpeper.] It is a fine cooling purge for 
any part of the body, and very gentle, it too much; you may take it in white wine, may be given (an ounce, or half an ounce and keep yourself warm. If you would at a time, according to the strength of the have my opinion of it, I do not like it. patient) in acute, or peracute diseases, for it gently loosens the belly, and adds strength, it helps infirmities of the liver and spleen, Myrobalans two ounces, Myrobalans, gouts of all sorts, quotidian, tertian, and Chebs and blacks, Violets, Colocynthis, quartan agues, as also head-aches. It is Polypodium of the Oak, of each one ounce usually given in clysters. If you like to and an half, Wormwood, 'Thyme, of each take it inwardly, you may take an onnce at half an ounce, the seeds of Annis, and night going to bed ; in the morning drink Fennel, the flowers of red Roses of each a draught of hot posset drink and go about your business.

\section{Electuarium de Citro Solutivum.}

Or Electuary of Citrons, solutive.

College.] Take of Citron pills preserved, conserves of the flowers of Violets and Bugloss, Diatragacanthum frigidum, Diacrydium, of each half an ounce, Turbith of each one pound, boil it to the thickness five drams, Ginger half a dram, Sena six of Honey, strewing in towards the end. drams, sweet Fennel seeds one dram, white Agarick trochiscaled, Sena of cach two sugar dissolved in Rose-water, and boiled ounces, Rhubarb one ounce and an half, according to art, ten ounces, make a solid Epithimum one ounce, Diacrydium six electuary according to art.

Culpeper.] Here are some things very cordial, others purge violently, both put together, make a composition no way pleasing to me; therefore $I$ acconut it a pretty receipt, good for nothing.

\section{Electuarium Elescoph.}

College.] Take of Diacrydium, Turbith, of each six drams, Cloves, Cinnamon, Ginger, Myrobalans, Emblicks, Nutmegs, Polypodium, of each two drams and an half, Sugar six ounces, clarified Honey ten ounces, make it into an electuary according to art.

Culpeper.] It purges choler and flegm, and wind from all parts of the body, helps pains of the joints and sides, the cholic, it cleanses the reins and bladder, yet $I$ advise you noi to take too much of it at a time, for it works pretty violently, let half an ounce be the most, for such whose bodies are strong, always remembering that you had better ten times take too little, than once drams, Cinnamon half an ounce, Ginger two drams, the seeds of Fumitory and Annis, Spikenard, of each one dram, make it into an electuary according to art.

Culpeper.] The receipt is chiefly appropriated as a purge for melancholy and salt flegm, and diseases thence arising, as scabs, itch, leprosies, cancers, infirmities of the skin, it purges adust humours, and is good against madness, melancholy, forgetfulness, vertigo. It purges very violently, and is not safe given alone. I would advise the unskilful not to meddle with it inwardly: You may give half an ounce of it in clysters, in melancholy diseases, which commonly have astringency a constant companion with them.

\section{Electuarium Lenitinum.}

Or Lenitive Electuary.

College.] Take of Raisins of the Sun stoned, Polypodium of the Oak, Sena, of each two ominces, Mercury one handful and an half, Jujubes, Sebestens, of each twenty, 
Maidenhair, Violets, French Barley, of each in powder, and so make it into an electuary one handful, Damask Prunes stoned, according to art.

Tamarinds of each six drams, Liquorice . Culpeper.] It purges choler, and is good half an ounce, boil them in ten pounds of in tertian agues, and diseases of the joints, water till two parts of the three be con- it purges violently, therefore let it be warily sumed; strain it, and dissolve in the decoc-? given.

tion, pulp of Cassia, Tamarinds, and fresh Pruncs, Sugar of Violets, of each six ounces, Sugar two pounds, at last ald powder of samum, or wood of Aloes, the roots of Sena leaves, one ounce and an half, Annis Asarabacca, Spikenard, Mastich, Saffron, seeds in powder, two drams to each pound of each six drams, Aloes not washed twelve of clectuary, and so bring it into the form ounces and an half, clarified Honey four of an clectuary according to art.

Hiera Picra simple.

College.] Take of Cinnamon, Xylobal-
mun, or wood of Aloes, the roots of
sarabacca, Spikenard, Mastich, Saffron,
each six drams, Aloes not washed twelve
mces and an half, clarified Honey four
ounds and three ounces, mix them into an pounds and three ounces, mix them into an
electuary according to art. Also you may keep the species by itself in your shops.

Culpeper.] It is an excellent remedy for vicious juices which he furning the tunicle of the stomach, and such idle fancies and symptoms which the brain suffers thereby, whereby sone think they see, others that they hear strange things, especially when they are in bed, and between sleeping and waking : besides this, it very gently purges the belly, and helps such women as are not sufficiently purged after their travail.

\section{Hiera with Agarick.}

College.] Take of species Hiera, simple without Aloes, Agarick trochiscated, of each half an ounce, Aloes not washed one ounce, clarified Honey six ounces, mix it, and make it into an electuary according to art.

Culpeper.] Look but to the virtues of Agarick and add them to the virtues of the former receipt, so is the business done without any further trouble.

\section{Hiera Logadii.}

College.] Take of Coloquintida, Polypochium, of each two drams, Euphorbium, Or Electuary of the Juice of Roses.

College.] Take of Sugar, the juice of Poley mountain, the seeds of Spurge, of red Roses clarified, of each a pound and each one dram and an hatf, and six grains, four ounces, the three sorts of Sanders of Wormwood, Myrrh, of each one dram and each six drams, Spodium three drams, twelve grains, Centaury the less, Agarick, Diacydonium twelve drams, Camphire a Gum Ammoniacum, Indian laaf or Mace, scruple, let the juice be boiled with the Spikenard, Squills preparfd, Diacrydium sugar to its just thickness, then add the rest " of each one dram, Alocs, Thyme, German$(33,34$. 
der, Cassia Lignea, Bılellum, Horehound, half an ounce, filings of steel prepared with of each one seruple and fourteen grains, Vinegar twenty drams, let the Myrobalans Cinnamon, Oppopanax, Castorium, long be roasted with fresh butter, let the rest, being Birthwort, the three sorts of Pepper, powdered, be sprinkled with oil of sweet Sagapen, Saffron, Parsley of each twodrams, Almonds, then add Musk one dram, and Hellebore black and white, of each six with their treble weight in Honey, malse it grains, clarified Honey a pound and a half, into an electuary according to art.

mix them, and make of them an electuary according to art. Let the species be kept dry in your shops.

Culpeper.] It takes away by the roots daily evils coming of melancholy, fallingsicliness, vertigo, convulsions, megrim, leprosies, and many other infirmities; for my part I should be loth to take it inwardly unless upon desperate occasions, or in clysters. It may well take away diseases by the roots, if it takes away life and all.

Hiera Diacolocynthidos.

College.] Take of Colucynthis, Agarick, Germander, white Horehound, Stochas, of each ten drams, Opopanax, Sagapen, Parsley sceds, round Birthwort roots, white Pepper of each five drams, Spikenard, Cinnamon, Myrrh, Indián leaf or Mace, Saffron, of each four drams, bruise the Gums in a mortar, sift the rest, and with three pounds of clarified honey, thrce ounces and five drans, make it into an electuary according to art.

Culpeper.] It helps the falling-sickness, madness, and the pain in the head called Kephalalgia, pains in the breastand stomach whether they come by sickness or bruises, pains in the loins or back-bone, hardness of womens breasts, putrefaction of meat in the stomach, and sour belchings. It is but used seldomi and therefore hard to be gotten. Triphera the greater.

College.] T'ake of Myrobalans, Chebs, Bellericks, Inds and Emblicks, Nutmegs,
of each five drams, Water-cress seeds, Asarabacca roots, Persian Origanum, or clse Dittany of Crete, black Pepper, Olibanum, Ammi, Ginger, Tamarisk, Indian and the bitings of venomous beasts, and
Nard, Squinanth, Cypress roots of each helps such whose meat putrifies in their

Culpeper.] It helps the immoderate flowing of the menses in women, and the haemorrhoids in men, it helps weakness of the stomach, and restores colour lost, it frees the body from crude humours, and strengthens the bladder, helps melanclioly, and rectifies the distempers of the spleen. You may talie a dram in the morning, or two if your body be any thing strong.

\section{Thiphera solutive.}

College.] Take of Diacrydium, ten drams, Turbith, an ounce and an half, Cardamoms the less, Cloves, Cinnamon, Honey, of each three drams, yellow Sanders, Liquorice, sweet Fennel seeds, of each half an ounce, Acorns, Schœnanth, of each a dram, red Roses, Citron pills preserved, of each three drams, Violets two drams, Penids four ounces, white Sugar half a pound, Honey clarified in juice of Apples one pound, make an electuary according to art.

Culpeper.] The Diacrydium and Turbith, are a couple of untoward purges, the rest are all cordials.

Athanasia Mithridatis. Galen.

College.] Take of Cinnamon, Cassia, Schonanth, of each an ounce and an half, Saffron, Myrrh, of each onc ounce, Custus, Spignel, (Meum,) Acorus, (Water-flag perhaps they mean. See the root in the Catalogue of Simples, Agarick, Scordium, Carrots, Parsley, of each half an ounce, white Pepper eleven grains, Honey so much as is sufficient to make it into an electuary according to art.

Culpeper.] It prevails against poison, 
stomach, stays vomiting of blood, helps Calamitis two drams and an half, Sugar old coughs, and cold diseases in the liver, dissolved in Hyssop water, and clarified spleen, bladder, and matrix. The dose is Honey, of each twice the weight of all the half a dram.

Electuarium scoriaferri. Rhasis.

College.] Take of the flakes of Iron inrest, make thern into an electuary according to art.

Culpeper.]. The electuary is chiefly apfused in Vinegar seven days and dried, propriated to the lungs, and helps cold in-
three drams, Indian Spikenard, Schœnanth, firmities of them, as asthmaes, coughs, dif-
Cypress, Ginger, Pepper, Bishop's weed, ficulty of breathing, \&c. You may take it fused in Vinegar seven days and dried, propriated to the lungs, and helps cold in-
three drams, Indian Spikenard, Schœnanth, firmities of them, as asthmaes, coughs, dif-
Cypress, Ginger, Pepper, Bishop's weed, ficulty of breathing, \&c. You may take it Frankincense, of each half an ounce, with a Liquorice stick, or on the point of Myrobalans, Indian Bellericks, and Em- a knife, a little of it at a time, and often. blicks, Honey boiled with the decoction of Emblicks, sixteen ounces, mix them together, and make of them an electuary.

Culpeper.] The medicine heats the spleen gently, purges melanclıoly, eases pains in the stomach and spleen, and strengthens digestion. People that are strong may take half an ounce in the morning fasting, and weak people thrce drams. It is a good remedy for pains and hardness of the splcen.

\section{Confectio Humain. Mesue.}

College.] 'Take of Eyebright two ounces, Fennel seeds five drams, Cloves, Cinnamon, Cubebs, long Pepper, Mace, of each one dram, beat them all into powder, and with clarified Honey one pound, in which boil juice of Fennel one ounce, juice of Celandine and Rue, of each half an ounce, and with the powders make it up into an electuary.

Culpeper.] It is chiefly appropriated to the brain and heart, quickens the senses, especially the sight, and resists the pestilence. You may take half a dram if your body be hot, a dran if cold, in the morning fasting.

\section{Diaireos Solomonis. Nicl.} Diasatyrion. Nich.

College.] 'Take of the roots of Satyrion fresh and sound, garden Parsnips, Eringo, Pine-nuts, Indian Nuts, or if Indian Nuts, be wanting, take the double quantity of Pine-nuts, Fistic-nuts, of each one ounce and an lialf, Cloves, Ginger, the seeds of Annis, Rocket, Ash Keys, of each five drams, Cinnamon, the tails and loins of Scincus, the seeds of Bulbus Nettles, of each two drams and an half, Musk seven grains, of the best sugar dissolved in Malaga Wine, three pounds, make it into an electuary according to art.

Culpeper.] It helps weakness of the reins and bladder, and such as make water with difficulty, it provokes lust exceedingly, and speedily helps such as are impotent in the acts of Vemus. You may take two drams or more at a time.

Matthiolus his great antidote againist Poison and Pestilence.

College.] Take of Rhubarb, Rhapontic, Valerian roots, the roots of Acorus, or Calamus Aromaticus, Cypress, Cinquefoyl, Tormentil, round Birthwort, male P'cony, Clecampane, Costus, Illirick, Orris, white Chamelion, or Avens, of each three drams, College.] Take of Orris ronts one ounce, the Roots of Galanga, Masterwort, white Pennyroyal, Hyssop, Liquorice, of each six Dictanni, Angelica, Yarrow, Fillipendula drams, 'Iragacanth, white Starch, bitter or Dropwort, Zedoary, Ginger, of each Almonds, Pine-nuts, Cinnamon, Ginger, two drams, Rosemary, Gentian, Duvil's-bit, Pepper, of each three drants, fat Figs, the of each two drams and an laalf, the sceds pulp of Raisins of the Sun, and Dates, of each three drains and an half, Styrax, of Kermes, the seeds of Ash-tree, Sorre], 
wild Parsnips, Navew, Nigella, Peony the choice Honey eight pounds six ounces. male, Bazil, Hedge Mustard, (Irio) Treacle? These being all chosen and prepared with Mustard, Fennel, Bishop's-weed, of each diligence and art, let them be made into an two drams, the berries of Bay, Juniper, electuary just as Treacle or Mithridate is. and Ivy, Sarsaparilla, (or for want of it the double weight of Cubebs, Cubebs, of each one dram and an half, the leaves of Scordium, Germander, Chamepitys, Centaury the less, Stochas, Celtic Spikenard, Calaminth, Rue, Mints, Betony, Vervain, Scabious, Carduus Benedictus, Bawm, of medicine, order your body thus: Take it in each one dram and an half, Dittany of bed, and cover yourself warm, in your Crete three drams, Marjoram, St. John's sweating, drink posset-drink as hot as you Wort, Schœnanth, Horehound, Goats Rue, can, if it be for a fever, boil Sorrel and red Savin, Burnet, of each two drams, Figs, Sage in posset-drink, sweat an hour or two if Walnuts, Fistic-nuts, of each three ounces, your strength will bcar it, then the chamber Emblicks, Myrobalans half an ounce, the being kept very warm, shift yourself all but flowers of Violets, Borrage, Bugloss, Roses, your head, about which (your cap which Lavender, Sage, Rosemary, of each four you sweat in being kept on) wrap a hot scruples, Saffron threedrams, Cassia Lignea, napkin, which will be a means to repel the ten drams, Cloves, Nutmegs, Mace, of each vapuurs back. 'This I hold the best method two drams and an half, hlack Pepper, long? for sweating in fevers and pestilences, in Pepper, all the three sorts of Sanders, wood which this electuary is very good. I an of Aloes, of each one dram and an half, very loth to leave out this medicine, which Hart's-horn half an ounce, Unicorn's-horn, if jt were stretched out, and cut in thongs, or in its stead, Bezoar stone, one dram, would reach round the world.

bone in a Stag's heart, Ivory, Stag's pizzle, Castoreum, of each four scruples, Earth of Lemnos three drams, Opium one dram and an half, Orient Pearls, Emeralds, Jacinth, red Coral, of each one drain and an half, Camphire two drams, Gum Arabic, Mastich, Frankincense, Styrax, Turpentine, Sagapenum, Opopanax, Laserpitium, or Myrrh, of each two drams and an half, Musk, Ambergris, of each one dram, oil of Vitriol half an ounce, species cordiales temperatæ, Diamargariton, Diamoscu, Diambra, Electuarij de Gemmis, Troches of Camphire, of Squills, of each two drams and an half, Troches of Vipers two nunces, the juice of Sorrel, Sow 'Thistles, Scordium, Vipers Bugloss, Borrage, Bawm, of each half a pound, Hypocistis two drams, of the best Treacle and Mithridate, of each six ounces, old Wine three pounds, of the best Sugar, or and Gromwell, juice of Liquorice, of each

College.] Take of red Rose leaves, the whites being cut off, blue Violets, of each three drams, Opium of 'Thebes, dissolved in Wine, the seeds of white Henbane, Poppies white and black, the ronts of Mandrakes, the seeds of Endive, Purslain, garden Lettuce, Psyllium, Spodium, Gum Tragacanth, of each two scruples and five grains, Nutmegs, Cimmamon, Ginger, of each a dram and an half, Sanders, yellow, white, and red, of each a dram and an half, Sugar three times their weight, dissolved in Rosewater: mix them together, and make of them an electuary according to art.

Culpeper.] I like not the receipt taken inwardly.

Electuarium Regince Colenions.

College.] 'Take of the seeds of Saxifrage 
half an ounce, the sceds of Caraway, Annis, Smallage, Fennel, Parsley of Macedonia, Broom, Carrots, Bruscus, Asparagus, Lovage, Cummin, Juniper, Rue, Siler Mountain, the sceds of Acorus, Pennyroyal, Cinquefoyl, Bayberrics, of each two drams, Indian Spikcnard, Schœenanth, Amber, Valcrian, Hog's Fennel, Lapis Lincis, of each a dram and an half, Galanga, Gingcr, 'T'urbith, of each two drams, Scna an ounce, Goat's blood prepared half an ounce, mix them together : first beat thein into powder, then make them into an elcctuary according to art, with three times their weight in Sugar dissolved in white Wine.

Culpeper.] It is an excellent remedy for the stone and wind chnlic, a dram of it taken every morning: I assure such as are troubled with such diseases, I commend it to them as a iewel.

\section{P I L L S.}

Culpeper.] Pills in Greek" are called, Katopotia, in Latin, Pitulce: which signifies little balls, because they arc made up in such a form, that thay may be the better swallowed down, by reason of the offensiveness of their taste.

\section{Pilulce de Agarico. \\ Or Pills of Agarick}

Cóllege.] 'Take of Agarick three chrams, our own blue Orris roots, Masticl, Horehound, of each one dram, Turbith fire drams, Species Hiera Picra half an otince, Colocynthis, Sarcocol, of each two drams, Myrrh onc dram, Sapa as much as is sufficient to makc it into a mass accorrling to art.

Culpeper.] It was invented to clcunse the brcast and lungs of flegm, it works. pretty strongly. Half a dram at a time (kecping yoursclf warm, ) cannot well do you harm, unless your body be very weak.

$(33,34$.
Pilulce Aggregatice.

College.] Takc of Citron, Myrobalans, Rhubarb, of each half an ounce, juice of Agrimony and Wormwood made thick, of each two drams, Diagridium five drams, Agarick, Colocynthis, Polypodium of each twodrams, Turbith, Aloes, of each six drams, Mastich, red Roses, Sal. Gem. Epitlyymum, Annis, Ginger, of cach a dram, with Syrup of Damask Roses, makc it into a mas" according to art.

Culpeper.] It purges the head of choler, flegm and molancholy, and that stoutly: it is good against quotidian agues, and faults in the stomach and liver, yet because it is well corrected if you take but half a dram at a time, and kecp yourself warm, I supposc you may take it without danger.

Piluke Alaphangine.

College.] Take of Cimnamon, Clores, Cardamoms the less, Nutmegs, Mace, Calamus Aromaticus, Carpobalsamum, or Juniper berries, Squinanth, Wood of Aloes, ycllow Sanders, red Roses dried, Wormwood, of each half an ounce, let the tincture be taken out of these, being grossly bruised in spirit of Wine, the resscl being close stopped ; in three pounds of this tincture, being strained dissolve Aloes one pound, which being dissolved, add Mastich, Myrrh, of cach half an ounce, Saffion two drams, Balsam of Peru one dram, the superfluous liquor being consumed, either orer hot ashes, or a bath, bring it into a mass of pills.

Culpeper.] It cleanses both stomach and brain of gross and putrified humours, and sets the senses fiee when they are thereby troubled, it cleanses the brain officided by ill humours, wind, \&c. helps rertigo and head-aches, and strengthens the brain cxceedingly, lielps concoction, and strengthens the stoniach, one dram taken at night going to bed, will work gently next day: it the party be weak, you may gire less, if strong more. If you take but half a dram, you may go abroad the next day: but if you take $4 \mathrm{~s}$ 
a dram, you may keep the house; there can be no harm in that.

Pilula de Aloe Lota.

Or Pills of washed Aloes.
College.] Take of Aloes washed with an half, Turbith, Stoechas, of each five juice of red Roses, one ounce, Agarick three drams, with a sufficient quantity of Syrup drams, Mastich two drams, Diamoscu of Stœchas, make it into a mass, according Dulce half a dram, Syrup of Damask-roses, to art.

so much as is sufficient to make it into a mass according to art.

Culpeper.] It purges both brain, stomach, bowels, and eyes of putrified humours, and also strengthens them. Use these as the succeeding.

\section{Aloe Rosatı.}

College.] Take of Aloes in powder four ounces, juice of Damask Roses clarified one pound, mix them and digest them in the sun, or in a bath, till the superfluous liquor art.

be drawn off, digest it, and evaporate it four times over, and keep the mass.

Culpeper.] It is a gallant gentle purger of choler, trees the stomach from superffuous humours, opens stoppings, and other infirmities of the body proceeding from choler and flegm, as yellow jaundice, \&c. and strengthens the body exceedingly. Take a scruple, or half a dram at night going to bed, you may walk abroad, for it will hardly work till next day in the afternoon.

\section{Pilule Aurece.}

College.] Take of Aloes, Diacrydium, of each five drams, red Roses, Smallage seeds, of each two drams and an half, the seeds of Annis and Fennel, of each one dram and an half, Mastich, Saffron, Troch, Alhandal, of each one dram, with a sufficient quantity of Honey Roses, make it into a mass according to art.

Culpeper.] They are held to purge the head, to quicken the senses, especially the sight, and to expel wind from the bowels, but works something harshly. Half a dram is the utmost dose, keep the fire, take them in the morning, and sleep after them, they will work before noon.
Pilulce Coctice, the greater.

College.] .Take of Species, Hiera Picra, ten drams, Troch, Alhandal, three drams and an half, Diacrydium two drams. and

Culpeper.] It is held to purge the head, but it is but a dogged purge at best, and must be given only to strong bodies, and but lialf a dram at a time, and yet with great care.

Pilula Cochice, the less.
College.] Take of Aloes, Scammony, Colocynthis, of each one ounce, with equal parts of Syrup of Wormwood, and of purging thorn, make it into a mass according to.

\section{Pilula de Cynoglosso.}

Or Pills of Hound's-tongue.

College.] 'Take of the Roots of Hound'stongue dried, white Henbane seed, Opium prepared, of each half an ounce, Myrrh six drams, Olibanum five drams, Saffron, Castoreun, Styrax, Calamitis, of each one dram and an half, with Syrup of Stoechas, make it into a mass.

Culpeper.] It stays hot rheums that fall down upon the lungs, therefore is good in phthisics, also it mitigates pain, a scruple is enough to take at a time going to bed, and too much if your body be weak: have a care of opiates for fear they make you steep your last.

\section{Piluke ex Duobus.}

Or Pills of two things.

College.] Take of Colocynthis, and Scamony, of each one ounce, oil of Cloves as much as is sufficient to malax them well, then with a little Syrup of purging'Thorn, make it into a mass.

Pilula de Eupatorio.

Or Pills of Eupatorium.

College.] Take of the juice of Maudlin, and Wormwood made thick, Citıon, Myro- 
balans, of each three drams, Rhubarb three dram and an latf, with the Syrup of the drams and an half, Mastieh one dram, Aloes juice of Coleworts made with honey, make it five drams, Saffron half a dram, Syrup of Sinto a mass aecording to art. the juice of Endive, as mueh as is suffieient to make it into a mass.

Culpeper.] 'They are good against the gout, and other eold afflietions of the joints.

Culpeper.] It is a gallant gentle purge, 'I'These are more moderate by half than and strengthening, fitted for sueh bodies as Pilula Fictide, and appropriated to the are muel weakned by disease of eholer. same diseases.

The author appropriates it to such as have tertian agues, the yellow jaundice, obstruetions or stoppings of the liver; half a dran taken at night going to bed, will work with an ordinary body, the next day by noon.

Pilule Fetider.

Or Stinking Pills.

College.] Take of Aloes, Coloeynthis, Ammoniacum, Sagapen, Myrrh, Rue-sceds, Epithymum, of each five drams, Scamony three drams, the roots of Turbith half an ounee, the roots of Spurge the less prepared, Hermodactils of eaeh two drams, Ginger one dram and an half, Spikenard, Cinnamon, Saffron, Castoreum, of each one dran, Euphorbium prepared two scruples, dissolve the Gums in juiee of Leeks, and with Syrup made with the juice of Leeks and Sugar, make it into a mass.

Culpeper.] They purge gross and raw flegm, and diseases thercof arising; gouts of all sorts, pains in the baek-bone, and other joints: it is good against leprosies, and other such like infirmities of the skin. I fancy not the receipt much.

Pilula de Hermodactilis.

Or Pills of Hermodaetils.

College.] Take of Sagapen six drams, Opopanax thrce drams, melt them in warm juice of Coleworts, so numeh as is sufficient, then strain it through a convenient rag, afterwards boil it to a mean thiekness, then take of Hernodaetils, Aloes, Citron, Myrobalans, Turbith, Coloquintida, soft Bdellium, of eaeh six drams, Euphorbium prepared, the sceds of Rue and Smallage, Castorcum, Sarcocol, of each three drams, Saffron one

Pilute de Hiera cum Agarico.

Or Pills of Hicra with Agarick.

College.] 'T'ake of Species Hiera Picra, Agarick, of each half an ounee, Aloes one ounce, Honcy Roses so much as is sufficient to make it into a mass according to art.

\section{Pilula Imperiales.}

Or Inuperial Pills.

College.] 'Tike of Aloes two ounces, Rhubarb one ounee and an half, Agariek, Sena, of each one ounce, Cinnamon three drams, Ginger two drams, Nutmegs, Clores, Spikenard, Mastich, of each one dram: with Syrup of Violets, make it into a mass aceording to art.

Culpeper.] It cleanses the body of mixt humours, and strengthens the stomach $\mathrm{ex}$ ecedingly, as also the bowels, liver, and natural spirits : it is good for cold natures, and checrs the spirits. The dose is a seruple, or half a dram, taken at night.

Pilula de Lapide Lazuli.

Or Pills of Lapis Lazuli.

College.] Take of Lapis Lazuli in powder and well washed, five drams, Epithymum, Polypodium, Agariek, of each an ounce, Seamony, blaek Hellebore roots, Sal. Gem. of caelı two drams and an. lialf, Cloves, Annis sceds, of cach half an ounice, Species Hiera simple fifteen drams, with Syrup of the juice of Fumitory, make it into a mass aceording to art.

Culpeper.] It purges melancholy very violently.

Pilutce Macri.
College.] I'ake of Aloes two ounces,
Lastich half an ounce, dried Marjoram two
rams, Salt of Wormwood one dram, make 
them all, being in powder, into a mass ac- the Gums being dissolved in clarified juice cording to art with juice of Coleworts and of Coleworts, with Syrup of the juice of Sugar, so much as is sufficient.

Culpeper.] It strengthens both stomach and brain, especially the nerves and muscles, and eases then of such humours as afflict them, and hinder the motion of the body, they open obstructions of the liver and spleen, and takes away diseases thence? coming.

Pilulc Mastichina.

Or Mastich Pills.

college.] Take of Mastich two ounces, Aloes four ounces, Agarick, Species Hiera sinple, of each one ounce and an half, with Syrup of Wormwood, make it into a mass according to art.

Culpeper] 'I'hey purge very gently, but strengthen much, both head, brain, eyes, belly, and reins.

\section{Pilule Mechoacance.}

Or Pills of Mechoacan.

College.] Take of Mechoacan roots half in ounce, Turbith three drams, the leaves of Spurge steeped in Vinegar and dried, the seeds of Walwort, A garick trochiscated, of each two drams, Spurge roots prepared, Mastich, of each one dram and an half, Mace, Cinnainon, Sal. Gem. of each two scruples, beat them into powder, and with
white Wine, bring them into a mass. When it is dry, beat it into powder, and with Syrup made with the juice of Orris roots and sugar, make it the second time into a mass for pills.

Culpeper.] They purge flegm very violently.

\section{Pilula de Opopanace.}

Or Pills of Opopanax.

College.] Take of Opopanax, Sagapen, Bdellium, Ammoniacum, Hermodactils, stomachs, and work so easily that you need Coloquintida, of each five drams, Saffron, not fear following your business the next Castoreum, Myrrh, Ginger, white Pepper, day.

Cassia Lignea, Citron, Myrobalans, of each one dram, Scamony two drams, Turbith half an ounce, Aloes an ounce and an half, ing to art.

Culpeper.] It helps tremblings, palsies, gouts of all sorts, cleanses the joints, and is helpful for such as are troubled with cold afflictions of the nerves. It works violently: Pilule Rudii.

College.] Take of Coloquintida six drams, Agarick, Scamony, the roots of black Hellebore, and Turbith, of each half an ounce, Aloes one ounce, Diarrhodon Abbatis half an ounce, let all of them (the Diarrh. Abbatis excepted) be grossly bruised, and infused eight days in the best spirits of Wine in a ressel close stopped, in the sun, so that the liquor may swim at top the breadtlı of six fingers: afterwards infuse the Diarrhodon Abbatis in the same manner four days in Aqua vitæe, then having strained aud pressed them hard, mix them both together, casting the dross away, and draw off the moisture in a glass Alembick, and let the thick matter remain in a mass.

Culpeper.] It cleanses both head and body of choler, flegm, and melancholy : it must not be taken in any great quantity, half a drain is sufficient for the strongest body.

Pilutice Russi.
College.] Take of Aloes two ounces, Myrrh one ounce, Saffion half an ounce, with Sylup of the juice of Lemons, make

it into a mass according to art.
Culpeper.] A scruple taken at night going to bed, is an excellent preservative in pestilential times; also they clearse the body of such humours as are gotten by surfeits, they strengthen the heart, and weak stomachs, and work so easily that you need
not fear following your business the next

$$
\text { half an ounce, Alocs an ounce and an hall, }
$$

Pilule sine Quibus.

Or Pills without which

College.] Take of washed Aloes fourtcen 
drans, Scammony prepared six drams, of each two drams, Aloes five drams, Agaric Agarick, Rhubarb, Sena, of each half an a dram and an half, long Birthwort half a ounce, Wormwood, red Roses exungulated, dram, with Syrup of Wormwood make it Violet flowers, Dodder, Mastich, of each into a mass.

one dram, salt of Wormwood, of each half a dram, with Syrup of the juice of Fennel made with Honey, make it into a mass according to art.

Culpeper.] It purges flegm, choler, and melancholy from the head, makes the sight and hearing good, and gives ease to a burdened brain.

\section{Pilule Stomachice.} Or Stomach Pills.

College.] T'ake of Aloes six drams, Mastich, red Roses, of each two drams, with Syrup of Wormwood, make it into a mass according to art.

Culpeper] They cleanseand strengthen the stomach, they cleanse but gently, strengthen much, help digestion.

\section{Pilule Stomachia cum Gummi.}

Or Stomach Pills with Gums.

College.] Take of Aloes an ounce, Sena five drains, Gum Amoniacum dissolved in Eldcr-Hower Vinegar half an ounce, Mistich, Myrrh, of each a dram and an half, Saffiron, salt of Wormwood, of each half a dram, with Syrup of pirging Thorn, make it into a mass according to art.

Culpeper.] They work more strongly than the former.

\section{Pilule e Styrace.}

Or Pills of Styrax.

College.] Take of Styrax Calamitis, Olibanum, Myrrh, juice of Licjuorice, Opium, of each half an ounce, with Syrup of white Poppies, make it into a mass according to art.

Culpeper.] They help such as are troubled with defluxion of troubled with defluxion of rheum, coughs, Ambergris, Musk, of each six grains, and provoke slcep to such as cannot sleep 'oil of Nutmegs ten drops, evaporate the ror coughing.

\section{Pilula de Succino.}

Or Pills of Amber

College.] Take of white Amber, Mastich, $\left(\begin{array}{ll}35 & 36 .\end{array}\right)$
Culpeper.] It amends the evil state of a woman's body, strengthens conception, and takes away what hinders it ; it gently purges choler and flegm, and leaves a binding strengthening quality behind it.

\section{Pilula ex Tribus.}

Or Pills of three things.

College.] Take of Mastich two ounces, Aloes four ounces, Agarick, Hiera simple, of each an ounce and an half, Rhubarb two ounces, Cinnamon two drams, with Syrup of Succory, make it into a mass according to art.

Culpeper.] They gently purge choler, scabs, wheals, \&c. They strengthen the stomach and liver, and open obstructions, as also help the yellow jaundice.

\section{Pilula Turpeti Aurece.}

College.] Take of Turbith two ounces, Aloes an ounce and an half, Citron Myrobalans ten drams, red Roses, Mastich, of each six drams, Saffron three drams, beat them all into powder, and with Syrup of Wornwood bring them into a inass.

Culpeper:] They purge choler and flegm, and that with as much gentleness as can be desired; also they strengthen the stomach and liver, and help digestion.

\section{Laudanum.}

College.] Take of Thebane Opium extracted in spirit of Wine, one ounce, Saffion alike extracted, a dram and an half, Castorium one dram: let them be taken in tincture of lialf an ounce of species Diambre moisture away in a bath, and leave the mass.

Culpeper.] It was invented (and a gallant invention it is) to mitigate violent $4 \mathrm{~T}$ and help diseases thence arising, as itch, 
pains, stop the fumes that trouble the brain seldom used, and therefore are hardly to be in fevers, (but heware of Opiates in the be- had.

ginning of fevers) to provoke sleep, take not above two grains of it at a time, going to bed; if that provoke not sleep, the next night you may makc bold with three. Have a care how you be too busy with such medicines, lest you make a man sleep to doom's-day.

\section{Nepenthes Opiatum.}

College.] Take of tincture of Opium made first with distilled Vinegar, then with spirit of Wine, Saffron extracted in spirit of Wine, of each an ounce, salt of Pearl and Coral, of each half an ounce, tincture of species Diambræ seven drams, Ambergris one dram: bring them into the form of Pills by the gentle lieat of a bath. former.

Culpeper.] The operation is like the

Pilula Assaireth. Avicenna.

College.] Take of Species Hitra Picra Galeni one ounce, Mastich, Citron Myrobalans, of each half an ounce, Aloes two ounces, the Syrup of Stoechas as much as is sufficient, make of them a mass according to art.

Culpeper.] It purges choler and flegm, and strengthens the whole body exceedingly, being very precious for such whose bodies are weakened by surfeits, or ill diet, to take half a dram or a scruple at night going to bed.

\section{Pills of Bdellium. Mesue.}

College.] Take of Bdellium ten drams, Myrobalans, Bellericks, Emblicks, and Blacks, of each five drams, flakes of Iron, Leek seeds, of each three drams, Choncula Veneris burnt, Coral burnt, Amber, of each a.dram and an half, Pearls half an ounce, dissolve the Bdellium in juice of Leeks and with so much Syrup of juice of Leeks as is sufficient, make it into a mass according to art.

Culpeper.」Both this and the former are? mass.

College.] 'Take of choice Rhubarb three drams, Citron Myrobalans, Trochisci Diarrhodon, of each three drams and an half, juice of Liquorice, and juice of Wormwood, Mastich, of each one dram, the seeds of Smallage and Fennel, of each half a dram, Species Hiera Picra simp. Galeni, ten drams, with juice of Fennel not clarified, and Honey so much as is sufficient, make it into a mass.

Culpeper.] It purges choler, opens obstructions of the liver, helps the yellow jaundice, and dropsies in the beginning, strengthens the stomach and lungs.

\section{Pilule Arabica. Nicholaus.}

College.] Take of the best Aloes four ounces, Briony roots, Myrobalans, Citrons, Chebs, Indian Bellerick, and Emblick, Mastich, Diagrydiuni, Asarabacca, Roses, of each an ounce, Castorium three drams, Saffron one dram, with Syrup of Wormwood, nake it into a mass according to art.

Culpeper.] It helps such women as are not sufficiently purged in their labour, helps to bring away what a careless midwife hath left behind, purges the head, helps headach, megrini, vertigo, and purges the stomach of vicious humours.

Pilula Arthritica. Nicholaus.

College.] Take of Hermodactils, Turbith, Agarick, of each half an ounce, Cassia Lignea, Indian Spikenard, Cloves, Xylobalsamum, or Wood of Aloes, Carpobalsamum or Cubebs, Mace, Galanga, Ginger, Mastich, Assafoetida, the seeds of Annis, Fennel, Saxifrage, Sparagus, Bruscus, Roses, Gromwell, Sal. Gem. of each two drams, Scammony one ounce, of the best Aloes, the weight of them all, juice of Chamepitys made thick with sugar, so much as is sufficient: or Syrup of the juice of the same, so much as is sufficient to make it into a 
Culpeper.] It helps the gout, and other age, Schænanthus, Mastich, Asarabacca pains in the jnints, comforts and strengthens r roots, Cloves, Cinnamon, Cassia Lignea, both brain and stomach, and consumes dis- Saffron, Mace, of each two drams, Myroeases whose original comes of flegm.

\section{Piluke Cochice with Helebore.}

College.] Take of the powder of the Pills before prescriberl, the powder of the bark of the roots of black Hellebore, one ? ounce: make it into a mass with Syrup of Stœechas according to art.

\section{Pills of Fumitory. Avicenna.}

College.] Take of Myrobalans, Citrons, Chebs, and Indian Diagrydium, of each five dranı, Aloes seven dranns; let all of them being bruised, be thrice moistened with juice of Funitory, and thrice suffered to dry, then brought into a mass with Syrup of Funitory.

Culpeper.] It purges melancholy. Be not too busy with it 1 beseech you.

Pilule Inda. Mesue out of Haly.

College.] 'Take of Indian Myrobalans, black Hellebore, Polypodium of the Oak, of each five drams, Epithymuin, Stoechas, of each six drams, Agarick, Lapis Lazuli i often washed troches Allandal, Sal Indi, of each half an ounce, juice of Maudlin made thick, Indian Spikenard, of each two drams, Cloves one dram, Species Hiera Picra simplex Galeni, twelve drams, with juice of Sinallage, make it into a mass according to art.

Culpeper.] It'wonderfully prevails against afflictions coming of melancholy, cancers which are not ulcerated, leprosy, evils of ? the mind coming of melancholy, as sadness, fear, \&c. quartan agues, jaundice, pains and infirmities of the spleen.

Pilule Lucis Majorcs. Mesue.

College.] Take of Roses, Violets, Wormwood, Colocynthis, Turbith, Cubebs, Calamus Aromaticus, Nutmegs, Indian Spike- art.

nard, Epithimum, Carpobalsamum, or Culpeper.] It is appropriated to such as instead thereof, Cardamoms, Xylabalsamum, have phthisicks, and such as spit blood, but or Wood of Aloes, the seeds of Seseli or ought to be newly made, a scruple is sufHartwort, Rue, Annis, Fennel and Small-ificient taken going to bed. 


\section{T R O C H E S.}

\section{Trochisci de Absinthio.}

Or.Troches of Wormwood.

College.] Take of red Ruses, Wormwood leaves, Annis seeds, of each two drams, juice of Maudlin made thick, the roots of Asarabacca, Rhubarb, Spikenard, Smallage seeds, bitter Almonds, Mastich, Mace, of cach one dram, juice of Succory so inuch as is sufficient to make it into troches according to art.

Culpeper.] They strengthen the stomach exceedingly, open obstructions, or stoppings of the belly and boweds: strengthen digestion, open the passages of the liver, help the yellow jaundice, and consume watery supertluities of the body. They arc somewhat bitter, and seldom taken alone; if your pallate affect bitter things, you may take a dram of them in the morning: 'They cleanse the body of choler, but purge not, or not to any purpose.

\section{Agaricus Trochiscatus.}

Or Agarick Trochiscated.

College.] 'Take of Agarick sifted and powdered, three ounces, steep it in a sufficient quantity of white Wine, in which? two drams of ginger have bern infused, and make it into troches.

\section{Trochisci Albi. Rhasis.}

Or white Troches.

College.] Take of Ceruss washed in Rosewater ten drams, Sarcocol three drams, white Starch two drams, Gum Arabic and Tragacanth, of each one dram, Camphire half a dram, either with Rosewater, or women's milk, or make it into troches according to art.

Trochisci Alexiterii.

College.] Take of Zedoary roots, powder of Crab's Claws, of each one dram, and an half, the outward Citron preserved and dried, Angelica seeds, Pills, of each one dram, Bole-amoniac half a dram, with their treble weight in sugar make them into powder, and with a sufficient quantity of Mussilage of Guin Tragacanth, made into treacle water distilled, make it into paste, of which make troches.

Culpeper.] This preserves the body from ill airs, and epidemical diseases, as the pestilence, small pox, \&c. and strengthens the heart exceedingly, eating now and then a little: you may safely keep any troches in your pocket, for the drier you keep tham, the better they are.

\section{Trochisci Alhandal.}

College.] Take of Coloquintida freed from the seeds and cut small, and rubbed with an ounce of oil of Roses, then beaten into fine powder, ten ounces, Gum Arabic, Tragacanth, Bdellium, of each six drams. Steep the Gums three or four days in a sufficient quantity of Rose-water till they be melted, then with the aforesaid pulp, and part of the said mussilage, let them be dried in the shadow, then beaten again, and with the rest of the mussilage, make it up again, dry them and keep them for use.

Culpeper.] They are too violent for a vulgar use.

\section{Trochisci Alipte Moschate.}

College.] Take of Labdanum bruised thrce ounces, Styrax Calamitis one ounce and an half, Benjamin one ounce, Wood of Aloes two drams, Ambergris one dram, Camphire half a dram, Musk half a scruple, with a sufficient quantity of Rose-water, make it into troches according to art.

Culpeper.] It is singularly good for such as are asthmatic, and can hardly fetch their breath; as also for young children, whose throat is so narrow that they can hardly swallow down their milk.

\section{'Trochisci Alkekengi.}

Or 'Iroches of Winter-cherries.

College.] 'Take of Winter Cherries three drams, Gum Arabic, 'Tragacanth, Olibanum, Dragon's-blood, Pine-nuts, bitter Almonds, white Styrax, juice of Liquorice, 
Bole-ammoniac, white Poppy seeds, of each half, Camphire half a dram ; with Mianna six drams, the seeds of Melons, Cucumbers, dissolved in juice of Barberries, make them Citruls, Gourds, of each three drams and an into troches according to art.

half, the seeds of Smallage and white Hen-

Culpeper.] 'They wonderfully' cool the bane, Amber, Earth of Lemnos, Opium, heat of the liver, reins, and bladder, breast, of each two drams, with juice of fresh and stomach, and stop looseness, cools the Winter-Cherries, make them into troches heat of fevers.

\section{according to art.}

Culpeper.] They potently provoke urine, and break the stone. Mix them with other medicine of that nature, half a dram at a time, or a dram if age permit.

Trochisci Bechici aloi, vel, Rotula pectorales. Or, Pectoral Rolls.

College.] 'Take of white Sugar one pound, white Sugar Candy, Penids, of each four ounces, Urris Florentine one ounce, Liquorice six drams, white Starch one ounce and an half, with a sufficient quantity of mussilage of Guin Tragacantl made in Rose Water, make them into small troches. You may add four grains of Ambergris, and three grains of Musk to them, if occasion serve.

\section{Trochisci Bechici nigri.}

College.] Take of juice of Licpuorice, white Sugar, of each one dram, Gum 'Tragacanth, sweet Almonds blanched, of each six drams, with a sufficient quantity of mussilage of Quince seeds, made thick with Rose Water. Make them into troches according to art.

Culpeper.] Both this and the former will melt in ones mouth, and in that mamer to be used by such as are troubled with coughs, cold, hoarseness, or want of voice. The former is most in use, but in my opinion, the latter is most effectual.

\section{Trochisce de Barberis.}

Or, Troches of Barberries.

College.] Take of juice of Barberries, and Liquorice made thick, Spodium, Purslain seeds, of each three drans, red Roses, six drams, Indian Spikenard, Saffron, white Starch, Gum Tragacanth, of each a dram, Citrul seeds cleansed three drams and an, $(35,36$.

\section{Trochisci de Camphora.}

Or, T'roches of Camphire.

College.] 'Take of Camphire half a dram, Saffron two drams, white Starch three drams, red Roses, Gum Arabic, and Tragacantl, Tvory, of each half an ounce, the seeds of Cucumbers husked, of Purslain, Liquorice, of each an ounce, with mussilage of the seeds of Fleawort, drawn in Rose-water, make them into troches.

Culpeper.] It is exceeding good in burning fevers, heat of blood and choler, together with hot distempers of the stomach and liver, and extreme thirst coming thereby, also it is good against the yellow jaundice, phthisics, and hectic fevers.

\section{Trochisci de Capparibus.}

Or, 'Troches of Capers.

College.] 'Take of the bark of Caper roots, the sceds of Agnus Castus, of each six drams, Ammoniacum half an ounce, the seeds of Water Cresses and Nigella, the leaves of Calaminth and Rue, the roots of Acorus and long Birthwort, the juice of Maudlin made thick, bitter Almonds, of each two drams, Hart's-tongue, the roots of round Cypress, Madder, Gum Lac. of each one dram : being bruised let them be made into troches according to art, with Ammoniacum dissolved in Vinegar, and boiled to the thickness of Honey.

Culpeper.] They open stoppings of the liver and spleen, and help diseases thereof coming; as rickets, hypochondriac melancholy, \&c. Men may take a dram, shildren a scruple in the morning.

Trochisci de Carabe.

Or, Troclies of Amber.

College.] 'Take of Amber an ounce, $4 \mathrm{U}$ 
Hart's-horn burnt, Gum Arabic burnt, in a mortar, add the powders, and with new red Coral burnt, Tragacanth, Acacia, Hypo- juice make it into troches.

cistis, Balaustines, Mastich, Gum Lacca Culpeper.] Obstructions, or stoppings, washed, black Poppy seeds roasted, of each and swelling above nature, both of the liver two drams and two scruples, Frankincense, and spleen, are cured by the inward taking Saffron, Opium, of each two drams, with a of these troches, and diseases thereof coming, sufficient quantity of mussilage of the seeds as yellow and black jaundice, the beginning of Fleawort drawn in Plantain Water, make them into troches according to art.

Culpeper.] They were invented to stop fluxes of blood in any part of the body, the menses, the hæmorrhoids or piles; they also help ulcers in the broast and lungs. The dose is from ten grains to a scruple.

\section{Trochisci Cypheos, for Mithridaie.}

College.] 'Take of pulp of Raisins of the Sun, Cypress, 'Turpentine, of each three ounces, Myrrh, Syuinanth, of each an ounce and an half, Cinnamon half an ounce, Calamus Aromaticus nine drams, the roots of round Cypress, and Indian Spikenard, Cassia Lignea, Juniper berries, Bdelliun, Aspalthus or Wood of Aloes, two drams and an half, Saffron one dram, clarified Honey as much as is sufficient, Canary Wine a little: let the Myrrh and Bdellium be ground in a mortar with the wine, to the thickness of liquid Honey, then add the Turpentine, then the pulp of Raisins, then the powders: at last with the Honey, let them all be made into troclies.

Culpeper.] It is excellently good against to art.

inward ulcers in what part of the body soever they be. It is chiefly used in compositions, as Treacle and Mithridate.

Trochisci de Eupatorio.

Or' Troches of Maudlin.

College.] Take of the juice of Maudlin made thick, Manna, of excil an ounce, red Roses half an ounce, Spodium three drams and an half, Spikenard three drams, Rhubarb, Asarabacca roots, Annis seeds, of each two drams. Let the Nard, Annis seeds, and Roses, be beaten together, the Spodium, Asarabacca, and Rhubarb by themselves, then mix the Manna and juice of Maudlin

Culpeper.] Thcy are held to be very good in ulcers of the bladder, and all other inward ulcers whatsoever, and ease fevers coming thereby, being of a fine cooling, slippery heating nature.

Trochisci Hedichroi, (Galen) for Treacle. College.] Take of Aspalthus, or yellow Sanders, the leaves of Mastich, the roots of Asarabacca, of each two drams, Rhupontic, Castus, Calamus Aromaticus, Wood of Aloes, Cinnamon, Squinantl, Opobalsamum or oil of Nutmegs by expression, of each three dranis, Cassia Lignea, Indian Leaf or Mace, Indian Spikenard, Myrrh, Saffron, 
of each six drams, Amomus, or Cardamoms Sagapen, Opopanax, of each two drams, the less, an ounce and an half, Mastich a dissolve the Gums in Wine wherein Mugdram, Canary Wine as much as is sufficient. wort hath been boiled, or else Juniper-berLet the Myrrh be dissolved in the wine, ries, then add the rest, and with juice of then add the Mastich and Saffron well Mugwort, make it into troches according beaten, then the Opobalsamum, then the to art.

rest in powder, and with the wine, make them up into troches, and dry them gently.

Culpeper.] They are very seldom or never used but in other compositions, yet of them beaten into powder, in a spoonful naturally they heat cold stomachs, help or two of Syrup of Mugwort, or any othes digestion, strengthen the heart and brain.

\section{Trochisci Hysterici.}

College.] Take of Asafoetida, Galbanum, of each two drams and an half, Myrrh two drams, Castoreunı a dram and an half, the roots of Asarabacca and long Birthwort, the leaves of Savin, Featherfew, Nep, of each one dram, Dittany half a dram, with either the juice or decoction of Rue, make it into troches according to art.

Culpeper.] These are applied to the forminine gender, help fits of the mother, expel both birth and after-birth, cleanse women after labour, and expel the relics of a careless mid wife.

\section{Trochisci de Ligno Aloes.}

Or Troches of Wood of Aloes.

College.] Take of Wood of Aloes, red Roses, of cacli two drams, Mastich, Cinnamon, Cloves, Indian Spikenard, Nutmegs,
Parsnip seed, Cardamoms the greater and lessen, Cubebs, Gallia Moschata, Citron Pills, Mace, of each one dram and an half, Ambergris, Musk, of each half a scruple, with Honey of Raisins make it into troches.

Culpeper.] It strengthens the heart, stomach, and liver, takes away heart-qualms,
faintings, and stinking breath, and resists the dropsy.

\section{Trochisci e Mirrha.}

Or Troches of Myrrh.

College.] Take of Myrrb three drams, the Meal of Lupines five drams, Madder roots, the leaves of Rue, wild Mints, Dittany of Crete, Cummin seeds, Asafotida, liver.

Culpeper.] They provoke the menses, and that with great ease to such as have them come down with pain. Take a dram composition tending to the same purpose. Sief de Plumbo.

Or Sief of Lead.

College.] Take of Lead burnt and washed, Brass burnt, Antimony, Tutty washed, Gum Arabic and Tragacanth of each an ounce, Opium half a dram, with Rose-water, make them, being beaten and sifted, into troches.

\section{Trochisci Polyide Androm.}

College.] Take of Pomegranate flowers twelve drams, Roach Album three drams, Frankincense, Myrrh, of each half an ounce, Chalcanthum two drams, Bull's gall six drams, Aloes an ounce, with austere Wine, or juice of Nightshade or Plantain, make them into troches according to art.

Culpeper.] They are very good they say, bcing outwardly applied, both in green wounds and ulcers. I fancy them not.

Trochisci de Rhubarbaro.

Or Troches of Rhubarb.

College.] Take of Rhubarb ten drams, juice of Maudlin madethick, bitter Almonds, of ,eacli half an ounce, red Roses three drams, the roots of Asarabacca, Madder, Indian Spikenard, the leaves of Wormwood, the seeds of Annis and Smallage, of each one dram, with Wine in which Wormwood hath been boiled, make them into troches according to art.

Culpeper.] 'They gently cleanse the liver, help the yellow jaundice, and other diseases coming of choler and stoppage of the 


\section{Trochisci de Santalis.}

Or 'Iroches of Sanders.
College.] Take of the three Sanders, of each one ounce, the seeds of Cucumbers, Gourds, Citruls, Purslain, Spodiun, of each half an ounce, red Roses seven drams, juice of Barberriessix drans, Bole-ammoniachalf an ounce, Camphire one dram, with Purslain Water make it into troches.

Culpeper.] 'The virtues are the same with troches of Spodium, both of them harmless.

Troclisci da Scilla ad Theriacam.

Or Troches of Squils, for 'T'reacle.

College.] T'ake a Squil gathered about the beginning of July, of a uriddle bigness, and the hard part to which the small roots stick, wrap it up in paste, and bake it in an oven, till the paste be dry, and the Squil tender, which you may know by piercing it with a wooden skewer, or a bodkin, then take it out and bruise it in a mortar, adding to every pound of the Squil, eight ounces of white Orobus, or red Cicers in powder, then make it into troches, of the weight of twu drams a piece, (yomr hands being anointed with Oil of Rosts) dry them on the top of the house, opening towards the South, in the shadow, often turning them till they be well dry, then keep them in a pewter or glass vessel.

\section{Troches of Spodium.}

College.] Take of red Roses twelve drams, Spodium ten drams, Sorrel seed six drams, the seeds of Purslain and Coriander, steeped in Vinegar and dried, pulp of Sumach, of each two drams and an half, white Starch roasted, Balaustines, Barberries, of each two dranns, Gum A rabic roasted one dram and an half, with juice of unripe Grapes, make it into troches.

Culpeper.] They are of a fine cooling binding nature, excellent in fevers coming of choler, especially if they be accompanied with a loosciess, they also quench thirst.

Trochisci de terra Lemnia.

Or Truches of Earth of Lemnos.
College.] Take of Earth of Lemnos, Bole-ainnioniac, Acacia, Hypocystis, Gum Arabic toasted, Dragon's blood, white Starch, red Roses, hose seeds, Lap. Hematitis, red Coral, Amber, Balaustines, Spodiun, Purslain seeds a little toasted, Olibanum, Hart's-horn burnt, Cypress Nuts, Saffron of each two drams, black Poppy seeds, Tragacanth, Pearls, of each one dram and an half, Opium prepared one dram, with juice of Plantain, make it into troches.

$$
\text { Sief de Thure. }
$$

Or Sief of Frankincense.

College.] Take of Frankincense, Lap. Calaminaris, Pompholix, of each ten drams, Cyrus forty drams, Gum Arabic, Opium, of each six drams, with fair water make it into balls: dry them and keep them for use.

Trochisci e Violis solutivi.

Or Troches of Violets solutive.

College.] Take of Violet flowers meanly dry, six drams, Turbith one ounce and an half, juice of Liquorice, Scammony, Manna, of each two drams, with Syrup of Violets, make it into troches.

Culpeper.] They are not worth talking of, much less worth cost, the cost and labour of making.

\section{Trochisci de Vipera ad Theriacum.}

Or 'Iroches of Vipers, for Treacle. College.] Take of the flesh of Vipers, the skin, entrails, head, fat, and tail being taken away, boiled in water with Dill, and a little salt, eight ounces, white bread twice baked, grated and sifted, two ounces, make it into troches, your hands being anointed with Opobalsamum, or Oil of Mutmegs by expression, dry them upon a sieve turned the bottom upwards in an open place, often turning them till they are well dried, then put tliem in a glass or stone pot glazed, stopped close, they will keep a year, yet is it far better to make Treacle, not long after you have made them.

Culpeper.] They expel poison, and arc 
excellently.good, by a certain sympathetical virtue, for such as are bitten by an adder.

Trochisci de Agno Casto.

Or Troches of Agnus Castus.

College.] 'Take of the seeds of Agnus Castus, Lettuce, red Rose flowers, Balaustins, of each a dram, Ivory, white Amber, Bole-ammoniac washed in Knotgrass Water two drams, Plantain seeds four scruples, Sassafras two scruples, with mussilage of Quince seeds, extracted in water of Waterlily flowers, let them be made into troches.

Culpeper.? Very prettv' troches and good for little

Trochisci Alexiterii. Renodrus.

College.] 'Take of the roots of Gentian, Tormentil, Orris Florentine, Zedoary, of each two drams, Cinnamon, Cloves, Mace, of each half a dram, Angelica roots three drams, Coriander seeds prepared, Roses, of each one dram, dried Citron pills two drams, beat thein all into powder, and with juice of Liquorice softened in Hippocras, six ounces, make them into soft paste, which you may form into either troches or small rolls, which you please.

Culpeper.] It preserves and strengthens the lieart exceedingly, helps faintings and failings of the vital spirits, resists poison and the pestilence, and is an excellent? medicine for such to carry about them whose occasions are to travel in pestilential places and corrupt air, only taking a very small quantity now and then.

Troches of Anris seed. Mesue.

College.] Take of Annis seeds, the juice of Maudlin made thick, of each two drams, the seeds of Dill, Spikenard, Mastich, Indian leuf or Mace, the leaves of Wormwood, Asarabacca, Smallage, bitter Almonds, of each half a dram, Aloes two drams, juice of Wormwood so much as is sufficient to make it into troches according to art.

Culpeper.] They open obstructions of ? the liver, and that very'gently; and therefore diseases coming thereof, help quartan agues. $(35,36$.
You can scarce do amiss in taking them if they please but your palate.

\section{Trochisci Diarhodon. Mesue.}

College.] Take of the flowers of red Roses six drams, Spikenard, Wood of Aloes, of each two diams, Liquorice three drams, Spodium one dram, Saffron half a dram, Mastich two drams, make them up into troches with white Wine according to art.

Culpeper.] They wonderfully ease fevers coming of flegm, as quotidian fevers, agues, epiatos, \&c. pains in the belly.

\section{Trochisci de Lacca. Mesue.}

College.] Take of Gum Lacca cleansed, the juice of Liquorice, Maudlin, Wormwood, and Barberries, all made thick, Rhubarb, long Birthwort, Costus, Asarabacca, bitter Almonds, Madder, Annis, Smallage, Schænanth, of each one dram, with the decoction of Birthwort, Schænanth, or the juice of Maudlin, or Wormwood, make them into troches according to art.

Cillpeper.] It helps stoppings of the liver and spleen, and ferers thence coming, it expels wind, purges by urine, and resists dropsies.

\section{Pastilli Adronis. Galen.}

College.] Take of Pomegranate flowers ten drams, Copperas twelve drams, unripe Galls, Birthwort, Frankincense, of each an ounce, Alum, Myrrh, of each half an ounce, Misy two drams, with eighteen ounces of austere Wine, make it into troches according to art.

Culpeper.] This also is appropriated to wounds, ulcers, and fistulas, it clears the ears, and represses all excressences of flesh, cleanses the filth of the bones.

Trochisci Musce. Galen.

College.] Take of Alum, Aloes, Copperas, Myrrh, of each six drams, Croconlagma, Saffron, of each three drams, Pomegranate Howers half an ounce, Wine and Honey, of each so much as is sufficient to make it up into troches according to art.

$4 \mathrm{x}$ 
Culpeper.] Their use is the same with the much as is sufficient to make it into troches former.

Crocomagma of Damocrates. Galen.

College.] Take of Saffron an hundred drams, red Roses, Myrrh. of each fifty drams, white Starch, Gum, of each thirty drams, Wine, so much as is sufficient to make it into troches.

Culpeper.] It is very expulsive, heats and strengthens the heart and stornach.

\section{Trochisci Ramich. Mesue.}

College.] Take of the juice of Sorrel sixteen ounces, red Rose Leaves, an ounce, Myrtle Berries two ounces, buil them a little together, and strain them, add to the decoction, Galls well beaten, three ounces, boil them again a little, then put in these following things, in fine powder: take of red Roses an ounce, yellow Sanders, ten drams, Gum Arabic an ounce and an half, Sumach, Spodium, of each an ounce, Myrtle berries four ounces, Wood of Aloes, Cloves, Mace, Nutmegs, of each half an ounce, sour Grapes sever. drams, mix them all together, and let them dry upon a stmne, and grind them again into powder, and make them into small troches with one dram of Camphire, and so much Rose Water as is suflicient, and pcrfume them with tifteen grains of Musk.

Culpeper.] They strengthen the stomach, heart, and liver, as also the bowels, they help the cholic, and fluxes of blood, as also bleeding at the nose if you snuff up the powder of them, disburden the body of salt, fretting, choleric humours. You may carry then about you, and take them at your pleasure.

\section{Troches of Roses. Mesuc.}

College.] Take of red Roses half an
ince, Wood of Aloes two drams, Mastich, ounce, Wood of Aloes two drams, Mastich, Cinnarnon, Indian Spikenard, Cassia Lignea, Schonanth, of each one dram, old Wine, and decoction of the five opening roots, so ?

according to art.

Culpeper.] They help pains in the stomach, and indigestion, the illiac passion, hectic fevers, and dropsies, in the beginning, and cause a good colour.

Trochisci Diacorallion. Galen.

College.] Take of Bole-ammoniac, red Coral, of each an ounce, Balaustines, Terra Lemnia, white Starch, of each half an ounce, Hypocistis, the seeds of Henbane, Opium, of eacli two drams, juice of Plantain so much as is sufficient to make them into troches according to art.

Culpeper.] These also stop blood, help the bloody flux, stop the menses, and are a great help to such whose stomachs loath their victuals. I fancy them not.

Trochisci Diaspermaton. Galen.

College.] Take of the seeds of Smallage, and Bishop's weed, of each an ounce, Annis and Fennel seeds, of each half an ounce, Opium, Cassia Lignea, of each two drams, with rain water, make it into troches according to art.

Culpeper.] These also bind, ease pain, help the pleurisy.

Hremoptoici Pastilli. Galen.

College.] T'ake of white Starch, Balaustines, Earth of Samos, juice of Hypocystis, Gum, Saffron, Opium, of each two drasns, with juice of Plantain, make them into troches according to art.

Culpeper.] The operation of this is like the former.

\section{Troches of Agarick.}

College.] Take of choice Agarick three ounces, Sal. Gem. six drams, Ginger twu drams, with Oxymel simplex, so much as is sufficient, make it into troches according to art. 


\section{O I I S.}

\section{SIMPLE OILS BY EXPRESSION.}

\section{Oil of Sreet Almonds.}

College.] Take of Sweet Almonds not corrupled, as many as you will, cast the shells away, and blanch them, beat them in a stone mortar, beat them in a double vessel, and press out the oil without heat.

Culpeper.] It helps roughness and soreness of the throat and stomach, helps pleurisies, encreases seed, eases coughs and hectic fevers, by injection it helps such whose water scalds them; ulcers in the bladder, reins, and inatrix. You may either take half an ounce of it by itself, or mix it with half an ounce of Syrup of Violets, and so take a spoonful at a time, still shaking them together when you take them: only take notice of this, if you take it inwardly, let it be new drawn, for it will be sour in three or four days.

\section{Oil of bitter Almonds.}

College.] It is made like Oil of sweet Almonds, but that you need not blanch them, nor have such a care of heat in pressing out the oil.

Culpeper.] It opens stoppings, helps such as are deaf, being dropped into their ears, it helps the hardness of the nerves, and takes away spots in the face. It is seldon. or never taken inwardly.

\section{Oil of Ḧazel Nuts.}

College.] It is made of the Kernels, cleansed, bruised, and beat, and pressed like Oil of sweet Almonds.

Culpeper.] You must put them in a vessel (viz. a glass, or some such thing) and stop them close that the water come not to them when you put them into the bath. The oil is good for cold afflictions of the nerves, the gout in the joints, \&c.
College.] So is Oil of Been, Oil of Nutmegs, and Oil of Mace drawn.

\section{Oleum Caryinum.}

College.] Is prepared of Walnut Kernels, in like manner, save only that in the making of this sometimes is required dried, old, and rank Nuts.

\section{Oleum Chrysomelinum,}

College.] Is prepared in the same manner of $A$ pricots, so is also Oils of the Kernels of Cherry stones, Peaches, Pine-nuts, Fistic Nuts, Prunes, the seeds of Oranges, Hemp, Bastard Saffron, Citrons, Cucumbers, Gourds, Citruls, Dwarf Elder, Henbane, Lettuce, Flax, Melons, Poppy, Parsley, Radishes, Rape, Ricinum, Sesani, Mustard seed, and Grape stones.

Culpeper] Because most of these Oils are out of use, I took not the pains to quote the virtues of them; if any wish to make them, let them look to the simples, and there they have them; if the simples be not to be found in this book, there are other plentiful medicines conducing to the cure of all usual diseases; which are-

\section{Oil of Bays.}

College.] Take of Bay-berries, fresh and ripe, so many as you please, bruise them sufficiently, then boil them in a sufficient quantity of water till the Oil swim at top, which separate from the water, and keep for your use.

Culpeper.] It helps the cholic, and is a sovereign remedy for any diseases in any part of the body coming either of wind or cold.

College.] Common Oil of Olives, is pressed out of ripe olives, not out of the stones. Oil of Olives omphacine, is pressed out of unripe olives.

\section{Oil of Yolks of Eggs.}

College.] Boil the yolks till they be hard, and bruise them with your hand or with a pestle and mortar; beat them in an earthen vessel glazed until they begin to froth, stirring them diligently that they burn not, 
being hot, put them in a linen bag, and away, cut, bruised, and the vessel covered sprinkle them with Aromatic Wine, and press out the oil according to art.

Culpeper.] It is profitable in fistulas, , and malignant ulcers, it causes the hair to grow, it clears the skin, and takes away deformities thereof, viz. tetters, ringworms, morphew, scabs.

\section{SIMPLE OILS BY INFUSION AND DECOCTION.}

\section{Oil of Rose's omphacine.}

College.] Take of red Roses before they be ripe, bruised in a stone mortar, four ounces, oil Omphacine one pound, set them in a hot sun, in a glass close stopped, a whole week, shaking them every day, then boil them gently in a bath, press them out, and put in others, use thern in like manner, do so a third time: then keep the Oil upon a pound of juice of Roses.

\section{Oil of Roses complete,}

Is made in the same manner, with sweet and ripe oil, often washed, and red Roses fully open, bruised, set in the sun, and boiled gently in a double vessel, only let the third infusion stand in the sun forty days, then keep the roses and oil together.

In the same manner is made Oil of Wormwood, of the tops of common Wormwood thrice repeated, four ounces, and three pounds of ripe oil; only, the sast time put in four ounces of the juice of Wornwood, which evaporale away by gentle boiling.

Dil of Dill: Of the flowers and leaves of Dill four nunces, complete oil, one pound, thrice repeated.

Oil of Castoreum : Of one ounce of Castoreun oil one pound, Wine four ounces, which must be consumed with the heat of a bath.

Oil of Chamomel (which more than one call Holy) of cumplete oil, and fresh Chamomel flowers, the little white leaves taken

with a thin linen cloth, set in the sun, pressed out, and three times repeated.

Oil of Wall-flowers, as oil of Dill.

Oil of Quinces: Of six parts of oil Omphacine, the meat and juice of Quinces one part, set them in the sun fifteen days in a glass, and afterwards boil them four hours in a double vessel, press them out, and renew them three times.

Oil of Elecampane: Of ripe oil, and the roots of Elecampane bruised, and their juice, of each one part, and of generous Wine half a part, which is to be evaporated away.

Oil of Euphorbium: Of six drams of Euphorbium, Oil of Wall-flowers, and sweet Wine, of each five ounces, boiling it in a double vessel till the Wine be consumed.

Oil of Ants: Of winged Ants infused in four times their weight of sweet oil, set in the sun in a glass forty days, and then strain it out.

Oil, or Balsam of St. John's Wort simple, is made of the oil of seeds beaten and pressed, and the flowers being added, and rightly set in the sun:

Oil of Jesmine, is made of the flowers of Jesmine, put in clear oil, and set in the sun and afterwards pressed out.

Oil of Orris, made of the roots of Orris Florentine one pound, purple Orris flowers half a pound : boil them in a double vessel in a sufficient quantity of decoction of Orris Florentine, and six pounds of sweet oil, puting fresh roots and flowers again and again; the former being cast away as in oil of Roses.

Oil of Earthworms, is made of half a pound of Earthworms washed in white Wine, ripe Oil two pounds, boiled in a clouble vessel with eight ounces of good white Wine till the Wine be consunied.

Oil of Marjoram is made with four ounces of the herb a little bruised, white Wine six ounces, ripe oil a pound, mixed 
together, let them be set in the sun repeated them be set in the sun, and after forty days three times; at last boiled to the consump- strained.

\section{tion of the Wine.}

Oil of Mastich, is made of oil of Roses Wine four ounces: boil them in a do
vessel to the consumption of the Wine.

Oil of Melilot is made with the tops of the herb like oil of Chamomel.

Oil of Mints is made of the herb and oil omphacine, as oil of Roses.

Oil of Mirtles, is made of Mirtle berries bruised and sprinkled with sharp Wine one part, oil omphacine three parts; set it in the sun twenty-four days, and in the interim thrice renewed, boiled, and the berries pressed out.

Oil of Daffodils is made as oil of Roses.

Nard Oil is made of three ounces of Spikenard, sweet oil one pound and an half, sweet white Wine and clear water, of each two ounces and an half, boiled to the consumption of the moisture.

Oil of Water-lilies, is made of fresh white Water-lily flowers, one part, oil omphacine three parts, repeating the flowers as in oil of Roses.

Oil of Tobacco is made of the juice of Tobacco, and common oil, of each equal? parts boiled in a bath.

Oil of Poppies, is made of the flowers, heads, and leaves of garden Poppies, and vil omphacine, as oil of Dill.

Oil of Poplars, is made of the buds of the Poplar tree three parts, rich white Wine four parts, sweet oil seven parts ; first let the buds be bruised, then infused in the Wine and oil seven days, then boiled, then pressed out.

Oil of Rue, is made of the herb bruised, and ripe oil, like oil of Roses.

Oil of Savin is made in the same manner.

So also is Oil of Elder flowers made.

Oil of Scorpions, is made of thirty live Scorpions, caught when the sun is in the and consolidate wounds, especially in the lion; oil of bitter Almonds two pounds, let head.

$(35,36$.
Oleum Cicyonium, is made of wild Cucumber roots, and their juice, of each equal parts; with twice as much ripe oil, boil it to the consumption of the juice.

Oil of Nightshade, is made of the berries of Nightshade ripe, and one part boiled in ripe oil, or oil of Roses three parts.

Oil of Styrax, is made of Styrax and sweet white Wine, of each one part, ripe oil four parts gently boiled till the Wine be consumed.

Oil of Violets, is made of oil omphacine, and Violet flowers, as oil of Roses.

Oil of Vervain, is made of the herb and oil, as oil of Mints

Culpeper.] That most of these Oils, if not all of them, are used only externally, is certain; and as certain that they retain the virtues of the simples whereof they are made, therefore the ingenious might help themselves.

\section{COMPOUND OILS BY INFUSION AND DECOC'IION.}

\section{Oleum Benedictum. Or Blessed Oil.}

College.] Take of the roots of Carduus and Valerian, of each one ounce, the flowers of St. John's Wort two ounces, Wheat one ounce and an half, old Oil four ounces, Cypress Turpentine eight ounces, Frankincense in powder two ounces, infuse the roots and flower's, being bruised, in so much white Vine as is sufficient to cover them, after two days' infusion put in the Oil with the Wheat, bruised, boil them together till the Wine be consumed ; then press it out, and add the Frankincense and 'Turpentine, then boil them a little, and keep it.

Cuipeper. $\mathrm{It}$ is appropriated to cleance 4 . 
Oleum de Capparibus.

Or, Oil of Capers.

College.] Take of the bark of Caper roots an ounce, bark of 'Tamarisk, the leaves of the same, the seeds of Agnus Castus, Cetrach, or Spleenwort, Cypress roots, of besides these, it strengthens the liver, it
each two dranis, Rue one dram, oil of ripe keeps the hairs from turning grey, and gives Olives one pound, white Wine Vinegar, and a good colour to the body. I pray you white Wine, of each two ounces, cut them take notice that this and the following oils, and steep them, and boil them (two days (till I give you warning to the contrary) being elapsed) gently in a bath, then the are not made to eat.

Wine and Vinegar being consumed, strain it, and keep il.

Culpeper.] The oil is opening, and heat-? ing, absolutely appropriated to the spleen, hardness and pains thereof, and diseases coming of stoppings there, as hypocondriac melancholy, the rickets, \&c.

Oil of Castoreum compound.

College.] Take of Castcreum, Styrax Calamitis, Galbanum, Euphorbium, Opopanax, Cassia Lignea, Saffron, Carpobalsamum or Cubebs, Spikenard, Costus, of each two drams, Cypress, S'fuinanth, Pepperlong and black, Savin, Pellitory of Spain, of each two drams and an half, ripe Oil four pounds, Spanish Wine two pounds, the five first excepted, let the rest be prepared as they ought to be, and gently boiled in the Oil and Wine, until the Wine be consumed, mean time the Galbanum, Opopanax, and Euphorbium beaten in fine powder, being dissolved in part of the Wine, and strained, let them be exquisitely mixed with it (while the oil is warm) by often stirring; the boiling being finished, put in the Styrax and Castoreum.

Culpeper.] The virtues are the same with the simple.

\section{Oleum Castinum.}

College.] Take of the roots of bitter Castus two ounces, Cassia Lignea one ounce, the tops of Marjoram eight ounces, being bruised, steep them two days in twelve? ounces of sweet white Wine; then with hree pounds os sallad oil washed in white
Wine, boil it in Balneo Maria till the Wine

Culpeper.] It heats, opens obstructions, strengthens the nerves, and all nervous parts, as muscles, tendons, ligaments, the ventricle; besides these, it strengthens the liver, it Oleum Crocinum, Or, Oil of Saffron.

College.] Tiake of Saffron, Calamus Aromaticus, of each one ounce, Myrrh, half an ounce, Cardamoms nine drams, steep them six days, (the Cardarnoms excepted, which are not to be put in till the last day, in nine ounces of Vinegar, the day after put in a pound and an half of washed oil, boil it gently according to art, till the Vinegar, be consumed, then strain it.

Culpeper.] It helps pains in the nerves, and strengthens them, mollifies their hardness, helps pains in the matrix, and causes a good colour.

\section{Oil of Euphorbium.}

College.] 'Take of Stavesacre, Sopewort, of each half an ounce, Pellitory of Spain six drams, dried Mountain Calamint one ounce and an half, Castus two drams, Castoreum five drains, being bruised, let them be three days steeped in three pounds and an half of Wine, boil thern with a pound and an half of Oil of Wall-flowers, adding half an ounce of Euphorbium, before the Wine be quite consumed, and so boil it according to art.

Culpeper.] It hath the same virtue, only something more effectual than the simple.
Oleum Excestrense,
Or, Oil of Exeter.

College.] Take of the leaves of Wormwood, Centaury the less, Eupatorium, Fennel, Hysscp, Bays, Marjoram, Bawm, Nep,
Pennyroyal, Savin, Sage, Thyme, of each be consumed. 
four ounces, Southernwood, Betony, Chamepitys, Lavender, of cach six ounces, Rosemary one pound, the flowers of Chamomel, Broom, white Lilies, Elders, the seeds of Cunmin, and Fenugreek, the roots of Hellebore black and white, the bark of Ash and Lemons, of each four uunces, Euphorbium, Mustard, Castoreum, Pellitory of Spain, of each an ounce, Oil sixteen pounds, Wine three pounds, the herbs, flowers, seeds, anc Euphorbium being bruised, the roots, barks, and Castoreum cut, all of them infused twelve hours in the Wine and Oil, in a warm bath, then boiled with a gentle fire, to the consurnption of the Wine and moisture, strain the Oil and keep it.

Culpeper.] Many people by catching nruises when they are young, come to feel it when they are old: others by catching cold, catch a lameness in their limbs, to botli which I commend this sovereign oil to bathe their grieved members with.

\section{Oleum Hirundinum,}

Or, Oil of Swallows.

College.] Take of whole Swallows sixteen, Chamomel, Rue, Plantain the greater and lesser, Bay leaves, Pennyroyal, Dill, Hyssop, Rosemary, Sage, Saint John's Wort, Costmary, of each one handful, common Oil four pounds, Spanish Wine one pound, make it up according to art.

Culpeper.] Both this and the former are appropriated to old bruises and pains thereof coming, as also to sprains.

\section{Oleum Hyperici compositum.}

Or, Oil of St. John's Wort compound.

Culiege.] Take of the tops of St. John's Wort four ounces, steep them three whole days in a pound of old Sallad Oil, in the heat either of a bath, or of the sun, then press them out, repeat the infusion the second or third time, then boil them till the wine be almost consumed, press them out, and by adding three ounces of Turpentine, and one scruple of Saffron, boil it a little and keep it.
Culpeper.] See the simple oil of $\mathrm{St}$. John's Wort, than which this is stronger.

Oleum Hyperici magis compositur.

Or, Oil of St. John's Wort more compound. College.] Take of white Wine three pounds, tops of St. Joln's Wort ripe and gently bruised, four handfuls, steep them two days in a glass, close stopped, boil them in a bath, and strain them strongly, repeat the infusion three times, having strained it the third time, add to every pound of decoction, old Oil four pounds, T'urpentine six ounces, oil of Wornwood three ounces, Dittany, Gentian, Carduus, Tormentil, Carline, or Cordus Maria, Calamus Aromaticus, all of them bruised, of each two drams, Earth-worms often washed in white Wine two ounces, set it in the sun five or six weeks, then keep it close stopped.

Culpeper.] Besides the virtue of the simple oil of St. Jolnn's Wort, which this performs more effectually, it is an excellent remedy for old bruises, aches, and sprains.

\section{Oleum Irinum, \\ Or, Oil of Orris.}

College.] Take of the roots of Orris Florentine, three pounds four ounces, the flowers of purple Orris fifteen ounces, Cypress roots six ounces, of Elecampane three ounces, of Alkanet two ounces, Cinnanıon, Spikenard, Benjamin, of each one ounce: let all of them, being bruised as they ought to be, be steeped in the sun, or other hot place, in fifteen pounds of old oil, and four pounds and an half of clear water, after the fourth day, boil them in Balneo Marix, the water being consumed, when it is cold, strain it and keep it.

Culpeper.] The effects are the same with the simple, only'tis stronger.

\section{Oleum Marjorana.}

Or, Oil of Marjor in.

College.] Take of Marjoram four handfuls, Mother of Thyme two handfuls, the leaves and berries of Myrtles one handful, Southernwood, Water Mints, of each half 
an handful, being cut, bruised, and put in a Cardarnonis, of each one ounce and an half, glass, three pounds of Oil Omphacine being bruise them all grossly, and steep them in put to it, let it stand eight days in the sun, water and wine, of each fourteen ounces, or in a bath, close stopped, then strain it Oil of Sesamin, or oil of Olives, four pounds out, in the oil putin fresh simples, do so the and an half, for one day: then perfect the third time, the oil may be perfected accord- oil by boiling it gently in a double vessel. ing to art.

Culpeper.] It helps weariness and disOleum Populeum. Nicholaus.

College.] 'Take of fiesh Poplar buds eases of the brain and nerves, coming of three pounds, Wine four pounds, common cold ; it helps the dead palsy, the back (viz: the region along the back bone) being anointed with it; being snuffed up in the nose, it helps Spasmus cymicus, which is a wrying the mouth aside; it helps noise in the ears being dropped into them, it provokes the menses, and helps the biting of venomous beasts; it is a:most gallant oil to strengthen the body, the back being anointed with it; strengthens the museles, they being chafer with it; helps head-ache, the forehead being rubbed with it.

\section{Moschelcum,}

Or, Oil of Musk.

College.] Take two Nutmegs, Musk one dram, Indian leaf or Mace, Spikenard, Costus, Mastich, of each six drams, Styrax Calanitis, Cassia Lignea, Myrrh, Saffron, Cinnamon, Cloves, Carpobalsanum, or Cubebs, Bdellium, of each two drams, pure Oil three pounds, Wine three ounces, bruise them as you ought to do, mix them and let them boil easily, till the Wine be consumed, the ,Musk being mixed according to art after it is strained.

Culpeper.] It is exceeding good against all diseases of cold, especially those of the stomach, it helps diseases of the sides, they being anointed with it, the stranguary, cholic, and vices of the nerves, and afflictions of the reins.

\section{Oleum Nardinum,}

Or, Oil of Nard.

College.] Take of Spikenard three ounces, Marjoram two ounces, Wood of Aloes; Calamus Aromaticus, Elecampane, Cypress, Bay leares, Indian leaf or Mace, Squinanth, takes away dead and proud flesh, and dries.

\section{OINTMENTS MORE SIMPLE.}

\section{Unguentum album, Or, white Ointment.}

College.] Take of Oil of Roses nine ounces, Ceruss washed in Rose-water and diligently sifted, three ounces, white Wax two ounces, after the wax is melted in the oil, put in the Ceruss, and make it into an ointment according to art, add two drams of Camphire, made into powder with, a few drops of oil of sweet Almonds, so will it be camphorated.

Culpeper.] It is a fine cooling, drying ointment, eases pains, and jtching in wounds and ulcers, and is an hundred times better with Camphire than without it.

\section{Vnguentum Egyptiacum.}

College.] Take of Verdigris finely powdered, five parts, Honey fourteen parts, sharp Vinegar seven parts, boil thein to a just thickness, and a reddish colour.

Cuipeper.] It cleanses filthy ulcers and fistulas forcibly, and not without pain, it 


\section{Unguentum Anodymum.}

Or, an Ointment to easc pain.

Callege.] Take of Oil of white Lilies, six ounces, Oil of Dill, and Chamomel, of each two ounces, Oil of sweet Almonds one ounce, Duck's grease, and Hen's grcase, of each two ounces, white Wax threc ounces, mix them according to art.

Culpeper.] Its use is to assuage pains in any part of the body, especially such as come by inflammations, whether in wounds or tumours, and for that it is admirable.

\section{Uneucutum ex Apio.}

\section{Or, Ointment of Sinallage.}

College.] 'T'ake of the juice of Smallage one pound, Honey nine ounces, Wheat flower thrce ounces, boil them to a just thickiress.

Culpeper.] It is a very fine, and rery gentle clcanser of wounds and ulcers.

\section{Liniment of Gum Eitemi.}

College.] Take of Gum Llemi, Turipentinc of the Fir-tree, of each one ounce and an half, old Sheep's Suct gleamed two ounces, old Hog's grease cleansed one ounce: mix them, and make them into an ointmert according to art.

Culpeper.] It gently cleanses and fills up an ulcer with flesh, it being of a mild nature, and friendly to the body.

\section{Unguentum Aureum.}

College.] Take of ycllow IVax half a pound, common Oil two pounds, Turpentine two ounces, Pine Rozin, Colophonia, of each onc ounce and an half, Frankincense, Mastich, of each one ounce, Saffron one dram, first melt the wax in the vil, then the Turpentine being added, let them boil together; having done boiling, put in the rest in fine powder, (let the Saftron be the last) and by diligent stiring, make them into an ointment according to art.

\section{Inasilicon, the greater.}

College.] Take of white Wax, Pines secels stceped, and boiled in eight pounds of Rozin, Heifcr's Suct, Greek Pitch, 'Tur-spring water, and then pressed out. 'See pentine, Olibanum, Myrrh, of each one the compound.

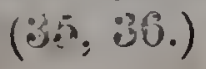

ounce, Oil five ounces, powder the Olibanum and Myrrh, and the rest being melted, make it into an ointment according to art. Basilicon, the less.

College.] Takc of yellow Wax, fat Rozin, Greck Pitch, of each half a pound, Oil nine ounces: mix them together, by melting them according to art.

Culpeper.] Both this and the former, heat, moisten, and digest, procure matter in wounds, I mean brings the filth or corrupted blood from green wounds: thcy clense and ease pain.

College.] Take of Bdelliun six drams, Euphorbium, Sagapen, of each four drams, Castoreum three drams, Wax fifteen drans, Oil of Filder or 'Wall-Howers, ten dranis, the Bdellium, and Sagapen being dissolved in water of wild Rue, lel the rest be united by the heat of a bath.

Unguentum de Calce.

Or, Ointment of Chalk.

College.] 'Take of Chalk washed, seven times at least, half a pound, Wax three ources, Oil of Roses one pound, stir them all together diligently in a leaden mortar, the wax being frest melted by a gentle fire in a sufficient quantity of the prescribed oil.

Culpeper.] It is exceeding good in burnings and scaldings.

\section{Unguentum Dialthe.}

Or, Ointment of Marsh-mallows.

Callege.]. Take of common Oil four pounds, mussilage of Marsh-nallow roots, Linsced, and Fenugreck seed two pounds: boil them together till the watry part of the mussilage be consumed, then add Wax half a pound, Rozin three ounces, sistence of an ointment, but let the mussilage be prepared of a pound of fresh roots bruised, and half a pound of 'each of the 'I'urpentine an ounce, boil them to the con- 
Unguentum Diapompholygos.

College.] Take of Oil of Nightshade sixteen ounces, white Wax, washed, Ceruss, of each four drams, Lead burnt and washed, Pompholix prepared, of each two ounces, pure Frankincense one ounce : bring them into the form of an ointment according to art.

Culpeper.] This much differing from the former, you shall have that inserted at latter end, and then you may use which you please.

\section{Ungiventum Enulatum.}

Or, Ointment of Elecampane.

College.] Take of Elecampane ruots boiled in Vinegar, bruised and pulped, one pound, Turpentine washed in their decoction, new Wax, of each two ounces, old Hog's grease salted ten ounces, old oil four ounces, common salt one ounce, add the Turpentine to the grease, wax, and oil, being melted, as also the pulp and salt being finely powdered, and so make it into an ointment according to art.

Unguentum Enulatum cum Mercurio. Or, Ointment of Elecampane with Quicksilver,

College.] Is made of the former oint- art ment, by adding two ounces of Quick-silver, killed by coutinual stirring, not only with spittle, or juice of Lemons, but with all the Turpentine kept for that intent, and part of the grease, in a stone mortar.

Culpeper.] My opinion of this ointment, is (brietly) this: It was invented for the itch, without quick-silver it will do no good, with quick-silver it may do harm.

Unguentum Laurinum commune.

Or, Ointment of Bays common.

Or, Ointment of Bays common. Crllege.] Take of Litharge of Gold
College.] Take of Bay leaves bruised finely powdered, half a pound, Vinegar College.] Take of Bay leaves bruised finely powdered, half a pound, Vinegar
one pound, Bay berries bruised half a one pound, Oil of Roses two pounds, grind pound, Cabbage leaves four ounces, Neat's- the Litharge in a mortar, pouring to it foot Oil five pounds, Bullock's suet two sometimes Oil, sumetimes Vinegar, till hy pounds, boil them togeller, and strain then, continual stiring, the Vinegar do no more that so it may be made into an ointment appear, and it come to a whitish ointment. according to art.

Unguentum de minio sive mbrum Camphora. Or, Ointment of red Lead.

College.] Take of Oil of Roses one pound and an half, red Lead three ounces, Litharge two ounces, Ceruss one ounce and an lialf, Tutty three drams, Camphire two drams, Wax one ounce and an half, make it into an ointment according to art, in a pestle and niortar inade of Lead.

Culpeper.] 'This ointment is as drying as a inan shall usually read of one, and withal cooling, therefore good for sores, and such as are troubled with defluctions.

Unguentum e Nicotiona, seu Peto.

Or, Ointment of Tobacco.

College.] Take of Tobacco leaves bruised, two pounds, steep them a whole night in red Wine, in the morning boil, it in fresh Hog's grease, diligently washed, one pound, till the Wine be consunied, strain it, and add half a pound of juice of Tobacco, Rozin four ounces, boil it to the corsumptiol. of the juice, adding towards the end, round Birthwort roots in powder, two ounces, new Wax as much as is sufficient to make it into an ointment according to Culpeper.] It would takea whole summer's day to write the particular virtues of this ointment, and my poor Genius is too weak to give it the hundredth. part of its due praise: It cures tumours, imposthumes, wounds, ulcers, 'guu-shot, stinging with nettles, bees, wasps, hornets, venomous \&c.

Unguentum Nutriturn, seu Trifarmacum. 
nature, good for itching of wounds, and hot and keep it for use ; then warm it a such like deformities of the skin.

\section{Unguentum Ophthalmicum.}

Or, An Ointment for the Eyes.

College.] Take of Bole-ainmuniac washed in Rose water, one ounce, Lapis Calaminaris washed in Eye-bright Water, Tutty prepared, of each two drams, Pearls in very fine powder half a dram, Camphire half a scruple, Opium five grains, fresl Butter washed in Plantain Water, as much as is sufficient to make it into an ointment according to art.

Culpeper.J It is exceeding good to stop hot rheums that fall down into the eyes, the eyelids being but anointed with it.

Unguentum ex Oxylapatho.

Or, Ointment of sharp-pointed Dock.

Collegre.] 'Take of the roots of sharppointed Dock boiled in Vinegar until they be soft, and then pulped, Brimstone washed in juice of Lemons, of each one ounce and an 'half, Hog's grease often washed in juicc of Scabious, half a pound, Unguentum Populeon washed in juice of Elecampane, half an ounce: make them into an ointment in a mortar.

Culpeper.] It is a wholesome, though troublesome medicine for scabs and itch.

Unguentum e Plumbo.

Or, Ointinent of Lead.

College.] Take of Lead burnt according to art, Litharge, of each two ounces, Ceruss, Antimony, of each one ounce, Oil of Roses as much as is sufficient: make i into an ointment according to art. Culpeper.] Take it one time with another, heat, as also the
it will go neer to to more harm than good. stoniach and liver.

\section{Uuguentum Pomatum.}

College.] Take of fresh Hog's grease three pounds, fresl Sheep's suet nine our.ces, Pomewater pared and cut, one pound and three pounds, fresh Sheep's suet nine our.ces, phaciue a pound, white Wax five ounces,
Pomewater pared and cut, one pound and phe punces, Damask Rose-water six ounces, which being melted and put in a leaden
nine ounces, the roots of Orris Florentine grossly bruised : mortar, put in the Earth of Lemnos or six drams, boil them in Balneo Marice till Bole-ammoniac, Lapis Calaminaris, of each the Apples be soft, then strain it, but press four ounces, Litharge of Gold, Ceruss, of water, adding to each pound twelve drops of oil of Lignum Rhodium.

Culpeper.] Its general use is, to soften and supple the roughness of the skin, and take away the chops of the lips, hands, face, or other parts.

\section{Unguentum Potabile.}

College.] Take of Butter without salt, a pound and an half, Spermaceti, Madder Tormentil roots, Castoreum, of each half an ounce: boil them as you ought in a sufficient yuantity of Wine, till the Wine be consumed, and become an ointment. of it.

Culpeper.] I know not what to make

\section{Unguentum Resinum.}

College.] Take of Pine Rozin, or Rozin of the Pine-tree, of the purest Turpentine, ycllow Wax washed, pure Oil, of each equal parts : melt them into an ointment ulpeper.] It is as pretty a Cerecioth for a new $s_{2}$,rain as most is, and cheap.

\section{Unguentum Rosatum.}

Or, Ointment of Roses.

College.] 'Take of fresh Hog's grease cleansed a pound, fresh red Roses half a pound, juice of the same three ounces, make it into an ointment according to art.

Culpeper.] It is of a fine cooling nature, exceeding useful in all gallings of the skin, and frettings, accompanied with choleric humours, angry pushes, tetters, ringworms, it mitigates diseases in the head coming of heat, as also the intemperate heat of the Desiccativum Rubrum.

Or, a drying Red Ointment.

College.] Take of the oil of Roses om-

little again and wash it with fresh Rose- 
each three ounces, Camphire one dram, make it into an ointment according to art.

Culpeper.] It binds and restrains fluxes of humours.

\section{Unguentum e Solano.}

Or, Ointment of Nightshade.

College.] Take of juice of Nightshade, Litharge washed, of each five ounces, Ceruss washed eight ounces, white Wax seven ounces, Frankincense in powder ten drams, oil of Roses often washed in water two pounds, make it into an ointment according to art.

Culpeper.] It was invented to take away inflammations from wounds, and to keep people from scratching of them when they are almost well.

\section{Or, Ointment of Tutty.}

College.] Take of Tutty prepared two ounces, Lapis Calaminaris often burnt and quenched in Plantain Water an ounce, make them, being finely powdered, into an ointment, with a pound and an half of ointment of Roses.

Culpeper.] It is a cooling, drying ointment, appropriated to the eyes, to dry up hot and salt humours that flow down thither, the eyelids being anointed with it.

\section{Valentia Scabiose.}

College.] Take of the juice of green Scabious, pressed out with a screw, and strained through a cloth, Hog's grease, of each as much as you will, heat the Hog's grease in a stone mortar, not grind it, putting in the juice by degrees for the more commodious mixture and tincture, afterwards set it in the sun in a convenient vessel, su as the juice may overtop the grease, nine days being passed, pour off the discoloured juice, and beat it again as before, putting in fresh juice, set it jn the sun again five days, which being elapsed, beat it again, put in more juice, after fifteen days more, do so again, do so five times, after which, keep it in a glass, or glazed vessel.

\section{Tapsivalentia.}

College.] Take of the juice of Mullen, Hog's grease, of each as much as you will, let the grease be cleansed and cut in pieces, and beat it with the juice, pressed and strained as you did the former ointment, then keep it in a convenient vessel nine or ten days, then beat it twice, once with fresh juice, until it be green, and the second time without juice beaten well, pouring off what is discoloured, and keep it for use.

\section{Tapsimel.}

College.] Take of the juice of Celandine and Mullen, of each one part, clarified Honey, two parts, boil them by degrees till the juice be consumed, adding (the physician prescribing) Vitriol, burnt Alum, burnt Ink, and boil it again to an ointment according to art.

\section{OINTMENTS MORE COMPOUND.}

\section{Unguentum Agrippa.}

College.] Take of Briony roots two pounds, the roots of wild Cucumbers one pound, Squills half a pound, fresh English Orris roots, three ounces, the roots of male Fern, dwarf Elder, water Caltrops, or Aaron, of each two ounces, bruise them all, being fresh, and steep them six or seven days in four pounds of old oil, the whitest, not rank, then boil them and press them out, and in the oil melt fifteen ounces of white Wax, and make it into an ointment according to art.

Culpeper.] It purges exceedingly, and is good to anoint the bellies of such as have dropsies, and if there be any humour of flegm in any part of the body that you know not how to remove (provided the part be not too tender) you may anoint it with this; but yet be not too busy with it, for I tell you plainly it is not very safe.

Unguentum Amarum.

Or, A bitter Ointment. 
College.] Take of Oil of Rue, Savin, Mints, Wormwood, bitter Almonds, of each one ounce and an half, juice of Peach flowers and leaves, and Wormwood, of each Camphire a dram, white Coral half an half an ounce, powder of Rue, Mints, ounce, Alum Plume an ounce, Unibilicus Centaury the less, Gentian, Tormentil, of Marinus, Tragacanth, white Starch, of each one dram, the seeds of Coleworts, the each three drams, Crystal, Dentalis Utalis, pulp of Colocynthis, of each two drams, Olibanum, Niter, white Marble, of each Aloes Hepatic, three drams, meal of Lupines two drams, Gersa Serpentaria an ounce, half an ounce, Myrrh washed in Grass Ceruss six ounces, Hog's grease not salted, water a dram and an half, Bull's Gall an a pound and an half, Goat's suet prepared, ounce and an half, with a sufficient quan- an ounce and an half, Hen's fat two ounces tity of juice of Lemons, and an ounce and and an half. Powder the things as you an half of Wax, make it into an ointment ought to do both together, and by themaccording to art.

Unguentum Apostolorum.

Or, Ointment of the Apostles.

College.] Take of Turpentine, yellow Wax, Ammoniacum, of each fourteen drams, long Birthwort roots, Olibanum, Bdellium, of each six drams, Myrrh, Gilbanum, of each half an ounce, Opopanax, Verd:gris, of each two drams, Litharge nine drams, Oil two pounds, Vinegar enough to dissolve the Gums, make it into an ointment according to art.

Culpeper.] It consumes corrupt and dead flesh, and makes flesh soft which is hard, it cleanses wounds, ulcers, and fistulas, and restores flesh where it is wanting.

\section{Unguentum Catapsoras.}

College.] Take of Ceruss washed in Purslain water, then in Vinegar wherein wild Rhadish roots have been steeped and pressed out, Lapis Calaminaris, Chácitis, of each six drams, burnt Lead, Goat's blood, of each half an ounce, Quick-silver sublinnated an ounce, the juice of Houseleek, Nightshade, Plantain, of each two ounces, Hog's grease cleansed three pounds, Oil of Violets, Poppies, Mandrakes, of each an ounce: first let the sublimate and exungia, then the oils, juices, and powders, be mixed, and so made into an ointment sart. according to art.

$(37,38$. selves, melt the fats being cleansed in a stone vessel, and steep in them two Citrons of a mean bigness cut in bits, in a warm bath, after a whole-week strain it, and put in the powders by degrees, amongst which let the Camphire and Borax be the last, stir them, and bring them into the form of

College. ] Take of fresh. Bay leaves three pounds, Garden Rue two pounds and an pound, Sage, Wormwood, Costmary, Bazil, of each half a pound, Sallad Oil twenty pounds, yellow Wax four pounds, Malaga Wine two pounds, of all of them being bruised, boiled, and pressed out as they ought, make an ointment according to art.

Culpeper.] - It is a great strengthener of the head, it being anointed with it; as also of all the parts of the body, especially the. nerves, muscles, and arteries.

\section{Unguentum Mastichimum.}

Or, An Ointment of Mastich.

College.] 'Take of the Oil of Mastich, Wormwood, and Nard, of each an ounce, Mastich, Mints, red Roses, red Coral, Cloves, Cinnamon, Wood of Aloes, Squinanth, of: each a dram, wax as much as is sufficient to make it into an cintment according to

Culpeper.]. This is like the former, and 5 I an ointment. half, Marjoram two pounds, Mints a 
not a whit inferior to it; it strengthens the stomacl being anointed with it, restores appetite and digestion. Before it was called a stomach ointment.

\section{Unguentum Neapolitanum.}

College.] Take of Hog's grease washed in juice of Sage a pound, Quick-silver strained through leather, four ounces, oil of Bays, Chamomel, and Earthworms, of each two ounces, Spirit of Wine an ounce, yellow Wax two ounces, Turpentine washed in juice of Elecampane three ounces, powder of Chamepitys and Sage, of each two drams, make them into an ointment according to art.

Culpeper.] A learned art to spoil people: hundreds are bound to curse such ointments, and those that appoint them.

\section{Unguentum Nervinum}

College.] Take of Cowslips with the flowers, Sage, Chamepitys, Rosemary, Lavender, Bay with the berries, Chamomel, Rue, Smallage, Melilut with the flowers, Wormwood, of each a handful, Mints, Betony, Pennyroyal, Parsley, Centaury the less, St. John's Wort, of each a handful, oil of Sheep's or Bullock's feet, five pounds, oil of Spike half an cunce; Sheep's or Bullock's Suet, or the Marrow of either, two pounds: the herbs being bruised and boiled with the oil and suet, make it into an ointment accordirng to art.

Culpeper.] It is appropriated to the nerves, and helps their infirmities coming of cold, as also old bruises; make use of it in dead palsies, chilliness or coldness of particular members, such as the arteries perform not their office to as the:y ought; for wind anoint your belly with it ; for want of digestion, your stomach; for the cholic, your belly; for whatever disease in any part of the body comes of cold, esteem this as a jewel.

Unguentum Pectorale.

Or, A Pectoral Ointment.

College.] Take of fresh Butter washed in Violet Water six ounces," oil of Sweet Almonds four ounces, oil of Chamomel and Violets, white Wax, of each three ounces, Hen's and Duck's greese, of each two ounces, Orris roots two drams, Saffron half a dram: The two last being finely powdered, the rest melted and often washed in Barley or Hyssop water, make an ointment of thiem according to art.

Culpeper.] It strengthens the breast and stomach, eases the pains thereof, helps pleurises and consumptions of the lungs, the breast being anointed with it.

College.] 'Take of Hog's grease three ounces, the griease of Hen's, Geese, and Ducks, of each two ounces, Oesipus half an ounce, oil of Violets, Chamoniel, and Dill, fresh Butter a pound, white Wax six ounces, mussilage of Gun Tragacanth, Arabic, Quince seeds, Lin-seeds, Marshmallow roots, of each half an ounce. Let the mussilages be made in Rose water, and adding the rest, make it into an ointment according to art.

Culpeper.] It mightily molifies without any manifest lieat, and is therefore a fit ointment for such as have agues, asthmas, hectic fevers, or consumptions. It is a good ointment to ease pains coming by inflammations of wounds or aposthumes, especially such as dryness accompanies, an infirmity wounded people are many times troubled with. In inward aposthumes, as pleurises, one of them to anoint the external region of the part, is very benefical.

\section{Unguentum Splanchicum.}

College.] Take of oil of Capers an ounce, oil of white Lillies, Chamomel, fresh Butter, juice of Briony and Sowbread, of each half an ounce, boil it to the consumption of the juice, add Ammoniacum dissolved in Vinegar, two drams and an half, Hen's grease, Oesypus, Marrow of a Calr's Leg, of each half an ounce, powder
of the bark of the roots of Tamaris and 
Capers, Fem roots, Cetrach, of each a dram, the seeds of Agnus Castuus, and Broom, tity, then dry them by a gentle fire, and of each a scruple, with a sufficicnt quantity with the oil and wax boil it into an ointof Wax, make it into an ointnent accord- ment.

ing to art.

Unguentum Splanchnicum Magistrale.

College.]. Take of the bark of Caper roots six drams, Briony roots, Orris Florentine, powder of sweet Fennel seeds, Ammoniacum dissolved in Vinegar, of each half an ounce, tops of Wormwood, Chainomel Howers, of each a drain, ointment of the juice and of flowers of Oranges, of each six drams, oil of Orris and Capers, of each an olnce and an half: the things which ought being powdered and sifted, the rest diligently mixed in a hot mortar, make it.into an ointment according to art.

Culpeper.] Both these ointments are appropriated to the splecn, and eases the pains thereof, the sides bcing anointed with them. I fancy not the former.'

Unguentum e Succis.

Or, Ointment of Juices.

College.] Take of the juice of DwarfElder cight ounces, of Smallage and Parsley, of each four ounces, Wormwood and Orris, of each five ounces, common Oil half a pound, oil of white Lilies ten ounces, of Wormwood and Chamomel, of each six ounces, the fat of Ducks and Hens, of each two ounces, boil them together with a gentle fire till the juice be consumed, then strain it, and with seven ounces of white Wax, and a little white Wine Vinegar, make it into an ointinent according to art.

See Unguentum ex Succis Aperitivis. Unguentum Sumach.

College.] Take of Sumach, unripe Galls, Myrtle leerries, Balaustines, Pomegranate Pills, Acorn Cups, Cypress Nuts, Acacia, Mastich, of each ten drams, white Wax five ounces, oil of Roses often washed in Alum water, a pound and ten ounces, make a fine powder of the things you can, and steep them four whole days in juice of Med-

Culpeper.] - It is a gallant drying and binding ointment. Besides, the stomach anointed with:it, stays vomiting, and the belly anointed, with it stays looseness, if the fiundament fall out, when you have put it up again anoint it with this ointment, and it will fall out no more. Do the like by the womb if that fall out.

\section{Ointment of Marsh-mallows, compoind Nicholaus.}

College.] Take of Marsh-mallow roots two pounds, the seeds of Flax and Fonugreek, of each one pound, pulp of Squills half a pound, Oil four pounds, Wax' one pound, 'I'urpentine, Gum of Ivy, Galbanum, of cach two ounces, Colophonia, Rozin, of each half a pound: Let the roots be well washed and bruised, as also the Linseed, Foenugreek seed, and Squills, then steep them three days in eight pints of watcr, the fourth day boil them a little upon the fire, and draw out the mussilage, of which take two pounds, and boil it with the oil to the consumption of the juice, afterwards add the Wax, Rozin, and Colophonia, when they are molted, add the Turpentine, afterwards the Galbanum and Gum of Ivy, dissolved in Vinegar, boil them a little, and having removed them from the firc, stir them till they are cold, that so they may be well incorporated.

Culpeper.] It heats and moistens, helps pains of the breast coming of cold and pleurises, old aches, and stitches, and softens hard swellings.

\section{Unguentum Diapompholigos nitili.} Nicholaus.

College.] Take of Oil of Roses sixteen ounces, juice of Nightshade six ounces, let them boil to the consumption of the juice, then add white Wax five ounces, Ccruss washed two ounces, Lead burnt and washed, 
Pompholix prepared, pure Frankincense, of each an ounce, let them be brought into the form of an ointment according to art.

Culpeper.] It cools and binds, drys, and stays fluxes, either of blood or humours in wounds, and fills hollow ulcers with flesh.

Unguentum Refrigerans. Galenus.

It is also called a Cerecloath.

College.] Take of white Wax four ounces, Oil of Roses omphacine one pound, melt it in a double vessel, then pour it out into another, by degrees putting in cold water, and often pouring it out of one vessel into another, stirring it till it be white, last of all wash it in Rose water, adding a little Rose Water, and Rose Vinegar.

Culpeper.] It is a fine cooling thing, to cure inflammations in wounds or tumours.

Unguentum e Succis Aperitivis primum. Fœsius.

College.] Take of the juice of Smaliage, Endive, Mints, Wormwood, common Parsley, Valerian, of each three ounces, oil of Wormwood and Mints, of each half a pound, yellow Wax three ounces, nix them together over the fire, and make of them an ointment.

Culpeper.] It opens stoppages of the stomach and spleen, eases the rickets, the breast and sides being anointed with it.

An Ointment for the Worms. Fœsius.

College.] Take of oil of Rue, Savin, Mints, Wormwood, and bitter Almonds, of each an ounce and an half, juice of the flowers or leaves of Peaches, and Wormwood, of each half an ounce, powder of Rue, Mints, Gentian, Centaury the less, Tormentil, of each one dram, the seeds of Coleworts, the pulp of Colocynthis, of each two drams, Aloes Hepatic, three drams, the meal of Lupines half an ounce, Myrrh washed in grass water a dram and an half, Bull's Galls an ounce and an half, with juice of Lemons, so much as is sufficient, and an ounce and an half of Wax, make it into an ointment according to art.
Culpeper.] The belly peing anointed with it kills the worms.

\section{CERECLOATHS.}

\section{Ceratum de Galbano.}

Or, Cerecloath of Galbanum.

College.] Take of Galbanum prepared, an ounce and an half, Assafœetida half an ounce, Bdellium a dram, Myrrh two drams, Wax two ounces, Carrot seeds a scruple, Featherfew, Mugwort, of each half a dram, dissolve the Gums in Vinegar, and make it a cerecloath according to art.

Culpeper.] Being applied to the belly of a woman after labour, it cleanses her of any relicts accidently left behind, helps the fits of the mother, and other accidents incident to women in that case.

\section{Ceratum Oesypatum.}

College.] Take of Oesypus ten ounces, Oil of Chamomel, and Orris, of each half a pound, yellow Wax two pounds, Rozin a pound, Mastich, Ammoniacum, Turpentine, of each an ounce, Spikenard two drams and an half, Saffron a dram and an half, Styrax Calamitis half an ounce, make them into a cerecloath according to art.

Culpeper.] It molifies and digests hard swellings of the liver, spleen, womb, nerves, joints, and other parts of the body, and is a great easer of pain.

\section{Ceratum Santalimum.}

College.] Take of red Sanders, ten drams, white and yellow Sanders, of each six drams, red Roses twelve drams, Boleanmoniac seven drams, Spodium four drams, Camphire two drams, white Wax washed thirty drams, Oil of Roses ompliacine six ounces: make it into a cerecloath according to art.

Culpeper.] It wonderfully helps hot infirmities of the stomach, liver, and other parts, being but applied to them. 


\section{PLAISTERS.}

Emplastrum ex Ammoniaco.

Or, A Plaister of Ammoniacum.

College.] Take of Ammoniacum, Bran well sifted, of each an ounce, Ointment of Marsh-mallows, Melilot plaister compound, roots of Briony, and Orris in powder, of ? each half an ounce, the fat of Ducks, Geese, and Hens, of each three drams, Bdellium, Galbanum, of each one dram and an half, Per-Rozin, Wax, of each five ounces, oil of Orris, Turpentine, of each half an ounce, boil the fats and oil with mussilage of Lin-seed, and Fenugreek seed, of each three ounces, to the consumption of the mussilage, strain it, and add the Wax, Rozin, and Turpentine, the ointment of Marsh-mallows with the plaister of Melilot; when it begins to be cold, put in the Ammoniacum, dissolved in Vinegar, then the Bdellium in powder, with the rest of the powders, and make it into a plaister according to art.

Culpeper.] It softens and assuages hard swellings, and scatters the humours offending, applied to the side it softens the hardness of the spleen, assuages pains thence arising.

\section{Emplastrum e Baccus Lauri.}

Or, A Plaister of Bay-berries.

College.] Take of Bay-berries husked, Turpentine, of each two ounces, Frankincense, Mastich, Myrrh, of each an ounce, Cypress, Costus, of each half an ounce, Honey warmed and not scummed, four ounces : make it into a plaister according to art.

Culpeper.] It is an excellent plaister to ease any pains coming of cold or wind, in any part of the body, whether stomach, liver, belly, reins, or bladder. It is an excellent remedy for the cholic and wind in the bowels.

$(37,38$.

\section{Emplastrum Barbarum Magnum.}

College.] Take of dry Pitch eight pounds, yellow Wax six pounds and eight ounces, Per-Rozin five pounds and four ounces, Bitumen, Judaicum, or Mummy, four pounds, Oil one pound and an half, Verdigris, Litharge, Ceruss, of each three ounces, Frankincense half a pound, Roach Alum not burnt, an ounce and an half, burnt, four ounces, Opopanax, scales of Brass, Galbanum, of each twelve diams, Aloes, Opium, Myrrh, of each half an ounce, Turpentine two pounds, juice of Mandrakes, or else dried bark of the root, six drams, Vinegar five pounds: Let the Litharge, Ceruss, and Oil, boil to the thickness of Honey, then incorporate with them the Pitch, being melted with Bitumen in powder; then add the rest, and boil them according to art, till the vinegar be consumed, and it stick not to your hands.

Culpeper.] It helps the bitings of men and beasts, eases inflammations of wounds, and helps infirmities of the joints, and gouts in the beginning.

\section{Emplastrum de Betonica. \\ Or; A Plaister of Betony.}

College.] Take of Betony, Burnet, Agrimony, Sage, Pennyroyal, Yarrow, Comfrey the greater, Clary, of each six ounces, Frankincense, Mastich, of each three drams, Orris, round Birthwort, of each six drams, white Wax, Turpentine, of each eight ounces, Per-Rozin six ounces, Gum Elemi, Oil of Fir, of each two ounces, white Wine three pounds: bruise the herbs, boil them in the Wine, then strain them, and add the rest, and make them into a plaister according to art.

Culpeper.] It is a good plaister to unite the skull when it is cracked, to draw out pieces of broken bones, and cover the bones with flesh: It draws filth from the bottom of deep ulcers, restores flesh lost, cleanses, digests, and drys. 


\section{Emplastrum Ceesarus.}

College.] T'ake of red Roses one ounce to art. and an half, Bistort roots, Cypress Nuts, all the Sanders, Mints, Coriander sceds, of each three drams, Mastich half an ounce, Hypocistis, Acacia, Dragon's blood, Earth of Lenmos, Bole-ammoniac, red Coral, of each two drams, Turpentine washed in Plantain water four ounces, Oil of Roses three ounces, white Wax twelve ounces, Per-Rozin ten ounces, Pitch six ounces, the juice of Plantain, Houseleek, and Orpine, of each an ounce, the Wax, Rozin, and Pitch being melted together, add the Turpentine and Oil, then the Hypocistis and Acacia dissolved in the juices, at last the powders, and make it into a plaister according to art.

Culpeper.] It is of a fine, cool, binding, strengthening nature, excellently good to repel hot rheums or vapours that ascend up to the head, the hair being shaved off, and it applied to the crown.

Emplastrum Catagmaticum the first.

College.] Take of juice of Marsh-mallow roots six ounces, bark of Ashtree roots, and their leaves, the roots of Comfrey the greater and smaller with their leaves, of each two ounces, Myrtle Berries an ounce and an half, the leaves of Willow, the tops of St. John's Wort, of each an handful and an half, having bruised them, boil them together in red Wine, and Smith's Water, of each two pound, till half be consumed, strain it, and add Oil of Myrtles, and Roses omphacine, of each one pound and an half, Goat's suet eight ounces, boil it again to the consumption of the decoction, strain it again, and add Litharge of Gold and Silver, red Iead, of each four ounces, yellow Wax one pound, Colophonia half a pound, boil it to the consistance of a plaister, then add Turpentine two ounces, Myrrh, Frankincense, Mastich, of each half an ounce, Boleammoniac, Earth of Lemnos, of each one cunce, stir them about well till they be boil- ed, and made into an emplaister according Catagmaticum the second.

College.] Take of the roots of Comfrey the greater, Marsh-mallows, Misselto of the Oak, of each two ounces, Platain, Chamepitys, St. John's Wort, of each a handful, boil them in equal parts of black Wine, and Smith's Water till half be consumed, strain it, and add mussilage of Quince seeds made in Tripe water, Oil of Mastich and Roses; of each four ounces, boil it to the consumption of the humidity, and having strained it, add Litharge of Gold four ounces, boil it to the consistence of an emplaister, then add yellow Wax four ounces, Turpentine three ounces, Colophonia six drams, Ship Pitch ten ounces, powders of Balaustines, Roses, Myrtles, Acacia, of each half an ounce, Mummy, Androsamum, Mastich, Amber, of each six drams, Boleammoniac fine flowers, Frankincense, of each twelve drams, Dragon's blood two ounces: make it into a plaister according to art.

Culpeper.] Both this and the former are binding and drying, the former rules will instruct you in the use.

\section{Emplastrum Cephalicum \\ Or, A Cephalic Plaister.}

College.] Take of Rozin two ounces, black Pitch one ounce, Labdanum, Turpentine, flower of Beans, and Orobus, Dove's dung, of each half an ounce, Myrrh, Mas.. tich, of each one dram and an half, Gum of Juniper, Nutmegs, of each two dranıs, dissolve the Myrrh and Labdanum in a hot mortar, and adding the rest, make it into a plaister according to art. If you will have it stronger, add the powders, Euphorbium, Pellitory of Spain, and black Pepper, of each two scruples.

Culpeper.] It is proper to strengthen the brain, and repel such vapours as annoy it, and those powders being added, it dries up the superfluous moisture thereof, and eases 
the eyes of hot scalding vapours that annoy them.

\section{Emplastrum de Cerussa.}

Or, A Plaister of Ceruss.

College.] Take of Ceruss in fine powder, white Wax, Sallad Oil, of each three ounees, add the Oil by degrees to the Ceruss, and boil it by eontinual stirring over a gentle fire, till it begin to swell, then add the Wax cut sinall by degrees, and boil it to its just consistence.

Culpeper.] It helps burns, dry scabs, and hot ulcers, and in gencral whatever sores abound with moisture.

Emplastrum ex Cicuta cum Ammoniaco.

Or, A Plaister of Hemlock with Ammoniaeum.

College.] Take of the juice of Hemlock four ounees, Vinegar, of Squills, and Ammoniacum, of eaeh eight ounces, dissolve the Gum in the juice and Vinegar; after a due infusion, then strain it into its just eonsistence aecording to art.

Culpeper.] I suppose it was invented to mitigate the extrene pains, and allay the inflammations of wounds, for which it is very good: let it not be applied to any principal part.

\section{Emplastrum e crusta Panis.}

Or, A Plaister of a crust of Breaci.

College.] T'ake of Mastich, Mints, Spodium, red Coral, all the Sanders, of each one dram, Oil of Mastieh and Quinces, of each one dram and an half, a erust of Bread toasted, and three times steeped in red Rose Vinegar, and as often dried, Labdanum, of each two ounces, Rozin four ounces, Styrax Calanitis half an ounee, Barley meal five drams : make them into a plaister according to art.

Culpeper.] I shall commend this for a good plaister to strengthen the brain as any is in the Dispensatory, the hair being shaved off, and it applied to the erown; also being applied to the stomach, it strengthens ? it, helps digestion, stays vomiting and putrefaction of the meat there.

\section{Eimplastrum e Cymino.}

Or, 'A Plaister of Cummin.

College.] Take of Cummin-seed, Bayberries, yellow Wax, of each one pound, Per-Rozin two pounds, eommon Rozin three pounds, Oil of Dill half a pound: mix them, and make them into a plaister.

Culpeper.] It assuages swellings, takes away old aches coming of bruises, and applied to the belly, is an excellent remedy for the wind eholie. This I have often proved, and always with good success.

Emplastrum Diacalciteos.

College.] 'Take of Hog's grease fresh and purged from the skins two pounds, oil of Olives omphaeine, Litharge of Gold beaten and sifted, of each three pounds, white Vitriol burnt and purged four ounces: let the Litharge, grease, and oil boil together with a gentle fire, with a little Plantain water, always stirring it, to the eonsistence of a plaister, into which (being removed from the fire) put in the Vitriol and make it into a plaister according to art.

Culpeper.] It is a very drying, binding plaister, profitable in green wounds to hinder putrefaction, as also in pestilential sores after they are broken, and ruptures, and also in burnings and scaldings.

\section{Diachylon simple.}

College.] Take of mussilage of Linseed, Fenugreek seed, Marsh-mallow roots, of each one pound, old Oil three pounds: boil it to the consumption of the mussilage, strain it, and add Litharge of Gold in fine powder, one pound and an lialf: boil them with a little water over a gentle fire always stirring them to a just thickness.

Culpeper.] It is an exceeding good remedy for all swellings without pain, it softens hardness of the liver and spleen, it is very gentle.

Diachylon Ireatum.
College.] Add one ounce of Orris in 
powder to every pound of Diachylon simple.

Crllege.] Take of mussilage of Raisins, fat Figs, Masticl, Mallow-roots, Linseeds; and Fenugreek-sceds, Bird-lime, the juice of Orris and Squills, of each twelve drams and an half, CEsypus or oil of Sheep's feet an ounce and an half, Oil of Orris, Chamomel, Dill, of each eight ounces, litharge of Gold in fine powder one pound, Turpentine three ounces, Per-Rozin, yellow Wax, of each two ounces, buil the oil with the mussilages and juices to the consumption of the humidity, strain the oil from the faces, and by adding the Litharge boil it to its consistence; then add the Rozin and Wax; lastly, it being removed from the fire, add the Turpentine, OEsypus and Birdlime, make of them a plaister by melting them according to art.

Culpeper.] It dissolves hardness and inflammations.

\section{Diachylon magnum cum Gummi.}

College.] Take of Bdellium, Sagapenum, Amoniacum, of each two ounces, dissolved in Wine, and added to the mass of Diachylon magnum : first boil the gums being dissolved, to the thickness of Honey.

Culpeper] This is the best to dissolve hard swellings of all the three.

Diachylon compositum, sive Emplaistrum e Mussilaginibus.

Or, A Plaister of Mussilages.

College.] Take of mussilages of the middle bark of Elm, Marsh-mallow roots, Linseed, and Fenugreek seed, of each four ounces and an half, oil of Chamomel, Lilies, and Dill, of each an ounce and an half, Ammoniacum, Galbanum, Sagapen, Opopanax, of each half an ounce, new Wax twenty ounces, Turpentine two ounces, art.

Saffron two drams, dissolve the Gums in Wine, anc make it into a plaister according to art. Culpeper.] It ripens swellings, and fluxes. art.

breaks them, and cleanses them when they are broken. It is of a most excellent ripening nature.

Emplaistrum Diaphenicon hot.

Take of yellow Wax two ounces, Per-Rozin, Pitch, of each four ounces, Oil of Roses and Nard, of each one ounce, melt them together, and add pulp of Dates made in Wine four ounces, flesh of Quinces boiled in red Wine an ounce, then the powders following: take of Bread twice baked, stceped in Wine and dried, two ounces, Mastich an ounce, Frankincense Wormwood, red Roses, Spikenard, of each two drams and an half, Wood of Aloes, Mace, Myrrh, washed Aloes, Acacia, Troches of Gallia Moschata, and Earth of Lemnos, Calamus Aromaticus, of each one dram, Labdanum three ounces, mix them and make them into a plaister according to

Culpeper.] It strengthens the stomach and liver exceedingly, helps fluxes, apply it to the places grieved.

\section{Diaphoenicon cold.}

College.] Take of Wax four ounces, Ship Pitch five ounces, Labdanum three ounces and an half, Turpentine an ounce and an half, Oil of Roses one ounce, melt these, and add pulp of Dates almost ripe, boiled in austere Wine four ounces, flesh of Quinces in like manner boiled, Bread twice baked often steeped in red Wine and dried, of each an ounce, Styrax Calamitis, Acacia, unripe Grapes, Balaustines, yellow Sanders, troclies of Terra Lemnia, Myrrh, Wood of Aloes, of each half an ounce, Mastich, red Roses, of each an ounce and an half, austere Wine as much as is sufficient to dissolve the juices, make it into a plaister according to Culpeper.] It strengthens the belly and liver, helps concoction in those parts, and distribution of humuurs, stays vomiting and 
Emplastrum Ditimum.

Or, A Divine Plaster.

College.] 'Take of Loadstone four ounces, Ammoniacum three ounces and three altered.

drams, Bdellium two ounces, Galbanum, Myrrh, of each ten drams, Olibanum nine drams, Opopanax, Mastich, long Birthwort, Verdigris, of each an ounce, Litharge, of each two ounces, Turpentine three common Oil, of each a pound and an halt, ounces and an half, Mallaga Wine so much new Wax eight ounces: let the Litharge in as is sufficient: boil it to the consumption fine powder be boiled with the oil to a of the Wine, then add the Ammoniacum thickness, then add the Wax, which being dissolved in Vinegar.

melted, take it from the fire, add the Gums dissolved in Wine and Vinegar, strain it, then add the Myrrh, Mastich, Frankincense, Birthwort, and Loadstone in powder, last of all the Verdigris in powder, and make it into a plaster according to art.

Culpeper.] It is of a cleansing nature, exceeding good against malignant ulcers, it consumes corruption, engenders new flesh, and brings them to a scar.

Emplastrum Epispasticum.

College.] 'Take of Mustard seed, Euphorbium, long Pepper, of each one dram and an half, Stavesacre, Pellitory of Spain of each two drams, Ammoniacun, Galbanum, Bdellium, Sagapen, of each three drams, whole Cantharides five drams, Ship Pitch, Rozin, yellow Wax, of each six drams, Turpentine as much as is sufficient to make it into a plaster.

Culpeper.] Many people use to draw blisters in their necks for the touth ache; or? for rheums in their eyes; if they please to lay a plaster of this there, it will do it.

Emplastrum a nostratibus, Flos Unguentorum Dictum.

Or, Flower of Ointments.

College.] Take of Rozin, Per Rozin, yellow Wax, Sheep's Suet, of each half a pound, Olibanum four ounces, Turpentine two ounces and an half, Myrrh, Mastich, of each an ounce, Camphire two drams, white Wine half a pound, boil them into a plaster.

$(37,38$.
Culpeper.]. I found this receipt in an old manuscript written in the year 1513. the quantity of the ingredients very little A Pluster of Gum Elemi.

College.] Take of Gum Elemi three Culpeper.] The operation is the same with Arceus Liniment.

A Plaister of Lapis Calaminaris.

College.] Take of Lapis Calaminaris prepared an ounce, Litharge two ounces, Ceruss half an ounce, Tutty a dram, Turpentine six drams, white Wax an ounce and an half, Stag's Suet two ounces, Frankincense five drams, Mastich three drams, Myrrh two drams, Camphire a dram and an half, make it up according to art.

\section{Emplastrum ad Herniam.}

College.] Take of Galls, Cypress. Nuts, Pomegranate Pills, Balaustines, Acacia, the seeds of Plantain, Fleawort, Watercresses, Acorn Cups, Beans torrified, Birthwort long and round, Myrtles of each half an ounce. Let these be powdered, and steeped in Rose. Vinegar four days, then torrified and dried, then take of Comfrey the greater and lesser, Horsetail, Woad, Cetrach, the roots of Osmond Royal, Fearn, of each an ounce, Frankincense, Myrrh, Aloes, Mastich, Mummy, of each two ounces, Bole-ammoniac washed in Vinegar, Lap, Calaminaris prepared, Litharge of Gold, Dragon's blood, of each three ounces, Ship Pitch two pounds, Turpentine six ounces, or as much as is sufficient to make it into a plaster according to art.

Culpeper.] The plaster is very binding and knitting, appropriated to ruptures or burstens, as the title of it specifies, it strengthens the reins and womb, stays $5 \mathrm{c}$ 
abortion, it consoliclates wounds, and helps all diseases coming of cold and moisture.

\section{Emiplastrum Hystericum.}

College.] Take of Bisturt roots one pound, Wood of Aloes, yellow Sanders, Nutmegr, Barberry Kernels, Rose seeds, of each one onnce, Cinnamon, Cloves, Squinanth, Chamomel flowers, of each half an ounce, Frankincense, Mastich, Alipta Moschata, Gallia Moschata, Styrax Calamitis, of each one dram, Mosch half a dram, yellow Wax one pound and an half, Tur pentine half a pound, Moschreum four ounces, Labdanum four pounds, Ship Pitch three pounds : let the Labdanum and Turpentine be added to the Pitch and Wax, being melted, then the Styrax, lastly the rest in powder, and sifted, that they may be made into a plaster according to art.

Culpeper.] The plaster being applied to the navel, is a means to withstand the fits of the mother in such women as are subject to them, by retaining the womb in its place.

Emplastrum de Mastich.

Or, A Plaster of Mastich.

College.] Take of Mastich three ounces, Bole-ammoniac washed in black Wine, an ounce and an half, red Roses six drams, Ivory, Myrtle Berries, red Coral, of cach half an ounce, Turpentine, Colophonia, Tachamahacca, Labdanum, of each two ounces, yellow Wax half a pound, Oil of Myrtles four ounces: make it into a plaster according to art.

Cuipeper.] It is a binding plaster, strengthens the stomach being applied to it, and helps such as loath their victuals, or cannot digest it, or retain it till it be digested.

\section{Emplastrum de Meliloto Simplex.}

Or, A Plaster of Melilot simple.

College.] Táke of Rozin eight pounds, yellow Wax four pounds, Sheep's Suet two pounds: these being' inclted, "add green Melilot cut small, five pounds: make it into a plaster according to art.
Emplastrum de Meliloto compositum.

Or, A Plaster of Melilot compound.

College.] Take of Melilot flowers six drams, Chamomel flowers, the secds of Fenugreek, Bay berries husked, Marshmallow roots, the tops of Wormwood and Marjoram, of each three drams, the seeds of Smallage, Ammi, Cardamoms, the roots of Orris, Cypress, Spikenard, Cassia Lignea, of each one dram and an half, Bdellium five drams : beat them all into fine powder, the pulp of twelve Figs, and incorporate them with a pound and an half of Melilot plaster simple, 'Turpentine an ounce and an half, Ammoniacum dissolved in Hemlock Vinegar, three ounces, Styrax five drams, oil of Marjoram, and Nard, of each half an ounce, or a sufficient quantity, make. it into a plaster with a hot mortar and pestle, without boiling.

Culpeper.] It mollifies the hardness of the stomach, liver, spleen, bowels, and other parts of the body: it wonderfully assuages pain, and eases hypochondriac melancholy, and the rickets.

\section{Emplastrum de minio compositum.}

Or, A Plaster of red Lead compound.

College.] Take of Oil of Roses ompliacine twenty ounces,' oil of Mastich two ounces, Suet of a Sheep and a Calf, of each half a pound, Litharge of Gold and Silver, red Lead, of each two ounces, a taster full of Wine: boil them by a gentle fire continually stirring it till it grow. black, let the fire be hottest towards the latter end, then add Turpentine half a pound, Mastich two ounces, Gum Elemi one ounce, white Wax as much as is sufficient: boil them a little, and make them into a plaster according to art.

Culpeper.] It potently cures wounds, old malignant ulcers, and is very drying.

\section{Emplastrum de minio Simplicius.}

Or, A Plaster of red Lead simple.

College.] Take of red Lead nine ounces, Oil of red Roses one pound and an half, white Wine Vinegar six ounces, boil it 
into the perfect body of a plaster. It is and make them into a plaster according to prepared without Vincgar thus, take of red art.

Lead one pound, Oil of Roses one pound Culpeper.] It strengthens the brain and and an half, Wax half a pound, make it nervcs, and then being applied to the back, into a plaster according to art.

Culpeper.] It is a fine cooling healing plaster, and very drying.

\section{Emplastrum Metroproptoticon.}

College.] Take of Mastich one ounce down along the bone, it must needs add strength to the body.

College.] Take of Saffron, Ship-pitch, Colophonia, yellow Wax, of each four ounces, Tứpentinc, Galbanum, Ammoniaand an half, Galbanum dissolved in red Wine and strained, six drams, Cypress Turpentine two drams, Cypress Nuts, Galls, of each one dram and an half, oil of Nutmegs by expression one dram, Musk two grains and an half, Pitch scraped off from old ships two drams and an half, beat the Galbanuml, Pitch, Turpentine, and Mastich gently in a hot mortar and pestle, towards the end, adding the Oil of Nutmegs, then the rest in powder, last of al.' the Musk mixed with a little Oil of Mastich upon a marble, and by exact mixture make them into a plaster.

\section{Emplastrum Nervenum.}

College.] Oake of Oil of Cliamomel and Roses, of each two ounces, of Mastich, Turpentine, and Linseeds, of each an ounce and an half, Turpentine boiled four ounces, Rosemary, Bettony, Horsetail, Centaury the less, of each a handful, Earth-worms washed and cleansed in Wine three ounces, tops of St. Jolın's Wort a handful, Mastich, Gum Elemi, Madder roots, of each ten drams, water half an ounce, the Rózin, Labdanum, Ship-pitch, Rozin, of each an ouncc and Juniper Gum, and Turpentine, being gcntly an half, Litharge of Gold and Silver, of beaten in a hot mortar, with a lot pestlc, each two ounces and an half, red Lead two sprinkling in a few drops of red Wine till ounces, Galbanum, Sagapen, Amoniacum, they are in a body ; then put in the powof each thrce drams, boil the roots, herbs, sers, and by diligent stirring make them and worms, in a pound and an laalf of Wine into an exact plaster.

till half. be consumed, then press them out, and boil the decoction again with the Oils, Emplastrum Sticticum.
Collesc.] 'Take of Oil of Olives six Sucts, Litharge, and red Lead, to the con- aunces, yellow Wax an ounce and an half, sumption of the Wine: then add the Gums Litharge in powder four ounces and an dissolved in Wine, afterwards the Turpen-? half, Ainmoniacum, Bdellium, of each half tine, Rozin, Pitch, and Mastich, in powder, an ounce, Galbanum, Opopanax, Oil of 
Bays, Lapis Calaminaris, both sorts of or faces of liquid Styrax, Bdellium, of each Birthwort, Myrrh, Frankincense, of each one dran, Litharge half a dram.

two drams, pure Turpentine an ounce. Let the Oil, Wax, and Litharge be boiled together till it stick not to your fingers, then the mass being removed from the fire and cooled a little, and the Gums dissolved in white Wine Vinegar, which evaporate away by boiling, strain it strongly, then add the powders, Turpentine, and Oil of Bays, that it may be made into a plaster according to art.

Cilpeper.] It strengthens the nerves, draws out corruption, takes away pains and aches, and restores strength to members that have lost it: the last is inost effectual.

Emplastrum Stomachicum Magistrale. Or, A Stomach Plaster.

College.] Take of Mints, Wormwood, Stochas, Bay leaves, of each a dram, Marjoram, red Roses, yellow Sanders, of each two drams, Calamus Aromaticus, Wood of Aloes, Lavender flowers, Nutmegs, Cubebs, Galanga, long Pepper, Mace, of each a dram, Mastich three drams, Cloves two drains and an half, Oil of Mints an.ounce and an half, Oil of Nard an ounce, Oil of? Spike a dram, Rozin, Wax, of each four ounces, Labdanum three ounces, Styrax half an ounce : make it into a plaster.

Culpeper.] Both this and the other of that name which you shall have by and by, strengthen the stomach exceedingly, help digestion and stay vomiting.

Emplastrum Ceroma, or, Ceroneum. Nich. Alex.

College.] Take of Pitch scraped from a Ship that hath been a long time at Sea, yellow Wax, of each seven drams, Sagapenum six drams, Ammoniacum, Turpen- niacum, burnt brass of each eight drams, tine, Colophonia, Saffron, of each four burnt Alum six drams, Aloes, Myrrh, Galdrams, Aloes, Olibanum, Myrrh, of each banum, of each an ounce and an half, old three drams, Styrax Calamitis, Mastich, Oil one pound, sharp Vinegar so much as Opopanax, Galbanum, Alum, the sceds of is sufficicnt. Let the metals be dissolved Fenugreek; of each two drams, the settlings in the sun with the Vinegar, then put in 
those things that may be melted, last of all Thurpentine, last of all the Colophonia, the powders, and make them all into an Mastich, Frankincense, Bdellium, Alum, emplaster.

Myrrh, and Fenugreek in powder: let Culpeper.] Galen appropriates it to the them be made into a plaster. head, and ulcers there. I know no reason Culpeper.] It strengthens the stomach, but why it may as well serve for other parts and helps digestion. of the body.

A Plaster of Mastich. Nich. Alex.

College.] Take of Mastich, Ship Pitch, Sagapenum, Wax, of each six drams, Ship Pitch, white Wax, roman Vitriol, Ammoniacum, Turpentine, Colophonia, Ceruss, Olibanum, Myrrh, of each eight Saffron, Aloes, Frankincense, Myrrh, of ounces, Oil of Roses seven ounces, Oil of each three drams, Opopanax, Galbanum, Juniper Berries three ounces, Oil of Eggs Styrax Calamitis, Alum, (Rondeletius ap- two ounces, Oil of Spick one ounce, white points, and we for him) Bitumen, Fenu- Vitriol, red Coral, Mummy, of each two greek, of - each two drams, the feces of ounces, Earth of Lemnos, Mastich, Dragon's Liquid Styrax, Bdellium, Litharge, of each blood, of each one ounce, the fat of an half a dram: Let the Litharge being beaten Heron one ounce, the fat of Pimullus three into powder, be boiled in a sufficient quan- ounces, Load stone prepared, two ounces, tity of water; then add the Pitch, which Earthworms prepared, Camphire, of each being melted, add the Wax and Ammoni- one ounce; make them into a plaster acacum, afterwards - let the Sagapenum, Opo- cording to art.

nanax, and Galbanum be put in; then the Culpeper.] It is very good in green Styrax and Feces heing mixed with the wounds and shootings. 


\title{
A KEY
}

\author{
TO
}

\section{GALEN'S METHOD OF PHYSIC.}

The general use of physic. I SIIALL desire thee, whoever thou art, abused) study of physic, to mind heedfully these following rules; which being well understood, sheiv thee the Key of Galen and Fippocrates their method of physic: he that useth their method, and is not heedful of these rules, may soon cure one disease, and cause another more desperate.

That thou mayest understand what I intend, it is to discover in a general way of the manifest virtues of medicines.

I say of the manifest virtues, and qualities, viz. Such as are obvious to the senses, especially to the taste and smell: for it hath been the practice of most Physicians, in these latter ages as well as ours, to say, when they cannol give, nor are minded to study a reason, why an herb, plant, \&c. hath such an operation, or produces such an effect in the body of man: It doth it by an hidden quality, for they not minding the whole creation, as one united body, not knowing what belongs to astral infuence, not regarding that excellent harmony the only wise God hath made in a composition of contraries (in the knowlcdge of which consists the whole ground and foundation of physic) are totally led astray by Tradition.

It is the manifest qualities of medicines that here I am to speak to, and you may be pleased to behold it in this order.

\section{Section. 1. Of the Temperature of Medicines. \\ SECTION. 2. Of the appropriation of Medicines.}

Section. 3. Of the Properties of Medicines.

\section{SECTION I.}

\section{Of the Temperature of Medicines.}

Herbs, plants, and olhcr medicines manifestly operate, either by heat, coldness, dryness, or moisture, for the world being composed of so many qualities, they and only they can be found in the world, and the mixtures of them one with another.

But that they may appear as clear as the sun when he is upon the meridian, I shall treat of them severally, and in this order. 
1. Of Medicines temperate.

2. Of Medicines hot.

3. Of Medicines cold.

4. Of Medicines moist.

5. Of Medicines dry.

\section{Of Medicines Temperate.}

If the world be composed of extremcs, then it acts by extrcmes, for as the man is, so is his work: thercfore it is impossible that any medicine can be temperate, but may be reduced to heat, cold, dryness, or moisture, and must operate, (I mean such as operate by manifest quality) by one of these, hecause there is no other to operate by, and that there should be such a temperate mixture, so exquisitcly of these qualities in any medicinc, that onc of them should not nianifestly excel the other, I doubt it is a system too rare to find.

'Thus then I conclude the matter to be, those Medicines are called temperatc (not because they have excess of tcmperature at all in them) which can neither be said, to heat nor cool so much as will amount to the first degrce of excess, for daily experience witnesscs that thcy being added to medicines, change not their qualities, they make them neither hotter nor coldcr.

Their use. They are used in such diseases where there is no manifest distemper of the first qualities, viz. heat and cold, for example; In obstruction of the bowcls, whcre cold medicines might make the obstruction greater, and lot medicines cause a fever.

In fercrs of flegm, whore the cause is cold and moist, and the effect hot and dry; in such, use tcmiperate mcdicines which nay neither encrease the fever by their heat, nor condensate the flegm by their coldness.

Besicles, because contrarics are taken away by their contraries, and cvery like maintained by its like, they are of great use, to prescrve the constitution of the body empcratc, and the body itsclf in, strength and vigour, and may be used without dangcr, or fear of danger, by considering whch part of the body' is weak, and using such temperate medicines as arc appropriated to that part.

\section{Of Medicines hot.}

The care of the ancient Physicians was such that they did not labour to hide from, but impart to posterity, not only the temperature of medicines in gencral, but also thcir degrees in temperature, that so the distempered part may be brought to its temperature, and no further ; for all things which are of a contrary temperature, conduce not to cure, but the strength of the contrariety must be observed, that so the medicine may be neither weaker nor stronger, than just to take avay the distemper; for if the distemper be but meanly hot, and you apply a medicine, cold in the fourth degree, it is true, you may soon remove that distemper of heat, and bring another of cold twice as bad. Galen, de simp. med. facul. lib. 3. cap. 12.

Then, secondly, Not only the distemper itself, but also the part of the body distempered must be hecded; for if the head be distempered by heat, and you give such medicines as cool thc heart or liver, you will bring another disease, and not eure the former.

The degrees then of temperature are to be diligently heeded, which antient physicians have concluded to be four in the qualities, viz. heat and cold, of eacl we shall speak a word or two sevcrally.

Of Medicines hot in the first degree.

Thosc are said to bc hot in the first degree, which induce a moderate and natural hicat to the body, and to the parts thereof; either cold by nature, or cooled by accident, by which natural hcat is cherished when weak, or restored when wanting.

Effect 1. The first effect then of medicines lot in the first degree, is, by their sweat and temperate heat to reduce the 
body to its natural heat, as the fire doth the external parts in cold weather, unless the affliction of cold be so great that such mild medicines will not serve the turn.

Effect 2. The second effect is, the mitigation of pain arising from such a distemper, and indeed this effect hatl other medicines, some that are cold, and some that are hotter than the first degree, they being rationally applied to the distemper. These medicines the Greeks call Anodyna, and shall be spoken of in their proper places. In this place let it suffice that medicines hot in the first degree, make the offending humours thin, and expel them by sweat, or insensible transpiration, and these of all others are most congruous or agrecable to the body of man, for there is no such equal temperature of heat and cold in a sound man, but heat exceeds, for we live by heat and moisture, and not by cold.

Aledicines then which are hot in the first degree, are such as just correspond to the natural heat of our bodies; such as are hotter ur colder, are more subject to do mischief, being administered by an unskilful hand, than these are, because of their contrariety to nature; whereas these are grateful to the body by their moderate heat.

Effect 3. Thirdly, These take away. weariness, and help fevers, being outwardly applied, because they open the pores of the skin, and by their gentle heat prepare the humours, and take away those fuliginous vapours that are caused by fevers.

Discommodities.] Yet may discommodities arise by heedless giving even of these, which I would have young students in physic to be very careful in, lest they do more mischief than they are aware of, viz. It is possible by too much use of them, to consume not only what is inimical in the body, but also the substance itself, and the strength of the spirits, whence comes faintings, and sometimes death: besides, by applying them to the parts of the body they are not appropriated to, or by not heeding well the complexion of the patient, or the natural temper of the part of the body afflicted, for the heart is hot, but the brain temperate.

Effect 4. Lastly, Medicines hot in the first degree, cherish heat in the internal parts, help concoction, breed good blood, and keep it good in temper, being bred.

Of Medicines hot in the second degree

These are something hotter than the natural temper of a man.

Use. Their use for such whose stomachs are filled with moisture, because their faculty is too hot and dry; they take away obstructions or stoppings, open the pores of the skin, but not in the same manner that such do as are hot in the first degree, for they do it without force, by a gentle heat, concocting, and expelling the humours, by strengthening and helping nature in the work; but these cut tough humours, and scatter them by their own force and power when nature cannot.

Of Medicines hot in the third degree.

Those which attain the third degree of heat, have the same faculties with those before mentioned; but as they are hotter, so are they more powerfut in their operations, for they are so powerful in heating and cutting, that if unadvisedly given they cause fevers. Use. Their use is to cut tough and compacted humours, to provoke sweat abundantly; hence it comes to pass they all of them resist poison.

Of Medicines hot in the fourth degree.

Those medicines obtain the highest degree of heat, which are so hot that they burn the body of a man, being outwardly applied to it, and cause inflammations, or raise blisters, as Crowfoot, Mustard-seed, Onions, \&c. Of these more hereafter.

\section{Of cooling Medicines.}

Physicians have also observed four degrees of coldness in medicines, which I shall briefly treat of in order. 
Of Medicines cold in the first degree. Those medicines which are least cold all, obtain the first degree of coldness; and I beseech you take notice of this, that seeing our bodies are nourished by heat, and we live by heat, therefore no cold medicines are friendly to the body, but what good they do our bodies, they do it by removing an unnatural heat, or the body heated above it natural temper.

The giving then of cold medicines to a man in his natural temper, the season of the year alsu being but moderately hot, extinguishes natural heat in the body of man.

Yet have these a necessary use in them too, though not so frequent as hot medicines have; and that may be the reason why an all wise God hath furnished us with far more hot herbs and plants, \&c. than cold.

Use 1. Their use is first, in nourishment, that so the heat of food may be qualified, and made for a weak stomach to digest.

Use 2. Secondly, To restrain and assuage the heat of the bowels, and to cool the blood in fevers.

Therefore if the distemper of heat be but gentle, medicines cold in the first degree wiri suffice; also children, and such people whose stomachs are weak, are easily hurt by cold medicines.

of Medicines cold in the second and third degree.

Usa 1. Such whose stomachs are strong, and livers hot, may easily bear such medicines as are cold in the second degree, and in cases of extremity find much help by them : as also by such as are cold in the thiid degree, the extremity of the disease considered, for by both these the unbridled heat of choler is assuaged.

Use 2. Also they are outwardly applied to hot swellings, due consideration heing to these, viz. To consume moisture, stop had, that if the inflammation be not great, fluxes, and make such parts dry as are use those that are less; if the inflammation slippery, they make the body and members be vehement, make use of medicines cold : firm, when they are weakened by too much

$(37,38$. in the second or third degree, always let the remedy correspond to the just proportion of the affliction.

Use 3. Thirdly, Sometimes the spirits are moved inordinately through heal, thence follows immoderate watchings, if not deprivation of the senses, this also must be remedied with cold medicines, for cold stops the pores of the skin, makes the humours thick, represses sweat, and lieeps up the spirits from fainting.

Of Medicines cold in the fourth degree. fourth degree, is, To mitigate desperate and rehement pains, stupifying the senses, when no other course can be taken to save life: of the use of which more hereafter

\section{Of moistening Medicines.}

There can be no such difference found amongst moistening medicines, that they should surpass the second degree. For seeing all medicines are either hot or cold, neither heat nor cold, seeing they are extremes, can consist with moisture, for the one dries it up, the other condensates it.

Use. Phylosophers therefore call moisture and dryness, passive qualities, yet have they their operation likewise; for noist medicines lenifies and make slippery, ease the cough, and help the roughness of the throat. These operations are proper to medicines moist in the first degree.

Those which are moister, take away naturally strength, help the sharpness of humours, make both blood and spirits thicker, looses the belly, and fits it for purgation.

The immoderate or indiscreet use of them. dulls the body, and makes it unfit for action. Of drying Medicines.

Drying medicines have contrary faculties $5 \mathrm{E}$
Lastly, The use of medicines cold in the 
moisture, that so they may perform their proper functions.

Yet although the members be strengthened by drying medicines, they have notwithstanding their own proper moisture in ? them, which ought to be conserved, and not destroyed, for without it they cannot consist: If then this moisture be consumed by using, or rather over use of drying medicines, the members can neither be nourished, nor yet perform their proper actions.

Such medicines as are dry in the third degree, being unadviscdly given, hinder the parts of the body they are appropriated to, of their nourishment, and by that means brings them into consumption.

Besides, 'There is a certain moisture in the body of man, which is called radical moisture, which being taken away, the parts must needs die, secing natural heat and life also consists in it, and this may be done by too frequent use of medicines dry in the fourth degree: And it may be this was the reason of Galen's writing, that things dry in the fourth degree, must of necessity burn; which is an effect of heat, and not of dryness, unless by burning, Galen means consuming the radical moisture.

The use then of drying medicines, is only to such bodies, and parts of the body, as abound with moisture, in which observe these rules.

1. If the moisture be not exteme, let not the medicine be extremely drying.

2. Let it be proper to the part of the body afflicter, for if the liver be afflicted by moisture, and you go about to dry the brain or heart, you may sooner kill than cure.

Thus have we briefly spoken of the first qualities of medicines, and in the general only, and but briefly, because we shall always touch upon them in the exposition of the other qualities, in which you must always have an eye to these.

\section{SECTION II.}

\section{Of the appropriation of.Medicines to the several parts of the body.}

That the qualities and use of these medicines may be found out, and understood by every one, and so my country reap the benefit of my labour, they shall find them presented to their view in this order.

Medicines appropriated.
1. To the head.
2. T'o the breast and lungs
3. To the heart.
4. To the stomach.
5. To the liver.
6. To the spleen.
7. To the reins and bladder.
8. To the womb.
9. To the joints.

\section{CHAP'TER I.}

Of Medicines appropriated to the head.

By [head] is usually understood all that part of the body which is between the top of the crown, and the uppermost joint of the neck, yet are those medicines properly called Cephalical, which are appropriated to the brain, not to the eyes, ears, nor teeth; neither are those medicines which are proper to the ears, proper also to the eyes, therefore (my intent being to write as plain as I can) I shall subdivide this chapter into these parts.

Medicines appropriated
1. To the brain.
2. To the eyes
3. To the mouth, and nostrils.
4. in the cars.
5. To the teeth.

For what medicines are appropriated to an unruly tongue, is not un my power at 3 present to determine. 
Of Medicines appropriated to the brain.

Before we treat of medicines appropriated to the brain, it is requisite that we describe what the nature and affection of the brain is.

The brain which is the seat of apprehension, juidgment, and memory, the original of sense and motion, is by nature temperate, and if so, then you will grant me that it may easily be afflicted both by heat and cold, and it is indeed more subject to affliction by either of them, than any other part of the hody, for if it be afflicted by heat, sense and reason, it is immoderately moved, if by cold, they languish, and are dulled, to pass by other symptoms which invarle the lread, if the brain be altered from its proper temper.

Also this is peculiar to the brain, that it is delighter or offended by smells, sights, and sounds, but I shall meddle no further with these here, because they are not medicines.

Cephalical Medicines may be found out from the affectiors of the brain itself. The brain is usually oppressed with moisture in such afflictions; therefore give such medicines as very gently warm, cleanse, cut, and dry: but withal, let them be such as are; appropriated to the head, such as physicians say (by an hidden quality) strengthen the brain.

Again, if you consider the situation of the brain, you shall find it placed in the highest part of the body, therefore it is casily afflicted with hot vapours: this punishes a man with watching and headache, as the former did with sottishness and sleepiness, in such cases use such Cephalecs as gently cool the brain.

'To make Cephalecs of Narcoticks, or stupifying medicines, is not my intent, for I am confident they are inimical both to the optick nerves which convey it to the brain and senses. Of these, and such ieyes (say Doctors) do it by an hidden medicines as also purge the brain, I shall virtue, into the reason which no man can speak by and by. 'lio return to my purpose. "dive, unless they should fetch it from the

Some Cephalics purge the brain, some heat it, some cool it, some strengthen it; but how they perform this office peculiarly to the brain, most physicians confess they could neither comprehend by reason, nor describe by precepts, only thus, they do it by an hidden quality, either by strengthening the brain, thereby descending it from diseases, or by a certain antipathy between them and the diseases incident to the brain. Lastly, For the use of Cephalics, observe, if the brain be much afflicted, you cannot well strengthen it before you liave purged it, neither can you well purge the brain before you have cleansed the rest of the body, it is so subject to receive the vapours up to it; give cooling Cephalics when the brain is too hot, and hot Cephalics wlıen it is too cold.

Beware of using cooling medicines to the brain when the crisis of a disease is near: how that time may be known, I shall (God assisting me) instruct you hereafter, let it suffice now, that according as the disease afflicting your head is, so let your remedy be.

Of Medicines appropriated to the eyes.

T'ake such medicines as are appropriated to the eyes under the name of (Ocular Medicines) I do it partly to avoid multiplicity of words, and partly to instruct my conntrymen in the terms of art belonging to physic, (I would lave called them [Ophthalmics] had not the word been troublesome to the reading, much more to the understanding of a countryman) as I even now called such medieines [C Cphalics] as were appropriated 10 the brain.

Ocular medicines are two-föld, viz. such as are $r:$ ferred to the visive virtues, and such as are referred to the eyes themselves.

Such as strengthen the visive virtue or 
similitude of the substance: And yet they' say a Goat's liver conduces much to make one see in the night, and they give this reason, because Goats see as well in the night as in the day. Yet is there no affinity in temperature nor substance between the liver and the eyes: However Astrologers know well enough that all herbs, plants, \&c. that are under the dominion of either sun or moon, and appropriated to the head, be they hot or cold they strengthen the visive virtue, as Eyebright, which is hot Lunaria, or Moonwort which is cold.

As for what appertains to the constitution of the eyes themselves, seeing they are exact in sense, they ${ }^{\circ}$ will not endure the least inconvenience, therefore such medicines as are outwardly applied to them (for such medicines as strengthen the visive virtues are always given inwardly) let them neithor hurt by their hardness nor gnawing quality, nor be so tough that they should stick to them. Therefore let ocular medicines be neither in powders nor ointments, because oil itself is offensive to the eyes, and how pleasing powders are to them, you may perceive yourself by just going into the dust.

Medicines appropriated to the mouth and nose. Apply no stinking medicine to a disease in the nose, for such offend not only. the nose, but also the brain; neither administer medicines of any ill taste to a disease in the mouth, for that subverts the stomach, because the tunicle of the mouth and of the stomach is the same: and because both mouth and nostrils are ways by which the brain is cleansed, therefore are they infected with such vices as need almost continual cleansing, and let the medicines you apply to them be either pleasant, or at least, not ingrateful.

Medicines appropriated to the ears.

The ears are easily afflicted by cold, because they are always open, therefore they require hot medicines. And because they are of themselves very dry, therefore they require medicines which dry much. Medicines appropriated to the teeth.

Vehement heat, and vehement cold, are inimical to the teeth, but they are most of all offended by sharp and sour things, and the reason is, because they have neither skin nor flesh to cover them, they delight in such medicines as are cleansing and binding, because they are troubled with defluxions and rheums upon every light occasion; and that's the reason the common use of fat and sweet things, soon rots the teeth.

\section{CHAPTER II.
Of Medicines appropriated to the breast
and lungs.}

The medicines appropriated to the breast and lungs, you shall find called all along by the name of [pectorals] that's the term Physicians give them, when you heat them talk of pectoral Syrups, pectoral rows, or pectoral Ointments.

the part afflicted, others the matter afflicting.

But although sometimes in ulcers of the lungs, we are forced to use binding medicines, to join the ulcer, yet are not these called pectorals, because binding medicines are extreme hurtful to the breast and lungs, both because they hinder one's fetching his breath, and also because they hinder the avoiding that flegm by which the breast is oppressed.

Such medicines are called pectorals, which are of a lenifying nature.

Besides, Those which make thin matter thicker are of two sorts, viz. Some are mild and gentle, which may safely be arlministed, be the matter hot or cold which offendeth; others are very cold, which are used only when the matter offending is sharp. 
But because such medicines as conduce will casily grant me, that it is the property to the cure of the phthisics (which is an of cordials to administer to the heart in these ulceration of the lungs, and the discase? priticulars.

usually called, the consumption of th $\quad$ Of Cordials, some cheer the mind, some lungs,) are also reckoned in amongst pec- strengthen the keart, and refresh the spirits toral,s it is not amiss to speak a word or thereof, being decayed.

two of them.

In the cure of this disease are three things to be regarded.

1. To cut and bring away the concreted blood.

2. To cherish and strengthen the lungs.

3. To conglutinate the ulcer.

And indeed some particular simples will perform all these, and physicians confess it; which shews the wonderful mystery the all-wise God hath made in the creation, that one and the same simple should perform two contrary operations on the same part of the body ; for the more a medicine cleanses, the more it conglutinates.

To conclude then, Pectoral Medicines are such as either cut and cleanse out the compacted humours from the arteries of the lungs, or make thin defluxions thick, or temper those that are sharp, help the roughness of the wind-pipe, or are generally lenitive and softening, being outwardly applied to the breast.

\section{CHAPTER III.}

Of Medicines appropriated to the heart.

These are they which are generally given under the notion of Cordials; take them under that name here.

The heart is the seal of the vital spirit, the fountain of life, the original of infused heat, and of the natural affections of man. heart.

So then these two things are proper to the

1. By its heat to cherish life throughout the body.

2. To add vigour to the affections.

Those which cheer the mind, are not one and the same ; for as the heart is variously disturbed, eitlier by anger, love, fear, hatred, sadness, \&c. So such things as flatter lovers or appease the angry, or comfort the fearful, or please the hateful, may well be called cordials; for the heart, seeing it is placed in the middle between the brain and the liver, is wrought upon by reason, as well as by digestion, yet these, because they are not medicines, are beside my present scope.

And although it is true, that mirth, love, \&c. are actions, or motions of the mind, not of the body; yet many have been induced to think such affections may be wrought in the body by medicines.

The heart is chietly afflicted by too muck heat, by poison, and by stinking vapours, and these are remedied by the second sort of cordials, and indeed chiefly belong to our present scope.

According to these three afflictions, viz.

1. Excessive heat.

2. Poison.

3. Melancholy vapours.

Are three kinds of remedies which succour the afflicted heart.

Such as

1. By their cooling nature mitigate the heat of fevers.

2. Resist poison.

3. Cherish the vital spirits when they curguinsh.

All these are called Cordials.

1. Such as cool the heart in fevers, yet is not every thing that cooleth cordial, for lead is colder than gold, yet is not lead cordial as gold is, some hold it cordial by a hidden quality, others by reason. 
2. Such as resist poison; there is a twofold resisting of poison.

1. By an antipathy between the medicine and poison.

2. By a sympathy betreen the medicine and the heart.

Of the first we shall speak anon, in a chapter by itself. The latter belongs to this chapter, and they are such medicines, whose nature is to strengthen the heart, and fortify it against the poison, as Rue, Angelica, \&c. For as the operation of the former is upon the poison, which afflicteth the heart, so the operation of the latter is upon the heart afflicted by the poison.

To this class may be referred all such medicines as strengthen the heart cither by astral influence, or by likeness of substance, if there be such a likeness in medicines, for a Bullock's heart is of like substance to man's, yet I question whether it be cordial or not.

3. And lastly, Such as refresh the spirits; and make them lively and active, both because they are appropriated to the office, and also because they drive stinking; and melancholy vapours from the, heart, for as the animal spirit be refreshed by fragrant smells, and the natural spirits by spices, so are the vital spirits refreshed by all such medicines as keep back melancholy vapours from the heart, as Borrage, Bugloss, Rosemary, Citron Pills, the compositions of them, and many others, which this treatise will amply furnish you with.

\section{CHAPTER IV:}

Of Medicines appropriated to the stomach. By stomach, I mean that ventricle which
contains the food till it be concocted into chyle.

Medicines appropriated to the stomacli are usually called stomachicals.

The infirmities usually incident to the stomach are three.
1. Appetite lost.

2. Digestion weakened.

3. The rctentive faculty corrupted.

When the appetitc is lost, the man feels no hunger when his body needs nourishment.

When digestion is weakcned it is not able to concoct the meat received into the stomach, but it putrifies there.

When the retentivc faculty is spoiled the stomach is not able to retain the food till it be digested, but either vomits it up again, or causes fluxes.

Such medicines then as remedy all these, are called stomachicals. And of them in order.

1. Such as provoke appetite are usually of a sharp or sourish taste, and yet withal of a grateful tastc to the palate, for although loss of appetitc may proceed from divers causes, as from choler in the stomach, or putiefied humours or the like, yet such things as purge this choler or humours, are properly called Orecticks, not stomachicals ; the former strengthen appetite after these are expelled.

2. Such medicines help digestion as strengthen the stomach, either by conrenient heat, or aromatic (viz. spicy) faculty, by hidden property, or congruity of nature.

3. The retentive faculty of the stomach is corrected by binding medicines, yet not by all binding medicines ncitlier, for some of them are adverse to the stomach, but by such binding medicines as are appropriated to the stomach.

For the use of these.

Use 1. Usé, not such medicincs as provoke appetite before you have cleansed the stomach of what hinders it.

Use. 2. Such medicines as help digestion, give them a good time before meat that sa they may pass to the bottom of the stomach, (for the digestive faculty lies there,) before the food come into it.

Use 3. Such as streugthen the retentive 
faculty, give them a little before meat, if to stay fluxes; a little after meat, if to stay vomiting.

\section{CHAPTER V.}

Of Medicines appropriated to the liver.

Be pleased to take these under the name of Hepatics, for that is the usual name physicians give them, and these also are of three sorts.

1. Some the liver is delighted in.

2. Others strengthen it.

3. Others help its vices.

The palate is the seat of taste, and its office is to judge what food is agreeable to the stomach, and what not, by that is both the quality and quantity of food for the stomach discerned: the very same office the meseraik veins perform to the liver.

Sometimes such food pleases the palate: which the liver likes not (but not often) and therefore the meseraik veins refuse it, and that is the reason some few men fancy such food as makes them sick after the eating thereof.

1. The liver is delighted exceedingly with sweet things, draws them greedily, and digests them as swiftly, and that is the reason honey is so soon turned into choler.

2. Such medicines strengthen the liver, as (beilg appropriated to it) very gently bind, for seeing the office of the liver is to concoct, it needs some adstriction, that so both the heat and the humour to be concocted may be stayed, that so the one sip not away, nor the other be scattered.

Yet to not hepatical medicines require so great a binding faculty as'stomachicals do, ? berause the passages of the stomach are more open than those of the liver by which it either takes in chyle, or sends out blood to the rest of the body, therefore medicines that are very binding are hurtful to the liver, and either causc obstructions, or hinder the distribution of the blood, or both.
And thus much for the liver, the office of which is to concoct chylc, (which is a white substance the stomach digests the food into) into blood, and distributes it, by the veins, to every part of the body, whereby the body is nourished, and deçaying flesh restored.

\section{CHAPTER VI.}

Of Medicines appropriated to the spleen.

In the breeding of blood, are three excrements most conspicuous, viz. urine, choler, and melancholy.

The proper seat of choler is in the gall.

The urine passeth down to the reins or kidneys, which is all one.

'The spleen takes the thickest or melancholy blood to itself.

This excrement of blood is twofold: for either by excessive heat, it is addust, and this is that the Latins call Atru Bilis: or else it is thick and earthly of itself, and this properly is called melancholy humour.

Hence then is the nature of splenical medicines to be found out, and by these two is the spleen usually afflicted for Atra bilis, (I know not what distinct English name to give it). many times causes madness, and pure melancholy causeth obstructions of the bowels, and tumours, whereby the concoction of the blood is vitiated, and dropsies many times follow.

Medicines then peculiar to the spleen must needs be twofold also, some appropriated to Atra bilis, others to pure melanclioly; but of purging either of them, $\Gamma$ shall omit till I come to treat of purging in a chapter by itself.

1. Such medicines are."splenical, which by cooling and moistening temper Atra bilis: let not these medicines be too cold neither, for there is no such heat in Atra bilis as there is in choler, and therefore it needs no such excessive cooling: among'st the number of these are such as we men- 
tioned amongst the cordials to repel melan- such, that they abhor all binding nedicines, choly vapours from the heart, such temper because they cause stoppage of urine.

and assuage the malice of Atra bilis.

T'ake notice, that the reins and bladder

2. Those medicines are also splenical, being subject to inflammintions endure not by which nelancholy humours are cor- very hot medicines.

rected and so prepared, that they may the more easily be evacuated: such medicines are cutting and opening, and they differ are, therefore it requires stronger medicines from hepaticals in this that they are no than the kidnics do, lest the strength of ways binding; for the spleen being no ways the medicine be spent before it be come to addicted to concoction, binding medicines the part afflicted.

do it harm, and not good.

3. Sometimes the spleen is not only ob-? structed, but also hardened by melancholy humours, and in such cases emolient medicines may be well called splenicals, not such as are taken inwardly, for they operate upon the stomach and bowels, but such as are outwardly applied to the region of the spleen.

And although sometimes medicines, are outwardly applied to hardness of the liver, yet they differ from splenicals, because they are binding, so are not splenicals.

CHAPTER VII.

\section{Of Medicines appropriated to the reins and bladder.}

The office of the reins is, to make a separation between the blood and the urine ; to receive this urine thus separated from the blood, is the bladder ordained, which is of a sufficient bigness to contain it.

Both these parts of the body officiating about the urine, they are both usually afflicted by the vices of the urine.

\section{By stones. \\ 2. By inflammation. \\ 3. By thick humours.}

Medicines appropriated to the reins and bladder are usually called Nephriticals, and are threefold; some cool, others cut gross humours, and a third sort breaks the stone.

In the use of all these, take notice, that the constitution of the reins and bladder is

Because the bladder is further remote from the centre of the body than the kidnies

\section{CHAP'ER VIII.}

Of Medicines appropriated to the womb.

These, physicians call Hystericals, and to avoid multiplicity of words, take them in this discourse under that notion.

'I'ake notice that such medicines as provoke the menses, or stop them 'when they flow immoderately, are properly hystericals, but shall be spoken to by and by in a chapter by themselves.

As for the nature of the womb, it seems to be much like the nature of the brain and stomach, for experience teacheth that it is delighted with sweet and arnmatical medicines, and flies from their contraries.

For example: a woman being troubled with the fits of the mother, which is drawing of the womb upward, apply sweet things, as Civet, or the like, to the place of :conception, it draws it down again; but apply stiuking things to the nose, as Assafoetida, or the like, it expels it from it, and sends it down to its proper place.

\section{CHAPTER IX.}

Of Medicines appropriated to the joints.

The joints are usually troubled with cephalic disease's, and then are to be cured by cephalic medicines.

Medicines appropriated to the joints, are called by the name Arthritical medicines. The joints, seeing they are very nervous, 
require medicines which are of a heating and drying nature, with a gentle binding, and withal, such as by peculiar virtue are appropriated to them, and add strength to them. It is true, most cephalics do so, yet because the joints are more remote from the centre, they require stronger medicines.

For removing pains in the joints this is the method of proceeding.

Pains is either taken away, or eased for the true cure is to take away the cause of the pain, sonetimes the vehemency of the pain is so great that you must be forced to use Anodines (for so physicians call such medicines as ease pain) before you can meddle with the cause, and this is usually when the part pained is intlamed, for those medicines which take away the cause of pain being very hot, if there be any inflammation in the part pained, you must abstain from them till the inflammation be taken away.

\section{SECTION II I.}

Of the propriety or operation of Medicines.

\section{CHAPTER I. \\ Of Emolient Medicines.}

The various mixtures of heat, cold, dryness, and moisture in simples, must of necessity produce variety of faculties, and operations in them, which now we come to treat of, beginning first at emolients.

What is hard, and what is soft, most men know, but few are able to express. Phylosophers define that to be hard which yields not to touching, and soft to be the contrary. An emolient, or softening medicine is one which reduceth a hard substance to its proper temperature.

But to leave phylosophy, and keep to physic: physicians describe hardness to be two-fold.

$(39,40$.
1. A distention or stretching of a part by too minch fulness.

2. Thick humours which are destitute of heat, growing hard in that part of the body into which they flow.

So many properties then ought emolient medicines to have, viz. To moisten what is dry, to discuss what is stretclied, to warm what is congealed by cold; yet properly, that only is said to mollify, which reduceth a hard substance to its proper temperature. Dryness and thickness of humours being the cause of hardness, emolient medicines must of necessity be hot and moist; and although you may peradventure find some of them dry in the second or third degrees, yet must this dryness be tempered and qualified with heat and moisture, for reason will tell you that dry medicines make hard parts harder.

Mollifying medicines are know, 1 . by their taste, 2. by their feeling.

1. In taste, they are near unto sweat, but fat and oily; they are neither sharp, nor austere, nor sour, nor salt, neither do they manifest either binding, or vehement heat, or cold to be in them.

2. In feeling you can perceive no roughness, neither do they stick to your fingers like Birdlime, for they ought to penetrate the parts to be mollified, and therefore many times if occasion be, are cutting medicines mixed with them.

\section{CHAPTER II.}

\section{Of hardening Medicines.}

Galen in Lib. 5. de Simple, Med. Facult. Cap. 10. determines hardening medicines to be cold and moist, and he brings some arguInents to prove it, against which other physicians contest.

I shall not here stand to quote the dispute, only take notice, that if softening medicines be hot and moist (as we shewed $5 \mathrm{G}$ 
even now) then hardening medicines must being useful, that it is obnoxious to the needs be cold and dry, because they are body of man. I pass, it without more contrary to them.

The universal course of nature will prove it, for dryness and moisture are passive qualities, neithcr can extremcties consist in moisture as you may know, if you do but consider that dryness is not attributed to the air, nor water, but to the fire, and earth.

2. The thing to be congealed must needs be moist, therefore the medicine congealing must of necessity be dry, for if cold be joined with dryness, it contracts the pores, that so the humours cannot be scattercd.

Yet you must observe a difference between medicincs drying, making thick, hardcning, and congealing, of which differences, a few words will not do amiss.

1. Such medicines are said to dry, which draw out, or drink up the moisture, as a spunge drinks up water.

2. Such medicines are said to make thick, as do not consume thc moisture, but add dryness to it, as you make syrups into a thick electuary by adding powders to them.

3. Such as congeal, neither draw out the moisture, nor make it thick by adding dryness to it, but contract it by vehement cold, as water is frozen into icc.

4. Hardncss differ's from all these, for the parts of the body swcll, and are filled with flegmatic humours, or melancholy blood, which at last grows hard.

That you may clearly understand this, obserre but these two things.

1. What it is which worketh.

2. What it worketh upon.

That which worketh is outwardly cold. That which is wrought upon, is a certain medicines be referred to heat, or coldness, thickness and dryness, of humours, for if or dryness, or moisture: but we speak not the humour wcre fluid as water is, it might here of the particular propertics of meciiproperly be said to be congealed by cold, cines, but of their joincd properties, as they but noi so properly hardencd. Thus you heat and moisten. see cold and dryness to be the cause of Others, they question how they can be dishardening. 'This hardening being so far from linguished from such as mollify, secing such 
as are loosening, and such as are emolient, are both of them hot and moist.

To that, thus: stretching and loosening are ascribed to the moveable parts of the body, as to the nuuscles and their tendons, to the ligaments and Membrance; but soft-? ness and hardness to such parts of the body as may be felt with the hand: I shall make clear by a similitude, Wax is softened, being hard, but Fiddle-strings are loosened being stretched. And if you say that the difference lying only in the parts of the body is no true difference, then take notice, that such medicines which loosen, are less hot, and more moistening, than such as soften, for they operate most by heat, these by moisture.

The truth is, I am of opinion the difference is not much, nay, scarce sensible, between emolient and loosening medicines; only I quoted this in a chapter by itself, not so much because some authors do, as because it conduceth to the increase of knowledge in physic, for want of which, this poor nation is almost spoiled.

The chief use of loosening medicines is in convulsions and cramps; and such like infirmities which cause distcrition or stretching.

I'ley are known by the very same marks and tokens that emolient medicines are.

\section{CHAPTER IV. \\ of drawing Medicines.}

The opinion of physicians is, concerning these, as it is concerning other medicines, viz. Sone draw by a manifest quality, some by a hidder, and so (quoth they) they draw to themselves both humours and thorns, or splinters that are gotten into the flesh; however this is certain, they are all of them hot, and of thin parts; hot because the nature of lieat is to draw off thin parts that so they may penetrate to the humours that are to to be drawn out.
'Their use is various, viz.

Use 1. That the bowels may be disburdened of corrupt humours.

2. Outwardly used, by them the offending humour (I should have said the peccant humour, had I written only to scholars, ) is called from the internal parts of the body. to the superfices.

3. By them the crisis of a disease is much helped forward.

4. They are exceedingly profitable to draw forth poison out of the body.

5. Parts of the body over cooled are cured by these medicines, viz. by applying: them outwardly to the place, not only because they heat, but also because they draw: the spirits by which life and heat are cherished, to the part of the body. which is destitute of them: you cannot but know: that many times parts of the body fall away. in flesh, and their strength decays, as in some persons arms or legs, or the like; the. usual reason is, because the vital spirit decays in those parts, to which use such plaister's or ointments as are attractive (which is the physical term for drawing medicines) for they do not only cherish the parts by their own proper heat, but draw the vital and natural spirits thither, whereby they? are both quickened and nourished:

They are known almost by the: same tokens that attenuating medicines are, seeing heat; and thinness of parts is in them both, they differ only in respect of quaritity; thinness of parts being most proper to attenuating medicines, but attractive medicines are hotter.

\section{CHAPTER $V$. \\ Of discussive Medicines.}

The nature of discussing (or sweating) medicines is almost the same with aftractive, for there are no discussive medicinss hut are attractive, nor scarce any allacive medicine but is in sone measure other 
discussing. The difference then is only this; that discussive medicines are hotter than attractive, and therefore nothing else need be written of their nature.

Use. Their use may be known even from their very name; for diseases that come by repletion or fulness, are cured by evacution or emptying; yet neither blood nor gross humours are to be expelled by sweating, or insensible transpiration (as they call it) but the one requires blood-letting, the other purgation, but.scrosus or thin humours and filthy vapours, and such like superfluities, are to be expelled by sweat, and be wary in this too, for many of them work violently, and violent medicines are not rashly to be given.

Caution 2. Besides;swellingsaresometimes made so hard by sweating medicines, that afterwards they can never be cured; for what is thin being by such medicines taken away, nothing but what is perfectly hard remains : If you fear such a thing, mix emolients with them.

Caut.3. Again, sometimes by using discussives, the: humours offending (which physicians usually call the peccant humours) is driven to some more noble part of the body, or else it draws more than it discussseth; in such cases, concoct and attenuate the matter offending before you go about to discuss it.

From hence may easily be gathered at what time of the disease discussive medicines are to be used, viz. about the declining of the disease, although in diseases arising from heat of blood, we sometimes use them in the encrease and state of them.

They are known by the same marks and tokens atteluating medicines are, viz. by their burning and biting quality, they being very hot, and of thin parts, void of any biting quality, therefore they contract not the tringue in tasting of them.

\section{CHAPTER VI.}

\section{Of repelling Medicines.}

Repelling medicines are of contrary operation to these three last inentioned, viz. altenuating, drawing, and discussive medicines: It is true, there is but little difference between these three, some hold none at all; and if you will be so nice, you may oppose them thus. And so medicines making thick, correspond to attenuating medicines, or such as make thin, repelling medicines are opposed to such as draw, and such as retain the humours and make them lough, are opposite to such as discuss, some hold this niceness needless.

2. The sentence of authors about repulsive medicines is various.

For seeing an influxion may be caused many ways, a repulsive hath got as many definitions.

For such things as cool, bind, stop, and make tlick, stay influxions, and therefore repulsives are by authors opposed, not only to attractives, but aiso to attenuating, and discussing medicines.

But properly such things are called repulsives, which do not only stay influxions, (for so do such medicines which stop and make thick) but such as drive the humours flowing to, or inherit in the place, to some other place.

The truth is, binding is inherent to repulsives, so is not coldness nor making thick : Yet surh as are binding, cold and thin in operation, are most effectual.

Your taste will find repulsives to be, tart, or sharp, or austere, with a certain binding which contracts the tongue.

Use 1. Their use is manifold, as in hot tumours, head-aches, or the like.

Use 2. By these in fevers are the vapours driven from the head, Vinegar of Roses is notable.

Time of giving. They are most commo- 
dious in the beginning and encrease of a disease, for then influxions most prevail.

But seeing that in the cure of tumours there are two scopes, 1. That that which flows to it may be repelled. 2. That that which is already in it may be discussed; repulsives are most commodiously used in the beginning, discussives in the latter end.

In the middle you may mix them, with this proviso, that repulsives exceed in the beginning, discussives in the latter end.

Caution 1. If the matter offending be of a venomous quality, either abstain from repulsives altogether, or use purging first, lest the matter tly to the bowels and prove dangerous, especially if the bowcls be weak.

2. Also forbear repulsives, if the pain be great.

3. Lastly, Have a care lest by repulsives you contract the pores so much, that the matter cannot be removed by discussives.

\section{CHAPTER VII. \\ Of cleansing Medicines.}

Cleansing medicines can neither be defined by lieat, nor coldness, because some of both sorts cleanse.

A cleansing medicine, then, is of a terrene quality, which takes away the filth with it, and carries it out.

Definition.] Here, to avoid confusion, a difference must be nade between washing : and cleansing.

A thing which washeth, carries away by fluxion, as a man washeth the dirt off from a thing.

A cleansing medicine by a certain roughness or nitrous quality, carries away the compacted filth with it.

This also is the difference between cleansing and discussing medicines, the one makes thick humours thin, and so scatters them, but a cleansing medicine takes the most tenacious humour along with it, without any alteration.

$(39,40$.
Besides, of cleansing medicines, some are of a gentler nature, some are more vehement. These are not known one and the same way; for some are sweet, some salt, and some bitter.

The use of cleansing is external, as the use of purges are internal.

They are used to cleanse the sanies and other filth of ulcers, yea, and to consume and eat away the flesh itself, as burnt Alum, precipitate, \&c.

When these must be used, not only the effects of the ulcers, but also the temperature of the body will tell you.

For if you see either a disease of fult ess, which our physicians call [Plethora] or corrupted humours which they call [Cacochyma] you must empty the body of these, viz. fulness by bleeding, and corrupt humours, or evil state of the body, by purging before you use cleansing medicines to the ulcer, else your cure will never proceed prosperously.

\section{CHAPTER VIII. \\ Of Emplasters.}

By Emplasters, here, I do mean things. glutinative, and they are quite contrary to things cleansing.

They are of a far more glutinous and tenacious substance.

'They differ from things stopping because they do not stop the pores so much, as stick to them like Birdlime.

They have a certain glutinous heat, tempered both with coldness and moisture.

From these plasters take their names.

Their taste is either none at all, or not discernable whether hot or cold, but fat, insipid, or without taste, or sweet, and viscous in feeling.

Their use is to stop flowing of blood, and other fluxes, to cause suppuration, to continue the heat, that so tumours may bo ripened. 
Also they are mixed with other medi- taste, for many things grateful to the taste cines, that they may the better be brought provokes vomiting, therefore why may not into the form of an emplaster, an may the contrary be?

stick the better to the members.

\section{CHAPTER IX. \\ Of suppurng Medicines.}

These have a great affinity with emolients, like to them in temperature, only emolients are somewhat hotter.

Yet is there a difference as apparent as the sun when he is upon the meridian, and the use is manifest. For,

Emolients are to make hard things soft, but what suppures, rather makes a generation than an alteration of the humour.

Natural heat is the efficient cause of suppuration, neither can it be done by any external means.

Therefore such things are said to suppure, which by a gentle heat cherish the inbred heat of man.

This is done by such medicines which are not only temperate in heat, but also by a gentle viscosity, fill up or stop the pores, that so the heat of the part affected be not scattered.

For althotigh such things as bind hinder the dissipation of the spirits, and internal heat, yet they retain not the moisture as suppuring medicines properly and especially do.

The heat then of suppuring medicines is like the internal heat of our bodies.

As things then very hot, are ingrateful either by biting, as Pepper, or bitterness: in suppuring medicines, no biting, no binding, no nitrous quality is perceived by the taste, (I shall give you better satisfaction both in this and others, by and by.)

For reason will tell a man, that sich things hinder rather than lielp the work of nature in maturation.

Yet it follows not from hence, that all suppuring medicines are grateful to the?
The most frequent use of suppuration is, to ripen Phlegmonce, a general term physicians give to all swellings proceeding of blood, because nature is very apt to help such cures, and physic is an art to help, not to linder nature.

The time of use is. usually in the height of the disease, when the flux is stayed, as also to ripen matter. that it may be the easier purged away.

\section{CHAPTER X.}

\section{Of Medicines provoking urine.}

The causes by which urine is suppressed are many.

1. By too much drying, or sweating, it may be consumind.

2. By heat or inflammation of the reins, or passages whereby it passes from the reins, it may be stopped by compression.

Urine is the thinnest part of blood, separated from the thickest part in the reins.

If then the blood be more thick and viscous than ordinary, it cannot easily be separated without cutting and cleansing medicines.

This is for certain, that blood can neitler be separated nor distributed without heat.

Yet amongst diureticks are some cold things, as the four greater cold seeds, Winter-cherries, and the like.

Although this seem a wonder, yet it may be, and doth stand with truth.

For cool diureticks, though they further not the separation of the blood one jot, yet they cleanse and purge the passages of the urine.

Diureticks then are of two sorts :

1. Such as conduce to the separation of the blood.

2. Such as open the urinal passages.

The former are biting fand are known by 
their taste) very hot and cuting, whence they penetrate to the reins, and cut the gross humours there.

Bitter things, although they be very hot, to the part wounded, which ought to be and cut gross humours, yet are they of a restored with the same flesh.

more dry and terrene substance than is convenicut to provoke urine.

Hence then we may safely gather, that bitter things are not so moist nor penetrating, as such as bite like Pepper.

\section{CHAPTER XI.}

\section{Of Medicines breeding flesh.}

There are many things diligently to be observed in the cures of wounds and ulcers, which incur and hinder that the cure cannot be speedily done, nor the separated parts reduced to their natural state.

$V_{i \sim}$. Fluxes of blood, inflammation, hardness, pain, and other things besides our present scope.

Our present scope is, to shew how the cavity of ulcers may be filled with flesh.

Such medicines are calied Sarcoticks.

This, llough it be the work of nature, yet it is helped forward with medicines, that the blood may be prepared, that it may the easier be turned into flesh.

These are not medicines which breed good blood, nor which correct the intemperature of the place afflicted, but. which defend the blood and the ulcer itself from corruption in breeding flesh.

For nature in breeding flesh produceth two sorts of excrements, viz. scrosus humours, and purulent dross.

Those medicines then which cleanse and consunc, these by drying are said to breed flesh, because by their helps nature performs that office.

Also take notice that these medicines are not so drying that they should consume the blood also as well as the sanies, nor so cleansing that they should consume the flesh with the dross.
Let them not then exceed the first degree unless the ulcer be very moist.

Their difference are various, according

The softer then, and tenderer the place is, the gentler let the medicines be.

\section{CHAP'TER, XII.}

\section{Of glutinative Medicines.}

That is the true cure of an ulcer which joins the mouth of it together.

That is a glutinative medicine, which couples together by drying and binding,

These require a greater drying faculty than the former, not only to consume what flows out, but what remains liquid in the flesh, for liquid flesh is more subject to flow abroad than stick to together.

The time of using them, any body may know without teaching, viz. when the ulcer is cleansed and filled with flesh, and such symptoms as hinder are taken away.

For many times ulcers must be kept open that the sanies, or fords that lie in them inay be purged out, whereas of thenselves they would heal before.

Only beware, lest by too much binding you cause pain in tender parts.

\section{CHAPTER XIII.}

\section{Of Medicines resisting poison.}

Such medicines are called Alexiteria, and Alexipharmaca, which resist poison. fluence, and some physicians (lhough but $f^{\prime} \mathrm{ew}$ ) can give a reason for it.

These they have sorted inio three ranks:

1. Such as strengthen nature, that so it may tame the poison the easicr.

2. Such as oppose the poison by a contrary quality. the sides of an ulcer before brought together.

Some of these resist poison by astral in- 
3. Such as violently thurst it out of doors.

Such as strengthen nature against poison, either do it to the body universally, or else strengthen soine particular part thereof.

For many times one particular part of the body is most afflicted by the poison, suppose the stomach, liver, brain, or any other part: such as cherish and strengthen those parts, being weakened, may be said to resist poison.

Such as strengthen the spirits, strengthen all the body.

Sometimes poisons kill by their quality, and then are they to be corrected by their contraries.

They which kill by cooling are to be remedied by heating, and the contrary; they which kill by corroding, are to be cured by lenitives, such as temper their acrimony.

Those which kill by induration, or coagulation, require cutting medicincs.

Also because all poisons are in motion, neither stay they in one till they have seized and oppressed the fountain of life, therefore they have invented another faculty to stay their motion, viz. terrene and emplastic.

For they judge, if the poison light upon these medicines, they embrace them round with a viscous quality.

Also they say the ways and passages are stopped by such means, to hinder their proceeding; take Terra Lemnia for one.

T'ruly if these reasons be good, which I leave to future time to determine, it may be done for little cost.

Some are of opinion that the safest way is to expel the poison nut of the body, so soon as may be, and that is done by vomit, or purge, or sweat.

You need not question the time, but do it as soon as may be; for there is no parlying with poison.

Let vomiting be the first, purging the next, and sweating the last. This is general. But,
If thou dost but observe the nature and motion of the venom, that will be thy best instructor.

In the stomach it requires vomiting, in the blood and spirits, sweating, if the body be plethoric, bleeding, if full of evil humours, purging.

Lastly, The cure being ended, strengthen the parts afflicied.

\section{CHAPTTER XIV.}

\section{Of purging Medicines.}

Much jarring hath becn amongst physicians about purging medicines, namely, whether they draw the humours to them by a hidden quality, which in plain English is, they know not how; or whether they pcrform their office by manifest quality, viz. by heat, dryness, coldness, or moisture: it is not my present scope to enter the lists of a dispute about the business, neither seem it such an hidden thing to me that every like should draw its like, only to make the matter as plain as I can, I subdivide this chapter into these following parts.

1. Cautions concerning purging.

2. Of the clioice of purging medicines.

3. Of the time of taking them.

4. Of the correcting of them.

5. Of the mamer of purging.

\section{Cautions concerning purging.}

In this, first consider diligently, and be excecding cautious in it too, what the matter offending is, what part of the body is afflicted by it, and which is the best way to bring it out.

Only hiere, by the way, first, have a care of giving vomits, for they usually work more violently, and afflict the body more than purges do, therefore are nol fit for weak bodies; be sure the matter offending lie in 
the tunicle of the stomach, else is a vomit given in vain.

Vomits are more dangerous for women than men, especially such as are either with child, or subject to the fits of the mother.

What medicine is appropriated to the purging of such a humour, for seeing the offending matter is not alike in all, the purging medicine ought not to be the same to all. I shall speak more of this anon. As also of the divers ways whereby medicines draw out or cast out humours, viz. by lenifying, cleansing, provokiug nature to expulsion, and (which is stranger than the doctor's hidden quality) some purge by binding, but indeed, and in truth, such as are properly called purging medicines, which, besides these faculties, have gotten another, by which they draw or call out the humours from the most remote parts of the body, whether these do it by heat or by an hidden quality, physicians are scarce every purge. able to determine, it being very well known to modern physicians, though the ancients denied it, that many cold. medicines purge. 'There is this faculty in all the purges of Galen's model, (because he gives the whole simple which must needs consist of diver's qualitics, because the creation is made up of and consists by an harmony of contraries) there is (I say) this faculty in all purges of that nature, that they contain in them a substance which is inimical both to the slomach and bowels, and some are of opinion this doth good, namely, provokes nature the more to expulsion; the reason might be good if the foundation of it were so, fur by this reason nature herself should purge, not the medicine, and a pliysician should help nature in her business and not hinder her. But to forbear being critical, this substance which I told you was inimical to the stomach, must be corrected in

\section{CULPEPER'S LAST LEGACIES.}

\section{Select Medicinal Aphorisms and Receipts, for many diseases our natures are incident to.}

1. A seneral Caution.

LET such as love their heads or brains, stomach clean. either forbar such things as are obnoxious 3. For a rheum in the Head, and the Palsy. to the brain, as Garlick, Leeks, Onions, Take a red Onion, and bruise it well, beware of surfeiting and drunkenness.

2. To purge the Head.

The head is purged by Gargarisms, of spoonful of good Mustard, when it is well which Mustard, in my opinion, is excel-boiled, raise the sick upright, and let him lent, and therefore a sponful of Mustard receive the sincll up his nose twice it day", put into the mouth, is excellent for one that whilst it is very lot. is troubled with the lethargy: also the head is purged by sueezing; but be sure if you $(39,40$.
Boil Pinpernel well in Wine, and drink 5 I 
a draught of the Wine in the evening, hot, but in the morning cold.

\section{Another.}

Stew Onions in a close pot, and bathe the head and mouth, and nose therewith.

6. For the falling off of the Hair.

Beat Linseeds very well, and mix them with Sallad-oil; and when you have well mixed them, anoint the head therewith, and in three or four times using it will belp you.

\section{To purge the Head.}

Chew the root of Pellitory of Spain, and chew it on both sides of thy mouth, and as the rheum falls down into thy mouth, spit it out, but retain the root there still, till you think the head is purged enough for that time.

\section{FOR THE EYES, AND THEIR IMPEDIMENTS.}

8. For Eyes that are blasteri.

Only wear a piece of black Sarcenet beforc thy eyes, and meddle wilh no medicine; only forbear wine and strong drink.

9. An excellent water to clear the Sight.

Take of Femnel, Eyebright, Roses, white, Celandine, Vervain and Rue, of each a handful, the liver of a Goat chopt small, infuse them woll in Eyebright-water, then distil them in an alembic, and you shall have a water will clear the sight beyond comparison.

10. For a hurt in the Eye with a stroke.

Take Agrimony, and bruise it very well, and temper it with white Wine, and the white of an egg: spread it pretty thick upon a cloth, like a plaster, and apply it to the outside of the eye-lid, and, although it be alnost out, it will cure it.

11. To draw rheum back from the E'yes.

Take an egg and roast it hard, then pull off the shell, and slit it in two, and apply it hot to the nape of the neck, and thou shalt find ease presently.

\section{For the web in the Eye.}

Tike the gall of a hare, and clarified honey, of each cqual proportions: mix them together, and lay it to the web.
FOR THE. EARS, AND THEIR IMPEDIMENTS.

13. For pain in the Ears.

Drop a little oil of sweet Almonds into the ear, and it easeth the pain instantly: (and yet oil of bitter Almonds is our doctor's commonl remedy.)

14. For an imposthume in the Ear.

Boil some milk, and put it into a stone pot with a narrow mouth, and hold the sore car over the pot whilst the milk is very hot, that the vapour of the milk may ascend in to the ear: this is an often approved remedy to take away the pain, and break the imposthume.

FOR 'THE NOSE, AND ITS INFIRMITIES.

15. For Polypus; or a fleshy substance grozeing in the Nose.

Take the juice of Ivy, and make a tent with a little cotton, the which dip in the juice and put it up in the nostril. 16. To cleanise the Nose.

Snuff up the juice of red Beet-root; it will cleanse not only the nose, but also the head, this is a singular remedy for such as are troubled with hard congealed stuff in their nostrils.

17. For bleeding at the Nose.

Bind the arms and Iegs as hard as you can with a piece of tape-ribboning; that, perliaps, may call back the blood. 18. For a Canker in the Nose.

Boil strong ale till it be thick, if the Canker be in the outside of the nose, spread it as a plaster, and apply it; if in the inside, make a tent of a linen rag, and put it up the nostril.

\section{Another for the Polypis.}

The water of Adder's-tongue snuffed up the nose, is very good: but it were better, in iny opinion, to keep a rag continually moistened with it in the nose.

20. For bleeding at the Nose.

Take Amber and bruise into gross powder, put it upon a cluafing-dish of coals, and receive the smoke up into the nose wi th a funnel. 21. Another.
When no other means will stop the 
bleeding at the nose, it has been known that it hath been slopped by opening a vein in the ear.

OF THE MOUTH, AND ITS DISEASES.

\section{A Caution.}

Whosoever would keep their mouth, or tongue, or nose, or eyes, or ears, or teeth, from pain or infirmities, let them often use sncezing, and such gargarisms as they were? instructed in a preceding chapter; for, indeed, most of the infirmities, if not all, which infest those parts, proceed from rheum.

23. For extreme heat of the Mouth.

Take Rib-wort, and boil it in red Wine, and hold the decoction as warm in your mouth as you can endure it.

24. For a Canker in the Mouth.

Wash the mouth often with Verjuice.

OF THE TEETH, AND THEIR MEDICINES.

\section{A Caution.}

If you will keep your teeth from rotting, or aching, wash your mouth continually every morning with juice of Lemons, and afterwards rub your teeth either with a Sage-leaf, or else with a little Nutmeg in powder; also wash your mouth with a little fair water: after meats; for the only way to keep teeth sound, and free from pain, is to keep them clean.

\section{To keep Teeth white.}

Dip a little picce of white cloth in Vinegar of Quinces, and rub your gums with it, for it is of a gallant binding quality, and not only makes the teeth white, but also strengthens the gums, fastens the teeth, and also causeth a sweet breath.

$$
\text { 27. To fasten the Teeth. }
$$

Seethe the roots of Vervain in old Wine, and wash your teeth often with them, and it ? will fistcn them.

\section{For the Tooth-ache.}

Take the inmer rind of an Elder-tree, and bruise it, and put thereto a little Pepper, and make it intu balls, and hold them between the teeth that ache.
OF THE GUMS, AND THEIR INFIRMITIES.

29. For a Scurvy in the gums.

'Take Cloves, and boil them in Rosewater, then dry them, and beat them to powder, and rub the gums with the powder, and drink the decoction in the morning fasting an hour after it. Use red Kosewater, for that is the best.

30. For rotting and consuming of the gums.

Take Sage-water, and wash your mouth with it' every morning, and afterwards rub your mouth with a Sage-leaf.

OF THE FACE, AND ITS INFIRMITIES.

\section{The cause.}

It is palpable, that the cause of redness and breaking out of the face, is a venomous matter, or filthy vapours ascending from the stomach towards the head; where meeting with a rheum or flegm thence descending, mix with it, and break out in the face. Therefore let the first intention of cure be to cleanse the stomach.

\section{Caution negative.}

Let such as are troubled with red faces, abstain from salt meats, salt fish and herrings, drinking of strong beer, strong waters or Wine, Garlick, Onions, and Mustard.

33. For a face full of red pimples.

Dissolve Camphire in Vinegar, and mix it, and the Vinegar with Celandine-water, and wash the face with it: this cured a maid in twenty days, that had 'been troubled with the infirmity half so many years. 34. To take away the marks of the small pox. 'Take the juice of Fennel, heat it lukewarm, and when the small Pox are well scabbed, anoint the face with it divers times in a day, three or four days together. OF TIE THROAT, AND ITS INFIRMITIES. 35. A cantion.

Diseases in the throat, most commonly proceed of rheum descending from the head upon the trachea arteria, or wind-pipe; in such cases there is many times no other cure than first to purge the body of flegm, and then the head of rheum, as you were taught in the first chapter. 


\section{For hodrseness.}

Take of sugar so nuch as will fill a common taster, then put so much rectified spirit of Wine to it as will just wet it, eat this up at night going to bed, use this three or four times together.

\section{Another.}

If the body be feverish, use the former medicine as before, only use Oil of sweet hot. Almonds, or for want of it, the best Salledoil instead of spirit of Wine.

38. Anolher.

Take Penny-royal, and seethe it in running waier, and drink a good draught of the decoction at night going to bed, with a little sugar in it.

\section{For the Quinsey.}

Take notice that bleeding is grood in all inflammations, therefore in this.

It were very convenient that a syrup, and an ointment. of Orpine were always ready in the house for such occasions; for I know no better remedy for the Quinsey, than to drink the one, and anoint the throat with the other.

\section{OF WOMEN'S BREASTS, THEIR INFIRMITIES} AND CURES.

40. For sore Breasts.

Y Take a handful of Figs, and stamp them well till the kernels are broken, then temper them with a little fresh grease, and apply them to the breast as hot as the patient can endure; it will presently take away the anguish, and if the breast will break, it will break it, else it will cure it without breaking. 41. An inward medicine for a sore Breast.

Let her drink either the juice or decoction of Vervain: it were fit that syrup were made of it to keep all the year.

OF THE STOMACH, AND I'TS INFIRMITIES. 42. A cuution.

Infirmities of the stomach usually proceed from surfeiting.
43. Another.

Let such as have weak stomachs, avoid all sweet things, as honey, sugar, and the like; milk, cheese and all fat meats: let him not eat till he-is hungry, nor drink before he is dry; Jet him avoid anger, sadness, much travel, and all fryed meats: let him not vomit by any meaus, nor eat when he is

\section{For moisture of the Stomach.}

Take : a drachm of Galanga, in powder, every morning in a. draught of that Wine you like best.

4õ. For heat of the Stomach.

Swallow four or five grains of Mastich every night going to bed.

\section{OF THE LIVER, AND ITS INFIRMITIES.}

\section{A caution.}

If the liver be too hot, it usually proceeds from too much blood; and is known by redness of urine, the pulse is'swift, the veins great and full, the spittle, mouth, and tongue, seem sweeter than they used to be: the cure is letting blood in the right arm.

47. To canse the Liver zell to digest.

Take Oil of Wormwood, and so much Mastich in powder as wil] make it into a poultice, lay it warm to your right side.

$$
\text { 48. A caution. }
$$

If the liver be stopped, the face will swell, and you shall be as sure to have a pain in your right side, as though you had it there already.

\section{For stoppage of the Lizer.}

Use Garden-thyme in all your drinks and broaths, it will prevent stoppages before they come, and cure them after they are come.

\section{For the liver.}

The liver of a Hare dryed, and beaten into powder, cures all the diseases of the liver of man 


